FORSCHUNGSERGEBNISSE DER WIRTSCHAFTSUNIVERSITÄT WIEN

Michael Weber

Die Generierung von Empfehlungen für zwischenbetriebliche Transaktionen als gesamtwirtschaftliche Infrastrukturleistung 


\section{Die Generierung von Empfehlungen für zwischenbetriebliche Transaktionen als gesamtwirtschaftliche Infrastrukturleistung}

Mit der allmählichen Wandlung des Internets und seiner Dienste zu einem Transaktionsmedium und dem damit einhergehenden Bedarf nach geschäftlichen Kollaborationsplattformen sowie der zugleich zunehmenden Durchdringung der betrieblichen Prozesse mit integrierten Informationssystemen ergeben sich Möglichkeiten, Leistungen von Unternehmen, insbesondere von kleinen und mittleren Unternehmen, gezielter miteinander $\mathrm{zu}$ verknüpfen und dabei typische Probleme des Zustandekommens von Transaktionen zu umgehen bzw. zu lindern. Es besteht das Potenzial, die (regionalen, mitunter grenzüberschreitenden) Verflechtungen zwischen Unternehmen $\mathrm{zu}$ erhöhen und dadurch deren betriebswirtschaftliche Situation $\mathrm{zu}$ vereinfachen. Die Ausschöpfung dieses Potenzials erscheint sowohl aus gesamtwirtschaftlicher als auch aus einzelwirtschaftlicher Sicht vorteilhaft. Diese Arbeit schlägt ein Modell zur Umsetzung einer dieser Zielsetzung entsprechenden Infrastrukturleistung vor und demonstriert die Generierung von Empfehlungen für das Schaffen, das Pflegen, das Beleben und den Ausbau von Transaktionen für die Teilnehmer einer Kollaborationsplattform zur Erhöhung der Transaktionsdynamik sowie zur Verdichtung der Transaktionsverflechtungen.

Michael Weber studierte Handelswissenschaft und Volkswirtschaft an der Wirtschaftsuniversität Wien sowie an der University of Technology Sydney. Er war vier Jahre im interdisziplinären Forschungsverein EC3 in Wien tätig, wo er seine Forschungsarbeit auf Innovationen im Spannungsfeld zwischen Ökonomie, Informatik und Statistik konzentrierte. Seit 2008 setzt er seine Forschungstätigkeit in diesem Bereich als Ökonom bei der Weltbank in Washington, D.C. fort. 
Die Generierung von Empfehlungen für zwischenbetriebliche Transaktionen als gesamtwirtschaftliche Infrastrukturleistung 


\section{Forschungsergebnisse der Wirtschaftsuniversität Wien}

Band 32

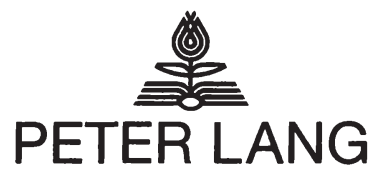

Frankfurt am Main · Berlin · Bern · Bruxelles · New York · Oxford · Wien

Michael Weber - 978-3-631-75376-7

Downloaded from PubFactory at 01/11/2019 05:44:37AM

via free access 
Michael Weber

\section{Die Generierung von Empfehlungen für zwischenbetriebliche Transaktionen als gesamtwirtschaftliche Infrastrukturleistung}

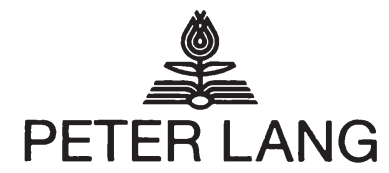

Internationaler Verlag der Wissenschaften 
Bibliografische Information der Deutschen Nationalbibliothek Die Deutsche Nationalbibliothek verzeichnet diese Publikation in der Deutschen Nationalbibliografie; detaillierte bibliografische Daten sind im Internet über http://dnb.d-nb.de abrufbar.

Open Access: The online version of this publication is published on www.peterlang.com and www.econstor.eu under the international Creative Commons License CC-BY 4.0. Learn more on how you can use and share this work: http://creativecommons.org/ licenses/by/4.0.

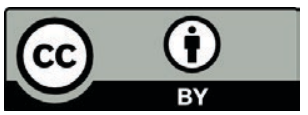

This book is available Open Access thanks to the kind support of ZBW - Leibniz-Informationszentrum Wirtschaft.

\author{
Umschlaggestaltung: \\ Atelier Platen, nach einem Entwurf \\ der Werbeagentur Publique. \\ Universitätslogo der Wirtschaftsuniversität Wien: \\ Abdruck mit freundlicher Genehmigung \\ der Wirtschaftsuniversität Wien. \\ Gefördert durch die Wirtschaftsuniversität Wien. \\ Gedruckt auf alterungsbeständigem, \\ säurefreiem Papier. \\ ISSN 1613-3056 \\ ISBN 978-3-631-58924-3 \\ ISBN 978-3-631-75376-7 (eBook) \\ (c) Peter Lang GmbH \\ Internationaler Verlag der Wissenschaften \\ Frankfurt am Main 2010 \\ Alle Rechte vorbehalten.
}

Das Werk einschließlich aller seiner Teile ist urheberrechtlich geschützt. Jede Verwertung außerhalb der engen Grenzen des

Urheberrechtsgesetzes ist ohne Zustimmung des Verlages unzulässig und strafbar. Das gilt insbesondere für Vervielfältigungen, Übersetzungen, Mikroverfilmungen und die Einspeicherung und Verarbeitung in elektronischen Systemen.

www.peterlang.de 


\section{Danksagung}

Noch bevor Sie, werter Leser bzw. werte Leserin, sich den folgenden Ausführungen zuwenden, möchte ich die Aufmerksamkeit auf jene lenken, ohne die diese Dissertation nicht möglich gewesen wäre oder sich aber schwieriger gestaltet hätte. An dieser Stelle gilt es, innen ein aufrichtiges Dankeschön für Ihre Unterstützung auszurichten.

Zuallererst möchte ich bei meiner Familie, insbesondere meinen Eltern und meiner Freundin, bedanken, die mich begleitet und mir ein positives Umfeld zur Entwicklung meiner Ideen bietet.

Michaela Denk danke ich herzlichst für ihre versierten Ratschläge und Anmerkungen, die einen wichtigen Beitrag für diese Arbeit darstellen. Ebenso möchte ich ihr für die Ermutigung und Unterstützung bei der Erschließung neuer Fachgebiete und Methoden danken. Es freut mich, dass unsere zahlreichen und zeitintensiven Diskussionen abseits dieser Arbeit zu gemeinsamen Publikationen sowie Einladungen zu Vorträgen geführt haben.

Auch möchte ich mich bei Erwin Kolleritsch und Josef Richter für ihre Diskussionsbeiträge und ihr Interesse bedanken.

Den Begutachtern meiner Dissertation, Alfred Taudes und Karl Fröschl, gebührt zu guter Letzt Dank für Ihre bereitwillige inhaltliche Unterstützung sowie Ihre fachkundigen Anregungen, die diese Arbeit bereichert haben. 
Michael Weber - 978-3-631-75376-7

Downloaded from PubFactory at 01/11/2019 05:44:37AM

via free access 


\section{Inhaltsverzeichnis}

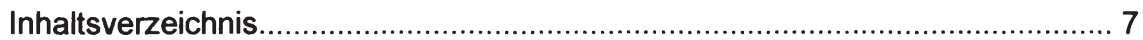

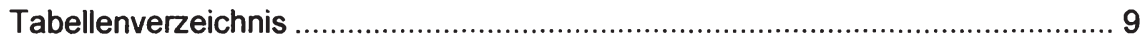

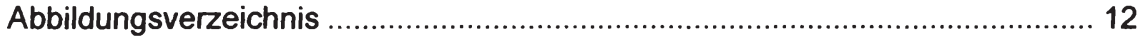

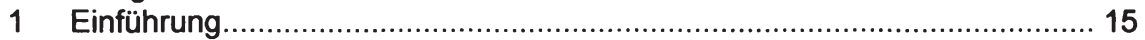

1.1 Überlegungen zu Infrastrukturleistungen ........................................ 15

1.2 Überlegungen zu Kollaborationsplattformen.................................... 17

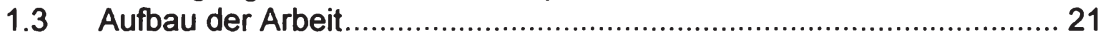

2 Volkswirtschaftstheoretische Betrachtung ............................................... 23

2.1 Wissen als ökonomische Kategorie .................................................. 24

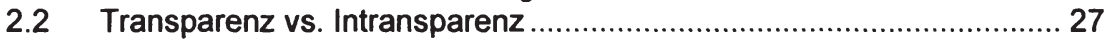

2.3 Ókonomie als evolutionärer Prozess ................................................ 31

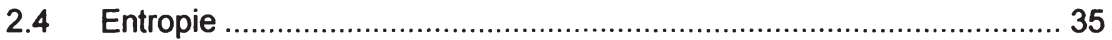

2.5 Kontextualisierung und Bedeutungskoordination .............................. 37

3 Überlegungen zu einem Transaktionssystemunterstützungsmodell............ 41

3.1 Input-Output-Tabellen als produktionsbezogene

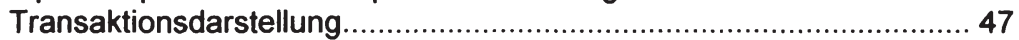

3.2 Zum Aufbau der Input-Output-Rechnung ......................................... 49

3.2.1 Zuordnungsprinzipien und Aufstellungsprobleme ........................57

3.2.2 Annahmen bei der Aufstellung von Input-Output-Tabellen ............ 59

3.2.3 Generierung von Güter $\times$ Güter IOT mit der

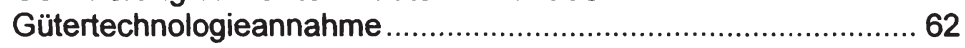

3.2.4 Negative Werte im Zuge der Gütertechnologieannahme ................68

3.3 Alternative Formen der Produktionsstrukturerhebung ....................... 73

$4 \mathrm{BCl}$ - Modell und Daten ........................................................................ 75

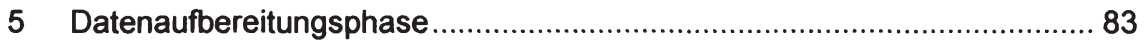

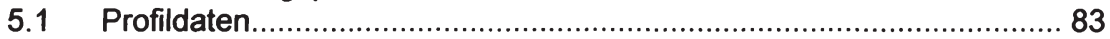

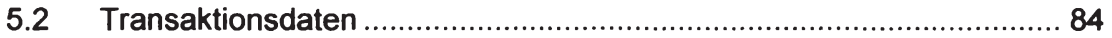

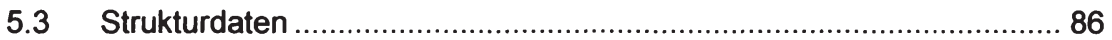

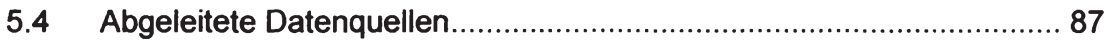

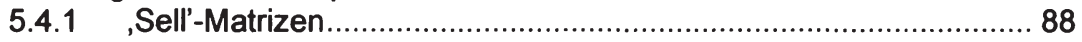

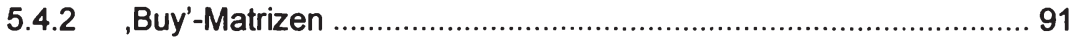

5.4.3 Erweiterungen der ,Buy'-Matrizen ............................................... 93

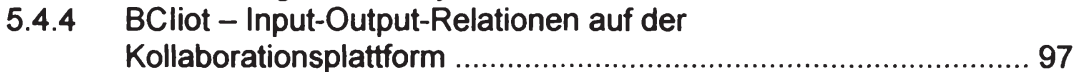

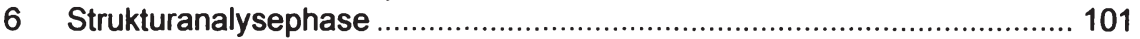

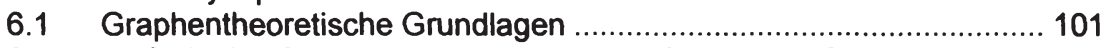

6.2 Einfache Verflechtungseigenschaften auf Basis der Graphentheorie 107

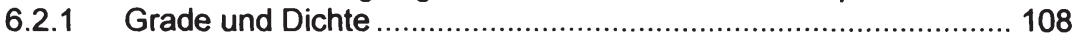

6.2.2 Erreichbarkeit und Distanzen .............................................. 110

6.2.3 Indexierte Knoteneigenschaften.............................................. 112

6.3 Knotenbetrachtung unter Einbezug direkter und indirekter

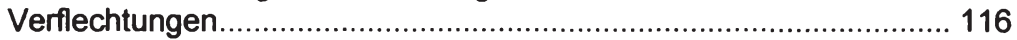

6.4 Berücksichtigung von Kantengewichten bei der Bewertung von Knoten 
6.4.1 Bedeutungsbestimmung mit der Leontief-Inverse..................... 122

6.4.2 Cliquenidentifikation und komplementärer Status nach Hubbell.. 126

6.4.3 Zwischen Perron-Frobenius und PageRank ${ }^{\mathrm{TM}}$............................ 132

6.5 Darstellung von Verflechtungsdaten .............................................. 135

6.6 Blockmodeling - Clustering und Partitionierung von relationalen

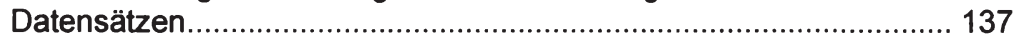

6.6.1 Direkte und indirekte Verfahren ............................................. 140

6.6.2 Äquivalenzrelationen und Blocktypen ..................................... 141

6.6.3 Optimierungsprozess ......................................................... 145

6.6.4 Two-mode Blockmodeling ............................................................ 148

6.6.5 Illustration des two-mode Blockmodeling.................................. 150

6.6.6 Blockmodellberechnung für gewichtete Relationen ................... 153

6.6.7 Illustration der Blockmodellberechnung für gewichtete Kanten.... 156

7 Strukturvergleichsphase .................................................................. 159

7.1 Lückentypologie ...................................................................... 159

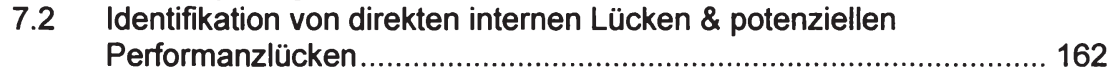

7.3 Identifikation von indirekten internen und externen Lücken................. 166

7.4 Identifikation von internen Lückenfolgen ......................................... 169

7.5 Identifikation von externen Lückenfolgen ...................................... 173

7.6 Deskriptive Auswertung der Lücken bzw. Lückenfolgen ................... 179

7.7 Kennzahlen zur Lückenstruktur .................................................. 183

7.8 Einsatz von Kantenlisten zur detaillierten Lückenbestimmung........... 186

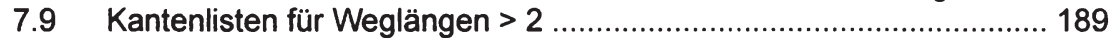

7.10 Graphentheoretische Vergleichskennzahlen ................................... 192

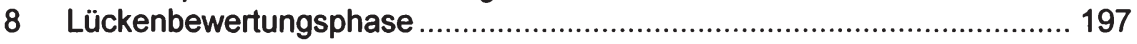

8.1 Performanzlückenbestimmung und -bewertung .............................. 197

8.1.1 Einfache Bewertungsverfahren für Performanzlücken ................. 197

8.1.2 Komplexe Bewertungsverfahren für Performanzlücken ............... 200

8.2 Identifikation von schwachen Knoten ......................................... 202

8.3 Zusätzliche Überlegungen zur Bedeutungsbestimmung ................... 204

8.3.1 Wirkungen über die W-Reihe ................................................. 204

8.3.2 Matrizenzerlegung und Gewichtung von Subgraphen ................. 209

8.4 Bewertung von direkten internen Lücken ....................................... 211

8.5 Bewertung und Zielwerte für externe Lücken .................................. 218

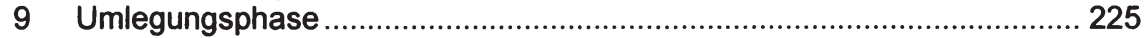

9.1 Generierung der Datenbasis....................................................... 225

9.2 Umlegung von Performanzlücken und internen strukturellen Lücken 228

9.3 Umlegung von externen Lücken ................................................... 234

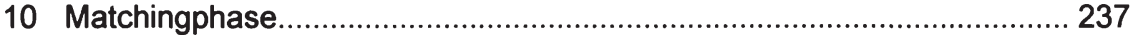

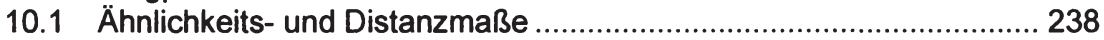

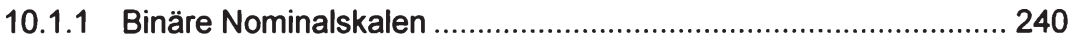

10.1.2 Mehrstufige Nominalskalen .................................................... 246

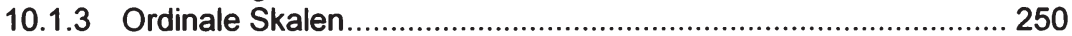

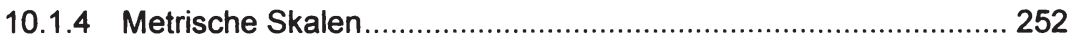

10.2 Referenzpräferenzvergleich - indirekter Vergleich zweier Objekte .... 255

10.3 Duale Präferenzen .........................Michael..Weber...97.8-3-631..7.537.6-7.... 262 


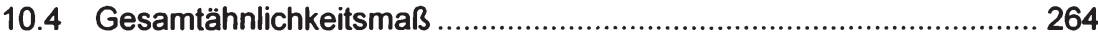

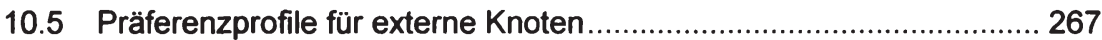

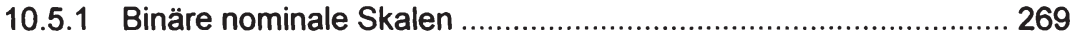

10.5.2 Mehrstufig nominale Skalen ................................................. 270

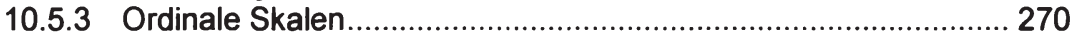

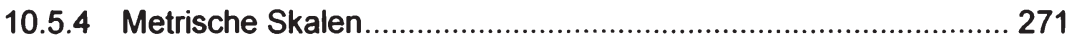

10.5.5 Referenzpräferenzbestimmung .............................................. 273

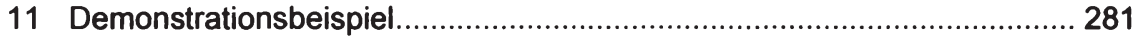

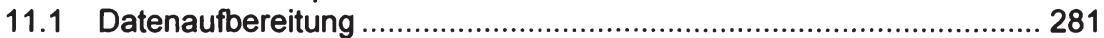

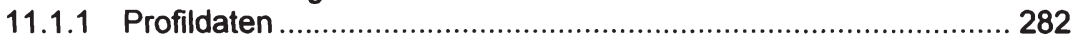

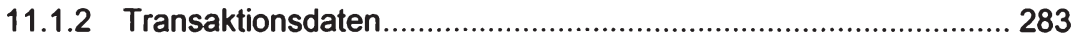

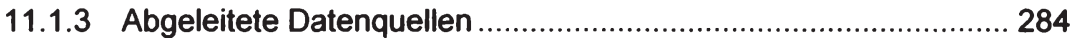

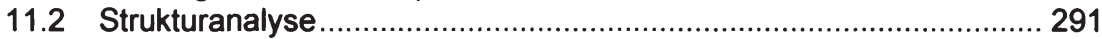

11.2.1 Erreichbarkeit und Distanzen ............................................... 294

11.2.2 Identifikation und Bewertung von Cliquen .................................. 295

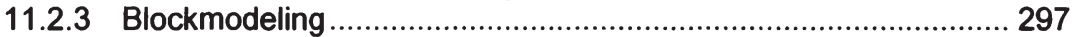

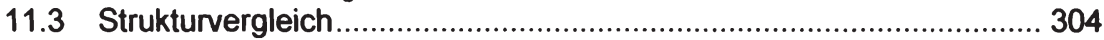

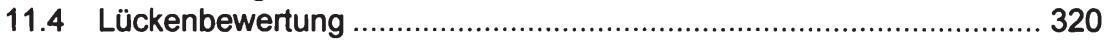

11.4.1 Identifikation und Bewertung von Performanzlücken ................... 320

11.4.2 Bewertung von direkten internen Lücken .................................... 324

11.4.3 Bewertung von externen Lücken ............................................ 329

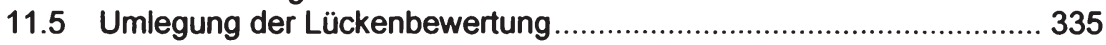

11.5.1 Umlegung der Bewertung interner Lücken................................ 335

11.5.2 Umlegung der Effekte externer Knoten ....................................... 343

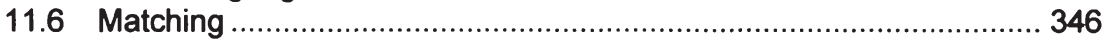

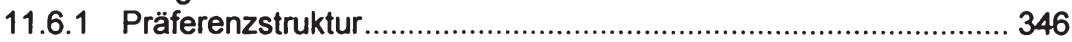

11.6.2 Gesamtähnlichkeitsmaß für Plattformteilnehmer .......................... 349

11.6.3 Präferenzprofil für potenzielle Teilnehmer ................................ 351

11.6.4 Erweiterung der Transaktionsempfehlungen .............................. 352

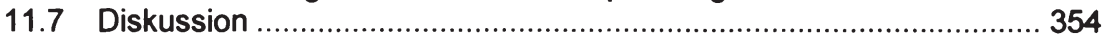

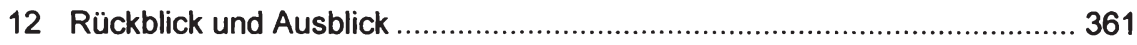

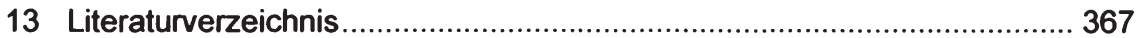

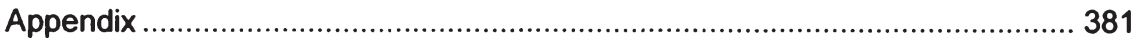

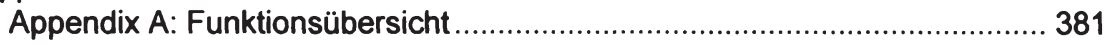

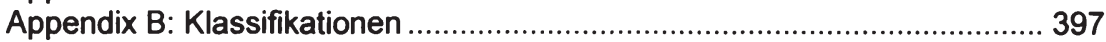

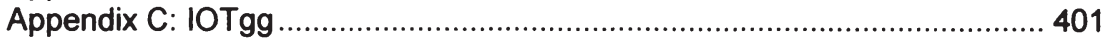

\section{Tabellenverzeichnis}

Tabelle 1: Schematisches Produktionskonto ................................................50

Tabelle 2: Schematisches Güterkonto ...................................................... 50

Tabelle 3: Auszug aus der Aufkommenstabelle (Make/Supply) für Gut i und

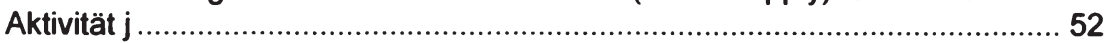

Tabelle 4: Auszug aus der Verwendungstabelle (Use) für Gut i und Aktivität j... 53

Tabelle 5: Struktur der Aufkommenstabelle ..................................................... 54

Tabelle 6: Struktur der Verwendungstabelle ............................................... 54

Tabelle 7: Das Make- und Use-System (Versign_B) V.eber...9.78-3-63.1-7.7537.-7...... 55 
Tabelle 8: Das Make- und Use-System (Version B) mit Formeln ...................... 56

Tabelle 9: Anteilsmatrizen aus der Make- und Use-Systematik ........................ 62

Tabelle 10: Matrix der Inputkoeffizienten (,Vorleistungskoeffizienten') .............. 63

Tabelle 11: Matrix der Importkoeffizienten .......................................................63

Tabelle 12: Matrix der Nachfrageanteile heimischer Güter.................................64

Tabelle 13: Matrix der Nachfrageanteile importierter Güter .............................. 64

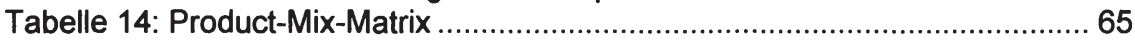

Tabelle 15: Matrix der Marktanteile ................................................................... 65

Tabelle 16: Summenbedingungen des Make- und Use-Systems ..................... 66

Tabelle 17: Berechnungsmethoden der Matrix der direkten Inputkoeffizienten .. 68

Tabelle 18: Erforderliche Datenbasis ........................................................... 79

Tabelle 19: Variablen- und Indexübersicht.................................................... 83

Tabelle 20: Gegenüberstellung der Spalten- und Zeilensummenvektoren von

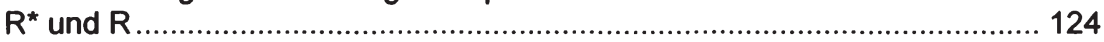

Tabelle 21: Ermittlung der Zusammenhangsmaßzahl des Graphen................ 130

Tabelle 22: Blocktypen für Blockmodelle basierend auf der Systematik von

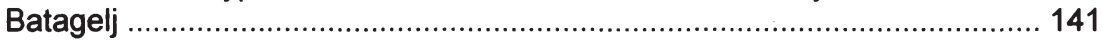

Tabelle 23: Beispiel für ein ideales Blockmodell ........................................... 145

Tabelle 24: Beispieldaten zur Blockmodellberechnung ................................. 151

Tabelle 25: Beispiele für redefinierte Blocktypen nach Žiberna ...................... 154

Tabelle 26: Typen von Lücken für einen Weg $\omega$ mit Weglänge $=1 \ldots \ldots \ldots \ldots \ldots \ldots . . .161$

Tabelle 27: Spezielle Knotentypen.............................................................. 162

Tabelle 28: Mögliche Transaktionszusammenhänge in

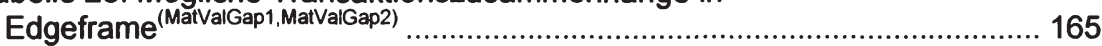

Tabelle 29: Typen von Lücken für einen Weg $\omega$ mit Weglänge $>1$................ 167

Tabelle 30: Klassifikation von internen Lückenfolgen ..................................... 171

Tabelle 31: Klassifikation von externen Lückenfolgen .................................. 176

Tabelle 32: Kontingenztafel für binäre Präferenzvariablen .............................. 241

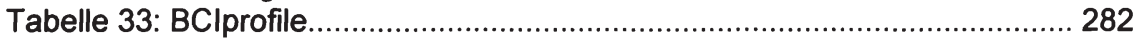

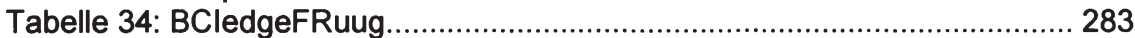

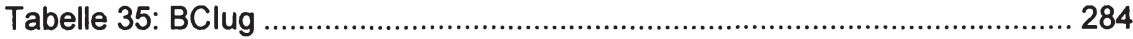

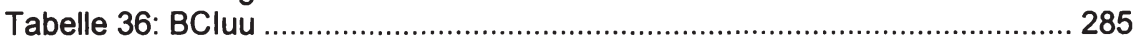

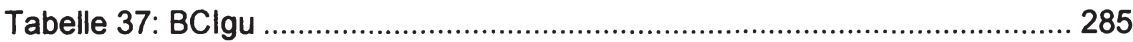

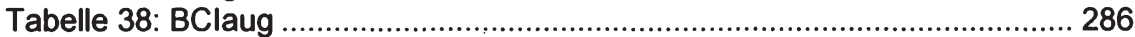

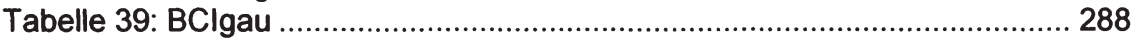

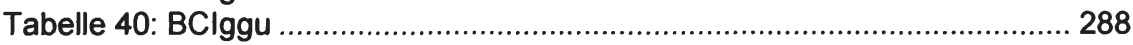

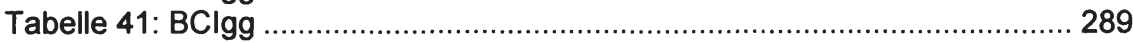

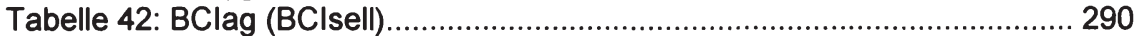

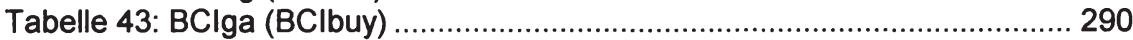

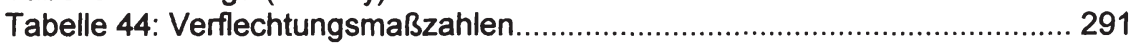

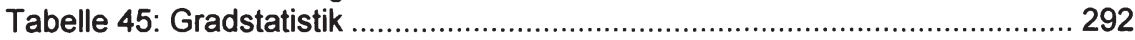

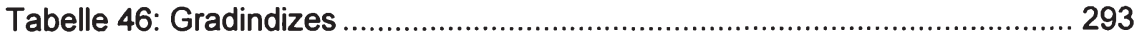

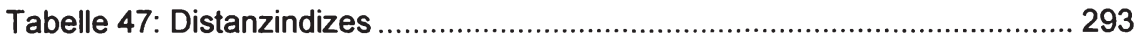

Tabelle 48: Distanzgraph zu BClgg in Adjazenzmatrixform ........................... 294

Tabelle 49: Distanzgraph zu rIOTgg in Adjazenzmatrixform............................ 295

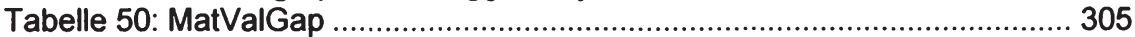


Tabelle 51: Typen von Lückenfolgen mit dazugehöriger Weglänge I .............. 306

Tabelle 52: Typen von Lückenfolgen mit dazugehöriger Weglänge II ............. 307

Tabelle 53: Deskriptive Auswertung von GapLiTot ......................................... 308

Tabelle 54: Kennzahlen auf Basis der Auswertung von GapLiTot................... 309

Tabelle 55: Beidseitig neue Kanten ............................................................. 311

Tabelle 56: Nur ausgangsseitig neue Kanten ............................................... 312

Tabelle 57: Nur zielseitig neue Kanten .................................................. 312

Tabelle 58: Ausgangs- zu Verbindungsknoten für beidseitig neue Kanten....... 314

Tabelle 59: Verbindungs- zu Zielknoten für beidseitig neue Kanten ................. 314

Tabelle 60: Ausgangs- zu Verbindungsknoten für ausgangsseitig neue

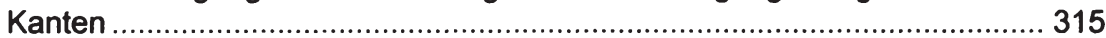

Tabelle 61: Verbindungs- zu Zielknoten für zielseitig neue Kanten .................. 315

Tabelle 62: Externe Verbindungsknoten ....................................................... 316

Tabelle 63: Ausgangs- zu externen Verbindungsknoten I ............................. 317

Tabelle 64: Ausgangs- zu externen Verbindungsknoten II ............................. 318

Tabelle 65: Externer Verbindungs- zu Zielknoten ......................................... 319

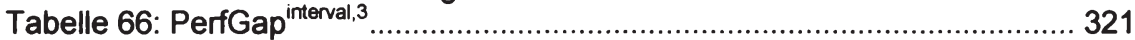

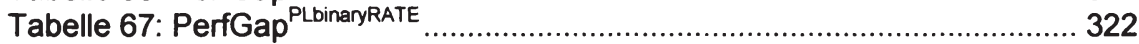

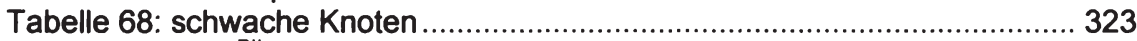

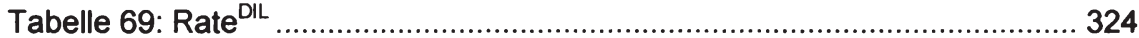

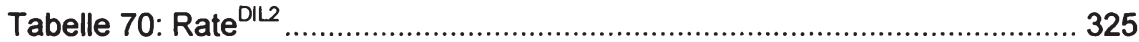

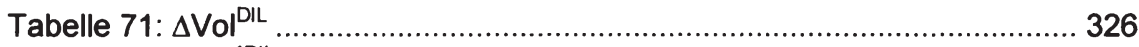

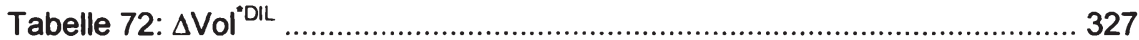

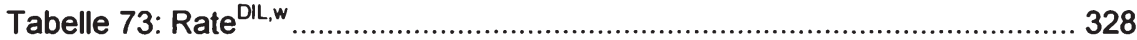

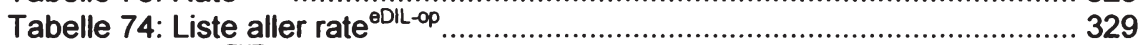

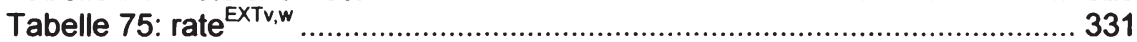

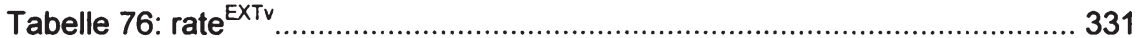

Tabelle 77: Gesamtbedeutung des externen Knoten 70A für die internen

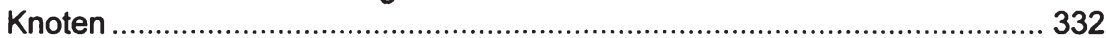

Tabelle 78: Verteilung der Bedeutung des externen Knoten 70A auf die

internen Knoten ..................................................................................... 332

Tabelle 79: Gesamtbedeutungen der externen Knoten für die internen

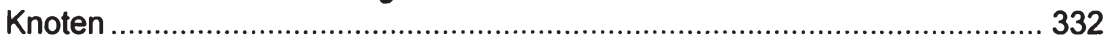

Tabelle 80: relativer Bedeutungsgewinn/-verlust durch den externen Knoten.. 334

Tabelle 81: Input- und Output-Änderungsbedarf der lokalen Struktur .............. 335

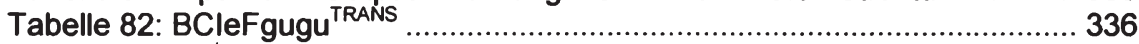

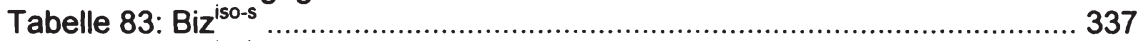

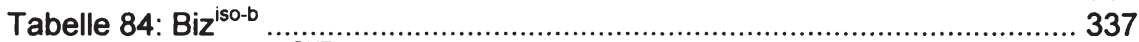

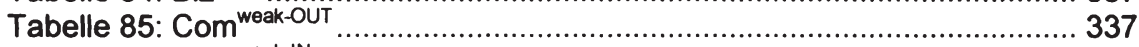

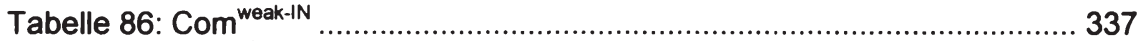

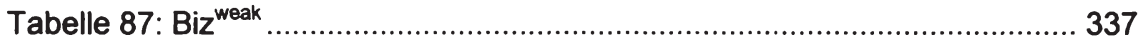

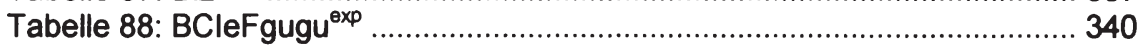

Tabelle 89: Bedeutung des externen Gutes $70 \mathrm{~A}$ für die Plattormteilnehmer ... 343

Tabelle 90: gewichtete Bedeutung des externen Gutes 70A (Variante 1) ........ 344

Tabelle 91: gewichtete Bedeutung des externen Gutes 70A (Variante 2) ........ 345

Tabelle 92: BClpreferences I .................................................................... 347 


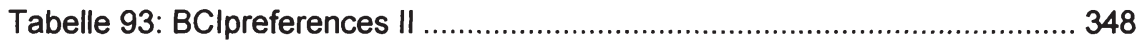

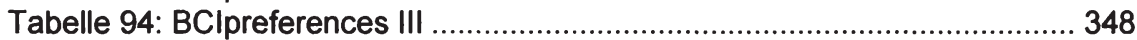

Tabelle 95: Gesamtähnlichkeitsmaß ......................................................... 350

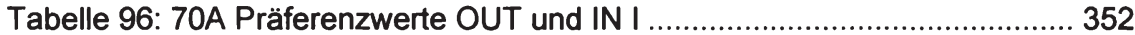

Tabelle 97: 70A Präferenzwerte OUT und IN II ........................................... 352

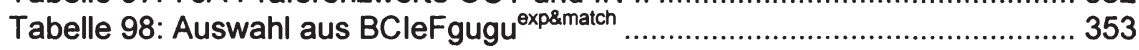

Tabelle 99: Funktionen für die Datenaufbereitung ........................................ 381

Tabelle 100: Funktionen für die Strukturanalyse........................................... 381

Tabelle 101: Funktionen für den Strukturvergleich ..................................... 384

Tabelle 102: Funktionen für die Lückenbewertung ...................................... 387

Tabelle 103: Funktionen für die Umlegung ................................................. 390

Tabelle 104: Funktionen für das Matching .................................................... 392

Tabelle 105: Aktivitäten nach der auf der ONACE aufbauenden IO-

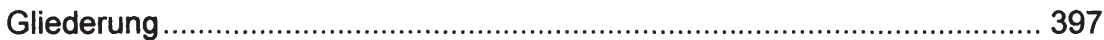

Tabelle 106: Güter nach der auf der OCPA aufbauenden IO-Gliederung ........ 399

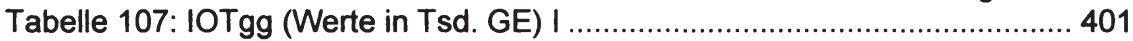

Tabelle 108: IOTgg (Werte in Tsd. GE) II ..................................................... 402

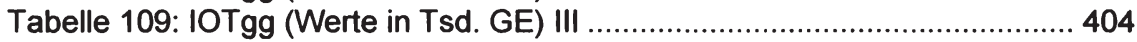

Tabelle 110: IOTgg (Werte in Tsd. GE) IV ................................................. 405

Tabelle 111: IOTgg (Werte in Tsd. GE) V ...................................................... 407

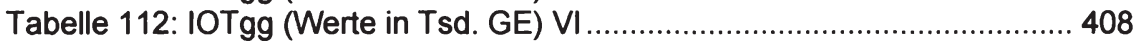

\section{Abbildungsverzeichnis}

Abbildung 1: Marktaustausch bei vollständigem (relevanten) Wissen ................ 43

Abbildung 2: Marktaustausch bei asymmetrischem Wissen .............................. 43

Abbildung 3: Unbeabsichtigte externe Effekte ............................................. 44

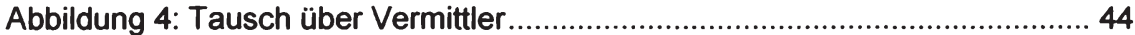

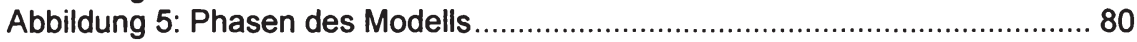

Abbildung 6: ursprüngliche Adjazenzmatrix K........................................... 128

Abbildung 7: Adjazenzmatrix K nach Cliquenidentifikation und Permutation.... 129

Abbildung 8: Elementares Schema des wirtschaftlichen Kreislaufs................. 137

Abbildung 9: Beziehungen zwischen den Blocktypen.................................. 144

Abbildung 10: Adjazenzmatrix vor und nach der Blockmodellberechnung ....... 152

Abbildung 11: Gewichtete Adjazenzmatrix vor und nach der

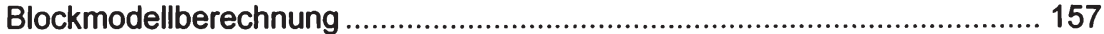

Abbildung 12: Typologie von Lücken für Vergleichszwecke .......................... 161

Abbildung 13: Hierarchie der Typen........................................................... 178

Abbildung 14: Maßzahlen für verschiedene Skalentypen .............................. 239

Abbildung 15: Beispiel für eine zusammengesetzte Distanzfunktion................ 260

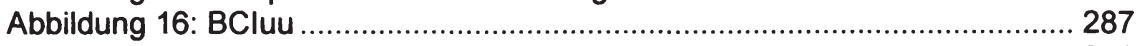

Abbildung 17: Cliquenidentifikation von BClgg …....................................... 296

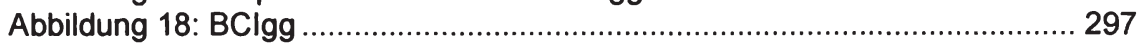

Abbildung 19: BM für BClgg mit 6 Vorgänger- und 6

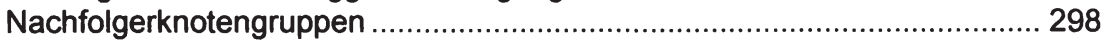

Abbildung 20: gewichtetes BM für BClgg 6 Vorgänger- und 6

Nachfolgerknotengruppen .......................Michael..Wveber...97.8-3-63.1-7.537.6-7..... 299 
Abbildung 21: BM für rlOTgg mit 6 Vorgänger- und 6

Nachfolgerknotengruppen

Abbildung 22: gewichtetes BM für rlOTgg 6 Vorgänger- und 6

Nachfolgerknotengruppen 302

Abbildung 23: Histogramm für das Gesamtähnlichkeitsmaß 351 Abbildung 24: Darstellung des Auszuges aus BCleFguguexp\&match 354 Abbildung 25: Überblick über die Modellphasen 362 
Michael Weber - 978-3-631-75376-7

Downloaded from PubFactory at 01/11/2019 05:44:37AM

via free access 


\section{Einführung}

\section{1 Überlegungen zu Infrastrukturleistungen}

Infrastrukturleistungen bieten wachstums-, versorgungs-, und integrationsnotwendige Basisfunktionen für eine Gesamtwirtschaft. Sie sind dabei nicht nur wachstumsbegleitend, sondern vielmehr wachstumsinduzierend und haben daher wirtschaftspolitisch betrachtet eine herausragende Bedeutung [Jochimsen \& Gustafsson 1977]. Im Zuge der Wandlung des Internets und seiner Dienste zu einem Transaktionsmedium und - damit einhergehend - des Aufkommens von geschäftlichen Kollaborationsplattformen verschiedenster Art und Ausrichtung verlagert sich die Notwendigkeit wirtschaftspolitischer Maßnahmen zur Unterstützung wirtschaftlicher Akteure zusehends auch auf dieses Medium. Ungeachtet der Förderung grundsätzlicher informations- und kommunikationstechnischer Neuerungen und Einrichtungen, die die Verbreitung des Mediums begünstigen, stellt sich hierbei die Frage, inwieweit und welche Maßnahmen innerhalb des Mediums geeignet sind, die Akteure in kritischen Fragen ihres wirtschaftlichen Alltags zu unterstützen, und zwar möglichst unter Ausreizung der Möglichkeiten des Mediums selbst, welches die Rahmenbedingungen setzt und zugleich Anlass der Überlegungen sowie Lösungsraum ist. Dementsprechend ist die technische Dimension bei der Suche nach (neuen) Antworten auf - womöglich altbekannte - wirtschaftliche Problemstellungen nicht außer Acht zu lassen.

Eine im Zusammenhang mit dieser Suche nennenswerte Vision, die sich mit Blick auf das Medium gewissermaßen aufdrängt, ist die des ,Ubiquitous Computing', welches die „durchgängige, überall eindringende, digitale Unterstützung vieler Personen mit Hilfe von personalisierten Diensten“ [Pfaff \& Skiera 2002, S. 2 nach Fleisch 2001, S. 178] zum Ziel hat und dabei die Idee verfolgt, "dass die menschliche Umgebung nicht in einem Computer abzubilden ist (Virtual Reality), sondern die Computer versteckt in die gewohnte Umgebung zu integrieren sind (Calm Technology)“ [Pfaff \& Skiera 2002, S. 3 nach Weiser 1991] Das_Medium sollte 
gemäß dieser Sichtweise den Benützer nicht diktieren, d.h. in diesem Kontext umfassend digitalisieren bzW. virtualisieren - anders formuliert ,maschinisieren' und dadurch letztlich Problemstellungen lediglich verlagern, sondern idealenweise dem Benützer bei der Bewältigung von Herausforderungen in dessen erster und einziger Realität hilfreich zur Seite stehen. Eine Idee, die verfolgenswert erscheint, in Anbetracht jüngerer Entwicklungen aber zumindest zum Zeitpunkt der Veröffentlichung dieser Arbeit etwas aus der Mode gekommen sein dürfte. Nichtsdestotrotz hat sich basierend auf der Vision des ,Ubiquitous Computing' ein Forschungsfeld namens ,Ubiquitous Commerce' etabliert, welches die wirtschaftlichen Aspekte des ,Ubiquitous Computing' zu behandeln versucht und im Wesentlichen diskutiert, wie bspw. Geschäftsprozesse speziell durch ,smarte Dinge' (mit Informations- und Kommunikationstechnologie (IKT) angereicherte Alltagsgegenstände) verbessert werden können ${ }^{1}$. Die Idee hinter der Vision des ,Ubiquitous Computing' und ihrem wirtschaftlichen Ableger bietet einen interessanten gedanklichen Anknüpfungspunkt für die Ausrichtung von geeigneten Maßnahmen. Die Fragestellung, welche Maßnahme innerhalb des Mediums zur Unterstützung von wirtschaftichen Akteuren bei deren wirtschaftlichen Problemstellungen bestmöglich passt, bleibt davon aber unberührt und kann für diese Arbeit erst mit Blick auf die wesentlichen wirtschaftlichen Auswirkungen der zunehmenden Vernetzung durch die Informations- und Kommunikationstechnologien in Folge der Diffusion und somit Popularisierung des Internets und seiner Dienste beantwortet werden. Wie Malone \& Rockart [1991] bereits 1991 hervorhoben, sind diese Technologien nämlich weniger in der Lage, die Form der Produktion, sondern vielmehr die der Koordination zu revolutionieren, weshalb dem Medium spezifische Potenziale und Herausforderungen im Bereich der Koordination sowie in weiterer Folge der Integration und Kommunikation zwischen wirtschaftlichen Akteuren unterstellt werden. Die

1 Es handelt sich hierbei einerseits um eine Weiterentwicklung des rein an Mobilitätsbedürfnissen ausgerichteten ,mobile commerce', der in diesem Kontext als eine Art Unterbegriff interpretiert werden kann, wie auch andererseits um eine Verbreiterung der Integrationstiefe des ,eBusiness' [Fleisoh_2001 i Pfaff \&.Skiera 2002]376-7 
Potenziale und Herausforderungen im Bereich der Koordination, Integration und Kommunikation stecken demgemäß den Lösungsraum $a b$, in welchem unterstützende Maßnahmen für wirtschaftliche Akteure im Sinne des Mediums gesetzt werden können und sind folglich der Fokus der weiteren Überlegungen zu wachstumsinduzierenden Infrastrukturleistungen auf und für diese Ebene.

\section{2 Überlegungen zu Kollaborationsplattformen}

Das Konzept des ,Business Networking' ist in einem engen Zusammenhang mit der Vision des Echtzeitunternehmens ${ }^{2}$ zu sehen, die bereits seit den $60 e r$ Jahren gemeinsam mit informationstechnischen Integrationsbestrebungen einen Leitgedanken der Wirtschaftsinformatik bildet und vielfach von diversen ERPAnbietern $^{3}$ als Verkaufsargument eingesetzt wurde [Alt \& Ósterle 2003]. ,Business Networking' bezeichnet umfassend auf Basis von Informations- und Kommunikationstechnologien vernetzte Geschäftsabläufe und kann auch als Kopplung interner und externer Prozesse sowie ihre Abstimmung mit den betroffenen Partnern (,eCollaboration') verstanden werden. Hierbei beschreibt ,eCollaboration' die koordinierte Zusammenarbeit von Geschäftseinheiten oder Personen zur Erbringung einer gemeinsam definierten Leistung mit oder durch Informationssysteme [Reichmayr 2003]. In den letzten Jahren hat sich Business Networking als ein bedeutender Trend herausgestellt, dem sich viele Unternehmen gewidmet haben, um im Zeitalter der Informationsgesellschaft und deren vielfältigen technischen Möglichkeiten wettbewerbsfähig zu bleiben. Dabei können nach Ósterle et al. [2000] zwei wesentliche Zielsetzungen unterschieden werden: (i) einerseits die sich aus dem Wettbewerb ergebende Notwendigkeit einer erhöhten Prozesseffizienz, die eine optimierte Einbindung der Kunden und

2 Dieser Begriff steht für den Wunsch von Unternehmen, Informationen zu unternehmerischen Abläufen auf Abfrage in ,Echtzeit' überall zur Verfügung zu haben, um Reaktionszeiten zu minimieren (time-to-X), Kundenwünsche rascher umzusetzen bzw. die Koordination zu optimieren. Der Fokus enweitert sich im Zuge des Netzwerkgedankens verstärkt von der reinen unternehmensinternen Betrachtung auf zwischenbetriebliche Aspekte.

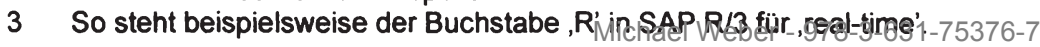


Lieferanten bzw. weiterer Stakeholder des Unternehmens fordert, sowie (ii) andererseits das Aufspüren und die Unterstützung von neuen Geschäftsfeldern bzw. innovativen Geschäftsmodellen. Hierbei wird von Österle et al. [2000] betont, dass das Konzept über eine isoliert betriebene eCommerce-, SCM- oder CRM-Strategie sowie über die technische Implementierung eines IT-Systems hinausgeht ${ }^{4}$, da es grundlegende Geschäftsziele unterstützt, die verschiedenen Strategien integrieren - und dabei eine isolierte Sichtweise überwinden - soll sowie eine erfolgreiche Umsetzung der unternehmerischen Vernetzung unter Berücksichtigung von organisatorischen, politischen und technischen Faktoren anstrebt. Im Zuge dessen wird hervorgehoben, dass Business Networking ein durch Technologie ermöglichtes, aber kein technologisches Konzept ist, und die Erfolgsfaktoren von Unternehmensnetzwerken in vieler Hinsicht im organisatorischen Bereich zu finden sind. Die Haupttreiber für ,Business Networking' liegen dabei in der steigenden Standardisierung, die Transaktionskosten reduziert und die zwischenbetriebliche Konnektivität fördert, der Interaktion von Netzwerkstrategien, dem exponentiell zunehmenden Geschäftsvolumen bzw. Geschäftspotenzial des Internets und der wachsenden Unterstützung von m:n Beziehungen durch Informationssysteme [Österle et al. 2000].

Eine notwendige Bedingung für die Realisierung einer $m: n$ Integration von zwischenbetrieblichen Abläufen, die die Phase der mehr oder weniger proprietären 1:1 Beziehungen überwindet, ist die Verfügbarkeit einer Art Netzwerkinfrastruktur, die die Rahmenbedingungen für den unternehmerischen Austausch - nicht nur aus einem informationstechnischen Blickwinkel - klärt. Der Begriff der ,Business Collaboration Infrastructure' (BCl) [Österle et al. 2000] erscheint hierfür gut geeignet, da er den wirtschaftichen Bezug, den Schwerpunkt auf eCollaboration sowie die Ausrichtung auf allgemein zugängliche, wirtschaft-

4 ,SCM' steht für Supply-Chain-Management, also die Handhabung der Lieferbeziehungen eines Unternehmens. Der Begriff ,CRM' steht für CustomerRelationship-Management und umfasst Aktivitäten zur Förderung der Kundenbeziehungen eines Unternehmens. IT' steht ff̈̈r Informatjepstę) 
lich notwendige bzw. wirtschaftspolitisch empfehlenswerte Leistungen in den Vordergrund rückt. Alternative bzw. allgemeinere Bezeichnungen wären beispielsweise ,Business Bus', ,Networking Infrastructure', ,E-Business Integration Hubs' oder nur ,Collaboration Infrastructure'. Es handelt sich bei $\mathrm{BCl}$ um multilaterale Plattformen oder - treffender formuliert - um eine Art ,elektronisches Verkehrssystem der vernetzten Wirtschaft', welches letztendlich die Interaktion von Unternehmen in digitalen Wertschöpfungsnetzwerken offen und flexibel begünstigen soll. Die Bedeutung solcher ,Verkehrssysteme' kann dabei direkt aus der bereits angeführten Veränderung der Koordination, die durch die Wandlung des Internets und seiner Dienste zu einem Transaktionsmedium zunehmend wirtschaftliche Implikationen aufweist, abgeleitet werden.

Unabhängig, ob man $\mathrm{BCl}$ nun als gedankliches Konstrukt zur Erleichterung der elektronischen Interaktion zwischen Unternehmen oder als eine Plattform, die branchenübergreifende und/oder branchenspezifische Koordinations-, Kommunikations- und Integrationsleistungen vereinfacht, verstehen möchte, erscheinen Infrastrukturleistungen, die die zwischenbetrieblichen Transaktionen unterstützen, im Rahmen des Business Networking Paradigmas erforderlich. In diesem Kontext wird beispielsweise von Alt \& Österle [2003] betont, dass eine Ursache für den fehlenden Erfolg mancher b2b-Marktplätze ${ }^{5}$ darauf zurückzuführen ist, dass diese eher nausgerichtet waren, Auktionen zu organisieren und unter Konkurrenten Transparenz zu schaffen als Geschäftsprozesse abzuwickeln“ [Alt \& Österle 2003, S. 99]. Demgemäß rückt das breiter als elektronische Marktplätze gefasste Konzept $\mathrm{BCl}$ die Bedeutung von Infrastrukturen im IKT-Bereich in den Vordergrund und fokussiert dabei auf Koordination, Integration und Kommunikation im Rahmen von zwischenbetrieblichen Transaktionsplattformen. Der Fokus liegt folglich nicht auf ,marketplace'-Funktionalitäten, sondern auf der Unterstützung von ,exchange'-Funktionen und somit eines offenen und flexiblen Austausches

5 Die Abkürzung ,b2b' steht für business-to-business und beschreibt Beziehungen zwischen Unternehmen oder auch zwischen Betrieben (im Sinne von Produktionsstätten). 
von Diensten und Informationen, die schließlich ,digitale Wertschöpfungsnetzwerke' wesentlich begünstigen könnten. Eine solche übergreifende Informationsinfrastruktur für Kooperationen, die zentral aber auch dezentral bereitgestellt werden kann, wurde vor Österle et al. [2000] bereits von Theoretikern der Netzwerkökonomie wie Malone \& Crowston [1994] oder Tapscott [1995] angedacht.

In der Literatur finden sich auch Beiträge, die mit dem Blick auf unternehmerische Innovationspotenziale betonen, dass neben der Marktorientierung und der Mitarbeiterentwicklung bzw. des Mitarbeiterempowerment die verschiedenen Formen des (unternehmens-)internen und externen Netzwerkens grundlegend für unternehmerische Innovationsstrategien sind [Wigand et al. 1997]. Dies ist ein weiterer Indikator dafür, dass Leistungen zur Erhöhung der Netzwerkfähigkeit von Unternehmen innerhalb des zuvor abgesteckten Lösungsraums zentrale Ansatzpunkte zur Ausnützung der Entwicklung des Internets zu einem Transaktionsmedium sind, wobei innen die Aufgabe zukommt, zwischenbetriebliche Probleme der Koordination zu lösen. Die Zielsetzung dieser Arbeit ist demnach die Entwicklung einer Infrastrukturleistung zur Optimierung bzw. Vereinfachung der Realisierung von Wertschöpfungsnetzen auf zwischenbetrieblichen Transaktionssystemen ${ }^{6}$, die sich auf der Grundlage des Internets bilden können, ohne die bereits vielfach diskutierten technischen Fragestellungen zu diesem Thema ein weiteres Mal zu erörtern. Hierbei sollen die Bedürfnisse von kleinen und mittleren Unternehmen (KMU) besondere Beachtung finden, nachdem diese in der sich entfaltenden Netzwerkökonomie spezifischen Potenzialen und Herausforderungen gegenüberstehen, die ihre vielfach ohnedies kritische Stellung in unterschiedlichsten Wertschöpfungsbeziehungen stark beeinflussen können. Eine wichtige Forderung bei der Entwicklung der Infrastrukturleistung ist daher, dass im Falle deren Realisierung auch die häufig stark regionalen, mitunter grenzüberschreitenden Transaktionsbeziehungen zwischen KMU, die das Po-

6 Solche Transaktionssysteme werden nachfolgend vorwiegend als (Kollaborations-) plattformen bezeichnet. 
tenzial des Internets und seiner Dienste als Transaktionsmedium nützen möchten, auf einer zwischenbetrieblichen Kollaborationsplattform verbessert werden können. Diese Forderung steht im Einklang mit der Forschungsvision der ,digitalen Ökosysteme' (,digital ecosystems') der Generaldirektion Informationsgesellschaft und Medien der Europäischen Kommission, die in das 7. Rahmenprogramm (2007-2013) der Europäischen Union aufgenommen wurde [Nachira 2007; Nachira et al. 2007].

"Thus, rather than relying on regulation and tariffs to control globalisation, it is wiser to create the structural conditions to support production in the regions, acknowledging the key role of the knowledge embedded in the regions and integrating SME business concepts with ICT solutions. The key is diversity. Standards can travel round the world, but if something can be explicitly rooted at the regional level - for example, food, art, the natural environment - it will tie economic development to the region." [Dini et al. 2005, S. 18]

\subsection{Aufbau der Arbeit}

Mit dieser Zielsetzung vor Augen wirft das Kapitel 2 im Anschluss einen theoriegeleiteten Blick auf Themenbereiche wie Daten, Kommunikation, Information, Kognition, (Un-)Wissen, Koordination und wirtschaftliche Dynamik und setzt diese aus einer (evolutions-)ökonomischen Perspektive in einen Kontext, um damit die Basis für eine Verfeinerung der Zielsetzung in Kapitel 3 zu legen. In Kapitel 3 erfolgt dementsprechend eine detaillierte und theoretisch fundierte Einschätzung der Notwendigkeit, der Chancen und der grundlegenden Vorbedingungen zur Unterstützung von Transaktionssystemen bzw. dessen Teilnehmern, wobei die bereits in Kapitel 2 eingeführte evolutionsökonomische Sichtweise weiter ausgebaut wird. Außerdem wird in Kapitel 3 ein wesentlicher Bestandteil des Lösungsansatzes vorgestellt: die Input-Output-Tabellen, deren Generierung und denkbare Alternativen. Daran anknüpfend präsentiert Kapitel 4 den Lösungsweg zur Erreichung der zuvor verfeinerten Zielsetzung, beschreibt die dafür erforderlichen Daten und skizziert die in den folgenden sechs Kapiteln (Kapitel 5 bis Kapitel 10) dargelegten Phasen des Modells, welches das Er- 
reichen der zuvor gesetzten Ziele ermöglicht und somit als Modell für die angestrebte Infrastrukturleistung für Kollaborationsplattformen dienen kann. Zur Illustration der Arbeitsweise des Modells wird nach den Kapiteln 5 bis 10 dessen Einsatz mittels der im Zuge der Modellerstellung entwickelten und implementierten Methoden anhand realitätsnaher, aber dennoch fiktiver Daten demonstriert und abschließend diskutiert (Kapitel 11). Kapitel 12 widmet sich zu guter Letzt dem Rückblick auf die hierin entwickelte Infrastrukturleistung und wagt der Hoffnung Ausdruck zu verleihen, dass die Ideen dieser Arbeit eines Tages tatsächlich das Licht der Welt erblicken dürfen. 


\section{Volkswirtschaftstheoretische Betrachtung ${ }^{7}$}

Die Wirkungsweise der Arbeitsteilung, ausgerichtet auf die Interessen der Individuen, ist nach Adam Smith [1776] der Schlüssel zur Erklärung des Wohlstandes von Nationen. Die Produktivitätssteigerung durch Spezialisierung und das daraus ermöglichte Anwachsen von spezifischem Wissen ${ }^{8}$ sowie Marktmechanismen, welche die Geschwindigkeit und Art der Informationsanhäufung (Wissensprozesse) regeln, sind dabei zentrale Triebfedern. Mit zunehmender Größe und Organisation von Märkten steigen dementsprechend die Möglichkeiten der Spezialisierung, des Austausches (,Handel') und konsequenterweise des Anwachsens von - sowohl markt- als auch produktionsbezogenem Wissen, um schließlich die Wohlstandsdynamik zu erhöhen.

Wenngleich also Wissen in allen Produktionsprozessen von fundamentaler Bedeutung ist, so tritt mit zunehmender Arbeitsteilung vor allem die Koordination der Akteure in den Vordergrund. Die Bedeutung von Koordination als zentrale Fragestellung der Wirtschaftswissenschaft wurde im 20. Jahrhundert insbesondere von dem österreichischen Nationalökonomen Friedrich A. Hayek hervorgehoben. Wie Adam Smith geht Hayek ebenfalls der Frage nach, wie Ordnung aus den Handlungen von Millionen von Wirtschaftsakteuren entsteht. Die Koordination von Spezialisierungen und dementsprechend von spezifischem Wissen ist demgemäß ein zentrales Anliegen der Wirtschaftswissenschaft als Instrument zur Steigerung des Wohlstandes, wobei Verfechter einer liberalen Marktwirtschaft (bzw. Marktordnung) wie Hayek die effizienteste Koordination in einem System dezentraler Austauschprozesse sehen und zentrale Planung daher ablehnen.

7 Die in diesem Kapitel angestellten Überlegungen flossen zum Teil in Weber \& Fröschl [2006] ein.

8 Die begriffliche Problematik hierzu wird weliterantenteagigegrifferb.31-75376-7 


\subsection{Wissen als ökonomische Kategorie}

Die Betonung der Rolle von wirtschaftlicher Koordination kann - technikgeschichtlich begleitet von Strömungen wie Kybernetik, Systemtheorie und einer allgemeinen ,Informatisierung' der Gesellschaft speziell ab der zweiten Hälfte des 20. Jahrhunderts - als Kritik an den Dogmen der neoklassischen Wirtschaftsmodelle gesehen werden: wie vor allem Hayek herausstreicht, lässt die neoklassische Theoriekonzeption die zentrale Bedeutung der Koordinationsprozesse erst gar nicht sichtbar werden, da in deren Modellen Vorbedingungen wie vollkommene Information am Markt, homogene Güter, keine Transaktionskosten und nicht vorhandene Markteintritts- und Austrittsbarrieren unterstellt werden - und somit Koordination ex ante obsolet machen. Demgegenüber - so Hayek [1937; 1945] - sind jene Prozesse zu hinterfragen, die dazu führen, dass Individuen überhaupt das erforderliche Wissen erhalten, welches in weiterer Folge in Richtung vollkommener Konkurrenz führen könnte. Die ,ökonomische Wissensproblematik' wäre also weniger an der Wirkung von Informationen über Preise sowie Preiserwartungen zu zentrieren, sondern schlösse vielmehr einen weit größeren Bereich ein, nämlich die Frage nach dem Wissen, wie unterschiedliche Güter erworben und verwendet werden könnten und unter welchen Bedingungen diese Wissensprozesse abliefen. ${ }^{9}$

Letztlich muss aus einer informationsökonomischen Perspektive überhaupt die Erklärungskraft von Gleichgewichtsanalysen bzw. (zeitlos gültigen) Gleichgewichtspostulaten der Wirtschaftswissenschaft ${ }^{10}$ bezüglich des wirtschaftlichen

9 Hayek geht hierbei noch weiter: für inn steht im Hintergrund dieser Problematik die allgemeine Fragestellung, wie von verschiedenen Personen subjektiv wahrgenommene Daten objektiven Fakten entsprechen können [Hayek 1937].

10 Beginnend mit Walras (Lausanner Schule) wurden mathematische Modelle formuliert, um das Gleichgewicht von Produktion und Konsum über ein Preissystem am Gesamtmarkt (und später auch von anderen Ökonomen in Teilmärkten) zu beweisen, u.a. um Adam Smiths marktliche Koordination über den Preismechanismus (,invisible hand') zu demonstrieren. Walras Ansatz konnte allerdings erst durch Arrow \& Debreu [1954] unter restriktiven Grundannahmen (z.B. keine Transaktions-

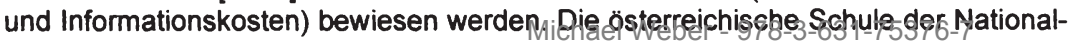


Kreislaufs und der Abstimmung von Angebot und Nachfrage - insbesondere in nicht zentral geregelten Wirtschaften - angezweifelt werden. Konsequent legt Hayek den Fokus seiner Analyse auf die Pläne interagierender Individuen, die sich aus den Erwartungen der Akteure ergeben und daher letztlich durch deren Wissen bestimmt werden [Hayek 1937]. Er unterstreicht seine Kritik an den Grundannahmen vollkommener Märkte, in dem er betont, dass die Unterstellung eines Gleichgewichts bei vollkommenem Wissen der Akteure tautologisch sei, da dies der Definition von Gleichgewicht entspreche, wobei der Weg zu diesem Gleichgewichtszustand in den Gleichgewichtsmodellen jedoch nicht spezifiziert würde ${ }^{11}$. Die Pläne und Erwartungen sowie, in weiterer Folge, das den Erwartungen der Akteure zugrunde liegende Wissen zu koordinieren, stellt demnach eine elementare Herausforderung an jedes Wirtschaftssystem dar, formuliert in der Frage:

"... how the combination of fragments of knowledge existing in different minds can bring about results which, if they were to be brought about deliberately, would require a knowledge on the part of the directing mind which no single person can possess." [Hayek 1937, S. 52]

Hayek, der sich in seiner systemischen Sicht der Ökonomie stark von einer sozialwissenschaftlichen Seite her nähert'12, trifft bei der Koordination von Plänen

ökonomie und auch Schumpeter sahen kurzfristige Gleichgewichte bzw. statische oder allgemeine Gleichgewichte aufgrund der Dynamik nicht als erreichbares Ziel und Merkmal eines freien Markts, sondern betonten vielmehr die Aufteilung von Ressourcen hinsichtlich der Bedürfnisse der Individuen und die Rolle von Entdeckung und Erfindung (v.a. Schumpeter) für wirtschaftliches Wachstum.

11 Hayek kritisiert dabei weniger die Möglichkeit der - etwas entschärfteren - Behauptung, dass eine Tendenz zu einem Gleichgewicht bestehen kann, weil sich sozusagen die Erwartungen der Akteure im Laufe der Zeit (ceteris paribus) verstärkt im Einklang befinden. Diese Behauptung, welche im Übrigen den Übergang der Wirtschaftswissenschaft von einem Konstrukt rein logischer Aussagen zu einer empirischen Disziplin demonstriert, trifft allerdings für Hayek noch keine Aussagen über die Bedingungen, unter denen diese Tendenz wirkt und erklärt auch nicht die Prozesse, durch die das Wissen der Individuen ausgetauscht wird [Hayek 1937].

12 Siehe dazu etwa Durkheim [1977] oder Luhmann [1988]; Hayek akzeptiert implizit marktrationales Verhalten der Akteure, lehnt aber das Konzept der vollständigen Rationalităt der Akteure explizit ab. 
Unterscheidungen hinsichtlich der lediglichen Kompatibilität individueller Pläne, d.h. der Widerspruchsfreiheit und folglich Anpassbarkeit nicht deckungsgleicher Pläne bspw. bezogen auf Angebot und Nachfrage, sowie deren Korrespondenz und betont die Rolle von externen Fakten (,objektive Daten'). Intersubjektives Einverständnis erfolge häufig auf Basis von - gemeinsam wahrgenommenen externen Fakten. Hayek zeigt dabei die Problematik von widersprechenden Erwartungen im Zuge der Koordination ebenso auf wie die exogenen und ungleich wirkenden Einflüsse auf u.U. ursprünglich kompatible oder gar korrespondierende Erwartungen. Überhaupt wäre zu klären, wem die verfügbaren Daten bereitgestellt werden sollen, etwa nur den beobachtenden Ökonomen, der breiten Bevölkerung oder nur bestimmten Marktakteuren. In diesem Zusammenhang ergeben sich Verteilungsfragen, die in Gesellschaften, welche das Kriterium Information bzw. Wissen zur Allokationsentscheidung einsetzen (,Informationsgesellschaften'), verstärkt an Brisanz gewinnen. Ebenso stellt sich die Frage, inwieweit bzw. über welche Prozesse subjektiv wahrgenommene Daten zu objektiven Daten bzw. Fakten werden können. Gleichzeitig erscheint die häufig implizite Optimalitätsbehauptung von Gleichgewichtspostulaten fragwürdig, wenn man bedenkt, dass diese erreicht werden durch relatives, d.h. v.a. auch relativ geringes, Wissen unter den Akteuren, welches auf dem Weg zur Durchführung der individuellen Originalpläne angereichert wird und ebenfalls zu korrespondierenden Plänen und (temporären) Gleichgewichten führen kann. Solche Gleichgewichte würden - so Hayek - eben gerade deswegen denkbar sein, weil die Akteure nicht über Dinge lernen könnten, die ihre Pläne änderten, insbesondere um diese besser an die individuellen Bedürfnisse anzupassen.

Zusammenfassend und in Hayeks Sinne formuliert sind theoretische Marktgleichgewichte Ausdruck von Koordinationsgleichgewichten, die durch die Wissensstände und daraus folgernde Erwartungshaltungen der Akteure charakterisiert werden. Dynamik erklärt sich folglich aus Art und Menge des verfügbaren Wissens bzw. der Verteilung desselben über die Einzelakteure; methodisch schwierig bleibt aber die Bestimmung eines für die Erklärung der Marktdynamik überhaupt erforderlichen Wissens: Weber - 978-3-631-75376-7 
"But what is this relevant knowledge? It can hardly mean simply the knowledge which actually influenced his [Anm.: des Akteurs] actions, because his decisions might have been different not only if, for instance, the knowledge he possessed had been correct instead of incorrect but also if he had possessed knowledge about altogether different fields." [Hayek 1937, S. 49]

und weiter:

"Clearly there is here [sic.] a problem of the division of knowledge which is quite analogous to, and at least as important as, the problem of the division of labour. But, while the latter has been one of the main subjects of investigation ever since the beginning of our science, the former has been [as] completely neglected, although it seems to me to be the really central problem of economics as a social science." [Hayek 1937, S. 49]

Die Verteilung von Information bzw. Wissen und dementsprechend die (informations- und kommunikations-)Technologien zur Unterstützung der Allokation von Wissen treten aus dieser Analyse als die entscheidenden Determinanten der wirtschaftlichen Dynamik hervor. Wissenszugang und Koordinationseffizienz der einzelnen Akteure bestimmen letztlich die Entscheidungen über die Allokation traditioneller ökonomischer Ressourcen wie Arbeit und Kapital, die darüber hinaus freilich durch Institutionen - allgemeine kulturelle und rechtliche Struktur en insbesondere zur Reduktion von Unsicherheit (mittels Sanktionierung) - beeinflusst werden, wobei unterschiedliche institutionelle Rahmenbedingungen auch unterschiedliche Formen der Koordination bewirken können. Als eine zentrale Herausforderung der Ökonomie einer Gesellschaft können also die Grenzen des Wissens bzw. der Informationsgrundlage des Individuums in Verbindung mit der optimalen Kooperation der individuellen Wirtschaftsakteure gesehen werden.

\subsection{Transparenz vs. Intransparenz}

Im Hinblick auf die Möglichkeiten des Einsatzes speziell von Informations- und Kommunikationstechnologien zur Unterstützung der Allokation von Wissen sowie der Koordination individueller Wirtschaftsakteuse drängt sich_rasch die-7rage auf, 
Volkswirtschaftstheoretische Betrachtung

ob und inwieweit - die für die Informationsverarbeitung erforderliche - Transparenz überhaupt realisiert werden kann und in weiterer Folge ,dem Wissen' (zumindest theoretisch) Grenzen gesetzt sind.

Kurt Gödel stand und steht als Begründer des Unvollständigkeitssatzes und somit der mathematischen Entsprechung von Paradoxien im Rampenlicht der Diskussion um Transparenz, Vollständigkeit, Wissen, Kontrollierbarkeit bzw. Berechenbarkeit und damit zusammenhängender (computergestützter) Automatisation und Zentralisation. Ihm gelang es, die Aussichten auf einen Beweis für die Vollständigkeit und Widerspruchsfreiheit formaler axiomatischer Systeme ${ }^{13}$ wie der Principia Mathematica von Whitehead \& Russel [1910] zu zerstören. Gödel zeigte das Gegenteil, nämlich die Existenz von ,formal unentscheidbaren Sätzen der Principia Mathematica und verwandter Systeme' [Gödel 1931], wobei letzterer Zusatz in dem Titel der Arbeit Gödels enorme Auswirkungen haben sollte, nicht nur in der Mathematik. Gödels Ausführungen zur Unentscheidbarkeit von mathematischen Aussagen initiierten in den unterschiedlichsten Disziplinen eine Vielzahl an Diskussionen, Interpretationen und Schlussfolgerungen. So wurde auch in der Wirtschaftswissenschaft Gödels Satz bei tendenziell individualistisch orientierten Theoretikern, die entsprechend dezentrale Koordination forcierten, bereitwillig als gewichtiges Argument gegen planwirtschaftliche Ansätze, bspw. in Abwandlung bzw. computational/zentralistischer Ablösung des

13 Die Bedeutung von Axiomen, also den als wahr angenommenen, nicht zu beweisenden Grundsätzen eines Systems, erhöhte sich mit der zunehmenden Komplexität mathematischer Theorien und Theoreme, insbesondere ab dem 19. Jahrhundert und relativierten die Rolle der Evidenz (lat. videre $=$ sehen). Während Axiomensysteme unter Euklid noch überschaubar und einsichtig waren und auch sein sollten, waren um 1900 die Systeme so mächtig, dass sie zusehends kompliziert, unanschaulich und unberechenbar wurden. Mit der Verdrängung der Evidenz, die von Mathematikern vereinzelt zu verteidigen versucht wurde, kam auch die gesicherte Widerspruchsfreiheit abhanden. Nun stellte sich die Frage, ob diese Axiome - man könnte sie auch als Spielregeln bezeichnen - letztlich ein gesichertes Fundament für die Mathematik ermöglichen würden. Diese Fragestellung findet man bereits im Jahr 1900 in Hilberts Liste von 23 mathematischen Problemen [Hilbert 1900] und wurde von Hilbert in den 1920er Jahren zume Prgarammerhgbeg:7 
Auktionärs von Léon Walras und dem dadurch zu erreichenden ,vollständigen Gleichgewicht', aufgenommen und eingesetzt (vgl. hierzu bspw. [Mirowski 2002]).

All diesen Folgerungen voran machte Gödels Beweis allerdings die Losung "Wir müssen wissen, Wir werden wissen“ [Hilbert 1930, S. 963] des Mathematikers David Hilbert, die dieser zuletzt 1930 bei einer naturwissenschaftlichen Tagung in Königsberg ${ }^{14}$ auch für den Rundfunk wiederholt hat, nicht mehr haltbar. Vielmehr wurde durch Gödel die - von Hilbert (für die Mathematik) abgelehnte - Vorstellung des ,(Ignoramus et) Ignorabimus', von Erich Heinrich du Bois-Reymond aus dem Jahr 1872 bekräftigt. Eine Vorstellung, die nicht nur aussagt, dass wir nicht wissen, sondern auch niemals wissen werden und die aus der Auseinandersetzung von du Bois-Reymond mit den ,Grenzen des Naturerkennens' [du Bois-Reymond 1912] folgt.

Abgesehen von irreführenden und vielfach widerlegbaren Trivialinterpretationen, die auf Basis der Veröffentlichung und Verbreitung von Gödels Aussagen auftraten, nahmen fundierte Folgerungen von Gödels Beweis bald Einfluss auf die Theoriebildung in der Wissenschaft und faszinierten vor allem auch aufgrund der weit reichenden philosophischen Schlussfolgerungen [Köhler 2002a]. In diesem Zusammenhang sei insbesondere auf die philosophische Position des Wiener Kreises ${ }^{15}$ unter Moritz Schlick hingewiesen, welcher Gödel förderte und inspirierte und zu dem Gödel mit seinem Satz einen wesentlichen Beitrag leisten konnte, ebenso wie zu dem geistigen Umfeld der österreichischen Schule der Nationalökonomie zu der es damals starke Querverbindungen gab.

14 Dies war die Tagung an der Gödel seine Erkenntnisse in einer Diskussion äußerte, an der John von Neumann, ein Schüler Hilberts, teilnahm. John von Neumann begriff die Bedeutung von Gödels Erkenntnis rasch und setzte Hilbert davon in Kenntnis. Hilberts Akzeptanz von Gödels Beitrag lässt sich am besten mit der Veröffentlichung von Gödels Argumentation in einem von David Hilbert und Paul Bernays herausgegebenen Buch über die ,Grundlagen der Mathematik II' dokumentieren (vgl. hierzu [Köhler 2002a] sowie [Smoryński 2002]).

15 Sowie dem daraus hervorgegangenen ,Mathematischem Kollogujisp' $75376-7$ 
Volkswirtschaftstheoretische Betrachtung

Der in Wien zu jener Zeit lehrende Wirtschaftswissenschafter und (spätere) Nachfolger von Friedrich A. Hayek am Institut für Konjunkturforschung, Oskar Morgenstern, beschäftigte sich ab den späten 1920er Jahren kritisch mit den ,Voraussetzungen und Möglichkeiten der Wirtschaftsprognose' [Morgenstern 1928] und den - damit zusammenhängenden - ,Grenzen der Wirtschaftspolitik' [Morgenstern 1934], wobei er speziell auf die Problematik prognostischer Entscheidungen über die Entwicklung der Wirtschaft bzw. allgemeine Probleme der Prognose hinwies ${ }^{16}$. Die Grenzen der Kognition ökonomischer Akteure im Hinblick auf wirtschaftliche Interaktionen waren ein zentrales Thema der österreichischen Schule der Nationalökonomie, das auch wesentlich zu deren Aussagen im Rahmen der Debatte über die sozialistische Wirtschaftsrechnung beitrug. Morgenstern, der sich über die negativ und positiv rückkoppelnde Wirkung von Vorhersagen (später ,selbsterfüllende Prophezeiungen') bewusst war, ging lange davon aus, dass wirtschaftliche Prognosen nicht möglich wären. Er folgte der Vorstellung einer grundsätzlichen Unbestimmtheit, die den meisten wirtschaftlichen Abläufen zu eigen waren (vgl. [Mirowski 1992; 2002]). Das Aufeinandertreffen Morgensterns mit dem Mathematiker John von Neumann in Princeton ${ }^{17}$ führte schließlich zu einer Wende in den Überlegungen Morgensterns. Von Neumann, der sich schon seit längerem mit Gesellschaftsspielen beschäftigt hatte (siehe z.B. [von Neumann 1928]), zeigte einen Weg zur Vorhersage von Verhaltensweisen von rationalen Akteuren und damit einen Ausweg aus der Problematik. Mittels einer Mischung aus Zufall und Wahrscheinlichkeit könnte man Prognosen über Handlungen von Akteuren erstellen, allerdings unter

16 Zum Verhältnis von Gödel zu Morgenstern ist an dieser Stelle anzumerken, dass sich beide bereits in Wien u.a. über den Mathematiker Karl Menger, Sohn des österreichischen Nationalökonomen Carl Menger, sowie den Mathematiker Abraham Wald wohl kannten, eine intensivere Auseinandersetzung ergab sich allerdings erst nach der Emigration nach Princeton, USA [Köhler 2002b, S.169].

17 Nach einem Vortrag im Wiener Kreis 1937 über die Prognoseproblematik, am Beispiel der Verfolgungsgeschichte von Sherlock Holmes und dem ebenso rationalen und wissenden Prof. Moriarty, wurde Morgenstern durch Eduard Cech bereits auf eine mögliche Formalisierung bzw. Herangehensweise in einer Arbeit von John von Neumann aus dem Jahr 1928 hingewiese M[Mirgmski b1992d.78-3-631-75376-7 
den Annahmen der vollständigen Informationsgrundlage und der ebenso vollständigen Rationalität der beteiligten Akteure. Die wirtschaftliche Anwendung der Überlegungen von Neumanns zu Gesellschaftsspielen sowie dessen MenschMaschine-Analogien führten somit gepaart mit Morgensterns Interesse für Wirtschaftsprognosen zur Entwicklung der Spieltheorie [von Neumann \& Morgenstern 1944]. Ungeachtet der zahlreichen Kritikpunkte bspw. betreffs der theoretischen Voraussetzungen oder der weiteren Entwicklung (alternativer) spieltheoretischer Konzepte, wie z.B. die Evolutionäre Spieltheorie, die die zentralen Prämissen der Spieltheorie durch Annahmen über Anpassungsprozesse ersetzt, sei darauf hingewiesen, dass diese als Spiel titulierten simulierten Entscheidungsumgebungen letztlich auf (Spiel-)regeln aufbauen, welche in der mathematischen Interpretation nichts anderes als Axiome darstellen. Im Sinne der Ausführungen zu Gödels Beweis sei daher an dieser Stelle nochmals darauf hingewiesen, dass Vollständigkeit und Widerspruchsfreiheit von, hinreichend mächtigen formalen' (aufgrund von Komplexität formalisierten), auf Axiomen basierenden Systemen (z.B. eine als Spiel bezeichnete simulierte Entscheidungsumgebung) laut Gödel nicht nachweisbar sind. Dies relativiert dementsprechend die Entscheidungsfindung und bedeutet auch, dass Transparenz nicht abschließend geschaffen werden kann. Gleichzeitig soll in diesem Zusammenhang aber die Gefahr des möglichen Missbrauchs dieser relativierenden Argumentationskette bspw. zur Abwehr von in ihrer Wirkung eingegrenzten, aber hilfreichen Maßnahmen zur Reduktion von Intransparenz oder allgemein in (tatsächlich) überschaubaren Entscheidungssituationen nicht unerwähnt bleiben.

\section{3 Ökonomie als evolutionärer Prozess}

In enger Verbindung mit der Tradition der österreichischen Schule der Nationalökonomie steht die Strömung der Evolutionären Ökonomie, welche (Un-)Wissen in das Zentrum der wirtschaftswissenschaftlichen Betrachtung rückt und dabei auf die evolutionären Prozesse Variation, Selektion und Bewahrung abstellt. Die Evolutionäre Ókonomie interpretiert sich dabei allerdings nicht als eine Form von ökonomischem Darwinismus, sondern führt die Wurzeln der Wahrnehmung 
dieser drei evolutionären Prozesse in Wirtschaft und Gesellschaft auf die Aufklärer des 18. Jahrhunderts zurück und hebt die evolutionären Aspekte der Aussagen u.a. auch von Adam Smith hervor. Ökonomische Evolution stellt gemäß dieser Strömung - wie bei Schumpeter und Hayek - das tatsächliche Wachstum von Wissen dar ${ }^{18}$, wobei die Mechanismen dieses Wachstums ein wichtiges Forschungsthema für die Evolutionäre Ökonomie sind. Diese Strömung trägt prinzipiell der Entwicklung der industrialisierten Gesellschaften in eine Informationsgesellschaft (oder gar ,Wissensgesellschaft' vgl. [Kreibich 1986] oder [Stehr 2001]) in Verbindung mit Informations- und Kommunikationstechnologien (IKT) Rechnung. Vertreter der Evolutionären Ökonomie definieren die Problematik der Wirtschaft(-swissenschaft) weniger - wie sie traditionell und weit verbreitet gesehen wird - in der Zuweisung knapper Ressourcen zu bestimmten (vorgegebenen) Zwecken, sondern vielmehr im optimalen Einsatz sowie der Erzeugung von Wissen bzw., in Anlehnung an Metcalfe, allgemein in der weit verbreiteten Ignoranz. Wirtschaft wird dabei als offenes, komplexes und dynamisches System interpretiert, welches mit zahlreichen Überraschungen und Neuheiten aufwarten kann und notwendigerweise instabil ist Witt 2003; Metcalfe 2003]. Die Aussage der Evolutionären Ökonomie ist in diesem Zusammenhang nicht, dass nur marktwirtschaftliche Gesellschaften auf Wissen basieren, sondern dass die spezielle Form der Dynamik des Wissens in solchen Systemen, die Dynamik von Innovation, Unternehmen und Wettbewerb, von besonderer Bedeutung für Wachstum ist. Schumpeter lieferte hierbei mit seinem Konzept der ,schöpferischen Zerstörung' (,creative destruction') [Schumpeter 1942] und der Sichtweise des Entrepreneurs als Innovator ${ }^{19}$ und konsequenterweise als Produzent von Ungleichgewichten einen wichtigen Ausgangspunkt für die

18 Erstmals analysierte Machlup empirisch den Beitrag und die Rolle von Wissen in einer Volkswirtschaft [Machlup 1962].

19 Eine Interpretation der Rolle des Entrepreneurs, die auf Baudeau im 18. Jahrhundert zurückgeht. 
Evolutionäre Ókonomie, wobei die Bedeutung von Unsicherheit ${ }^{20}$, wie sie bereits bei Vorklassikern wie Cantillon oder etwas später von Say Berücksichtigung fand, federführend von Knight [1921] in diese Perspektive integriert wurde. Dieser Schritt half einerseits die Rolle der Investoren und deren Profite bzw. Verluste zu erklären und trug andererseits zum Verständnis von Konkurrenz und dem Aufkommen von Motivation zur Innovation bei.

Die Instabilität bzw. Dynamik des Wirtschaftssystems erklärt sich nun für evolutionäre OKonomen in dem Auftreten und der Verbreitung von Neuheiten (neue bedeutungstragende Unterscheidungen) im Rahmen unternehmerischer Tätigkeit, die durch das Anwachsen von Wissen hervorgerufen werden. Dieses Wachstum resultiert wiederum aus der Lösung einer Problemstellung, die allerdings häufig neue Fragen und Möglichkeiten aufwirft, welche abermals eine Kombination verschiedenster Ideen und Entdeckungen induzieren. Im Laufe der Zeit führen diese Prozesse zu einem explosiven Anstieg von Wissen, was zu der Behauptung führt, dass eine Wirtschaft nicht im Gleichgewicht sein kann, wenn Wissen nicht zu einem Gleichgewicht kommen kann [Witt 2003; Metcalfe 2002]. In diesem Zusammenhang wird von der Evolutionären Ökonomie unterstrichen, dass die Erzeugung und Verbreitung von Neuheiten zutiefst ökonomische Prozesse darstellen, wobei Unsicherheit und der Wettbewerb um neue, bessere Ideen den Hintergrund und die Motivation für diese Prozesse bilden.

Die rationale Bewirtschaftung der physikalischen Faktoren Raum, Zeit und Energie bei gleichzeitiger Optimierung der Nutzung von - der Gesellschaft zugrundeliegenden bzw. verfügbaren - Daten bewirkt, kurz gesagt, den Entwicklungspfad, welcher der Gesellschaft historisch gesehen unterstellt werden kann. Die fortlaufend optimierte Verwertung dieser Datengrundlage führt über Informationsextraktion und kognitive sowie behavioristische Prozesse in Kombination mit Vorwissen, Werten und Emotionen der Individuen schlussendlich zu 
Wissen bzw. Zuwachs an Wissen [Boisot \& Canals 2004]. Wohlstand sieht die Evolutionäre Okonomie in weiterer Folge als ein Produkt der Integration von spezifischem Wissen und folgt daher nicht direkt aus dem Ausnutzen natürlicher Ressourcen. Dabei wird nicht lediglich auf das Wissen von Eliten abgestellt, sondern vielmehr auf das Wissen jedes Menschen. Jeder Wirtschaftsakteur stellt sozusagen eine Komponente mit speziellem Wissen dar, wobei sich hierbei die zentrale ökonomische Frage stellt, wie man dieses spezielle Wissen effektiv koordiniert.

Die Rolle von IKT zur Unterstützung einer solchen (marktlichen bzw. gesellschaftlichen) Koordination ergibt sich im Zuge der rasanten technologischen Entwicklung beginnend mit dem Zeitalter der Spätaufklärung und des darin stattfindenden naturwissenschaftlichen und technischen Erkenntnisfortschritts. $\mathrm{Zu}$ Beginn war allerdings in vielen Ländern die zentrale Staatsmacht alleinig im Besitz der aufkommenden Kommunikationstechnologien wie bspw. die optischen und später elektrischen Telegrafennetze. Erst allmählich und unter Druck wohlhabender Bevölkerungsschichten mit vorwiegend wirtschaftlichen Interessen gelang es, die vergleichsweise geringe staatliche Kommunikation, die kommunikationstechnische Innovationen aufgrund fehlender Märkte lange Zeit be- bzw. verhinderte, für private Kommunikation zu öffnen, wobei Maßnahmen zur Ergänzung des Eisenbahnsystems und die Konkurrenz zwischen den Nationalstaaten ebenfalls zur Öfnung der Netze beitrugen. Börse und Handel waren nach dieser Liberalisierung federführend in der Adoption der telegrafischen Kommunikationstechnologie, da ein rascher Informationsfluss in diesen Domänen Voraussetzung für das Angleichen und Bestimmen der Preise ist und Informationen im Allgemeinen eine zentrale Stellung bei der Herausbildung von Märkten spielen, wie Jevons ${ }^{21}$ bereits 1871 festhielt. Eine rasche Abfolge weiterer kommunikationstechnischer Erfindungen und vielfach auch Innovationen in den

21 Jevons bemerkte in diesem Zusammenhang weiters, dass unter der Voraussetzung von engen Kommunikationsbeziehungen zwischen Geschäftspartnern Märkte auch ohne festen Marktort existieren können (vg|idflichy, 19941) 978-3-631-75376-7 
folgenden Jahrzehnten und Jahrhunderten gab einer verstärkt privaten Kommunikation weiteren Auftrieb, begleitet von der Entwicklung der Fotografie, des Phonographs, des Telefons, der Funktechnologie und des Rundfunks, später dann vom Aufkommen der globalen Kommunikation mit Automatisation, Digitalisierung und neuen Übertragungstechniken sowie dem Einzug des Fernsehens und der Datenverarbeitung (vgl. [Flichy 1994])22. Die allmähliche Verschmelzung von Audio- und Videotechnologien, Telekommunikation und Datenverarbeitung, die mittlerweile zu einer irreversibel ,ubiquitären' Durchdringung aller Lebens- und Gesellschaftsbereiche geführt hat und in ihrer Extrapolation absehbar auf eine universale ,Kommunikationsprothetik' hinauszulaufen scheint, begleitet dabei eine weiter zunehmende Individualisierung der Kommunikation bis hin zur geradezu folgerichtigen Entwicklung auf bestimmte Interaktionsziele programmierter, selbstgesteuerter ,Kommunikationsagenten' [Kimbrough \& Wu 2005]. Die zeitgeistige Koinzidenz wirtschaftsliberaler Konzeptionen der zitierten Ökonomen mit der dezentral-privaten Kommunikationstopologie der digitalen Kommunikationsinfrastrukturen muss dabei - in Gegenüberstellung etwa zu staatsinterventionistischen, planwirtschaftlichen oder sonst kollektivistischen Ansätzen in Ökonomie und Kommunikationsorganisation - besonders deutlich herausgestrichen werden.

\subsection{Entropie}

Naturwissenschaftlich zeichnen sich großtechnische Kommunikationssysteme mit hoher Entropie dadurch aus, dass sie Zeichen bzw. Symbole in hoher Bandbreite übermitteln können. Der Begriff der Entropie entstammt hierbei ursprünglich der Thermodynamik und wurde von Informationstheoretikern (insbesondere

22 Flichy [1994] betont in seinen Ausführungen, dass die Telekommunikation in unterschiedlichen Ländern (wie England und Frankreich) jeweils von jenen Geistesströmungen gefördert wurde, die dem Freihandel positiv gegenüber standen und sieht die Aufklärung bzw. den Gebrauch der Vernunft als eigentliche Basis für die zahlreichen Entdeckungen und Erfindungen im Bereich der Telekommunikation, die abhängig von den Wechselwirkungen zwischen Technologie und Gesellschaft vielfach zu Innovationen führten. 
Shannon [1948], aufbauend auf frühere Überlegungen von Nyquist, Hartley und von Neumann) als Maß für den Informationsgehalt von übermittelten Signalen (Daten) eingeführt. Daten entspringen unterscheidbaren (d.h.: wahrnehmbaren) physischen Zuständen [Rosen 1991] und können demgemäß - je nach Entropie - mehr oder weniger Information beinhalten. Hierbei kann zwischen einer objektiven (intersubjektiven) und einer subjektiven Betrachtung unterschieden werden, wobei ,objektiv' die Menge der potenziell ableitbaren Information bezeichnet und ,subjektiv' die Menge an Information umfasst, die ein Akteur in (s)einer bestimmten Situation aus einer Menge von Daten ableiten kann [Boisot \& Canals 2004]. Diese subjektive Sichtweise, die - gemäß der Interpretation von ,objektiv' als intersubjektiv - über die Agglomeration des subjektiven zu einer ,objektiven' Sichtweise führt, unterstreicht hierbei die Definition von Information gemäß Bateson [1971] als Unterschied, der einen Unterschied für jemanden macht, d.h. relational gesehen wird: Daten tragen unterschiedliche Bedeutung, abhängig von den Charakteristika (u.a. Vergangenheit, Wertesystem, emotionaler Zustand, Erwartungen [Damásio 1999]) des jeweils die Daten interpretierenden Individuums. Qualitativ gesprochen drückt sich der Informationsgehalt einer Nachricht durch das Verhältnis der a posteriori verbleibenden (internen) Zustandsalternativen gegenüber den vor dem Empfang einer Botschaft gegebenen Zustandsalternativen aus: diese Neg-Entropie entspricht einer Reduktion von Unsicherheit, wohingegen die Auslöschung eines solchen ,Unterschieds' wiederum zu einer korrespondierenden Steigerung der Entropie (Unsicherheit, Undeterminiertheit) führt. Formal wird der potenzielle Informationsgewinn (eines Akteurs) durch die - theoretische - Gesamtmenge der in einem Kommunikationssystem darstellbaren Zustandsmenge ${ }^{23}$ beschränkt.

Die bisher entwickelten Kommunikationssysteme - einschließlich des Internets bzw. WWW - sind darauf ausgerichtet, Daten möglichst, rauschfrei' zu über- 
mitteln, d.h. dafür zu sorgen, dass die Daten einer Nachricht beim Empfänger identisch mit den ursprünglich versendeten Daten sind. Diese Kommunikationsebene stellt allerdings nur die erste von insgesamt drei Stufen der Semiotik dar, die helfen, den Wirkungsgrad der Kommunikation zu differenzieren. Während die erste Ebene (Syntax) vorwiegend technische Probleme der Übermittlung von Nachrichten betrachtet, stellt sich auf der nächsten Stufe die Problematik des Verständnisses der Nachricht, die semantische Ebene, wohingegen auf der letzten Ebene dieser Dreiteilung Fragen der Auswirkung der Nachricht im Kontext, die pragmatische Ebene, stehen.

\subsection{Kontextualisierung und Bedeutungskoordination}

Eine denkbare nächste Ausbaustufe des Internets, das ,Semantic Web' [Minsky 1968; Berners-Lee et al. 2001; Handschuh \& Staab 2003; Visser 2004; Stuckenschmidt \& van Harmelen 2004], stellt einen ambitionierten Ansatz dar, sich der semantischen Ebene anzunähern und das Internet als technisches Kommunikationssystem so weiterzuentwickeln, dass Information zielgerechter verteilt werden kann - damit diese bei den Empfängern tatsächlich ,einen Unterschied macht', d.h. subjektiv (bzw. individuell) Nutzen generiert und pragmatisch Effekte nach sich zieht, wie von Bateson definiert und gefordert. Eine Annäherung an diese Stufe der Kommunikation erweist sich allerdings in vieler Hinsicht als problematisch, nachdem sich die Aufgabenstellung für die datenbasierenden Kommunikationssysteme insofern ändert, als auf Ebenen 2 und 3 zur informationsgewinnenden Interpretation von Nachrichten (Daten) Wissen bzw. ,Vorwissen' erforderlich ist: im Unterschied zur Dinghaftigkeit von Daten entsteht Information (erst) aus dem Abgleich von empfangenen Daten mit bestehendem Wissen, wobei Wissen seinerseits eine Menge von - nicht zuletzt auch sozial geprägten bzw. konventionalisierten ${ }^{24}$ - Erwartungen (eines Individuums nach außen) darstellt, welche ihrerseits durch das Eintreffen von 
Informationen beeinflusst werden [Arrow 1984]. Wissen steuert letztendlich (kognitiv) die Aktionen des Individuums, insbesondere auch in der Gewinnung von Information (,information gain') durch Ausfilterung ,bedeutungstragender Strukturen' aus den Daten [Boisot \& Canals 2004] ${ }^{25}$.

Information kann - auf der Grundlage gemeinsamen Wissens - graduell zwischen Akteuren geteilt (bzw. verteilt) werden; im Gegensatz dazu ist Wissen grundsätzlich niemals zwischen Akteuren teilbar. Nur bei Daten kann man von einer allgemeinen Teilbarkeit (Kopierbarkeit) sprechen, weshalb Ebene 1, in der die spezifischen Eigenschaften der kommunizierenden Akteure irrelevant sind und in der Konventionen über Symbole und Grammatik die Basis bilden, technisch relativ (!) einfach gelöst werden kann.

Boisot \& Canals [2004] schlagen in ihrer informationsökonomischen Analyse vor, das Konzept der Entropie auf alle drei Stufen der Kommunikation anzuwenden, wobei jeweils für Daten, Information und Wissen verschiedene Formen von Entropie definiert werden. Auf dem Niveau der Daten wäre von ,Entropie 1' zu sprechen, die sich mit den physikalischen Zuständen beschäftigt, wobei eine Erhöhung dieser Entropie eine entsprechende Verringerung der (Wahrnehmungs) Unterschiede zwischen den Zuständen bewirkt. Im Bereich der Information beschreibt ,Entropie 2' (wie von Shannon [1948] für die Informationstheorie eingeführt) nun die Variation der symbolischen Unterschiede, die mit steigender Entropie 2 nachlässt - und zwar auch bei gleichbleibender Entropie 1. Schließlich kann auch Wissen in das Konzept der Entropie, nun ,Entropie 3', eingebettet werden, welche bei dessen Anstieg die Unterschiede zwischen möglichen Kontexten, in denen Bedeutungszuweisungen vorgenommen bzw. Symbole interpretiert werden, verringern lässt (und somit Mehrdeutigkeit bewirkt). Entropie 1 und 2 lassen sich nun nach Boisot und Canals der ersten Ebene der Kommunikation (Syntax) zuordnen, während Entropie 3 auf der semantisch-

25 Boisot \& Canals sprechen von "significant regularities residing in the data" [Boisot \& Canals 2004, S. 47]. 
pragmatischen Ebene anzusiedeln wäre, nachdem hinsichtlich der Semantik u.U. der Empfänger Symbole nicht kennt, also innen keine Bedeutung zuweisen kann, bzw. hinsichtlich der Pragmatik der Empfänger die gesamte Nachricht mitunter nicht in einen ,passenden' Kontext einbinden kann [Boisot \& Canals 2004].

Die Bemühungen, Kommunikationssysteme wie das Semantic Web nun an die höheren, semantisch-pragmatischen Ebenen auszurichten, bedingen konsequenterweise, dass Entropie - konkret Entropie 3 - verringert werden muss, um die Kontexte der Dateninterpretation voneinander klar(er) unterscheidbar zu machen. Im Sinne der Entwicklung kollektiver, kulturell objektivierter Erwartungshaltungen (d.h. kommunikativen Handelns [Habermas 1981]) bedarf es dazu zunächst einmal einer entsprechenden meta-kommunikativen Koordination über die Herstellung derartiger kognitiver Referenzsysteme, eine Vorstellung, die zu einem stufenweisen Prozess der Konventionalisierung bzw. Entropie-Reduktion führt. Durch die Amalgamierung von Kommunikation und Meta-Kommunikation treten dabei naturgemäß Interferenzen zwischen den Entropie-Ebenen auf, da etwa die definitorisch intendierten Begriffsunterscheidungen - Verabredungen über bestimmte Bedeutungszuweisungen zu Symbolen - materiell selbst wiederum für die kommunikative Praxis auf Unterschiede in den Codierungen (Symbolen) reduziert werden bzw. werden müssen.

Die wirtschaftlichen Effekte (Wohlstandseffekte) solcher ,semantisch angereicherter' gesellschaftlicher Kommunikationssysteme hängen in ihrer Realisierung also stark davon ab, inwieweit entsprechende Bedeutungskoordination durch die Etablierung, relevanter' lokaler Repräsentationen von (insbesondere auch spezialisiertem) Weltwissen bei den einzelnen Akteuren (vgl. Hayek) zur Koordinationsoptimierung gelingt, sodass - unbeschadet interpersoneller Präferenzen oder Repräsentationsvarianten - sich der Grad der effektiven Informationsverteilung verbessern lässt und, ganz spezifisch, in Richtung einer zunehmenden Disposition physischer (d.h. Zeit, Raum, Energie) durch informationelle (d.h. Daten und deren Analyse) Produktionsfaktoren wirkt. 
Teilnehmer von Transaktionssystemen, d.h. Systemen, deren Zweck die Abwicklung von (wirtschaftlichen) Transaktionen sind, können wesentlich von den Möglichkeiten der Bedeutungskoordination und Kontextualisierung bzw. allgemein einer effektiveren Informationsverteilung profitieren, nachdem diese Entwicklungen ressourcenschonend wirken, aber auch neue Potenziale eröffnen und folglich ökonomische Vorteile durch die Disposition von Produktionsfaktoren lukrierbar machen. Der Notwendigkeit, den Chancen und den grundlegenden Vorbedingungen zur Unterstützung von Transaktionssystemen bzw. dessen Teilnehmern widmet sich das nachfolgende Kapitel. 


\section{3 Überlegungen zu einem \\ Transaktionssystemunterstützungsmodell}

Für ein möglichst umfassendes Verständnis der unternehmerischen Verflechtungen in einem Transaktionssystem genügt die Analyse von nur oberflächlich wahrnehmbaren Austauschvorgängen zum einen bestimmten Zeitpunkt alleine nicht. Vielmehr müssen die Perspektiven der Akteure bzw. Unternehmen, die zu den Transaktionsbeziehungen geführt haben, miteinbezogen werden, und zwar hinsichtlich der eigenen Beweggründe (Zustandsräume), der Beweggründe beteiligter Akteure bzw. Unternehmen, sowie deren Wahrnehmung diesbezüglicher Perspektiven anderer. Mit steigender Anzahl an Akteuren bzw. Unternehmen und somit auch potenzieller Transaktionspartner gestaltet sich die Analyse der Transaktionsgeflechte zunehmend schwierig, was unmittelbar auf die Ermittlung optimaler Transaktionen und Partner innerhalb des Transaktionssystems rückwirkt. Der Unternehmer (Entrepreneur), dem in der wirtschaftswissenschaftlichen Sichtweise eine besondere Rolle bei der Optimierung der Konfiguration von Transaktionsbeziehungen zukommt, nachdem er erfolgreiche Transaktionen entdeckt und damit Neuheit innerhalb des Systems generiert, erfährt durch diese Umstände eine komplexere Aufgabenstellung und zugleich eine erhöhte Bedeutung. Die Erfüllung der unternehmerischen Rolle und damit die Bewahrung einer gewissen Übersicht wird allerdings durch die Komplexitätsschübe insbesondere für an Ressourcen ärmere Akteure neben deren eigentlichen Produktionsleistung problematisch, weshalb der Bedarf an Unterstützungsleistungen innerhalb des Transaktionssystems anwächst. Wie digitale Infrastrukturleistungen zur Verbesserung von Transaktionsverflechtungen eingesetzt werden können, um Transaktionsbeziehungen zu unterstützen bzw. zu optimieren, soll in dieser Arbeit ausgehend von diesem Kapitel beschrieben werden.

Zuvor sei allerdings noch die Unterscheidung und der Zusammenhang zwischen Wahrnehmung, Kommunikation und Transaktion näher erläutert. Diese drei 
Typen von Ereignissen können gepaart mit dem individuellen Wissen als die Bestimmungsfaktoren der Handlungen und Handlungskompetenzen von (wirtschaftlichen) Akteuren gesehen werden, wie bspw. Herrmann-Pillath [2002] hervorhebt. Die (Interpretation der) Wahrnehmung im Wechselspiel mit der Kommunikation und dem bestehenden Wissen der Akteure bilden dabei den Ausgangspunkt für die Realisierung von Transaktionen ${ }^{26}$ zwischen Akteuren und ermöglichen sozusagen eine ,Koordination des Wissens'. Wesentlich ist hierbei die - wenig spektakuläre, aber folgenreiche - Feststellung, dass die Felder der Wahrnehmung, die Netze der Kommunikation und letztlich die Konfigurationen der Transaktionsbeziehungen vielfach nicht kongruent sind. In Anbetracht (wirtschaftlicher) Austauschverhältnisse können vier grundsätzliche Konstellationen der (In-)Kongruenz unterschieden werden [Herrmann-Pillath 2002].

Abbildung 1, die den marktlichen Austausch bei vollständigem (relevanten) Wissen darstellt, beschreibt eine Situation der optimalen Übereinstimmung der handlungsbestimmenden Faktoren zwischen zwei Handelspartnern $U_{x}$ und $U_{y}$ in einem Transaktionssystem TS, welche - neoklassisch gesehen - einem Marktgleichgewicht gleichkommt und die Rolle des Wissens in den Hintergrund drängt. Abbildung 2 entspricht hingegen dem Bild der asymmetrischen Informationsverteilung der Institutionenökonomik, die zu einer einseitig unbefriedigenden Transaktion führt, nachdem ein prinzipielles Vertrauensproblem aufgrund der Asymmetrie (letztlich eine Asymmetrie des relevanten Wissens) vorherrscht. Oftmals können solche Situationen auch als Gleichgewicht (Abbildung 1) erscheinen. Abbildung 3 zeigt eine weitere wichtige Konstellation von Wahrnehmung, Kommunikation und Transaktion, die in der ökonomischen Begriffsbildung als ,externer Effekt' bekannt ist und sich über die unbeabsichtigten Auswirkungen eines Akteurs zu Gunsten oder Ungunsten eines anderen manifestiert. Ein weiterer weit verbreiteter Typus wird in Abbildung 4 dargestellt. Es handelt sich

26 Der Begriff ,Konfiguration' wird in diesem Zusammenhang für grundlegende Transaktionsmuster eingesetzt. 
hierbei um den Austausch über die Vermittlung eines Dritten (der auch außerhalb des Transaktionssystems stehen kann), der die Wahrnehmung der Transaktionspartner erweitert und oftmals auch benötigt wird, um Vertrauen herzustellen.

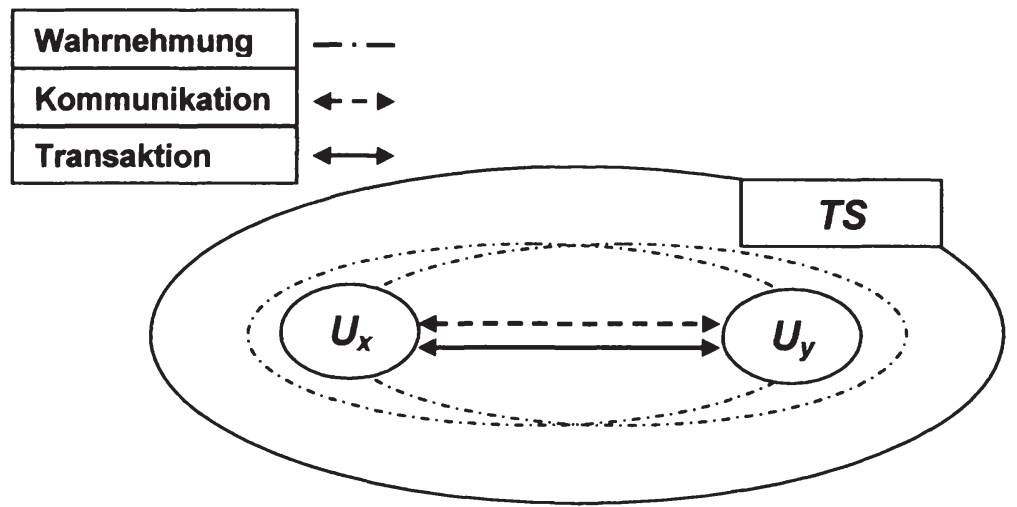

Abbildung 1: Marktaustausch bei vollständigem (relevanten) Wissen Quelle: modifizierte Darstellung basierend auf Hermann-Pillath [2002, S. 72]

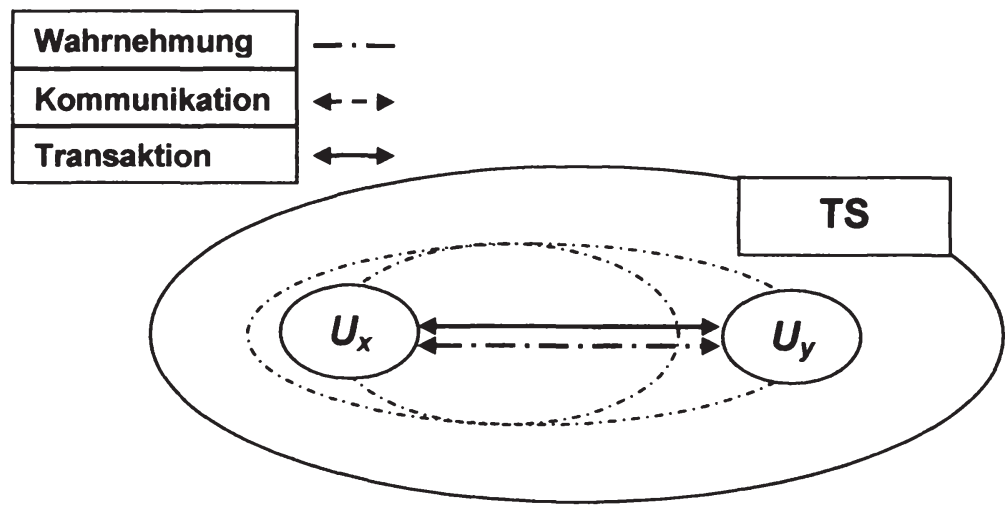

Abbildung 2: Marktaustausch bei asymmetrischem Wissen Quelle: modifizierte Darstellung basierend auf Hermann-Pillath [2002, S. 72] 


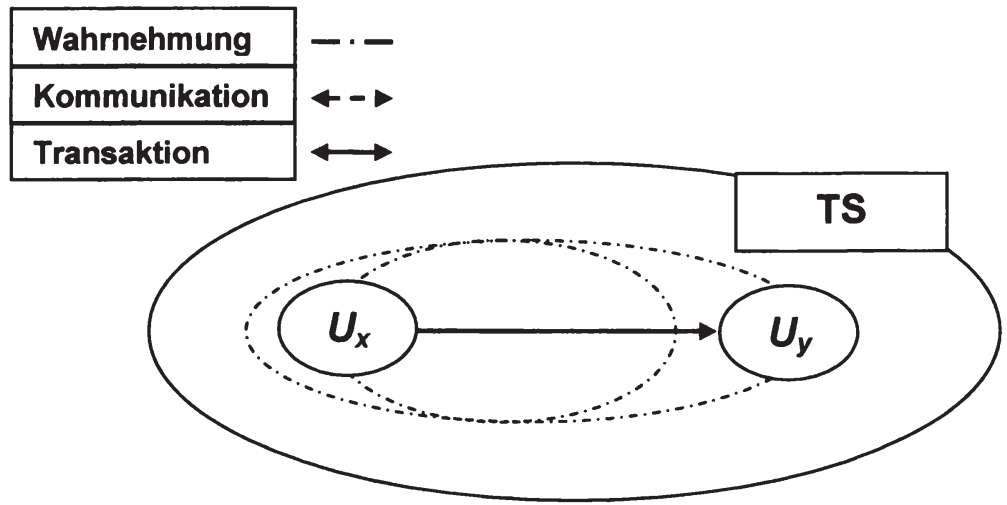

Abbildung 3: Unbeabsichtigte externe Effekte

Quelle: modifizierte Darstellung basierend auf Hermann-Pillath [2002, S. 72]

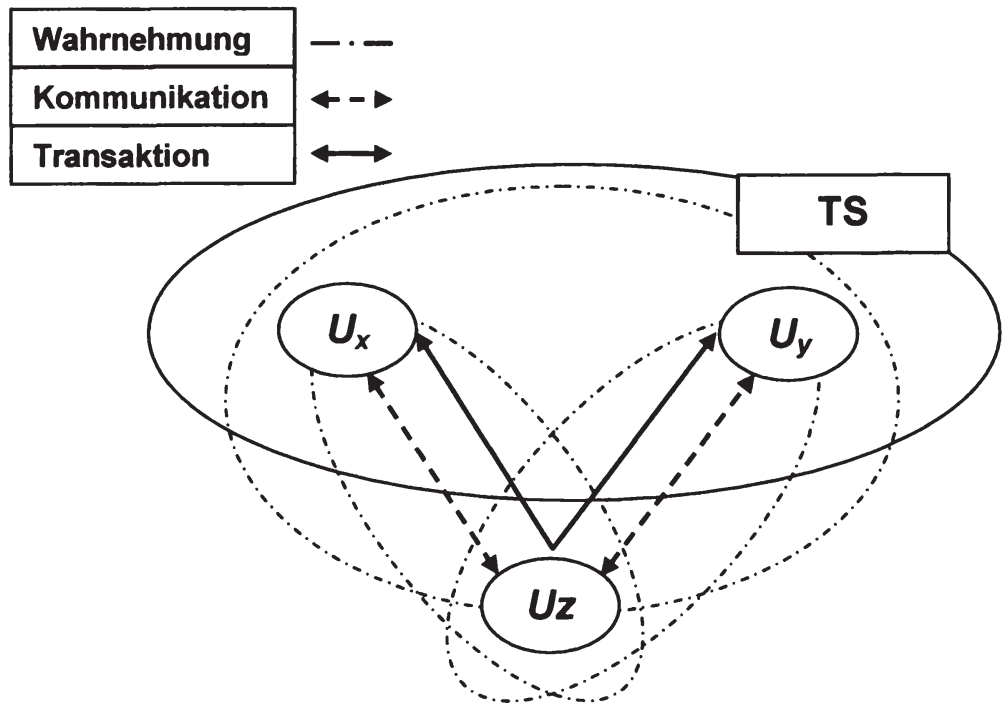

Abbildung 4: Tausch über Vermittler

Quelle: modifizierte Darstellung basierend auf Hermann-Pillath [2002, S. 72]

Es zeigt sich, dass Konfigurationen von Transaktionsgefügen sehr unterschiedlich ausfallen können und stark von der gegenseitigen Wahrnehmung der Beteiligten sowie der Kommunikationsverbindungenvabhängigosind, açer @uch von 
der Verteilung von Informationen und letztlich von Wissensbeständen unter den Akteuren wesentlich mitbestimmt werden. Dementsprechend überrascht es wenig, dass in der Praxis die Konstellationen der Bestimmungsfaktoren für das Setzen von Handlungen selten so übereinstimmen, dass die möglichen Transaktionen auch zur Gänze umgesetzt werden [Herrmann-Pillath 2002]. Das Potenzial wird folglich nicht ausgeschöpft, weil Wahrnehmungsfelder und Kommunikationsbeziehungen lückenhaft sind und letztlich Transaktionen nicht stattfinden können. In der neoklassischen Perspektive wird zur Umgehung dieser alltäglichen Problematik die Annahme vollständiger Information eingesetzt, eine Spielart dieser Annahme ist bspw. Walras' Auktionär, der erst nach Verarbeitung sämtlicher relevanten Informationen am Markt Entscheidungen trifft bzw. Transaktionen durchführt.

Gemeinhin können Strukturen, in denen alle möglichen Transaktionen auch realisiert werden, als, integrale Strukturen' bezeichnet werden. Vertrauen, d.h. die Antizipation (,der Glaube an') eine/r angestrebte/n Zustandsänderung, ist in solchen Strukturen bei jeder Transaktion gerechtfertigt. Vertrauen verliert dadurch den Charakter des auf wahres oder falsches Wissen gestützten Glaubens an bestimmte Zustände und wird damit als Begriff unbedeutend, nachdem das Glaubenscharakteristikum wegfällt. Ex ante Erwartungen entsprechen vollständig den ex post Realisierungen aller an der Transaktion beteiligten Aktoren, ein Gleichgewicht wird erreicht. Im Gegensatz treten ,nicht-integrale Strukturen' aufgrund der Problematik von nicht-kongruenten Wahrnehmungsfeldern, Kommunikationsverflechtungen und Transaktionskonfigurationen auf. Vertrauen kann, muss aber nicht gerechtfertigt sein. Gleichgewichte sind denkbar, ihre Wahrscheinlichkeit nimmt aber mit der Inkongruenz von Wahrnehmung, Kommunikation und Transaktion in einem System ab, weshalb sie in der Regel nicht auftreten.

Zentral für jedes nicht-integral strukturierte System mit dem Erfolgskriterium der Realisierung von Transaktionen zwischen Beteiligten ist das Schließen von Wahrnehmungs-, Kommunikations- und letztendlich Transaktionslücken, die sich 
allerdings dynamisch bilden und verändern. Diese Dynamik von nicht-integralen Strukturen erklärt sich dabei - gemäß einer evolutionsökonomischen Sichtweise (siehe Kapitel 2.3) - über die Informationsstände sowie in weiterer Folge dem Wissen der beteiligten Akteure, d.h. über Einflussfaktoren, die selbst der Fluktuation unterliegen, u.a. weil die Akteure in unterschiedlichen Austauschbeziehungen stehen. Nicht zuletzt aufgrund dessen werden Akteure in der Evolutionsökonomik als singulär angesehen. Die ,Singularität' ${ }^{27}$ (gewissermaßen die Einzigartigkeit bzw. implizit die Unterschiedlichkeit), der Akteure ergibt sich dabei - sozio-ökonomisch betrachtet - aus der Interpretation von Information durch einen Akteur relativ zu dessen bestehendem Wissen (siehe Kapitel 2, insbesondere 2.4). Die Entwicklung der Konfiguration der Verflechtungen zwischen den Akteuren (oder aber zwischen selbst handlungsfähigen und/oder wissensspeichernden Gefügen von Akteuren, so genannten Elementen) - bspw. innerhalb eines Transaktionssystems - wird entsprechend von der Entwicklung des Wissens der Akteure (Elemente) über die Verflechtungsmöglichkeiten des Systems entscheidend mitbestimmt.

Im Falle einer nicht-integralen Struktur von Systemen und somit auch der nichtintegralen Verflechtungen der Akteure (Elemente) innerhalb dieser Systeme gilt aufgrund der Singularität der Akteure (sowie der Elemente) im System, dass die Verflechtungen ebenfalls singulär sind. In weiterer Folge kann auch die Dynamik in den nicht-integralen Strukturen und somit die Entwicklung der Konfiguration der Verflechtungen als singulär verstanden werden [Herrmann-Pillath 2002]. Diese evolutionsökonomische Perspektive unterstreicht die Historizität von ex post Betrachtungen und - bei gleichzeitiger Beachtung der Ausführungen aus Kapitel 2.2 - die allgemein anerkannten Probleme und Unabwägbarkeiten von Prognosen, insbesondere bei singulären Phänomenen.

27 Singularität ist eine Grundannahme von Prozessen der Variation, Selektion und Bewahrung in der Evolutionsökonomik und bezeichnet die Eigenschaft eines Phänomens nicht (genauer: nie) vollständig anderen Phänomenen zu entsprechen [Hermann-Pillath 2002]. 
Für Transaktionssysteme - also Systeme, deren Zweck die Förderung und Ermöglichung der wechselseitigen Aktionen zwischen Akteuren (Elementen) ist stellt sich die Frage, inwieweit transaktionsrelevante und -fördernde Informationen generiert und so verteilt werden können, dass die Lücken der Transaktionsgeflechte, die in Folge der nicht-integralen Struktur auftreten, identifiziert und geschlossen werden. Transaktionsrelevante Informationen umfassen dabei Informationen, die die Wahrnehmungsfelder, Kommunikationsabläufe und letztlich die realisierten Transaktionen von Akteuren (Elementen) im System betreffen. Institutionen bzw. Infrastrukturleistungen sollten hierbei typischerweise das Schließen der Lücken unterstützen.

\subsection{Input-Output-Tabellen als produktionsbezogene Transaktionsdarstellung}

Prinzipiell ist das Wissen über die Transaktionsbeziehungen singuläres Wissen der an der Transaktion beteiligten Akteure (Elemente). Dieses Wissen ist - wie Wissen im Allgemeinen - nur teilweise explizierbar, wobei die Extraktion des explizierbaren Teils nur bedingt (freiwillig) erfolgt [Wilson 2002; Polanyi 1958].

Trotz oder gerade aufgrund dieser Tatsache beschäftigt die Suche nach Näherungslösungen zu Transaktionsbeziehungen wirtschaftlicher Akteure, wenn auch auf einem weit höher aggregierten Niveau, die Wissenschaft spätestens seit der Erstellung des ,Tableau économique' durch den Physiokraten und Arzt Fransois Quesnay im Jahr 1758 [Screpanti \& Zamagni 1995]. Quesnay veranschaulichte darin den damals - zumindest am französischen Hof - wahrgenommenen wirtschaftlichen Kreislauf zwischen gesellschaftlichen Klassen ${ }^{28}$, und brachte damit die Interpretation der Wirtschaft als Kreislauf zu einem - wenn

28 Genauer gesagt zwischen der ,Classe Productive', der ,Classe Stérile' und der ,Classe des Propriétaires', also der produktiven Klasse (v.a. Landwirte), der sterilen Klasse (Händler und handwerklich Tătige) und der besitzenden Klasse (Grundeigentum, Adel und Klerus). Die Klasse der Besitzlosen wird von Quesnay erwähnt, aber nicht in den Kreislauf inkludiert, was u.a. mit der Zuweisung einer rein konsumptiven Rolle erklärt werden könnte [Holub \& Schnabl| 1985] leber - 978-3-631-75376-7 
auch verzögert einsetzenden - Durchbruch. Das Kreislaufmodell wurde später u.a. von politischen Ökonomen wie Karl Marx oder Léon Walras gewürdigt. Hierbei konnte insbesondere Léon Walras auf der Suche nach einem allgemeinen Gleichgewichtsmodell auf der Idee Quesnays aufbauen und über die Berücksichtigung der Interdependenzen wirtschaftlicher Vorgänge in seinen linearen Gleichungen auch einen wesentlichen Beitrag zu deren Weiterentwicklung in Richtung einer Input-Output-Rechnung leisten. Die Problematik der unzureichenden empirischen Datenbasis konnte in jener frühen Entwicklungsphase allerdings nicht annähernd befriedigend gelöst werden, weshalb viele theoretische Überlegungen zu dem Einbezug von wirtschaftlichen Austauschbeziehungen in ökonomische Berechnungen noch mehrere Jahrzehnte auf eine Umsetzung warten mussten (und teilweise noch immer müssen). Erst staatliche Planungsprobleme, speziell in Planwirtschaften wie bspw. der UdSSR, führten ab den 1920er Jahren zu umfangreicheren Datenerhebungen zur Identifikation von wirtschaftlichen Zusammenhängen [Holub \& Schnabl 1985]. Die Initialzündung erfuhr die Input-Output-Rechnung schließlich aber erst durch die Ausführungen von Wassily Leontief zu den Input-Output-Beziehungen des Wirtschaftssystems der USA [Leontief 1936; 1941], in denen er zeigte, wie man neben den direkten wirtschaftlichen Zusammenhängen zwischen den wirtschaftlichen Akteuren auch die indirekten und damit nicht vordergründig sichtbaren, aber tatsächlich ebenso wirkenden Zusammenhänge aufzeigen bzw. errechnen kann. Leontief bezog sich dabei auf Quesnays ,Tableau économique' ebenso wie auf Walras' Modell, allerdings weniger unter Betonung dessen Aspekts des allgemeinen Gleichgewichts, sondern vielmehr der allgemeinen Interdependenz [Leontief 1941].

Ausgehend von Leontiefs Ansatz entwickelte sich die Input-Output-Rechnung in ihre heute gültige Form, die im Kern Fragestellungen rund um die sich in wirtschaftlichen Transaktionen manifestierende Struktur einer Volkswirtschaft zu beantworten versucht. Dabei stehen die für die Produktion erforderlichen Inputund Output-Ströme im Zentrum der Betrachtung (,Vorleistungen' oder auch ,intermediärer Verbrauch') und werden von den Endnachfrage- und Wertschöpfungs- 
komponenten komplettiert, um letztlich einen Gesamteindruck über die wirtschaftliche Struktur zu vermitteln.

\subsection{Zum Aufbau der Input-Output-Rechnung}

Grundsätzlich lässt sich die Input-Output-Rechnung (IOR) zur Beantwortung struktureller Fragestellungen in zwei Bereiche gliedern:

1. in den Bereich der Input-Output-Tabellen (IOT), der die ex post erfolgende Generierung der Tabellen und die damit zusammenhängende deskriptive Datenauswertung umfasst und

2. in den Bereich der Input-Output-Analyse (IOA), der die auf den Tabellen begründeten Analyseschritte und Modellrechnungen beinhaltet.

Diese beiden Bereiche sind über eine Rückkoppelungsschleife miteinander verbunden, die aufgrund divergierender Zwecke bzw. Fragestellungen der InputOutput-Analyse auch zu Zielkonflikten bei der Erstellung der Input-OutputTabellen führen kann. Das vermehrte Aufkommen weiterer Anwendungsfelder der an die Input-Output-Rechnung angelehnten Strukturanalyse hat nicht zuletzt aus dieser Problematik heraus mehrere Satellitensysteme, wie bspw. im Umweltbereich, im Energiebereich oder (ansatzweise) auch im Tourismus, hervorgebracht. Unabhängig von diversen Satellitensystemen und weiteren Anwendungen löste die Vielfalt an unterschiedlich ausgerichteten nationalen InputOutput-Rechnungen Probleme bei internationalen Vergleichen aus, die über das ,System of National Accounts' [United Nations 1993; 2004] der Vereinten Nationen für - im Kern - international vergleichbarere Messungen und Darstellungen der Input-Output-Beziehungen gelindert werden sollen. Auf Basis des ,System of National Accounts' der Vereinten Nationen [United Nations 1993] ist mit der Einführung des Europäischen Systems der Volkswirtschaftlichen Gesamtrechnung (ESVG95) [Eurostat 1995] die Input-Output-Rechnung in die volkswirtschaftlichen Berechnungen der nationalen Statistikämter der EU integriert worden, was ihren Status unterstreicht. Durch die Harmonisierung mit der Volkswirtschaftlichen Gesamtrechnung (VGR) kann die/Input-Qytput-Tabelle, in der EU 
seitdem als weiterführende und mit der VGR konsistente Quelle für Informationen gesehen werden, die bei der Erstellung der volkswirtschaftlichen Produktionskonten der VGR in Folge der Datenaggregation üblicherweise verloren gehen. Zur Veranschaulichung der Verbindung der Input-Output-Tabelle zu den Konten der VGR können die schematischen Darstellungen aus Tabelle 1 und Tabelle 2 herangezogen werden.

Tabelle 1: Schematisches Produktionskonto

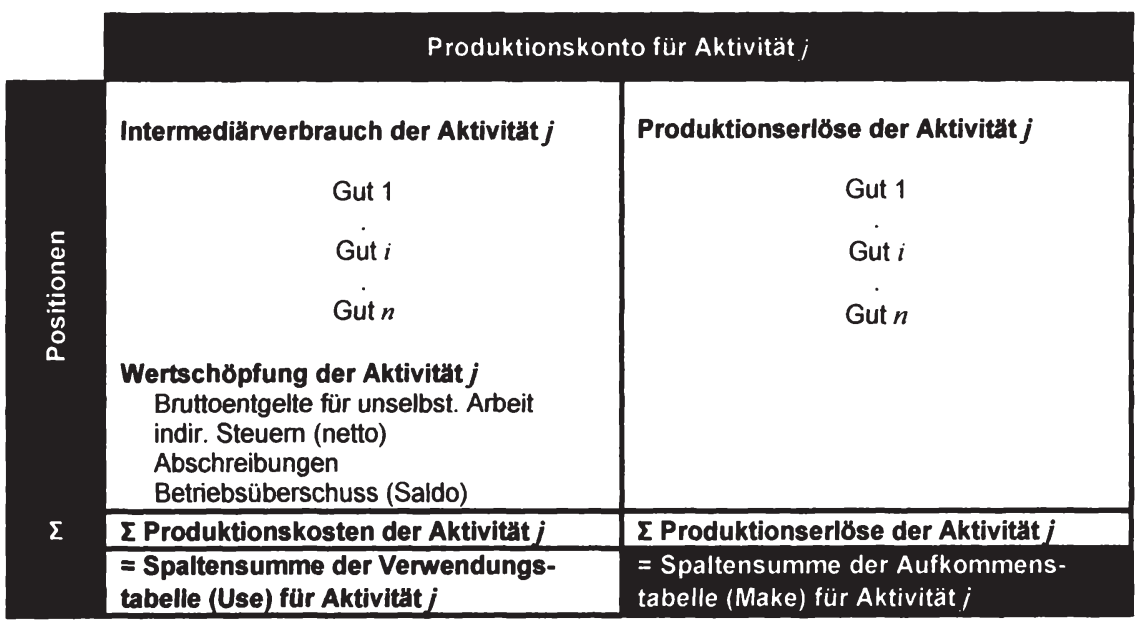

Tabelle 2: Schematisches Güterkonto

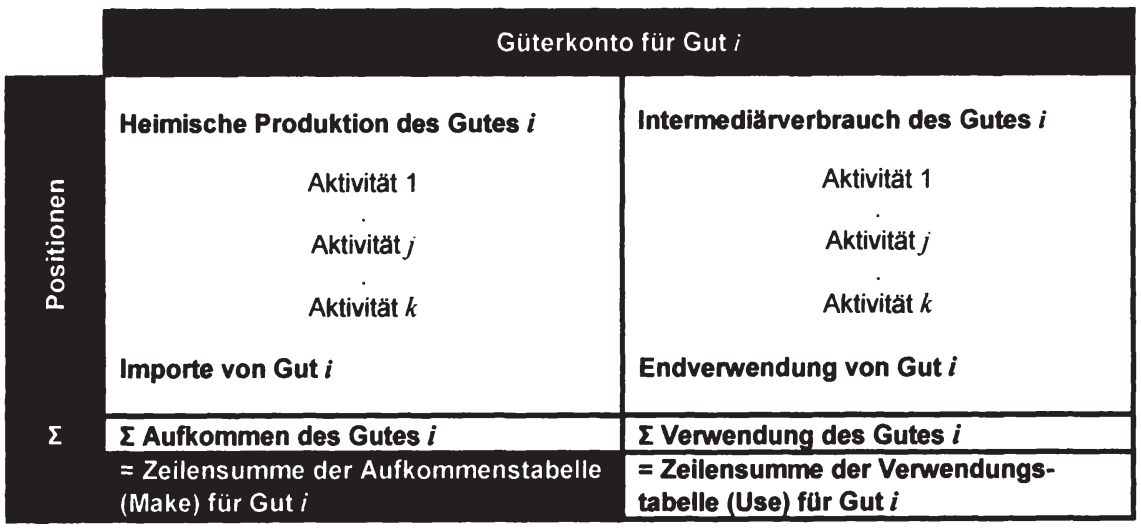


,Güter' und ,Aktivitäten' sind zwei Grundbegriffe der wirtschaftsstatistischen Systematik und zentral bei der Erfassung der wirtschaftlichen Verflechtungen ${ }^{29}$. ,Güter' beinhaltet in unterschiedlicher Tiefe spezifizierbare Gütergruppen, die auf Basis der jeweils gültigen Version der $\mathrm{CPA}^{30}$ (bzw. deren nationalen Variante wie z.B. OCPA [Statistik Austria 2004]) definiert sind. Der Begriff ,Aktivitäten' beschreibt hingegen wirtschaftliche Tätigkeitsbereiche. Ihre Definitionen entstammen der jeweiligen Version der NACE ${ }^{31}$ (bzw. deren nationalen Spielart wie die ONACE [Statistik Austria 2003]) und liegen für verschiedene Aggregationsniveaus vor.

Das Produktionskonto (Tabelle 1) einer solchen Aktivität (z.B. Aktivität $j$ aus der Menge der Aktivitäten 1:k) hält im Soll sowohl den Intermediärverbrauch, d.h. die Vorleistungen, als auch die Wertschöpfungskomponenten der Aktivität, die in die Produktion einfließen, fest, während im Haben die Erlöse aus der Güterproduktion der Aktivität erfasst werden. Demgegenüber gibt das Güterkonto (Tabelle 2) auf der Soll-Seite Auskunft über die Aktivitäten, durch die ein Gut (z.B. Gut $i$ aus der Menge von Gütern 1:n) im Inland hergestellt wird, dessen Importanteil sowie - auf der Haben-Seite - über die Aktivitäten, die das Gut als Vorleistung einsetzen, ebenso wie die Endverwendungszwecke des Gutes. Das Produktions- und das Güterkonto enthalten gemeinsam mit dem Einkommensentstehungskonto jene wirtschaftlichen Ströme, die auch den Tabellen zu dem Aufkommen und der Verwendung wirtschaftlicher Leistungen, den Ausgangspunkten zur Generierung der Input-Output-Tabelle, zugrunde liegen. Auf-

29 Erwăhnenswert in diesem Zusammenhang ist, dass die Eignung der wirtschaftstatistischen Systematik für die Zwecke der Input-Output-Analyse nicht grundsätzlich gegeben ist, weshalb mitunter Adaptionen der gewählten Klassifikation für die InputOutput-Analyse vorzunehmen sind.

30 CPA steht für ,Classification of products by activities in the European Economic Community', und beschreibt die statistische Güterklassifikation in Verbindung mit den wirtschaftlichen Tätigkeitsbereichen in der Europäischen Gemeinschaft.

31 NACE ist das Akronym für ,Nomenclature générale des activités économiques dans les communautés européennes' und liefert dementsprechend die statistische Systematik der wirtschaftlichen Tätigkeitsbereiche in der Europăischen Gemeinschaft. 
kommenstabellen (,Make-' oder ,Supply'-Matrizen) zeigen dabei die Produktionswerte der unterschiedlichen Aktivitäten aufgeteilt auf die Güter, die diese Werte generieren (dies entspricht der heimischen Produktion der jeweiligen Güter), und halten auch die jeweiligen Güterimporte fest. Verwendungstabellen (,Use'Matrizen) dokumentieren den Intermediärverbrauch der Aktivitäten bezogen auf die Güter, die Wertschöpfungskomponenten, die in die Produktion der jeweiligen Aktivitäten fließen, und zeigen darüber hinaus die Verteilung der Güter auf die Endverwendungskategorien.

Tabelle 3 und Tabelle 4 illustrieren die Verbindung zwischen der Soll- bzw. Habenseite von Produktions- und Güterkonto einerseits und der Aufkommenssowie der Verwendungstabelle andererseits. Es wird nun von der Darstellung in Kontenform abgegangen.

Tabelle 3: Auszug aus der Aufkommenstabelle (Make/Supply) für Gut $i$ und Aktivität $j$

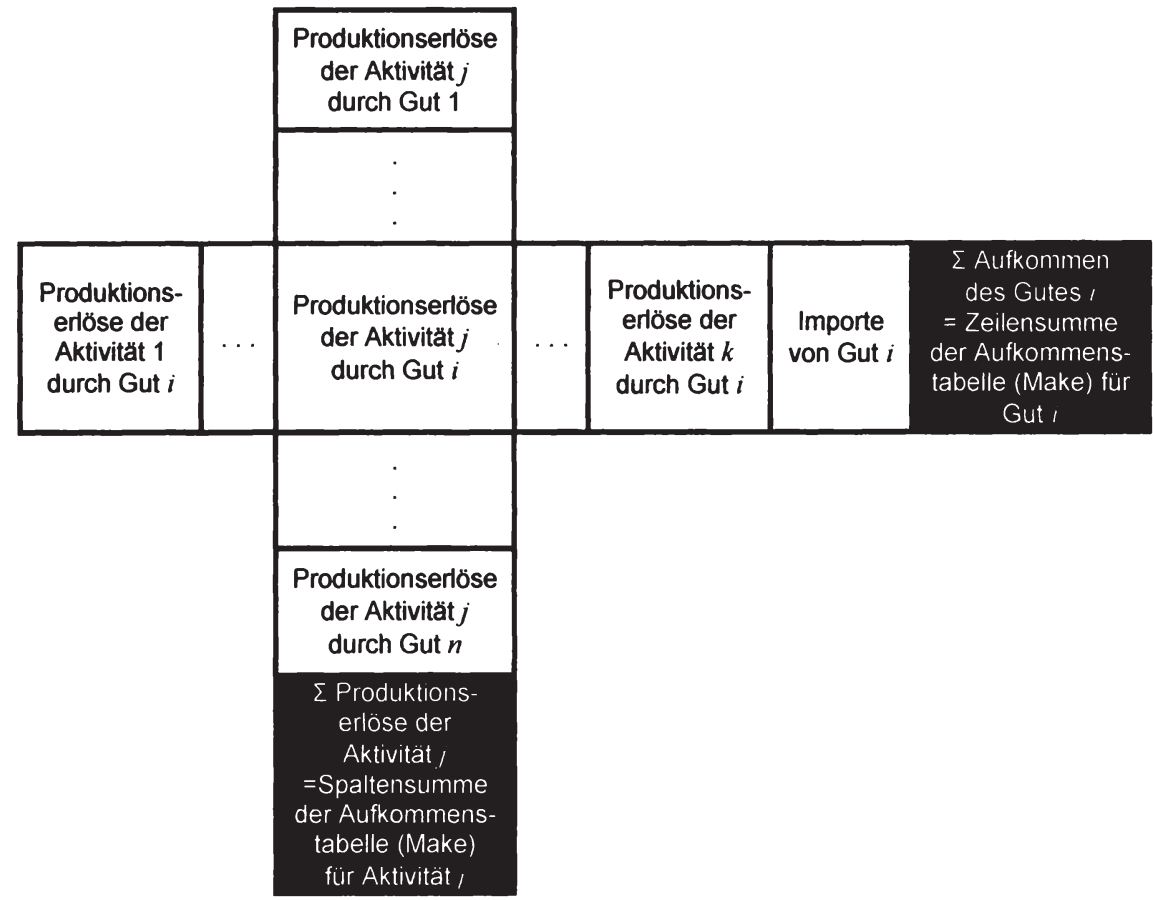


Tabelle 4: Auszug aus der Verwendungstabelle (Use) für Gut $i$ und Aktivität $j$

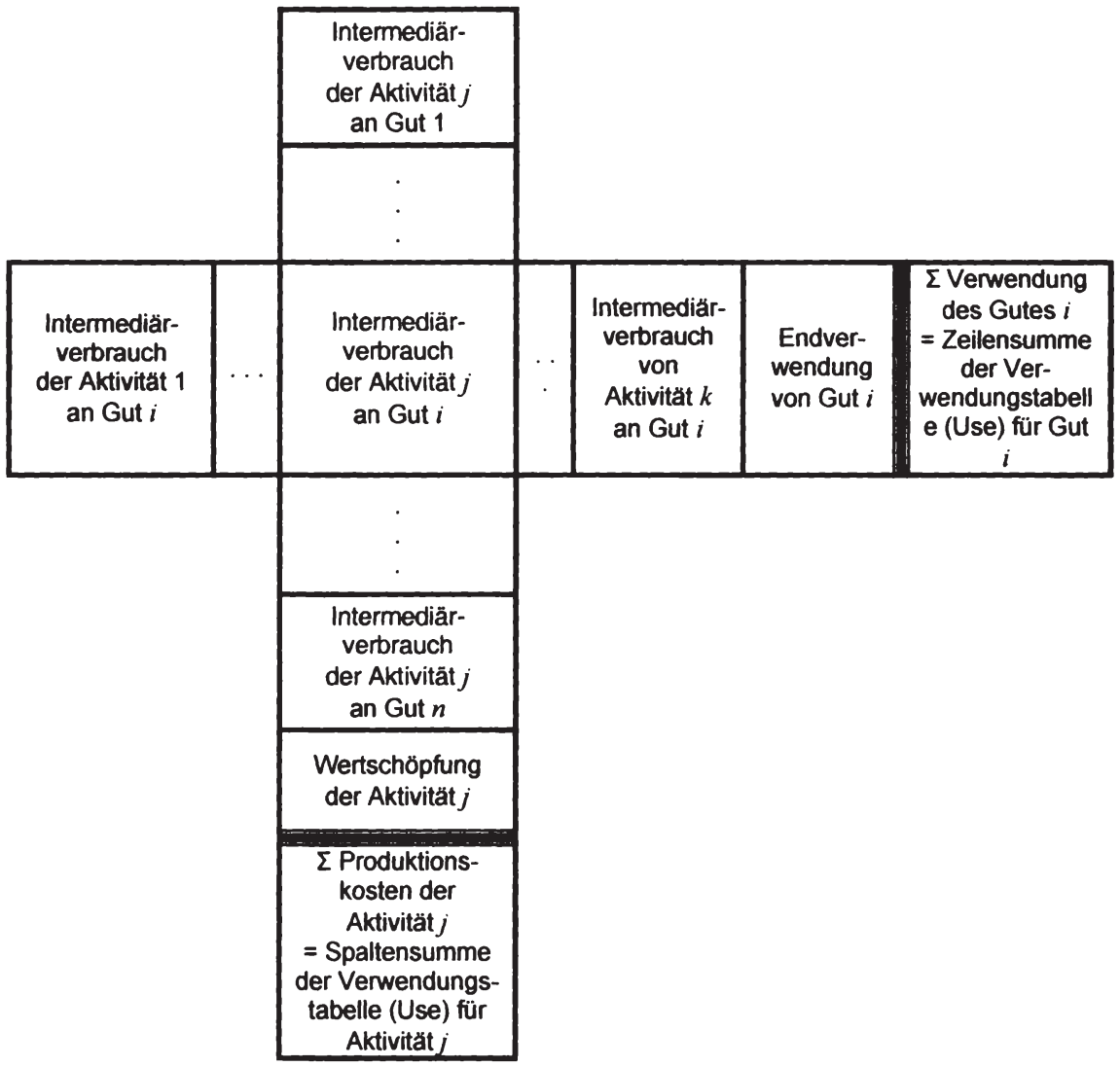

Summiert man über alle Güter und Aktivitäten sowie über alle Wertschöpfungskomponenten und die Endverwendungskategorien, so kann man die Struktur der Aufkommens- und Verwendungstabelle wie in Tabelle 5 und Tabelle 6 skizzieren. Die Grundstruktur der in diesen beiden Tabellen gezeigten Aufkommens- und Verwendungstabellen basiert dabei auf einer Verbuchung der heimischen Güter und deren Importe in einem gemeinsamen Güterkonto (dieser Vorgang generiert die so genannte ,Version $A(2)$ ' der Aufkommens- und Verwendungstabellen). Verteilt man hingegen heimische und importierte Güter auf jeweils getrennte Güterkonten, so gelangt man zu ,Version B' der Aufkommens- und Verwendungstabellen. 
Tabelle 5: Struktur der Aufkommenstabelle

Quelle: Statistik Austria [2005, S. 13]

\begin{tabular}{|c|c|c|c|}
\hline & Aktivitäten & Importe & Gesamtaufkommen \\
\hline Güter & $\begin{array}{l}\text { Produktionswerte nach } \\
\text { Aktivitäten und Gütern }\end{array}$ & Importe nach Gütern & $\begin{array}{l}\text { Gesamtaufkommen } \\
\text { nach Gütern }\end{array}$ \\
\hline Summe & $\begin{array}{c}\text { Produktionswerte nach } \\
\text { Aktivitäten }\end{array}$ & Importe Gesamt & Gesamtaufkommen \\
\hline
\end{tabular}

Tabelle 6: Struktur der Verwendungstabelle

Quelle: Statistik Austria [2005, S. 14]

Aktivitäten
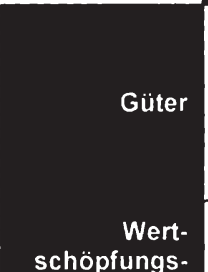

komponenten

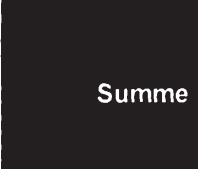

Produktionswerte nach Aktivităten

Wertschöpfung nach Aktivitäten
Endnachfrage

Gesamtverwendung

Man kann nun die beschriebenen Aufkommens- und Verwendungstabellen so verknüpfen, dass ein konsistentes System wirtschaftlicher Zusammenhänge entsteht. Tabelle 7 zeigt das ,Make- und Use-System'32 in Version B. Diese Darstellung unterscheidet sich von Version $A$ durch eine zusätzliche Zeile und Spalte ,importierte Güter', welche die Use-Matrix ebenso wie die Endnachfragematrix zerlegen.

\begin{tabular}{|c|c|c|}
\hline Konsum- & Brutto- & Exporte \\
ausgaben & investitionen & nach \\
nach & nach Gütern & Gütern \\
Gütern & & \\
\hline & & \\
\hline
\end{tabular}

Gesamtverwendung nach Gütern

32 Ausgehend von den Vorschlägen der Vereinten Nationen zu dem ,System of National Accounts' aus dem Jahr 1968 sowie (konkreter) 1973 [Holub \& Schnabl 1985]. 
Tabelle 7: Das Make- und Use-System (Version B)

Quelle: Holub \& Schnabl [1994, S. 56]

\begin{tabular}{|c|c|c|c|c|c|}
\hline & heim. Güter & imp. Güter & Aktivitäten & Endnachfrage & Summe \\
\hline $\begin{array}{l}\text { heim. } \\
\text { Güter }\end{array}$ & & & $\begin{array}{l}\text { Use-Matrix } \\
\text { heim. Güter } \\
U^{d}\end{array}$ & $\begin{array}{c}\text { Endnachfrage- } \\
\text { matrix } \\
\text { heim. Güter } \\
Y^{d t}\end{array}$ & $\begin{array}{l}\text { heim. Guter- } \\
\text { verwendung } \\
q^{d}\end{array}$ \\
\hline imp. Güter & & & $\begin{array}{l}\text { Use-Matrix } \\
\text { imp. Güter } \\
\qquad U^{m}\end{array}$ & $\begin{array}{c}\text { Endnachfrage- } \\
\text { matrix } \\
\text { imp. Güter } \\
Y^{m}\end{array}$ & $\begin{array}{c}\text { imp. Güter- } \\
\text { verwendung } \\
q^{m}\end{array}$ \\
\hline Aktivitäten & $\begin{array}{c}\text { Make-Matrix } \\
V\end{array}$ & & & & $\begin{array}{c}\text { Produktions- } \\
\text { erlöse } \\
:\end{array}$ \\
\hline Importe & & $\begin{array}{c}\text { Importe } \\
m^{\prime}\end{array}$ & & & \\
\hline $\begin{array}{r}\text { Wert- } \\
\text { schöpfung }\end{array}$ & & & $\begin{array}{l}\text { Wert- } \\
\text { schöpfung } \\
W\end{array}$ & & Primärinput \\
\hline Summe & $\begin{array}{c}\text { heim. Güter- } \\
\text { aufkommen } \\
q^{d^{\prime}}\end{array}$ & $\begin{array}{c}\text { imp. Güter- } \\
\text { aufkommen } \\
q^{m,}\end{array}$ & $\begin{array}{c}\text { Produktions } \\
\text { kosten } \\
\end{array}$ & Endnachfrage & \\
\hline
\end{tabular}

Das Make- und Use-System besteht gemäß Tabelle 7 aus folgenden Matrizen:

- der Make-Matrix $V$ in der Dimension Aktivitäten $\mathrm{x}$ heimische Güter,

- dem Importvektor $m^{\prime}$ in der Dimension Importe x importierte Güter,

- der Wertschöpfungsmatrix $W$ in der Dimension Wertschöpfung(-skomponenten) $\times$ Aktivitäten,

- der Use-Matrix für heimische Güter $U^{d}$ in der Dimension heimische Güter x Aktivitäten,

- der Use-Matrix für importierte Güter $U^{m}$ in der Dimension importierte Güter x Aktivitäten,

- der Matrix der Endnachfrage für heimische Güter $Y^{d}$ in der Dimension heimische Güter $x$ Endnachfragekategorien und

- der Matrix der Endnachfrage für importierte Güter $Y^{m}$ in der Dimension importierte Güter x Endnachfragekategorien ael Weber - 978-3-631-75376-7 
Die formalisierten Zusammenhänge und Summenbedingungen der Aufkommensund Verwendungstabellen bzw. des Make- und Use-Systems, welches die Basis für die weiteren Ausführungen bildet, sind in Tabelle 8 dargestellt.

Tabelle 8: Das Make- und Use-System (Version B) mit Formeln Quelle: erweiterte Darstellung aufbauend auf Holub \& Schnabl [1994, S. 56]

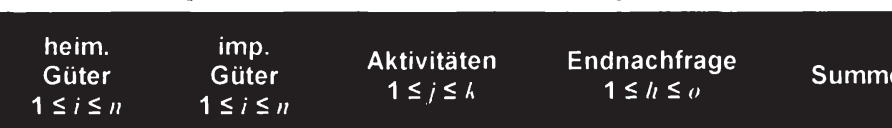

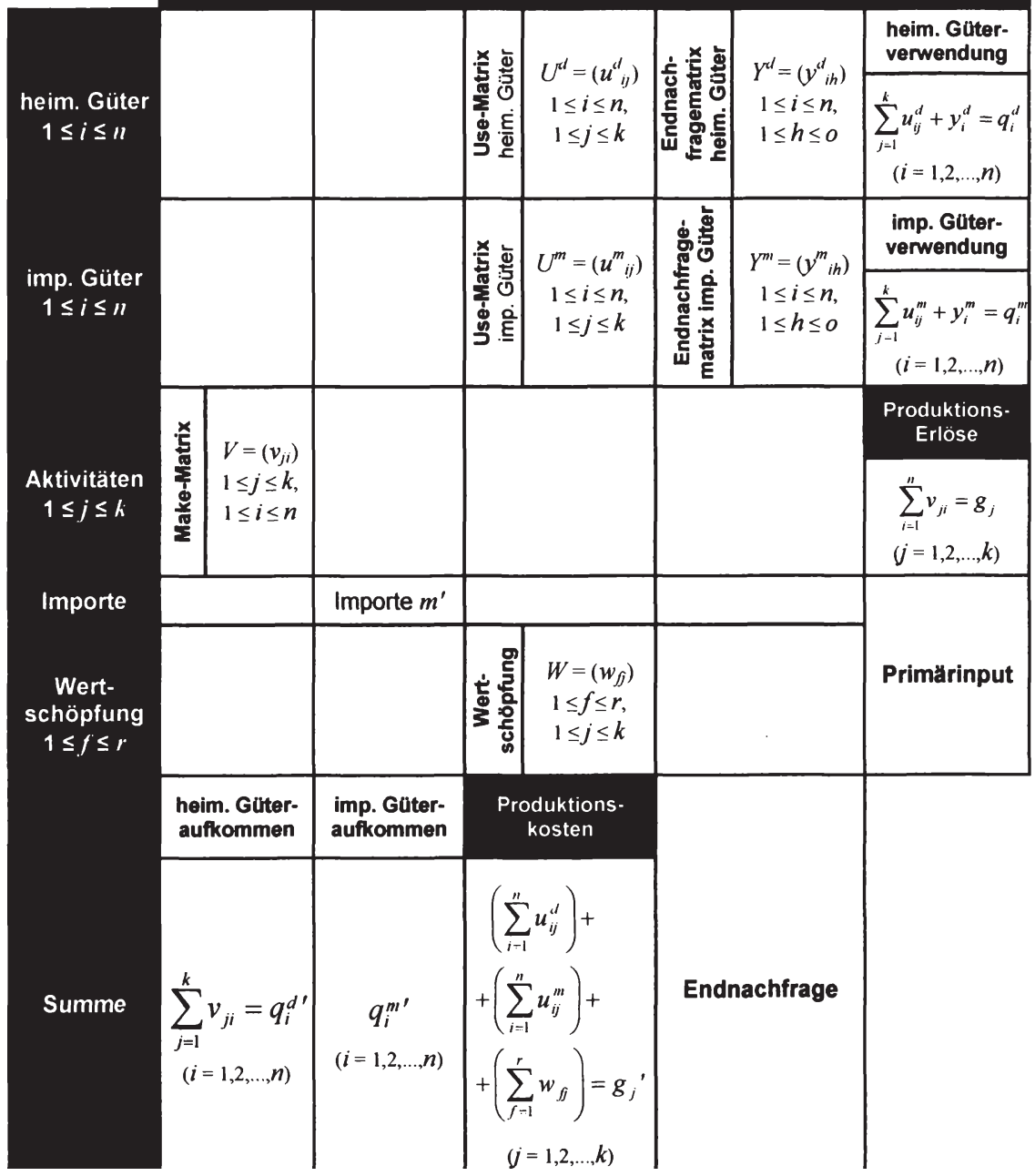


Die in der Formelnotation in Tabelle 8 eingesetzten Indizes sind für:

- Güter: $1, \ldots, i, \ldots, n$,

- Aktivitäten: $1, \ldots, j, \ldots, k$,

- Wertschöpfungskomponenten: $1, \ldots, f, \ldots, r$ und

- Endnachfragekategorien: $1, \ldots, h, \ldots, o$.

Wichtige Summenbedingungen, die in Tabelle 8 hervorgehoben sind, lauten:

$q^{d}$ : heimisches Güteraufkommen (Make) = heimische Güterverwendung (Use)

(Vektor der Spaltensummen der Make- bzw. der Zeilensummen der Use-

Matrix bezogen auf heimische Güter)

$q^{m}$ : importiertes Güteraufkommen (Make) = importierte Güterverwendung (Use) (Vektor der Spaltensummen der Make- bzw. Zeilensummen der Use-Matrix bezogen auf importierte Güter)

g. Produktionserlöse $($ Make) $=$ Produktionskosten (Use); ,Produktionswerte' (Vektor der Zeilensummen der Make- bzw. der Spaltensummender UseMatrix)

\subsubsection{Zuordnungsprinzipien und Aufstellungsprobleme}

In der Praxis ist die Erstellung der Aufkommens- und Verwendungstabellen und der (,symmetrischen') Input-Output-Tabellen mit einer Reihe von konzeptuellen Problemen konfrontiert, die viele theoretische Forderungen an Aufkommens- und Verwendungstabellen bzw. die Make- und Use-Systematik relativieren. Eine zentrale Fragestellung ergibt sich bspw. im Hinblick auf den Einsatz bestimmter Zuordnungsprinzipien, d.h. Prinzipien, die die Zuordnung der dargestellten Einheiten zu den Güter bzw. Gütergruppen sowie den Aktivitäten regeln, vor dem Hintergrund des jeweiligen Verwendungszwecks. Hier unterscheidet man eine Zuordnung nach dem ,institutionellen' und dem ,funktionellen' Prinzip, wobei ersteres sozusagen als Vorstufe des letzteren verstanden werden kann. 
Das ,institutionelle Prinzip' konzentriert sich auf die institutionelle Darstellungseinheit von sogenannten ,örtlichen fachlichen Einheiten' (engl. ,local kind-ofactivity units'), welche entsprechend ihrer jeweiligen Haupttätigkeit mit Bezug auf eine bestimmte Gliederungsebene innerhalb der passenden NACE-Klassifikation zusammengefasst werden. Ortliche fachliche Einheiten sind operativ tätige Teile von ,institutionellen Einheiten', d.h. - nach ESVG95 - von wirtschaftlichen Entscheidungsträgern, die ein einheitliches Verhalten aufweisen und Entscheidungsfreiheit genießen [Eurostat 1995]. Die Aufkommens- und Verwendungstabellen, die gemäß dem ,institutionellen Prinzip' angelegt werden, spiegeln somit beobachtbare Zusammenhänge auf Basis von örtlichen fachlichen Einheiten wider. Vorteilhaft ist dieses praxisnahe Zuordnungsprinzip insoweit, als das Datenmaterial meist ohne spezielle Manipulationen zugänglich ist und die örtlichen fachlichen Einheit demgemäß ,nur' über das Informationssystem der institutionellen Einheit zu etwaigen anderen örtlichen fachlichen Einheiten abgegrenzt werden muss. Die aufgrund der Zuteilung der örtlichen fachlichen Einheiten gemäß deren Haupttätigkeit erzielte Heterogenität kann als gewichtiger Nachteil angeführt werden.

Das ,funktionelle Prinzip' fokussiert hingegen auf produktionstechnisch homogene Einheiten (,homogene Produktionseinheiten'; engl. ,unit of homogeneous production'), die den Anforderungen der Produktionsanalyse optimal entsprechen, nachdem diese Einheiten den Bezugspunkt für den eigentlichen Produktionsvorgang, d.h. den für die Produktion erforderlichen Tätigkeiten inklusive den diese unterstützenden Hilfstätigkeiten aber exklusive diverser Nebentätigkeiten z.B. der örtlichen fachlichen Einheit, darstellen [Eurostat 1995]. Dieses Prinzip ermöglicht Input-Output-Tabellen, die die (technisch determinierte) Produktionsverflechtung beschreiben, wobei auch interne Leistungsströme ausgewiesen werden können. Ein Vorteil dieser Vorgehensweise ist insbesondere die vergleichsweise hohe Homogenität der festgehaltenen Transaktionen, nach- 
teilig ist v.a. die aufwendige und schwierige Beschaffung der erforderlichen Daten ${ }^{33}$, die mitunter auch Ungenauigkeiten bewirkt [Holub \& Schnabl 1985].

Während also die Aufkommens- und Verwendungstabellen dem ,institutionellen Prinzip' folgen und dementsprechend örtliche fachliche Einheiten als Darstellungseinheit aufweisen, die zu ,Wirtschaftsbereichen' gruppiert werden, stellen die (symmetrischen) Input-Output-Tabellen auf die Darstellungseinheit der homogenen Produktionseinheiten $a b$, die zusammengefasst als ,homogene Produktionsbereiche' bezeichnet werden. Nachdem es gemeinhin nicht möglich ist, homogene Produktionseinheiten direkt zu beobachten, ist es allerdings erforderlich, diese aus dem vorhandenen Datenmaterial zu eruieren. Konkret werden zu diesem Zweck die Aufkommens- und Verwendungstabelle üblicherweise in der Bewertung zu Herstellungspreisen unter Berücksichtigung weiterer statistischer, aber auch produktionstechnischer Datenquellen herangezogen.

\subsubsection{Annahmen bei der Aufstellung von Input-Output-Tabellen}

Die Überleitung zu einer (symmetrischen) Input-Output-Tabelle bedingt jedoch zusätzlich zu diesen Daten auch eine Festlegung auf ein bestimmtes Überleitungsverfahren, welches bestimmte Annahmen (Imputationsannahmen) über die Produktionstechnologie impliziert. Diese Annahmen unterscheiden sich einerseits in dem Rechenvorgang und dem erzielten Endergebnis und andererseits in dessen Plausibilität je nachdem ob die IOT in der Dimension Güter $\times$ Güter oder Aktivitäten $\times$ Aktivitäten erstellt werden soll und werden nachfolgend beschrieben.

\section{Dimension Güter x Güter}

\section{- Industrietechnologieannahme}

Die Industrietechnologieannahme geht davon aus, dass alle Güter einer Aktivität (unabhängig davon, ob die Güter charakteristisch oder nicht-

33 Diese müssen oftmals ausgehend von der institutionellen Einheit aufgespalten werden, wobei statistisches Sekundärmaterial und begleitende Expertengespräche häufig zusätzlich notwendig sind. 
charakteristisch für diese Aktivität sind) mit ein und derselben Inputstruktur hergestellt werden.

\section{- Gütertechnologieannahme}

Die Gütertechnologieannahme setzt hingegen für die Erstellung eines Gutes, abermals unabhängig von dessen Eigenschaft als charakteristisches oder nicht-charakteristisches Gut einer Aktivität, aktivitätsübergreifend die gleiche Inputstruktur voraus.

\section{Dimension Aktivitäten x Aktivitäten}

- Annahme fixer, güterbezogener Absatzstrukturen

Gemäß dieser Annahme ist die Struktur des Absatzes jedes Gutes unabhängig von der das Gut produzierenden Aktivität immer gleich. Dies bedeutet, dass die produktspezifische Absatzstruktur über alle (das Gut produzierenden) Aktivitäten als gleich angenommen wird. Dieser Ansatz wurde früher als eine Spielart der Industrietechnologieannahme bei der Erstellung von IOT in der Dimension Aktivitäten x Aktivitäten interpretiert. Dieser Vergleich ist allerdings aufgrund der fehlenden Technologieannahme nicht zulässig [Eurostat 2007].

- Annahme fixer, aktivitätsbezogener Absatzstrukturen

Diese Annahme sieht hingegen den Absatz von Gütern in Abhängigkeit von der die Güter jeweils produzierenden Aktivität, d.h. die nichtcharakteristische Produktion einer Aktivität wird im gleichen Verhältnis wie die charakteristische Produktion einer Aktivität aufgeteilt. Diese Annahme wurde lange als Variante der Gütertechnologieannahme für IOT in der Dimension Aktivität $\times$ Aktivität interpretiert, tatsächlich ist auch dieser Vergleich aufgrund des Fehlens einer Technologieannahme unpassend [Eurostat 2007].

Obwohl die Gütertechnologieannahme für die Erstellung von IOT in der Dimension Güter $\times$ Güter durchwegs plausibler erscheint (vgl. hierzu bspw. 
[Almon 2000]), wurde in der Vergangenheit häufig die Industrietechnologieannahme und das dazugehörige Rechenverfahren zur Erstellung quadratischer IOT in der Dimension Güter x Güter oder Aktivitäten x Aktivitäten eingesetzt. Dieser Schritt wurde dabei lange Zeit damit begründet, dass in Folge des Berechnungsverfahrens für die Gütertechnologieannahme negative Koeffizienten in der quadratischen IOT auftreten können, die ökonomisch nicht interpretierbar sind. Dieses Problem wird im Wesentlichen auf (i) Inhomogenitäten der eingesetzten VGR-Klassifikationen im Hinblick auf die Zwecke der Input-OutputRechnung, (ii) konzeptive Probleme, die bspw. dazu führen, dass die innerbetriebliche Leistungserstellung (vertikale Integration) nicht berücksichtigt werden kann, und (iii) Lücken in der Gültigkeit der Gütertechnologieannahme zurückgeführt. Inzwischen konnte die Problematik des Auftretens negativer Koeffizienten durch einen speziellen Algorithmus von Almon [2000], welcher weiter unten genauer erläutert wird, weitgehend entschärft werden. Die Gütertechnologieannahme ist auf Basis dieser Lösung aufgrund ihres - in vielen Aktivitäten höheren Realitätsbezugs zur vorherrschenden Annahme geworden. Abgesehen von den beiden grundlegend unterschiedlichen Annahmen zur Erstellung von IOT in der Dimension Güter $\times$ Güter sei in diesem Zusammenhang noch auf die Existenz von Mischverfahren (,Hybridtechnologieannahmen') hingewiesen, die über eine Zerlegung der Make-Matrix beide Annahmen entsprechend einem bestimmten Mischverhältnis berücksichtigen [Holub \& Schnabl 1994].

Bei der Generierung von IOT in der Dimension Aktivitäten $\times$ Aktivitäten bietet die Annahme fixer, güterbezogener Absatzstrukturen gegenüber der Annahme fixer, aktivitätsbezogener Absatzstrukturen einen höheren Realitätsgrad. Dies ist auf die größere Plausibilität von unterschiedlichen Absatzwegen und -gruppen für unterschiedliche charakteristische und nicht-charakteristische Güter von Aktivitäten zurückzuführen. Mischvarianten der beiden Annahmen für die Erstellung von IOT in der Dimension Aktivitäten $\times$ Aktivitäten sind darüber hinaus ebenso denkbar. 
Zur besseren Nachvollziehbarkeit der weiteren Ausführungen werden nachfolgend die erforderlichen Verfahrensschritte zur Berechnung von Input-OutputTabellen auf Basis der Gütertechnologieannahme beschrieben.

\subsubsection{Generierung von Güter x Güter IOT mit der Gütertechnologieannahme}

\section{Vorbereitende Schritte}

Ausgehend von der Aufkommens- und Verwendungstabelle und deren Zerlegung entsprechend der Make- und Use-Systematik werden zunächst Anteilsmatrizen bezogen auf die Spalten- und Zeilensummen der Make- und Use-Matrizen berechnet. Tabelle 9 zeigt die entstehenden Anteilsmatrizen, die aus einem Makeund Use-System in Version B, d.h. bei Trennung zwischen heimischen und importierten Gütern, entstehen.

Tabelle 9: Anteilsmatrizen aus der Make- und Use-Systematik

$l^{\prime \prime}$ - Use heimisch (Dimension

Güter x Aktivitäten)

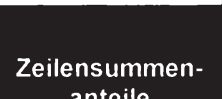

anteile

Spalten-

summenanteile
$C^{\text {' }}$ - Use importiert (Dimension

Güter x Aktivitäten) anteile heimischer Güter

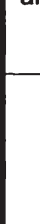

\begin{tabular}{c|c} 
Matrix der Nachfrage- & $\begin{array}{c}\text { Matrix der Nachfrage- } \\
\text { anteile importierter }\end{array}$
\end{tabular}

\begin{tabular}{c|c} 
Matrix der Nachfrage- & $\begin{array}{c}\text { Matrix der Nachfrage- } \\
\text { anteile importierter }\end{array}$
\end{tabular} $N^{\prime}$

Matrix der Inputkoeffizienten $B^{\prime}$ Güter $N^{m}$

Matrix der Importkoeffizienten $B^{m}$
I - Make

(Dimension

Güter x Aktivitäten)

(transponierte) Matrix der Marktanteile

D

Product-Mix-Matrix

C

Im Folgenden wird auf die Berechnung der Anteilsmatrizen und deren ökonomische Interpretation eingegangen. Grundlage für die folgenden Formeln zu Anteilsmatrizen bilden die Definitionen in Holub \& Schnabl [1994].

Element $b_{i j}^{d}$ der Matrix der Inputkoeffizienten $B^{d}$ gibt an, mit welchem Anteil das heimisch erzeugte Gut $i$ an der Herstellung einer Einheit der Produktion von Aktivität $j$ (gemessen an den Produktionskosten, die definitorisch den Produktionserlösen entsprechen) beteiligt ist. Elsciwied lalsoe degralativel Aateif-von Gut 
$i$ gemessen am Gesamtinput für Aktivität $j$ (inkl. Wertschöpfung) in dieser Matrix festgehalten.

Tabelle 10: Matrix der Inputkoeffizienten (, Vorleistungskoeffizienten')

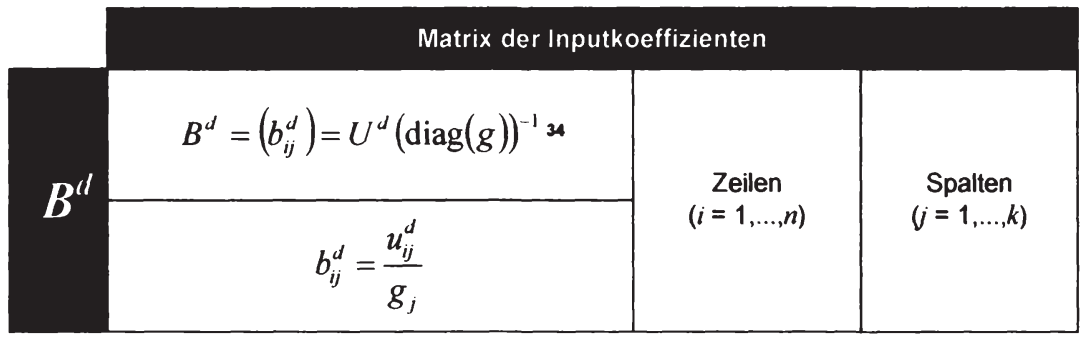

Fragen, die über die Matrix $B^{d}$ beantwortet werden können sind:

- rein deskriptiv:

Welche Vorleistungen dominieren die Produktion in Aktivität $j$ ?

- hypothesenbehaftet und modellgebunden:

Bei einer Erhöhung der Produktionswerte (Produktionskosten bzw. -

erlöse) der Aktivität $j$ sind welche Vorleistungen (Güter) in welchem Umfang zusätzlich (direkt) erforderlich?

Tabelle 11: Matrix der Importkoeffizienten

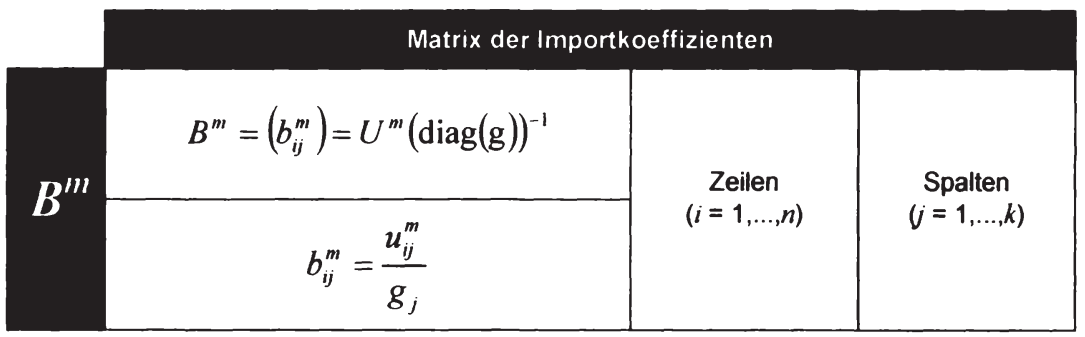

Element $b_{i j}^{m}$ der Matrix der Importkoeffizienten $B^{m}$ gibt an, wie hoch der Anteil des importierten Guts $i$ an der Herstellung einer Einheit der Produktion von Aktivität $j$

34 Die Funktion, diag' wird zur Erstellung einer Diagonalmatrix mit den Elementen des

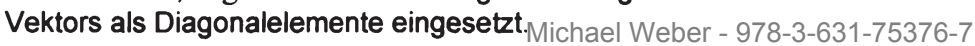


(abermals gemessen an den Produktionskosten, die definitorisch den Produktionserlösen entsprechen) ist. Die Interpretation und die mittels dieser Matrix beantwortbaren Fragen der Matrix der Importkoeffizienten $B^{m}$ sind analog zu der Matrix der Inputkoeffizienten, allerdings im Bezug auf importierte anstatt auf heimische Güter.

Tabelle 12: Matrix der Nachfrageanteile heimischer Güter

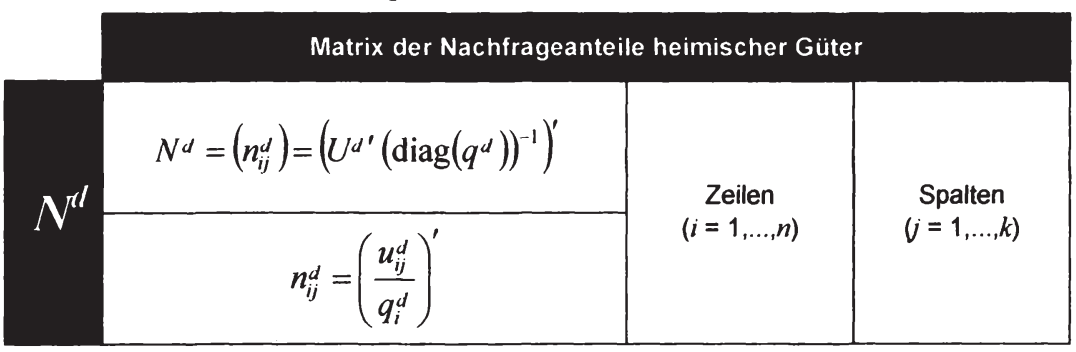

Element $n_{i j}^{d}$ der Matrix der Nachfrageanteile heimischer Güter $N^{d}$ gibt an, in welchem Ausmaß Gut $i$ von Aktivität $j$ relativ zu den anderen Aktivitäten eingesetzt bzw. nachgefragt wird. Der Wert gibt also den Anteil der Nachfrage von Aktivität $j$ an der Gesamtnachfrage nach Gut $i$, gemessen an der heimischen Güterverwendung, die auch die Endnachfrage nach heimischen Gütern einschließt, an. Fragen, die mit dieser Matrix beantwortet werden können, betreffen bspw. die Bedeutung von bestimmten Aktivitäten für ein Gut.

Tabelle 13: Matrix der Nachfrageanteile importierter Güter

Matrix der Nachfrageanteile importierter Güter

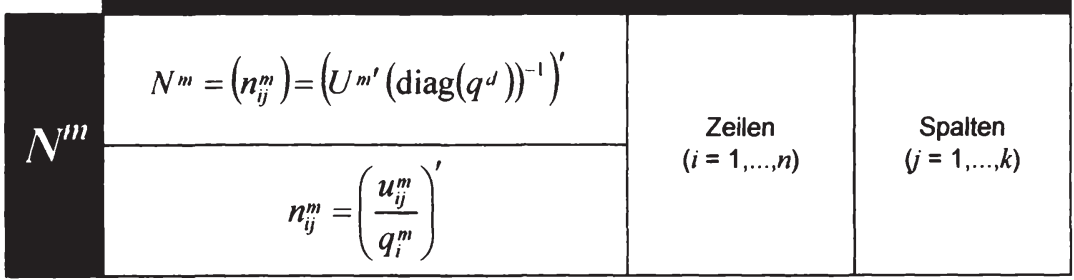

Element $n_{i j}^{m}$ der Matrix der Nachfrageanteile importierter Güter $N^{m}$ gibt an, wie stark das importierte Gut $i$ von Aktivität $j$ relativ zu den anderen Aktivitäten nach- 
gefragt bzw. eingesetzt wird. Die Interpretation und die über diese Matrix adressierbaren Fragen sind analog zu der Matrix der Nachfrageanteile heimischer Güter im Hinblick auf importierte Güter. Dies gilt auch für die Güterverwendung sowie die darin enthaltene Endnachfrage.

Tabelle 14: Product-Mix-Matrix

Product-Mix-Matrix

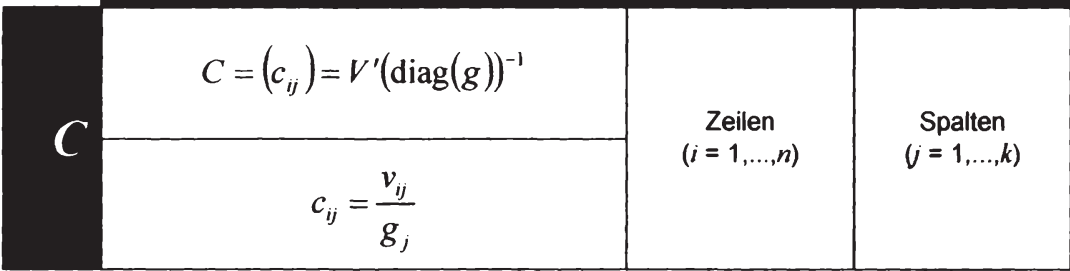

Element $c_{i j}$ der Product-Mix-Matrix zeigt den Anteil des Gutes $i$ am Gesamtoutput der Aktivität $j$, gemessen an dem Produktionswert (Produktionskosten bzw. -erlöse). Mit dieser Matrix kann die Frage beantwortet werden, welche Produkte (Güter) die Produktion der Aktivität $j$ dominieren bzw. wie hoch der Anteil von Gut $i$ am gesamten Output der Aktivität $j$ ist. Ebenso können auch die charakteristischen Güter einer Aktivität identifiziert werden.

Tabelle 15: Matrix der Marktanteile

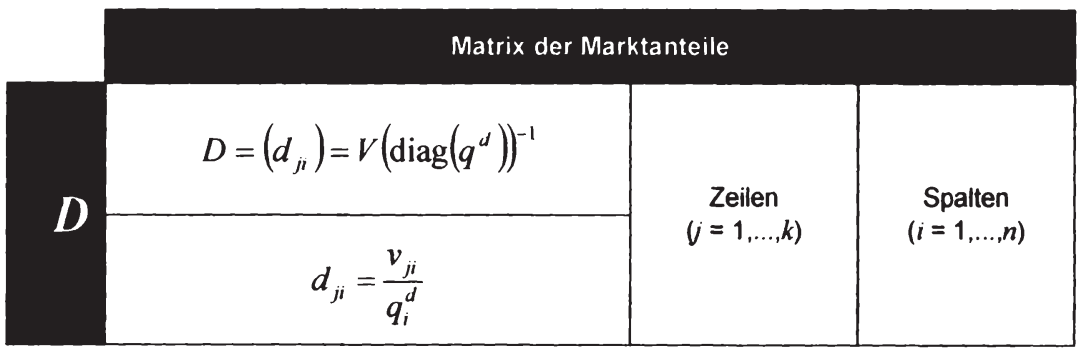

Element $d_{j i}$ der Matrix der Marktanteile $D$ - üblicherweise angegeben in der Dimension Aktivitäten $\times$ Güter - gibt den ,Marktanteil' der Aktivität $j$ am Gesamtaufkommen des Gutes $i$ an. Die Matrix beantwortet somit die Frage, wie hoch der Anteil der Aktivität $j$ an der heimischen Produktion von Gut $i$ ist. 
Aufgrund der in Tabelle 8 dargestellten Zusammenhänge des Make- und UseSystems ergeben sich ausgehend von den Anteilsmatrizen folgende Summenbedingungen [Holub \& Schnabl 1994].

Tabelle 16: Summenbedingungen des Make- und Use-Systems

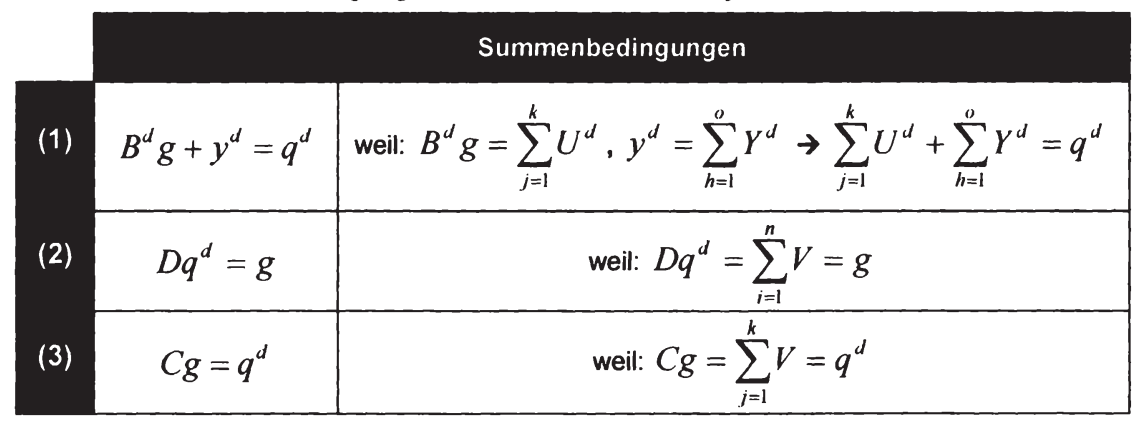

(1) Matrix der heimischen Inputkoeffizienten * Vektor der Produktionserlöse

+ Vektor der Endnachfrage nach heimischen Gütern

= Vektor der heimischen Güterverwendung

(2) Matrix der Marktanteile * Vektor der heimischen Güterverwendung $=$ Vektor der Produktionserlöse

(3) Product-Mix-Matrix * Vektor der Produktionserlöse $=$ Vektor der heimischen Güterverwendung

\section{Berechnungsverfahren}

Bei der Berechnung von Güter x Güter IOT auf Basis der Gütertechnologieannahme wird aufbauend auf den Gleichungen (1) und (3) durch deren Auflösung nach $y^{d}$

$$
\begin{array}{rlrl}
C g & =q^{d} & & \mid \rightarrow C^{-1} \\
g & =C^{-1} q^{d} & & \mid \text { substituiere } g \text { in }(1) \\
B^{d} C^{-1} q^{d}+y^{d} & =q^{d} & & \mid-B^{d} C^{-1} q^{d} \\
y^{d} & =q^{d}-B^{d} C^{-1} q^{d} & & \\
& =(I-\underbrace{B^{d} C^{-1}}_{A_{C}})_{\text {Michael Weber - 978-3-631-7 }}^{q^{d}} &
\end{array}
$$


die Matrix der direkten Inputkoeffizienten $A_{C}$ (Formel (5)) in der Dimension Güter $x$ Güter gewonnen. Voraussetzung für diese Berechnungsschritte ist aufgrund der erforderlichen Invertierung die Gleichheit der Dimensionen $n$ und $k$, d.h. der Einsatz von Matrizen mit der gleichen Anzahl an Gütern und Aktivitäten.

$$
A_{C}=B^{d} C^{-1}=\left(a_{C, i l}\right)_{i, l=1, \ldots, n}
$$

Die Elemente $a_{C, i l}$ der Matrix der direkten Inputkoeffizienten $A_{C}$ beschreiben die direkt erforderliche Erhöhung der Produktion (bzw. des Einsatzes) des Inputfaktors Gut $i$ für die Bereitstellung einer weiteren Einheit des Gutes l (Output). Diese Interpretation der Elemente von $A_{C}$ deutet bereits auf den eigentlichen Zweck der Input-Output-Tabellen hin, für die in weiterer Folge kumulative Inputkoeffizienten - eine Matrix, die auch als Leontief-Inverse bekannt ist - für die Abschätzung der Auswirkung von direkten und indirekten Effekten auf die Produktionsstruktur berechnet werden. Der Vollständigkeit halber sei kurz die Berechnung dieser Matrix $R_{C}$ (Dimension Güter $\times$ Güter) auf Basis der Gütertechnologieannahme im offenen statischen Mengenmodell aufgezeigt. Die Elemente $r_{C, i l}$ der Matrix $R_{C}$ bezeichnen hierbei die indirekt und direkt erforderlichen Änderungen des Outputs des Inputfaktors Gut $i$ für die Generierung einer weiteren Einheit von Gut $l$ [Leontief 1937].

$$
\begin{gathered}
q^{d}=\underbrace{\left(I-B^{d} C^{-1}\right)^{-1}}_{R_{C}} y^{d} \\
R_{C}=\left(I-A_{C}\right)^{-1}=\left(r_{C, i l}\right)_{i, l=1, \ldots, n}
\end{gathered}
$$

Einen Überblick über die - abhängig von den gewählten Annahmen und der angestrebten Dimensionierung variierenden - Ansätze zur Berechnung der Matrix der direkten Inputkoeffizienten $A$ (genauer: $A_{C}$ oder $A_{l}$ ) bietet Tabelle 17. 
Tabelle 17: Berechnungsmethoden der Matrix der direkten Inputkoeffizienten

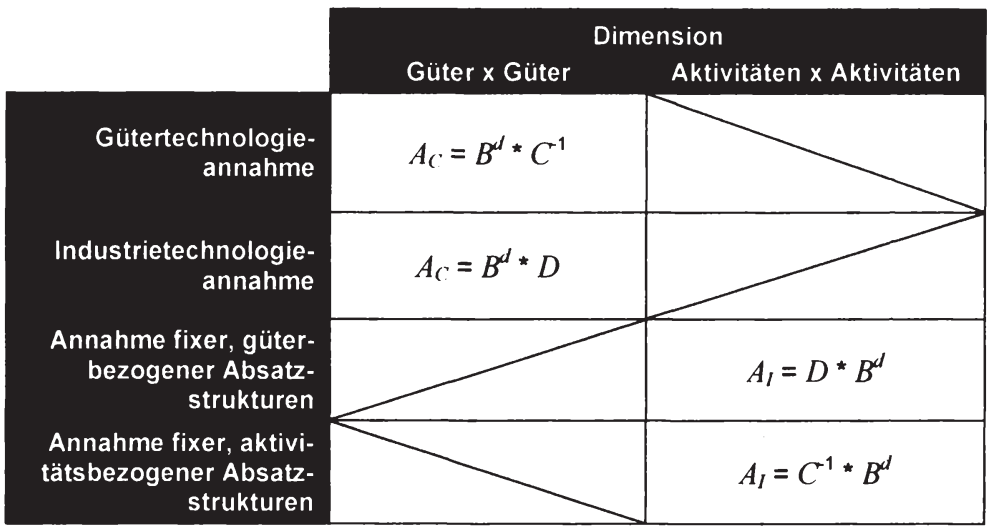

\subsubsection{Negative Werte im Zuge der Gütertechnologieannahme}

Die bereits erwähnte Problematik des Auftretens von negativen Koeffizienten bei der Berechnung der Matrix der direkten Inputkoeffizienten, hervorgerufen durch die Multiplikation der Matrix der Inputkoeffizienten $(B)$ mit der invertierten Product-Mix-Matrix $(C)$, hat verschiedenste Verfahren zur Linderung hervorgerufen, die von rein algorithmischen bis zu inhaltlichen Lösungsansätzen reichen [Rainer \& Richter 1992; Steenge \& Konijn 1992]. Im Folgenden wird eine rein algorithmische Herangehensweise von Almon [2000] vorgestellt, die bei der Erstellung der Matrix der direkten Inputkoeffizienten Einsatz findet, häufig unter zusätzlicher Beachtung inhaltlicher Auswirkungen dieser mathematisch getriebenen Vorgehensweise. Der Algorithmus orientiert sich an der Gütertechnologieannahme und kann auch verwendet werden, um Problemfelder von Makeund Use-Matrizen zu identifizieren (Funktion ALMON; eine Übersicht über alle für diese Arbeit implementierten Funktionen bietet Appendix A).

Der Einsatz der Gauss-Seidel-Methode, einem iterativen Verfahren zur Lösung linearer Gleichungssysteme, erlaubt hierbei den Eingriff in die Berechnung der Matrix der direkten Inputwerte. Im Anschluss wird auf die Vorgehensweise des Algorithmus genauer eingegangen, einerseits, um Fehler und Unklarheiten in 
dem Beitrag von Almon [2000] auszuräumen und andererseits, um den Einsatz des Algorithmus im Rahmen der Überlegungen dieser Arbeit zu ermöglichen.

Die erforderlichen Matrizen für den Algorithmus von Almon sind $D^{35}$, eine quadratische Matrix der Marktanteile in der Dimension Aktivitäten x Güter, deren Spaltensummen auf 1 normiert sind, sowie eine quadratische Use-Matrix $U$ in der Dimension Güter $\times$ Aktivitäten. $R$ ist die zu errechnende, nichtnegative Ergebnismatrix in der Dimension Güter $\times$ Güter. Es sei darauf hingewiesen, dass $R$ in dem Algorithmus von Almon nicht die Leontief-Inverse bezeichnet.

Die Konvergenz des Verfahrens wird von Almon mit folgendem Theorem begründet. Die Matrix I sei die Einheitsmatrix zu $D$. Ist der Anteil der Gesamtproduktion eines Gutes in dessen charakteristischen Herstellungsaktivität (folglich in der Diagonalzelle von $D$ ) $x$, dann entspricht dessen Absolutbetrag in der Diagonalzelle der Matrix $(I-D), 1-x$. Dieser Absolutbetrag ist gleich der Summe aller Absolutbeträge abseits der Diagonale in der Spalte des Gutes, also ebenfalls $1-x$. Dies ist jener Produktionsanteil des Gutes, welcher von anderen Aktivitäten beigesteuert wird. Die Summe der Güterspalte der Matrix $(I-D)$ beträgt $2^{*}(1-x)$, wobei die Summe kleiner als 1 ist, wenn $x>0,5$. Dieser Fall ist gleichzeitig die zentrale Voraussetzung für die Konvergenz des Iterationsverfahrens, d.h. die charakteristische Produktion einer Aktivität muss einen Produktionsanteil von über $50 \%$ in der Anteilsmatrix $D$ der Make-Matrix aufweisen [Almon 2000].

Formel (8) zeigt die Berechnung der Ergebnismatrix $R$ mittels der üblichen Matrixmanipulation ausgehend von $U$ und $D$ an.

$$
U^{\prime}=D R^{\prime} \Leftrightarrow R=U\left(D^{\prime}\right)^{-1}
$$


In diesem Zusammenhang ist hervorzuheben, dass die Spaltensummenanteile der Ergebnismatrix $R$ der Matrix der direkten Inputkoeffizienten gemäß der Gütertechnologieannahme in der Dimension Güter x Güter entsprechen.

$$
R \cdot \operatorname{diag}\left(\sum_{i=1}^{n} R_{i j}\right)^{-1} \hat{=} B \cdot C^{-1}
$$

Für die Berechnung des Beitrags von Gut $i$ für die Produktion von Gütern wird zur besseren Veranschaulichung von der $i$-ten Zeile der Use-Matrix in der Dimension Güter $\times$ Aktivitäten ausgegangen. Über mehrere Iterationsschritte soll von der Use-Matrix ausgehend die $i$-te Zeile der Matrix $R$ in der Dimension Güter $\mathrm{x}$ Güter errechnet werden.

$$
U_{i}^{\prime}=D R_{i}^{\prime}
$$

Zur besseren Illustration werden die $i$-ten Spalten der transponierten Matrizen $U^{\prime}$ und $R^{\prime}$ als Vektoren $u$ und $r$ angeschrieben.

$$
\begin{aligned}
& u=D r \\
& r=D^{-1} u
\end{aligned}
$$

Unter Anlehnung an die Gauss-Seidel Methode ist nun folgende Umformulierung möglich:

$$
r=(I-D) r+u
$$

Beginnend mit $u$ wird dabei $r$ schrittweise (mit dem Iterationszähler $k$ ) berechnet:

$$
\begin{aligned}
r^{0} & =u \\
r^{(k+1)} & =(I-D) r^{(k)}+u
\end{aligned}
$$

Die Formel für die elementweise, iterative Berechnung des Beitrags von Gut $i$ zur Produktion von Gut $j$ lautet folglich: 


$$
\begin{aligned}
r_{j}^{0} & =u_{j} \\
r_{j}^{(k+1)} & =\left(1-d_{j j}\right) r_{j}^{(k)}-\sum_{\substack{h=1 \\
h \neq j}}^{n} d_{j h} r_{h}^{(k)}+u_{j}
\end{aligned}
$$

oder anders angeordnet:

$$
r_{j}^{(k+1)}=u_{j}-\sum_{\substack{h=1 \\ h \neq j}}^{n} d_{j h} r_{h}^{(k)}+\left(1-d_{j j}\right) r_{j}^{(k)}
$$

Der Zellenwert $R_{i j}$, oder entsprechend obiger Notation $r_{j}$, beschreibt den direkten Einsatz von Gut $i$ in der Produktion von Gut $j$. Ausgegangen wird bei der Berechnung von $u_{j}$ (Zellwert $U_{i j}$ ), dem Einsatz von Gut $i$ in der Aktivität $j$. Im zweiten Term der Formel (15) werden die Produkte der Anteile von Aktivität $j$ an der Produktion der Güter $h$, wobei $h=1, \ldots, n$, und $h \neq j$, aus der Matrix $D$ mit den $h$ ten Werten der bisherigen iterativen Berechnung des Beitrags von Gut $i\left(r_{h}\right)$, ermittelt, über alle $h$ summiert und von $u_{j}$ (Zellwert $U_{i j}$ ) abgezogen. Über diesen Schritt kann ermittelt werden, in welchem Ausmaß Aktivität $j$ Gut $i$ für die Produktion von anderen - aus Sicht von Aktivität $j$ nicht-charakteristischen Gütern $^{36}$ (Güter $h$ ) einsetzt, nachdem der Vektor $d_{j h}$ jene Produktionswerte beinhaltet, die von Aktivität $j$ abseits des Gutes $i$ erstellt werden und $r_{h}$ den letzten Kenntnisstand (die $k$-te Iteration) über den für die Produktion der Güter $h$ jeweils erforderlichen Einsatz von Gut $i$ festhält. Mit dem dritten Term von Formel (15) wird schließlich der nicht-charakteristische Produktionsanteil der Aktivität $j$ mit dem Beitrag von Gut $i$ für die Produktion von Gut $j$ (dem charakteristischen Produkt der Aktivität $j$ ), $r_{j}$ gemäß der vorangegangenen Iteration, multipliziert und dem bisherigen Ergebnis hinzuaddiert. Dadurch wird der Einsatz von Gut $i$ für die

36 Diese Vorgehensweise setzt eine entsprechend adaptierte Make-Matrix voraus, bei der Diagonalelemente Auskunft über die charakteristische Güterproduktion der Aktivitäten geben. 
nichtcharakteristische Produktion des Gutes $j$, d.h. der Produktion von Gut $j$ in sämtlichen von Aktivität $j$ verschiedenen Aktivitäten, zu $r_{j}$ (Zellwert $R_{i j}$ ) hinzugefügt.

Wie Almon [2000] hervorhebt, entspricht der Beitrag von Gut $i$, der durch den dritten Term zu $r_{j}$ (Zellwert $R_{i j}$ ) hinzugefügt wird, genau dem Einsatz an Gut $i$, der über die zweiten Terme aller anderen Aktivitäten $h$, wobei $h=1, \ldots, n$, und $h \neq j$, über deren Produktionsanteil an Gut $j$ abgezogen wird. Die folgende Gleichung veranschaulicht dies.

$$
\left(1-d_{j j}\right) r_{j}^{(k)}=\sum_{\substack{h=1 \\ h \neq j}}^{n} d_{h j} r_{j}^{(k)}, \text { nachdem } \sum_{h=1}^{n} d_{h j}=1
$$

Zur Vermeidung von negativen Werten in der Ergebnismatrix $R$ führt Almon nun Skalierungsfaktoren $s_{j}$ ein, die ein Anwachsen des zweiten Terms über den Ausgangswert der Use-Matrix vermeiden sollen, indem rechtzeitig die Werte für den zweiten und dritten Term der jeweiligen Iteration skaliert werden. Der Skalierungsvektor für den dritten Term schränkt hierbei den möglichen Wertzuwachs des Beitrags von Gut $i$ für Gut $j$ aufgrund des nicht-charakteristischen Einsatzes von Gut $i$ für Gut $j$ ein und bewirkt damit einen Ausgleich. Angesichts der Tatsache, dass die für diesen Schritt zentralen Gleichungen (Gleichungen (15) und (16) in Almon [2000, S. 33]) zur Berechnung des Skalierungsfaktors $s_{j}$ (bzw. des Vektors der Skalierungsfaktoren für die jeweils zu iterierende Zeile $i$ ohne $s_{j}, s_{h}$ ) fehlerhaft sind, werden nun die korrigierten Versionen dieser beiden Gleichungen angeschrieben:

$$
s_{j}^{(k)}= \begin{cases}1 & \text { wenn } u_{j} \geq \sum_{\substack{h=1 \\ h \neq i}}^{n} d_{j h} r_{h}^{(k)} \\ \frac{u_{j}}{\sum_{\substack{h=1 \\ h \neq j}}^{n} d_{j h} r_{h}^{(k)}} & \text { ansonsten }\end{cases}
$$


Baut man die Skalierungsfaktoren nun in die obige Gleichung ein, so erhält man schließlich den Algorithmus von Almon unter der Bedingung der Nichtnegativität:

$$
\begin{aligned}
r_{j}^{0} & =u_{j} \\
r^{(k+1)} & =u_{j}-s_{j}^{(k)} \sum_{\substack{h=1 \\
h \neq j}}^{n} d_{j h} r_{h}^{(k)}+\sum_{\substack{h=1 \\
h \neq j}}^{n} s_{h}^{(k)} d_{h j} r_{j}^{(k)}
\end{aligned}
$$

Der Gesamteinsatz des Gutes $i$ für alle Güter in der Ergebnismatrix $R$ gleicht dabei der Gesamtverwendung des Gutes $i$ in allen Aktivitäten der Use-Matrix $U$, oder - anders formuliert - die Zeilensummen der Use-Matrix gleichen den Zeilensummen der Ergebnismatrix $R$, wie in folgender Gleichung dargestellt.

$$
\sum_{j=1}^{n} r_{j}^{(k+1)}=\sum_{j=1}^{n} u_{j}
$$

Der Algorithmus von Almon hat - teilweise enweitert bzw. verfeinert - eine breite Akzeptanz bei der Erstellung von Input-Output-Tabellen unter der Gütertechnologieannahme und der Bedingung der Nichtnegativität gefunden. Der Algorithmus lässt sich auch einsetzen, um Abweichungen von der - dem Gütertechnologieansatz zugrundeliegenden - Annahme der Homogenität der Güterströme oder Inkonsistenzen aufgrund unpassender Klassifikation sowohl bei der Make- als auch Use-Matrix aufzudecken. So kann z.B. die generierte Ergebnismatrix $(R)$ gemeinsam mit der Make-Matrix zur Berechnung einer ,New-Use'-Matrix herangezogen werden, um diese dann mit der ursprünglichen Use-Matrix zu vergleichen.

\subsection{Alternative Formen der Produktionsstrukturerhebung}

Die Produktionsstruktur von Gütern, d.h. der Zusammenhang zwischen bspw. einem Gut $i$ und den Gütern, die in die Erzeugung dieses Gutes $i$ in einem bestimmten Verhältnis einfließen, kann alternativ zu der Erfassung im Zuge der Generierung von Input-Output-Tabellen im Kontext der Volkswirtschaftlichen Gesamtrechnung auch direkt über eine (umfassende) Stücklistenerhebung er- 
mittelt werden. Stücklisten (engl. bill-of-materials), die insbesondere in der Sachgüterproduktion eine zentrale Datenquelle für Fertigungsprozesse bilden, können - je nach Ausformung der Erhebung und der eingesetzten Klassifikation Produktionsrelationen mit einer hohen Detaillierungsstufe festhalten und erscheinen daher als eine verfolgenswerte Option für die Datenbeschaffung in dem Transaktionssystemunterstützungsmodell. Besonders ist hierbei auf den Vorteil hinzuweisen, dass Stücklisten auch über Warenwirtschafts- bzw. ERP-Systeme abgerufen werden könnten, was die Datenbeschaffung erheblich vereinfachen würde. Dieser Ansatz einer automatisierten, direkten Datenanbindung zur ,Rohdatenübermittlung', der in Deutschland bereits testhalber für ausgewählte Statistiken der statistischen Ämter [Destatis 2005] verfolgt wird, unterstreicht die Realisierbarkeit von hoch detaillierten Stücklisten zur Analyse der Produktionsstruktur und ist gleichzeitig eine wichtige Maßnahme, um den Befragungsaufwand bei den Respondenten zu reduzieren. Gleichzeitig werden dadurch allerdings Fragen bezüglich der Auswirkungen des Ansatzes auf Geschäftsmodelle, Fragen der Erwünschtheit von Transparenz und Fragen des Datenschutzes aufgeworfen, die abseits von der Erhebungsmethodik, Datenmodellierung und -aufbereitung weitere spannende Forschungsthemen garantieren. Neben diesem zukunftsweisenden Erhebungsansatz sind Experteninterviews und vergleichbare Befragungsmethoden, die bereits aus der Input-OutputTabellenerstellung bekannt sind, als weitere wertvolle Quellen für die Bestimmung und Aggregation von Stücklisten zu nennen, die speziell auch für den Bereich der Dienstleistungen von Relevanz sind. 


\section{$4 \mathrm{BCl}$ - Modell und Daten}

Die Input-Output-Tabellen bzw. Stücklisten dienen als Informationsgrundlage über die charakteristischen Verflechtungen innerhalb aber auch zwischen den Wirtschaftsbereichen, in der die Daten erhoben bzw. unter Zuhilfenahme sekundärer Statistiken geschätzt wurden. Sie sind der Ausgangspunkt für die nun folgenden Überlegungen zur gezielteren Wahrnehmung, effizienteren Kommunikation, letztlich aber zur Unterstützung und Erhöhung von Transaktionen, die insbesondere über elektronisch gestützte zwischenbetriebliche Kollaborationsplattformen (bzw. Transaktionssysteme) vermittelt werden können.

Gemäß dieser grundsätzlichen Zielsetzung wird in den anschließenden Kapiteln ein Modell vorgestellt, welches das Verflechtungsmuster als Indikator für potenzielle Transaktionen zwischen wirtschaftlichen Akteuren auf einer Kollaborationsplattform, die gewisse Aktivitäten setzen und über diese Aktivitäten spezifische Güter bereitstellen, interpretiert. Über das Modell soll eine Möglichkeit aufgezeigt werden, wie Teilnehmern einer solchen Plattform qualifizierte Empfehlungen zu Transaktionsmöglichkeiten mit anderen Teilnehmern unterbreitet werden können, um diese zusätzlich zur selbstständigen Suche auf der Plattform durch direkte oder indirekte Vermittlung bei der Transaktionsgenerierung zu unterstützen. Eine weitere wichtige Eigenschaft des Modells, die sich aus der obigen Zielsetzung ergibt, ist, dass auf der Ebene der Plattform die Zusammensetzung der von den Teilnehmern angebotenen Aktivitäten bzw. Güter besser organisiert bzw. gelenkt werden kann, um die realisierten Transaktionen ebenso wie die wirtschaftlichen Verflechtungen zwischen den Teilnehmern auf der gemeinsamen Plattform zu fördern. Die über die bereits diskutierten Erhebungsformen gewonnenen wirtschaftlichen Strukturinformationen sind dabei der zentrale Baustein. Diese Informationen bilden gemeinsam mit dem Modell die Basis für die Realisierung einer Selbstorganisation von Wertschöpfungsketten auf einer Kollaborationsplattform, nachdem die Aktivitäten der Teilnehmer an dem Transaktionssystem, deren Leistungen wirtschaftlich gesehen Glieder einer Wertschöpfungskette darstellen, besser koordiniert werden können. Weitere Wessentliche Yoraussetzungen, die in 
diesem Zusammenhang zu nennen sind, sind Informationen zu den Aktivitäten und Gütern der Teilnehmer sowie - idealerweise - auch zu den Transaktionsverläufen auf der Kollaborationsplattform. Letztlich zielt das Modell darauf ab, aus einer gesamtsystemischen Perspektive eine integrale Struktur etablieren und in weiterer Folge aufrechterhalten zu können. Nachhaltigkeit soll dabei durch die Ausrichtung des Modells - genauer dessen Datenbasis - auf charakteristische Verflechtungen der Produktionsstruktur gewährleistet werden.

Ausgangspunkt für die Überlegungen zu einem Transaktionsförderungsmodell sind demnach Kenntnisse über die Güter und Aktivitäten der Teilnehmer an einer zwischenbetrieblichen Kollaborationsplattform zumindest auf Betriebsebene und (idealenweise) über die Transaktionsverläufe zwischen den Teilnehmern an der Plattform sowie Daten über allgemeine bzw. grundsätzliche Strukturen des wirtschaftlichen Austausches bezogen auf den Wirtschaftsraum, in dem die Teilnehmer ihren wirtschaftlichen Schwerpunkt haben. Diese Daten können prinzipiell aus Stücklisten ebenso stammen wie aus - auf hohem Detaillierungsgrad vorliegenden - Make- und Use-Matrizen oder sogar Input-Output-Tabellen.

Ein wesentlicher Bestandteil des Modells sind folglich Daten zu dem Profil der Teilnehmer an der Kollaborationsplattform. Zum einen zählen hierzu v.a. Informationen zu der Anzahl, der Gliederung und dem Zweck der Betriebe des Unternehmens (die Ausgangs- oder Zielpunkt der getätigten Transaktionen sind) und deren schwerpunktmäßige Zuordnung zu Aktivitäten gemäß NACE-Klassifikation. Zum anderen ist es für das Profil wichtig, die in den jeweiligen Aktivitäten hergestellten Güter - Sachgüter und/oder Dienstleistungen, die bspw. aus dem (elektronischen) ${ }^{37}$ Produktkatalog der Betriebe der Unternehmen entnommen werden können, - entsprechend deren Klassifikation laut CPA anzuführen. Darüber hinaus können im Profil - neben allgemeinen Informationen zu den teil-

37 Quellen hierfür könnten u.a. das ERP-System, d.h. das Informationssystem zur elektronischen Ressourcenplanung, oder auch der ,eShop' (die elektronischen Filiale) des Unternehmens sein. 
nehmenden Unternehmen bzw. Betrieben - bspw. auch die Präferenzen der jeweiligen Entscheidungsträger auf unterschiedlichsten Ebenen festgehalten werden. Solche Präferenzen können die Zahlungsmodalitäten, die Zahlungsart, allgemeine Lieferbedingungen und -zeiten u.v.m. betreffen. Kapitel 11 präsentiert im Anschluss an die Ausführungen zu den Modellphasen ein Demonstrationsbeispiel zwecks Illustration der Empfehlungsgenerierung. Tabelle 33 in Kapitel 11.1.1 zeigt hierbei ein Beispiel für Profilinformationen zu den Teilnehmern einer solchen Plattorm, die in dem Modell in der Profiltabelle BCIprofile abgelegt werden. Tabelle 92, Tabelle 93 und Tabelle 94 in Kapitel 11.6 enthalten hingegen die Präferenzdaten (BCIpreferences) für das Demonstrationsbeispiel.

Transaktionsdaten sind nach den Teilnehmerprofilen die zweite Säule, auf der die Überlegungen zu dem Transaktionsförderungsmodell für zwischenbetriebliche Kollaborationsplattformen beruhen. Sie sind für ein solches Modell zwar nicht unbedingt erforderlich, können aber Vorschläge für die Erhöhung der zwischenbetrieblichen Interaktionen erheblich zielsicherer machen. Eine qualitative Unterscheidung kann dabei bei gleicher Detaillierungsstufe abhängig von dem Transaktionsbezug getroffen werden. In ihrer Aussagekraft umfassendere und somit qualitativ höherwertige Schlüsse können aus Transaktionsdaten gezogen werden, die die Güterströme zwischen den Unternehmen auf der Kollaborationsplattform abbilden. Eingeschränkte bzw. gröbere Schlussfolgerungen können hingegen gewonnen werden, wenn nur die Aktivität, die Grundlage der realisierten Transaktion ist, aufgezeichnet werden kann. Tabelle 34 in Kapitel 11.1.2 zeigt beispielhaft Transaktionsdaten bezogen auf Güterströme zwischen Unternehmen, die als BCledgeFRuug bezeichnet werden. BCledgeFRuua beschreibt im Gegensatz Transaktionsdaten zwischen Unternehmen bezogen auf die der Transaktion zugrunde liegenden Aktivität.

Die dritte wesentliche Säule des Modells sind Daten über die (u.U. regional spezifische) Struktur der Verflechtungen zwischen Gütern, zwischen Aktivitäten und/oder zwischen Gütern und Aktivitäten. Datenquellen hierfür können in Statistiken zur Struktur und zu den Verflechtungen einer Volkswirtschaft ebenso identi- 
fiziert werden, wie in Stücklisten für Güter (insbesondere Sachgüter) oder aber auch für Aktivitäten. Eine wichtige Forderung für das Modell ist, dass neben der Klassifikation ${ }^{38}$ auch der Detaillierungsgrad der gesamtwirtschaftlichen Verflechtungsdaten mit den Transaktionsdaten der Kollaborationsplattform übereinstimmt. In diesem Zusammenhang ist zu betonen, dass die Detailtiefe die bestimmende Determinante für die Nutzbarkeit und Aussagekraft des Modells ist. Kapitel 11.1 geht auf den Einsatz und die Adaption dieser grundlegenden Datenquellen im Zuge des Demonstrationsbeispiels im Hinblick auf Verflechtungen zwischen Gütern (IOTgg) ein.

Eine Übersicht über die für das Modell erforderliche Datenbasis bietet Tabelle 18, wobei je nach Notwendigkeit der jeweiligen Informationen drei Klassen unterschieden werden. Die Einteilung der Tabelle erfolgt einerseits mit Blick auf die drei zuvor beschriebenen Säulen des Modells und andererseits bezogen auf die möglichen Rollen der Teilnehmer in einer Transaktionsbeziehung. Grundvoraussetzungen für das Modell sind demgemäß Informationen zu den an der Kollaborationsplattform teilnehmenden Unternehmen, die Klassifikation der Aktivitäten der Teilnehmer, der Transaktionsgegenstand (Gut bzw. Aktivität) sowie die (gesamtwirtschaftliche) Input-Output-Tabelle oder die diese konstituierenden Make- und Use-Matrizen, falls die IOT nicht bereits vorliegt. Wichtige weitere Informationen beziehen sich auf die Klassifikation der Güter, die über die Aktivitäten der Teilnehmer bereitgestellt werden, sowie den Transaktionswert der über die Plattform abgewickelten Transaktionen. Während das Vorliegen einer Güterklassifikation eine Vorbedingung für Transaktionsempfehlungen auf Güterebene darstellt, ermöglicht die Berücksichtigung der Transaktionshöhe die später erläuterte Bestimmung des zusätzlichen Potenzials bestimmter Transaktionsbeziehungen oder Teilnehmer. Auch das Ziel der Transaktionsgegenstände bei den jeweiligen Abnehmern stellt eine wichtige Information dar, die allerdings

38 Für jene Fälle, in denen die Klassifikationen voneinander abweichen, wäre es möglich, mittels Methoden des Ontologieabgleichs eine Übereinstimmung herbeizuführen [Denk \& Fröschl 2004; Hulliger 1998] ]chael Weber - 978-3-631-75376-7 
unter Einsatz bestimmter Annahmen auch zuschätzbar ist. Die Präferenzstruktur, Teil des erweiterten Teilnehmerprofils, stellt eine interessante zusätzliche Informationsquelle dar, die die Eignung von Transaktionspartnerpaarungen nach Maßgabe der jeweiligen spezifischen Rollen in weiterer Folge quantifizierbar macht und zur Erhöhung der Erfolgsaussichten von Empfehlungen in das Modell miteinbezogen wird. Schließlich sei in diesem Zusammenhang angemerkt, dass es theoretisch auch möglich wäre, ohne tatsächliche Kenntnis des Transaktionsgegenstandes Empfehlungen abzugeben. Dies ist insofern möglich, als auf Basis der Profildaten zweier Transaktionspartner sowie der Strukturdaten der Transaktionsgegenstand zugeschätzt werden kann. Dieser Ansatz wird allerdings aufgrund der damit verbundenen Unschärfe in dem Modell nicht weiter verfolgt.

Tabelle 18: Erforderliche Datenbasis

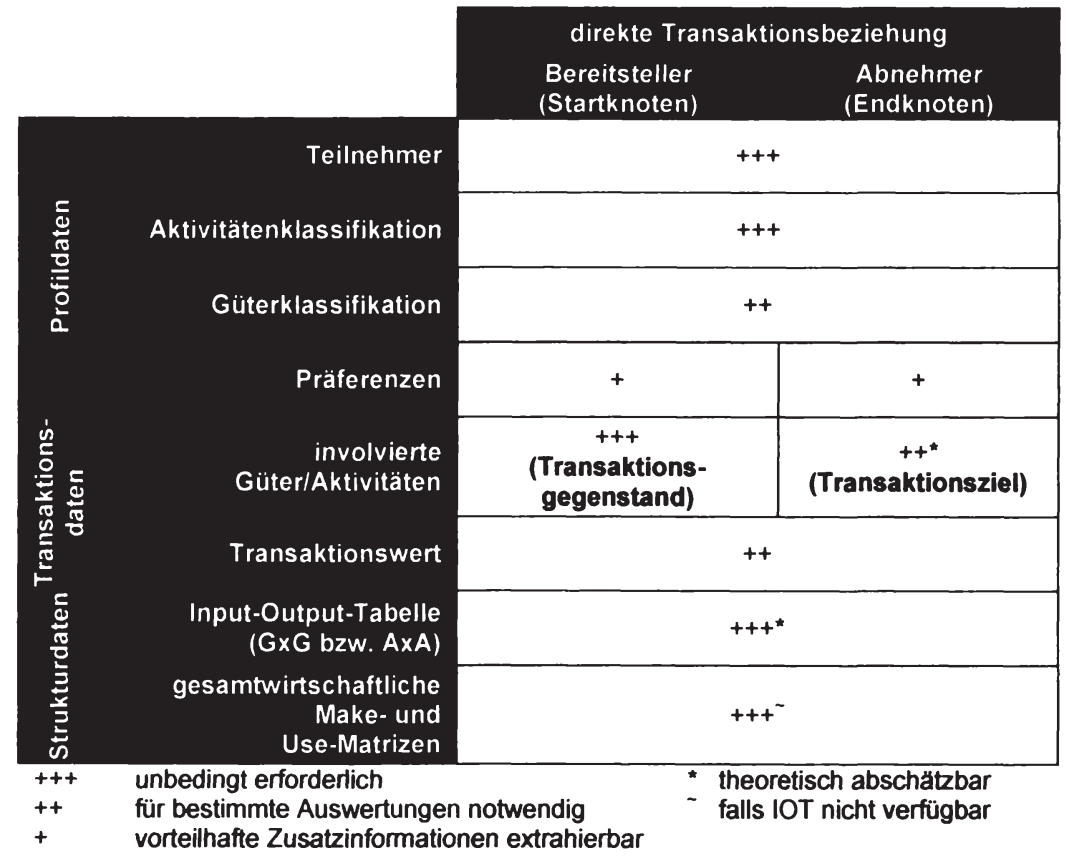

Zur Übersicht über die im Anschluss an dieses Kapitel erfolgende Erläuterung des Modells zur Transaktionssystemunterstützung stellt Abbildung 5 die sechs Modellphasen grafisch dar. 


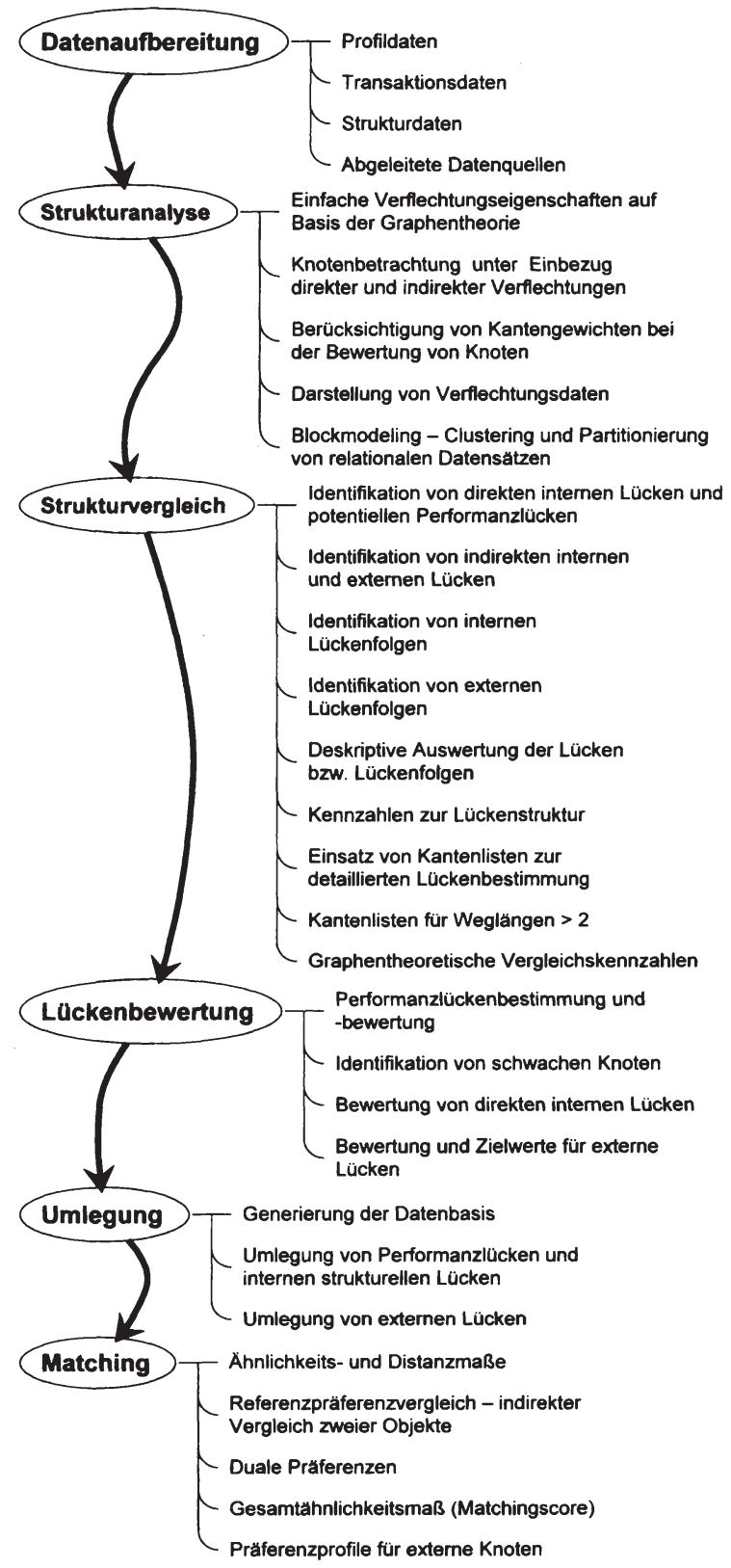

Abbildung 5: Phasen des Modells 
Ausgehend von der Phase der Datenaufbereitung (Kapitel 5) werden in der Strukturanalysephase (Kapitel 6) grundsätzliche Eigenschaften der Transaktionsbeziehungen untersucht und visualisiert. Danach erfolgt in der Strukturvergleichsphase (Kapitel 7) eine Gegenüberstellung der gesamtwirtschaftlichen Verflechtungsdaten mit den Transaktionsdaten der Plattform, die die Identifikation von strukturellen Lücken in den Transaktionsbeziehungen auf der Ebene der Plattform (güter- oder aktivitätsbezogen) ermöglicht. In der Lückenbewertungsphase (Kapitel 8) wird darauf aufbauend die Bedeutung der identifizierten Lücken bzw. die Priorität der Schließung dieser Lücken ermittelt. In der Umlegungsphase (Kapitel 9) werden die gewonnen Erkenntnisse von der Ebene der Plattorm bezogen auf Güter oder Aktivitäten auf die Ebene der Plattformteilnehmer transferiert, um auch teilnehmerbezogene Empfehlungen ableiten zu können. Abschließend wird in der Matchingphase (Kapitel 10) unter Einsatz der Präferenzdaten für jede generierte Transaktionsempfehlung zusätzlich ein Maß für die Transaktions(n)eig(n)ung der jeweiligen Transaktionspartner ermittelt. Jede dieser sechs Phasen wird in den folgenden sechs Kapiteln detailliert beschrieben. Zusätzlich bietet Appendix A eine Übersicht über die für das Modell implementierten Funktionen, während Appendix B eine Kurzbeschreibung der verwendeten Aktivitäten- sowie Güteridentifikatoren bereitstellt. 
Michael Weber - 978-3-631-75376-7

Downloaded from PubFactory at 01/11/2019 05:44:37AM

via free access 


\section{Datenaufbereitungsphase}

Die interne Datensammlung und die externe Datenerhebung sind die Grundvoraussetzungen für die Anwendbarkeit des Modells und bilden dementsprechend eine kritische Eingangsphase, die die Aussagekraft der über das Modell zu generierenden Empfehlungen entscheidend prägt. Vorkehrungen zur Beschaffung von validen und reliablen Daten sowie Prozeduren zur Beibehaltung einer möglichst hohen Datenqualität sind daher - wie bei vielen anderen Modellen auch - unerlässlich, werden allerdings bei der nun folgenden Beschreibung des Modells nicht weiter behandelt, nachdem hierzu bereits verschiedenste Literaturbeiträge vorliegen [Groves et al. 2004; Lyberg et al. 1997; Kish 1965].

Aufbauend auf der Datenbeschaffung kann die Aufbereitung der Daten beginnen. Die Profildaten, Transaktionsdaten, Produktionsstrukturdaten sowie abgeleitete Datenquellen, die in das Modell einfließen, werden nachfolgend beschrieben. Tabelle 19 fasst die für die Datendefinition zentralen Variablen und Indizes zwecks Übersicht zusammen.

Tabelle 19: Variablen- und Indexübersicht

\begin{tabular}{|c|c|c|c|}
\hline & Transaktionsdaten & Strukturdaten & Variablenname \\
\hline Aktivitäten & $a=1, \ldots, n a$ & $y=1, \ldots, z a$ & $A c t / A c t V W$ \\
\hline Güter & $c=1, \ldots, n g$ & $x=1, \ldots, z g$ & Com/ComVW \\
\hline Unternehmen & $b=1, \ldots, n u$ & - & Biz (Buy, Sell) \\
\hline
\end{tabular}

\subsection{Profildaten}

Die Profildaten der Teilnehmer an einer Kollaborationsplattform werden in der Tabelle BCIprofile (Formel (22)) abgelegt. BCIprofile besteht aus je einer Spalte für Aktivitäten, Güter und Unternehmen (Teilnehmer). Die Aktivitäten sind dabei den Unternehmen und die Güter wiederum den dazugehörigen Aktivitäten zugeordnet und können dementsprechend mehrmals pro Teilnehmer vorkommen. Die Klassifikation der Aktivitäten und Güter ist an der Klassifikation der Aktivitäten 
und Güter in der Produktionsstruktur ausgerichtet. Die in Formel (20) definierten Listen ComVW bzw. ActVW beinhalten sämtliche klassifizierten Güter bzw. Aktivitäten der betrachteten Produktionsstruktur. Com und Act (Formel (21)) sind die entsprechenden Güter- bzw. Aktivitätenlisten der Kollaborationsplattform die als Ausschnitt der Klassifikation auf Produktionsstrukturebene definiert sind. Biz ist ein Verzeichnis aller an der Plattform teilnehmenden Unternehmen.

$$
\begin{aligned}
& C o m V W=\left(c o m v w_{x}\right)_{x=1, \ldots, z g} \\
& A c t V W=\left(a c t v w_{y}\right)_{y=1, \ldots, 2 a} \\
& A c t=\left(a c t_{a}\right)_{a=1, \ldots, n a}, A c t \subseteq A c t V W \\
& \operatorname{Com}=\left(\operatorname{com}_{c}\right)_{c=1, \ldots, n g}, \mathrm{Com} \subseteq \mathrm{ComVW} \\
& B i z=\left(b i z_{b}\right)_{b=1, \ldots, n u} \\
& \text { BCIprofile }=\left(\text { act }_{h, a}, \operatorname{com}_{h, c}, b i z_{h, b}\right)_{h=1, \ldots, a g u} \\
& \text { wobei } \\
& \operatorname{act}_{h, a}=a_{c t} \in A c t \\
& \operatorname{com}_{h, c}=\operatorname{com}_{c} \in C o m \\
& b_{h, b}=b i z_{b} \in B i z \\
& \forall a \in\{1, \ldots, n a\}:\left(\exists h \in\{1, \ldots, a g u\}:\left(\text { act }_{h, a}, \operatorname{com}_{h, c}, \text { biz }_{h, b}\right) \in \text { BCIprofile }\right) \\
& \forall c \in\{1, \ldots, n g\}:\left(\exists h \in\{1, \ldots, a g u\}:\left(\text { act }_{h, a}, \text { com }_{h, c}, \text { biz }_{h, b}\right) \in \text { BCIprofile }\right) \\
& \forall b \in\{1, \ldots, n u\}:\left(\exists h \in\{1, \ldots, a g u\}:\left(\text { act }_{h, a}, \operatorname{com}_{h, c}, \text { biz }_{h, b}\right) \in \text { BCIprofile }\right)
\end{aligned}
$$

\subsection{Transaktionsdaten}

Die Transaktionsdaten der Kollaborationsplattform werden mit Bezugnahme auf Güter in der Transaktionsliste BCledgeFRuug (Formel (23)) bzw. bezogen auf Aktivitäten in der Transaktionsliste BCledgeFRuua (Formel (24)) festgehalten. Diese Tabellen enthalten eine Spalte entweder für Aktivitäten oder für Güter und weiters je eine Spalte für den Käufer, den Transaktionswert und den Verkäufer. 
Jeder Eintrag in BCledgeFRuug bzw. BCledgeFRuua entspricht den kumulierten Transaktionen zwischen einem Käufer und einem Verkäufer im Hinblick auf ein Gut bzw. eine Aktivität auf der Kollaborationsplattform für den betrachteten Zeitraum. Bedingungen, die für beide Transaktionslisten gelten, sind, dass das Profil eines Teilnehmers alle Güter bzw. Aktivitäten umfasst, zu denen Transaktionen des Teilnehmers vorliegen und dass der Wert einer Gütertransaktion den Wert der korrespondierenden Aktivitätentransaktion nicht überschreiten kann. Für jene Fälle, in denen die Transaktionsinhalte nicht erhoben und über BCledgeFRuug bzw. BCledgeFRuua verfügbar gemacht werden können, wäre es denkbar, dass unter gewissen Annahmen mittels der Profildaten und womöglich auch der Strukturdaten eine Schätzung der Transaktionsinhalte auf der Kollaborationsplattform erfolgt. Eine Voraussetzung für diese Zuschätzung ist, dass zumindest die Transaktionsbeziehungen zwischen den Teilnehmern (im Sinne der weiter unten vorgestellten Matrix BCIuu) bekannt sind.

$$
\begin{aligned}
& \text { BCledgeFRuug }=\left(\text { bciedgefruug }_{\mathrm{gg}}\right)_{\mathrm{tg}=1, \ldots, \mathrm{G}} \\
& \text { bciedgefruug }_{\mathrm{tg}}=\left(\text { com }_{\mathrm{tg}, \mathrm{g}}, \text { buy }_{\mathrm{tg}, \mathrm{k}}, \text { val }_{\mathrm{tg}}, \text { sell }_{\mathrm{tg}, \mathrm{v}}\right) \\
& \text { wobei } \\
& \text { com }_{\mathrm{tg}, \mathrm{g}}=\text { com }_{\mathrm{g}} \in \mathrm{Com} \\
& b u y_{\mathrm{tg}, k}=\mathrm{biz}_{k} \in B i z, \text { sell }_{\mathrm{tg}, v}=b i z_{v} \in B i z, b u y_{\mathrm{tg}, k} \neq \text { sell }_{\mathrm{tg}, v} \\
& \text { val }_{\text {tg }}>0 \\
& \forall \text { bciedgefruug }{ }_{\mathrm{tg}}:\left(\exists l \in\{1, \ldots, a g u\}:\left(\operatorname{com}_{\mathrm{tg}, g}=\operatorname{com}_{l, g}\right) \wedge\left(\operatorname{sell}_{\mathrm{tg}, v}=\operatorname{biz}_{l, v}\right)\right. \\
& \left.\wedge\left(\left(\text { act }_{l, a}, \operatorname{com}_{l, g}, \text { biz }_{l, v}\right) \in \text { BCIprofile }\right)\right) \\
& \forall \text { bciedgefruug }_{\mathrm{tg}}:\left(\exists \text { bciedgefruua }_{t a}:\left(\text { buy }_{\mathrm{tg}, \mathrm{k}}=\text { buy }_{\mathrm{ta}, \mathrm{k}}\right) \wedge\left(\text { sell }_{\mathrm{tg}, v}=\operatorname{sell}_{\mathrm{a}, v}\right)\right. \\
& \left.\wedge\left(v a l_{t g} \leq v a l_{t a}\right)\right)
\end{aligned}
$$




$$
\begin{aligned}
& \text { BCledgeFRuua }=\left(\text { bciedgefruua }_{t a}\right)_{t a=1, \ldots, t A} \\
& \text { bciedgefruua }_{t a}=\left(\text { act }_{t a, a}, \text { buy }_{t a, k}, v_{a l}, \text { sell }_{t a, v}\right) \\
& \text { wobei } \\
& a c t_{t a, a}=a c t_{a} \in A c t \\
& \text { buy }_{t a, k}=b i z_{k} \in B i z, \text { sell }_{t a, v}=b i z_{v} \in B i z, b u y_{t a, k} \neq \text { sell }_{t a, v} \\
& v_{a l}>0 \\
& \forall b \text { ciedgefruua } a:\left(\exists l \in\{1, \ldots, a g u\}:\left(\text { act }_{t a, a}=\operatorname{act}_{l, a}\right) \wedge\left(\operatorname{sell}_{t a, v}=b i z_{l, v}\right)\right. \\
& \left.\wedge\left(\left(\text { act }_{l, a}, \operatorname{com}_{l, c}, \text { biz }_{l, v}\right) \in \text { BCIprofile }\right)\right)
\end{aligned}
$$

\subsection{Strukturdaten}

Je nach gewählter Quelle können Daten zur Produktionsstruktur in verschiedensten Formen vorliegen. Als Make-Matrix VWmake, als Use-Matrix VWuse, oder als Input-Output-Tabelle bezogen auf Güter IOTgg (über die Funktion ALMON) bzw. auf Aktivitäten IOTaa. Die beiden letztgenannten Tabellen können über die Make- und Use-Matrix generiert werden. Ebenso wäre es aber auch denkbar, dass IOTgg bzw. IOTaa Ergebnis von Stücklistenerhebungen bezogen auf Güter bzw. Aktivitäten sind, für die eine passende Klassifikation eingesetzt wird. Es ist darauf hinzuweisen, dass auf Basis der Produktionsstrukturdaten die Anteilsmatrizen der Make- und Use-Systematik sowie die Matrix der Inputkoeffizienten und die Leontief-Inverse entsprechend der obigen Ausführungen berechnet werden können. Darüber hinaus ist an dieser Stelle zu betonen, dass bei der Verwendung von Daten aus der Volkswirtschaftsstatistik eine Modifikation der grundlegenden Tabellen im Hinblick auf die Zwecke dieser Arbeit, in der Produktionsstrukturdaten für den Vergleich mit und zur Optimierung von realen Transaktionsdaten herangezogen werden, stattfinden muss. Dies bedeutet, dass z.B. Bewertungskonzepte, spezifische Annahmen oder unterstellte Güter bzw. Aktivitäten in den in Frage kommenden Input-Output-Statistiken sorg- 
fältig auf ihre Eignung hin überprüft und aus- oder abgewählt werden müssen. In diesem Zusammenhang ist weiters auf die Notwendigkeit des spezifischen Umgangs mit Teilnehmern einer Kollaborationsplattform, die eine Sonderrolle im Produktionsablauf einnehmen, - insbesondere Handelsunternehmen - bei der Transaktionsunterstützung bzw. Vorschlagsgenerierung hinzuweisen. Dieser Rolle kann bspw. durch eine ausschließliche Zuweisung der generierten inputseitigen Empfehlungen auf die Produzenten und der outputseitigen Empfehlungen auf die Händler eines Gutes Rechnung getragen werden, wobei die wegfallenden Empfehlungen zu out- bzw. inputseitigen Transaktionen durch spezifische Empfehlungen zu Händlern bzw. Produzenten eines Gutes kompensiert werden müssten.

$$
\begin{gathered}
\text { VWmake }=\left(\text { vwmake }_{y x}\right)_{\substack{y=1, \ldots, z a \\
x=1, \ldots, z g}} \text { VWuse }=\left(\text { vwuse }_{x y}\right)_{\substack{x=1, \ldots, z g \\
y=1, \ldots, z a}} \\
\text { IOTgg }=\left(\text { iotgg }_{x y}\right)_{x, y=1, \ldots, z g} \\
\text { IOTaa }=\left(\text { iotaa }_{x y}\right)_{x, y=1, \ldots, z a}
\end{gathered}
$$

\subsection{Abgeleitete Datenquellen}

Aus den Teilnehmerprofilen, den Transaktionsdaten und den Daten zu der Produktionsstruktur werden nachfolgend weitere für das Modell erforderliche Datenquellen abgeleitet.

\section{BCluu - Matrix der Teilnehmerverflechtungen}

BCluu ist die Matrix der Verflechtungen zwischen den Teilnehmern der Kollaborationsplattform und liegt in der Dimension Unternehmen $x$ Unternehmen vor. BCluu zeigt sozusagen einen auf die Plattform bezogenen Teilausschnitt der Marktstruktur und abstrahiert von den Informationen zu Gütern oder Aktivitäten. Die Matrix kann über die Transaktionsdaten generiert werden, indem über die 
Güter bzw. Aktivitäten, die Gegenstand der Transaktionen zwischen zwei Unternehmen sind, aggregiert wird. Formel (29) zeigt die Bestimmung von BCIuU anhand von BCledgeFRuug. Alternativ kann BCIuu auch über BCledgeFRuua nach einer dementsprechenden Adaption von Formel (29) ermittelt werden. Voraussetzung zur Bestimmung der Matrix BCluu sind neben BCledgeFRuug bzw. BCledgeFRuna die Profildaten der Teilnehmer aus BCIprofile. Sollten keine Daten zu den Transaktionsinhalten vorhanden sein, können die beobachteten Verflechtungen zwischen den Teilnehmern unter Verwendung der Profildaten und mitunter auch der Strukturdaten Ausgangspunkt für die Generierung von BCledgeFRuug bzw. BCledgeFRuua sein. Die Funktion, die zur Berechnung von BCluu gemäß Formel (29) aufgerufen werden kann, heißt MAKEBCIMAT.

$$
\begin{aligned}
\text { BCIuu } & =\left(\text { bciuu }_{r s}\right)_{r, s=1, \ldots, m u} \\
\text { bciuu }_{r s} & =\sum_{t=1}^{g r s} \text { val }_{t} \\
\text { mit val } & \in \text { bciedgefruug }_{t}^{a g g(i, r s} \in \text { BCledgeFRuug } \\
\text { wobei für } r, s & =1, \ldots, \text { nu gilt }: \\
\text { BCledgeFRuug } & =\left(\text { bciedgefruug }_{t}^{a g g(i, r s}\right)_{t=1, \ldots, g r s}= \\
& =\left(\text { bciedgefruug }_{t}: \text { buy }_{t, r}=b_{i z} \wedge \text { sell }_{t, s}=\text { biz }_{s}\right)_{t=1, \ldots, g r s}= \\
& =\left(\text { com }_{t, g}, \text { buy }_{t, r}, \text { val }_{t}, \text { sell }_{t, s}\right)_{t=1, \ldots, g r s} \\
\text { biz }_{r}, \text { biz }_{s} & \in \text { Biz }^{\text {agi }}
\end{aligned}
$$

\subsection{1 ,Sell'-Matrizen}

Die zu der Idee der ,Make'-Matrix analogen ,Sell'-Matrizen, die die Herkunft der auf der Kollaborationsplattform angebotenen Leistungen beschreiben, können auf der Ebene der Kollaborationsplattform unter Berücksichtigung der anbietenden Teilnehmer bestimmt werden. Hierzu wird in den Formeln (30), (31) und (32) über die Käufer von Gütern bzw. die Abnehmer von Leistungen aus bestimmten Aktivitäten auf Basis der Transaktionsdaten aggregiert. Für das Modell wichtige ,Sell'-Matrizen sind BCIug, BCIaug und BCIua. Voraussetzung zu deren Ge- 
nerierung sind die Transaktionsdaten aus BCledgeFRuug bzw. BCledgeFRuua sowie die Profildaten aus BCIprofile.

\section{BClug - Matrix der abgesetzten Güter}

BCIug bringt in Matrixform die auf der Plattorm als Verkäufer agierenden Teilnehmer mit den von innen verkauften Gütern in Verbindung. Sie liegt in der Dimension Unternehmen $\times$ Güter vor und ist folglich eine Sell-Matrix für die Güterperspektive. BClug kann über die Funktion MAKEBCIMAT gemäß Formel (30) ermittelt werden.

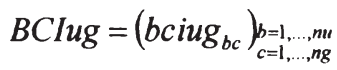

$$
\begin{aligned}
& \text { bciug }_{b c}=\sum_{i=1}^{b b c} v a l_{,} \\
& \text {mit val, } \in \text { bciedgefruug }{ }_{1}^{a g g B, b c} \in B \text { CledgeFRung }{ }^{a g g B, b c} \\
& \text { wobei für } b=1, \ldots, \text { nu und } c=1, \ldots, \text { ng gilt : } \\
& \text { BCledgeFRuug }{ }^{a g g B, b c}=\left(\text { bciedgefruug } g_{t}^{a g g B, b c}\right)_{t=1, \ldots, b b c}= \\
& =\left(\text { bciedgefruug }_{,}: \text {sell }_{t, b}=\text { biz }_{b} \wedge \operatorname{com}_{t, c}=\operatorname{com}_{c}\right)= \\
& =\left(\text { com }_{t, c}, \text { buy }_{t, k}, \text { val }_{t}, \text { sell }_{t, b}\right)_{t=1, \ldots, b b c} \\
& b i z_{b} \in B i z \\
& \operatorname{com}_{c} \in \mathrm{Com}
\end{aligned}
$$

\section{BClaug - Matrix der abgesetzten Güter mit Aktivitätsbezug}

BCIaug ist eine Erweiterung der Matrix BClug. Sie zeigt die Verkäufe von Gütern ebenso an wie die Aktivität, aus der das jeweilige, vom Plattformteilnehmer verkaufte Gut entstammt. Die Dimensionierung der Matrix BClaug lautet demzufolge Aktivitäten-Unternehmen x Güter. Hinter der in Formel (32) dargestellten Ermittlung von BClaug steckt die Annahme, dass jedes Unternehmen ein Gut nur in der dazugehörigen Aktivität herstellt. Sollte diese Annahme nicht zutreffen, so wäre Formel (32) insoweit anzupassen, als eine - bspw. proportionale - Verteilung des betrachteten Gutes auf dieMdaseGuebbereitstellendén 7 Aktivitäten 
erfolgen würde. Grundsätzlich sollte diese Anpassung allerdings nicht notwendig sein. Für jene Fälle, in denen ein Gut nicht über die Profildaten des das Gut bereitstellenden Teilnehmers einer Aktivität zugeordnet werden kann, besteht auch die Möglichkeit, die Strukturdaten zur Zuweisung eines Gutes heranzuziehen. Die Funktion MAKEBCIAUG kann zur Generierung von BClaug eingesetzt werden.

$$
\begin{aligned}
& \text { BClaug }=\left(\text { bciaug }_{u c}\right)_{\substack{u=1, \ldots, \text { nau } \\
c=1, \ldots, \text {, }}} \\
& \text { bciaug }_{u c}=\sum_{t=1}^{b u c} v a l_{1}
\end{aligned}
$$

mit val,$\in$ bciedgefruug, ${ }_{1}^{a g g B, P, u c} \in$ BCledgeFRuug ${ }^{a g g B, P, u c}$ wobei für $u=1, \ldots$, nau und $c=1, \ldots$, ng gilt :

BCledgeFRuugaggB,P,uc $=\left(\text { bciedgefruug }{ }_{t}^{a g g B, P, u c}\right)_{t=1, \ldots, b u c}=$

$$
\begin{aligned}
=( & \left(\text { com }_{t, c}, \text { buy }_{t, k}, \text { val }_{t}, \text { sell }_{t, b}, \text { sellact }_{t, a}\right): \\
& \left(\text { com }_{t, c}, \text { buy }_{t, k}, \text { val }_{t}, \text { sell }_{t, b}\right) \in \text { BCledgeFRuug } \\
& \wedge \operatorname{sell}_{t, b}=\text { biz }_{b}=\text { biz }_{h, b} \wedge \operatorname{com}_{t, c}=\operatorname{com}_{c}=\operatorname{com}_{h, c} \\
& \wedge\left(\text { sellact }_{t, a}, \text { sell }_{t, b}\right)=\left(\text { act }_{a}, \text { biz }_{b}\right)_{u}=\left(\text { act }_{h, a}, \text { biz }_{h, b}\right) \\
& \left.\wedge\left(\text { act }_{h, a}, \operatorname{com}_{h, c}, \text { biz }_{h, b}\right) \in \text { BCIprofile }\right)
\end{aligned}
$$

$$
\begin{aligned}
\text { biz }_{b} \in & \text { Biz } \\
\text { ActBiz }= & \left(\text { act }_{a}, \text { biz }_{b}\right)_{u=1, ., \text { nau }} \\
= & \left(\left(\text { act }_{a}, \text { biz }_{b}\right):\left(\text { act }_{a}, \text { biz }_{b}\right)=\left(\text { act }_{h, a}, \text { biz }_{h, b}\right)\right. \\
& \left.\wedge\left(\text { act }_{h, a}, \text { com }_{h, c}, \text { biz }_{h, b}\right) \in \text { BCIprofile }\right)
\end{aligned}
$$

\section{BClua - Matrix der beanspruchten Aktivitäten}

Die Matrix BCIua zeigt welchen Aktivitäten die Leistungen der Teilnehmer, die über die Kollaborationsplattform abgewickelt werden, zuzuordnen sind. Die 
Dimension dieser Sell-Matrix in der Aktivitätsbetrachtung ist Unternehmen $\mathbf{x}$ Aktivitäten. Die Funktion MAKEBCIMAT dient auch zur Ermittlung von BClua entsprechend der Formel (32).

$$
\begin{aligned}
& \text { BCIua }=\left(\text { bciua }_{b a}\right)_{\substack{b=1, \ldots, n u \\
a=1, \ldots, n a}} \\
& \text { bciua }_{b a}=\sum_{i=1}^{b b a} v a l_{t} \\
& \text { mit val }, \in \text { bciedgefruua } a_{t}^{a g g B, b a} \in B C \text { IedgeFRuna } a^{a g g B, b a} \\
& \text { wobei für } b=1, \ldots, \text { nu und } a=1, \ldots, \text { na gilt : } \\
& \text { BCledgeFRuua }{ }^{a g g B, b a}=\left(\text { bciedgefruua } a^{a g g B, b a}\right)_{1=1, \ldots, b b a}= \\
& =\left(\text { bciedgefruua }_{t}: \text { sell }_{t, b}=\text { biz }_{b} \wedge \text { act }_{t, a}=a c t_{a}\right)= \\
& =\left(\text { act }_{t, c}, \text { buy }_{t, k}, \text { val }_{t}, \text { sell }_{t, b}\right)_{t=1, \ldots, b a} \\
& b i z_{b} \in B i z \\
& a c t_{a} \in A c t
\end{aligned}
$$

\subsection{2 ,Buy'-Matrizen}

Komplementär zu den Sell-Matrizen ist auch die Betrachtung der Ziele der über die Kollaborationsplattform bezogenen Leistungen für das Modell von Interesse. Diese in Analogie zur Use-Matrix definierten ,Buy'-Matrizen stellen den Kauf von Gütern bzw. den Bezug von Leistungen aus Aktivitäten in den Vordergrund. Zur Erstellung dieser Matrizen werden die Transaktionsdaten über die Verkäufer aggregiert und der Bezug von Gütern bzw. die in Anspruch genommenen Aktivitäten der an der Plattform teilnehmenden Unternehmen festgehalten. In diesem Zusammenhang wichtige Buy-Matrizen sind BCIgu sowie BClau. Voraussetzung zur Berechnung dieser Matrizen sind Transaktionsdaten (BCledgeFRuug bzw. BCledgeFRuua) und Profildaten (BCIprofile).

\section{BClgu - Matrix der Güterabnahme}

BCIgu stellt die Abnehmer (Unternehmen) der auf der Kollaborationsplattform angebotenen Güter sowie den jeweiligen Transaktionswert dar und hat die Di-

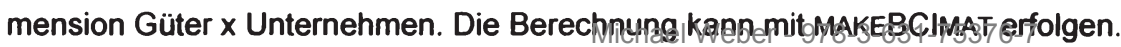




$$
\begin{aligned}
B C I g u & =\left(b c i g u_{c b}\right)_{\substack{c=1, \ldots, \ldots, n \\
b=1, \ldots, n u}} \\
b c i g u_{c b} & =\sum_{t=1}^{s c b} v a l_{t}
\end{aligned}
$$

mit val, $\in$ bciedgefruug, ${ }^{a g g S, c b} \in B$ CledgeFRung ${ }^{a g g S, c b}$

wobei für $c=1, \ldots, n g$ und $b=1, \ldots, n u$ gilt :

$$
\begin{aligned}
\text { BCledgeFRuug }{ }^{a g g S, c b} & =\left(\text { bciedgefruug }_{t}^{a g g S, c b}\right)_{t=1, \ldots, s c b}= \\
& =\left(\text { bciedgefruug }_{t}: \text { buy }_{t, b}=\text { biz }_{b} \wedge \text { com }_{t, c}=\text { com }_{c}\right)= \\
& =\left(\text { com }_{t, c}, \text { buy }_{t, b}, \text { val }_{t}, \text { sell }_{t, v}\right)_{t=1, \ldots, s c b} \\
\operatorname{biz}_{b} & \in \text { Biz } \\
\operatorname{com}_{c} & \in \text { Com }
\end{aligned}
$$

\section{BClau - Matrix der Inanspruchnahme von Aktivitäten}

Während BCIgu die Güterbetrachtung ermöglicht, liefert BCIau die Aktivitätenperspektive zu den über die Verkäufer aggregierten Verkäufen auf Basis der Transaktionsdaten der Kollaborationsplattform. Sie zeigt also, von welchen Aktivitäten Leistungen in welchem Ausmaß auf der Plattform von deren Teilnehmern bezogen wurden. Die Dimension der Matrix BCIau ist Aktivitäten $\mathrm{x}$ Unternehmen. Die Funktion MAKEBCIMAT ermöglicht die Generierung von BCIau.

$$
\begin{aligned}
\text { BClau } & =\left(\text { bciau }_{a b}\right)_{\substack{a=1, \ldots, \ldots a \\
b=1, \ldots, m}} \\
\text { bciau }_{a b} & =\sum_{t=1}^{s a b} v_{t} a l_{t} \\
\text { mit val }_{t} & \in \text { bciedgefruua }_{t}^{a g g S, a b} \in \text { BCledgeFRuua }^{a g g S, a b}
\end{aligned}
$$

$$
\text { wobei für } a=1, \ldots, \text { na und } b=1, \ldots, \text {, nu gilt : }
$$

$$
\begin{aligned}
\text { BCledgeFRuna }^{a g g S, a b} & =\left(\text { bciedgefruua }_{t}^{a g g s, a b}\right)_{t=1, \ldots, \text { sab }}= \\
& =\left(\text { bciedgefruua }_{t}: \text { buy }_{t, b}=\text { biz }_{b} \wedge \text { act }_{t, a}=\text { act }_{a}\right)= \\
& =\left(\text { act }_{t, a}, \text { buy }_{t, b}, \text { val }_{t}, \text { sell }_{t, v}\right)_{t=1, \ldots, \text { sab }} \\
\operatorname{biz}_{b} & \in \text { Biz } \\
\operatorname{act}_{a} & \in \text { Act }
\end{aligned}
$$




\subsubsection{Erweiterungen der ,Buy’-Matrizen}

Anschließend an die Erstellung der grundlegenden Buy-Matrizen stellt sich im Hinblick auf die Produktionsstruktur der Teilnehmer an der Kollaborationsplattform die Frage, in welche Güter bzw. Aktivitäten die erworbenen Leistungen eingeflossen sind. Zur Beantwortung dieser Fragestellung wird in dieser Arbeit der Ansatz einer zur Produktionsstruktur proportionalen Verteilung verfolgt. Dies bedeutet, dass auf Basis von IOTgg bzw. IOTaa oder aber von VWuse die bezogenen Leistungen auf die Güter bzw. Aktivitäten der an der Plattform teilnehmenden Unternehmen proportional zu deren Produktionsanteil aufgeteilt werden. Die Güter bzw. Aktivitäten, die zur Verteilung herangezogen werden, entstammen dabei aus den Profildaten. Alternativ wäre aber auch eine ausschließliche bzw. stärkere Berücksichtigung der Transaktionen des Teilnehmers auf der Plattform oder - falls entsprechende Zusatzinformationen vorliegen auch der Relationen zwischen den tatsächlichen Produktionsvolumina des Teilnehmers zur Gewichtung der Verteilung einsetzbar. Matrizen, die über diesen Verteilungsansatz gewonnen werden, sind BCIggu, BCIgau und BCIaau. Voraussetzungen zu deren Generierung sind neben IOTgg, VWuse, IOTaa und BCIprofile auch BCIgu sowie BCIau. Alle drei Matrizen benötigen des Weiteren einen auf den jeweiligen Teilnehmer zugeschnittenen Auszug aus den Profildaten (Formel (35)).

$$
\begin{aligned}
{\text { BCIprofile } U_{u n t}} & =\left(\text { bciprofile }_{u n t, s}\right)_{s=1, \ldots, n u p_{u n t}} \quad \text { für unt }=1, \ldots, n u \\
& =\left(\text { biprofile }_{h}: \text { biz }_{h, u n t}=\text { ciz }_{u n t}\right) \\
\text { mit nup }_{u n t} & =\mid \text { CIprofile }_{u n t} \mid
\end{aligned}
$$

\section{BClggu - Güter-Teilnehmer-Güter Matrix}

$B C I g g u$ aus Formel (38) zeigt aufbauend auf den Formeln (35), (36) und (37) die Zuweisung der in BCIgu enthaltenen Transaktionsvolumina auf die von dem jeweiligen Teilnehmer der Kollaborationsplattform (Käufer) bereitgestellten Güter gemäß den Profildaten und der Relationen aus IOTgg. BCIggu hat die Dimen- 
sion Güter x Güter-Unternehmen, wobei die zweite Dimension abhängig von dem Profil des teilnehmenden Unternehmens und der von diesem bereitgestellten Güter ist. Die Funktion ESTIMATEGOA4U dient zur Ermittlung von BCIggu.

$$
\begin{aligned}
& \operatorname{IOTgg}_{u n t}=\left(\operatorname{iotgg}_{u n t, k v}\right)_{\substack{k=1, \ldots, n g \\
o=1, \ldots, n p_{u n t}}} \text { für unt }=1, \ldots, n u \\
& \operatorname{iotgg}_{u n t, k v}=\left\{\begin{array}{cc}
\operatorname{iotgg}_{x y} & \begin{array}{c}
\text { wenn bcigu } \\
\text { kunt }
\end{array} \\
\wedge \operatorname{com}_{o . z}=\operatorname{comvw}_{y} \wedge \operatorname{com}_{k}=\operatorname{comv} w_{x} \\
0 \quad \text { andernfalls }
\end{array}\right. \\
& \text { wobei } \\
& \left(\text { act }_{o, a}, \text { com }_{o, z}, \text { biz }_{o, u n t}\right) \in \text { BCIprofile }_{u n t} \\
& I O T g g_{u n t}^{z n o r m}=\left(\operatorname{iotgg}_{u n t, k o}^{z n o r m}\right)_{\substack{k=1, \ldots, \ldots g \\
o=1, \ldots, n u p_{u n t}}} \text { für unt }=1, \ldots, n u \\
& \operatorname{iotgg}_{u n t, k o}^{z n o m m}=\frac{\operatorname{iotgg}_{u n t, k o}}{\sum_{v=1}^{n u p_{u n t}} \operatorname{iotgg}_{u n t, k v}}
\end{aligned}
$$

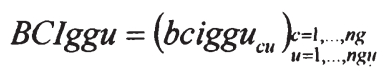

$$
\begin{aligned}
& n g u=\mid\left\{\operatorname{com}_{c} \in \text { Com }: \exists s \in\left\{1, \ldots, \text { nup }_{u n t}\right\}: \text { com }_{c} \in \text { bciprofile }_{u n t, s}\right\} \mid \\
& b c i g g u_{c u}=b c i g u_{c u n t} \cdot \operatorname{iotgg}_{u n t, c l}^{z n o m} \\
& \text { wobei gilt: } \begin{array}{ll}
u n t=1 \text { und } l=u & \text { wenn } u<n u p \\
u=\sum_{b=1}^{u n t-1} \text { nup }_{b}+l & \text { ansonsten }
\end{array}
\end{aligned}
$$

\section{BClgau - Güter-Teilnehmer-Aktivitäten Matrix}

Die Aufteilung der Güter auf die Aktivitäten des jeweils die Güter beziehenden Unternehmens zeigt die Matrix BCIgau aus Formel (41), die aufbauend auf den Formeln (35), (39) und (40) generiert wird. BCIgau wird im Gegensatz zu BCIggu unter Einsatz von VWuse ermittelt. VWuse ermöglicht dabei eine einfache (proportionale) Zuordnung der bezogenen Güter zu den jeweils in Frage kommenden Aktivitäten. Diesem Vorteil steht allerdings ein möglicherweise auftretender Nach-

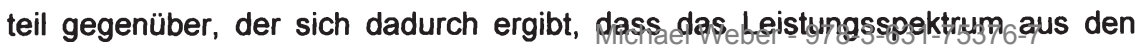


Aktivitäten des Unternehmens sich nicht notwendigerweise gänzlich mit dem Leistungsspektrum der Aktivitäten aus VWuse deckt. Dies bewirkt die Möglichkeit einer gewissen Unschärfe in den Relationen zwischen den zu dem Unternehmen gehörigen Aktivitäten, die insbesondere bei einem geringeren Detaillierungsgrad der Klassifikationen zunimmt und in solchen Fällen als problematisch anzusehen ist. Ein höherer Detaillierungsgrad würde im Gegensatz die Wahrscheinlichkeit der Kongruenz von Aktivitäten erhöhen. In diesem Zusammenhang ist auch hervorzuheben, dass grundsätzlich immer überlegt werden sollte, ob die durch die Wahl einer Methode implizit getroffenen Annahmen (z.B. Technologieannahmen) mit dem jeweiligen Einsatzbereich im Einklang stehen. BCIgau hat die Dimension Güter x Aktivitäten-Unternehmen. Die letztgenannte Dimension wird hierbei von den Aktivitäten der Unternehmen laut Profil bestimmt. BCIgau kann durch den Aufruf der Funktion von ESTIMATEGOA4U generiert werden.

$$
\begin{aligned}
& \text { VWuse }_{\text {unt }}=\left(\text { vwuse }_{\text {unt,ko }}\right)_{\substack{k=1, \ldots, \ldots, o=1, \ldots, n u p_{u n t}}} \text { für unt }=1, \ldots, n u \\
& \text { mit nuap }_{u n t}=\mid\left\{\text { act }_{r, s}:\left(\text { act }_{r, s}, \operatorname{com}_{r, i}, \text { biz }_{r, u n t}\right) \in \text { BCIprofile } U_{u n t}\right\} \mid \\
& \text { vwuse }_{u n t, k o}=\left\{\begin{array}{cc}
\text { wwuse }_{x y} & \text { wenn bciau }_{k u n t}>0 \wedge \operatorname{com}_{k}=c o m v w_{x} \\
& \wedge a c t_{o, z}=a c t v w_{y} \wedge b_{o, u n t}=b i z_{u n t} \\
0 & \text { andernfalls }
\end{array}\right. \\
& \text { wobei } \\
& \left(\text { act }_{o, z}, \text { com }_{o, c}, \text { biz }_{o, u n t}\right) \in{\text { BCIprofile } U_{u n t}} \\
& V W u s e_{u n t}^{z n o r m}=\left(\text { vwuse }_{u n t, k o}^{z n o r m}\right)_{\substack{k=1, \ldots, \ldots \\
o=1, \ldots, \text { map }}} \text { für unt }=1, \ldots, n u \\
& v w u s e_{u n t, k o}^{z n o r m}=\frac{\text { vwuse }_{u n t, k o}}{\sum_{v=1}^{\text {muap }} v_{u n t} v w u s e_{u n t, k v}}
\end{aligned}
$$




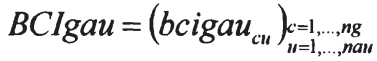

$$
\begin{aligned}
& \text { bcigau }_{c u}=b c i a u_{c u n t} \cdot v w u s e_{u n t, c l}^{z n o r m} \\
& \text { wobei gilt: } \text { unt }=1 \text { und } l=u \quad \text { wenn } u<\text { nuap }_{1} \\
& u=\sum_{b=1}^{u n t-1} \text { nuap }_{b}+l \text { ansonsten }
\end{aligned}
$$

\section{BClaau - Aktivitäten-Teilnehmer-Aktivitäten Matrix}

Analog zu der Ermittlung der Matrix BClggu für die Güterperspektive ist das Vorgehen bei der Ermittlung von BClaau für die Aktivitätenbetrachtung, bei der gemäß den Formeln (42), (43) und (44) über IOTaa die Relationen zwischen den Aktivitäten festgestellt werden. BClaau hat die Dimension Aktivitäten $\times$ Aktivitäten-Unternehmen, wobei letztere von den Aktivitäten, die den Teilnehmern über deren Profil zuordenbar sind, abhängt. BClaau kann über die Funktion ESTIMATEGOA4U berechnet werden.

$$
\begin{aligned}
& \operatorname{IOTaa}_{\text {unt }}=\left(\text { iotaa }_{\text {unt }, k o}\right)_{\substack{k=1, \ldots, \text {, na } \\
o=1, \ldots, \text { naap }_{\text {unt }}}} \text { für unt }=1, \ldots, n u
\end{aligned}
$$

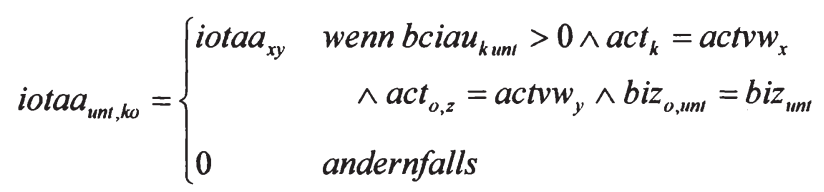

$$
\begin{aligned}
& \text { wobei } \\
& \left(\text { act }_{o, z}, \text { com }_{o, c}, \text { biz }_{o, u n t}\right) \in \text { BCIprofile }_{u n t} \\
& I O T a a_{u n t}^{z n o r m}=\left(\text { iotaa }_{u n t, k o}^{z n o m m}\right)_{\substack{k=1, \ldots, n a \\
o=1, \ldots, \text { nuap }}} \text { für unt }=1, \ldots, n u \\
& \operatorname{iotaa}_{u n t, k o}^{z n o r m}=\frac{\operatorname{iotaa}_{u n t, k o}}{\sum_{v=1}^{\text {nuap }} \text { iotant }_{u n t, k v}}
\end{aligned}
$$




$$
\begin{aligned}
& \text { BCIaau }=\left(\text { bciaau }_{a u}\right)_{\substack{a=1, \ldots, n a \\
u=1, \ldots, n a u}} \\
& n a u=\mid\left\{\text { act }_{a} \in \text { Act }: \exists s \in\left\{1, \ldots, \text { nup }_{u n t}\right\}: \text { act }_{a} \in \text { bciprofile }_{u n t, s}\right\} \mid \\
& \text { bciaau }_{a u}=b c i a u_{a u n t} \cdot \operatorname{iotaa}_{u n t, a l}^{\text {znorm }} \\
& \text { wobei gilt: } \text { unt }=1 \text { und } l=u \quad \text { wenn } u<\text { nuap }_{1} \\
& u=\sum_{b=1}^{u n t-1} \text { nuap }_{b}+l \text { ansonsten }
\end{aligned}
$$

\subsubsection{BCliot - Input-Output-Relationen auf der Kollaborationsplattform}

Zur Darstellung von Input-Output-Beziehungen auf der Kollaborationsplattform zwischen Gütern, zwischen Aktivitäten aber auch zwischen Gütern und Aktivitäten, die als Grundlage für einen späteren Vergleich mit dazu passenden gesamtwirtschaftlichen Produktionsstrukturen dienen sollen, ist eine weitere Aggregation von BCIggu, BClaau aber auch BClaug und BCIgau erforderlich. Die Aggregation erfolgt hierbei über die an der Kollaborationsplattform teilnehmenden Unternehmen und führt zu den Matrizen BCIgg, BCIaa, BClsell und BCIbuy.

\section{BClgg}

BCIgg zeigt die - zum Teil im Zuge der Aufteilung gemäß der Produktionsstruktur unterstellten - Input-Output-Beziehungen zwischen den Gütern, die über die teilnehmenden Unternehmen auf der Kollaborationsplattform gehandelt werden können. Diese Matrix liegt in der Dimension Güter $x$ Güter vor und kann über den Aufruf der Funktion ESTIMATEGOA4U bestimmt werden.

$$
\begin{gathered}
B C l g g=\left(\text { ccigg }_{c g}\right)_{\substack{c=1, \ldots, n g \\
g=1, \ldots, n g}} \\
b c i g g_{c g}=\sum_{u m t=1}^{n u} b c i g u_{c u n t} \cdot \operatorname{iotgg}_{u n t, c g}^{\text {znorm }}
\end{gathered}
$$




\section{BClaa}

BCIaa liefert im Gegensatz zu BCIgg die Aktivitätsbetrachtung und stellt somit die - teils auf Basis der aktivitätsbezogenen Produktionsstruktur unterstellten Input-Output-Relationen zwischen den Aktivitäten, die den an der Plattform teilnehmenden Unternehmen zugeordnet werden können. Die Dimension der Matrix ist folglich Aktivitäten $\times$ Aktivitäten. Die Funktion ESTIMATEGOA4U wird verwendet um BClaa zu erzeugen.

$$
\begin{aligned}
& \text { BCIaa }=\left(\text { bciaa }_{a k}\right)_{\substack{a=1, \ldots, n a \\
k=1, \ldots, n a}} \\
& b_{c i a a_{a k}}=\sum_{u n t=1}^{n u} b \operatorname{ciau}_{a u n t} \cdot \operatorname{iotad}_{u n t, a k}^{z n o m m}
\end{aligned}
$$

\section{BClsell}

Eine Übersicht über die Beziehungen zwischen den Aktivitäten und Gütern auf der Kollaborationsplattform unter Ausblendung der Teilnehmer bietet BCIsell. Diese Matrix, die die Dimension Aktivitäten x Güter hat, zeigt die von den an der Plattform teilnehmenden Unternehmen bereitgestellten Güter, deren ursprüngliche Aktivität und den dazugehörigen kumulierten Transaktionswert in der betrachteten Periode. BCIsell kann durch Aufruf der Funktion mAKEBClaug bestimmt werden.

$$
\begin{aligned}
\text { BCledgeFRuug }{ }^{a g g B S, P, c}= & \left(\text { cciedgefruug }_{u}^{a g g B S, P, c}\right)_{u=1, \ldots, \text { nau }} \quad \text { für } c=1, \ldots, \text { ng } \\
= & \left(\left(\text { com }_{u, c}, \text { sval }_{u, u c}, \text { sell }_{u, v}, \text { sellact }_{u, a}\right):\right. \\
& \left(\text { com }_{u, c}, \text { buy }_{u, b}, \text { val }_{u}, \text { sell }_{u, v}, \text { sellact }_{u, a}\right)=\text { bciedgefruug }_{1}^{a g g B, P, u c} \\
& \left.\wedge \text { sval }_{u, u c}=\text { bciaug }_{u c}\right)
\end{aligned}
$$

$$
\begin{aligned}
& \text { BCledgeFRuug }{ }^{a g g B S, P, a c}=\left(\text { bciedgefruug }{ }_{k}^{a g g B S, P, a c}\right)_{k=1, \ldots, n u_{\triangleleft c}} \text { für } a=1, \ldots, n a, \\
& c=1, \ldots, n g \\
& =\left(\text { bciedgefruug }_{u}^{a g g B S, P, c}: \text { sellact }_{u, a}=\text { act }_{a}\right)
\end{aligned}
$$




$$
\begin{aligned}
\text { BCIsell } & =\left(\text { bcisell }_{a g}\right)_{\substack{a=1, \ldots, \ldots a \\
g=1, \ldots, n g}} \\
\text { bcisell }_{a g} & =\sum_{k=1}^{n u_{o g}} \text { sval }_{k, k g} \quad \text { mit sval } \\
k, a & \in \text { bciedgefruug }_{k}^{a g g B S, P, a g}
\end{aligned}
$$

\section{BClbuy}

Einen Einblick in die - über die Use-Matrix teilweise abgeschätzten - Beziehungen zwischen den Gütern und Aktivitäten auf der Plattform aggregiert über die teilnehmenden Unternehmen soll BCIbuy auf Grundlage von BCIgau ermöglichen. Die Funktion ESTIMATEGOA4U liefert auch diese Matrix in der Dimension Güter $\times$ Aktivitäten als Ergebnis.

$$
\begin{aligned}
\text { BCIbuy } & =\left(\text { bcibuy }_{g a}\right)_{\substack{g=1, \ldots, \ldots g \\
a=1, \ldots, n a}} \\
\text { ccibuy }_{g a} & =\sum_{u n t=1}^{n u} \text { cciau }_{g u n t} \cdot v w u s e_{u n n, g a}^{2 n o r m}
\end{aligned}
$$

Nach der Sammlung von Strukturdaten sowie der Ermittlung bzw. Ableitung von Input-Output-Relationen auf der Kollaborationsplattform kann zu den nächsten Phasen des Modells übergegangen werden, die sich der Analyse, dem Vergleich und der Bewertung der zuvor aufbereiteten Verflechtungsdaten widmet, um in weiterer Folge konkrete Maßnahmen und Empfehlungen für identifizierte Problembereiche und Potenziale zu ermöglichen. 
Michael Weber - 978-3-631-75376-7

Downloaded from PubFactory at 01/11/2019 05:44:37AM

via free access 


\section{Strukturanalysephase}

In der Strukturanalysephase des Modells werden die Verflechtungen, die sich in den Strukturdaten widerspiegeln, mit graphentheoretischen und netzwerkanaIytischen Konzepten konfrontiert, um Eigenschaften und Muster der Beziehungsgeflechte erkennen zu können. Hierzu werden verschiedene theoretische Konstrukte eingeführt und beschrieben, die ein erhöhtes Abstraktionsniveau zur umfassenden Darstellung und Betonung der Allgemeinheit des Modells verlangen. Zur Interpretation der Konzepte im Sinne der bisherigen Ausführungen kann der nachfolgend eingeführte Begriff der, globalen Struktur' mit den Daten zur Produktionsstruktur gleichgesetzt werden, während der Begriff ,lokale Struktur' als die Input-Output-Beziehungen auf einer Kollaborationsplattform verstanden werden kann. Weiters können Güter, Aktivitäten bzw. Unternehmen (Teilnehmer) als ,Knoten' und deren Beziehungen als ,Kanten' gesehen werden.

\subsection{Graphentheoretische Grundlagen}

Für die Analyse der Struktur von Verflechtungen oder genauer formuliert von relationalen Daten, welche Verflechtungen repräsentieren, bietet die Graphentheorie ein umfassendes Instrumentarium an strukturanalytischen Methoden. Dieses Teilgebiet der Mathematik eignet sich aufgrund dessen Auseinandersetzung mit Relationen zwischen Objekten besonders für Fragestellungen rund um die Input-Output-Rechnung und wurde bspw. von Katzenbeisser [1978] oder Holub \& Schnabl [1985] eingesetzt, um Probleme der Input-Output-Rechnung zu adressieren und letztlich gezieltere wirtschaftspolitische Empfehlungen zu ermöglichen. Für die Zwecke der Input-Output-Rechnung sind in diesem Zusammenhang im Wesentlichen Kennzahlen zu gerichteten Graphen ${ }^{39}$ von Interesse. In diesen gerichteten Graphen stellen Aktivitäten oder Güter Knoten dar, die über (u.U. ,gewichtete' oder, gefärbte') Kanten paarweise verbunden und geordnet 
werden können. Ungerichtete Graphen weisen im Gegensatz keine paarweise Ordnung der Knoten mittels der Kanten auf. Sie können als eine Spezialform von gerichteten Graphen verstanden werden, nachdem verbundene Knoten immer gleichwertig in beide Richtungen - folglich ungerichtet - in Beziehung gesetzt werden.

Formal sind Graphen ein Tupel, also eine geordnete Zusammenstellung von Objekten, aus einer Menge von Knoten (engl. vertices) $V$ und einer Menge von Kanten (engl. edges) $E$.

$$
G=(V, E) \quad \text { wobei } E \subseteq V \times V \text { und } V \cap E=\varnothing
$$

Für eine gerichtete Kante $e \in E$ mit dem zugeordneten Knotenpaar $e=(v, w)$ mit $v, w \in V$ wird $v$ als Startknoten und $w$ als Endknoten von $e$ bezeichnet. Grafisch wird diese Beziehung durch einen Pfeil von $v$ nach $w$ dargestellt. Ein Spezialfall tritt dabei bei dem Vorliegen von Selbstbezüglichkeit eines Knotens auf. Selbstbezüglichkeit wird als (direkte) Schleife in einem Graphen abgebildet und mathematisch als $e=(v, v)$, d.h. Kante $e$ verweist (gerichtet oder ungerichtet) ausgehend von dem Knoten $v$ zurück zu eben diesem Knoten, definiert.

Die Repräsentation eines (ungewichteten) Graphen mit $n$ Knoten kann über eine Matrix in der Dimension $n \times n$ mit den Zellwerten 0 oder 1 erfolgen. Eine solche Matrix wird als (binäre) Adjazenzmatrix ${ }^{40}$ bezeichnet, da sie sozusagen die (unmittelbare) Nachbarschaft von Knoten festhält. Für die Darstellung einer gerichteten Beziehung zwischen dem $i$-ten und $j$-ten Knoten des Graphen wird in der Zelle der Matrix, die durch Zeile $i$ und Spalte $j$ der Matrix definiert wird, eine 1 eingetragen, ansonsten bleibt der Initialwert 0 bestehen. Selbstbezüglichkeit der Knoten ist bei gleich geordneten Zeilen und Spalten der Matrix entlang der Hauptdiagonale feststellbar. Die Adjazenzmatrix eines ungerichteten Graphen ist

40 Adjazenz leitet sich aus dem lateinischen adiacēns, dem Partizip Präsens von adiacēre ab, wobei ad bei und iacēre liegeni bequêtet ber - 978-3-631-75376-7 
symmetrisch, nachdem für die Beziehung zwischen Knoten $i$ und $j$ in den Zellen für beide Richtungen, d.h. Zeile $i$ und Spalte $j$ sowie Zeile $j$ und Spalte $i$, der gleiche Wert eingetragen wird. Auch bei gerichteten Graphen stellt sich die Frage, inwieweit Selbstbezüglichkeit und damit Schleifenbildung für die Berechnungen gewünscht bzw. erforderlich sind. Nachdem die Verflechtungen zwischen den Knoten im Zentrum der relationalen Analyse stehen und mögliche Bezüge unter mehrmaliger Einbindung des gleichen Knoten für viele Anwendungen verzerrend wirken, sollte die Selbstbezüglichkeit in der Ausgangsmatrix der Berechnungen und zusätzlich auch in den Zwischenberechnungen zumindest optional eliminiert werden können. In der Literatur werden daher üblicherweise auch die Diagonalelemente der Adjazenzmatrix von gerichteten Graphen nullgesetzt. Bei Zwischenergebnissen von Matrixberechnungen erscheint dieser Schritt ebenfalls angebracht, um Schleifen vorzubeugen und wird daher in dieser Arbeit verfolgt. OKonomisch betrachtet - bspw. im Sinne der Input-Output-Rechnung - können die Werte einer Adjazenzmatrix als Manifestation von Vorleistungsbeziehungen der betrachteten Knoten interpretiert werden. Vorleistende Knoten werden dabei über die Zeilen und empfangende Knoten über die Spalten repräsentiert. Die Interpretation bei ungerichteten Graphen beschränkt sich auf das Festhalten des Vorliegens einer (Leistungs-)Beziehung zwischen den verbundenen Knoten.

Relationen in Graphen können auch in Listenform dargestellt werden. Die so genannte Adjazenzliste, die alle Knoten des Graphen in Listenform beschreibt, wird in der Praxis bspw. als Alternative zur Adjazenzmatrix eingesetzt. Eine Adjazenzliste besteht aus einer Aneinanderkettung von Knoteneinträgen, die wiederum Verweise zu den - im Falle eines ungerichteten Graphen - ,benachbarten' oder - bei gerichteten Graphen - nachfolgenden und/oder vorangehenden Knoten enthalten. Vorteile der Adjazenzliste gegenüber der Adjazenzmatrix sind insbesondere im geringeren, linearen Speicherbedarf und in den linearen Berechnungszeiten von graphentheoretischen Problemen im Gegensatz $\mathrm{zu}$ dem quadratischem Speicherbedarf und den quadratischen Berechnungszeiten bei dem Einsatz von Adjazenzmatrizenezw/findern7Diese 1 Vortede wirken 
sich insbesondere bei Graphen mit einer hohen Knotenanzahl bei einer gleichzeitig relativ geringen Kantenzahl, d.h. bei geringer Dichte, aus. Als Nachteile gegenüber Adjazenzmatrizen sind die kompliziertere Handhabbarkeit und die verhältnismäßig größere Unübersichtlichkeit v.a. bei einer höheren Knotenzahl anzuführen.

Inzidenzmatrizen ${ }^{41}$ und Inzidenzlisten sind alternative Darstellungsformen für Graphen. Bei Inzidenzmatrizen wird die Beziehung zwischen Knoten über Kanten in einer Matrix in der Dimension Knoten $x$ Kanten repräsentiert. Bei einem schleifenfreien, gerichteten Graphen wird bei Vorliegen einer direkten Beziehung zwischen den Knoten $v$ und $w \in V$ über eine Kante $e \in E$ mit $e=(v, w)$ in der Inzidenzmatrix $B$ der positive Wert 1 in der Zelle $b_{v e}$ des Startknotens $v$ und der Kante $e$ eingetragen und der negative Wert -1 in der Zelle $b_{w e}$ des Endknotens $w$ und der Kante $e$. Falls keine Beziehung vorliegt, bleibt der Initialwert 0 bestehen. Bei ungerichteten Kanten wird nicht zwischen Start- und Endknoten unterschieden und dementsprechend wird nur der positiven Wert 1 in die Zellen $b_{v e}$ und $b_{w e}$ der Inzidenzmatrix $B$ bei Vorliegen einer direkten Beziehung zwischen den Knoten $v$ und $w$ über Kante $e$ eingetragen. Die Repräsentation dieser Darstellungsvariante in Listenform nennt man Inzidenzlisten.

Weitere wichtige, teilweise triviale sowie geläufige Begriffe aus der Graphentheorie sind unter anderem Grade (Ein- und Ausgangsgrade), Wege, Pfade, Zyklen und Kreise sowie Erreichbarkeit, Durchmesser, kürzester Pfad, Distanzgraph und Dichte. Eingangs- bzw. Ausgangsgrade von Knoten geben bei einfachen, gerichteten Graphen die Anzahl an direkten Vorgängern bzw. Nachfolgern eines Knoten an. Im Gegensatz hierzu beschreibt bei ungerichteten, einfachen Graphen der Begriff ,Grad' die Anzahl der direkt verbundenen Knoten

41 Während der Begriff ,Adjazenz' in der Graphentheorie die Beziehung(en) zwischen Elementen einer Menge bezeichnet, beschreibt ,Inzidenz' die Beziehung zwischen Elementen zweier verschiedener Mengen (der Menge der Knoten und der der Kanten). Inzidenz leitet sich aus dem lateinischen incidēns, dem Partizip Perfekt von

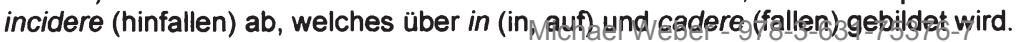


(Nachbarn). Ein Weg $W=\left(v_{l}, \ldots, v_{n}\right)$ ist eine Folge von Knoten $v \in V$ eines Graphen $G=(V, E)$ mit dem Ausgangsknoten $v_{I}$ und dem Zielknoten $v_{n}$. Wege können auch als Kantenfolgen verstanden werden, nachdem die Knoten $v_{k}$ und $v_{k+1}$ eines Weges $W$ definitionsgemäß über eine ${ }^{42}$ Kante verbunden werden. Abhängig davon, ob es sich um Kanten eines gerichteten oder ungerichteten Graphen handelt, unterscheidet man zwischen gerichteten und ungerichteten Wegen. Ein Pfad ist ein Weg, dessen Knoten sich im Verlauf des Weges nicht wiederholen, d.h. sämtliche Knoten eines Pfades sind voneinander verschieden und die maximale Länge eines Pfades ist $n-1$. Ein Zyklus ist ein Weg, dessen Ausgangs- und Zielknoten identisch sind. Ein Kreis ist ein Spezialfall eines Zyklus bei dem ausschließlich Ausgangs- und Zielknoten identisch sind. Azyklische gerichtete Graphen können topologisch sortiert werden, d.h. es ist möglich eine Reihenfolge der Knoten festzulegen, da keine gegenseitige Abhängigkeiten zwischen den Knoten vorliegen. Ein ungerichteter, einfacher Graph ist ein Wald, wenn zwei beliebige Knoten über maximal einen Pfad verbunden sind. Ist dieser Graph zusammenhängend, so ist er auch ein Baum. Ein ungerichteter, einfacher Graph wird als (ungerichteter) Baum bezeichnet, wenn jedes Knotenpaar des Graphen über genau einen Pfad verbunden wird. Ein gerichteter oder,gewurzelter' Baum ist ein Baum, der einen sogenannten Wurzelknoten enthält, d.h. einen Knoten, zu dem alle anderen Knoten verweisen oder von dem auf alle anderen Knoten verwiesen wird. Sind alle Knoten eines Graphen (unabhängig von der Richtung) untereinander erreichbar, so ist der Graph zusammenhängend. Stark zusammenhängend wird ein gerichteter Graph bezeichnet, bei dem jeder Knoten sämtliche anderen Knoten über einen gerichteten Weg erreichen kann. Das Problem der Erreichbarkeit von zwei beliebigen Knoten eines Graphen über einen Weg ist dementsprechend für zusammenhängende ungerichtete Graphen bzw. stark zusammenhängende gerichtete Graphen nicht gegeben. Der Durchmesser eines Graphen bezeichnet den längsten Abstand 
zwischen zwei (verbundenen) Knotenpaaren. Die Distanz zwischen zwei Knoten $v$ und $w$ ist die Länge des kürzesten Pfades zwischen diesen beiden Knoten innerhalb des Graphen. Die Distanz ist $\infty$ wenn zwei Knoten unverbunden sind und 0 im Falle eines Selbstbezugs. Der Distanzgraph eines Graphen zeigt die Distanzen sämtlicher Knotenpaare des Graphen auf. Dementsprechend ist ein Distanzgraph ein vollständiger Graph, der als Kantengewicht die Distanzen zwischen den Knoten verwendet. Eine weitere Eigenschaft eines Graphen ist dessen ,Dichte'. Diese Dichte ist das Verhältnis der Anzahl der Kanten eines Graphen zu der maximal möglichen Anzahl an Kanten (basierend auf den Knoten des Graphen) und kann als Quotient zwischen den von Null verschiedenen Einträgen einer Adjazenzmatrix und der Anzahl der Zellen der Adjazenzmatrix abzüglich der Diagonalzellen bestimmt werden.

Es ist möglich, Graphen mit zusätzlicher Information anzureichern, indem Knoten oder Kanten gewichtet bzw. ,gefärbt' werden, d.h. auf die Menge der reellen bzw. natürlichen Zahlen abgebildet werden. Hierbei wird das Tupel $G=(V, E)$ bspw. um die Abbildung $g(v)$ oder $g(e)$ zu dem Tripel $G=(V, G, g)$ erweitert. Die Färbung ist in diesem Zusammenhang ein Spezialfall der Gewichtung. Darüber hinaus können Knoten und Kanten auch in einen Namensraum abgebildet werden, eine Vorgehensweise, die sich insbesondere zu lllustrationszwecken eignet.

Neben dem bisher beschriebenen Typus des einfachen Graphen existieren auch komplexere Typen von Graphen wie Multigraphen oder Hypergraphen. Multigraphen können im Gegensatz zu einfachen Graphen zwei Knoten statt über eine Kante auch über mehrere Kanten verbinden. Die Kanten von Multigraphen können dabei ebenso wie einfache Graphen gerichtet oder ungerichtet sein. Hypergraphen hingegen sind dadurch gekennzeichnet, dass sie über eine Kante mehrere Knoten gleichzeitig verbinden können. Grafisch ergeben sich allerdings Darstellungsprobleme, wenn Knoten über mehrere unterschiedliche ,Hyperkanten' verbunden werden. Falls dieses Problem nicht auftritt, können die durch die Hyperkante verknüpften Knoten bspw. als eine Punktwolke dargestellt werden, die über eine Linie eingegrenzt und/oder farblich unterlegt werden kann. 


\subsection{Einfache Verflechtungseigenschaften auf Basis der Graphentheorie}

Im Folgenden werden einfache, grundsätzliche Kennzahlen der Graphentheorie [Harary 1969; Diestel 1997], die in den vergangenen Jahrzehnten auch in dem sich entwickelnden Feld der sozialen Netzwerkanalyse Eingang gefunden haben [Scott 1991; Wasserman \& Faust 1994], vorgestellt. Diese Kennzahlen können einen Überblick über die Eigenschaften der Verflechtungen auf einer Kollaborationsplattform aus Knoten- und auch Gesamtbetrachtung geben sowie für den Vergleich zwischen einer solchen Plattform und der dazu passenden Produktionsstruktur herangezogen werden. Hierbei ergeben sich mehrere Möglichkeiten, die bereits eingehend in der Literatur behandelten Kennzahlen für den spezifischen Zweck dieser Arbeit zu enweitern, um Handlungsempfehlungen für die Weiterentwicklung der Kollaborationsplattform zu geben.

$$
\text { MatVal }=\left(\text { matval }_{i j}\right)_{i, j=1, \ldots, n} \quad \text { wobei i.A. gilt }: \text { matval }_{i j} \neq \text { matval }_{j i} \text { für } i \neq j
$$

Ausgehend von MatVal (Formel (52)), der Matrixdarstellung eines einfachen, gerichteten Graphen mit Knotenbenennung und Kantengewichten, welche dem Transaktionsvolumen zwischen den Knoten im Sinne einer Input-OutputBeziehung entsprechen, soll nun die Berechnung von grundlegenden Verflechtungseigenschaften aufgezeigt werden. Hierzu wird in einem ersten Schritt die binäre Adjazenzmatrix Mat (Formel (53)) der in quadratischer Form vorliegenden Matrix MatVal mit gleich geordneter Zeilen- und Spaltenbezeichnung gewonnen. Diagonalelemente sollten in allen für die Berechnungen eingesetzten Matrizen auf 0 gesetzt werden, um Selbstbezüglichkeit zu vermeiden.

$$
\begin{aligned}
& \text { Mat }=\left(\text { mat }_{i j}\right)_{i, j=1, \ldots, n}
\end{aligned}
$$

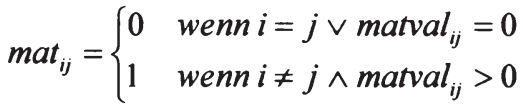

Im Folgenden gibt $n$ die Anzahl der Knoten der binären Adjazenzmatrix Mat sowie der Ausgangsmatrix MatVal an. Die Kantenanzahl narcs (Formel (54)) der 
Adjazenzmatrix Mat entspricht der Anzahl der Zellen mit einem Wert von 1 oder - anders formuliert - der Summe der Werte der Adjazenzmatrix Mat, nachdem definitionsgemäß nur die Werte 1 oder 0 in dieser Adjazenzmatrix vorkommen. Knoten werden mit $v$ bezeichnet, wobei der Index $i$ für die Zeilenbetrachtung (Beziehung zu den Nachfolgerknoten) und der Index $j$ für die Spaltenbetrachtung (Beziehung zu den Vorgängerknoten) der Eigenschaften eines Knoten $v$ steht.

$$
n \operatorname{arcs}=\sum_{i, j=1}^{n} \text { mat }_{i j}
$$

\subsubsection{Grade und Dichte}

In einem gerichteten Graphen können Eingangsgrade (engl. indegrees) und Ausgangsgrade (engl. outdegrees) auf Knotenebene unterschieden werden. Diese Grade geben Auskunft über die Anzahl der direkten Vorgänger bzw. Nachfolger von Knoten und werden auch für Gesamtstatistiken über den Graphen eingesetzt. Knoten ohne Vorgänger oder Nachfolger $\left(d_{j}^{I N}=d_{j}^{\text {OUT }}=0\right)$ sind isolierte Knoten. Formel (55) zeigt die Berechnung des Eingangsgrades $d_{j}^{I N}$ eines Knoten $v_{j}$, während Formel (56) die Berechnung des Ausgangsgrades $d_{i}^{\text {OIT }}$ eines Knoten $v_{i}$ definiert.

$$
\begin{aligned}
& d_{j}^{I N}=\sum_{i=1}^{n} \text { mat }_{i j} \quad \text { für } j=1, \ldots, n \\
& d_{i}^{\text {OUT }}=\sum_{j=1}^{n} \text { mat }_{i j} \quad \text { für } i=1, \ldots, n
\end{aligned}
$$

Den Grad $d_{k}$ aller direkt verbundenen Knoten des Knoten $v_{k}$ erhält man durch simples Aufsummieren des Eingangs- und Ausgangsgrades dieses Knoten (Formel (57)).

$$
d_{k}=d_{k}^{I N}+d_{k}^{\text {Olt }}=\sum_{i=1}^{n} m a t_{i k}+\sum_{\text {Mjehael Weber }-978-3-631-7}^{n} \text { mat }_{k j} \quad \text { für } k=1, \ldots, n
$$


Der Durchschnittsgrad der Eingangsgrade $\vec{d}^{N}$ entspricht dem Durchschnittsgrad der Ausgangsgrade $\bar{d}^{\text {OUT }}$ der Knoten (Formel (58)).

$$
\bar{d}^{I N}=\frac{\sum_{j=1}^{n} d_{j}^{I N}}{n}=\bar{d}^{O I T T}=\frac{\sum_{i=1}^{n} d_{i}^{O U T}}{n}
$$

Aufbauend auf den Knotengraden $d_{k}$ zeigt Formel (59) den Durchschnittsgrad des gesamten Graphen $\bar{d}$.

$$
\bar{d}=\frac{\sum_{k=1}^{n} d_{k}}{n}=2 \bar{d}^{I N}=2 \bar{d}^{\text {OuT }}
$$

Nachdem es sich bei den erhobenen Werten des Graphen um keine Stichproben handelt, werden die Standardabweichungen zu dem Eingangs- und Ausgangsgrad sowie deren Summe entsprechend der Formeln (60) bis (62) ermittelt.

$$
\begin{gathered}
s_{d^{I N}}=\sqrt{\frac{\sum_{j=1}^{n}\left(d_{j}^{I N}-\bar{d}^{I N}\right)^{2}}{n}} \\
s_{d^{O L T}}=\sqrt{\frac{\sum_{i=1}^{n}\left(d_{i}^{O U T}-\bar{d}^{O U T}\right)^{2}}{n}} \\
s_{d}=\sqrt{\frac{\sum_{k=1}^{n}\left(d_{k}-\bar{d}\right)^{2}}{n}}
\end{gathered}
$$

Eine Alternative zur Bestimmung der tatsächlichen Kantenzahl narcs ist die Aufsummierung der Eingangs- oder Ausgangsgrade der Knoten des Graphen (Formel (63)).

$$
\operatorname{narcs}=\sum_{j=1}^{n} d_{j}^{I N}=\sum_{i=1}^{n} d_{i}^{O U T}
$$

Die maximal mögliche Kantenzahl eines gerichteten Graphen narcs $\max _{\max }$ kann über Formel (64) bestimmt werden. 


$$
\operatorname{narcs}_{\max }=n(n-1)
$$

Aufbauend auf der tatsächlichen Kantenzahl narcs und der maximal möglichen Kantenzahl narcs $_{\max }$ ist es möglich, die Dichte $o$ eines gerichteten Graphen zu definieren (Formel (65)).

$$
o=\frac{\text { narcs }}{\text { narcs }_{\max }}
$$

Formel (66) zeigt am Beispiel der Wertedichte $o^{V A L}$ der gewichteten Matrix MatVal die alternative, allgemeine Formel für die Dichte eines gerichteten oder ungerichteten, gewichteten oder ungewichteten einfachen Graphen.

$$
o^{V A L}=\frac{\sum_{i=1}^{n} \sum_{j=1}^{n} \text { matval }_{i j}}{n(n-1)}
$$

Setzt man die Summe über alle Kantengewichte anstatt zur maximal möglichen Kantenzahl zur tatsächlichen Kantenzahl in Beziehung, erhält man den Kantendurchschnittswert $\overline{\text { matval }}$ eines kantengewichteten Graphen, Formel (67) entnommen werden kann.

$$
\overline{\text { matval }}=\frac{\sum_{i=1}^{n} \sum_{j=1}^{n} \text { matval }_{i j}}{\text { narcs }}
$$

Die in den Formeln (54) bis (67) vorgestellten Kennzahlen sind über die im Anhang gelistete Funktion GSTAT generierbar.

\subsubsection{Erreichbarkeit und Distanzen}

Aussagen über die prinzipielle Erreichbarkeit von Knotenpaaren einer Adjazenzmatrix Mat, d.h. die Erreichbarkeit entlang eines Pfades mit der maximalen Pfadlänge von $n-1$, erhält man über $S M a t$, der Summe der bis zur $(n-1)$ ten Potenz potenzierten Matrizen von Mat (Formel (68)). Schleifen werden dabei ausgeschlossen, indem die Diagonalelemente in den Zwischenergebnissen sowie im 
Endergebnis der einfachen Matrixmultiplikationen eliminiert werden (Funktion SUMMAT). Formel (69) demonstriert dies, wobei anzumerken ist, dass zirkuläre Bezüge durch Beibehalten der Diagonale in der Ergebnismatrix (Formel (70)) in das Resultat einfließen (Funktion MATPOWER). Die Berechnung von SMat erfolgt in diesem Fall durch Aufsummieren von Mat $^{p}$, anstatt Mat in Formel (68). Die Erreichbarkeitsmatrix RMat aus Formel (71) (Funktion REACH) ergibt sich durch Ersetzen von Zellwerten aus $S M a t \geq 1$ mit 1 .

$$
\begin{aligned}
& \text { SMat }=\sum_{p=1}^{n-1} \operatorname{Mat}^{p} \\
& M a t^{<>>}=n o D i a g(M a t)=\left(\operatorname{mat}_{i j}^{<1>}\right)_{i, j=1, \ldots, n} \\
& \text { mat }_{i j}^{<>>}= \begin{cases}m t_{i j} & \text { wenn } i \neq j \\
0 & \text { andernfalls }\end{cases} \\
& M a t^{<p>}=n o D i a g\left(M^{<a t}{ }^{<p-1>} \cdot M^{<t^{<>>}}\right) \\
& M a t^{<p>c i r c}=M a t^{<p-1>} \cdot M a t \\
& \text { wobei } \text { Mat }^{<>>c i r c}=\text { Mat } \\
& R M a t=\left(r m a t_{i j}\right)_{i, j=1, ., n} \\
& \text { rmat }_{i j}= \begin{cases}1 & \text { wenn smat }_{i j} \geq 1 \\
0 & \text { andernfalls }\end{cases}
\end{aligned}
$$

DIST, der Distanzgraph zu Mat in Matrixform, hält die kürzesten Pfade zwischen den Knotenpaaren fest.

$$
\begin{aligned}
\text { DIST } & =\left(\text { dist }_{i j}\right)_{i, j=1, \ldots, n} \\
\text { dist }_{i j} & = \begin{cases}\min _{p}\{p \in P\} & |P|>0 \\
0 & |P|=0 \wedge i=j \\
\infty & |P|=0 \wedge i \neq j\end{cases} \\
\text { wobei } P & =\left\{p:\left(\text { Mat }^{p}\right)_{i j}>0\right\}
\end{aligned}
$$


Formel (72) stellt die Ermittlung von DIST dar. Für nicht verbundene Knotenpaare abseits der Hauptdiagonale wird $\infty$, in der Hauptdiagonale 0 eingetragen.

Aufbauend auf der Matrix DIST kann die Anzahl der Wege, die das jeweilige Knotenpaar über den kürzesten Pfad verbinden, aus DISTc (Formel (73)) entnommen werden.

$$
\begin{aligned}
D I S T c & =\left(\operatorname{distc}_{i j}\right)_{i, j=1, \ldots, n} \\
\operatorname{distc}_{i j} & =\left(\operatorname{Mat}^{\text {dist }_{1 j}}\right)_{i j}
\end{aligned}
$$

Der Durchmesser $\tau$ eines Graphen, der als längster Pfad bzw. größter Abstand zwischen zwei Knoten eines Graphen verstanden wird, kann ebenfalls auf Basis der Matrix DIST über

$$
\tau=\max _{i, j=1, \ldots, n}\left(\text { dist }_{i j}\right)
$$

ermittelt werden. Im Falle von nicht zusammenhängenden Graphen ist der Durchmesser nicht definiert, nachdem nicht verbundene Knotenpaare eine Distanz von $\infty$ haben. Alternativ kann dann der Durchmesser als maximale, nichtunendliche Distanz ermittelt werden. Zu diesem Zweck setzt man den Zellwert für unverbundene Knotenpaare 0.

Die im Anhang beschriebenen Funktionen GEOD zur Distanzgraphermittlung und GEODCOUNT zur Ermittlung der Anzahl an kürzesten Pfaden pro Knotenpaar (aufbauend auf der Distanzmatrix), folgen diesem alternativen Ansatz und setzen Zellen von nicht verbundenen Knotenpaaren ebenso wie Zellen der Hauptdiagonale der jeweiligen Matrix 0.

\subsubsection{Indexierte Knoteneigenschaften}

Die Indexierung der Ausgangs- und Eingangsgrade sowie der Distanzwerte eines Graphen auf Knoten- und Graphenebene vereinfacht die relative Vergleichbarkeit dieser Werte. Die Ausgabe der indexierten Knoteneigenschaften erfolgt über die Funktion GSTAT. 


\section{Betrachtung der indexierten Knoteneigenschaften bezogen auf Nachfolgerknoten}

Der Ausgangsgradindex eines Knoten $\operatorname{dind}_{i}^{\text {OUT }}$ (Formel (75)) ist ein auf 1 normierter, relativer Ausgangsgradwert pro Knoten $v_{i}$ in einem gerichteten Graphen. Der Wert beträgt (maximal) 1 , wenn $v_{i}$ alle anderen Knoten $v_{j}, j=1, \ldots, n, j \neq i$, als Nachfolger hat und 0 , wenn der Knoten Vorgänger keines anderen Knoten ist.

$$
\operatorname{dind}_{i}^{\text {OUT }}=\frac{\sum_{j=1}^{n} \text { mat }_{i j}}{n-1} \text { für } i=1, \ldots, n
$$

Formel (76) definiert den Ausgangsgradindex auf Graphenebene dind ${ }^{\text {OlT }}$ für einen gerichteten Graphen [Wasserman \& Faust 1994, S. 192 nach Freeman 1979; 1977].

$$
\operatorname{dind}^{\text {OUT }}=\frac{\sum_{i=1}^{n}\left[\max _{i=1, \ldots, n}\left(\operatorname{dind}_{i}^{\text {OUT }}\right)-\operatorname{dind}_{i}^{\text {OUT }}\right]}{n-1}
$$

Der Indexwert beträgt maximal 1 , wenn ein Knoten $v_{i}$ alle anderen Knoten $v_{j}$, $j=1, \ldots, n, j \neq i$ als Nachfolger hat und die anderen Knoten $v_{j}$ ausschließlich $v_{i}$ als Vorgänger aufweisen, im umgekehrten Extremfall beträgt der Wert 0 .

Der Indexwert für die Distanz distind $_{i}^{* O U T}$ der Vorläuferknoten zu den Nachfolgeknoten kann über die Formel (77) ermittelt werden.

$$
\text { distind }_{i}^{* \text { OUT }}=\frac{n-1}{\sum_{j=1}^{n} \text { dist }_{i j}} \text { für } i=1, \ldots, n
$$

Der Wertebereich für diesen Index reicht von 0 bis 1 . Tritt der Fall ein, dass ein Vorläuferknoten keine Verbindung zu einem Nachfolgerknoten hat (d.h. eine Distanz von $\infty$ zu diesem Knoten hat), so ist der Wert nicht definiert. Alternativ kann über den ausschließlichen Einbezug von positiven Nachfolgewerten pro Vorläuferknoten (konkreter: deren Anzahl), wie in Formel (78) unter Einsatz der 
Erreichbarkeitsmatrix RMat demonstriert, der Indexwert für alle Vorläuferknoten errechnet werden [Wasserman \& Faust 1994, S. 200 nach Lin 1976].

$$
\begin{aligned}
\text { distind }_{i}^{\text {OuT }} & =\frac{\sum_{j=1}^{n} \text { rmat }_{i j} /(n-1)}{\sum_{j \in F_{i}} \text { dist }_{i j} / \sum_{j=1}^{n} \text { rmat }_{i j}} \text { für } i=1, \ldots, n \\
\text { wobei } F_{i} & =\left\{j: \text { dist }_{i j}>0 \wedge \text { dist }_{i j} \neq \infty\right\}
\end{aligned}
$$

Der Indexwert beträgt (maximal) 1 für alle Knoten, die direkt, d.h. über einen ,Schritt', an alle anderen Knoten Leistungen erbringen (z.B. Waren liefern) und 0 bei allen Knoten, für die dies bei keinem anderen (Nachfolger-)Knoten zutrifft. Über

$$
\sum_{j=1}^{n} r m a t_{i j} \quad \text { für } i=1, \ldots, n
$$

kann dabei die Anzahl der Knoten im direkten und indirekten Nachfolgebereich der Vorläuferknoten festgestellt werden.

Auf Graphenebene ermöglicht der Durchschnittswert $\overline{\text { distind }}^{\text {olt }}$ dieses Indexwertes gemeinsam mit der Standardabweichung $s_{\text {distind } d^{\text {oli }}}$ die standardisierte Interpretation der Beziehungen der Vorläuferknoten des Graphen.

$$
\begin{aligned}
& \overline{\text { distind }}^{\text {out }}=\frac{\sum_{i=1}^{n} \text { distind }_{i}^{\text {OUT }}}{n} \\
& S_{\text {distind OCT }}=\sqrt{\frac{\sum_{i=1}^{n}\left(\text { distind }_{i}^{\text {OUT }}-\overline{\text { distind }}^{\text {OUT }}\right)^{2}}{n}}
\end{aligned}
$$

\section{Betrachtung der indexierten Knoteneigenschaften bezogen auf Vorgängerknoten}

Analog bzw. ,transponiert' zur Betrachtung des Ausgangsgradindex ist auch der Eingangsgradindex eines Knoten dind ${ }_{j}^{I N}$ (Formel (82)) ein auf 1 standardisierter, 
relativer Eingangsgradwert pro Knoten $v_{j}$ in einem gerichteten Graphen. Der Wert beträgt (maximal) 1 , wenn $v_{j}$ von allen anderen Knoten $v_{i}, i=1, \ldots, n$, mit $i \neq j$ direkter Nachfolger ist und 0 wenn $v_{j}$ keinen anderen Knoten als Vorläufer hat.

$$
\operatorname{dind}_{j}^{I N}=\frac{\sum_{i=1}^{n} \text { mat }_{i j}}{n-1} \text { für } i=1, \ldots, n
$$

Auf Basis des Ergebnisses aus Formel (82) kann der Eingangsgradindex auf Graphenebene dind $^{I N}$ für einen gerichteten Graphen ermittelt werden (Formel (83)).

$$
\operatorname{dind}^{I N}=\frac{\sum_{j=1}^{n}\left[\max _{j=1, \ldots, n}\left(\operatorname{dind}_{j}^{I N}\right)-\operatorname{dind}_{j}^{I N}\right]}{n-1}
$$

Der Indexwert beträgt - angelehnt an die obigen Ausführungen - maximal 1, wenn ein Knoten $v_{j}$ alle anderen Knoten $v_{i}, i=1, \ldots, n, i \neq j$, als Vorgänger hat und die anderen Knoten ausschließlich $v_{j}$ als Nachfolger haben, im umgekehrten Extremfall beträgt der Wert 0 .

Wie bei den Eingangsgradindizes gilt auch für die Distanzindizes der Nachfolgerknoten die Analogie zur Betrachtung der Vorläuferknoten (Formel (84)).

$$
\operatorname{distind}_{j}^{* N}=\frac{n-1}{\sum_{i=1}^{n} \operatorname{dist}_{i j}} \text { für } j=1, \ldots, n
$$

Für Nachfolgerknoten ohne Vorläufer ist dieser Index abermals nicht definiert, weshalb eine verbesserte Indexformel - wie auch bei den Distanzindizes für Vorläuferknoten - notwendig ist (Formel (85)).

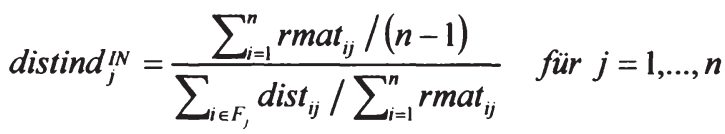

$$
\begin{aligned}
& \text { wobei } F_{j}=\left\{i: \text { dist }_{i j}>0 \wedge \text { dist }_{i j} \neq \infty\right\}
\end{aligned}
$$


Der Index beträgt 1 für alle Knoten, die von sämtlichen anderen Knoten direkt Leistungen beziehen und 0 wenn dies für keinen Vorläuferknoten (direkt oder indirekt) zutrifft. Die Anzahl der Knoten im direkten und indirekten ,Zulieferbereich' erhält man pro Nachfolgerknoten über Formel (86).

$$
\sum_{i=1}^{n} r^{\prime} m a t_{i j} \quad \text { für } j=1, \ldots, n
$$

Auf Graphenebene können abermals Durchschnittswert und Standardabweichung, in diesem Fall $\overline{\text { distind }}^{I N}$ (Formel (87)) und $s_{\text {distind } d^{l N}}$ (Formel (88)), zur Interpretation der Verflechtungen aus der Bezugsperspektive herangezogen werden.

$$
\begin{gathered}
\overline{\operatorname{distind}}^{I N}=\frac{\sum_{j=1}^{n} \operatorname{distind}_{j}^{I N}}{n} \\
S_{{\text {distind } \mathbb{N}^{N}}}=\sqrt{\frac{\sum_{j=1}^{n}\left(\text { distind }_{j}^{I N}-\overline{\text { distind }}^{I N}\right)^{2}}{n}}
\end{gathered}
$$

\subsection{Knotenbetrachtung unter Einbezug direkter und indirekter Verflechtungen}

Die Frage nach der Einbeziehbarkeit der Verbindungen zu den direkten sowie den indirekten Vorgänger- bzw. Nachfolgerknoten in die Betrachtung eines Knoten hat v.a. in der Soziometrie zu einer Reihe von Lösungsvorschlägen beginnend mit Seeley [1949] geführt, die versuchen, den ,Status' oder den ,Rang' eines Knoten über einen unendlichen Regress auf die Vorläuferknoten bzw. deren ,Status' rein mathematisch auf Basis von relationalen Daten zu eruieren. Diese Lösungsvorschläge können umgemünzt werden, um den komplementären ,Status' eines Knoten bezogen auf die Nachfolgerknoten zu erhalten. In diesem Zusammenhang ist auf die Beiträge von Wassily Leontief zur Ermittlung von Inputkoeffizienten zur Beschreibung der Struktur einer Volkswirtschaft hinzu-

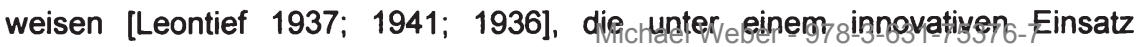


mathematischer bzw. ökonometrischer Methoden Quesnays Tableau Économique auf ein breites Fundament stellten. Der zentrale Beitrag von Leontief [1937] war die Berechnung der Leontief-Inverse, welche nicht nur die relative direkte, sondern auch die indirekte Bedeutung der Kanten (Transaktionsrelationen) quantifiziert und über einen unendlichen Regress aller Knoten auf die jeweiligen direkten und indirekten Vorleisterknoten ermittelt wird. Leontiefs $\mathrm{Er}$ kenntnisse flossen später in das (statische) Input-Output-Modell ein [Leontief 1944; 1946], welches über die Leontief-Inverse bspw. die direkten und indirekten Effekte einer (exogenen) Nachfrageerhöhung auf Basis der Modellannahmen errechnen kann. Dem Einfluss der ökonometrischen Errungenschaften Leontiefs auf die (später einsetzenden) soziometrischen Entwicklungen wird in einschlägigen Publikationen, die sich mit der Bedeutung (,Status' oder ,Rang') eines Knoten beschäftigen, allerdings kaum bis gar nicht Rechnung getragen (eine Ausnahme ist Hubbell [1965]).

Eine Weiterentwicklung des soziometrischen Ansatzes von Seeley brachte die Arbeit von Katz [1953], der u.a. einen (jeweils festzulegenden) Dämpfungsparameter einführte, um den Einfluss entfernt liegender (Vorläufer- bzw. Nachfolger-) Knoten auf die Bedeutung eines Knoten zu relativieren. Darüber hinaus betonte Katz in seinen Ausführungen die Rolle der Normierung der Verflechtungsdaten (in Matrixform) auf einheitliche Spalten- oder Zeilensummen für die Lösbarkeit des Gleichungssystems zur Berechnung der Statuswerte ${ }^{43}$.

Nachdem der ,Status' eines Knoten $v_{j}$ von den jeweiligen Vor- oder Nachfolgerknoten $v_{i}$ abhängig ist, kann nun folgendes lineares Gleichungssystem zur Wertbestimmung auf Basis der Adjazenzmatrix Mat angeschrieben werden.

$$
\operatorname{status}\left(v_{j}\right)=\sum_{i=1}^{n}\left(\text { mat }_{i j} \cdot \operatorname{status}\left(v_{i}\right)\right) \text { für } j=1, \ldots, n
$$


In Matrixnotation stellt sich dieses Gleichungssystem für die Statusberechnung bezogen auf die Vorläuferknoten wie folgt dar (Mat wird in diesem Fall transponiert).

$$
\begin{gathered}
\text { status }=\text { Mat }^{\prime} \cdot \text { status } \\
\text { wobei status }=\left(\operatorname{status}\left(v_{1}\right), \ldots, \text { status }\left(v_{n}\right)\right)^{\prime} \\
\text { bzw. } \\
\left(I-\text { Mat }^{\prime}\right) \cdot \text { status }=0
\end{gathered}
$$

Diese Schreibweise entspricht einem Gleichungssystem zur Ermittlung der Eigenvektoren einer Matrix Mat'. Der Statusvektor status ist dann der zum größten Eigenwert gehörende Eigenvektor von Mat'. Das Gleichungssystem ist nur dann lösbar, wenn man zusätzliche Bedingungen in Bezug auf Mat' einführt. In diesem Kontext sei auf Wasserman \& Faust [1994] verwiesen, die in ihrem Literaturüberblick u.a. auch zu diesem Thema mehrere Publikationen anführen, die sich mit Bedingungen zur Lösbarkeit auseinandersetzen.

Die bereits erwähnte Normierung von Katz [1953], die ursprünglich aufgrund des Dämpfungsparameters $a$ vorgeschlagen wurde, ist eine anerkannte Vorgehensweise, um die Lösbarkeit des Gleichungssystems (auch ohne Dämpfungsparameter) zu gewährleisten. Unter Bezugnahme auf das Gleichungssystem mit Dämpfungsparameter nach Katz [1953] kann die Normierung folgendermaßen begründet werden. Ausgehend von der Potenzreihe

$$
P=a M a t+a^{2} M a t^{2}+\ldots+a^{k} M a t^{k}+\ldots=(I-a M a t)^{-1}-I
$$

kann das Gleichungssystem mit Dämpfungsparameter mittels

$$
\begin{aligned}
u & =(1, \ldots, 1)^{\prime} \text { mit } \operatorname{dim}(u)=n \\
P u & =\left(\sum_{j=1}^{n} P_{i j}\right)_{i=1, \ldots, n}
\end{aligned}
$$


durch die Umformung

$$
\begin{aligned}
(P u)^{\prime} & =u^{\prime} \cdot\left((I-a M a t)^{-1}-I\right) \\
(P u)^{\prime} \cdot(I-a M a t) & =u^{\prime}-u^{\prime} \cdot(I-a M a t)=a u^{\prime} M a t \\
\left(I-a M a t^{\prime}\right) \cdot P u & =a M a t^{\prime} u \\
\left(\frac{1}{a} I-M a t^{\prime}\right) \cdot P u & =M a t^{\prime} u=\left(\sum_{i=1}^{n} m a t_{i j}\right)_{j=1, \ldots, n}
\end{aligned}
$$

als

$$
\left(\frac{1}{a} I-\text { Mat }^{\prime}\right) \cdot \text { status }^{\#}=\left(\sum_{i=1}^{n} m a t_{i j}\right)_{j=1, \ldots, n}
$$

dargestellt werden.

Der Lösungsvektor status ${ }^{t}$ des Gleichungssystems wird in diesem Verfahren abschließend durch eine spezielle Prozedur (vgl. [Katz 1953, S. 42]) in den Statusvektor status umgewandelt.

Mit Verweis auf Ferrar [1951] stellt Katz für die Lösbarkeit obiger Gleichung die Bedingung, dass der Kehrwert von a größer als der größte Eigenwert der Adjazenzmatrix Mat (bzw. bei umgekehrter Betrachtung Mat') ist. Eine obere Schranke für den größten Eigenwert von nicht-negativen Matrizen (wie bspw. Adjazenzmatrizen) ist das Maximum der Zeilen- bzw. Spaltensummen. Der größte Eigenwert erreicht diese Schranke, wenn alle Zeilen- bzw. Spaltensummen gleich sind. Eine Normierung der Zeilen- bzw. Spaltensummen auf 1 bedeutet dementsprechend ein Erreichen der Schranke, die dann ebenfalls gleich 1 ist. Daraus folgt gemäß der Bedingung für die Lösbarkeit, dass der Kehrwert von $a$ größer als 1 sein muss. Dies trifft dann zu, wenn der Dämpfungsparameter $a$ zwischen 0 und 1 liegt. Per Definition ist dies für Dämpfungsparameter der Fall. Das bedeutet, dass bei normierter Matrix Mat (bzw. Mat') diese Lösbarkeitsbedingung immer erfüllt ist. 
Generalisiert man nun den Ansatz von Katz, d.h. setzt man den Dämpfungsparameter $a$ auf 1 und entledigt man sich somit auch der nun nicht mehr erforderlichen Umformung, so besitzt die Normierungsbedingung für die Lösbarkeit der Ausgangsgleichung grundsätzlich ebenso Gültigkeit. Eine weitere durch die Normierung erzielte Eigenschaft ist, dass der größte Eigenwert des Gleichungssystems 1 entspricht. Der Eigenvektor zu diesem Eigenwert soll gemäß Wasserman \& Faust [1994] der Vektor der Statuswerte sein. In der Praxis ist diese Vorgehensweise allerdings problembehaftet und das Ergebnis schwierig zu interpretieren, nachdem auch komplexe Zahlen in dem Eigenvektor vorkommen können.

In zahlreichen auf die Herangehensweise von Katz folgenden Publikationen setzten sich Wissenschaftler mit Modifikationen und erweiterten Ansätzen auseinander, um die direkten und indirekten Einflüsse in der Betrachtung von Knoten in einem Beziehungsgeflecht zu quantifizieren. Taylor [1969] versuchte bspw. die Einflüsse der Vorgänger ebenso wie die Einflussnahme auf die Nachfolger eines Knoten in einer Maßzahl auszudrücken und wurde dabei auch durch Harary [1959] inspiriert, der in seinen unterschiedlich definierten Statusmaßzahlen Beziehungen zu Vorgänger- bzw. Nachfolgerknoten betrachtet und gesondert vergleicht. Eine Publikation, die später und ebenso wie Katz [1953] - wenn auch indirekt - beträchtliche Auswirkungen auf die Allokation des ,Produktionsfaktors' Information über das Internet hatte (und noch immer hat), lieferte Hubbell [1965]. Hubbel, der den Konnex zu den Methoden der Input-Output-Analyse von Leontief (wieder-)herstellte, beschrieb in seinem Beitrag eine Methode, die stark zusammenhängende Subgraphen, sogenannte Cliquen, auch für anteilsgewichtete und/oder negative Relationen in gerichteten Graphen identifizieren und für Vergleichszwecke bewerten lässt. Dieser, wie Hubbell schreibt, ,Input-Output Ansatz', den Hubbell schon in seiner Dissertation in Michigan, unweit der (ehemaligen) Wirkungsstätten von Katz und Harary verfolgte, floss später in den PageRank $^{\mathrm{TM}}$ Algorithmus [Page et al. 1998] der Google ${ }^{\mathrm{TM}}$-Gründer Page und Brin [Brin \& Page 1998] ein. Explizit erwähnt wird die Arbeit von Katz und Hubbell in dem ,United States Patent 6,285,999 - Patent Method for node ranking in a 
linked database' des ,Erfinders' Page [2001], der in Michigan geboren wurde und dort auch studiert hatt4.

\subsection{Berücksichtigung von Kantengewichten bei der Bewertung von Knoten}

Will man unter Einbezug direkter und indirekter Relationen die Bedeutung (auch ,Einfluss', ,Status' oder ,Rang') der Knoten eines gerichteten und gewichteten Graphen bewerten, so erscheint die bereits in Formel (7) auf Seite 67 hergeleitete Leontief-Inverse $R=(I-A)^{-1}$ - basierend auf der Koeffizientenmatrix $A$, die im Input-Outputmodell entweder die Matrix der direkten Inputkoeffizienten für Aktivitäten $\left(A_{I}\right)$ oder Güter $\left(A_{C}\right)$ ist, - als ein viel versprechender Ansatzpunkt. In ökonomischer Interpretation beschreiben die Elemente der auch als ,statischer Leontief-Multiplikator' bekannten Inversen, die direkt und indirekt bewirkte Erhöhung der Nachfrage nach dem Output des Vorgängerknoten $v_{i}$ für die $\mathrm{Er}$ zeugung einer weiteren Einheit an Output von Knoten $v_{j}$. Würde statt der spaltennormierten Koeffizientenmatrix $A$ eine zeilennormierte Koeffizientenmatrix $A^{*}$ in die Formel für die Leontief-Inverse eingesetzt werden, so könnte man die Koeffizienten der Ergebnismatrix $R^{*}$ als Indikator für die direkte und indirekte $\mathrm{Ab}$ hängigkeit des Outputs von Knoten $v_{i}$ von der Nachfrage nach dem Output von Knoten $v_{j}$ verstehen. Diese neuartige Berechnung (und Interpretation) einer Variation der Leontief-Inverse soll nun in Kombination mit der Berechnungsmethode und den Folgerungen auf Basis der klassischen Leontief-Inverse ein-

44 Neben den thematischen Berührungspunkten sind auch die geografischen Zusammenhänge interessant. Leo Katz promovierte an der University of Michigan und schrieb seinen Beitrag 1953 am Michigan State College (heute: Michigan State University), wo er von 1946 bis 1976 tätig war. Frank Harary arbeitete von 1948 bis 1986 an der University of Michigan, wo auch Charles H. Hubbell 1962 seine Dissertation verfasste. Lawrence Page wurde in East Lansing, dem Standort der Michigan State University, geboren und studierte an der University of Michigan, bis er an die Stanford University wechselte. Ein wenig weiter entfernt in der an Michigan angrenzenden kanadischen Provinz Ontario liegt die einstige Arbeitsstătte von John R. Seeley, die University of Toronto. 
gesetzt werden, um die Fragestellung nach der ,Bedeutung' eines Knoten für seine Vorgänger- bzw. Nachfolgerknoten zu beantworten. Dieses Vorgehen wird dabei durch die auf die Leontief-Inverse zeitlich nachgeordneten Entwicklungen zur Ermittlung von ,Status' etc. in der Soziometrie bestärkt.

\subsubsection{Bedeutungsbestimmung mit der Leontief-Inverse}

Die Grundidee der Festlegung der Bedeutung der Knoten einer gerichteten und gewichteten Matrix liegt in der Interpretation der Elemente der Leontief-Inverse zu den Koeffizientenmatrizen $A$ und $A^{*}$ als Indikatoren für die gegenseitige Bedeutung der über die Elemente jeweils verbundenen Knotenpaare. Dementsprechend würden die Koeffizienten der Matrix $R^{*}$ abzüglich der dazugehörigen Einheitsmatrix I die direkte und indirekte Bedeutung des Nachfolgerknotens $v_{j}$ für den Vorgängerknoten $v_{i}$ zeigen, während die Koeffizienten der Matrix $R$ unter Abzug der Einheitsmatrix $I$ die Bedeutung des Vorgängers $v_{i}$ für dessen Nachfolgerknoten $v_{j}$ darstellen. Möchte man die Bedeutungsbestimmung ohne Selbstbezüglichkeit durchführen, so wären vor der Normierung der Matrizen $A$ und $A^{*}$, die für die Matrix MatVal in Formel (96) gezeigt wird, die jeweiligen Diagonalelemente nullzusetzen (siehe auch Formel (69)). Mit diesem Schritt werden die direkten Effekte eines Knoten auf sich selbst ausgeschlossen. Indirekte Effekte, die über Zirkelbeziehungen auf die Knoten rückwirken - und daher in der Diagonale der Ergebnismatrizen zu finden sind -, werden durch die Entfernung der Selbstbezüglichkeit nicht berührt.

Die Bestimmung der Bedeutung der Knoten für deren Nachfolger und Vorgänger und damit deren relative Stellung (Rangreihung) kann schließlich durch die Summierung über alle Vorgänger- bzw. Nachfolgerknoten der Ergebnismatrizen, d.h. auch über den jeweils zu betrachtenden Knoten selbst, erreicht werden.45 Wie Formel (97) und Formel (98) zeigen, können die Spaltensummenvektoren $s 1$

45 Ein Abzug der Einheitsmatrix $I$ von den Ergebnismatrizen $R$ und $R^{*}$ bleibt für die Rangreihung der Knoten nach ihrer Bedeutungehne Effekt)78-3-631-75376-7 
und $s 2$ bzw. die Zeilensummenvektoren $z l$ und $z 2$ von $R^{*}$ und $R$ grundsätzlich als Vektoren der ,Bedeutung' der Knoten bezogen auf die Vorläuferknoten bzw. die Nachfolgerknoten gesehen werden. Tabelle 20 beschreibt die verschiedenen Kenngrößen, die aus den Spalten- und Zeilensummenvektoren für die Knoten entnommen werden können und einen Einblick in die ,Sogwirkung' (die Wirkung eines Knoten im Hinblick auf die Vorgängerknoten) bzw. die ,Folgewirkung' (die Wirkung hinsichtlich der Nachfolgerknoten) der Knoten gewähren.

$$
\begin{gathered}
A^{*}=\left(a_{i j}^{*}\right)_{i, j=1, \ldots, n} \quad A=\left(a_{i j}\right)_{i, j=1, \ldots, n} \\
a_{i j}^{*}=\frac{\text { matval }_{i j}}{\sum_{j=1}^{n} \text { matval }_{i j}} a_{i j}=\frac{\text { matval }_{i j}}{\sum_{i=1}^{n} \text { matval }_{i j}} \\
R^{*}=\left(r_{i j}^{*}\right)_{i, j=1, \ldots, n}=\left(I-A^{*}\right)^{-1} \\
s 1=\left(\sum_{i=1}^{n} r_{i j}^{*}-1\right)_{j=1, \ldots, n}^{\prime} \\
z I=\left(\sum_{j=1}^{n} r_{i j}^{*}-1\right)_{i=1, \ldots, n}^{\prime} \\
R=\left(r_{i j}\right)_{i, j=1, \ldots, n}=(I-A)^{-1} \\
s 2=\left(\sum_{i=1}^{n} r_{i j}-1\right)_{j=1, \ldots, n}^{\prime} \\
z 2=\left(\sum_{j=1}^{n} r_{i j}-1\right)_{i=1, \ldots, n}^{\prime}
\end{gathered}
$$


Tabelle 20: Gegenüberstellung der Spalten- und Zeilensummenvektoren von $R^{*}$ und $R$

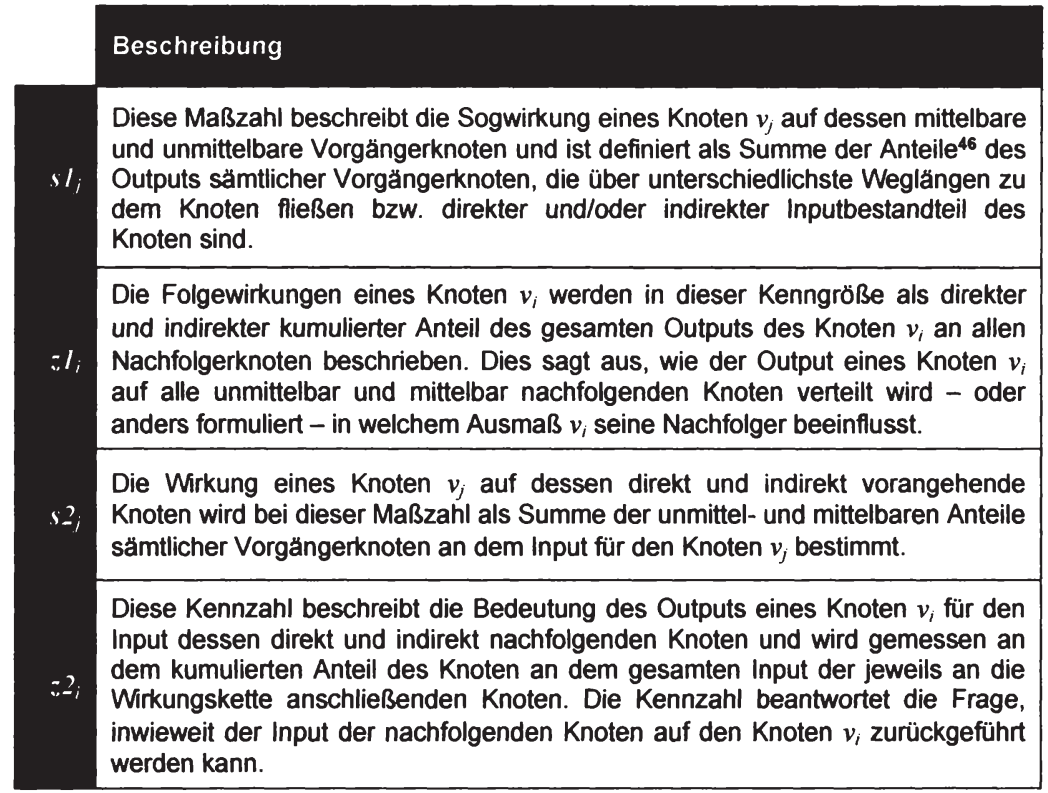

Aus einer ökonomischen Sichtweise bezeichnet $s l_{j}$ die Summe der direkten und indirekten Anteile eines Knoten $v_{j}$ am Konsum der Produktion (des Outputs) der Vorleisterknoten, während $s 2_{j}$ die Summe der mittelbaren und unmittelbaren Anteile der Produktion der Vorleisterknoten an der Produktion von $v_{j}$ anzeigt. Demzufolge beschreibt der Spaltensummenvektor $s 1$, welche Bedeutung ein Knoten $v_{j}$ als Abnehmer für all seine Vorgänger hat. Dies steht im Kontrast zu der Aussage des Spaltensummenvektors $s 2$, der die Bedeutung der Vorgängerknoten als Zulieferer für den Knoten $v_{j}$ festhält. Im Gegensatz zu $s 2$ stellen die Elemente des Zeilensummenvektors $z 1$ jeweils die Summe der unmittelbaren und mittelbaren Verteilung des Outputs eines Knoten $v_{i}$ auf dessen Nachfolger dar. Der Zeilensummenvektor $z 2$ hingegen gibt die Summe der direkten und in-

46 Für jene Fälle in denen über verschiedene Weglängen addiert wird, ist die Summe der Anteile von 1 verschieden. 
direkten Anteile eines Knoten $v_{i}$ an den Inputs für die Produktion der Nachfolgerknoten an. Während $z 2$ also die unmittel- und mittelbaren Effekte eines Knoten $v_{i}$ bezogen auf die Zusammensetzung des Inputs für die anderen Knoten widerspiegelt, zeigt der Zeilensummenvektor $z l$ die Aufteilung der Produktion eines Knoten auf dessen direkte und indirekte Nachfolger. Diese Gegenüberstellung der Spalten- bzw. Zeilensummenvektoren zeigt, dass der Spaltensummenvektor $s 1$ bzw. der Zeilensummenvektor $z 2$ die primäre Quelle für die Bedeutung der Knoten bezogen auf die Vorgänger bzw. Nachfolger sind, nachdem die jeweiligen Maßzahlen Bezug auf den Output der Vorgänger bzw. den Input der Nachfolgerknoten nehmen und sich nicht wie die Kennzahlen der Vektoren $s 2$ bzw. $z 1$ auf den Input bzw. Output des zu bewertenden Knoten beziehen. Nachdem die Knotenbedeutung im Verflechtungskontext zu analysieren ist, sind dementsprechend $s l$ und $z 2$ als Bewertungsgrößen heranzuziehen. Dies gilt nicht nur für gewichtete Graphen, die bspw. für ökonomische Anwendungen relevant sind, sondern auch für nicht gewichtete Graphen, die bspw. bei soziometrischen Anwendungen eingesetzt werden. Zur Lösbarkeit ist in diesem Zusammenhang zu betonen, dass die Koeffizientenmatrizen $A$ und $A^{*}$ definitionsgemäß normiert sind. Darüber hinaus ist für die Konvergenz der in Formel (99) dargestellten unendlichen geometrischen Reihe erforderlich, dass die Matrix nicht nur normiert, sondern auch nicht-zerlegbar ist [Frobenius 1912 aufbauend auf Perron 1907 (Beweise zu diesen Arbeiten werden in Wielandt 1950 auf kürzerem Wege erbracht)]. In der Sprache der Graphentheorie formuliert bedeutet NichtZerlegbarkeit, dass alle Knotenpaare wenigstens in eine Richtung über zumindest eine (beliebige) Weglänge miteinander verbunden sind.

Möglichkeiten der Modifikation der Bedeutungsbestimmung ergeben sich über eine Abschwächung oder Verstärkung der indirekten Effekte gemäß dem Ansatz von Katz durch eine Multiplikation der Einheitsmatrix der Leontief-Inverse mit einem entsprechend gewählten Faktor oder aber auch über eine Beschränkung auf bestimmte Weglängen, wie später bei der Bewertung von Lücken vorgeschlagen wird. Als eine weitere Variation könnten die indirekten Effekte auf 
den jeweiligen Knoten selbst aus der Kalkulation entfernt werden, was aufgrund des erforderlichen Eingriffs in die Inversion allerdings rechnerisch aufwendiger ist, für Vergleichszwecke aber interessant sein könnte. Hierfür müssten in der hinter der Leontief-Inverse liegenden geometrischen Reihe

$$
\left(I+A^{1}+A^{2}+A^{3}+\ldots+A^{n}\right)=(I-A)^{-1}
$$

die Diagonalelemente jeder Potenz der Koeffizientenmatrix $A$ nullgesetzt werden. Das heißt, anstatt der Potenz $A^{p}$ wäre die bereits eingeführte Variante $A^{p}$ (Formel (69)) einzusetzen.

\subsubsection{Cliquenidentifikation und komplementärer Status nach Hubbell}

Die in den obigen Ausführungen beschriebene alternative Interpretation der Leontief-Inverse wird unterstützt durch die Arbeit von Hubbell [1965], der im Zuge der Identifikation und Bewertung von stark zusammenhängenden Subgraphen (Cliquen) eines Graphen den ,komplementären Status' der Knoten (anders formuliert: die Bedeutung der Knoten bezogen auf deren Nachfolgerknoten) anhand der Koeffizienten einer Inversen misst. Darüber hinaus führt Hubbell exogene Einflussfaktoren ein, die die Statuswerte der Knoten mitbestimmen können.

Unter Erweiterung von Hubbells Notation und Formalisierung sei nun dessen Vorgehensweise kurz dargestellt. Ausgehend von einer symmetrischen Assoziationsmatrix $M$ (Formel $\left.(100)^{47}\right)$, die für jedes Knotenpaar das Minimum der jeweiligen Inversekoeffizienten enthält, werden die Knotenpaare als starkzusammenhängend (,major') oder schwach-zusammenhängend (,minor') mittels der Schranke $\Theta$ klassifiziert. Matrix $K$ aus Formel (101) fasst diese Klassifikation zusammen. Stark zusammenhängende Knotenpaare werden dabei der gleichen Clique zugeordnet. Die Anzahl der Cliquen ist von der Wahl von $\Theta$ abhängig. Die

47 Je nach Anwendungszweck kann anstatt - wie von Hubbell vorgeschlagen - der

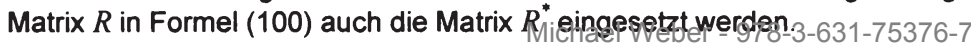


Variation der Schranke $\Theta$ erzeugt eine Hierarchie von Gruppierungen. Dieses Prozedere ist vergleichbar mit der Vorgangsweise in der hierarchischen Clusteranalyse.

$$
\begin{gathered}
M=\left(m_{i j}\right)_{i, j=1, \ldots, n} \\
m_{i j}=m_{j i}=\min \left(r_{i j}, r_{j i}\right) \\
K=\left(k_{i j}\right)_{i, j=1, \ldots, n} \\
k_{i j}= \begin{cases}\text { major } & \text { wenn } m_{i j} \geq \Theta \\
\text { minor } & \text { wenn } m_{i j}<\Theta\end{cases}
\end{gathered}
$$

Dieser Cliquenidentifikationsansatz von Hubbell könnte nun folgendermaßen umgesetzt werden. Ausgehend von der Matrix $K$ (ohne Selbstbezüglichkeit) wäre zeilen- oder spaltenweise ein Vektor klik zu befüllen, der letztlich für jeden Knoten die Cliquenzugehörigkeit angibt. Beginnend mit einer Basiszuordnung auf Grundlage der 1. Zeile oder Spalte würden dabei über eine zeilen- oder spaltenweise Iteration Knoten im Falle der Zusammengehörigkeit in einer Clique vereinigt werden. Zur weiteren Veranschaulichung dieses Berechnungsvorschlags siehe die Formeln (102) bis (104) (Funktion COMPKLIK).

$$
k l i k=\left(k l i k_{j}\right)_{j=1, \ldots, n}
$$

$k l i k_{1}=1$

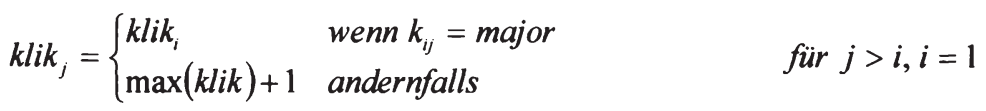

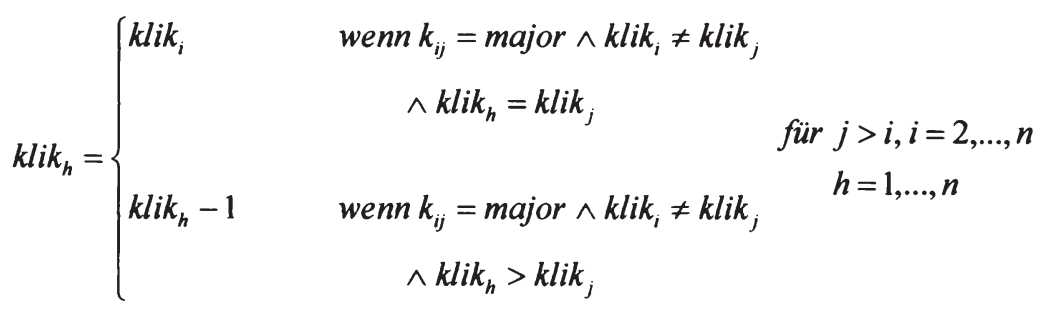


Auf Basis dieses Algorithmus kann für die Ausgangsmatrix $K$ - in Abbildung 6 grafisch in Adjazenzmatrixform dargestellt - für ein bestimmtes $\Theta$ über die Cliquenidentifikation eine Matrixpermutation durchgeführt werden. Das Ergebnis dieser Permutation zeigt Abbildung 7 (Funktion ORDEREDPLOTKLIK).

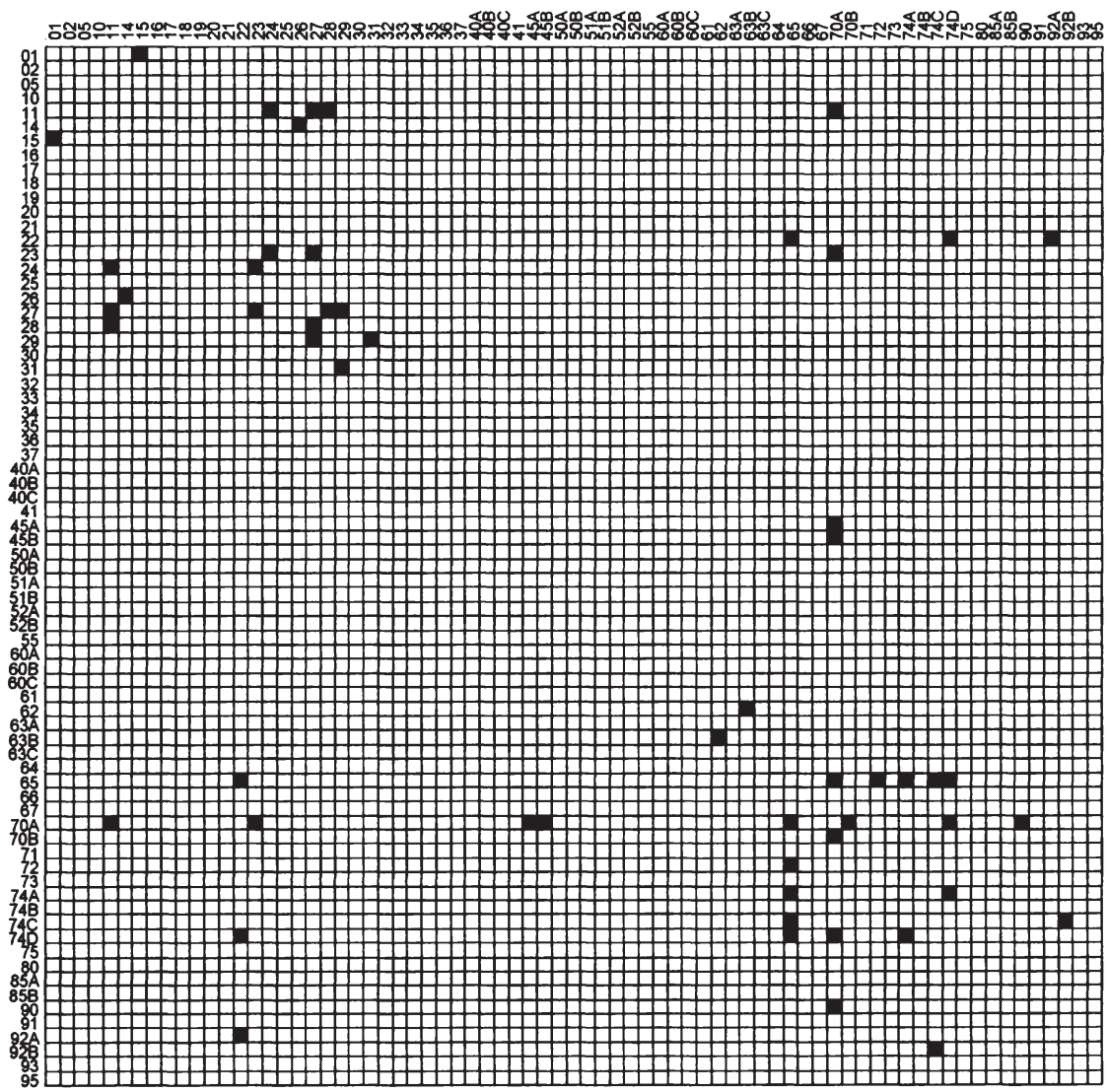

Abbildung 6: ursprüngliche Adjazenzmatrix $K$ 


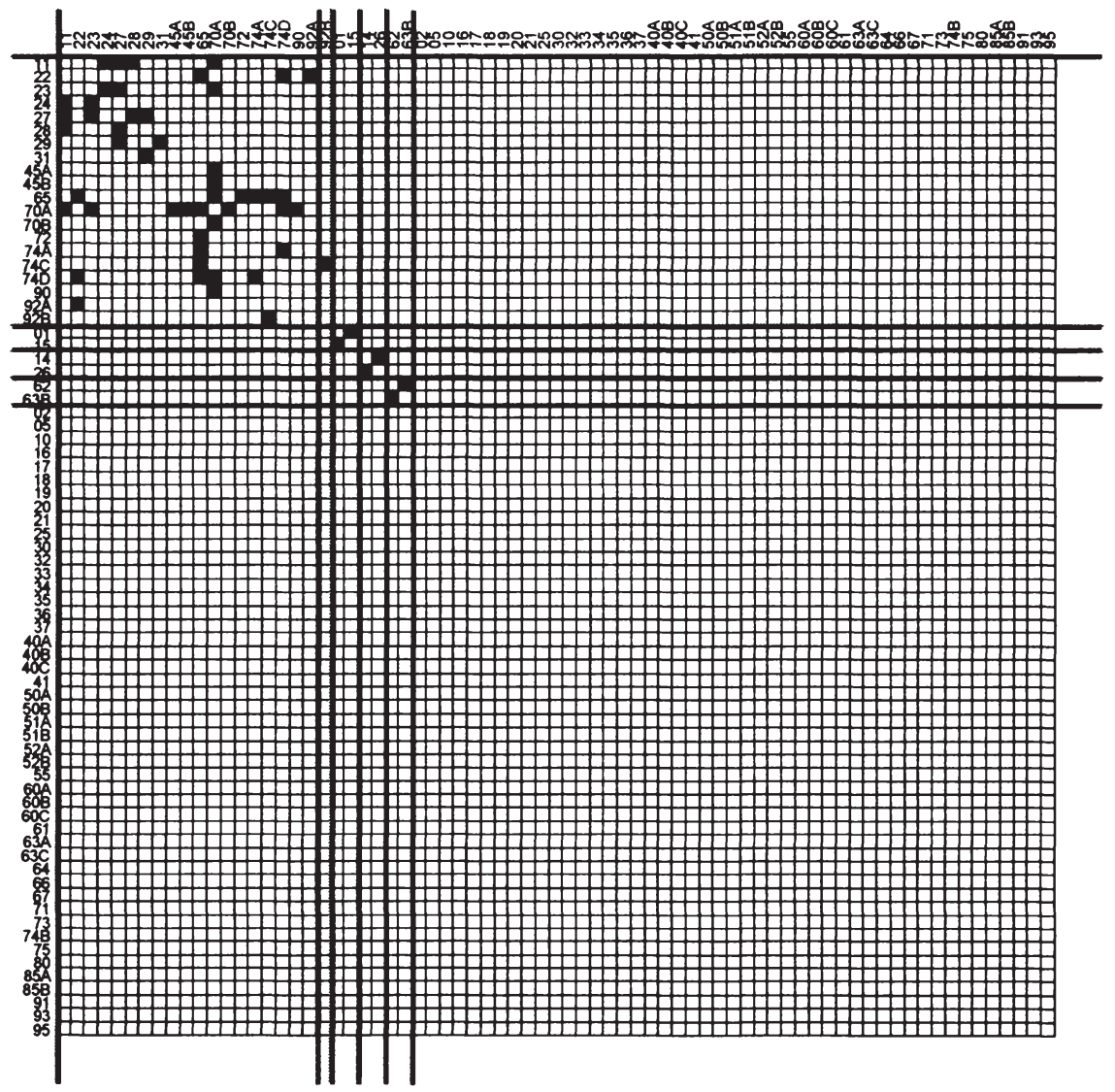

Abbildung 7: Adjazenzmatrix $K$ nach Cliquenidentifikation und Permutation

Eine Zusammenhangsmaßzahl für den Graphen ist in einem weiteren Schritt ermittelbar (Funktion COMPZMG), wobei im Allgemeinen selbstbezügliche Kanten auszuschließen sind. Diese von Hubbell vorgeschlagene Maßzahl ist natürlich auch für die identifizierten Subgraphen (d.h. die Cliquen) eruierbar und könnte bspw. für den Vergleich von Cliquen herangezogen werden. Zunächst sind für die Berechnung die Knotenpaare eines Graphen nach dem jeweiligen Assoziationsindex $m_{i j}$ aufsteigend zu ordnen. Darauf folgend wird die Gesamtknotenverbindungsstärke $\xi$ des Graphen ermittelt, die als Summe der absoluten Koeffizienten der Knotenpaare aus Matrix $A$ (exklusive Selbstbezüglichkeit) definiert ist. 
Für jedes Knotenpaar wird die Knotenverbindungsstärke durch Addition der absoluten Koeffizienten der das Knotenpaar verbindenden Kanten berechnet. Die relative Knotenverbindungsstärke $\xi_{i j}$ erhält man durch Division dieses Wertes durch die Gesamtknotenverbindungsstärke $\xi$, wie in Formel (105) und Formel (106) gezeigt.

$$
\begin{gathered}
\xi=\sum_{\substack{i, j=1 \\
i \neq j}}^{n}\left|a_{i j}\right| \\
\xi_{i j}=\frac{\left|a_{i j}\right|+\left|a_{j i}\right|}{\xi} \text { wobei } i, j=1, \ldots, n, i \neq j
\end{gathered}
$$

Die Zusammenhangsmaßzahl des Graphen $\Theta_{(1-\alpha)}$ ist nun das $(1-\alpha)$-Perzentil des Assoziationsindex $m_{i j}$. Im Allgemeinen wird ein $\alpha$ von $5 \%$ festgelegt. Die Bestimmung des Perzentils wird in Tabelle 21 skizziert, die die nach dem Assoziationsindex aufsteigend sortierten Knotenpaare $\left(v_{i}, v_{j}\right)$ mit $i<j$, deren relative Knotenverbindungsstärke $\xi_{i j}$ und die jeweilige kumulierte relative Knotenverbindungsstärke enthält.

Tabelle 21: Ermittlung der Zusammenhangsmaßzahl des Graphen

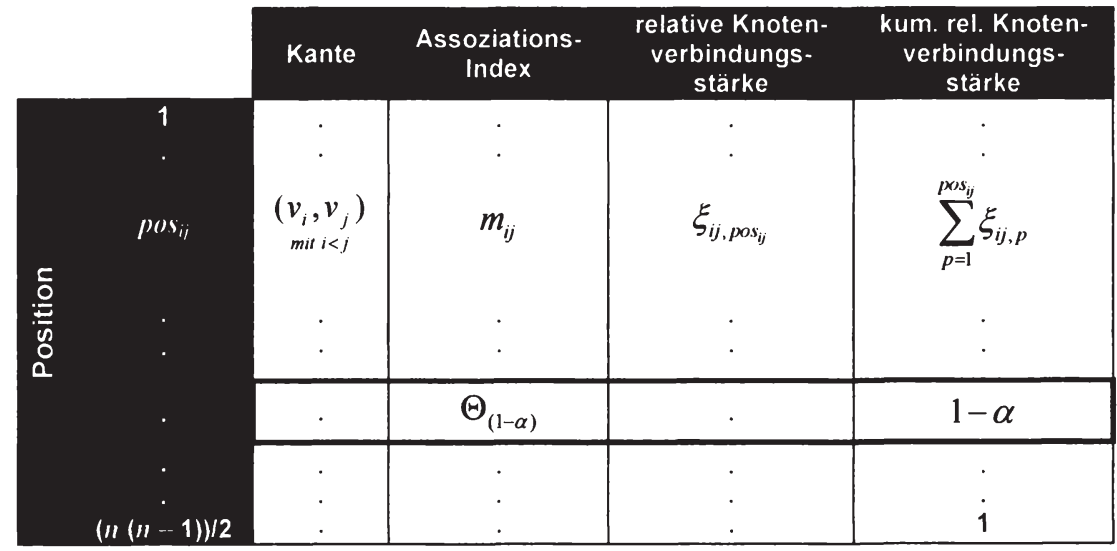


Die am stärksten verbundenen Knotenpaare, die gemeinsam $\alpha$ (z.B. $5 \%$ ) der gesamten Knotenverbindungsstärke des Graphen ausmachen, haben einen Assoziationsindex von über $\Theta_{(1-\alpha)}$. Die Zusammenhangsmaßzahl $\Theta_{(1-\alpha)}$ gibt damit eine untere Schranke des Assoziationsindex für die am stärksten verbundenen Knotenpaare an. Der Indikator kann als Vergleichswert zwischen Graphen (bzw. den ermittelten Subgraphen) eingesetzt werden.

Mit explizitem Bezug auf die Leontief-Inverse $R$ berechnet Hubbell einen ,komplementären Statuswert' (Zeilenvektor status ${ }^{N K}$ ) und berücksichtigt auch exogene Faktoren, die den Status der Knoten beeinflussen (Spaltenvektor $e^{N K}$ ) in der Kalkulation. Aus verschiedenen Perspektiven wird im Folgenden eine auf Hubbells Vorschlag beruhende Berechnung eines komplementären ,Status' bezogen auf Nachfolger- aber auch auf Vorläuferknoten unter Einbezug von externen Beiträgen beschrieben. Das Zusammenspiel der exogenen Faktoren mit der Leontief-Inverse wird in Formel (107) und Formel (108) bzw. Formel (111) und Formel (112) aufgezeigt. Formel (109) und Formel (110) bzw. Formel (113) und Formel (114) stellen den Zusammenhang zwischen den exogenen Faktoren und der Koeffizientenmatrix dar. Betreffs möglicherweise auftretender Selbstbezüglichkeit, die bei Hubbell aufrechterhalten bleibt, wird auf obige Ausführungen zur alternativen Berechnung der Inverse verwiesen.

Die Bedeutung, der Einfluss oder der ,komplementäre Status' eines Knoten im Bezug auf die Nachfolgerknoten, der Spaltenvektor status ${ }^{N K}$, ermittelt sich nach Hubbell demnach wie folgt:

$$
\begin{gathered}
\text { status }^{N K}=(I-A)^{-1} e^{N K}=R \cdot e^{N K} \\
\text { status }_{i}^{N K}=r_{i 1} e_{1}^{N K}+r_{i 2} e_{2}^{N K}+\ldots+r_{i n} e_{n}^{N K} \quad \text { wobei } i=1, \ldots, n \\
\text { status }^{N K}=e^{N K}+A \cdot \text { status }^{N K} \\
\text { status }_{i}^{N K}=e_{i}^{N K}+\left(a_{i 1} \text { status }_{1}^{N K}+a_{i 2} \text { status }_{2}^{N K}+\ldots+a_{i n} \text { status }_{n}^{N K}\right) \\
\text { wobei } i=\text { Mịchinal Weber }-978-3-631-75376-7_{\text {Downloaded from PubFactory at 01/11/2019 05:44:37AM }} \\
\text { via free access }
\end{gathered}
$$


In umgekehrter Betrachtung kann die Bedeutung eines Knoten bezogen auf die Vorläuferknoten, der Zeilenvektor status $^{V K}$, folgendermaßen errechnet werden:

$$
\begin{gathered}
\text { status }^{V K}=e^{V K}(I-A)^{-1}=e^{V K} \cdot R \\
\text { status }_{j}^{V K}=r_{1 j} e_{1}^{V K}+r_{2 j} e_{2}^{V K}+\ldots+r_{n j} e_{n}^{V K} \quad \text { wobei } j=1, \ldots, n \\
\text { status }^{V K}=e^{V K}+\text { status }^{V K} \cdot A \\
\text { status }_{j}^{V K}=e_{j}^{V K}+\left(a_{1 j} \text { status }_{1}^{V K}+a_{2 j} \text { status }_{2}^{V K}+\ldots+a_{n j} \text { status }_{n}^{V K}\right) \\
\text { wobei } j=1, \ldots, n
\end{gathered}
$$

Für den Fall, dass die exogenen Statusbeiträge $e^{V K}\left(\mathrm{bzW} . e^{N K}\right)$ oder deren relatives Verhältnis zueinander nicht bekannt sind, betont Hubbell, dass die Statusberechnung stattdessen mit identischen exogenen Statusbeiträgen pro Knoten durchgeführt werden kann. Ohne Beschränkung der Allgemeinheit kann daher bspw. 1 als exogener Beitrag angenommen werden. Dies läuft nun letztlich aber darauf hinaus, dass der (komplementäre) ,Status' nur über die Spalten- bzw. Zeilensumme der Leontief-Inverse bestimmt wird - allerdings bei Hubbell mit Selbstbezüglichkeit der Knoten -, was der Bedeutungsbestimmung von Knoten aus Formel (98), $s 2$ bzw. $z 2$ (ohne Abzug der Identitätsmatrix), entspricht.

\subsubsection{Zwischen Perron-Frobenius und PageRank ${ }^{\mathrm{TM}}$}

Auch wenn Hubbell in seinem Beitrag neben Leontief intensiv auf soziometrische Ansätze wie jene von Forsyth \& Katz [1946], Festinger [1949], Luce \& Perry [1949] und insbesondere Katz [1953] hinweist, muss an dieser Stelle betont werden, dass die Gewichtung der Kanten durch die Koeffizientenmatrix $A$ im Modell von Leontief bereits inkludiert ist. Die zitierten soziometrischen Ansätze bauen auf binären Adjazenzmatrizen auf, welche zu einer Informationsverdichtung und somit zu Informationsverlust führen, nachdem alle Kanten mit 1 ,gewichtet' werden. Erst Katz [1953] führte - vor dem Hintergrund der Regulierung des Einflusses von weiter entfernten Knoten - einen Dämpfungs- 
parameter ein, der jedoch auch nur eine (mit der Entfernung abnehmende) Gleichgewichtung aller Kanten bewirkt. Hubbells Verdienst ist es, die Aufmerksamkeit (zurück) ${ }^{48}$ auf Leontiefs Inverse zu lenken, wobei er auch negative Koeffizienten bzw. Gewichte in die Berechnung einbeziehbar machte. Die Auswirkungen lassen sich in folgenden, überaus einflussreichen Formeln (Formel (115) und Formel (116)) veranschaulichen. Die Notation in beiden Formeln ist nicht in Verbindung mit der bisherigen Notation zu sehen, dies gilt insbesondere auch für $R$.

$$
\begin{gathered}
R(u)=c \cdot \sum_{v \in B_{u}} \frac{R(v)}{N_{v}} \\
R^{\prime}(u)=c \cdot \sum_{v \in B_{u}} \frac{R^{\prime}(v)}{N_{v}}+c \cdot E(u)
\end{gathered}
$$

Diese zu Demonstrationszwecken wiedergegebenen Formeln (115) und (116) sind nichts anderes als der Kern des PageRank ${ }^{\top M}$ Algorithmus von Google ${ }^{\mathrm{TM}}$, der in Page et al. [1998] vorgestellt wird, ohne Referenz (,Link') auf Hubbell [1965] oder Katz [1953]. Formel (115) gibt die Statusberechnung (den Rang) für einen Knoten auf Basis der Bewertung der Vorläuferknoten wieder, Formel (116) stellt eine Erweiterung dieser Berechnung um exogene Faktoren dar. Vergleicht man nun die Grundidee des PageRank ${ }^{\top M}$ Algorithmus mit der Berechnung der Leontief-Inverse, so zeigt sich, dass seit der Veröffentlichung von Leontief [1937], der sich schon während seines Doktoratsstudiums in Deutschland in den späteren 1920er Jahren mit Wirtschaftskreisläufen und wirtschaftlichen Verflechtungen beschäftigte ${ }^{49}$, keine nennenswerte Änderungen des auf dem Perron-Frobenius

48 Wie bereits erwähnt, wurde in den vorangegangenen einschlägigen Publikationen nicht auf Leontief referenziert.

49 Wassily Leontiefs 1928 an der heutigen Humboldt-Universität zu Berlin approbierte

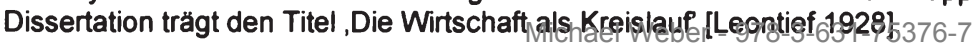


Theorem ${ }^{50}$ beruhenden Berechnungsansatzes erfolgt sind. Dies soll nun veranschaulicht werden.

Der ,Status' oder ,Rang' $R$ eines Knoten $u$ (bspw. eine Internetseite) ist gemäß Formel (115) das Produkt der Normierungskonstante $c$ mit der Summe der Quotienten der Ränge $R$ der Vorgängerknoten $v \in B_{u}$ des Knoten $u$ und der Anzahl $N$ der Nachfolger aller Vorgänger $v$. In Formel (116) wird zusätzlich ein mit $c$ normierter exogener Statusbeitrag $E$ für den Knoten $u$ addiert. Vergleicht man Formel (116) mit Formel (113), so sieht man, dass die Koeffizienten der Matrix $A$ im PageRank ${ }^{\mathrm{TM}}$ Algorithmus durch

$$
a_{i j}=\frac{c}{\sum_{k=1}^{n} m^{2} t_{i k}} \text { für } i, j=1, \ldots, n, \text { wobeic }<1
$$

gegeben sind, wobei Mat die binäre Adjazenzmatrix zu $A$ ist. Umgekehrt interpretiert enthalten die Koeffizienten der Matrix $A$ die Gewichte für die Elemente der Adjazenzmatrix Mat. Allerdings führt die Definition der Gewichte aus Formel (116) bzw. (117) zu einer zeilennormierten Matrix $A$, d.h. gemäß der bisherigen Notation handelt es sich eigentlich um $A^{*} . R(u)$ steht für $\operatorname{status}_{j}^{V K}$ und $R(v)$ für status $_{i}{ }^{K}$ mit $i:$ mat $_{i j}>0$. Der, Status' $R$ entspricht somit dem bereits beschriebenen Vektor zur Bedeutung eines Knoten bezogen auf dessen Vorläuferknoten $s 1$ aus Formel (97) (ohne Abzug der Identitätsmatrix). Die Normierungs-

50 Das Perron-Frobenius Theorem, das auf die Mathematiker Oskar Perron [1907] und Ferdinand Georg Frobenius [1912] zurückgeht, trifft Aussagen über die Eigenwerte und Eigenvektoren einer reellwertigen, nicht negativen quadratischen Matrix. Einsatzbereiche des Theorems reichen von der Leontief-Inverse über Markov-Ketten bis hin zu Bewertungsansätzen für Teilnehmer an sportlichen Wettkämpfen. Konkret besagt das Theorem, dass es für eine nicht zerlegbare Matrix $P$ mit nicht negativen Elementen, deren Potenz $P^{k}$ ausschließlich positive Werte aufweist, einen reellen, positiven und einfachen Eigenwert $\lambda$ gibt, der größer als alle anderen Eigenwerte der Matrix $P$ ist. $Z u$ diesem Eigenwert $\lambda$ existiert ein Eigenvektor, der nur positive Elemente enthalt. 
konstante $c$ ist der größte Eigenwert einer adaptierten Adjazenzmatrix, die anstelle der Einträge $>0$ jeweils den Kehrwert der entsprechenden Zeilensumme der Adjazenzmatrix Mat enthält. Sie wird benötigt, um die Summe der Statuswerte $\Sigma R(u)$ über alle Knoten $u$ selbst bei variierender Knotenanzahl gleich zu halten. Der exogene Statusbeitrag $e$ für den Knoten $u$ mit dem (Spalten-)index $j$ aus Formel (113) - mit $A^{*}$ anstatt $A$ - entspricht im PageRank ${ }^{\top M}$ einem mit der Normierungskonstante $c$ multiplizierten exogenen Beitrag $E(u)^{51}$.

$$
e_{j}=c \cdot E(u) \text { für } i, j=1, \ldots, n
$$

Eine vergleichende Betrachtung der hier vorgestellten Ansätze zeigt, dass im PageRank $^{\top M}$ Algorithmus einzig die Gewichtung unter Verwendung einer Normierungskonstante neu definiert wird, während die Formeln zur Status- oder Rangberechnung bei Hubbell und Page et al. identisch sind und von Leontiefs Berechnung nur aufgrund der exogenen Beiträge abweichen. Die Gewichtung bei Leontief ist, wie zuvor dargelegt, über die Koeffizientenmatrix $A$ bereits vorgegeben. Die Wahl von $A^{\star}$ zur Bestimmung der Knotenbedeutung bezogen auf die Vorgängerknoten über $s I$ wurde in dieser Arbeit auf Seite 124f. begründet und erfährt durch obige Ausführungen zusätzliche Bestätigung. Auch Wassily Leontiefs Einfluss auf die heutige Informationsallokation über das Internet konnte hiermit demonstriert werden.

\subsection{Darstellung von Verflechtungsdaten}

Eine Beschränkung der Auswertung von gerichteten, gewichteten Verflechtungsdaten auf bestimmte Transaktionsströme, bspw. zur Identifikation von maßgeblichen Vorläuferknoten (Lieferanten) oder Nachfolgerknoten (Nachfragern) in der deskriptiven Input-Output-Analyse oder allgemein zur Extraktion von Relationen

51 Auch wenn der exogene Beitrag $E$ im PageRank ${ }^{\mathrm{m}}$ Algorithmus groß geschrieben wird, ist er ein Vektor, dementsprechend ist. Ex(y) ein Skgalar.78-3-631-75376-7 
in einem bestimmten Wertebereich, ermöglicht eine höhere Übersichtlichkeit in der weiteren Verarbeitung und Darstellung der Daten. Abhängig von der jeweiligen Fragestellung kann zu diesem Zweck ein Bereichskriterium bzw. ein Schwellenwert als Filter für die Verflechtungsdaten gesetzt werden. Die gefilterte Matrix FMatVal kann ausgehend von einer (anteils-)gewichteten Matrix MatVal mittels der unteren Filterschranke und/oder der oberen Filterschranke $f s^{\circ}$, gemäß Formel (119) berechnet werden (Funktion FS). FMatVal kann dann als Ausgangspunkt für die Analyse der Adjazenzmatrix $(F)$ Mat dienen.

$$
\begin{aligned}
\text { FMatVal } & =\left(\text { fmatval }_{i j}\right)_{i, j=1, \ldots, n} \\
\text { fmatval }_{i j} & = \begin{cases}\text { matval }_{i j} & \text { wenn } f s^{u} \leq \text { matval }_{i j} \leq f s^{o} \\
0 & \text { andernfalls }\end{cases}
\end{aligned}
$$

Alternativ wäre es auch denkbar rein zeilen- oder spaltenbezogene Filter zu setzen. Will man bspw. die Vorläuferknoten im Wertebereich der $x$ höchsten Inputwerte pro Knoten ermitteln, so wäre folgendes simples Vorgehen unter Verwendung einer absteigenden Sortierung über sort ${ }^{\text {desc }}$ zweckmäßig (Funktion SHRINK).

$$
\begin{aligned}
f s_{j}^{u}(x) & =\left(\underset{i=1, ., n}{\operatorname{sortdesc}}\left(\text { matval }_{i j}\right)\right)_{x} \\
\text { fmatval }_{i j} & = \begin{cases}0 & \text { wenn } \text { matval }_{i j}<f s_{j}^{\prime \prime}(x) \\
\text { matval }_{i j} & \text { andernfalls }\end{cases}
\end{aligned}
$$

Die Visualisierung bietet neben der Identifikation von maßgeblichen Relationen in Verflechtungsdaten eine weitere Möglichkeit zur Identifikation von wesentlichen Zusammenhängen, die sich auch für ungewichtete Graphen eignet. Abbildung 8 zeigt die schematische Darstellung des wirtschaftlichen Kreislaufs von Wassily Leontief [1928, S. 587], die im Kern nicht von der heute gebräuchlichen grafischen Darstellungsform von relationalen Datenbeständen abweicht. Die visuelle Aufbereitung bietet den Vorteil einer einfachen Interpretationsmöglichkeit der Daten, welcher allerdings nur für kleinere bzw. gefilterte Datensätze gilt 
(Funktion PLOTIO basierend auf der Funktion GPLOT aus dem R-Paket,sna' [Butts 2007]). Zu dem Zweck der Analyse und Interpretation von Zusammenhängen in größeren relationalen Datenbeständen sind daher andere Alternativen zu verfolgen. Ein interessanter und viel versprechender Ansatz, der auch für das Transaktionsunterstützungsmodell dieser Arbeit von großem Nutzen sein kann, sei daher nachfolgend beschrieben.

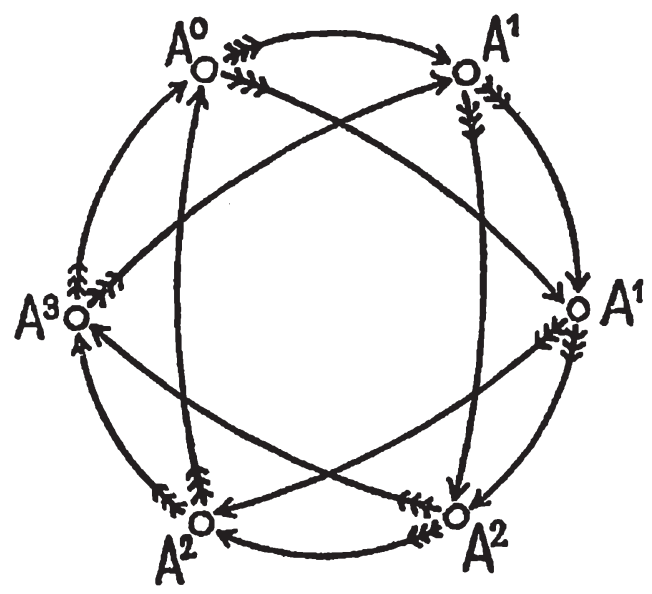

Abbildung 8: Elementares Schema des wirtschaftlichen Kreislaufs Quelle: Leontief [1928, S. 587]

\subsection{Blockmodeling - Clustering und Partitionierung von relationalen Datensätzens2}

Die Identifikation und Kategorisierung von Verflechtungsmustern in relationalen Datenbeständen ist ein weiterer wichtiger Analyseschritt, der speziell im Umgang mit Graphen mit hoher Knotenanzahl die Interpretation der Daten erleichtert bzw. deren Übersichtlichkeit erhöht und auch der Input-Output-Analyse interessante Auswertungsmöglichkeiten eröffnet. Im Umfeld der sozialen Netzwerkanalyse 
(SNA) konnten hierzu in den letzten Jahren Fortschritte bei dem Clustering ${ }^{53}$ von Knoten eines Graphen bei gleichzeitiger Partitionierung der Beziehungen zwischen den Knoten in sogenannte ,Blöcke' erzielt werden. Diese ,Blockmodellberechnung' (,blockmodeling') [Breiger et al. 1975; White et al. 1976] entwickelte sich ausgehend von indirekten Clustering-Ansätzen im Hinblick auf die strukturelle Äquivalenz ${ }^{54}$ von Knoten bzW. Knotengruppen [Lorrain \& White 1971; Burt 1976], über eine Generalisierung auf reguläre Äquivalenz ${ }^{55}$ [White \& Reitz 1983] inzwischen zu einer Strömung, die neben indirekten v.a. auch direkte Verfahren für Blockmodellberechnungen auf Basis struktureller und regulärer Äquivalenz von Knoten [Batagelj et al. 1992a; 1992b] bietet. In jüngster Zeit wurde hierbei insbesondere der direkte Ansatz zur Berechnung von ,Blockmodellen' für die soziale Netzwerkanalyse durch [Doreian et al. 2004] bzw. [Doreian et al. 2005] im Rahmen des so genannten, generalized blockmodeling' [Batagelj et al. 1992a; 1992b; Doreian et al. 1994; Batagelj 1997] ausgebaut bzw. verdichtet.

Doreian et al. [2004] erscheint in diesem Zusammenhang besonders geeignet für

53 Die Clusteranalyse ist ein strukturuntersuchendes, multivariates Analyseverfahren, welches zur Ermittlung von (in sich) möglichst homogenen Gruppen (Clustern) von beobachteten Fällen anhand von Ähnlichkeiten bzw. Unähnlichkeiten zwischen den beobachteten Merkmalen dieser Fälle eingesetzt wird. Für eine Vertiefung in die Clusteranalyse und deren Verfahren siehe bspw. Everitt [1974], Backhaus et al. [2000] oder Fahrmeir et al. [1996].

54 In einem (gerichteten) Graph $G(V, E)$ sind zwei Knoten $v, w \in V$ strukturell äquivalent, wenn für jede (gerichtete) Beziehung des Knoten $v$ zu irgendeinem anderen Knoten $x \in V$ auch eine (gleichgerichtete) Beziehung des Knoten $w$ zu diesem anderen Knoten $x$ besteht. D.h., wenn eine (gerichtete) Kante $(v, x)$ und/oder $(x, v)$ existiert, so gibt es auch $(w, x)$ und/oder $(x, w)$, wobei $v \neq w$. In dem Blockmodell zu dem (gerichteten) Graph werden die strukturell äquivalenten Knoten zusammengefasst dargestellt.

55 Reguläre Äquivalenz ist eine Generalisierung von struktureller Äquivalenz. In einem (gerichteten) Graph $G(V, E)$ sind zwei Knoten $v, w \in V$ regulär äquivalent, wenn für jede (gerichtete) Beziehung von $v$ zu einem Knoten $x \in V$ immer auch eine (gleichgerichtete) Beziehung von $w$ zu einem Knoten $y \in V$ existiert, d.h. besteht eine (gerichtete) Kante $(v, x)$ und/oder $(x, v)$, so existiert auch $(w, y)$ und/oder $(y, w)$, wobei $v \neq w$. Für die Strukturanalyse bietet die reguläre Äquivalenz somit einen Ausgangspunkt zur Bestimmung von Knotengruppen. So könnte bspw. eine Gruppe der Knoten $x$ und $y$ sowie eine Gruppe der Knoten $v$ und $w$ gebildet und beide Gruppen

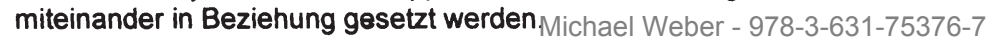


den Einsatz im Kontext der hier behandelten Fragestellung. Der Beitrag stellt eine Erweiterung des (direkten) Ansatzes um die getrennte Betrachtung bzw. Klassifikation der Vorläufer- und Nachfolgerbeziehungen von Knoten eines Graphen vor ${ }^{56}$, die unterschiedliche Vorläufer- und Nachfolgerknotengruppierungen sowie Partitionierungen der Kanten bewirkt. Dieser Ansatz ermöglicht einerseits die Modellierung von Graphen mit disjunkten Vorläufer- und Nachfolgerknoten im SNA Jargon ,two-mode network data' - und kann andererseits auch eingesetzt werden, um die unterschiedlichen Vorläufer- und Nachfolgerbeziehungen einer Menge von Knoten (,one-mode network data') differenziert zu gruppieren und dabei gleichzeitig die Kanten in Blöcke zu partitionieren. Zentrale Herausforderung für Verfahren der direkten ,two-mode' Blockmodellberechnung ist dabei die Notwendigkeit, Struktur über Vor- und Nachfolgerknoten zu entdecken, wobei nur eine variable Teilmenge der jeweils anderen Knotenmenge (Nachfolger- bzw. Vorläuferknoten) strukturbestimmend ist und somit die jeweilige Gruppenzugehörigkeit (Cluster) festlegt. Dies ist ein wesentlicher Unterschied zu konventionellen Clustering-Verfahren, die sozusagen - um in der bisherigen Terminologie zu bleiben - von (zwei) disjunkten Knotenmengen und deren Relationen (,two-mode') ausgehen, wobei eine Knotenmenge die beobachteten Fälle umfasst, während die andere Knotenmenge die beobachteten Merkmale enthält. Diese ,two-mode' Daten werden daraufhin durch die Berechnung der Ähnlichkeiten bzw. Unähnlichkeiten zwischen den beobachteten Fällen unter Berücksichtigung aller Merkmale in eine Matrix in der Dimension beobachtete Fälle $x$ beobachtete Fälle - die so genannte Distanzmatrix ${ }^{57}$ - transformiert. Die Distanzmatrix hat somit den Charakter einer ,one-mode' Datenstruktur und ist Ausgangspunkt für das jeweils gewählte Clustering-Verfahren.

56 Die grundsätzliche Idee einer differenzierten Betrachtung der Vorläufer- und Nachfolgerknotengruppen bei der Erstellung von Blockmodellen wurde bereits von Borgatti \& Everett [1992] diskutiert.

57 Die Distanzmatrix, die für Zwecke des Clustering Ähnlichkeits- bzw. Unähnlichkeitsmaße für die beobachteten Fälle beinhaltet, soll hierbei nicht mit den Distanzen zwischen Knoten eines Graphen icferwe Ghselt werdeg-631-75376-7 


\subsubsection{Direkte und indirekte Verfahren}

Indirekte Verfahren der Berechnung von Blockmodellen (z.B. Breiger et al. [1975]) bestimmen für jeden Knoten gewisse Merkmale, meist strukturelle Eigenschaften wie Grade, Reichweiten von Knoten oder Distanzindizes zu den Vorläufern und Nachfolgern, die als Basis für die Ermittlung einer Distanzmatrix dienen. Diese Vorgangsweise entspricht einer Transformation der ursprünglichen ,one-mode' Daten zunächst in ,two-mode' Daten (Knoten und deren Merkmale), um diese wiederum in ,one-mode' Daten umzuwandeln (Distanzmatrix). Auf Basis der Distanzmatrix werden konventionelle Clustering-Verfahren eingesetzt.

Direkte Verfahren zur Ermittlung von Blockmodellen (beginnend mit Batagelj et al. [1992a]) vermeiden den soeben geschilderten Umweg. Ausgehend von der Spezifikation der Struktur eines idealen Blockmodells unter gleichzeitiger Berücksichtigung verschiedener Blocktypen, die abhängig von der gewünschten Äquivalenz (pro Block) variieren, wird in einem iterativen Optimierungsprozess die Abweichung des zu ermittelnden, empirischen Blockmodells zu der idealen Struktur minimiert. Zwei Möglichkeiten können bei der Spezifikation des idealen Blockmodells unterschieden werden. In einer explorativen Vorgangsweise legt man die zulässigen Blocktypen und die Clusteranzahl ohne konkrete Anordnung der Blocktypen fest, während in einer konfirmatorischen Datenanalyse die Anordnung zumindest für einen Teil der gewählten Blocktypen vordefiniert werden muss. Letztere Herangehensweise ermöglicht das Prüfen von Hypothesen über die Struktur des relationalen Datenbestands. In beiden Fällen ${ }^{58}$ erfolgt die Vorgabe einer idealen Struktur an welche die Daten angepasst werden, womit ein wesentlicher Unterschied zu indirekten Verfahren besteht, bei denen Knotengruppierungen (Cluster) und Blöcke rein datengetrieben festgelegt werden.

58 Doreian et al. [2005] bezeichnen das explorative Verfahren als ,induktiv' und das konfirmatorische Verfahren als ,deduktiv'. Nachdem diese Benennung irreführend ist, wird davon abgesehen. 


\subsection{2 Äquivalenzrelationen und Blocktypen}

Wesentliche Voraussetzung zur Bestimmung des idealen Blockmodells ist die Festlegung der Äquivalenzrelation(en), durch die eine Einschränkung der zulässigen Blocktypen erfolgt. Diese Blocktypen dienen als Vorlage für die Idealblöcke des Modells. Blocktypen für strukturelle Äquivalenz sind dabei Nullblöcke (,null blocks') oder vollständige Blöcke (,complete blocks'), für reguläre Äquivalenz sind neben den Nullblöcken die regulären Blöcke (,regular blocks') zu nennen. In einem generalisierten Blockmodell können zusätzliche Blocktypen definiert werden [Doreian et al. 1994; Batagelj 1997]. Auf Basis der Ausführungen in Batagelj [1997] erläutert Tabelle 22 die Blocktypen sowohl für strukturelle und reguläre Äquivalenz als auch für generalisierte Blockmodelle.

Tabelle 22: Blocktypen für Blockmodelle basierend auf der Systematik von Batagelj Quelle: eigene Darstellung beruhend auf Batagelj [1997]

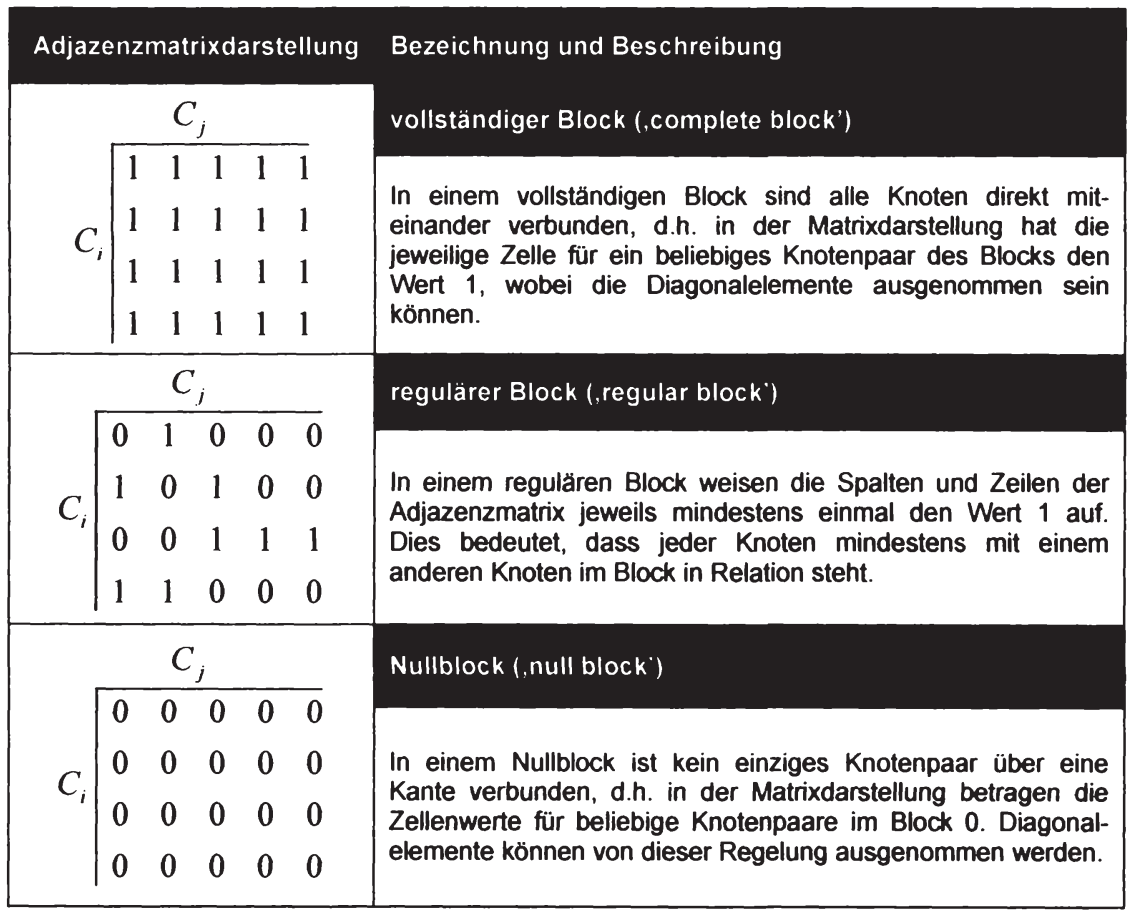




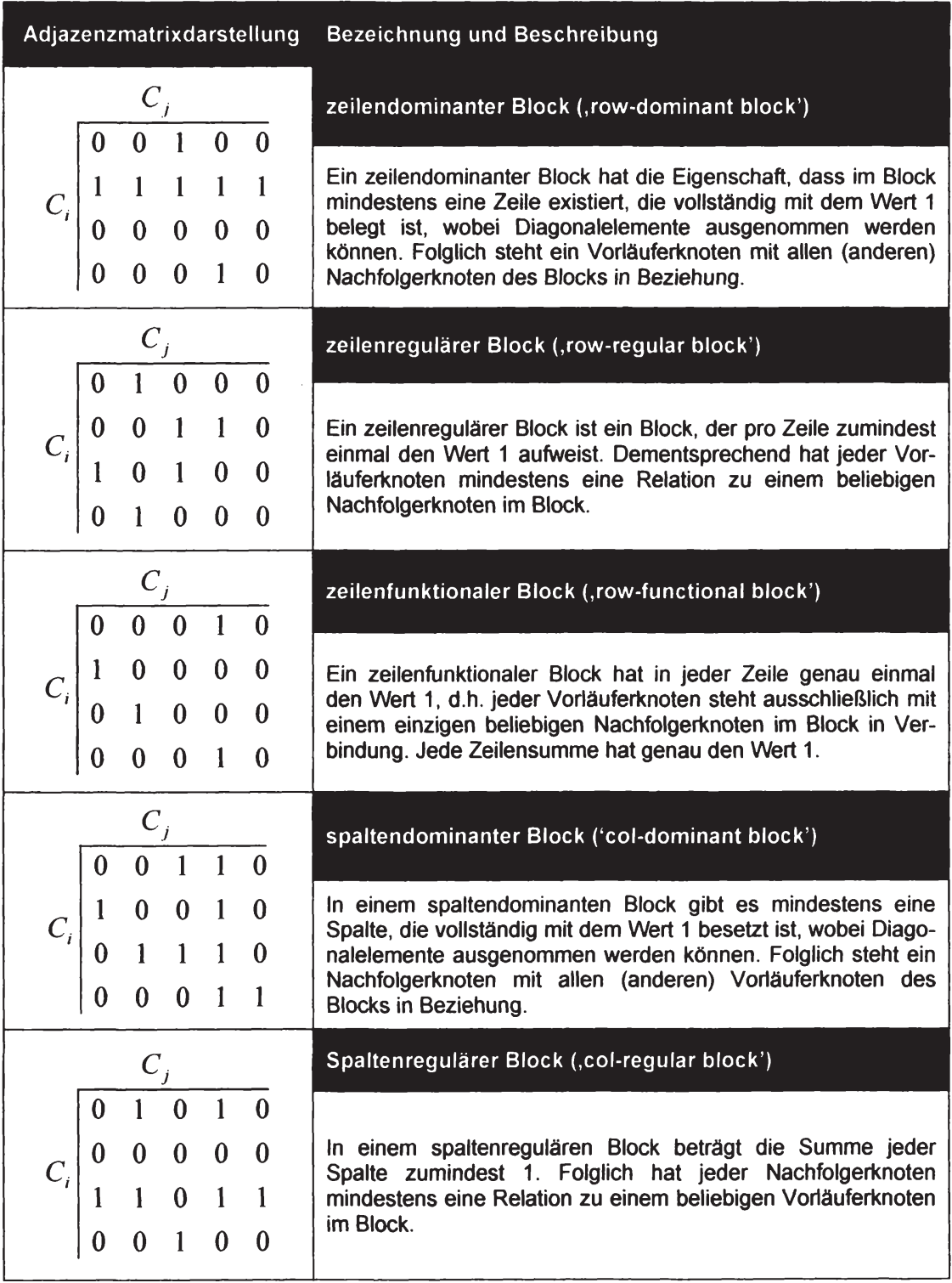




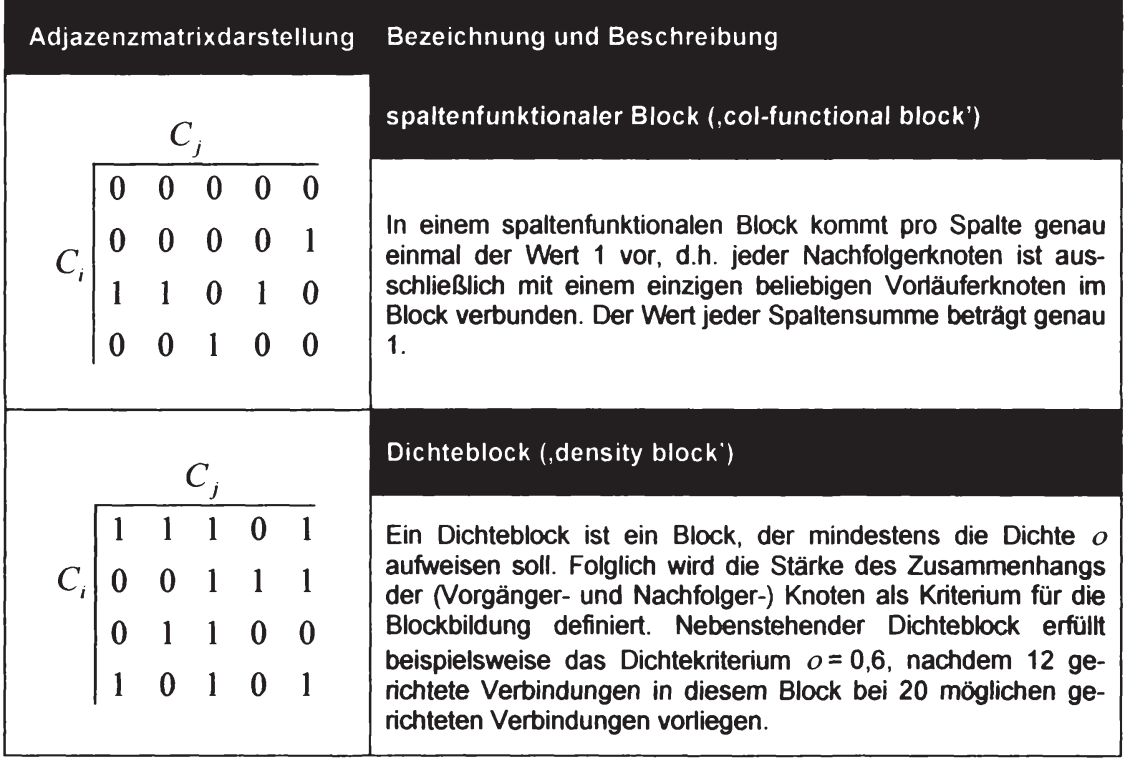

Das Verhältnis der Blocktypen untereinander für einen Block $b$, der - abgesehen vom Nullblock - nicht gleichzeitig einen (oder mehrere) Nullzeilen- und Nullspaltenvektor(en) enthält und somit in die Typologie einordenbar ist, wird in Abbildung 9 dargestellt. Es zeigt sich, dass die Definition der Typen zu einer Art Typenhierarchie führen.

Unter den Blocktypen für reguläre Äquivalenz ermöglichen die Typen ,zeilendominant' und/oder ,spaltenfunktional' bzw. ,spaltendominant' und/oder ,zeilenfunktional' die feinsten Unterscheidungen. Diese vier Blocktypen können demnach in die dritte Ebene einer fiktiven Blocktyphierarchie eingeordnet werden. Eine gleichzeitige Klassifikation eines Blocks als zeilendominant und zeilenfunktional ist aufgrund der Definitionen ebenso auszuschließen wie die Zuordnung der Blocktypen spaltendominant und spaltenfunktional zu einem Block. Die Typen ,spaltenregulär' und ,zeilenregulär' der zweiten Ebene können sich einerseits aus den Typen der dritten Ebene ergeben oder treten eigenständig auf. So ist ein zeilendominanter oder ein spaltenfunktionaler Block gleichzeitig auch ein spaltenregulärer Block, ein spaltendominanter Block oder ein zeilen- 
funktionaler Block ist ebenso zwingend auch ein zeilenregulärer Block. Nicht notwendigerweise ist allerdings ein zeilendominanter Block auch ein zeilenregulärer Block. Ebenfalls ist ein spaltendominanter Block nicht in jedem Fall gleichzeitig spaltenregulär. Ein spalten- und zeilenregulärer Block ist zwangsläufig auch regulär.

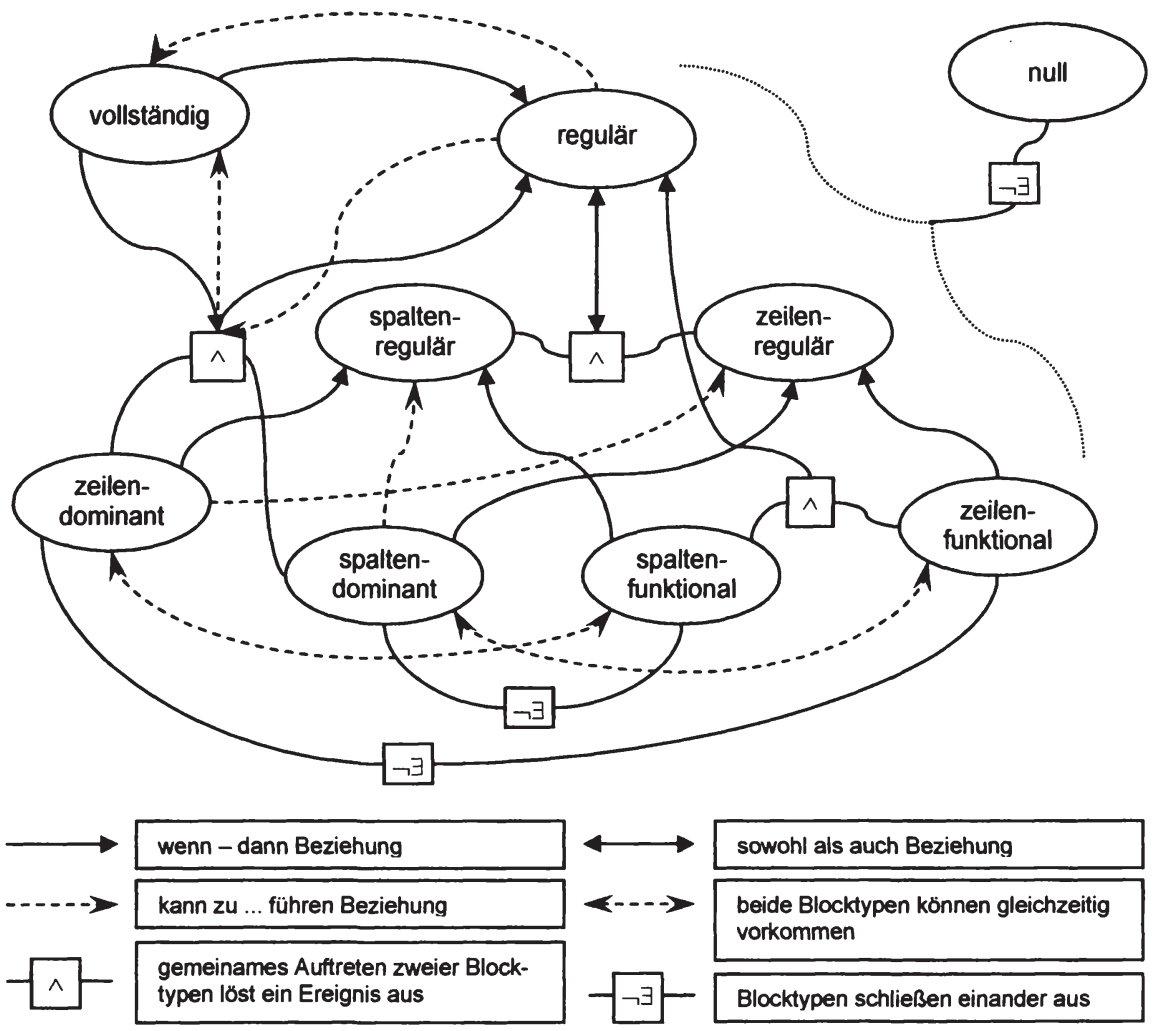

Abbildung 9: Beziehungen zwischen den Blocktypen

Der Typ ,regulär' gehört zu der obersten Ebene der Typenhierarchie. Dieser Typ kann auch indirekt aus der dritten Ebene abgeleitet werden. Ein spaltendominanter und zeilendominanter Block ist beispielsweise spaltenregulär und zeilenregulär und in weiterer Konsequenz regulär. Ein spaltenfunktionaler und zeilenfunktionaler Block ist sowohl spaltenregulär als auch zeilenregulär und demgemäß regulär. Ein Spezialfall ist der fürdie strukturelle Äquivalengz erforder- 
liche vollständige Block, der auch als komplett zeilendominant oder - gleichbedeutend - komplett spaltendominant interpretiert werden kann und definitionsgemäß regulär ist. Der Nullblock steht konträr zu den genannten Blocktypen. Einen Algorithmus für die Zuordnung eines Blocks zu Blocktypen bietet die Funktion CHECKBT.

Ein ideales Blockmodell für einen relationalen Datensatz könnte unter Kenntnis der Äquivalenzrelationen und der daraus bestimmbaren Blocktypen sowie der Anzahl der Knotengruppen beispielsweise wie Tabelle 23 aussehen.

Tabelle 23: Beispiel für ein ideales Blockmodell

\begin{tabular}{|c|c|c|c|c|c|}
\hline & ( ; & r: & ( & $c_{4}$ & ( \\
\hline ( & vollständig & regulär & spaltenregulär & zeilenregulär & null \\
\hline ( & null & vollständig & zeilenfunktional & null & spaltenregulär \\
\hline t & regulär & null & null & null & zeilenfunktional \\
\hline$C_{+}$ & zeilenregulär & zeilenregulär & null & zeilenfunktional & null \\
\hline c & spaltenregulär & null & zeilenregulär & zeilenfunktional & null \\
\hline
\end{tabular}

\subsubsection{Optimierungsprozess}

Nach der Bestimmung des idealen Blockmodells auf Basis der Blocktypen erfolgt die Definition der Zielfunktion für den Optimierungsprozess im direkten Verfahren entsprechend Formel (121) [Batagelj et al. 1992a].

$$
Z(C)=\sum_{i, j=1}^{k} z\left(C_{i}, C_{j}\right) \text { wobei } C_{i}, C_{j} \in C
$$

Konkret misst die Zielfunktion $Z(C)$ die Abweichung zwischen dem ermittelten, empirischen Blockmodell $R(C)$ und dem Idealmodell $B(C)^{59}$, wobei $C=\left\{C_{i}\right\}_{i=1,, k}$ die Menge der dem empirischen Blockmodell zugrunde liegenden paarweise dis-

59 Zunächst definiert sich das Idealmodell nur aus der Anzahl der zu ermittelnden Knotengruppen (Cluster) und den Blocktypen. Erst über die Festlegung der Gruppenzugehörigkeit der Knoten ergeben sich die Idealblöcke, deren Vorlage die jeweiligen (zulässigen) Blocktypen sind. 
junkten Knotengruppen (Cluster) $C_{i} \subseteq V$ ist, d.h. $\forall C_{i}, C_{j} \in C: C_{i} \cap C_{j}=\varnothing$. Die Anzahl der Knotengruppen $k=|C|$ wird über das vordefinierte, ideale Blockmodell festgelegt. Die Zielfunktion wird über die Summe der Abweichungen $z\left(C_{i}, C_{j}\right)$ der einzelnen empirischen Blöcke $r\left(C_{i}, C_{j}\right) \in R(C)$ vom jeweils korrespondierenden Idealblock $b$ bzw. (wie unten formuliert) von der Menge der möglichen Idealblöcke $B\left(C_{i}, C_{j}\right)$ ermittelt. Diese Idealblöcke folgen aus der Definition der Blocktypen. Wird die Abweichung $z\left(C_{i}, C_{j}\right)$ eines empirischen Blocks $r\left(C_{i}, C_{j}\right) \mathrm{zu}$ einem korrespondierenden Idealblock $b$ ermittelt, so entspricht diese einfach dem definierten Maß der Diskrepanz $\delta$ zwischen den beiden Blöcken (konfirmatorische Analyse). Erfolgt die Berechnung der Abweichung $z\left(C_{i}, C_{j}\right)$ hingegen in Bezug auf die Menge der möglichen Idealblöcke $B\left(C_{i}, C_{j}\right)$, so geschieht dies durch Ermittlung der minimalen Diskrepanz $\delta$ zwischen dem empirischen Block $r\left(C_{i}, C_{j}\right)$ und allen Idealblöcken $b \in B\left(C_{i}, C_{j}\right)$ (explorative Analyse; Formel (122)).

$$
z\left(C_{i}, C_{j}\right)=\min _{b \in B\left(C_{i}, C_{j}\right)} \delta\left(r\left(C_{i}, C_{j}\right), b\right) \quad \text { wobei } C_{i}, C_{j} \in C
$$

Nachstehend werden zwei Varianten zur Berechnung der Diskrepanz $\delta$ beispielhaft angeführt. Diese Vorschläge folgen den Ideen von Batagelj et al. [1992a] und Batagelj et al. [1992b].

$$
\delta\left(r\left(C_{i}, C_{j}\right), b\right)=\sum_{v \in C_{i}, w \in C,}\left|r_{v w}-b_{v w}\right| \quad \text { wobei } r_{v w} \in r\left(C_{i}, C_{j}\right), b_{v w} \in b
$$

Formel (123) zeigt die Bestimmung eines Blockmodells bei rein struktureller Äquivalenz. Hierbei wird die Summe der absoluten Differenzen zwischen den Elementen $r_{v w}$ im empirischen Block und den korrespondierenden Elementen $b_{v w}$ im Idealblock ermittelt. Dies impliziert die gleiche Gewichtung von positiven und negativen Abweichungen (d.h. Null-Elemente im empirischen Block, die idealerweise 1 sein sollten bzw. umgekehrt). Alternativ können unterschiedliche Gewichtungsfaktoren berücksichtigt werden. 
Bei rein regulärer Äquivalenz kann folgende Formel (124) zur Anwendung kommen.

$$
\begin{aligned}
& \delta\left(r\left(C_{i}, C_{j}\right), b\right)= \begin{cases}n_{s 0} n_{z}+n_{z 0} n_{s} & \text { wenn b regulär } \\
\sum_{v \in C_{i}, w \in C_{C},} r_{w v} & \text { wenn b null }\end{cases} \\
& \text { wobei } \\
& n_{z 0}=\left|\left\{v \in C_{i}: r_{v w}=0 \forall w \in C_{j}\right\}\right| \\
& n_{z}=\left|C_{i}\right| \\
& n_{s 0}=\left|\left\{w \in C_{j}: r_{w w}=0 \forall v \in C_{i}\right\}\right| \\
& n_{s}=\left|C_{j}\right| \\
& r_{v w} \in r\left(C_{i}, C_{j}\right)
\end{aligned}
$$

Abhängig von dem Blocktyp für $b$ wird gemäß Formel (124) die Diskrepanz $\delta$ entweder als Summe der Elemente $r_{w w}$ im empirischen Block (bei Blocktyp null für den (dealblock $b$ ) oder als Summe des Produkts der Anzahl der Nullspalten mit der Anzahl der Zeilen und des Produkts der Anzahl der Nullzeilen mit der Anzahl der Spalten (wenn der Blocktyp für $b$ regulär ist) errechnet.

Das Optimierungsproblem kann nun wie folgt dargestellt werden. Für einen Graph $G(V, E)$ sollen die Knoten $v \in V$ in Knotengruppen (Cluster) $C_{i} \in C^{*}$ (unter gleichzeitiger Partitionierung der Beziehungen $e \in E$ zwischen den Knoten) zusammengefasst werden, so dass die Zielfunktion $Z(C)$ minimiert wird (Formel (125)).

$$
Z\left(C^{*}\right)=\min _{(}(Z(C))
$$

Über einen iterativen Optimierungsprozess kann diese Problemstellung ausgehend von einem initialen Clustering $C$ gelöst werden. Falls sich in der Nach-

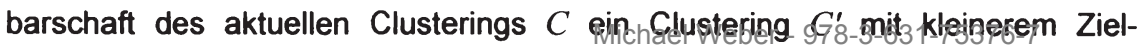


funktionswert befindet, dann wird die aktuelle Lösung gleich $C^{\prime}$ gesetzt. Zur Bestimmung der Nachbarschaft empfehlen Doreian et al. [1994] zwei denkbare Transformationen, einerseits die Verschiebung eines Knoten von einer Knotengruppe in eine andere oder andererseits den Austausch zweier Knoten zwischen den jeweiligen Knotengruppen. Dieses Vorgehen wird solange wiederholt, bis keine wesentliche Verringerung des Zielfunktionswerts erreicht werden kann. Üblicherweise wird der Prozess mehrmals mit unterschiedlichen initialen Clusterings durchgeführt, um lokale Optima möglichst zu vermeiden.

\subsubsection{Two-mode Blockmodeling}

Für Input-Output-Relationen bieten direkte Verfahren der Erstellung und Berechnung von (generalisierten) Blockmodellen vielversprechende Möglichkeiten zur Strukturentdeckung und zur empirischen Überprüfung von Annahmen über die Produktionsstruktur der zugrunde liegenden Verflechtungsdaten, die weit mehr analytisches Potenzial haben als bspw. Verfahren der Triangulation ${ }^{60}$ oder einfache graphentheoretische Kennzahlen. Dies gilt insbesondere dann, wenn die Modelle Knoten unterschiedlich in ihren Eigenschaften als Vorgänger- und Nachfolgerknoten - gemeinsam mit der Partitionierung der Relationen - klassifizieren können. Zu diesem Zweck ist ein direktes Verfahren erforderlich, das für den Umgang mit ,two-mode' Daten, d.h. relationalen Daten, die unterschiedliche bzw. unterschiedlich interpretierbare ${ }^{61}$ Knoten in Beziehung setzen, geeignet ist.

60 Triangulationsverfahren können zur Identifikation der Produktionshierarchie (Produktionsstufen) unter Einbezug der Transaktionshöhe entsprechend den Daten der Input-Output-Tabelle eingesetzt werden und dienen somit der (quantitativen) Strukturanalyse. Gewichtiger Nachteil der Triangulation ist, dass die Reihenfolge der Produktionsstufen bei nicht linear verlaufender Produktionsverflechtung instabil wird, wobei die Instabilităt mit zunehmender Nichtlinearităt ansteigt. Zudem werden nicht direkt verflochtene ,Produktionsknoten' über das Triangulationsverfahren aneinandergereiht (näheres hierzu siehe [Holub \& Schnabl 1985]).

61 Ein Beispiel für solche ,unterschiedlich interpretierbare' Knoten ist dementsprechend die Differenzierung in die Vorgänger- und Nachfolgerbeziebugge日 von Kngten. 
Für die Erstellung eines ,two-mode' Blockmodells ändert sich die Definition der Zielfunktion für den Optimierungsprozess im direkten Verfahren wie folgt (Formel (126)) [Doreian et al. 2004].

$$
Z(C)=\sum_{i=1}^{k} \sum_{j=1}^{h} z\left(C_{i}^{Z}, C_{j}^{s}\right) \text { wobei } C_{i}^{Z} \in C^{Z}, C_{j}^{s} \in C^{s}, C=\left(C^{Z}, C^{s}\right)
$$

Im Unterschied zur ,one-mode' Betrachtung werden hier die Vorläufer- bzw. Nachfolgerknoten unterschiedlich in Knotengruppen (Cluster) zusammengefasst, wobei $C^{Z}$ die Menge der (empirischen) Vorläuferknotengruppen und $C^{S}$ die Menge der (empirischen) Nachfolgerknotengruppen enthält. $C$ ist das Tupel von $C^{Z}$ und $C^{S}$. Die Kardinalitäten der Knotengruppen $k=\left|C^{Z}\right|$ und $h=\left|C^{S}\right|$ werden über das vordefinierte, ideale Blockmodell festgelegt. Basierend auf der Diskrepanz $\delta$ zwischen den empirischen Blöcken $r\left(C_{i}^{Z}, C_{j}^{S}\right) \in R(C)$ und den Idealblöcken $b \in B\left(C_{i}^{Z}, C_{j}^{S}\right)$ werden die Zielfunktionsbeiträge $=\left(C_{i}^{Z}, C_{j}^{S}\right)$ über die Formel (127) ermittelt.

$$
z\left(C_{i}^{z}, C_{j}^{s}\right)=\min _{b \in B\left(C_{,}^{z}, C_{j}^{s}\right)} \delta\left(r\left(C_{i}^{z}, C_{j}^{s}\right), b\right) \text { wobei } C_{i}^{z} \in C^{z}, C_{j}^{s} \in C^{s}
$$

Die Berechnung der Diskrepanz $\delta$ erfolgt analog zu den Ausführungen der ,onemode' Betrachtung, $C_{i}$ wird dabei durch $C_{i}^{Z}$ und $C_{j}$ durch $C_{j}^{S}$ ersetzt.

Das Optimierungsproblem kann nun folgendermaßen beschrieben werden. Für einen Graph $G(V, E)$ mit $V=\left(V_{l}, V_{2}\right)$ und $e \in E$ mit $e=(v, w)$, wobei $v \in V_{l}, w \in V_{2}$, sollen die Knoten in Knotengruppen $C_{i}^{Z} \in C^{Z^{*}}$ und $C_{j}^{S} \in C^{S^{*}}$, mit $C_{i}^{Z} \subseteq V_{1}$, $C_{j}^{S} \subseteq V_{2}$ und $C^{*}=\left(C^{z^{*}}, C^{s^{*}}\right)$, (unter gleichzeitiger Partitionierung der Beziehungen $e \in E$ zwischen den Knoten) zusammengefasst werden, so dass die Zielfunktion $Z(C)$ minimiert wird (Formel (128)).

$$
Z\left(C^{*}\right)=\min _{C^{\prime}}(Z(C))
$$


Die Ausführungen zu dem iterativen Optimierungsprozess gelten auch bei der Erstellung eines ,two-mode' Blockmodells.

Annahmen über das ideale Blockmodell setzen - zumindest partielle - Vorkenntnisse über die Struktur der relationalen Daten, d.h. die zulässigen Blocktypen und die Anzahl der Knotengruppen, voraus. Sind diese a priori Informationen allerdings nicht verfügbar, so erscheinen Ansätze zur Identifikation eines idealen Blockmodells, wie bspw. Brusco \& Steinley [2006], verfolgenswert. Brusco und Steinley präsentieren in ihrer Arbeit ein Verfahren, das kein Strukturwissen voraussetzt und dessen Ergebnis Grundlage für das Erstellen eines Blockmodells sowohl für Analysen von ,one'- als auch von ,two-mode' Verflechtungsdaten dienen kann. Dieses Verfahren setzt auf eine zeilen- und spaltenweise Permutation einer quadratischen oder rechteckigen Adjazenzmatrix, die auf eine Partitionierung in möglichst homogene Blöcke abzielt. Knotengruppen werden dadurch nicht direkt identifiziert, können aber bspw. über die Visualisierung der Ergebnismatrix gebildet werden. Gleiches gilt für die Bestimmung von Äquivalenzrelationen bzw. Blocktypen. Die Performanz dieses auf der Branch-andBound Methode beruhenden Verfahrens ist für Graphen mit mehr als 40 Knoten allerdings nicht gegeben, wie Brusco \& Steinley [2006] unter Verweis auf alternative, heuristische Methoden hervorheben.

\subsubsection{Illustration des two-mode Blockmodeling}

Die Berechnung eines Blockmodells wird nun anhand eines Beispiels zur strukturellen Äquivalenzuntersuchung über die zu diesem Zweck in $R$ [R 2007] implementierte Funktion BLOCKMODELING demonstriert. Entsprechend den obigen Ausführungen erfolgt die explorative Ermittlung eines two-mode Blockmodells über eine iterative Knotenverschiebeprozedur. Den Ausgangspunkt für die $\mathrm{Be}$ rechnung bildet eine Zufallsstartlösung, die aus einem initialen Clustering der Zeilen- und Spaltenknoten sowie dem daraus resultierenden initialen empirischen Blockmodell - d.h. der entsprechend dem initialen Clustering angeordneten Adjazenzmatrix - besteht (Funktion PUTVINC). Die Anzahl der Zeilen- und Spaltencluster ist dabei vorzugeben. Zunächst wird/ein ideales, Bloqkmodell/ayf Bassis der 
(Start-)Lösung identifiziert auf dessen Basis der Zielfunktionswert ermittelt wird (Funktion EVALUATECLUSTERING). In einem iterativen Prozess (Funktion ITERATE) wird darauf folgend die Nachbarschaft der aktuellen Lösung untersucht (Funktion EXPLORESHIFT). Nachbarschaft definiert sich dabei als Lösungsraum, der durch Verschiebung der (Zeilen- oder Spalten-)Knoten unabhängig voneinander in alle anderen Knotengruppen entsteht. Kann eine Lösung mit geringerem Zielfunktionswert errechnet werden, so gilt diese Lösung als Ausgangsbasis für den nächsten Iterationsschritt. Andernfalls kann die Lösung beibehalten werden. Diese entspricht dann einem lokalen Optimum. Zur Vermeidung des Verharrens in lokalen Optima ermöglicht der Algorithmus die wiederholte Durchführung des Iterationsprozesses mit unterschiedlichen zufälligen Startlösungen. Zur Veranschaulichung des Ergebnisses kann das bestimmte empirische Blockmodell grafisch dargestellt werden (Funktion DRAWCLU). Diese Visualisierung erleichtert auch die Vorgabe der Anzahl von Knotengruppen aus Zeilen- und Spaltensicht. Je nach zu beantwortender Fragestellung können die im Anhang dargelegten Funktionen zur explorativen Ermittlung eines empirischen Blockmodells für relationale Daten um weitere Äquivalenzarten bzw. Blocktypen erweitert werden. Ebenso wäre es denkbar, die optimale Knotengruppenanzahl über den Algorithmus zu bestimmen oder die Funktionen für die konfirmatorische Datenanalyse nutzbar zu machen. Auf den computationalen Aufwand auch für diese Ziele sei in diesem Kontext hingewiesen.

Ein kleines Testbeispiel soll abschließend zu den Ausführungen zur Blockmodellberechnung die Wirkungsweise des vorgestellten Ansatzes illustrieren.

Tabelle 24: Beispieldaten zur Blockmodellberechnung

\begin{tabular}{|c|c|c|c|c|c|}
\hline & \multicolumn{2}{c}{ A } & \multicolumn{2}{c}{ B } & \multicolumn{2}{c}{ C } & \multicolumn{2}{c|}{ D } & E \\
\hline a & 0 & 0 & 0 & 0 & 0 \\
\hline b & 0 & 0 & 1 & 0 & 1 \\
\hline c & 0 & 0 & 0 & 0 & 0 \\
\hline d & 1 & 0 & 0 & 1 & 0 \\
\hline e & 0 & 0 & 1 & 0 & 1 \\
\hline f & 1 & 0 & 0 & 1 & 0 ALabe \\
\hline
\end{tabular}


Für eine Matrix mit den Ausgangs- oder Zeilenknoten $V_{l}=\{a, b, c, d, e, f\}$ und den Eingangs- oder Spaltenknoten $V_{2}=\{A, B, C, D, E\}$ soll ein Blockmodell auf Basis der in Tabelle 24 festgehaltenen Relationen erstellt werden.

Zur Untersuchung der strukturellen Äquivalenz dieser Adjazenzmatrix werden nun bspw. 3 Zeilenknotengruppen und 3 Spaltenknotengruppen unterstellt. Die linke Grafik in Abbildung 10 zeigt eine zufällige Startlösung für den Algorithmus, der letztlich über mehrere Iterationen und möglicherweise neue Zufallsstartlösungen zu dem in der rechten Grafik von Abbildung 10 dargestellten Ergebnis führt. In diesem Beispiel entspricht das ermittelte empirische Blockmodell dem errechneten idealen Blockmodell, d.h. es besteht keine Diskrepanz, der Zielfunktionswert ist folglich 0 .
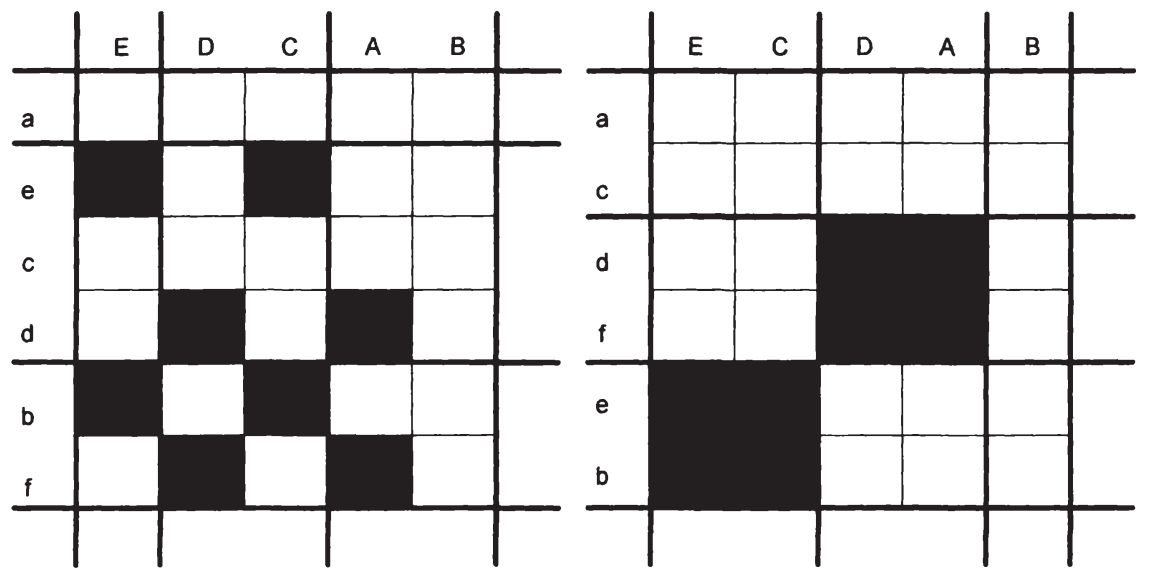

Abbildung 10: Adjazenzmatrix vor und nach der Blockmodellberechnung

Im Schnittpunkt der Cluster $C_{2}^{Z}=\{d, f\}$ und $C_{2}^{S}=\{D, A\}$ ergibt sich ebenso wie im Schnittpunkt der Knotengruppen $C_{3}^{Z}=\{e, b\}$ und $C_{1}^{S}=\{E, C\}$ jeweils ein vollständiger Block. Dies bedeutet, dass die Knoten dieser Knotengruppen komplett miteinander verbunden und demgemäß innerhalb der Cluster strukturell äquivalent sind. Die verbleibenden Blöcke sind Nullblöcke, d.h. die Knoten in den jeweiligen Clustern weisen keinerlei Zusammenhang auf. Es zeigt sich, dass die für das Beispiel getroffene Annahme von 3 Zeilenknotengruppen und 3 Spalten- 
Blockmodeling - Clustering und Partitionierung von relationalen Datensätzen 153

knotengruppen korrekt war. In der Praxis sind allerdings oftmals mehrere Anläufe notwendig um zur optimalen Knotengruppenanzahl zu gelangen.

\subsubsection{Blockmodellberechnung für gewichtete Relationen}

Zweifelsohne kann in Einsatzgebieten in denen überwiegend mit bewerteten relationalen Daten (gewichteten Graphen) gearbeitet wird, wie bspw. die InputOutput-Analyse, die oben vorgestellte binäre Blockmodellberechnung zu keinem zufrieden stellenden Ergebnis führen, nachdem die ,Binarisierung' der Daten einen erheblichen Informationsverlust bewirken würde. Darüber hinaus wäre es äußerst schwierig einen geeigneten Parameter zur Transformation der gewichteten zu binären relationalen Daten (z.B. größer als 0 oder der Mittelwert der Kantengewichte) zu definieren, ohne gleichzeitig das Ergebnis über die Parameterwahl maßgeblich mitzubestimmen. Nichtsdestotrotz kann die Blockmodellberechnung, die ursprünglich für den Einsatz mit binären Daten entwickelt wurde [Breiger et al. 1975], auch die Analyse von gewichteten relationalen Daten bereichern bzw. erleichtern. Diese Aussage gewinnt angesichts der jüngeren Beiträge zur gewichteten (generalisierten) Blockmodellerstellung ohne signifikanten Informationsverlust wie Žiberna [2007] oder Weber \& Denk [2007] an Bedeutung. Aufbauend auf der Arbeit von Doreian et al. [2005] entwickelt bspw. Žiberna [2007] zwei alternative Ansätze zur Erstellung von Blockmodellen für gewichtete relationale Daten. Diese hierin als $f$-Wert- (Žiberna nennt es ,valued blockmodeling') und Homogenitätsblockmodellberechnung (,homogeneity blockmodeling') bezeichneten Alternativen knüpfen direkt an der binären (generalisierten) one-mode Blockmodellgenerierung an und können jeweils auch zu einem twomode Ansatz ausgebaut werden. Insbesondere die $f$-Wert Blockmodellberechnung ist eng an die Überlegungen zu (generalisierten) one-mode Blockmodellen angelehnt. So entstehen bspw. die Äquivalenzrelationen und folglich die Blocktypdefinitionen im Wesentlichen durch einen Austausch der Forderungen für den binären Wert 1 mit analogen Forderungen für einen Parameter $m$ unter Berücksichtigung einer Funktion $f$ der Zellwerte im jeweiligen Block, die die Bedingung für $m$ erfüllen sollte. Beispiele für solche veränderten Block- 
typdefinitionen können der Tabelle 25 entnommen werden. Die Funktionen $f$, die für die gewichteten Blöcke eingesetzt werden können, sind bspw. $\min (), \max ()$, sum(), oder mean(). Eine weitere Modifikation, die die $f$-Wert Blockmodellerstellung mit sich bringt, ist die Berechnung der Diskrepanz zwischen dem empirischen und den idealen Blocktypen. Žiberna [2007] stellt für jeden der abgeänderten Blocktypen ein eigenes Diskrepanzkriterium vor. So wird bspw. die Diskrepanz für einen $f$-regulärer Block über die Abweichung der Funktion $f$ von dem Parameter $m$ spalten- und zeilenweise berechnet. Konkret werden für jedes Paar einer Zeile eines Knoten $v_{k}$ und einer Spalte eines Knoten $v_{l}$ die Differenzen $d_{k}=m-f\left(\left(r\left(C_{i}, C_{j}\right)\right)_{k}\right)$ und $d_{l}=m-f\left(\left(r\left(C_{i}, C_{j}\right)\right)_{l}\right)$ gebildet. Die Blockdiskrepanz wird daran anschließend bestimmt als die Summe der paarweisen Maxima von $d_{k}$. und $d_{l}$ über alle Paare $\left(v_{k}, v_{l}\right)$, für die die Funktion $f$ der betrachteten Zeile $k$ und der betrachteten Spalte $l$ größer als der Parameter $m$ ist.

Tabelle 25: Beispiele für redefinierte Blocktypen nach Žiberna Quelle: eigene Darstellung nach Žiberna [2007]

$f$-Wert Blockmodellberechnung

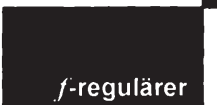
Block

rollstandiger

vollständiger

Block

zeilen- $f$ :

regulärer

Block

zeilen-

dominanter

Block

zeilen-

funktionaler

Block
In einem $f$-regulären Block ergibt die Funktion $f$ pro Zeile und Spalte des Blocks (einer gewichteten Adjazenzmatrix) zumindest den Wert $m$.

Jede Zelle des vollständigen Blocks hat zumindest den Wert $m$.

In einem zeilen- $f$-regulären Block ist das Ergebnis der Funktion $f$ pro Zeile zumindest vom Wert $m$. Ein zeilendominanter Block besitzt zumindest eine Zeile, deren Zellwerte zumindest den Wert $m$ aufweisen.

Ein zeilenfunktionaler Block weist in jeder Zeile den Wert $m$ genau einmal auf. Die Zeilensummen des Blocks sind $m$.
Homogenitätsblockmodellberechnung

In einem $f$-regulăren Block liefert die Funktion $f$ für jede Zeile und ebenso auch für jede Spalte des Blocks den gleichen Ergebniswert. Die Ergebnisse für die Zeilen und Spalten müssen nicht notwendigerweise gleich sein.

Alle Zellwerte des vollständigen Blocks sind gleich.

Ergibt die Funktion $f$ in jeder Zeile des das gleiche Resultat, so handelt es sich um einen zeilen-f-regulären Block.

In einem zeilendominanten Block gibt es zumindest eine Zeile, die die gleichen Zellwerte aufweist.

Jede Zeile eines zeilenfunktionalen Blocks besitzt genau eine Zelle, die den von 0 verschieden Werten der anderen Zeilen entspricht. Alle anderen Zellwerte jeder Zeile sind 0. 
Blockmodeling - Clustering und Partitionierung von relationalen Datensätzen 155

Im Kern sind dies die wichtigsten Änderungen bei der $f$-Wert Blockmodellberechnung. Es zeigt sich, dass im Gegensatz zu den binären Blockmodellberechnungsverfahren nun zusätzlich die Frage zu beantworten ist, wie der Parameter $m$ bestimmt werden kann. Hierfür könnten ergänzende Verfahren als Hilfestellung zur Bestimmung von $m$ eingeführt werde, es sei denn, man kann auf Vorkenntnisse zurückgreifen.

Als eine Alternative zu $f$-Wert Blockmodellberechnung schlägt Žiberna [2007] vor, die Blöcke anhand deren maximaler Homogenität oder minimaler Variabilität zu bestimmen. Hierzu muss eine Maßzahl eingeführt werden, die die Homogenität bzw. Variabilität innerhalb eines Blocks misst, wie die Summe der quadratischen Abweichungen vom Mittelwert oder die Summe der absoluten Abweichungen vom Median. Weiters ist die Definition der idealen Blocktypen so anzupassen, dass die Homogenität (Gleichwertigkeit) des Hauptcharakteristikums des jeweiligen Blocktyps in den Vordergrund der Definition rückt (Tabelle 25). Dieses Erfordernis bedingt auch eine Adaptierung des Diskrepanzkriteriums pro Blocktyp. Im Vergleich zur $f$-Wert Blockmodellberechnung sowie zur binären Blockmodellberechnung für binarisierte gewichtete relationale Daten sind keine weiteren Parameter für diese ,Homogenitätsblockmodellberechnung' erforderlich und der durch die Binarisierung hervorgerufene Informationsverlust kann vermieden werden. Dies sind bedeutende Vorteile dieser Methode, die zur Zeit allerdings noch nicht vollständig ausgereift ist, wie Žiberna [2007] bemerkt. Folglich schlägt er vor, das Ergebnis der Homogenitätsblockmodellberechnung (zunächst) als Startlösung für die anderen Berechnungsverfahren einzusetzen.

Weber \& Denk [2007] schlagen einen two-mode Ansatz für die Berechnung von Blockmodellen für gewichtete relationale Daten vor. Sie setzen dabei Blocktypdefinitionen ein, die die gegenseitige Bedeutung der Knoten füreinander berücksichtigen und demgemäß das Ausmaß der vorgänger- und nachfolgerbezogenen Wirkungen der Knoten simultan in die Blockgenerierung einbeziehen. Die Eigenschaften der Blöcke und die Beziehungen zwischen den Knotengruppen werden über diesen Ansatz stärker beachtet als die Eigenschaften und 
Beziehungen der einzelnen Knoten. Dies führt dazu, dass diese Vorgehensweise komplementär zu den Ideen der verallgemeinerten Blockmodellberechung (,generalized blockmodeling') von Doreian et al. [2005] ist. Wie auch bei Žibernas [2007] Vorschlag stehen allerdings auch für den Ansatz von Weber \& Denk [2007] vergleichende und bewertende empirische Studien aus, nachdem dieser sich noch in der Entwicklungsphase befindet.

\subsubsection{Illustration der Blockmodellberechnung für gewichtete Kanten}

Abbildung 11 zeigt ein Demonstrationsbeispiel einer gewichteten two-mode Blockmodellberechnung, die den Homogenitätsansatz verfolgt und mit Žibernas R-Paket ,blockmodeling' [Žiberna 2006] durchgeführt wurde. Aus einer InputOutput-Betrachtung können die Vorläuferknoten als eine Menge von Gütern (,a', $\left.\ldots, j^{\prime}\right)$, die Nachfolgerknoten als eine Menge von Aktivitäten $\left(A^{\prime}, \ldots, H^{\prime}\right)$ verstanden werden. Augenscheinlich zeigen die Kanten die gewichteten InputOutputbeziehungen zwischen diesen beiden Knotenmengen. Ausgehend von einer gewichteten Adjazenzmatrix auf der linken Seite der Abbildung 11 werden homogene Blöcke über den Einsatz der Summe der absoluten Abweichung vom Median des Blocks als Maßzahl der Blockvariabilität und die Verwendung von $\max ()$ als Funktion $f$ für die gesuchten $f$-regulären Blöcke identifiziert. Ein maxregulärer Block in einem Homogenitätsblockmodell sollte für jede Zeile und Spalte des Blocks den gleichen maximalen Zellwert aufweisen.

Nach Experimenten mit unterschiedlichen Werten für die Anzahl von Zeilen- und Spaltenknotengruppen zeigte sich für dieses Beispiel die Eignung eines Blockmodells mit 3 Zeilenknotengruppen und 4 Spaltenknotengruppen. Die rechte Seite der Abbildung 11 zeigt das Ergebnis der Optimierung, das empirische Blockmodell. Neben vier Nullblöcken, die unverbundene Güter und Aktivitäten anzeigen, konnte ein vollständiger Block identifiziert werden, deren Güter für die Blockaktivität jeweils das gleiche Kantengewicht aufweisen. Die restlichen Blöcke sind vom Typ max-regulär mit unterschiedlichen Niveaus von Kantengewichten und Diskrepanzen. 


\begin{tabular}{|c|c|c|c|c|c|c|c|c|}
\hline & A & B & $C$ & $\mathrm{D}$ & $\mathrm{E}$ & $\mathrm{F}$ & G & $\mathrm{H}$ \\
\hline & 0 & 26 & 0 & 18 & 0 & 4 & 0 & 35 \\
\hline & 0 & 0 & 45 & 3 & 49 & 0 & 47 & 0 \\
\hline & 0 & 1 & 0 & 2 & 44 & 0 & 48 & 0 \\
\hline & 63 & 5 & 18 & 0 & 21 & 0 & 21 & 58 \\
\hline & 55 & 4 & 17 & $y$ & 0 & 0 & 18 & 62 \\
\hline & 60 & 7 & 20 & 0 & 17 & 0 & 19 & 56 \\
\hline & 33 & 23 & 0 & 27 & 0 & 4 & 0 & 30 \\
\hline & 56 & 0 & 18 & 6 & 19 & 0 & 16 & 0 \\
\hline & 0 & 2 & 53 & 1 & 46 & 0 & 44 & 0 \\
\hline & 31 & 24 & 0 & 22 & 0 & 4 & 0 & 34 \\
\hline
\end{tabular}

\begin{tabular}{|c|c|c|c|c|c|c|c|c|}
\hline & C & $\mathrm{E}$ & G & $F$ & B & D & A & $\mathrm{H}$ \\
\hline$a$ & 0 & 0 & 0 & 4 & 26 & 18 & 0 & 35 \\
\hline $\mathrm{g}$ & 0 & 0 & 0 & 4 & 23 & 27 & 33 & 30 \\
\hline j & 0 & 0 & 0 & 4 & 24 & 22 & 31 & 34 \\
\hline d & 18 & 21 & 21 & 0 & 5 & 0 & 63 & 58 \\
\hline e & 17 & 0 & 18 & 0 & 4 & 9 & 55 & 62 \\
\hline$f$ & 20 & 17 & 19 & 0 & 7 & 5 & 60 & 56 \\
\hline $\mathrm{h}$ & 18 & 19 & 16 & 0 & 8 & 6 & 56 & 0 \\
\hline b & 45 & 49 & 47 & 0 & 0 & 3 & 0 & 0 \\
\hline c & 0 & 44 & 48 & 0 & 1 & 2 & 0 & 0 \\
\hline $\mathrm{i}$ & 53 & 46 & 44 & 0 & 2 & 1 & 0 & 0 \\
\hline
\end{tabular}

Abbildung 11: Gewichtete Adjazenzmatrix vor und nach der Blockmodellberechnung

Die Extraktion von strukturellen Informationen aus relationalen Datensätzen kann in der Strukturanalysephase mittels - insbesondere jüngeren - Methoden der (generalisierten) Blockmodellberechnung sowohl für explorative als auch für konfirmatorische Analysezwecke unterstützt werden. Die Blockmodellberechnungsverfahren ermöglichen hierbei die Verringerung von Komplexität bzw. einen Einblick in die komplexen Verflechtungen von relationalen Daten, die - in Anbetracht der steigenden und global wirkenden Differenzierung von Wertschöpfungsprozessen sowie der Datenerfassungsleistungen heutiger (und v.a. auch zukünftiger) Informationssysteme - zunehmend unüberschaubarer werden. Methoden der Blockmodellgenerierung können demnach einen Beitrag zur Identifikation von relevanten Informationen auf Basis der Datensammlungen leisten, um bspw. die Koordination von Wirtschaftssubjekten zur Wertgenerierung oder die Input-Output-Analyse i.A. zu unterstützen. Nichtsdestotrotz ist anzumerken, dass die Methoden speziell für ökonomische Anwendungen noch in einer frühen Entwicklungsphase stecken, weshalb weitere Forschungsergebnisse zur Optimierung der (Performanz der) Algorithmen für gewichtete Verflechtungsdaten oder auch zur Unterstützung der Spezifikation von Blockmodellen notwendig sind, um das Potenzial der (generalisierten) Blockmodellberechnung ausschöpfen zu können. 
Michael Weber - 978-3-631-75376-7

Downloaded from PubFactory at 01/11/2019 05:44:37AM

via free access 


\section{Strukturvergleichsphase}

Nach der Analyse der Knoten und deren Relationen, d.h. im Kontext dieser Arbeit Güter, Aktivitäten oder Plattformteilnehmer bzw. deren Transaktionen, wird in dieser Phase aufgezeigt, wie zwei gewichtete Graphen verglichen werden können, um weitere Aussagen über strukturelle Eigenschaften, Potenziale und Defizite der Graphen bzw. deren Knoten und Kanten zu generieren. Für den Zweck des Transaktionssystemunterstützungsmodells erfolgt daher ein Vergleich der Transaktionsdaten der Kollaborationsplattform, der lokalen Struktur, mit den relationalen Daten der übergeordneten Produktionsstruktur, der globalen Struktur, bei gleichzeitiger Ermittlung zusätzlicher Vergleichskennzahlen. Der Vergleich dient in weiterer Folge zur Identifikation und Bewertung von ,strukturellen Lücken' ebenso wie von ,Performanzlücken' und zur Bestimmung der Priorität von entsprechenden Gegenmaßnahmen. Voraussetzung für diese Phase ist, wie bereits bei der Datenaufbereitung hervorgehoben, die Herstellung einer Vergleichbarkeit von Transaktionsdaten, die die Marktstruktur wiedergeben, mit den Daten zur Produktionsstruktur. Informationen zu den ,strukturellen Lücken' sowie ,Performanzlücken' der lokalen Struktur sind erforderlich, um das Transaktionsgefüge in weiterer Folge gezielt optimieren zu können. Diese Informationen können über nachfolgende Vergleichsoperationen zur Identifikation und Klassifikation von Lücken generiert werden. Zuvor sind allerdings die hierin verwendeten Bezeichnungen für das leichtere Verständnis der Vorgangsweise näher zu erläutern.

\subsection{Lückentypologie}

Unter dem Begriff, strukturelle Lücken' werden die Bezeichnungen ,interne strukturelle Lücken' und ,externe (strukturelle) Lücken' zusammengefasst. Eine interne strukturelle Lücke in einem Graph tritt auf, wenn zwischen zwei Knoten des Graphen eine Verbindung (Kante) fehlt, diese allerdings in der Vergleichsstruktur existiert. Eine interne strukturelle Lücke besteht demnach bspw. zwischen zwei Gütern, zwei Aktivitäten oder zwei Betrieben, wenn diese direkt 
oder indirekt miteinander in Beziehung stehen könnten, dies aber im Gegensatz zur globalen Vergleichsstruktur nicht tun. Indirekte strukturelle Lücken sind insofern interessant, als man durch ihre Erfassung Folgewirkungen einer möglichen Lückenschließung zuordenbar macht. Abhängig von den Kanten zwischen den Knoten die zur Bestimmung der Folgewirkungen betrachtet werden, kann man zwischen ,partiellen' und ,kompletten indirekten internen Lücken' unterscheiden. Während bei partiellen internen indirekten Lücken mindestens eine Kante in der lokalen Struktur nicht existiert, sind bei einer kompletten indirekten internen Lücke sämtliche Kanten nicht in der Kantemenge der lokalen Struktur enthalten. Dieser Spezialfall beschreibt somit eine Folge von direkten internen Lücken. Eine externe (strukturelle) Lücke bezeichnet demgegenüber einen Knoten, der nur in der globalen Struktur vorhanden ist, aber auch in der lokalen Struktur bestimmte Verbindungen herstellen könnte. Dementsprechend ist bspw. ein Gut dann eine externe strukturelle Lücke, wenn es auf der Kollaborationsplattform nicht vorzufinden ist, aber zusätzliche Verbindungsmöglichkeiten zwischen den bestehenden Gütern eröffnen würde.

Ein weiterer wesentlicher Begriff, der für die folgenden Vergleiche eingeführt wird, ist ,Performanzlücke'. Eine Performanzlücke beschreibt eine verhältnismäßig schwache Verbindung (Kante) zwischen bestehenden Knoten. Eine solche Lücke entsteht beispielsweise in Folge unzureichender Transaktionsvolumina bezogen auf den Austausch zwischen Gütern, Aktivitäten oder Plattformteilnehmer und birgt demzufolge Verbesserungspotenzial. In diesem Zusammenhang soll der Begriff ,schwacher Knoten' für Knoten verwendet werden, die als Ursache für Performanzlücken identifiziert werden können. Darüber hinaus sind ,isolierte Knoten' zu berücksichtigen. Dies sind Knoten, die über keine Verbindungen verfügen, nachdem sie keine anderen Knoten direkt adressieren bzw. von diesen direkt adressiert werden können. Abbildung 12 stellt den soeben geschaffenen Begriffsraum grafisch dar. Die Typen von Lücken für einen Weg mit Länge 1 werden in Tabelle 26 definiert, während in Tabelle 27 die beiden speziellen Knotentypen erläutert werden. 


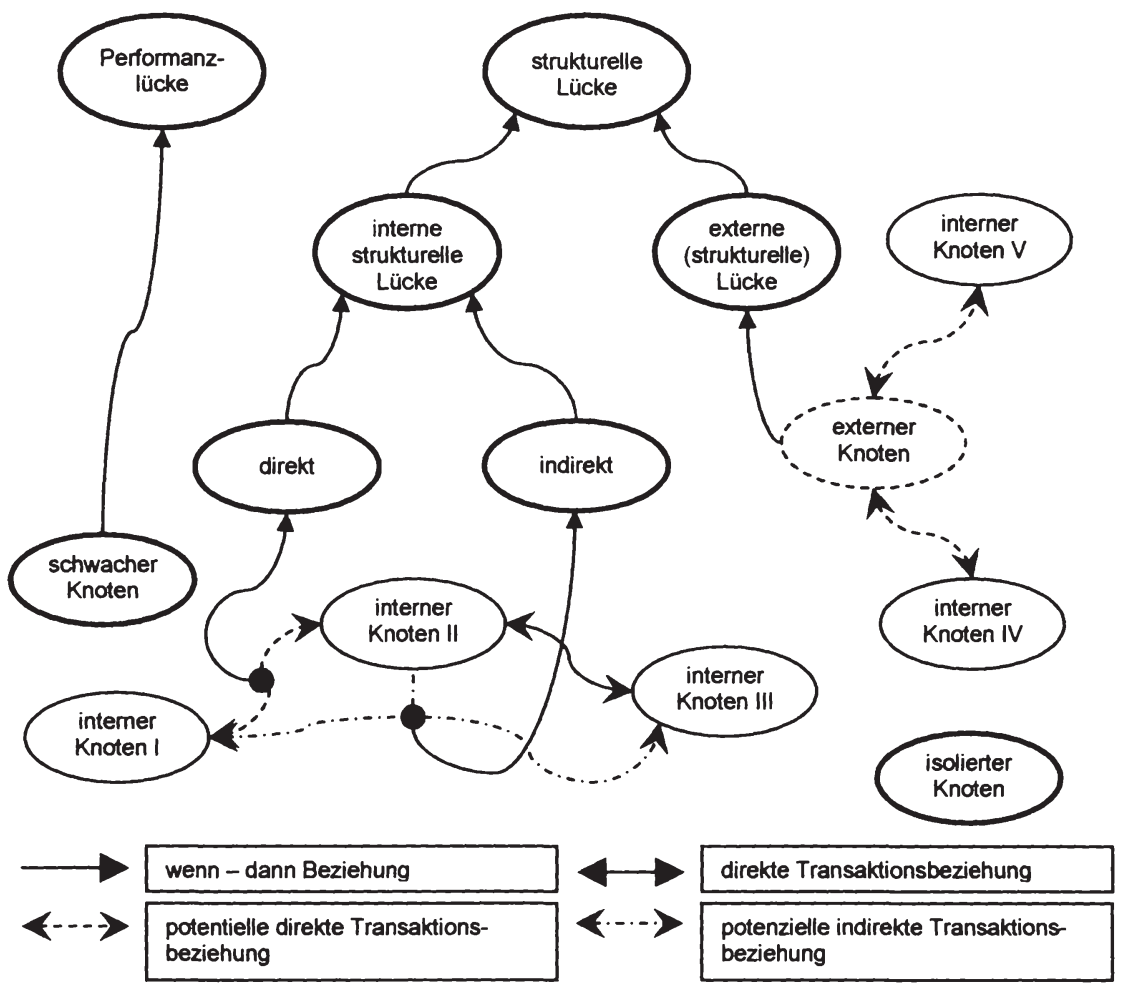

Abbildung 12: Typologie von Lücken für Vergleichszwecke

Tabelle 26: Typen von Lücken für einen Weg $\omega$ mit Weglänge $=1$

\begin{tabular}{|c|c|c|}
\hline & Bedingung & Beschreibung \\
\hline $\begin{array}{c}\text { Perfor- } \\
\text { manz- } \\
\text { lücke }\end{array}$ & $\begin{array}{l}v_{i}, w_{j} \in V^{L O C}, v_{i}, w_{j} \in V^{(i L O} \\
\left(v_{i}, w_{j}\right) \in E^{L O C},\left(v_{i}, w_{j}\right) \in E^{G L O} \\
\text { matval }_{i j}^{L O C}<\operatorname{th}\left(\text { matval }_{i j}^{G L O}\right)\end{array}$ & $\begin{array}{l}\text { Die lokalen Knoten } v_{i} \text { und } w_{j} \text { sind } \\
\text { über eine, im Vergleich zur globalen } \\
\text { Struktur relativ gering gewichtete, } \\
\text { Kante miteinander verknüpft. Dies } \\
\text { wird im Allgemeinen über einen } \\
\text { Schwellwert definiert. }\end{array}$ \\
\hline $\begin{array}{l}\text { direkte } \\
\text { interne } \\
\text { Lücke }\end{array}$ & $\begin{array}{l}v_{i}, w_{j} \in V^{L O C}, v_{i}, w_{j} \in V^{G L O} \\
\left(v_{i}, w_{j}\right) \notin E^{L O C},\left(v_{i}, w_{j}\right) \in E^{G L O}\end{array}$ & $\begin{array}{l}\text { Nach Maßgabe der globalen Struktur } \\
\text { könnten die unverbundenen lokalen } \\
\text { Knoten } v_{i} \text { und } w_{j} \text { in Beziehung ge- } \\
\text { setzt werden. }\end{array}$ \\
\hline $\begin{array}{l}\text { externe } \\
\text { Lücke }\end{array}$ & $\begin{array}{l}v_{i}, w_{j} \in V^{L O C}, v_{x} \in V^{G L O}, v_{x} \notin V \\
\left(v_{i}, v_{x}\right),\left(v_{x}, w_{j}\right) \in E^{G L O}\end{array}$ & $\begin{array}{l}\text { Über einen externen, d.h. einen in } \\
\text { der globalen, jedoch nicht in der } \\
\text { lokalen Struktur vorhandenen, Kno- } \\
\text { ten können die lokalen Knoten } v_{i} \text { und } \\
w_{j} \text { verbunden werden. } \\
\text { lichael Weber - 978-3-631-75376-7 }\end{array}$ \\
\hline
\end{tabular}


Tabelle 27: Spezielle Knotentypen

\begin{tabular}{|c|c|c|}
\hline & Bedingung & Beschreibung \\
\hline $\begin{array}{r}\text { schwacher } \\
\text { Knoten }\end{array}$ & vgl. Formeln (208) bis (212) & $\begin{array}{l}\text { Ein schwacher Knoten } v_{i} \text { ist hauptsäch- } \\
\text { lich über Performanzlücken mit anderen } \\
\text { lokalen Knoten verbunden. }\end{array}$ \\
\hline $\begin{array}{r}\text { isolierter } \\
\text { Knoten }\end{array}$ & $\forall w_{j} \in V^{L O C}:\left(v_{i}, w_{j}\right) \notin E^{L O C}$ & $\begin{array}{l}\text { Ein isolierter Knoten } v_{i} \text { verfügt über } \\
\text { keine Beziehung zu einem anderen } \\
\text { Knoten in der lokalen Struktur. }\end{array}$ \\
\hline
\end{tabular}

\subsection{Identifikation von direkten internen Lücken \& potenziellen Performanzlücken}

Die internen strukturellen Lücken einer lokalen Struktur können über einen Vergleich der lokalen Verflechtungsdaten, repräsentiert durch $\mathrm{MatVal}^{L(O C}$, mit den global gültigen Zusammenhängen, die in $\mathrm{MatVal}^{(i L / O)}$ festgehalten sind, bestimmt werden. Formel (129) zeigt die Berechnung der Matrix MatValGap, die direkte interne strukturelle Lücken ebenso wie potenzielle Performanzlücken hervorhebt. Gemäß Formel (129) stehen die positiven Werte dieser Matrix für direkte strukturelle Lücken der lokalen Struktur, während negative Werte deren potenzielle Performanzlücken kennzeichnen. Die Beträge für positive Werte werden direkt aus den jeweiligen Zellen von $\mathrm{MatVal}^{G L O}$ entnommen, ebenso wie für potenzielle Performanzlücken, wobei für diese Zellen die Beträge aus MatVal ${ }^{G L O}$ mit negativem Vorzeichen versehen werden. Inkonsistenzen treten auf, wenn die lokale Struktur Relationen aufweist, die global nicht vorgesehen sind. In solchen Fällen wird für die betreffende Zelle ,NA' ausgegeben. Eine wichtige Fragestellung betrifft die Dimensionen der Matrizen MatVal ${ }^{L O C}$ und MatVal ${ }^{G L O}$. Diese können, müssen aber nicht gleich sein. Zentral ist, dass die Knoten von MatVal $^{L(C)}$, d.h. die Zeilen- $i=1, \ldots, n$ und Spaltenknoten $j=1, \ldots, m$, die folglich eine Dimension von $n \times m$ für MatVal ${ }^{L O C}$ bewirken, in $\mathrm{MatVal}^{G L O}$ enthalten sind. Es wird empfohlen, die Dimension von $\mathrm{MatVal}^{(i L)}$ für die spätere Bewertung an $\mathrm{MatVal}^{L O C}$ anzupassen, hierfür wird das Superskript $r$ an $\mathrm{MatVal}^{(i L O)}$ und dessen Derivate angefügt. Die Ausgangsmatrizen sollten bereits um etwaige Selbst- 
bezüglichkeit bereinigt worden sein. Funktion STRUK zeigt eine Möglichkeit, wie MatValGap erstellt werden kann.

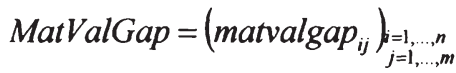

$$
\begin{aligned}
& \text { matvalgap }_{i j}= \begin{cases}\text { matval }_{i j}^{G L . O r} & \text { wenn matval }_{i j}^{\text {GLOr }}>0 \wedge \text { matval }_{i j}^{L . O C}=0 \\
-\left(\text { matval }_{i j}^{G L O r}\right) & \text { wenn matval }_{i j}^{G L O r}>0 \wedge \text { matval }_{i j}^{L(\mathcal{O}}>0 \\
0 & \text { wenn matval }_{i j}^{G L O r}=0 \wedge \text { matval }_{i j}^{L O C}=0 \\
N A & \text { wenn matval }_{i j}^{G L O r}=0 \wedge \text { matval }_{i j}^{L O C}>0\end{cases}
\end{aligned}
$$

Wie bereits in den Ausführungen über die Einsatzmöglichkeiten von Adjazenzlisten anstatt Adjazenzmatrizen hervorgehoben, hat die Darstellung von relationalen Daten in Listenform gegenüber der Matrixform eine Reihe von Vorteilen bei der computergestützten Verarbeitung. Darüber hinaus ermöglicht die Listenform auch eine einfache Anreicherung der Kanten mit Zusatzinformationen sowie allgemein ein unkompliziertes Festhalten von Zwischenergebnissen, die bei der Matrixrechnung verloren gingen. Der spezielle Listentyp ,data frame' der statistischen Programmierumgebung R [R 2007] liefert eine Möglichkeit, unterschiedliche Vektortypen (wie integer, double, logical oder character) von gleicher Länge in einer Datenstruktur abzulegen und erscheint daher als eine geeignete Form der Repräsentation der Verflechtungsdaten für weitere Manipulationen. Die Umwandlung von Matrix- in Listenform wird in Formel (130) aufgezeigt und kann über die Funktion EDGEFRAME erfolgen. Für die weiteren Schritte soll auch die Matrix MatValGap in die Form dieses speziellen Listentyps gebracht werden (Edgeframe MalValiap). $^{\text {(E) }}$.

$$
\text { Edgeframe }^{\text {Marlal }}=\left(v_{i}, w_{j}, \text { matval }_{i j}\right)_{\substack{=1, \ldots, n \\ j=1, \ldots, m}} \text { für } \text { matval }_{i j} \neq 0
$$

\section{Spezialfall: Vergleich von Graphen mit disjunkten Knotenmengen}

Eine spezielle Problemstellung tritt auf, wenn zur lokalen wie auch zur globalen Verflechtungsstruktur jeweils zwei disjunkte Knotenmengen $V_{1}$ und $V_{2}$ vorliegen, deren Zusammenhänge nur über zwei Graphen bestimmt werden können, welche die unterschiedlichen Rollen der Knoten fiesthalten,-Währeadieine Kno- 
tenmenge in einem der beiden Graphen als Vorläuferknoten auftritt, notiert die andere Knotenmenge in diesem Graphen als Nachfolgerknotenmenge. Im dazugehörigen zweiten Graph ist die Rollenverteilung zwischen den beiden Knotenmengen genau umgekehrt. Diese Reziprozität der Graphen ermöglicht eine Verknüpfung der Knotenmengen. Ein anschauliches Beispiel aus der Input-Ouptut Rechnung bieten Make- und Use-Matrizen, die jeweils zwei disjunkte Knotenmengen enthalten, deren jeweilige Knoten in den Matrizen abwechselnd als Vorläufer- bzw. als Nachfolgerknoten auftreten. Eine Kombination der beiden Matrizen, wie in Formel (131) dargestellt, gibt einen Einblick in den Gesamtzusammenhang der Knotenmengen. Eine solchermaßen kombinierte Matrix kann vielfach in den nachstehend vorgestellten Vergleichsoperationen für Graphen mit einheitlichen Knotenmengen eingesetzt werden. Zu beachten wäre hierbei, dass die Weglängen in einem solchen Fall einer speziellen Interpretation bedürfen, nachdem die Potenzierung der kombinierten Matrix alternierende Lösungen ergibt. Ungerade Weglängen führen von einer Knotenmenge in die jeweils andere, während bei geraden Weglängen die Ausgangs- und Zielknotenmenge identisch ist. Für den Vergleich von Graphen mit disjunkten Knotenmengen sind demgemäß nur ungerade Weglängen von Relevanz. Prinzipiell ist bei der Potenzierung einer kombinierten Matrix zu prüfen, welche Annahmen für den jeweiligen Anwendungszweck implizit getroffen werden und ob diese zulässig sind.

$$
\begin{aligned}
& \text { MatVal }^{d i s K M}=\left(\text { matval }_{i j}^{d i s K M}\right)_{\substack{i=1, \ldots, \ldots+m \\
j=1, \ldots, m+n}} \\
& \text { matval }_{i j}^{d i s K M}= \begin{cases}\text { matval }_{i j} & \text { für } i=1, \ldots, n, j=m+1, \ldots, m+n \\
\text { matval }_{i j} & \text { für } i=n+1, \ldots, n+m, j=1, \ldots, m \\
0 & \text { andernfalls }\end{cases}
\end{aligned}
$$

Will man die Relationen der disjunkten Knotenmengen einer lokalen und einer globalen Ebene vergleichen, so kann dies über eine Formel (131) entsprechende Integration der MatValGap-Matrizen (MatValGapl in der Dimension $n \times m$, MatValGap2 in der Dimension $m \times n$; in Listenform Edgeframe $e^{\text {MatValGapl }}$ und Edgeframe ${ }^{\text {MatValGap2 }^{2}}$ für beide (vergleichbare) Rollen erfolgen. Hierbei ist hervor- 
zuheben, dass die direkten internen Lücken zwischen den disjunkten Knotenmengen bereits über die Berechnung der jeweiligen MatValGap-Matrizen identifiziert werden. Formel (132) zeigt die Zusammenführung der aus den Kantenlisten Edgeframe MalValGapl und Edgeframe $e^{\text {MatValGap2 entstehenden, enweiterten }}$ Kantenliste Edgeframe ${ }^{(\text {MatValGiapl,MatValGap2) }}$ (Funktion EDGEMIX). Eine wichtige Entscheidung bei der Erstellung dieser zusammengeführten Kantenliste ist, welche der beiden Knotenmengen als Bindeglied der Vorläufer- und Nachfolgerknoten dienen soll, die entsprechend aus der anderen Knotenmenge stammen.

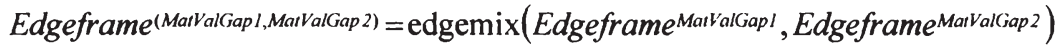

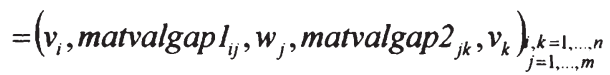

$$
\begin{aligned}
& \left(v_{i}, w_{j}, \text { matvalgap } I_{i j}\right) \in \text { Edgeframe MalvalGap } 1^{\prime} \\
& \left(w_{j}, v_{k}, \text { matvalgap } 2_{j k}\right) \in \text { Edgeframe MaiValiap } 2^{2} \\
& v_{i}, v_{k} \in V_{1} \\
& w_{j} \in V_{2}
\end{aligned}
$$

Für das bessere Verständnis der Ergebnisse dieser erweiterten Kantenliste gibt Tabelle 28 eine Übersicht über die möglichen Kombinationen zwischen Ausgangsknoten $v_{i}$, Verbindungsknoten $w_{j}$ und Zielknoten $v_{k}$. Die positiven bzw. negativen Vorzeichen, die sich aus der Berechnung der MatValGap-Matrizen ergeben und direkte Lücken bzw. potenzielle Performanzlücken bezeichnen, dienen hierbei als Unterscheidungskriterium, ,NA' gibt fehlende Knoten in der lokalen Struktur an.

Tabelle 28: Mögliche Transaktionszusammenhänge in Edgeframe (MatVal(Gap1.Martal(jap2)

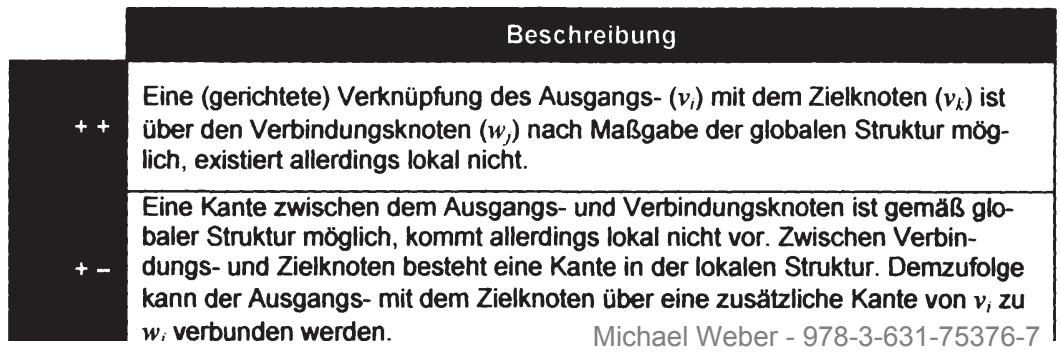




\section{Beschreibung}

Eine Kante zwischen dem Ausgangs- und Verbindungsknoten existiert in der lokalen Struktur. Zwischen Verbindungs- und Zielknoten gibt es jedoch lokal keine Kante, obwohl eine solche entsprechend der globalen Struktur möglich wäre. Der Ausgangsknoten könnte also mit dem Zielknoten über eine zusätzliche Kante von $w_{i} z u v_{k}$ in Beziehung gesetzt werden.

Eine (gerichtete) Verknüpfung des Ausgangs- mit dem Zielknoten über einen Verbindungsknoten ist global möglich und existiert lokal bereits.

Global gibt es einen gerichteten Zusammenhang zwischen dem Verbindungsund dem Zielknoten, nicht aber lokal. Gleichzeitig fehlt lokal nicht nur eine Kante zwischen dem Ausgangs- und dem Verbindungsknoten, sondern selbst der Ausgangsknoten existiert im Gegensatz zur globalen Struktur nicht. (Der Ausgangsknoten entspricht in einer solchen Situation nicht der Definition einer externen Lücke.)

Es besteht eine gerichtete Beziehung zwischen dem Ausgangs- und dem Verbindungsknoten nur in der globalen, nicht aber in der lokalen Struktur.

+ NA Darüber hinaus fehlt lokal nicht nur eine Kante zwischen dem Verbindungsund dem Zielknoten, sondern auch der Zielknoten selbst. (Der Zielknoten ist aufgrund dessen nicht als externen Lücke zu klassifizieren.)

Lokal existiert bereits eine Kante zwischen dem Verbindungsknoten und dem Zielknoten. Eine Kante zwischen dem Verbindungs- und dem Ausgangsknoten

NA - ist allerdings lokal genauso wenig vorhanden wie der Ausgangsknoten selbst, der global existiert. (Auch in diesem Fall ist der Ausgangsknoten keine externe Lücke.)

Eine Verbindung zwischen dem Ausgangs- und dem Verbindungsknoten be-

- NA steht bereits. Eine Kante zwischen dem Verbindungsknoten und dem Zielknoten kommt ebenso wie der Zielknoten selbst in der lokalen Struktur nicht vor. (Der Zielknoten ist allein deshalb keine externe Lücke.)

In der globalen Struktur stehen Ausgangs- und Zielknoten über den Ver-

NA NA bindungsknoten in Zusammenhang, allerdings sind Ausgangs- und Zielknoten nicht in der lokalen Struktur vorhanden. Der Verbindungsknoten hingegen ist Teil der lokalen Struktur.

\subsection{Identifikation von indirekten internen und externen Lücken}

Nach der Identifikation von internen direkten Lücken sowie Performanzlücken stellt sich die Frage, wie interne indirekte Lücken und externe Lücken gefunden werden können. Zu diesem Zweck ist es erforderlich, die vorhandenen relationalen Datenbestände zu verbinden, um fehlende Knoten einer potenziellen Knotenfolge zu ermitteln oder um Knotenmengen zu identifizieren, die durch Schließung von direkten Lücken indirekt profitieren, d.h. positive Externalitäten lukrieren. Mittels einer solchen Verbindung kann sozusagen der Raum der Wege zwischen einer bestimmten Ausgangsknotenmenge und einer bestimmten Zielknotenmenge gebildet werden, wobei die Knoten bzw. Kanten zwischen diesen 
beiden Mengen, also die Menge der Knoten bzw. Kanten, die die Ausgangs- und Zielknotenmenge verbinden, oder auch nur deren Anzahl, gesucht sind. Eine Verfeinerung der bereits eingeführten Typologie zu Lücken, wie in Tabelle 29 vorgestellt, erscheint dabei hilfreich, um auftretende Konstellationen von Lücken für einen Weg $\omega$ mit einer Weglänge > 1 zu klassifizieren.

Abhängig von der Weglänge und der gewünschten Detaillierung der Lückenidentifikation kann eine potenzierte Adjazenzmatrix herangezogen werden, um zu erkennen, bei welcher Weglänge eine Verbindung zwischen der jeweiligen Ausgangs- und Zielknotenmenge möglich ist und wie viele unterschiedliche Wege für diese Verbindung existieren. Das Ergebnis dieser Potenzierung gibt einen Überblick über Verbindungsmöglichkeiten, kann jedoch ohne weitere Manipulation nicht zeigen, ob Wege, die zwischen einem Ausgangs- und Zielknoten bei einer bestimmten Weglänge möglich sind, Knoten mehrmals enthalten oder worin sich diese Wege unterscheiden, d.h. welche Folgen von Knoten und Kanten die zu vergleichenden Wege ausmachen. Eine detaillierte Auskunft über die Knoten bzw. Kanten, die für die lokale Struktur Lücken darstellen, kann daher eine Verknüpfung der bereits eingeführten Kantenlisten geben. Bevor auf diese Verknüpfung eingegangen wird, soll nun gezeigt werden, wie und welche Informationen aus einem Vergleich der potenzierten Adjazenzmatrizen gewonnen werden können.

Tabelle 29: Typen von Lücken für einen Weg $\omega$ mit Weglänge $>1$

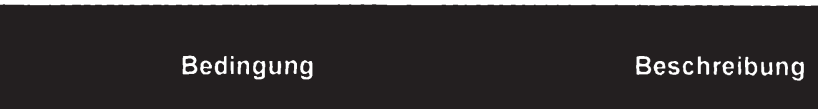

$\forall\left(v_{k}, v_{h}\right) \in \omega:\left(v_{k}, v_{h}\right) \in E^{L O C}$
$\forall\left(v_{k}, v_{h}\right) \in \omega:$
$\left(v_{k}, v_{h}\right) \notin E^{L\left(\mathcal{K}^{\prime}\right.} \wedge v_{k}, v_{h} \in V^{L .\left(\mathcal{C}^{\prime}\right.}$

Die lokalen Knoten $v_{i}$ und $w$, können über eine Folge von Performanzlücken miteinander verknüpft werden.

Der lokale Ausgangsknoten $v_{i}$ und der lokale Zielknoten $w_{j}$ sind über eine Folge von direkten internen Lücken verbunden. 


\begin{tabular}{|c|c|c|}
\hline $\begin{array}{r}\text { partielle } \\
\text { indirekte } \\
\text { interne } \\
\text { Lücke }\end{array}$ & $\begin{array}{l}\left(\forall v_{k} \in \omega: v_{k} \in V^{L O C}\right) \wedge \\
\left(\exists\left(v_{g}, v_{h}\right) \in \omega:\left(v_{g}, v_{h}\right) \in E^{L O C}\right) \wedge \\
\left(\exists\left(v_{a}, v_{b}\right) \in \omega:\left(v_{a}, v_{b}\right) \notin E^{L O C}\right)\end{array}$ & $\begin{array}{l}\text { Die Knoten } v_{i} \text { und } w_{j} \text { aus der lokalen } \\
\text { Knotenmenge stehen über eine Folge } \\
\text { von direkten internen Lücken und } \\
\text { Performanzlücken miteinander in } \\
\text { Beziehung. }\end{array}$ \\
\hline $\begin{array}{r}\text { komplette } \\
\text { externe } \\
\text { Lücke }\end{array}$ & $\begin{array}{l}\forall\left(\left(v_{k}, v_{h}\right) \in \omega \wedge v_{k} \neq v_{i} \wedge v_{h} \neq w_{j}\right): \\
\left(v_{k}, v_{h}\right) \notin V^{L O C}\end{array}$ & $\begin{array}{l}\text { Der lokale Knoten } w_{j} \text { kann von dem } \\
\text { lokalen Knoten } v_{i} \text { über eine Folge von } \\
\text { ausschließlich externen Knoten er- } \\
\text { reicht werden. }\end{array}$ \\
\hline $\begin{array}{r}\text { partielle } \\
\text { externe } \\
\text { Lücke mit } \\
\text { kompletten } \\
\text { (in-)direkten } \\
\text { internen } \\
\text { Lücken }\end{array}$ & $\begin{array}{l}\left(\left\{\left(v_{h} \in \omega\right):\left(v_{h} \in V^{L O C}\right)\right\} \mid>3\right) \wedge \\
\left(\exists\left(v_{k} \in \omega\right):\left(v_{k} \notin V L O C\right)\right) \wedge \\
\left(\exists\left(v_{h}, v_{k}\right) \in \omega: v_{h}, v_{k} \in V^{L O C}\right) \wedge \\
\left(\forall\left(\left(v_{a}, v_{b}\right) \in \omega \wedge v_{a}, v_{b} \in V^{L O C}\right):\right. \\
\left.\left(v_{a}, v_{b}\right) \notin E^{L O C}\right)\end{array}$ & $\begin{array}{l}\text { Der lokale Knoten } v_{i} \text { ist mit dem loka- } \\
\text { len Knoten } w_{j} \text { über eine Folge von } \\
\text { externen und internen (d.h. lokalen) } \\
\text { Knoten verbunden. Die internen Kno- } \\
\text { ten stehen hierbei aus Sicht der loka- } \\
\text { len Struktur in keiner gegenseitigen } \\
\text { Beziehung. Ihre Kanten sind, falls die } \\
\text { internen Knoten in einer Teilfolge } \\
\text { aneinandergereiht sind, als komplette } \\
\text { interne indirekte Lücke (Subweglänge } \\
\text { ist mindestens 2) bzw. als interne } \\
\text { direkte Lücke (Subweglänge 1) } \\
\text { klassifiziert. Enthält die Folge ab- } \\
\text { gesehen von externen Knoten nur } \\
\text { interne Knoten, die keine Teilfolgen } \\
\text { bilden - d.h. einzeln zwischen den } \\
\text { externen Knoten (-folgen) angeordnet } \\
\text { sind - so ist sie als partielle externe } \\
\text { Lücke mit singulären internen Knoten } \\
\text { zu klassifizieren. }\end{array}$ \\
\hline $\begin{array}{r}\text { partielle } \\
\text { externe } \\
\text { Lücke mit } \\
\text { Performanz- } \\
\text { lückenfolgen }\end{array}$ & $\begin{array}{l}\left(\left|\left\{\left(v_{h} \in \omega\right):\left(v_{h} \in V L O C\right)\right\}\right|>3\right) \wedge \\
\left(\exists\left(v_{k} \in \omega\right):\left(v_{k} \notin V L O C\right)\right) \wedge \\
\left(\exists\left(v_{h}, v_{k}\right) \in \omega: v_{h}, v_{k} \in V L O C\right) \wedge \\
\left(\forall\left(\left(v_{a}, v_{b}\right) \in \omega \wedge v_{a}, v_{b} \in V^{L O C}\right):\right. \\
\left.\left(v_{a}, v_{b}\right) \in E^{L O C}\right)\end{array}$ & $\begin{array}{l}\text { Zwischen dem lokalen Knoten } v_{i} \text { und } \\
\text { dem lokalen Knoten } w_{i} \text { besteht ein } \\
\text { Weg, der eine Folge von externen } \\
\text { Knoten in Kombination mit internen } \\
\text { Knoten ist. Die wechselseitigen Be- } \\
\text { ziehungen der internen Knoten sind } \\
\text { für den Fall, dass diese in einer (bzw. } \\
\text { mehreren) Teilfolgen aneinander- } \\
\text { gereiht sind, potenzielle Performanz- } \\
\text { lücken (Subweglänge 1) oder eine } \\
\text { Folge von potenziellen Performanz- } \\
\text { lücken (Subweglänge >1). Wie } \\
\text { bereits festgehalten, wird eine Folge, } \\
\text { die neben den externen Knoten nur } \\
\text { interne Knoten enthält, die einzeln } \\
\text { zwischen den externen Knoten(- } \\
\text { folgen) vorkommen, als partielle ex- } \\
\text { terne Lücke mit singulären internen }\end{array}$ \\
\hline
\end{tabular}




\begin{tabular}{|c|c|c|}
\hline $\begin{array}{r}\text { partielle } \\
\text { externe } \\
\text { Lücke mit } \\
\text { partiellen } \\
\text { indirekten } \\
\text { internen } \\
\text { Lücken }\end{array}$ & $\begin{array}{l}\left(\left|\left\{\left(v_{h} \in \omega\right):\left(v_{h} \in V^{L O C}\right)\right\}\right|>3\right) \wedge \\
\left(\exists\left(v_{k} \in \omega\right):\left(v_{k} \notin V^{L O K^{\prime}}\right)\right) \wedge \\
\left(\exists\left(\left(v_{a}, v_{b}\right) \in \omega \wedge v_{a}, v_{b} \in V^{L O C}\right):\right. \\
\left.\left(v_{a}, v_{b}\right) \in E^{L O C}\right) \wedge \\
\left(\exists\left(\left(v_{c}, v_{d}\right) \in \omega \wedge v_{c}, v_{d} \in V^{L O K^{\prime}}\right):\right. \\
\left.\left(v_{c}, v_{d}\right) \notin E^{L O C}\right)\end{array}$ & $\begin{array}{l}\text { Der lokale Knoten } v \text {, ist mit dem loka- } \\
\text { len Knoten } w_{j} \text { über eine Folge von } \\
\text { externen Knoten in Kombination mit } \\
\text { internen (d.h. lokalen) Knoten ver- } \\
\text { bunden. Sind die lokalen Knoten } \\
\text { aneinandergereiht, so stellen diese } \\
\text { Teilfolgen partielle indirekte Lücken } \\
\text { dar. Diese haben gemäß der all- } \\
\text { gemeinen Definition für indirekte } \\
\text { interne Lücken mindestens die Sub- } \\
\text { weglänge 2. Partielle externe Lücken } \\
\text { mit singulären internen Knoten bilden } \\
\text { eine eigene Kategorie. }\end{array}$ \\
\hline $\begin{array}{r}\text { partielle } \\
\text { externe } \\
\text { Lücke mit } \\
\text { singulären } \\
\text { internen } \\
\text { Knoten }\end{array}$ & $\begin{array}{l}\left(\left|\left\{\left(v_{h} \in \omega\right):\left(v_{h} \in V^{L O C}\right)\right\}\right|>2\right) \wedge \\
\left(\exists\left(v_{k} \in \omega\right):\left(v_{k} \notin V^{L} L \mathcal{C}\right)\right) \wedge \\
\left(\neg \exists\left(\left(v_{a}, v_{b}\right) \in \omega\right):\right. \\
\left.\left(v_{a} \in V^{L O C}\right) \wedge\left(v_{b} \in V^{L O C}\right)\right)\end{array}$ & $\begin{array}{l}\text { Der lokale Knoten } w_{j} \text { ist vom lokalen } \\
\text { Knoten } v_{i} \text { aus durch eine Folgen von } \\
\text { jeweils externen Knoten und internen } \\
\text { (d.h. lokalen) Knoten erreichbar. Der } \\
\text { Weg enthält zwar inteme Knoten, } \\
\text { jedoch keine Teilfolgen von internen } \\
\text { Knoten. Der Weg entspricht dem- } \\
\text { gemäß einer Verkettung von } \\
\text { kompletten externen Lücken. Eine } \\
\text { Folge, die abwechselnd aus internen } \\
\text { und externen Knoten besteht, d.h. } \\
\text { aus kompletten externen Lücken mit } \\
\text { Subweglänge } 2 \text { aufgebaut ist, stellt } \\
\text { einen für die Bewertung interessanten } \\
\text { Spezialfall dar. }\end{array}$ \\
\hline
\end{tabular}

\subsection{Identifikation von internen Lückenfolgen}

Bei der Analyse indirekter Wirkungsketten über einen Vergleich potenzierter Adjazenzmatrizen stehen zunächst interne Effekte im Vordergrund der Betrachtung. Zur Bestimmung von internen indirekten Lücken kann dabei MatValGap als Ausgangspunkt für die zu Vergleichszwecken erforderlichen Adjazenzmatrizen dienen, nachdem aus MatValGap die internen direkten Lücken, die potenziellen Performanzlücken sowie - in Kombination - alle Kanten der globalen Struktur für die Knotenmenge der lokalen Struktur einfach extrahierbar sind, wie die Formeln (133) bis (135) zeigen. Eine Voraussetzung für die Umwandlung in Adjazenzmatrizen und deren Potenzierung ist, dass MatValGap eine quadratische Matrix mit übereinstimmenden Zeilen- und Spaltenbezeichnungen ist. Dies ergibt sich, wenn sämtliche Knoten des, Graphen aus ein und 
derselben Menge entstammen. Während MatGap ${ }^{\text {edgeLOC }}$ alle Kanten der globalen Struktur bezogen auf die lokale Knotenmenge in binärer Form beinhaltet, stellt MatGap ${ }^{\text {'edgeLOC }}$ die direkten Lücken der lokalen Struktur dar. MatGap edgel.OC? umfasst hingegen die potenziellen Performanzlücken der lokalen Struktur.

$$
\begin{aligned}
& \text { MatGap }{ }^{\text {edgel.OC }}=\left(\text { matgap }_{i j}^{\text {edgel }(\mathcal{O})}\right)_{i, j=1, \ldots, n} \\
& \text { matgap }_{i j}^{\text {edgeloc }}= \begin{cases}1 & \text { wenn } \text { matvalgap }_{i j} \neq 0 \\
0 & \text { andernfalls }\end{cases} \\
& \text { MatGap }{ }^{+ \text {edgeLOC }}=\left(\text { matgap }_{i j}^{+ \text {edgeloC }}\right)_{i, j=1, \ldots, n} \\
& \text { matgap }_{i j}^{+ \text {edgeLOC }}= \begin{cases}1 & \text { wenn matvalgap } \\
0 & \text { andernfalls }\end{cases} \\
& \text { MatGap }{ }^{- \text {edgel. } O C}=\left(\text { matgap }_{i j}^{- \text {edgel.oC }}\right)_{i, j=1, \ldots, n} \\
& \text { matgap }_{i j}^{\text {-edgel. }:}= \begin{cases}1 & \text { wenn matvalgap } \\
0 & \text { andernfalls }\end{cases}
\end{aligned}
$$

Für die Weglänge $p$ kann die (potenzielle) Erreichbarkeit auf Basis jeder dieser drei Adjazenzmatrizen durch Potenzierung gemäß dem Potenzierungsansatz aus Formel (69) festgestellt werden. Formel (136) stellt dies am Beispiel von MatGap edgeLOC dar. Analog werden aus MatGap edgeLOC und MatGap ${ }^{\text {edgeLOCC }}$ die potenzierten Matrizen PMatGap edgel. ${ }^{\text {C, }}$ p und PmatGap edgeLOC, $p$ gebildet.

$$
\begin{aligned}
& P M a t G a p^{\text {edgeLOC },<p>}=\left(\text { pmatgap }_{i j}^{\text {edgel } O C,<p>}\right)_{i, j=1, \ldots, n} \\
& \text { pmatgap }_{i j}^{\text {edgeLOC, }<p>}=\left(\left(\text { MatGap }{ }^{\text {edgeLOC }}\right)^{\langle p\rangle}\right)_{i j}
\end{aligned}
$$

Auf Basis dieser drei Matrizen für die Weglänge $p$ können nun Vergleiche durchgeführt werden, die Aufschluss über Verbindungsmöglichkeiten durch Folgen von direkten internen Lücken, Folgen von Performanzlücken oder durch eine Kombination beider geben. Diese Lückenfolgen können abhängig von den Vergleichsoperationen klassifiziert werden. Tabelle 30 verschafft einen Überblick über die 
entstehende Klassifikation, welche die Typen ,PER', ,KIL', ,PIL', ,KIP', ,PPL', ,KPP', ,KPI', und ,NOI' umfasst.

Tabelle 30: Klassifikation von internen Lückenfolgen

\begin{tabular}{|c|c|c|}
\hline & Bedingung & Beschreibung \\
\hline & 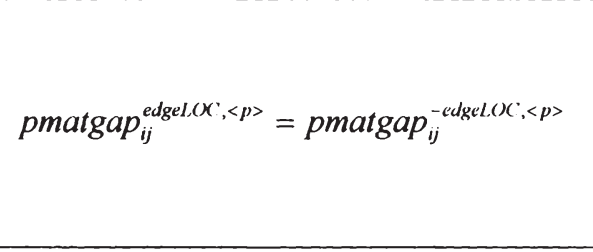 & $\begin{array}{l}\text { Der lokale Ausgangsknoten } v_{i} \text { ist in } \\
\text { jedem der Wege gleicher Länge } p \text { mit } \\
\text { dem lokalen Zielknoten } w_{j} \text { über eine } \\
\text { Folge von in der lokalen Struktur } \\
\text { bestehenden Kanten verbunden. } \\
\text { Demzufolge sind sämtliche Wege } \\
\text { Folgen von potenziellen Performanz- } \\
\text { lücken. }\end{array}$ \\
\hline KI & pmatgap $_{i j}^{\text {edgel.oc },<p>}=$ pmatgap $_{i j}^{+ \text {edgel.oC, },<p>}$ & 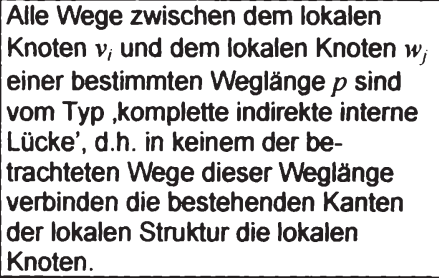 \\
\hline PIL & 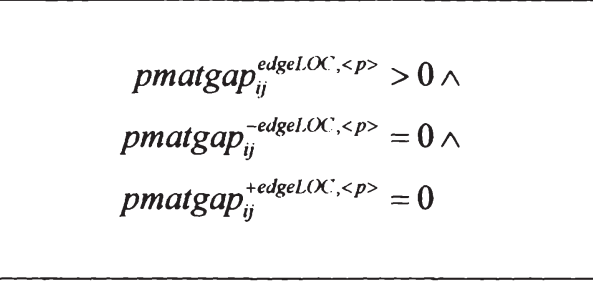 & $\begin{array}{l}\text { Sämtliche Wege gleicher Länge } p \\
\text { von dem lokalen Knoten } v_{i} \text { zu dem } \\
\text { lokalen Knoten } w_{\text {s sind über Folgen, }} \text {, } \\
\text { die aus internen (d.h. lokalen) Knoten } \\
\text { mit in der lokalen Struktur möglichen } \\
\text { und bereits bestehenden Kanten } \\
\text { zusammengesetzt sind, verbunden. } \\
\text { Jeder dieser Wege ist daher vom Typ } \\
\text {,partielle indirekte interme Lücke'. }\end{array}$ \\
\hline PF & $\begin{array}{l}\text { pmatgap }_{i j}^{\text {edgelOC },<p>}>\text { pmatgap }_{i j}^{\text {edgeLOC, }<p>} \wedge \\
\text { pmatgap }_{i j}^{\text {edgelOC, },<p>}>0 \wedge \\
\text { pmatgap }_{i j}^{\text {edgel } O C_{,}<p>}=0\end{array}$ & $\begin{array}{l}\text { Die Klassifikation ,PPL' klassifiziert } \\
\text { Wege gleicher Länge } p \text { zwischen } \\
\text { zwei lokalen Knoten, die entweder } \\
\text { von dem Typ ,potenzielle Per- } \\
\text { formanzlücke' oder, partielle indirekte } \\
\text { interne Lücke' sind. } \\
\end{array}$ \\
\hline KPP & 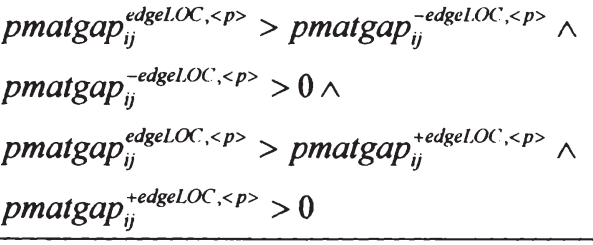 & $\begin{array}{l}\text {,KPP' klassifiziert jene Fälle, in denen } \\
\text { die Typen, komplette indirekte interne } \\
\text { Lücke', ,potenzielle Performanzlücke' } \\
\text { und ,partielle indirekte inteme Lücke' } \\
\text { als Verbindungsmóglichkeiten zwi- } \\
\text { schen zwei lokalen Knoten bei fixier- } \\
\text { ter Weglänge } p \text { zugleich auftreten. } \\
\end{array}$ \\
\hline KIP & 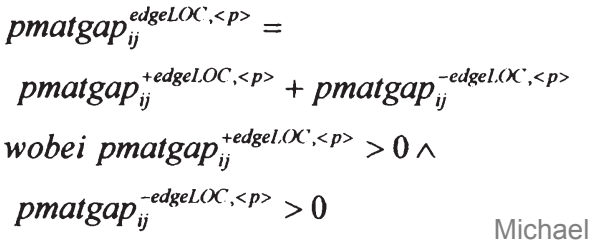 & $\begin{array}{l}\text { KIP' besagt, dass zwei Arten von } \\
\text { Wegen für eine bestimmte Weglänge } \\
p \text { nebeneinander existieren. Dies } \\
\text { sind Wege vom Typ ,komplette in- } \\
\text { direkte interne Lücke' und Wege vom } \\
\text { Typ ,potenzielle Performanzlücke'. } \\
\text { Weber - 978-3-631-75376-7 }\end{array}$ \\
\hline
\end{tabular}




\begin{tabular}{|c|c|c|}
\hline KP & 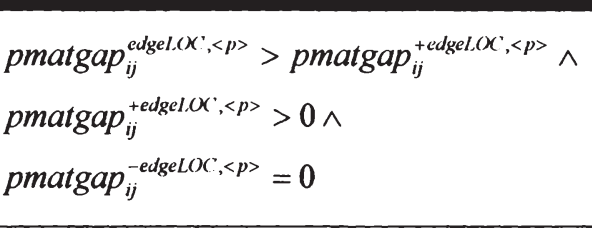 & $\begin{array}{l}\text {,KPl' trifft zu wenn die Wege gleicher } \\
\text { Länge } p \text { zwischen dem lokalen Kno- } \\
\text { ten } v_{i} \text { und dem lokalen Knoten } w_{j} \\
\text { entweder als ,komplette indirekte } \\
\text { interne Lücke' oder als ,partielle in- } \\
\text { direkte interne Lücke' zu klassi- } \\
\text { fizieren sind. }\end{array}$ \\
\hline NO & pmatgap $_{i j}^{\text {edgel. } O C,<p>}=0$ & $\begin{array}{l}\text { Es existiert kein Weg der be- } \\
\text { trachteten Weglänge } p \text {, der Knoten } v_{i} \\
\text { mit Knoten } w_{j} \text { über interne Lücken- } \\
\text { folgen verbindet. }\end{array}$ \\
\hline
\end{tabular}

MatGapComp $p^{p}$ ist die Übersichtsmatrix der Ergebnisse dieser Vergleichsoperationen gemäß Formel (137), deren Bedingungen in Tabelle 30 definiert werden (Funktion MATGAPCOMP).

$$
\begin{aligned}
& \text { MatGapComp }=(\text { matgapcomp } \\
& i j)_{i, j=1, \ldots, n} \\
& \text { matgapcomp } p \\
& \text { Cat }=(\text { Cat } \\
&\text { CaR", "PPL", "KPP", "KIP", "PIL", "KPI", "KIL", "NOI" })
\end{aligned}
$$

In Erweiterung des Vergleichs der potenzierten Matrizen für eine Weglänge können zusätzlich unterschiedliche Weglängen gegenübergestellt werden. Dieses Vorgehen kann ähnlich wie bei der Erstellung von Distanzmatrizen eingesetzt werden, um für bestimmte Weglängenbereiche festzuhalten, welche Typen von Lückenfolgen bei welcher Weglänge zum ersten Mal auftreten. Diese Information kann in weiterer Folge beispielsweise für die Zuordnung oder Bewertung von Verbindungen herangezogen werden. Formel (138) zeigt, wie diese Spezialform einer Distanzmatrix namens Stafette gebildet wird (Funktion STAFETTE).

$$
\begin{aligned}
& \text { Stafette }=\left(\text { stafette }_{i j}\right)_{i, j=1, \ldots, n} \\
& \text { stafette }_{i j}=\operatorname{sort}_{k}^{\text {asc }_{l^{\prime} k}}\left(\left(\text { cat }_{k}, l_{i j k}\right)_{\text {cat } t_{k} \in(a \mathrm{at}}\right) \text { wobei } k=1, \ldots, \mid \text { Cat } \mid \\
& l_{i j k}=\min _{p}\left\{p: \text { matgapcomp }_{i j}^{p}=\text { cat }_{k}\right\} \text { für } i, j=1, \ldots, n, k=1, \ldots, \mid \text { Cat } \mid
\end{aligned}
$$




\subsection{Identifikation von externen Lückenfolgen}

Zur Vervollständigung der Untersuchung von indirekten Wirkungsketten und deren Lücken sind anschließend an die Erfassung von indirekten internen Lücken Effekte von externen Lücken zu behandeln. Hierfür wird zunächst der Potenzierungsansatz aus Formel (69) auf die Adjazenzmatrix der nicht reduzierten globalen Struktur mit $\left(\mathrm{Mat}^{G L O}\right)$ oder ohne (Mat $\left.{ }^{G L O L O C}\right)$ den Kanten der lokalen Struktur für eine Weglänge von $p$ angewendet, wobei die Kanten der lokalen Struktur bei den Zwischenergebnissen der Potenzierung von Mat ${ }^{\text {GLOLLOC }}$ jeweils nullzusetzen sind, nicht aber bei dem Endergebnis. Formel (139) bzw. (140) und (144) demonstrieren diese Vorgehensweise. Anschließend kann eine Matrix der potenziellen Erreichbarkeiten zu dieser Weglänge unter Einbezug der externen Knoten für die lokale Knotenmenge $V^{L O C}$ den Ergebnissen jeweils entnommen werden (Formel (145)). Während PMat ${ }^{G L O, V L O C, p}$ Beziehungen zwischen lokalen - und somit internen - Knoten unter Einschluss von externen und internen (d.h. lokalen) Knoten und Kanten aufzeigt, hält PMat GLOLOC,VLOC, p nur Wege fest, die über externe Kanten verlaufen, d.h. Kanten, die nicht in der lokalen Struktur enthalten sind. Diese Wege schließen Folgen von externen und internen Knoten ein. Für eine Analyse der internen Lücken bzw. Lückenfolgen wäre es vielfach aber interessant(er), Wege zu identifizieren, welche abgesehen von dem lokalen Ausgangs- und dem lokalen Zielknoten ausschließlich externe Knoten enthalten. Dieser Analyseschritt erfordert zusätzlich zu Mat ${ }^{\text {GLOLOC }}$ eine Adjazenzmatrix, die die nicht reduzierte globale Struktur ohne Kanten, die lokale Knoten beinhalten, wiedergibt. Formel (143) definiert diese Adjazenzmatrix $M a t^{C R O S S}$, die gemeinsam mit Mat ${ }^{G L O L C C}$ gemäß Formel (146) und Formel (147) Pmat ${ }^{E X T, V L O C, ~} p$ generiert, welche zur Bestimmung von kompletten externen Lücken eingesetzt werden kann. Will man im Gegensatz hierzu Wege zwischen den lokalen Ausgangs- und Zielknoten identifizieren, die partielle externe Lücken darstellen, also eine Mischform aus externen und internen Kanten sind

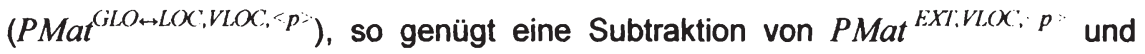

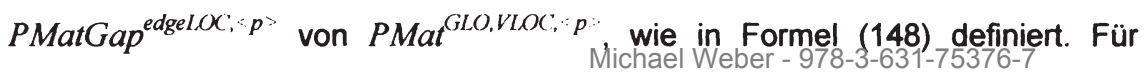


$P M a t^{\text {(iLO } \leftrightarrow L O C, V L O C, p}$ existieren Verbindungsmöglichkeiten und somit Ergebnisse größer 0 erst ab einer Weglänge größer 2 - das sind Wege, die mehr als 3 Knoten involvieren, - während bei PMat ${ }^{\text {GLOLOC,VLOC, } p}$ und PMat EXT,VLOC, p eine Weglänge größer 1 bereits zu Ergebnissen über 0 führen kann. Die beiden letztgenannten Matrizen sind bei Weglänge 2 identisch.

Für die auf $M a t^{G L O}$ beruhenden Berechnungen, d.h. für Berechnungen inklusive den Kanten der lokalen Struktur, ist anzumerken, dass je nach Fragestellung nur

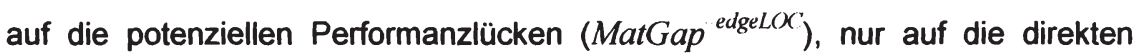
internen Lücken (MatGap ${ }^{+e d g e L O C}$ ) oder eben auf deren Kombination MatGap edgeLOC , die bereits in $\mathrm{Mat}^{(G L O}$ enthalten ist und daher nicht gesondert definiert wird, - zurückgegriffen werden kann. Formel (141) und (142) zeigen wie diese Versionen von $\mathrm{Mat}^{G L O}, \mathrm{Mat}^{G L O}$ und $\mathrm{Mat}^{+G L O}$, gebildet werden, für welche die Formel (144) und die Formel (145) analog gelten. Darüber hinaus ist auch eine Gegenüberstellung der jeweiligen Ergebnisse auf Basis von verschiedenen Versionen der Matrix $M a t^{G L O}$, die die unterschiedlichen Lücken der lokalen Struktur berücksichtigen, möglich und abhängig von der zu beantwortenden Fragestellung zweckmäßig. Die Funktion MATEXTCOMP kann zur Ermittlung von externen Lückenfolgen eingesetzt werden.

$$
\begin{aligned}
& \operatorname{Mat}^{G L O}=\left(\operatorname{mat}_{x y}^{G L O}\right)_{x, y=1, \ldots, z} \text { wobei }\left|V^{G L O}\right|=z>n=\left|V^{L O K}\right| \\
& \text { mat }_{x y}^{G L O}= \begin{cases}1 & \text { wenn matval } \\
0 & \text { andernfalls }\end{cases} \\
& M a t^{G L O \backslash L O C}=\left(m a t_{x y}^{G L O L L O C}\right)_{x, y=1, \ldots, z} \\
& m a t_{x y}^{G L O L O C}= \begin{cases}0 & \text { wenn }\left(v_{x}, w_{y} \in V^{L O C}\right) \vee m a t_{x y}^{G L O}=0 \\
1 & \text { andernfalls }\end{cases} \\
& M a t^{-G L O}=\left(m a t_{x y}^{-G L O}\right)_{x, y=1, \ldots, z} \\
& \text { mat }_{x y}^{-G L O}= \begin{cases}\operatorname{matgap}_{x y}^{-e d g e L O C} & \text { wenn } v_{x}, w_{y} \in V^{L, O C} \\
\operatorname{mat}_{x y}^{G L O} & \text { andernfalls }\end{cases}
\end{aligned}
$$




$$
\begin{aligned}
& \mathrm{Mat}^{+(i L O}=\left(\mathrm{mat}_{x y}^{+(i L O}\right)_{x, y=1, \ldots, z} \\
& \operatorname{mat}_{x y}^{+G L O}= \begin{cases}\operatorname{matgap}_{x y}^{+e d g e l O C}: & \text { wenn } v_{x}, w_{y} \in V^{L O C} \\
\operatorname{mat}_{x y}^{G L O} & \text { andernfalls }\end{cases} \\
& M a t^{C R O S S}=\left(\operatorname{mat}_{x y}^{C R O S S}\right)_{x, y=1, \ldots, z} \\
& \text { mat }_{x y}^{C R O S S}= \begin{cases}0 & \text { wenn }\left(v_{x} \in V^{L O C}\right) \vee\left(w_{y} \in V^{L O C}\right) \vee m a t_{x y}^{G L O}=0 \\
1 & \text { andernfalls }\end{cases} \\
& P M a t^{G L O,<p>}=\left(p m a t_{x y}^{G L O,<p>}\right)_{x, y=1, \ldots, z} \\
& p m a t_{x y}^{G L O,<p>}=\left(\left(\text { Mat }^{\text {GLO }}\right)^{<p>}\right)_{x, y=1, \ldots, z}
\end{aligned}
$$

Analog zu PMat ${ }^{G L O, p}$ in Formel (144) werden PMat ${ }^{+G L O, p}, P M a t^{-G L O ;} \cdot$ sowie PMat ${ }^{\text {GLOLOC: } p \text { errechnet. }}$

$$
\begin{aligned}
& P M a t^{G L O, \mathrm{VLOC},<p>}=\left(p^{2} \operatorname{mat}_{i j}^{G L O, \mathrm{VLOC},<p>}\right)_{i, j=1, \ldots, n} \\
& \text { pmat }_{i j}^{G L O, \text { VLOC, }<p>}=\text { pmat }_{x y}^{(i L O),<p>} \text { für } v_{i}=v_{x} \wedge w_{j}=w_{y}
\end{aligned}
$$

Formel (145) zeigt wie $P M a t^{G L O, V L O C,<p}$ aus $P M a t^{G L O,<p}$ extrahiert werden kann.

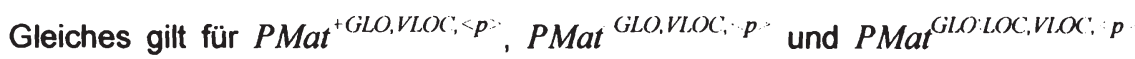
die aus $\mathrm{PMat}^{+ \text {GLO,VLOC, } \mathrm{p}, \text { PMat }}{ }^{\text {GLO,VLOC, p bzw. PMat }}{ }^{\text {GLOLOC,VLOC, p }}$ generiert werden können.

$$
\begin{aligned}
& P M a t^{E X T,<p>}=\left(p^{t} a t_{x y}^{t X T,<p>}\right)_{x, y=1, \ldots, z} \\
& P M a t^{E X T,<p>}=\left(\text { Mat }^{\text {GLOLLOC }}\right)^{<1>}\left(\text { Mat }^{\text {CROSS }}\right)^{<p-2>}\left(\text { Mat }^{\text {GLOLLOC }}\right)^{<\mid>}
\end{aligned}
$$

Sollen Zirkulärbezüge erhalten bleiben, so wäre $\left(\text { Mat }^{\text {GLOLLOC }}\right)^{<l>}$ in Formel (146) auf der rechten Seite durch Mat ${ }^{\text {GLO:LOC }}$ zu ersetzen.

$$
\begin{aligned}
& \text { PMat }^{\text {EXT, LLOC,<p> }}=\left(\text { pmat }_{x y}^{E X T, V L O C,<p>}\right)_{x, y=1, \ldots, z} \\
& \text { pmat }_{i j}^{E X T, \text { VLOC, }<p>}=\text { pmat }_{x y}^{G L O,<p>} \text { für } v_{i}=v_{x} \wedge w_{j}=w_{y}
\end{aligned}
$$




$$
\begin{aligned}
& P M a t t^{G L O \leftrightarrow L O C, \mathrm{VLOC},<p>}=\left(p m a t_{i j}^{G L O \leftrightarrow L O C, \mathrm{VLOC},<p>}\right)_{i, j=1, \ldots, n}
\end{aligned}
$$

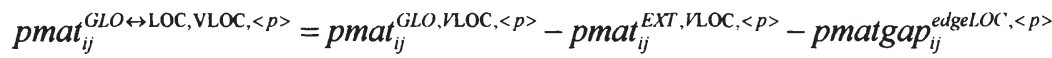

Tabelle 31: Klassifikation von externen Lückenfolgen

\begin{tabular}{|c|c|c|}
\hline & Bedingung & Beschreibung \\
\hline El1 & $\begin{array}{l}\text { pmat }_{i j}^{-G L O, V L O C,<p>}> \\
\left(\text { pmat }_{i j}^{E X T, V L O C,<p>}+\text { pmatgap }_{i j}^{-e d g e L(O C,<p>}\right)\end{array}$ & $\begin{array}{l}\text { Zwischen dem lokalen Knoten } v_{i} \text { und } \\
\text { dem lokalen Knoten } w_{i} \text { besteht zu- } \\
\text { mindest ein Weg der Länge } p \text { aus ex- } \\
\text { ternen Knoten und internen (d.h. } \\
\text { lokalen) Knoten, der als partielle ex- } \\
\text { terne Lücke mit Performanzlücken- } \\
\text { folgen klassifiziert ist. }\end{array}$ \\
\hline EI2 & $\begin{array}{l}p m a t_{i j}^{(L L O, V L O C,<p>}> \\
\left(\text { pmat }_{i j}^{\text {EXT,VLOC, },<p>}+\text { pmatgap }_{i j}^{\text {edgel.OC, },<p>}\right)\end{array}$ & $\begin{array}{l}\text { Mindestens eine Folge der Länge } p \text { von } \\
\text { externen Knoten und internen (d.h. } \\
\text { lokalen) Knoten, die als partielle externe } \\
\text { Lücke mit partiellen indirekten internen } \\
\text { Lücken klassifiziert ist, verbindet den } \\
\text { lokalen Knoten } v_{i} \text { mit dem lokalen Kno- } \\
\text { ten } w_{i} \text {. }\end{array}$ \\
\hline EI3 & $\begin{array}{l}\text { pmat }_{i j}^{+G L O, V L(\mathcal{C},<p>}> \\
\left(\text { pmat }_{i j}^{E X T, V L O C,<p>}+\text { pmatgap }_{i j}^{+e d g e L O C,<p>}\right)\end{array}$ & $\begin{array}{l}\text { Der lokale Knoten } v_{i} \text { ist mit dem lokalen } \\
\text { Knoten } w_{j} \text { über mindestens eine Folge } \\
\text { der Länge } p \text { von externen Knoten in } \\
\text { Kombination mit internen (d.h. lokalen) } \\
\text { Knoten verbunden, die vom Typ } \\
\text { partielle externe Lücke mit kompletten } \\
\text { indirekten internen Lücken' ist. }\end{array}$ \\
\hline El4 & $p m a t_{i j}^{\mathrm{GLO} I L O C, \mathrm{VLOC},<p>}>p \operatorname{mat}_{i j}^{\mathrm{LXT}, V L O C,<p>}$ & $\begin{array}{l}\text { Der lokale Knoten } w_{j} \text { ist vom lokalen } \\
\text { Knoten } v_{i} \text { aus zumindest durch eine } \\
\text { Folge der Länge } p \text { von extermen Knoten } \\
\text { und internen (d.h. lokalen) Knoten, die } \\
\text { eine partielle externe Lücke mit } \\
\text { singulären internen Knoten entspricht, } \\
\text { erreichbar. }\end{array}$ \\
\hline EXT & $p m a t_{i j}^{E X T, V L O C^{\prime},<p>}=p m a t_{i j}^{G L O, V L O C,<p>}$ & $\begin{array}{l}\text { Der lokale Knoten } w_{j} \text { kann von dem } \\
\text { lokalen Knoten } v_{i} \text { nur über eine oder } \\
\text { mehrere Folgen der Länge } p \text { von aus- } \\
\text { schließlich externen Knoten erreicht } \\
\text { werden. Diese Folge oder Folgen sind } \\
\text { komplette externe Lücken. }\end{array}$ \\
\hline NOW & $p m a t_{i j}^{G L O, V L O C,<p>}=0$ & $\begin{array}{l}\text { Es existiert kein Weg der Länge } p, \text { der } \\
\text { Knoten } v_{i} \text { mit Knoten } w_{j} \text { - wobei } v_{i}, w_{j} \in \\
V^{L O C^{\prime}} \text { - über die vorliegenden Typen } \\
\text { verbindet. }\end{array}$ \\
\hline
\end{tabular}

Auf Basis der in den Formeln (144) bis (148) zusätzlich gewonnenen Informationen zu den Verbindungsmöglichkeiten zwischen lokalen Knoten können verschiedene Typen von externen Lückenfolgen über einen Vergleich klassifiziert werden. Tabelle 31 beschreibt mögliche Typenevonbexternen-sückerfolgen, die 
im Gegensatz zu den Typen aus Tabelle 30 teilweise zugleich auftreten können und nicht sämtliche aus dem Vergleich denkbaren Kombinationsmöglichkeiten aus Gründen der Übersichtlichkeit beinhalten. Wie man sieht, stehen die Typen aus Tabelle 31 in einem hierarchischen Verhältnis zueinander, wobei das Ausmaß des Vorkommens von in der lokalen Struktur bereits bestehenden Kanten bzw. Knoten für die Rangfolge ausschlaggebend ist. Grundlage für die Klassifikation aller Wege zwischen zwei lokalen Knoten bilden die bereits vorgestellten Typen von externen Lücken für einen Weg $\omega$ mit Weglänge $p>1$ aus Tabelle 29.

Ebenso wie bei den internen indirekten Lücken können auch die ermittelten Typen von externen Lückenfolgen für eine oder mehrere aber auch für unterschiedliche Weglängen in einer Überblicksmatrix oder -liste dargestellt werden (Funktion DETEREXT). Eine solche Übersicht ist darüber hinaus auch für eine Kombination aus internen und externen Lücken möglich und sollte das Verhältnis zwischen den in Tabelle 30 und Tabelle 31 eingeführten Typen berücksichtigen (Funktion DETERINTEXT). Abbildung 13 gibt einen Einblick in die Rangfolge dieser Typen, an deren erster Stelle Folgen stehen, die ausschließlich bereits bestehende Kanten der lokalen Struktur beinhalten (potenzielle Performanzlückenfolgen). Wie schon für rein interne Lückenfolgen können darauf basierende Überblicksmatrizen erstellt werden, die zeigen, ab welcher Weglänge die jeweiligen Lückentypen ein Knotenpaar in Beziehung setzen (Funktion STAFETTE). Diese Überblicksmatrizen können auch als Kantenlisten GapLi bzw. GapLiTot dargestellt werden, wobei neben Ausgangs- und Zielknoten für jeden (internen) Lückentyp die kürzestmögliche Weglänge angegeben wird. Formel (149) spezifiziert die Kantenliste GapLi für interne Lückenfolgentypen, Formel (150) die Kantenliste GapLiTot für die gesamte Typenhierarchie. Für GapLi ist Cat wie in Formel (137) definiert. 


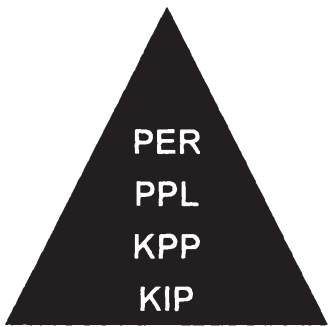

PIL

KPI

KIL

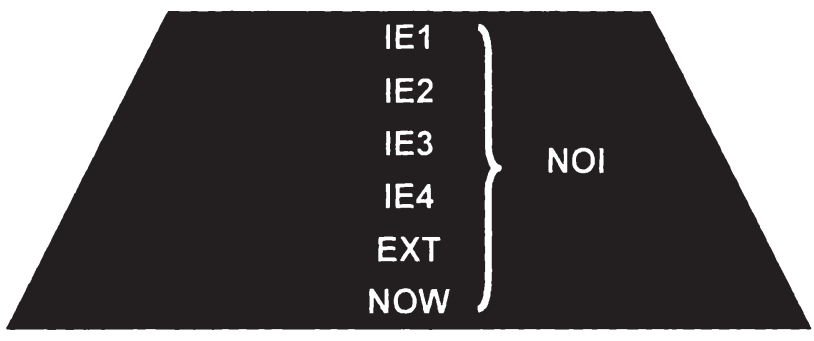

Abbildung 13: Hierarchie der Typen

$$
\begin{aligned}
& \operatorname{GapLi}=\left(v_{i}, w_{j}, \text { stafette }_{i j}^{\text {hierarch }}\right)_{i, j=1, \ldots, n} \\
& \text { wobei } \text { stafette }_{i j}^{\text {hierarch }}=\left(\text { stafette }_{i j}^{c a t_{k}}\right)_{k=1, \ldots, \mid}|c a t| \\
& \text { und } \text { stafette }_{i j}^{\text {cat }}{ }_{k}=\left\{\begin{array}{lc}
\left(\text { stafette }_{i j}\right)_{q} & {\text { wenn }\left(\text { stafette }_{i j}\right)_{q 1}=\text { cat }_{k}}, \\
\text { NA } & \begin{array}{c}
q=1, \ldots, \mid \text { stafette }_{i j} \mid \\
\text { ansonsten }
\end{array}
\end{array}\right. \\
& \text { GapLiTot }=\left(v_{i}, w_{j}, \text { stafette }_{i j}^{\text {hierarchTot }}\right)_{i, j=1, \ldots, n} \\
& \text { wobei } \text { stafette }_{i j}^{\text {hierarchTor }}=\left(\text { stafette }_{i j}^{\text {cattot }}\right)_{k=1, \ldots, \mid \text { cat }^{\text {tot }} \mid} \\
& \text { und } \text { stafette }_{i j}^{\text {cat tor }}=\left\{\begin{array}{lc}
\left(\text { stafette }_{i j}\right)_{q} & \text { wenn }\left(\text { stafette }_{i j}\right)_{q 1}=\text { cat tot }_{k}, \\
& q=1, \ldots ., \mid \text { stafette }_{i j} \mid \\
N A & \text { ansonsten }
\end{array}\right.
\end{aligned}
$$




$$
\begin{aligned}
C a t^{t o t}= & \left(\text { cat }{ }_{k}^{\text {tot }}\right)_{k=1, \ldots, 13} \\
= & \left(" P E R^{\prime \prime}, " P P L^{\prime},, " K P P^{\prime \prime},\right. \text { "KIP", "PIL", "KPI", "KIL", } \\
& \text { "IE1", "IE2", "IE3", "IE4", "EXT", "NOW") }
\end{aligned}
$$

\subsection{Deskriptive Auswertung der Lücken bzw. Lückenfolgen}

Eine Gegenüberstellung der Anzahl der verschiedenen Verbindungsmöglichkeiten bzw. -typen kann zusätzlich zu der Zusammenfassung der internen und externen Lückenfolgentypen bei unterschiedlichen Weglängen zu Analysezwecken erfolgen (Funktion TYPESTAT). Dies ermöglicht auch die Schaffung von Indikatoren für Vergleiche und für die Abschätzung des Erweiterungspotenzials. Abgesehen von der über MatValGap bereits identifizierten Anzahl an direkten internen Lücken $\left|G a p L i^{K I L I}\right|$ (Formel (152)) und der ohnedies offensichtlichen potenziellen Performanzlücken $\left|G a p L i^{P E R I}\right|$ (Formel (153)) kann eine solche Zählung bzw. Gegenüberstellung aufzeigen, wie viele und welche Knotenpaarungen indirekt primär, d.h. bei kürzester Distanz, über bestehende Verbindungen möglich wären $\left(\left|G a p L i^{\text {indirGroupl }}\right|\right.$ aus Formel (156) aufbauend auf Formel (154) und Formel (155)), wie groß die Zahl von Verbindungsmöglichkeiten ist, die sich primär über zusätzliche Kanten indirekt ergeben könnten, wenn die direkten internen Lücken geschlossen würden $\left(\mid\right.$ GapL $i^{\text {indirGroup } 2} \mid$ aus Formel (159)), wie viele und welche Knoten prinzipiell - womöglich bis zu einer bestimmten maximalen Weglänge (z.B. die Anzahl der Knoten minus 1) - über bestehende und/oder zusätzliche Verbindungen in Beziehung gesetzt werden können (|GapLindirGrouplv2 $\mid$ aus Formel (160)) und bei welchen dies nicht der Fall ist $\left(\mid\right.$ GapLi $^{\urcorner^{(G r o u p l \backslash G r o u p 2)} \mid}$ aus Formel (161)). Weiters kann ermittelt werden, inwieweit die Hinzunahme von externen Knoten eine Verbesserung der Erreichbarkeiten bewirkt $\left(\left|G_{\text {GapLi }}^{\text {Group3only }}\right|\right.$ aus Formel (163) aufbauend auf Formeln (161) und (162) mit Unterteilungsmöglichkeiten gemäß Formel (164) und Formel (166)) sowie wie viele dieser indirekten externen Verbindungen primäre komplette $\left(\left|G a p L i^{\text {completeExiprime }}\right|\right.$ aus Formel (167)) oder aber primäre partielle 
(|GapL $t^{\text {partialkxtprime }} \mid$ aus Formel (165)) externe Knotenfolgen darstellen, bzw. ob und wie viele primäre komplette (|GapLl ${ }^{\text {completeExtprime } 2} \mid$ aus Formel (169)) oder primäre partielle $\left(\left|G a p L i^{\text {partialExtprime2 }}\right|\right.$ aus Formel (168)) externe Knotenfolgen existieren, die allgemein kürzere indirekte Verbindungen zwischen lokalen Knoten bewirken sowie die Anzahl der Knotenpaare, die trotz des Einbezugs externer Knoten in keinerlei Zusammenhang stehen $\left(\left|G a p L i^{\text {no(iroup }}\right|\right.$ auf Basis von Formel (162)).

$$
\begin{aligned}
& \text { GapLi }^{\text {KILI }} \subset \text { GapLiTot } \\
& \text { GapLi }^{K L I L I}=\left\{\text { gaplitot }_{i j}: \text { stafette }_{i j} K I L "=(" K I L ", 1)\right\} \\
& \text { GapLi }{ }^{\text {PERI }} \subset \text { GapLiTot } \\
& \text { GapLi }^{\text {PERI }}=\left\{\text { gaplitot }_{i j}: \text { stafette }_{i j}^{\text {"PER" }}=(" P E R ", 1)\right\}
\end{aligned}
$$

GapLi Grouplonly $\subset$ GapLiTot GapLiGrouplonly $=\left\{\right.$ gaplitot $_{i j}$ :

$$
\begin{aligned}
& \mid\left\{\text { stafette }_{i j}^{t y p}: \text { typ } \in \text { Cat }^{\text {Groupl } 1} \wedge \text { stafette }_{i j}^{t y p} \neq N A\right\} \mid>0 \wedge \\
& \left.\mid\left\{\text { stafette }_{i j}^{t y p}: \text { typ } \in \text { Cat }^{\text {Group2 }} \wedge \text { stafette }_{i j}^{t y p} \neq N A\right\} \mid=0\right\}
\end{aligned}
$$

wobei Cat ${ }^{\text {Groupl }}=($ "PER", "PPL", "KPP", "KIP") und

$$
\text { CatGroup }=(" P I L ", \text { "KPI", "KIL") }
$$

GapLi ${ }^{\text {Grouplprime }} \subset$ GapLiTot

GapLi Grouplprime $=\left\{\right.$ gaplitot $_{i j}$ :

$$
\begin{aligned}
& \mid\left\{\text { stafette }_{i j}^{\text {typ }}: \text { typ } \in \text { Cat }^{\text {Groupl }} \wedge \text { stafette }_{i j}^{n p} \neq N A\right\} \mid>0 \wedge \\
& \mid\left\{\text { stafette }_{i j}^{\text {typ }}: \text { typ } \in \text { Cat }^{\text {Group2 }} \wedge \text { stafette }_{i j}^{\text {vp }} \neq N A\right\} \mid>0 \wedge
\end{aligned}
$$

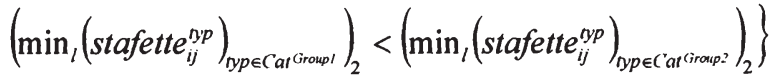

GapLindirGroupl $\subset$ GapLiTot

$$
G a p L i^{\text {indirGroupl }}=(\text { GapLi Grouplonly } \cup \text { GapLi Grouplprime }) \backslash G a p L i^{\text {PERI }}
$$




$$
\begin{aligned}
& \text { GapLi }^{\text {Group2only }} \subset \text { GapLiTot } \\
& \text { GapLi }^{\text {Group2only }}=\left\{\text { gaplitot }_{i j}:\right. \\
& \mid\left\{\text { stafette }_{i j}^{l y p}: \text { typ } \in \text { Cat }^{\text {iroup } 2} \wedge \text { stafette }_{i j}^{l y p} \neq N A\right\} \mid>0 \wedge \\
&\left.\mid\left\{\text { stafette }_{i j}^{t y p}: \text { typ } \in \text { Cat }^{\text {Groupl }} \wedge \text { stafette }_{i j}^{l y p} \neq N A\right\} \mid=0\right\}
\end{aligned}
$$

GapLi ${ }^{\text {Group2prime }} \subset$ GapLiTot

GapLiGroup2prime $=\left\{\right.$ gaplitot $_{i j}$ :

$$
\begin{aligned}
& \mid\left\{\text { stafette }_{i j}^{t p p}: \text { typ } \in \text { Cat }^{\text {Groupl }} \wedge \text { stafette }_{i j}^{t y p} \neq N A\right\} \mid>0 \wedge \\
& \mid\left\{\text { stafette }_{i j}^{t y p}: \text { typ } \in \text { Cat }^{\text {Group } 2} \wedge \text { stafette }_{i j}^{t y p} \neq N A\right\} \mid>0 \wedge
\end{aligned}
$$

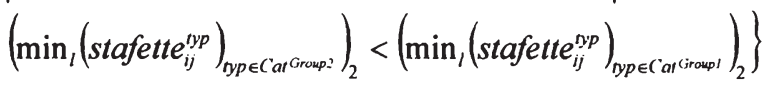

$$
\begin{aligned}
& G a p L i^{\text {indirGioup2 }} \subset G a p L i T o t \\
& G a p L i^{\text {indir(iroup2 }}=\left(G a p L i^{\text {Group2only }} \cup G a p L i^{\text {Group2prime }}\right) \backslash G a p L i^{K L L I}
\end{aligned}
$$

$$
\begin{aligned}
& G a p L i^{\text {indirGroup } / v 2} \subset G a p L i T o t \\
& G a p L i^{\text {indirGiroup } / v 2}=\left(G a p L i^{\text {indirGroup1 }} \cup G a p L i^{\text {indirGiroup2 }}\right)
\end{aligned}
$$

$$
\begin{aligned}
& \text { GapLi-(GrouplvGroup2) } \subset \text { GapLiTot } \\
& \text { GapLi-(GroupIVGroup2) }=\left\{\text { gaplitot }_{i j}\right. \text { : } \\
& \mid\left\{\text { stafette }_{i j}^{i p p}:\right.
\end{aligned}
$$

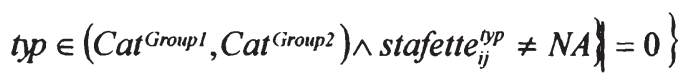

GapLinoGroup $\subset$ GapLiTot GapLinoGroup $^{\text {naplitot }}{ }_{i j}:$

$$
\begin{aligned}
& \mid\left\{\text { stafette }_{i j}^{n p}: \text { typ } \in\left(\text { Cat }^{\text {Groupl } 1}, \text { Cat }^{\text {Group } 2}, \text { Cat }^{\text {Group } 3}\right) \wedge\right. \\
& \text { stafette } \left.\left._{i j}^{\eta p p} \neq N A=0\right\} \mid\right\}
\end{aligned}
$$

wobei $\mathrm{Cat}^{\text {Group } 3}=$ ("IE1", "IE2", "IE3", "IE4", "EXT") 


$$
\begin{aligned}
& \text { GapLi } i^{\text {Group3only }} \subset \text { GapLiTot } \\
& \text { GapLi }^{\text {Group3only }}=G a p L i^{\neg(\text { Group } \backslash \text { Group2) }} \backslash G a p L i^{\text {no(iroup }}
\end{aligned}
$$

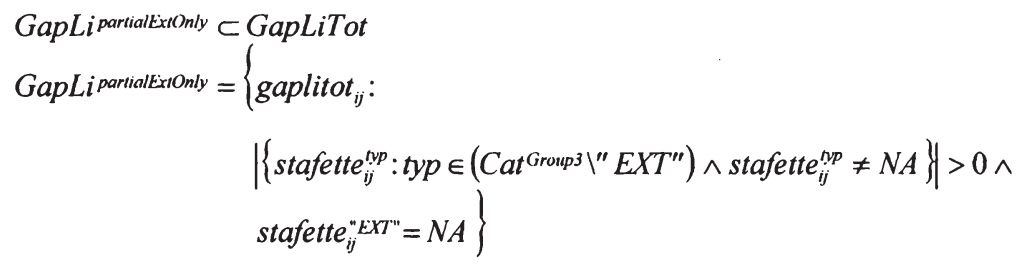

GapLiparrialExtPrime $\subset$ GapLiTot

GapLiparialExtPrime $=\left\{\right.$ gaplitot $_{i j}:$

$$
\begin{aligned}
& \mid\left\{\text { stafette }_{i j}^{i v p}: \text { typ } \in\left(\text { Cat }^{\text {Group } 3} \backslash " E X T^{\prime \prime}\right) \wedge \text { stafette }_{i j}^{n p p} \neq N A\right\} \mid>0 \wedge \\
& {\left[\text { stafette }_{i j}^{* E I T "}=N A \vee\right.} \\
& {\left[\text { stafette }_{i j}^{* \mathrm{E} I T "} \neq N A \wedge\right.}
\end{aligned}
$$

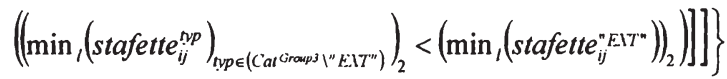

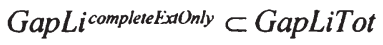

GapLicompleteExomly $=\left\{\right.$ gaplitot $_{i j}:$ stafette $_{i j} E^{\prime \prime \prime} \neq N A \wedge$

$$
\left.\mid\left\{\text { stafette }_{i j}^{i p p}: \text { typ } \in\left(\text { Cat }^{\text {Group } 3} \backslash^{\prime \prime} E X T^{\prime \prime}\right) \wedge \text { stafette }_{i j}^{i v p} \neq N A\right\} \mid=0\right\}
$$

GapLi completeErlPrime $\subset$ GapLiTot

GapLicompleteEx Prime $=\left\{\right.$ gaplitot $_{i j}:$ stafette $_{i j} E^{E I T "} \neq N A \wedge$

$$
\begin{aligned}
& {\left[\mid\left\{\text { stafette }_{i j}^{\text {tip }}: \text { typ } \in\left(\text { Cat }^{\text {Group }} \backslash^{\prime \prime} E X T^{\prime \prime}\right) \wedge \text { stafette }_{i j}^{\text {vp }} \neq N A\right\} \mid=0 \vee\right.} \\
& \left(\mid\left\{\text { stafette }_{i j}^{n p}: \text { typ } \in\left(\text { Cat }^{\text {Group } 3} \backslash " E X T^{\prime \prime}\right) \wedge \text { stafette }_{i j}^{N p p} \neq N A\right\} \mid>0 \wedge\right.
\end{aligned}
$$

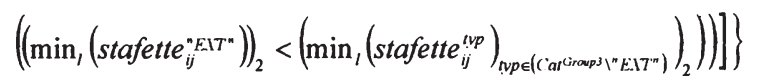


GapLi partialEriPrime? $\subset$ GapLiTot

GapLi partialkxtPrime $2^{2}=\left\{\right.$ gaplitot $_{i j}$ :

$$
\begin{aligned}
& \left\{\left\{\text { stafette }_{i j}^{t y p}: \text { typ } \in\left(\text { Cat }^{\text {to }} \backslash\left(" E X T^{\prime \prime}, " N O W^{\prime \prime}\right)\right) \wedge\right.\right. \\
& \text { stafette }_{i j}^{i p p} \neq N A \mid>0 \wedge \\
& {\left[\text { stafette }_{i j}^{\cdot{ }^{-E T T^{*}}}=N A \vee\right.} \\
& {\left[\text { stafette }_{i j}^{-E I 7^{\prime \prime}} \neq N A \wedge\right.}
\end{aligned}
$$

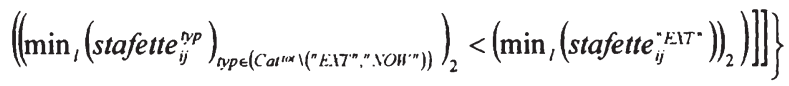

GapLicompleteExPrime2 $\subset$ GapLiTot

GapLi $^{\text {completef } ₫ \text { Prime2 }}=\left\{\right.$ gaplitot $_{i j}$ : stafette $_{i j}$ EXT" $\neq N A \wedge$

$$
\begin{aligned}
& {\left[\mid\left\{\text { stafette }_{i j}^{t p p}:\right.\right.} \\
& \text { typ } \left.\in\left(\text { Cat }^{t o t} \backslash\left(" E X T^{\prime \prime}, " N O W^{\prime \prime}\right)\right) \wedge \text { stafette }_{i j}^{i v p} \neq N A\right\}=0 \vee \\
& \left(\mid \text { stafette }_{i j}^{t y p}:\right. \\
& \text { typ } \left.\in\left(\text { Cat }^{t o t} \backslash\left(" E X T^{\prime \prime}, " N O W^{\prime \prime}\right)\right) \wedge \text { stafette }_{i j}^{t v p} \neq N A\right\}>0 \wedge \\
& \left(\left(\min ,\left(\text { stafette }_{i j} E x T^{\prime \prime}\right)\right)_{2}<\right. \\
& \left.\left.\left.\left.\left(\min ,\left(\operatorname{stafette}_{i j}^{i v p}\right)_{t v p \in\left(\left(\operatorname{ag} t^{\prime a x} \backslash\left({ }^{\prime \prime} E X T^{\prime \prime} . " N O W^{\prime \prime}\right)\right)\right.}\right)\right)\right)\right]\right\}
\end{aligned}
$$

\subsection{Kennzahlen zur Lückenstruktur}

Kennzahlen, die die Relationen zwischen den diversen Teilmengen wiedergeben, können auf Basis der ermittelten Kardinalitäten der Teilmengen (Formel (152) bis Formel (169)) gebildet werden. Zunächst sind der Anteil der lokal bestehenden Verbindungen (Formel (170)) und der lokal zusätzlich vorstellbaren Verbindungen (Formel (171)) an den gemäß der globalen Struktur allgemein mög- 
lichen Kanten zu ermitteln, um einen Überblick über das direkte Verbindungspotenzial zu schaffen.

$$
\begin{aligned}
\text { share }^{\text {PERI }} & =\frac{\left|G a p L i^{\text {PERI }}\right|}{\left|G a p L i^{P E R I}\right|+\left|G a p L i^{K L I}\right|} \\
\text { share }^{K I L I} & =\frac{\left|G a p L i^{K L I}\right|}{\left|G a p L i^{P E R I}\right|+\left|G a p L i^{K I I I}\right|}
\end{aligned}
$$

Daran anschließend können Kennzahlen zu den indirekten Wirkungsketten definiert werden, die einerseits Auskunft über den Anteil der in der lokalen Struktur bestehenden primären indirekten Relationen zwischen lokalen Knoten (Formel (172)) und andererseits über den Anteil der durch Schließen interner direkter Lücken ebenfalls auf kürzestmöglicher Distanz zusätzlich denkbaren indirekten Verbindungen (Formel (173)) an der Summe von bestehenden und zusätzlichen indirekten Beziehungen geben. Für eine Zusammenführung der Betrachtung von direkten und indirekten Anteilen können weiters die Zähler und Nenner aus Formeln (170) und (172) bzw. (171) und (173) addiert werden, um jeweils ein Gesamtanteilsmaß zu erhalten.

$$
\begin{aligned}
& \text { share }^{\text {indirGroupl }}=\frac{\left|G a p L i^{\text {indirGroupl } 1}\right|}{\left|G a p L i^{\text {indirGroupl } \mid}\right|+\left|G a p L i^{\text {indirGroup2 }}\right|} \\
& \text { share }^{\text {indirGroup2 }}=\frac{\left|G a p L i^{\text {indirGroup2 }}\right|}{\left|G a p L i^{\text {indirGroupl } 1}\right|+\mid G a p L i^{\text {indirGiroup2 } \mid}}
\end{aligned}
$$

In einem weiteren Schritt kann der relative Anteil jener Knotenpaare ermittelt werden, welche nur über den Einbezug externer Knoten indirekt in Beziehung gesetzt werden können (Formel (174)). Komplementär hierzu steht die Kennzahl aus Formel (175), die den Anteil der Knotenpaare berechnet, welche über bestehende und zusätzliche interne Kanten indirekt verbunden sind. Alternativ könnten auch direkte Verbindungen in die Kennzahlenermittlung miteinbezogen 
werden. Zur Feststellung des Anteils an Zusammenhängen, die nur über Hinzunahme von externen Knoten herstellbar sind, wären bspw. im Nenner der Formel (174) $\mid$ GapLi $^{K I L I} \mid$ sowie $\left|G a p L i^{P E R I}\right|$ aus den Formel (152) und (153) hinzuzuaddieren.

$$
\begin{aligned}
& \text { share }^{\text {indirGroup3 }}=\frac{\left|\operatorname{GapLi}^{\text {Group3only }}\right|}{\left|G a p L i^{\text {Group3only }}\right|+\left|G a p L i^{\text {indirGrouplV2 }}\right|} \\
& \text { share }^{\text {indirGroup } / \mathrm{v} 2}=\frac{\left|G a p L i^{\text {indirGroup } / \mathrm{v} 2}\right|}{\left|G a p L i^{\text {Group3only }}\right|+\left|G a p L i^{\text {indirGroup } / \mathrm{v} 2}\right|}
\end{aligned}
$$

Für die ausschließlich über externe Knotenfolgen verbindbaren Knoten können des Weiteren die Anteile der primären partiellen externen Knotenfolgen und der primären kompletten externen Knotenfolgen ermittelt werden, wie Formeln (176) und (177) aufzeigen. Dehnt man die Untersuchung der Wirkungsweise von externen Knotenfolgen auf alle indirekten Verbindungen aus, so ist zusätzlich ermittelbar wie hoch der Anteil der indirekten bestehenden und zusätzlich möglichen Verbindungen über den Einbezug externer Knoten bei kürzestmöglicher Weglänge in Zusammenhang gebracht werden können. Formel (178) und Formel (179) ermitteln diesen Indikator für primäre Verbindungsmöglichkeiten für partielle bzw. komplette Lückenfolgen. Zur Feststellung des gesamten Verkürzungspotenzials bezogen auf die Verbindungen über bestehende und zusätzlich mögliche Kanten ist die Summe der beiden letztgenannten Kennzahlen zu bilden.

$$
\begin{aligned}
& \text { share }^{\text {partialExtPrime }} \frac{\left|G a p L i^{\text {partialExiPrime }}\right|}{\mid G a p L i^{\text {Group3only } \mid}}
\end{aligned}
$$

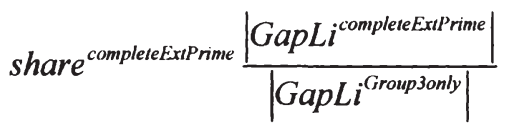




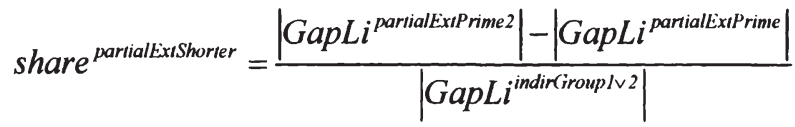

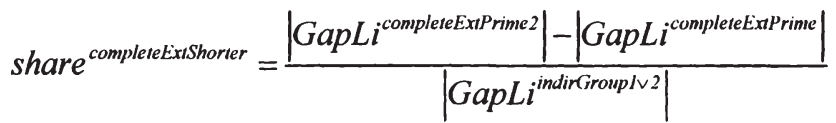

Schließlich kann auch der Anteil der nicht verbindbaren Knotenpaare (Formel (180)) bzw. komplementär hierzu der Anteil der Knotenpaare, die miteinander verflochten werden können oder es bereits sind, berechnet werden. Zu den ermittelten Anteilskennzahlen ist zu bemerken, dass sich diese vielfach zusätzlich auch jeweils als Anteil an der Kardinalität der Gesamtmenge |GapLiTot $\mid$ angeben lassen (Funktion TYPESTAT).

$$
\text { share }^{\text {noway }}=\frac{\mid \text { GapLi }}{\mid \text { no(iroup } \mid} \mid
$$

\subsection{Einsatz von Kantenlisten zur detaillierten Lückenbestimmung}

Ergänzend zu dem grundlegenden Überblick über das Auftreten von Lücken bzw. Lückenfolgen ist eine detaillierte Kenntnis der Zusammensetzung dieser Lücken(-folgen) erforderlich, um konkrete Maßnahmen zur Lückenschließung ergreifen zu können. Hierzu erscheinen Kantenlisten, wie in Formel (130) eingeführt, als ein idealer Ausgangspunkt. Zur detaillierten Lückenermittlung wie in Formel (183) für die Weglänge 2 beschrieben dient die Knotenmenge der lokalen Struktur $V^{L O C}$ als Ausgangs- und Zielknotenmenge, während die Knotenmenge der globalen Struktur $V^{G L O}$ die Menge der verbindenden Knoten bildet. Dementsprechend sind zur Vorbereitung von Formel (183) zwei spezielle Kantenlisten anzufertigen: eine Kantenliste, die von der Menge der lokalen Knoten ausgehend alle Kanten zu den Knoten der globalen Struktur enthält - wie in Formel (181) dargelegt - und eine zweite Kantenliste, die alle Kanten beinhaltet, die ausgehend von sämtlichen Knoten der globalen Struktur zu den Knoten der lokalen

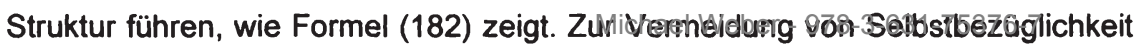


wären die Kanten in den Graphen der lokalen und globalen Struktur vor den Berechnungen entsprechend zu bereinigen. Die Ermittlung der Kantenlisten, die in den Formeln (181) bis (183) angeführt sind, erfolgt mit der im Anhang gelisteten Funktion STRUHO.

$$
\begin{aligned}
& \text { Edgeframe }_{\text {starLOC }}^{G L O}=\left(v_{i}, w_{j}, \text { matval }_{i j}^{G L O}\right)_{\substack{i=1, \ldots, n^{L L C} \\
j=1, \ldots, m^{(L L O O}}} \\
& v_{i} \in V^{L O C} \\
& w_{j} \in V^{(i L O)} \\
& \text { Edgeframe }_{\text {endLOC: }}^{G L O O}=\left(w_{h}, v_{k}, \text { matval }_{h k}^{G L O}\right)_{\substack{h=1, \ldots, m_{k}\left(\mathcal{L} L O \\
k=1, \ldots, n^{L O C}\right.}} \\
& w_{h} \in V^{G L O} \\
& v_{k} \in V^{L O C} \\
& E_{\text {dgeframe }}^{\text {oneho }}=\operatorname{struho}\left(\text { Edgeframe }_{\text {starl.OC }}^{G L O}, \text { Edgeframe }_{\text {endL } \mathcal{C O C}}^{(i L C)}\right) \\
& =\left(v_{i}, \text { matval }_{i j}{ }^{G L O}, w_{j=h}, \text { matval } l_{j k}^{G L O}, v_{k}\right)_{\substack{i, k=1, \ldots, \ldots \\
j, h=1, \ldots, m^{L I L C O}}} \\
& \left(v_{i}, w_{j}, \text { matval }_{i j}^{G L O}\right) \in \text { Edgeframe }_{\text {slariLOC }}^{G L O} \\
& \left(w_{j}, v_{k}, \text { matval }_{j k}^{G L O}\right) \in \text { Edgeframe }_{\text {endLCK: }}^{G L O}
\end{aligned}
$$

Das Ergebnis von Formel (183) ist eine Kantenliste, die Wege der Länge 2 mit dementsprechend 3 Knoten pro Listeneintrag aufweist. Die vermittelnden oder verbindenden Knoten dieser Kantenliste entstammen der Menge der globalen Knoten, die als Teilmenge die lokalen Knoten enthält. Für die Suche nach externen Lücken oder indirekten internen Lücken wäre diese Kantenliste daher zu allgemein und muss für spezifische Fragestellungen angepasst werden. Richtet sich die Fragestellung bspw. auf externe Lücken, d.h. eine verbindende Knotenmenge die keine Knoten enthält, die bereits in der lokalen Struktur vorkommen, so wäre - wie in Formel (184) gezeigt - eine diesbezügliche Auswahl zu treffen.

$$
\begin{aligned}
& \text { Edgeframe }_{e x i}^{o n e h o} \subset \text { Edgeframe oneho } \\
& \left.{\text { Edgeframe } e_{e x t}^{o n e h o}=\{\text { edgeframe }}_{i j k}^{o n e h o}: w_{j} \notin V \text { LOC }\right\}
\end{aligned}
$$


Sind hingegen interne indirekte Lücken zu eruieren, so wären nur verbindende Knoten von Interesse, die in der lokalen Struktur vorkommen. Bei Kantenlisten mit Weglängen von 2 dürfte zwischen den Ausgangs- und Vermittlungsknoten oder zwischen den Vermittlungs- und Zielknoten keine Verbindung in der lokalen Struktur bestehen, d.h. eine der beiden Kanten ist nicht in der Menge der lokalen Kanten $E^{L C C}$ enthalten oder aber beide Kanten sind nicht Teil von $E^{L O C}$. Im ersten Fall handelt es sich um eine partielle indirekte Lücke, im zweiten Fall würde man von einer kompletten indirekten Lücke sprechen. Die in Formel (185) definierte Bedingung bezieht sich auf sämtliche indirekte Lücken. Wollte man komplette indirekte Lücken finden, so wäre anstelle des logischen ODER $(v)$ zwischen den Kanten ein logisches UND $(\wedge)$ zu schreiben, wären hingegen partielle indirekte Lücken von Interesse, so müsste ein exklusives ODER (XOR) an diese Stelle in Formel (185) rücken.

$$
\begin{aligned}
& \text { Edgeframe }_{\text {indir }}^{\text {oneho }} \subset \text { Edgeframe oneho }^{\text {ond }} \\
& \text { Edgeframe }_{\text {indir }}^{\text {indir }}=\left\{\text { edgeframe }_{i j k}^{\text {oneho }}: w_{j} \in V^{L O C} \wedge\left(\left(v_{i}, w_{j}\right) \notin E^{L O C} \vee\left(w_{j}, v_{i}\right) \notin E^{L O C}\right)\right\}
\end{aligned}
$$

Einer gesonderten Betrachtung können Zyklen, d.h. Wege mit gleichem Ausgangs- und Zielknoten, oder Kreise, also - wie ebenfalls bereits bei der Einführung von Begriffen der Graphentheorie vorgestellt - Zyklen mit ausschließlich identischen Anfangs- und Zielknoten ohne weitere Redundanzen, unterzogen werden. Listeneinträge die Zyklen oder Kreise darstellen könnten je nach Fragestellung aus der Analyse ausgeschlossen oder besonders hervorgehoben bzw. bewertet werden. Dies gilt für die allgemeine Kantenliste ebenso wie für die Kantenliste der externen Lücken oder der indirekten Lücken. So zeigt bspw. ein Kreis - v.a. bei geringer Weglänge - einen relativ stärkeren Zusammenhang zwischen dem Ausgangs- oder Zielknoten und dem oder den jeweiligen Verbindungsknoten. Dies beeinflusst die Bedeutung einer externen oder einer indirekten internen Lücke. Formel (186) zeigt für die allgemeine Kantenliste von Weglänge 2 die Definition von Zyklen, die - im Falle des Ausschlusses von Selbstbezüglichkeit im zugrunde liegenden relationalen Datensatz - bei Weglänge 2 automatisch Kreise darstellen. 


$$
\begin{aligned}
& \text { Edgeframe }_{\text {circ }}^{\text {ono }} \subset \text { Edgeframe oneho } \\
& \text { Edgeframe }_{\text {circ }}^{\text {one }}=\{\text { edgeframe } \\
& \text { injk } \left._{\text {ono }}^{\text {od }}: v_{i}=v_{k}\right\}
\end{aligned}
$$

\subsection{Kantenlisten für Weglängen > 2}

Eine Identifikation von Lücken über eine Weglänge größer 2 kann über Kantenlisten erfolgen, die die bereits beschriebenen Ausgangs- und Zielkantenliste über weitere Kantenlisten der globale Struktur verknüpfen, wobei die Anzahl der verbindenden Kantenlisten von der angestrebten Weglänge abhängt. Ein Beispiel für die Weglänge 3 bietet Formel (187), die Redundanzen unter den verbindenden Knoten pro Listeneintrag ausschließt.

$$
\begin{aligned}
& \text { Edgeframe }^{\text {twoho }}=\operatorname{struho}\left(\text { Edgeframe }_{\text {slarloOC }}^{G L O}, \text { Edgeframe }^{G L O}, \text { Edgeframe }_{\text {endLOC }}^{G L O}\right)
\end{aligned}
$$

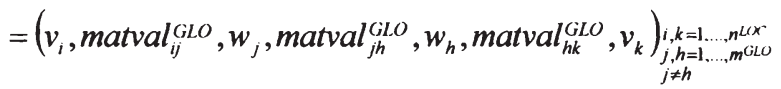

$$
\begin{aligned}
& \left(v_{i}, w_{j}, \text { matval }_{i j}^{G L O}\right) \in \text { Edgeframe }_{\text {start }}^{\text {GLO }} \text {.OC } \\
& \left(w_{j}, w_{h}, \text { matval }_{j h}^{G L O}\right) \in \text { Edgeframe }^{G L O} \\
& \left(w_{h}, v_{k}, \text { matval }_{j k}^{G I O}\right) \in \text { Edgeframe }_{\text {endLOC }}^{\text {GLO }}
\end{aligned}
$$

Zur Bestimmung von externen Lücken sind Einträge mit vermittelnden Knoten, die in der lokalen Struktur bereits enthalten sind, zu entfernen.

$$
\begin{aligned}
& \text { Edgeframe } e_{e x i}^{\text {wwoho }} \subset \text { Edgeframe } e^{\text {wwoho }} \\
& \text { Edgeframe }_{e x t}^{\text {nwoho }}=\left\{\text { edgeframe }_{i j h k}^{\text {nioho }}: w_{j}, w_{h} \notin V \text { LOC }\right\}
\end{aligned}
$$

Nur geringe Änderungen gibt es auch bei der Identifikation von internen indirekten Lücken. Formel (189) umfasst alle Arten von internen indirekten Lücken. Ein alleiniger Bezug auf komplette interne indirekte Lücken würde jeweils ein logisches UND $(\wedge)$ an die Stelle der logischen ODER $(v)$ in Formel (189) setzen, nachdem keine der drei Kanten in der lokalen Struktur existieren sollte. Für partielle indirekte Lücken, bei denen definitionsgemäß eine oder zwei der Kanten einer Kantenliste mit Weglänge 3 nicht vorhanden sein dürften, wäre die Bedingung aus Formel (190) anzuwenden. 


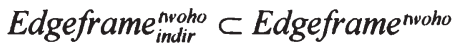

$$
\begin{aligned}
& \text { Edgeframe }_{i \text { indir }}^{\text {mwoho }}=\left\{\text { edgeframe }_{i j h k}^{\text {iwoho }}: w_{j}, w_{h} \in V^{L O C} \wedge\right. \\
& \left.\left(\left(v_{i}, w_{j}\right) \notin E^{L O C} \vee\left(w_{j}, w_{h}\right) \notin E^{L O C} \vee\left(w_{h}, v_{k}\right) \notin E^{L O C}\right)\right\} \\
& 0<\left|\left\{e_{x} \in E^{i j h k}: e_{x} \notin E^{L . O C}\right\}\right|<3 \quad \text { wobei } E^{i j h k}=\left\{\left(v_{i}, w_{j}\right),\left(w_{j}, w_{h}\right)\left(w_{h}, v_{k}\right)\right\}
\end{aligned}
$$

Zur Bestimmung von Zyklen und Kreisen gilt die Bedingung aus Formel (186) ebenso für Kantenlisten mit Weglänge 3. In den Fällen in denen Selbstbezüglichkeit eliminiert wurde, stellen sämtliche Zyklen Kreise dar, nachdem Redundanzen in Formel (187) ausgeschlossen werden.

Die deskriptive Analyse der Lücken bzw. Lückenfolgen kann auf Basis der detaillierten Kantenlisten durch einfache Häufigkeitstabellen bezogen auf bestimmte Rollen bzw. Positionen von Kanten und/oder Knoten in diesen Lücken(-folgen) erweitert werden (Funktionen EDGESTATONEHO und EDGESTATONEHOTAB). Dies gilt auch für detaillierte Kantenlisten die für disjunkte Knotenmengen generiert wurden.

Spezialfall: Erstellung von Kantenlisten für disjunkte Knotenmengen bei Weglänge $>1$

Will man für disjunkte Knotenmengen ebenfalls die indirekten internen sowie die externen Lücken erfassen, so bietet sich eine Vorgehensweise wie in Formeln (191) bis (196) beschrieben an. $V_{l}^{L C C}$ stellt sowohl die Ausgangsknotenmenge als auch die Zielknotenmenge eines Weges, der als Verbindungsknotenmengen eine alternierende Folge der Knotenmengen $V_{2}^{G L O}$ und $V_{1}^{G L O}$ aufweist, die mit $V_{2}{ }^{G L O O}$ endet. Den Ausgangspunkt für die Verknüpfung von Kantenlisten, die disjunkte Knotenmengen verbinden, zeigt Formel (191), den Zielpunkt Formel (192). Eine Kombination der unterschiedlichen Kantenlisten zur singulären Lückenidentifikation wird in Formel (193) demonstriert, dabei wird das Vorkommen der Ausgangs- und Zielknoten als Verbindungsknoten ausgeschlossen. Welche Knotenmenge die jeweilige Vorläufer- bzw. Nachfolgerknotenmenge bildet wird

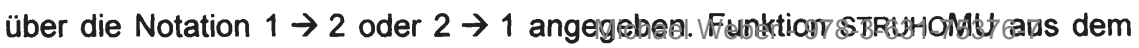


Anhang setzt die in Formeln (191) bis (193) angeführten Berechnungen um. Die Vorgehensweise kann auch für Weglängen $>2$ adaptiert werden.

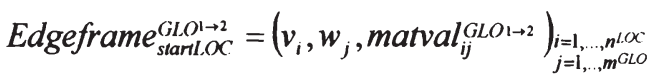

$$
\begin{aligned}
& v_{i} \in V_{1}^{L O S} \\
& w_{j} \in V_{2}^{G L O}
\end{aligned}
$$

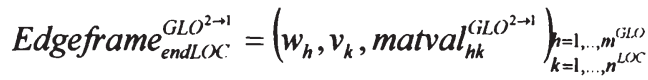

$$
\begin{aligned}
& w_{h} \in V_{2}^{G L O} \\
& v_{k} \in V_{1}^{L O C} \\
& \text { Edgeframe }_{\text {disjKM }}^{\text {oneho }}=\operatorname{struhoC}\left(\text { Edgeframe }_{\text {starl } L O C}^{(L L O 1 \rightarrow 2}, \text { Edgeframe }^{G L\left(O^{2 \rightarrow 1}\right.},\right. \\
& \text { Edgeframe } \left.^{G L O O^{\rightarrow 2}}, \text { Edgeframe }_{\text {endl.OC }}^{G L O 2 \rightarrow 1}\right) \\
& =\left(v_{i}, \text { matval }_{i j}^{G L O H \rightarrow 2}, w_{j}, \text { matval }_{j l}^{G L O O^{2 \rightarrow 1}}\right. \text {, }
\end{aligned}
$$

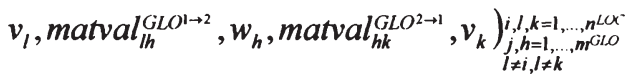

$$
\begin{aligned}
& \left(v_{i}, w_{j}, \text { matval }_{i j}^{G L O O^{\rightarrow 2}}\right) \in \text { Edgeframe }_{\text {stanLOC }}^{G L O 1 \rightarrow 2} \\
& \left(w_{j}, v_{l}, \text { matval }_{j l}^{G L O^{2 \rightarrow 1}}\right) \in \text { Edgeframe }^{G L O^{2 \rightarrow 1}} \\
& \left(v_{l}, w_{h}, \text { matval }_{l h}^{G L O^{1 \rightarrow 2}}\right) \in \text { Edgeframe }^{\text {GILOl } \rightarrow 2} \\
& \left(w_{h}, v_{k}, \text { matval }_{h k}^{G L O^{2 \rightarrow 1}}\right) \in \text { Edgeframe }_{\text {endl } L O C}^{G L O^{2 \rightarrow 1}}
\end{aligned}
$$

Externe Lücken können wie in Formel (194) beschrieben aus dem Ergebnis von Formel (193) gefiltert werden. Externe Lücken definieren sich hierbei als Verbindungsknoten, die aus der Differenzmenge von $V_{l}^{G L O}$ und $V_{l}{ }^{L O C}$ entstammen, d.h. folglich nicht in der lokalen Struktur vorhanden sind. Zusätzlich darf entweder der Vorläuferknoten oder der Nachfolgerknoten dieses Verbindungsknotens nicht in der lokalen Knotenmenge $V_{2}{ }^{L O C}$ enthalten sein.

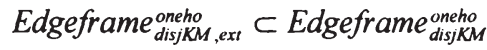

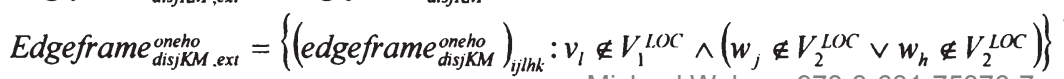


Formel (195) zeigt, wie interne indirekte Lücken in dem Ergebnis der Formel (193) gefunden werden können. Es gilt, dass diese Lücken auftreten, wenn maximal zwei der vier Kanten eines Weges auch in der lokalen Struktur vorkommen. Anders formuliert ist die Anzahl der Kanten, die nicht in der lokalen Struktur enthalten sind, größer oder gleich 2. $E^{i j h k}$ ist diesem Zusammenhang die Menge der Kanten eines bestimmten Weges aus der Ausgangsknotenmenge in die Zielknotenmenge. $E_{d i s j K M}^{L O C}$ ist die Menge aller Kanten der lokalen Struktur. Komplette interne indirekte Lücken haben die Eigenschaft, dass keine der Kanten in der lokalen Struktur vorzufinden ist. Trifft diese Eigenschaft nicht zu, so handelt es sich um partielle interne indirekte Lücken.

$$
\text { Edgeframe }_{\text {disjKM, indir }}^{\text {oneho }} \subset \text { Edgeframe } \text { disikM } K M^{\text {oneho }}
$$

$$
\begin{aligned}
& \text { Edgeframe } e_{\text {disjKM, indir }}^{\text {oneho }}=\left\{\left(\text { edgeframe }_{\text {disjKM }}^{\text {oneho }}\right)_{i j l h k}:\left\{\left\{e_{x} \in E^{i j h k}: e_{x} \notin E_{\text {disjKM }}^{L(O)}\right\} \mid \geq 2\right\}\right. \\
& \text { wobei } \\
& E^{i j h k}=\left\{\left(v_{i}, w_{j}\right)\left(w_{j}, v_{l}\right),\left(v_{l}, w_{h}\right),\left(w_{h}, v_{k}\right)\right\} \\
& E_{\text {disjKM }}^{L O C}=E^{L O C^{(1 \rightarrow 2}} \cup E^{L O C^{2 \rightarrow 1}}
\end{aligned}
$$

Wie auch in den vorangegangenen Verfahren zur Lückenidentifikation können bei zwei zusammenhängenden Graphen mit jeweils disjunkten Knotenmengen Zyklen bzw. Kreise durch deren Verknüpfung entstehen (Formel (196)). Diese sind abhängig von der Fragestellung besonders zu behandeln.

$$
\begin{aligned}
& \text { Edgeframe }_{\text {disjKM , circ }}^{\text {one }} \subset \text { Edgeframe }_{\text {disjKM }}^{\text {oneho }} \\
& \text { Edgeframe }_{\text {disjKM , irc }}^{\text {oneho }}=\left\{\left(\text { edgeframe } e_{\text {disjKM }}^{\text {oneho }}\right)_{i j h h k}: v_{i}=v_{k}\right\}
\end{aligned}
$$

\subsection{Graphentheoretische Vergleichskennzahlen}

Auf Basis der in der Strukturanalysephase behandelten graphentheoretischen Kennzahlen ist es möglich, spezielle Kennzahlen zum Vergleich der lokalen und globalen Struktur, also zweier gerichteter Graphen, die in einem hierarchischen Verhältnis zueinander stehen, zu entwickeln. Diese Maßzahlen dienen unter 
anderem zur Bestimmung von tatsächlichen Performanzlücken aus der Menge der potenziellen Performanzlücken, welche bislang alle Kanten der lokalen Struktur umfasste. Darüber hinaus eröffnen die Kennzahlen eine Möglichkeit, die identifizierten internen und externen Lücken sowie allgemein die Knoten der lokalen Struktur zu bewerten.

Während das herkömmliche Dichtemaß für gerichtete und ungerichtete Graphen die Gesamtzahl aller möglichen Kanten des Graphen auf Basis der Knotenanzahl als Dichtekriterium heranzieht, ist dieser Wert bei einem komparativen Dichtemaß $o_{\text {comp }}$ von der Anzahl der Kanten in dem übergeordneten Vergleichsgraphen abhängig. Dieses Dichtemaß ist der Quotient der Anzahl der gerichteten Kanten der lokalen Struktur und der Anzahl der gerichteten Kanten der globalen Struktur, die auf die Knotenmenge der lokalen Struktur reduziert wurde. Das komparative Dichtemaß $o_{c o m p}$ ist ein Indikator für das Ausmaß an direkten Lücken in der lokalen Struktur und beträgt maximal 1, wenn keine direkten internen Lücken existieren. Die Anzahl der direkten Lücken erhält man über die Subtraktion der Anzahl der Kanten in der lokalen Struktur von der Anzahl der Kanten der reduzierten globalen Struktur.

$$
o_{c o m p}=\frac{\sum_{i=1}^{n} \sum_{j=1}^{m} m a t_{i j}^{L O C}}{\sum_{i=1}^{n} \sum_{j=1}^{m} m a t_{i j}^{G L O r}}
$$

Ein komparatives Wertedichtemaß $o_{\text {comp }}^{V \mu *^{*}}$ kann in Anlehnung an das komparative Dichtemaß $o_{\text {comp }}$ formuliert werden. Die Grundlage für diese Kennzahl bildet ein verbessertes Wertedichtemaß $O^{V A L^{*}}$. Wie in Formel (198) beschrieben, entspricht dieses Wertedichtemaß dem durchschnittlichen Kantengewicht bezogen auf die Anzahl der gemäß der globalen Struktur lokal möglichen Kanten anstatt der über die Knotenanzahl theoretisch möglichen Kantenzahl. Zur Bestimmung des komparativen Wertedichtemaßes $o_{c o m p}^{V A *^{*}}$ wird der Quotient von $o^{V A L^{*}}$ und dem durchschnittlichen globalen Kantengewicht - abermals bezogen auf die Knotenmenge der lokalen Struktur - gebildet. Alternativ kann wie in Formel $(200)$ gezeigt, ein 
komparatives Wertedichtemaß $o_{\text {compl.E }}^{\text {VAL* }}$ in Bezug auf die Kantenmenge der lokalen Struktur berechnet werden. Hierfür ist der Bezugspunkt anstatt der auf die lokalen Knoten reduzierten Matrix für die globale Struktur MatVal ${ }^{\text {Glor }}$ die auf die lokalen Kanten reduzierte Matrix für die globale Struktur MatVal ${ }^{\text {iLOrLE }}$. Beide Maßzahlen sind nach oben durch 1 beschränkt.

$$
o^{V A L^{*}}=\frac{\sum_{i=1}^{n} \sum_{j=1}^{m} \operatorname{matval}_{i j}^{L O C}}{\sum_{i=1}^{n} \sum_{j=1}^{m} \operatorname{mat}_{i j}^{G L O r}}
$$

Das auf die lokale Knotenmenge abstellende komparative Wertedichtemaß $o_{\text {comp }}^{\text {VAL* }}$ aus Formel (199) kann auch als komparatives Gesamtgewichtemaß interpretiert werden. In dieser Interpretation quantifiziert die Maßzahl das Verhältnis aller lokalen Kantengewichte zu der Summe der globalen Kantengewichte bezogen auf die Knotenmenge der lokalen Struktur. Die Maßzahl $o_{\text {compLE }}^{\text {VAL* }}$, die im Gegensatz hierzu im Hinblick auf die Kantenmenge der lokalen Struktur berechnet wird, kann insbesondere zur Nivellierung der Kantengewichte der globalen Struktur eingesetzt werden, um bspw. die Kantengewichte der globalen Struktur zur Bewertung von Performanzlücken der lokalen Struktur heranziehen zu können.

$$
\begin{aligned}
& o_{c o m p}^{\text {VAL* }}=\frac{o^{\text {VAL* }}}{\frac{\sum_{i=1}^{n} \sum_{j=1}^{m} \operatorname{matval}_{i j}^{G L O r}}{\sum_{i=1}^{n} \sum_{j=1}^{m} \operatorname{mat}_{i j}^{\text {GLOr }}}}=\frac{\sum_{i=1}^{n} \sum_{j=1}^{m} \text { matval }_{i j}^{L(O C}}{\sum_{i=1}^{n} \sum_{j=1}^{m} \text { matval }_{i j}^{\text {GLOr }}} \\
& o_{c o m p L E}^{V A L^{*}}=\frac{\sum_{i=1}^{n} \sum_{j=1}^{m} \text { matval }_{i j}^{L O C}}{\sum_{i=1}^{n} \sum_{j=1}^{m} \operatorname{matval}_{i j}^{G L O r L E}}
\end{aligned}
$$

Die Kennzahlen können sowohl für Graphen mit disjunkten Knotenmengen $V_{I}$ und $V_{2}$ mit $\left|V_{l}\right|=n$ und $\left|V_{2}\right|=m$ als auch für Graphen mit einer Knotenmenge $V$ für Vorläufer- und Nachfolgerknoten (d.h. $V_{l}=V_{2}$ und $n=m$ ) anhand der angegebenen Formeln ermittelt werden. 
Zusammengefasst können die in den Formeln (197) bis (200) eingeführten Kennzahlen (Funktion GSTAT) als Indikatoren für das Verbesserungspotenzial der lokalen Struktur gesehen werden. Während das komparative Dichtemaß auf die direkten internen Lücken abzielt, gibt das komparative Wertedichtemaß eine Richtschnur für die Bewertung von Performanzlücken vor. 
Michael Weber - 978-3-631-75376-7

Downloaded from PubFactory at 01/11/2019 05:44:37AM

via free access 


\section{Lückenbewertungsphase}

Neben den zuvor behandelten allgemeinen Maßzahlen sind für weitere Bewertungsschritte Kennzahlen erforderlich, die zur Klassifikation von Lücken auf der Knoten- bzw. Kantenebene eingesetzt werden können. Nachstehend werden daher verschiedene Kennzahlen vorgestellt, die

- Performanzlücken bestimmen und gleichzeitig bewerten,

- direkte und indirekte interne Lücken gegeneinander abwägen und priorisieren sowie

- externe Lücken in ihrem potenziellen Beitrag für die lokale Struktur quantifizieren.

\subsection{Performanzlückenbestimmung und -bewertung}

Zur Auswahl und Reihung von tatsächlichen Performanzlücken aus der Menge der potenziellen Performanzlücken, die alle Kanten der lokalen Struktur enthält, können einfache und komplexe Verfahren unterschieden werden. Im Gegensatz zu einfachen Verfahren haben komplexe Verfahren den Vorteil, dass sie zusätzlich zu den direkten auch indirekte Effekte, die in einem Zusammenhang mit potenziellen Performanzlücken stehen, in die Kalkulation miteinbeziehen können.

\subsubsection{Einfache Bewertungsverfahren für Performanzlücken}

Ein simples Vorgehen zur Klassifikation der Kanten der lokalen Struktur wäre, die Gewichte der Kanten der globalen Struktur, die auch in der lokalen Struktur existieren, über das komparative Gesamtgewichtemaß zu nivellieren (Formel (201)). Dieses Vorgehen gewährleistet eine Vergleichbarkeit der lokalen und globalen Kantengewichte durch Reduktion der globalen Gewichte auf das lokale Niveau und bewirkt die Vorgabe eines Schwellenwerts für jede lokale Kante und deren Kantengewicht. Der Schwellenwert bestimmt bei dieser Methode ob der zu dem jeweiligen Schwellenwert gehörenden - Kante die Eigenschaft ,Performanzlücke' zugewiesen wird, wie in den Formeln (202) und (203) dargestellt 
(Funktion SIMPLEPERFGAP). Zur Berücksichtigung von möglicherweise angepeilten Wachstumszielen erscheint es sinnvoll, die Schwellenwerte um einen Steigerungsfaktor zu erhöhen, um den Spielraum für das Wachstum von Kantengewichten zu vergrößern. Ein alternatives, aber gleichbedeutendes Ermittlungsverfahren wäre die Division des lokalen Kantengewichts einer Kante mit dessen korrespondierendem globalen Kantengewicht und der Vergleich des Ergebnisses mit dem komparativen Gesamtgewichtemaß. Eine weitere Möglichkeit der Berechnung wäre, einen Quotient aus dem lokalen Kantengewicht mit dem korrespondierenden nivellierten globalen Kantengewicht zu bilden und mit einer zuvor für alle Kantengewichte festgelegten Schranke zu vergleichen. Der Vorteil dieses Ansatzes, die Möglichkeit einer flexiblen Schrankenbildung, unterliegt zugleich dem Vorwurf, dass die Schranke arbiträr zu setzen ist.

$$
\begin{aligned}
& \text { MatValGap }{ }^{\text {potPerf }}=(\text { matvalgap pooperf })_{\substack{i=1, \ldots, n \\
j=1, \ldots, m}} \\
& \text { matvalgap }_{i j}^{\text {porperf }}= \begin{cases}\mid \text { matvalgap }_{i j} \mid & \text { wenn matvalgap } \\
0 & \text { andernfalls }\end{cases} \\
& \text { MatValGap }{ }^{\text {porPerf, niv }}=\text { MatValGap }{ }^{\text {potPerf }} \cdot \delta_{\text {comp }}^{\text {VAl* }} \\
& \text { MatDist }=\left(\text { matdist } t_{i j}\right)_{\substack{i=1, \ldots, \ldots, j=1, \ldots, m}} \\
& \text { matdist }_{i j}=\text { matvalgap }_{i j}^{\text {potPerf }, n i v}-\text { matval }_{i j}^{L O C} \\
& \text { PerfGap }{ }^{\text {inary }}=\left(\text { perfgap }_{i j}^{\text {binan }}\right)_{\substack{i=1, \ldots, \ldots \\
j=1, \ldots, m}} \\
& \text { perfgap }_{i j}^{\text {binary }}= \begin{cases}1 & \text { wenn matdist } \\
i j & >0 \\
0 & \text { andernfalls }\end{cases}
\end{aligned}
$$

Die pauschale Nivellierung der zu den lokalen Kanten korrespondierenden globalen Kantengewichte mit dem komparativen Gesamtgewichtemaß, welches alle, nicht nur korrespondierende Kantengewichte der reduzierten globalen Struktur miteinbezieht, kann u.U. nicht erwünscht sein. In diesen Fällen wären alternative Nivellierungsschritte heranzuziehen. Eine weitere Möglichkeit wäre 
bspw. den Anteil der Summe der lokalen Kantengewichte eines Knoten - entweder bezogen auf dessen ausgehende Kanten oder auf dessen eingehende Kanten - an der korrespondierenden Summe der globalen Struktur als Nivellierungsmaß heranzuziehen. Erscheint die Summierung über alle entweder ausgehenden oder eingehenden Kantengewichte der reduzierten globalen Struktur weniger ratsam, so wäre als weitere Alternative nur die Summierung über die Kantengewichte der zu den lokalen Kanten korrespondierenden globalen Kantengewichte denkbar. Eine andere, simple Berechnungsmethode, welche keine Nivellierung erfordert, wäre die Division der Gewichtsanteile der lokalen Kantengewichte bezogen auf das lokale Gesamtgewicht mit den Gewichtsanteilen der korrespondierenden Kanten der globalen Struktur. Die lokalen Kanten würden bei dieser Methode auf Basis der jeweiligen Vergleichswerte über einen - arbiträr festzulegenden - Schwellenwert als Performanzlücke klassifiziert werden.

Eine Reihung der Performanzlücken erfolgt bei allen genannten Verfahren in Abhängigkeit der Abstände zwischen den Gewichten bzw. Gewichtsanteilen der als Performanzlücke klassifizierten Kanten der lokalen Struktur und der dazugehörigen Schranke. Zur differenzierteren Betrachtung oder auch zur Erhöhung der Übersichtlichkeit können Intervalle gebildet werden (Funktion SIMPLEPERFGAP). Ein Beispiel hierzu bietet Formel (204).

$$
\begin{aligned}
& \text { PerfGap }{ }^{\text {interval. } 3}=\left(\text { perfgap }_{i j}^{\text {interval. } 3}\right)_{\substack{i=1, \ldots, n \\
j=1, \ldots, m}} \\
& \text { perfgap }_{i j}^{\text {interval }, 3}=\left\{\begin{array}{ll}
1 & \text { wenn } 0<\text { matdist }_{i j} \leq t_{1} \\
2 & \text { wenn } t_{1}<\text { matdist }_{i j} \leq t_{2} \\
3 & \text { wenn matdist } t_{i j}>t_{2} \\
0 & \text { andernfalls }
\end{array} \quad \text { mit } 0<t_{1}<t_{2}\right.
\end{aligned}
$$

Sind die Kantengewichte der globalen oder etwa auch der lokalen Struktur normiert, d.h. die Summe aller Kanten die entweder in einen Vorgänger- oder Nachfolgerknoten fließen beträgt bspw. 1, so ändern sich die Bedingungen für den Vergleich und somit auch die Nivellierung. Eine spezielle Nivellierung für 
solche Anteilswerte - bspw. Gewichte von Kanten der globalen Struktur - kann durch eine abermalige spalten- bzw. zeilensummenbezogene Normierung dieser Gewichte bezogen auf zur lokalen Struktur korrespondierende Kanten erfolgen. Diese renormierten Gewichte sind dann mit Kantengewichten einer entsprechend normierten lokalen Struktur zu vergleichen. Eine allgemeine Vorgehensweise zur Bewertung von normierten Kanten zur Lückenbestimmung bieten komplexe Bewertungsverfahren, die im Folgenden erläutert werden.

\subsubsection{Komplexe Bewertungsverfahren für Performanzlücken}

Eine Normierung der Ausgangsdaten ermöglicht die Bestimmung der Bedeutung der Kanten analog zu der Bestimmung der Bedeutung der Knoten eines gerichteten und gewichteten Graphen, sofern die dazugehörige spaltennormierte Matrix $A$ bzw. die zeilennormierte Matrix $A^{*}$ quadratisch und nicht-zerlegbar ist. Die über Formel (97) bzw. Formel (98) errechenbaren Vektoren liefern gemäß der in dieser Arbeit bereits vorgestellten Interpretation die direkte und indirekte Bedeutung der Knoten bezogen auf die Vorgängerknoten, $s$, bzw. auf die Nachfolgerknoten, $z 2$. Dementsprechend zeigen die Zellwerte einer um die Einheitsmatrix verringerten Matrix $R^{*}$ die Bedeutung der direkten und indirekten Verbindungen zwischen dem Knoten $v_{j}$ und dessen Vorgänger $v_{i}$ für den Vorgänger, während die um die Einheitsmatrix verringerte Matrix $R$ die Bedeutung der direkten und indirekten Beziehungen zwischen dem Vorgängerknoten $v_{i}$ und dessen Nachfolger $v_{j}$ für den Nachfolger festhält. Bei der Wahl von $s 2$ und $z l$ als Bedeutungsvektoren wäre diese Interpretation entsprechend umzukehren.

Die Informationen über die unmittel- und mittelbare Bedeutung der Kanten kann nun auch zur Performanzlückenbestimmung unter zusätzlicher Berücksichtigung von indirekten Effekten eingesetzt werden, wenn sowohl die Matrix der zur lokalen Struktur korrespondierenden globalen Kantengewichte MatValGap potPerf als auch die Matrix $\mathrm{MatVal}^{L, C^{\prime}}$, die die Kantengewichte der lokalen Struktur

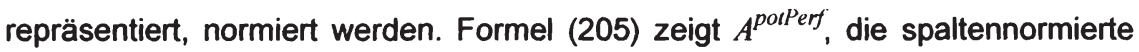

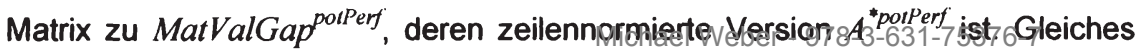


gilt für $A^{* L C C}$ und $A^{L O C}$, die aus MatVal ${ }^{L O C}$ generiert werden. Auf die Normierung folgend können die gemäß Formel (97) und Formel (98) ermittelten Inversen $R^{* \text { potPerf }}$ und $R^{* L O C}$ bzw. $R^{\text {potPerf }}$ und $R^{L O C}$ verglichen werden, von denen zuvor jeweils die Einheitsmatrix abzuziehen ist. Formel (206) und Formel (207) zeigen einen möglichen Vergleichsansatz, der im Ergebnis über die Matrix PerfGap ${ }^{\text {binany }}$ bzw. PerfGap ${ }^{*}$ inary anzeigt, ob eine Kante der lokalen Struktur unter zusätzlichem Einbezug indirekter Wirkungen eine Performanzlücke bezogen auf den Nachfolger- bzw. Vorgängerknoten darstellt. Alternativ könnten in Formel (206) auch eine Matrix $R^{- \text {potPerf }}$, die durch Addition von $R^{\text {potPerf }}$ mit $R^{* \text { potPerf }}$, und eine Matrix $R^{+L O C}$, die durch Addition von $R^{* I O C}$ mit $R^{L O C}$ entsteht, ebenfalls unter jeweiligem Abzug der Einheitsmatrix eingesetzt werden. Analog ändert sich das Superskript von Rate ${ }^{\text {potPerf }}$ und PerfGap ${ }^{\text {binary }}$. Dieses alternative Vorgehen würde eine Gegenüberstellung der Rolle der Kanten für Vorgänger- sowie Nachfolgerknoten in der lokalen Struktur mit der diesbezüglichen Rolle der Kanten in der korrespondierenden globalen Struktur ermöglichen. Ein Nachteil dieses Vorgehens wäre, dass im Ergebnis Performanzlücken weniger differenziert bestimmt würden, nachdem beide Richtungen, in denen die Kanten wirken bzw. für die die Kanten (mehr oder weniger) Bedeutung haben, kumuliert in die Betrachtung einfließen.

Der Vergleichsansatz in den Formeln (206) und (207) klassifiziert Kanten mit überproportional hoher Bedeutung in der lokalen Struktur ebenso wie Kanten mit gleich hoher Bedeutung in der Vergleichsstruktur nicht als Performanzlücken

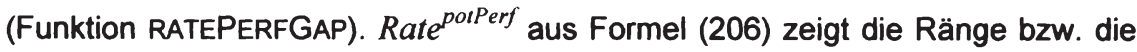
relative Bewertung der in der lokalen Struktur bestehenden Kanten.

$$
\begin{aligned}
& A^{\text {potPerf }}=\left(a_{i j}^{\text {potPerf }}\right)_{i, j=1, \ldots, n} \\
& a_{i j}^{\text {potPerf }}=\frac{\text { matvalgap potPerf }}{\sum_{i=1}^{n} \text { matvalgap potPerf }_{i j}^{\text {pot }}}
\end{aligned}
$$




$$
\begin{aligned}
& \text { Rate }^{\text {polPerf }}=\left(\text { rate }_{i j}^{\text {porPerf }}\right)_{i, j=1, \ldots, n} \\
& \text { rate }_{i j}^{\text {polPerf }}=r_{i j}^{\text {potPerf }}-r_{i j}^{L . O C} \\
& \operatorname{PerfGap}^{\text {binary }}=\left(\text { perfgap }_{i j}^{\text {binary }}\right)_{i, j=1, \ldots, n} \\
& \text { perfgap }_{i j}^{\text {binary }}= \begin{cases}1 & \text { wenn rate }_{i j}^{\text {potPerf }} \\
0 & \text { andernfalls }\end{cases}
\end{aligned}
$$

\subsection{Identifikation von schwachen Knoten}

Die Identifikation von schwachen Knoten, d.h. von Knoten, welche vergleichsweise stark mit Performanzlücken in direkter Verbindung stehen, bietet sich im Anschluss an die Performanzlückenbestimmung über nivellierte Absolutwerte oder Anteilswerte an. Diese Knoten sind mitunter in ursächlichen Zusammenhang mit dem Auftreten von Performanzlücken zu stellen und können nach deren Bestimmung als Ansatzpunkt für Verbesserungsmaßnahmen gewählt werden. Sie sind nicht mit isolierten Knoten zu verwechseln, welche keinerlei Verbindungen aufweisen und daher außerhalb dieser Betrachtung liegen. Ein Bestimmungsverfahren für schwache Knoten wird in den Formeln (208) und (209) vorgeschlagen, wobei der Vektor pfgNode ${ }^{I N}$ aus Formel (208) pro Knoten den gesamten Anteil der Performanzlücken, die zu dem jeweiligen Knoten führen, misst, während der Vektor pfgNode ${ }^{O U T}$ aus Formel (209) den Gesamtanteil jener Performanzlücken festhält, die pro Knoten ausgehen. Die Wahl von Rate ${ }^{* p o t P e r f}$ und PerfGap ${ }^{\text {*binary }}$ bzw. Rate potPerf $^{\text {und PerfGap }}{ }^{\text {binary }}$ in Formel (208) bzw. (209) ist auf die bereits erläuterte Bedeutungsfestlegung von Nachfolger- bzw. Vorgängerknoten, $s l$ aus Formel (97) bzw. $z 2$ aus Formel (98), zurückzuführen. Eine kombinierte Betrachtung der Anteile der Performanzlücken eines Knoten in beide Richtungen kann über eine Addition der Vektoren $p f g N o d e^{I N}$ und $p f g N o d e^{O U T} \mathrm{zu}$ pfgNode ${ }^{T O T}$ erfolgen. Das in den Formeln (208) und (209) vorgestellte Verfahren eignet sich nicht nur für Anteilswerte, sondern auch für (nivellierte) Absolutwerte. In einer solchen Berechnungsvariation müsste allerdings matdist $t_{i j}$ die Multi- 
plikation der Elemente der Matrizen Rate ${ }^{\text {potPerf }}$ und PerfGap ${ }^{* \text { binary }}$ bzw. Rate potPerf $^{\text {pzen }}$ und PerfGap ${ }^{\text {binary }}$ in Formel (208) bzw. (209) ersetzen, d.h. die Matrix MatDist wäre als Substitut heranzuziehen.

Nach der Generierung der erforderlichen Varianten von pfgNode kann die Bestimmung der schwachen Knoten über einen Schwellenwert erfolgen. Dieser Schwellenwert th könnte das 3. Quartil des zu betrachtenden Vektors der Performanzlückenanteile der Knoten oder auch eine andere Verteilungskennzahl sein. Die Bestimmung eines Vektors weakNode, der Auskunft über die Klassifikation als schwacher Knoten gibt, erfolgt in Formel (210) für den Vektor pfgNode $e^{I N}$ anhand des Schwellenwerts $t h$.

Die Zuordnung der Eigenschaft ,schwacher Knoten' ist ein Indikator für das Verbesserungspotenzial auf Knotenebene, welcher über die jeweilige Variante der Matrix Rate auch indirekte Effekte berücksichtigen kann. Für eine gesamtheitlichere Betrachtung der Knoten erscheint es zweckmäßig, diesen Indikator auch mit weiteren Kennzahlen der Knoten in der Rolle als Nachfolger oder Vorgänger zu verbinden. Ein Beispiel für eine solche Verknüpfung wäre die Relativierung des Vektors der Performanzlückenanteile der Knoten durch die Bedeutung der jeweiligen Knoten in der entsprechenden Rolle in der lokalen Struktur. Dieser Schritt erscheint bspw. dann empfehlenswert, wenn Knoten mit hohem Performanzlückenanteil und gleichzeitig geringer Bedeutung in der lokalen Struktur für Verbesserungsmaßnahmen priorisiert werden sollen. Ebenso könnte der Vektor der Performanzlückenanteile der Knoten mit dem Anteil der einzelnen Knoten an dem Gesamtvolumen (Absolutwerte) der lokalen Struktur gegenübergestellt werden, um Knoten mit geringem Anteil am Gesamtvolumen aber hohem Performanzlückenanteil in den Vordergrund zu stellen. Eine Demonstration für diesen Ansatz bieten die Formeln (211) und (212), wobei ein Wert $w h N o d e_{j}^{I N}>1$ als Indikator für einen zu priorisierenden Knoten $v_{j}$ gesehen werden kann. Die in den Formeln (208) bis (212) dargestellten Methoden werden in der Funktion WEAKNODES implementiert. 


$$
\begin{aligned}
& \operatorname{pfgNode}^{I N}=\left(\text { pfgnode }_{j}^{I N}\right)_{j=1, \ldots, n} \\
& \text { pfgnode }_{j}^{I N}=\frac{\sum_{i=1}^{n} \text { perfgap }_{i j}^{* \text { binary }} \cdot \text { rate }_{i j}^{* \text { polPerf }}}{\sum_{i, j=1}^{n} \operatorname{perfgap}_{i j}^{* \text { binary }} \cdot \text { rate }_{i j}^{* \text { potPerf }}} \\
& p f g N o d e^{\text {ouT }}=\left(\text { pfgnode }_{i}^{\text {ouT }}\right)_{i=1, \ldots, n} \\
& \text { pfgnode }_{i}^{O U T}=\frac{\sum_{j=1}^{n} \text { perfgap }_{i j}^{\text {binary }} \cdot \text { rate }_{i j}^{\text {potPerf }}}{\sum_{i, j=1}^{n} \text { perfgap }_{i j}^{\text {binary }} \cdot \text { rate }_{i j}^{\text {potPerf }}} \\
& \text { weakNode }^{I N}=\left(\text { weaknode }_{j}^{I N}\right)_{i=1, \ldots, n} \\
& \text { weaknode }_{j}^{I N}= \begin{cases}1 & \text { pfgnode } \\
0 & \text { andernfalls }\end{cases} \\
& \operatorname{shNode}^{I N}=\left(\operatorname{shnode}_{j}^{I N}\right)_{j=1, \ldots, n} \\
& \text { shnode }_{j}^{I N}=\frac{\sum_{i=1}^{n} \text { matval }_{i j}^{L O C}}{\sum_{i, j=1}^{n} \operatorname{matval}_{i j}^{L O C}} \\
& w_{\text {hNode }}^{I N}=\left(\text { whnode }_{j}^{I N}\right)_{j=1, \ldots, n} \\
& \text { whnode }_{j}^{I N}=\frac{\text { pfgnode }_{j}^{I N}}{\text { shnode }_{j}^{I N}}
\end{aligned}
$$

\subsection{Zusätzliche Überlegungen zur Bedeutungsbestimmung}

Die in den beiden nachfolgenden Subkapiteln angestellten Überlegungen zur Lückenbewertung stellen weitere Spezifizierungen der Berechnungsansätze im Rahmen der Lückenbewertung im Hinblick auf indirekte Wirkungsketten dar, die speziell die praktische Einsetzbarkeit der vorgestellten Metriken erhöhen.

\subsubsection{Wirkungen über die W-Reihe}

Das Verhältnis zwischen den direkten und sämtlichen indirekten Effekten, die die Bedeutungsbestimmung und in der Folge die Bewertung ausmachen, ist je nach 
Maßgabe des Verwendungszwecks anzupassen, nachdem bei bestimmten Anwendungen Effekte über längere Wegstrecken vergleichsweise noch stärker abgeschwächt oder aber auch verstärkt werden sollten. Darüber hinaus ist es auch möglich, nur die direkten Effekte in die Bewertung und den Vergleich einzubeziehen. Dies wird dadurch erreicht, dass in Formel (206) $R^{* \text { potPerf }}$ und $R^{*} L O C$ durch $A^{* \text { potPerf }}$ und $A^{* L O C}$ bzw. $R^{\text {potPerf }}$ und $R^{L O C}$ durch $A^{\text {potPerf }}$ und $A^{L O C}$ ersetzt werden. Ebenfalls können analog zur obigen Ausführung auch die Matrizen $A^{\text {-potPerf }}$ und $A^{+L O C}$ gebildet und in Formel (206) verwendet werden, um die direkte Bedeutung der Kanten in beide Richtungen - für deren jeweiligen Vorgänger- und Nachfolgerknoten - abzubilden. Sind hingegen die indirekten Effekte nur relativ schwächer oder stärker zu berücksichtigen, so ist die Einheitsmatrix in der Berechnung der Inversen mit einem entsprechenden Faktor größer bzw. kleiner $1 \mathrm{zu}$ multiplizieren. $\mathrm{Zu}$ bedenken bleibt bei einer solchen Weglängengewichtung nach Katz, dass für den Kantenvergleich von der Inversen auch die mit dem Faktor multiplizierte Einheitsmatrix abzuziehen ist, und nicht die Einheitsmatrix selbst. Eine weitere interessante Möglichkeit, die bei der allgemeinen Vorstellung der Variationen der Bewertung von Knoten aufgezeigt wurde, ist, indirekte Effekte nur in einem beschränkten Ausmaß miteinzubeziehen. Dies kann auch zusätzlich zu der Weglängengewichtung erfolgen. Mittels einer Schranke werden dabei die mittelbaren Wirkungen nur bis zu einer gewissen Weglänge begrenzt berücksichtigt, wobei sich die Frage stellt, welche Schranke zu wählen ist. Eine Variante wäre z.B. die maximale Pfadlänge als Schranke festzulegen und indirekte Effekte nur bis zu dieser speziellen Weglänge zusätzlich zu beachten. Dieses Vorgehen würde die multiplikativen Wirkungen - wie jedes andere beschränkende Verfahren - zwar eingrenzen, könnte aber sämtliche Hauptwirkungen ${ }^{62} z$ wischen den prinzipiell erreichbaren Knoten sowie gewisse Nebenwirkungen in die Berechnung einfließen lassen. Als eine weitere verfolgenswerte Variante könnten nur die indirekten Effekte der kürzesten

62 Zur konkreten Spezifikation von Haupt- und Nebenwirkungen siehe die Ausführungen zu Formel (214). 
Distanzen zu den anderen indirekt erreichbaren Knoten zusätzlich zu den direkten Wirkungen berücksichtigt werden. Diese Alternative würde Nebenwirkungen ausschließen und Hauptwirkungen nur bei ihrem erstmaligen Auftreten zulassen. Nachdem bei jeder Weglänge auch mehrere Wege von einem Knoten zu einem anderen führen können, wäre es bei diesem ebenso wie bei dem zuvor genannten Verfahren darüber hinaus zusätzlich möglich, entweder nur die durchschnittliche Wirkung der Weglänge bis zu der erstmaligen Erreichbarkeit oder aber die durchschnittliche Wirkung jeder Weglänge in das Ergebnis aufzunehmen. Des Weiteren ist es denkbar, bspw. ein Vielfaches der maximalen Pfadlänge als Schranke einzusetzen oder einen Schwellwert für die absolute Wirkungserhöhung pro Kante als Abbruchkriterium zu definieren.

Allgemein wäre die Matrix $W^{w /}$, die anstatt der modifizierten oder klassischen Leontief-Inverse für die lokale und globale Struktur in Formel (206) einzusetzen ist und Effekte bis zur Weglänge $w l$ festhält, gemäß Formel (213) zu berechnen (Funktion $\mathrm{WM}$ ). Zur Vermeidung des Einbezugs von zirkulären Wirkungen wird empfohlen, die mit $p$ potenzierte Matrix $A^{p}$ gemäß Formel (69) als $A^{p}$ (bzw. in Formel (218) als $A^{(p)}$ ) zu ermitteln. Dies gilt analog auch für Mat aus Formel (217) oder Formel (218).

$$
\begin{aligned}
W^{w l} & =\left(w_{i j}^{w l}\right)_{i, j=1, \ldots, n} \\
w_{i j}^{w l} & =\sum_{p=1}^{w l}\left(A^{p}\right)_{i j}
\end{aligned}
$$

Eine Beschränkung der Weglängen auf $n-1$ lässt sich demnach als

$$
W^{n-1}=\left(w_{i j}^{n-1}\right)_{i, j=1, \ldots, n}
$$

anschreiben. Die Matrix $W^{n-1}$ hält dabei alle Haupt-, jedoch nicht alle Nebenwirkungen fest. Als Hauptwirkungen werden dabei Effekte verstanden, die über einen Pfad auftreten, d.h. Effekte die nur bis zur maximalen Pfadlänge $n-1$ möglich sind und die über Wege zustande kommen, die keinen Knoten mehrmals

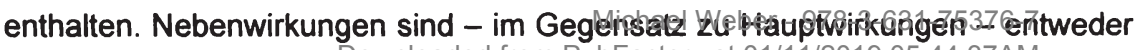


Effekte von Wegen, die als Zyklus oder - spezifischer - Kreis klassifiziert werden können, oder von Wegen, die zwar kein Zyklus sind, aber trotzdem Knotenpaare (Kanten) mehrmals enthalten und daher ebenfalls nicht als Pfad zu bezeichnen sind. Es ist hervorzuheben, dass auch Selbstbezüglichkeit, die selbstverstärkend wirken kann, im Kontext dieser Arbeit als Nebenwirkung zu sehen ist. Zyklen und folglich auch Kreise können ebenso wie Selbstbezüglichkeit durch den Einsatz von Formel (69) bei der Matrixpotenzierung einfach ausgeschlossen werden. Für die alleinige Identifikation von Hauptwirkungen erscheint die Erstellung von Kantenlisten die maximal bis zur Weglänge $n-1$ reichen und aus denen Einträge entfernt werden, die Knoten mehrfach enthalten, in der Durchführung vorteilhafter als eine Matrixberechnung.

Sind nur die indirekten und direkten Effekte bis zur ersten Erreichbarkeit der Knotenpaare einzubeziehen, so ist eine Matrix $W^{D I S T}$ zu bilden, die für jede Kante den Effekt bezogen auf die erstmalige Verbindung festhält. Dieser Effekt kann wie bereits ausgeführt - auf mehrere Wege gleicher Weglänge zurückzuführen sein. Die Ermittlung der Matrix $W^{\text {DIST }}$ basiert auf Formel (72), über die die Distanzmatrix DIST zur Ausgangsmatrix $A$ (bzw. $A^{*}$ ) berechnet werden kann. Die Elemente von DIST geben den Wert an, mit dem die Matrix $A$ (bzw. $A^{*}$ ) potenziert werden muss. Der Zellwert oder die Zellwerte der jeweiligen Ergebnismatrix $W^{p}$ die zu den Elementen von DIST korrespondieren sind daran anschließend für $W^{D I S T}$ zu entnehmen. Die genaue Definition von $W^{\text {DIST }}$ findet sich in Formel (215). Die Ermittlung der Matrix kann mittels der Funktion WDIST erfolgen.

$$
\begin{aligned}
& W^{D I S T}=\left(w_{i j}^{D I S T}\right)_{i, j=1, \ldots, n} \\
& w_{i j}^{D I S T}=\left(W^{d i t_{1}}\right)_{i j}
\end{aligned}
$$

Die Quantifizierung der durchschnittlichen erstmaligen Wirkung von Knoten aufeinander, d.h. die Berechnung des durchschnittlichen Kantengewichts bei erstmaliger Erreichbarkeit (kürzester Distanz) von Knotenpaaren, ist über die Matrix 
DISTc zur Anzahl der Wege, die die Knotenpaare auf kürzestem Pfad verbinden (Formel (73)), wie in Formel (216) beschrieben, möglich (Funktion WDIST).

$$
\begin{aligned}
& W^{D I S T, \text { aver }}=\left(w_{i j}^{\text {DIST,aver }}\right)_{i, j=1, \ldots, n} \\
& w_{i j}^{D I S T, a v e r}=\sum_{p=1}^{\text {dist }_{i j}} \frac{\left(A^{p}\right)_{i j}}{\operatorname{distc}_{i j}}=\frac{w_{i j}^{D I S T}}{\operatorname{distc}_{i j}}
\end{aligned}
$$

Verallgemeinert man diese Durchschnittsberechnung für mehrere Weglängen, so wäre einerseits (Formel (217)) eine Berechnung möglich, bei der pro Weglänge das Kantendurchschnittsgewicht durch Division der jeweiligen Potenz der Koeffizientenmatrix $A$ (bzw. $A^{*}$ ) mit der dazugehörigen Matrix der Weganzahl ermittelt (Formel (217) unter Verwendung der binären Adjazenzmatrix Mat) und daran anschließend aufsummiert wird. Andererseits wäre eine Variante denkbar, bei der die Berechnung der bis zur betrachteten Weglänge aufsummierten durchschnittlichen Kantengewichte pro Weglänge auf Basis der Durchschnittswertberechnung der vorangehenden Weglänge sowie der Matrix der Weganzahl für die aktuelle Weglänge erfolgt (Formel (218) und Formel (219)). Beide Berechnungsmethoden relativieren den Einfluss mehrerer Wege pro Weglänge, wobei bei der zweiten Vorgehensweise zusätzlich auch der Einfluss von mehrfachen Wegmöglichkeiten auf an den Weg anschließende Strecken ausgeschaltet werden kann. Die Umsetzung dieser beiden Methoden erfolgt durch die Funktion WW.

$$
\begin{aligned}
W^{w l, a v e r} & =\left(w_{i j}^{w l, a v e r}\right)_{i, j=1, \ldots, n} \\
w_{i j}^{w l, a v e r} & =\sum_{p=1}^{w l} \frac{\left(A^{p}\right)_{i j}}{\left(\operatorname{Mat}^{p}\right)_{i j}} \\
A^{\{1\}} & =\left(a_{i j}^{\{1\}}\right)_{i, j=1, \ldots, n} \\
a_{i j}^{\{1\}} & =a_{i j} \\
A^{\{p\}} & =\left(a_{i j}^{\{p\}}\right)_{i, j=1, \ldots, n} \\
a_{i j}^{\{p\}} & =\frac{\left(A^{\{p-1\}} A\right)_{i j}}{\left(\text { Mat }^{p}\right)_{i j}}
\end{aligned}
$$




$$
\begin{aligned}
W^{w l, a v e r 2} & =\left(w_{i j}^{w l, a v e r 2}\right)_{i, j=1, \ldots, n} \\
w_{i j}^{w l, a v e r ~} 2 & =\sum_{p=1}^{w l} A^{\{p\}}
\end{aligned}
$$

Soll die Unterschreitung eines Schwellwerts th die Schranke $b v(t h)$ bestimmen, die als Weglänge in Formel (213) dient, so ist wie in Formel (220) beschrieben vorzugehen (Funktion BVTH). Diese Berechnungsmethode erscheint besonders dann empfehlenswert, wenn die geometrische Reihe hinter der Leontief-Inverse (Potenzreihe, siehe Formel (99)), die entsprechend der bereits erläuterten Lösbarkeitsbedingungen konvergiert, für bestimmte Eingriffe, wie bspw. den Ausschluss von Zyklen gemäß Formel (69), abgeändert werden muss.

$$
\begin{aligned}
& W^{b v(t h)}=\left(w_{i j}^{b v(t h)}\right)_{i, j=1, \ldots, n} \\
& b v(t h)=\min _{p}\left\{p:\left|\left\{(i, j):\left(A^{p}\right)_{i j}<t h\right\}\right|=n^{2}\right\}
\end{aligned}
$$

\subsubsection{Matrizenzerlegung und Gewichtung von Subgraphen}

Eine Frage, die sich bei der Bedeutungsbestimmung und der vergleichenden Bewertung ergibt, ist, inwieweit bei nicht unilateral zusammenhängenden Graphen, d.h. Graphen die zerlegbare Matrizen ergeben, die Kanten- und folglich Knotenbedeutungen der unilateral zusammenhängenden Subgraphen so gewichtet werden können, dass alle Kanten und Knoten innerhalb des Graphen vergleichbar sind. Die Ermittlung der unilateral zusammenhängenden Subgraphen eines nicht unilateral zusammenhängenden Graphen kann dabei analog zu dem Cliquenidentifikationsansatz von Hubbell, der auf Seite 127ff. beschrieben wurde, erfolgen. Als Schranke $\Theta$ müsste hierbei 0 gewählt werden, wobei zuvor in Formel (100) das Minimum durch das Maximum zu ersetzen ist sowie in Formel (101) die Bedingung $m_{i j} \geq \Theta \mathrm{zu} m_{i j}>\Theta$ und die Bedingung $m_{i j}<\Theta \mathrm{zu} m_{i j} \leq \Theta$ umgeändert werden müssen. Es sei darauf hingewiesen, dass über diese Methode auch isolierte Knoten, d.h. Knoten, die in keiner Beziehung zu anderen Knoten stehen, als Subgraph bestimmt werden. Falls dies nicht erwünscht ist, 
können die isolierten Knoten über einen Filter eliminiert werden, wobei anzumerken ist, dass bei Ausschluss von Selbstbezüglichkeit der Wert der Kante ohnedies 0 beträgt. Die Ermittlung von Subgraphen kann über die Funktion COMPKLIK erfolgen.

Eine triviale Vorgehensweise zur Subgraphengewichtung wäre nun, die relative Knoten- oder aber auch die relative Kantenanzahl der Subgraphen gemessen am gesamten Graphen als Gewichtungsfaktor heranzuziehen. Nachdem diese beiden Varianten allerdings keine Wertrelationen zwischen den Subgraphen berücksichtigen, erscheint eine Gewichtung anhand von Wertrelationen - bezogen auf die Absolutwerte der lokalen bzw. globalen Struktur - vorteilhafter, da dadurch eine feinere Differenzierung zwischen den Subgraphen möglich wird. In diesem Zusammenhang ist zu betonen, dass bei einem Mangel an Absolutwerten für die globale Struktur eine Gewichtung aufgrund der Wertrelationen der lokalen Struktur argumentierbar wäre. Für jene Fälle, in denen die Größe des Subgraphen (gemessen an der Kantenanzahl) weniger relevant zur Bestimmung der Bedeutung einer Kante oder eines Knoten ist, wäre die Summe der Absolutwerte der Kanten des Subgraphen durch die jeweilige Kantenanzahl zu dividieren und danach erst der Anteil zu bestimmen. Eine von Absolutwerten unabhängige Subgraphengewichtung kann des Weiteren von der Leontief-Inverse der Subgraphen auf Basis der dazugehörigen Koeffizientenmatrix $A^{*}$ bzw. $A$ (abzüglich der Einheitsmatrix) ausgehen (Funktion SMATWEIGHTING). Bei diesem alleinig auf Anteilswerte abstellenden Ansatz würde die Summe der Inverse des Subgraphen als Anteil an der Gesamtsumme aller Inversen der Subgraphen die Gewichtung bestimmen. Eine vom Verwendungszweck abhängige Variationsmöglichkeit hierzu ist, die Anteile über die Addition der um die Einheitsmatrix reduzierten Leontief-Inverse für $A^{*}$ und $A$ des Subgraphen zu errechnen. Als Merkmal dieses auf Anteilen basierenden Gewichtungsansatzes ist die Höhergewichtung von möglichst eng verflochtenen Subgraphen mit verhältnismäßig hoher Knotenanzahl zu nennen. Zur Dämpfung des Einflusses der Größe des Subgraphen könnten die Werte der Inverse noch durch die Kantenzahl des Subgraphen dividiert werden. Will man die Verflochtenheit des Subgraphen_weniger stars-berücksichtigen, so 
ist eine Gewichtung über Absolutwertanteile oder auch eine Gewichtung über die relative Kantenzahl zu wählen. Bei einer Gewichtung über die durch die jeweilige Kantenanzahl relativierten Absolutwertanteile werden stärker verflochtene bzw. größere Subgraphen verhältnismäßig abgeschwächt. Bei der Entscheidung für die Knotenzahl als Gewichtungsmaß würden die Verflechtungen gänzlich unberücksichtigt bleiben und größere Graphen gestärkt werden.

\subsection{Bewertung von direkten internen Lücken}

Die Bewertung und Rangreihung von direkten internen Lücken, d.h. von Kanten, die grundsätzlich in der lokalen Struktur existieren könnten, orientiert sich an der Dringlichkeit der Einbettung der direkten internen Lücke in die lokale Struktur und somit an der Bedeutung der direkten internen Lücke für die Kanten und folglich auch für die Knoten der lokalen Struktur. Es sei hierbei betont, dass aufgrund der Fragestellung nach den erwarteten Effekten einer Hinzunahme einer noch nicht vorhandenen Kante in die lokale Struktur nicht auf indirekte interne Lücken abgestellt wird, die Bewertungsmethode aber sehr wohl die Rolle von direkten internen Lücken in Weglängen $>1$ einbeziehen können sollte. Für das Bewertungsverfahren wird daher vorgeschlagen, eine Bedeutung der direkten internen Lücken bezogen auf die Kanten der lokalen Struktur so zu ermitteln, dass auch die möglichen Sog- und Folgewirkungen des Schließens der direkten internen Lücke auf die lokale Struktur für eine bestimmte oder unendliche Weglänge berücksichtigt werden können. Dementsprechend definiert der $\mathrm{Be}$ rechnungsansatz aus Formel (221) die diesbezügliche Bedeutung einer direkten internen Lücke $e_{i j}=\left(v_{i}, v_{j}\right)$ für unendlich lange Weglängen als Summe der Bedeutung des Knoten $v_{i}$ für seine direkten Vorgänger und sämtliche indirekten Vorgänger und der Bedeutung des Knoten $v_{j}$ für dessen unmittelbare und mittelbare Nachfolger. Die Ergebnismatrix Rate ${ }^{I I L}$ gibt schließlich die gesuchte Bedeutung der direkten internen Lücken an. Wie auch bereits bei den vorangegangenen Bedeutungsbestimmungsmethoden betont, können $W^{*} L C^{\prime}$ bzW. $W^{L O C}$ für bestimmte Weglängen sowie $A^{* L C C}$ bzw. $A^{L O C}$ für Weglänge 1 statt 
$R^{* L O C}$ bzw. $R^{L O C}$ (jeweils abzüglich der Einheitsmatrix) in Formel (221) eingesetzt werden. Des Weiteren wurden in Formel (221) die Diagonalelemente der Matrix Rate $^{D I L}$ zur Demonstration der allgemeinen Gültigkeit der Herangehensweise nicht entfernt. Nachdem diese allerdings für die Bewertung von direkten internen Lücken verzerrend wirken können, sollten die Diagonalelemente gemeinhin aus Rate $^{\text {DIL }}$ eliminiert werden.

$$
\begin{aligned}
& \text { Rate }^{D L L}=\left(\text { rate }_{i j}^{D L L}\right)_{i, j=1, \ldots, n} \\
& \text { rate }_{i j}^{D L L}= \begin{cases}\sum_{k=1}^{n} r_{k i}^{* L O C}+\sum_{l=1}^{n} r_{j l}^{L O C} & \text { wenn matvalgap } \\
0 & \text { ansonsten }\end{cases}
\end{aligned}
$$

Eine Enweiterung dieses Berechnungsansatzes wäre, die Bedeutung der direkten internen Lücken ausschließlich im Hinblick auf Performanzlückenfolgen sowie Folgen, die Performanzlücken enthalten, zu bestimmen. Eine solche Berechnung erscheint vorteilhaft, nachdem die Bedeutung der Einbettung zusätzlicher Kanten in die lokale Struktur für bestehende Performanzlücken - bei längeren Wegstrecken für reine und gemischte Performanzlückenfolgen - besonders relevant ist und daher hervorgehoben werden sollte. Aufbauend auf MatVal ${ }^{L O C \text {, }}$ Perf , einer Matrix, die die gewichteten Kanten der lokalen Struktur als Anteile oder Absolutwerte enthält und über Formel (222) definiert wird, kann die Ermittlung dieser speziellen Bedeutung erfolgen.

$$
\begin{aligned}
\text { MatVal }^{L O C, \neg P e r f} & =\left(\text { matval }_{i j}^{L O C, \neg P \text { erf }}\right)_{i, j=1, . ., n} \\
\text { matval }_{i j}^{L, O C, \neg \text { Perf }} & = \begin{cases}0 & \text { wenn perfgap } \\
\text { matval }_{i j}^{L O C} & \text { ansonsten }\end{cases}
\end{aligned}
$$

Hierbei wird zunächst durch Zeilen- bzw. Spaltennormierung von MatVal ${ }^{L O C,- \text { Perf }}$ die Matrix $A^{* L O C, \neg \text { Perf }} \mathrm{bzw}$. die Matrix $A^{L O C, \neg \text { Perf }}$ berechnet. Abhängig von der zu betrachtenden maximalen Wirkungskette können anschließend $A^{* L C C},-$ Perf und $A^{L O C, \sim P e r f}$ oder aufbauend auf diesen Matrizeel entspreghend 
gestellten Berechnungsmethoden $R^{* L O C,-P \text { erf }}$ bzw. $R^{L(\mathcal{C},- \text { Perf }}$ oder aber $W^{* L O C,- \text { Perf }}$ bzw. $W^{L O C, P P e r f} z$ ur Berechnung einer Matrix Rate ${ }^{\text {DLL, }, \text { Perf }}$ analog zu Formel (221) verwendet werden. Während Rate ${ }^{D I L}$ grundsätzlich die Bedeutung der direkten internen Lücken für alle Performanzlücken bzw. reinen oder gemischten Performanzlückenfolgen bereits inkludiert, gibt Rate ${ }^{D I L, \neg P e r f}$ Auskunft über die Bedeutung der direkten internen Lücken ausschließlich für alle ,Nicht-Performanzlücken' bzw. Folgen, die nur aus ,Nicht-Performanzlücken' bestehen. Zur Feststellung der Bedeutung der direkten internen Lücken für reine oder gemischte Performanzlückenfolgen bzw. Performanzlücken Rate ${ }^{D I L 2}$ ist nun einfach die Differenz der Matrizen Rate ${ }^{\text {DIL }}$ und Rate $e^{\text {IIL, }- \text { Perf }}$ zu bilden (Formel (223)). Das Auftreten von negativen Werten ist bei der Differenzbildung nicht auszuschließen und auf die Verletzung der hinter dieser Berechnung liegenden Additivitätsannahme zurückzuführen. Negative Werte ermöglichen keine Aussagen über die Bedeutung der direkten internen Lücken und können daher durch Nullsetzen ausgeblendet werden. Die Berechnung von Rate ${ }^{D I L}$ und Rate ${ }^{D I L 2}$ erfolgt mittels der Funktion RATEDIGAP.

$$
\begin{aligned}
& \text { Rate }^{\text {DLL2 }}=\left(\text { rate }_{i j}^{D L L 2}\right)_{i, j=1, \ldots, n} \\
& \text { rate }_{i j}^{D L L 2}=\text { rate }_{i j}^{D L L}-\text { rate }_{i j}^{D L,, \sim P e r f}
\end{aligned}
$$

Für jene Fälle, in denen die Bedeutung der direkten internen Lücken nur zusätzlich, nicht aber ausschließlich durch die Bedeutung dieser für Performanzlücken bzw. reinen und gemischten Performanzlückenfolgen bestimmt werden soll, wird empfohlen, die Matrizen Rate $e^{D l L}$ und Rate ${ }^{D I L 2}$ (u.U. gewichtet) zu addieren. Eine weitere Möglichkeit zur Bewertung der direkten internen Lücken eröffnet sich, wenn für die Lücken Absolutbeträge in der globalen Struktur bekannt sind. Diese Beträge können dann als Anteil aller zu den direkten internen Lücken korrespondierenden Kanten der globalen Struktur als Gewichtung für die Kantenbedeutung der direkten internen Lücken für die lokale Struktur eingesetzt werden. 
Will man über eine Matrix DiGap ${ }^{\text {binary }}$ jene direkten internen Lücken identifizieren, die aufgrund ihrer verhältnismäßig hohen Bedeutung vorrangig zu behandeln sind, so kann dies durch den Vergleich der Matrix Rate ${ }^{D I L}$ (oder Rate ${ }^{I I L 2}$ ) mit einem Schwellwert th erreicht werden (Formel (224); Funktion RATEDIGAP). Der konkrete Wert dieser Schranke th kann über Verteilungskennzahlen des Bedeutungsmaßes wie z.B. dem Median oder dem 3. Quartil bestimmt werden. Dies entspräche dann einer Auswahl der wichtigsten 50\% bzw. 25\% der direkten internen Lücken.

$$
\begin{aligned}
& \operatorname{DiGap}^{\text {binary }}=\left(\text { digap }_{i j}^{\text {binary }}\right)_{i, j=1, \ldots, n}
\end{aligned}
$$

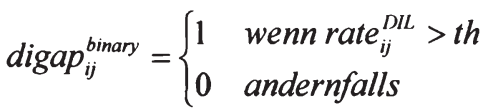

Soll ein Zielwert für jede der direkten internen Lücken definiert werden, so bietet sich für dessen Wahl entweder ein nivellierter Absolutwert aus dem Graph der globalen Struktur (soweit ein solcher existiert) oder - je nachdem ob der Bezugspunkt der Nachfolger- oder Vorgängerknoten (bzw. dessen Volumen) sein soll der entsprechende Anteilswert aus der auf die Knoten der lokalen Struktur reduzierten zeilen- bzw. spaltennormierten Matrizen der globalen Struktur $A^{*}$ GLOr bzW. $A^{G L O r}$ an. Die erforderlichen relativen Erhöhungen der Volumina des Nachfolger- bzw. Vorgängerknotens, d.h. deren Inputs bzw. Outputs, können aus der Matrix $\Delta V o l^{D I L}$ bzw. $\Delta V o l^{* D I L}$ (basierend auf $A^{G L O r}$ bzw. $A^{* G L O r}$ wie in Formel (225)) abgelesen werden (ebenfalls Funktion RATEDIGAP). Diese Interpretation gilt nur solange der Nachfolger- bzw. Vorgängerknoten in der lokalen Struktur bereits ein positives Input- bzw. Outputvolumen vorweisen kann. Andernfalls sind die Werte ein weiterer Indikator für die relative Bedeutung der Schließung der Lücke für den Nachfolger- bzw. Vorgängerknoten. In jenen Fällen, in denen nur eine Kante in der reduzierten globalen Struktur zu dem Nachfolgerknoten hin$\left(\Delta V o l^{I I L}\right)$ bzw. von dem Vorgängerknoten wegführt $\left(\Delta V o l^{*} D I L\right)$, die gleichzeitig eine direkte interne Lücke darstellt, ist der Zielwert unbestimmt. Die beiden

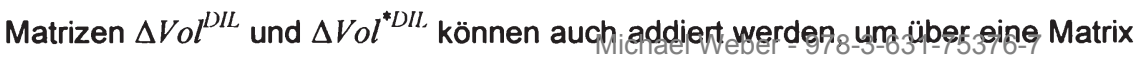


$\Delta \mathrm{Vol}^{+D I L}$ die Kanten mit dem höchsten Input- und Outputsteigerungserfordernis der dazugehörigen Nachfolger- und Vorgängerknoten bezogen auf die lokale Struktur auszuweisen. Über diese Matrix könnten bspw. die bedeutendsten direkten internen Lücken der lokalen Struktur mit dem geringsten oder höchsten (maximalen) lokalen Input- und Outputänderungsbedarf identifiziert werden. Für die Bestimmung solcher Knoten mit ausschließlichem Bezug auf den (maximalen) Input- bzw. Outputänderungsbedarf sind $\Delta V o l^{D I L}$ bzw. $\Delta V o l^{*} D L L$ heranzuziehen.

$$
\begin{aligned}
& \Delta \operatorname{Vol}^{\text {Dll }}=\left(\Delta \operatorname{vol}_{i j}^{D L L}\right)_{i, j=1, \ldots, n} \\
& \Delta v \operatorname{vol}_{i j}^{\text {DIL }}= \begin{cases}\frac{a_{i j}^{G L O r}}{\left(1-a_{i j}^{G L O r}\right)} & \text { wenn digap } p_{i j}^{\text {binary }}=1 \\
0 & \text { ansonsten }\end{cases}
\end{aligned}
$$

Eine Variation der Formel (221), die den Zielwert, d.h. den global vorgegebenen Anteil der zu bewertenden Kante, ausgerichtet auf den Input des Nachfolgerknotens bzw. den Output des Vorgängerknotens, in die Bedeutungsbestimmung miteinbezieht, bietet Formel (226) an. Dieser erweiterte Bestimmungsansatz gewichtet die in die Matrix der Kantenbewertungen der direkten internen Lücken Rate $^{D I L}$ einfließenden Bedeutungen der Start- und Endknoten der Kanten zusätzlich mittels des entsprechenden Zielwertes. Dies ermöglicht, dass die maximalen Zielwerte die Relationen der Kantenbedeutungen wesentlich beeinflussen und über diesen Ansatz mitberücksichtigt werden können. Ebenso wie bei den vorangegangenen Formeln können auch in Formel (226) anstatt der - eigentlich um die Einheitsmatrix zu verringernden - Matrizen $R^{* L C C}$ bzw. $R^{L O C}$ je nach Anwendung auch $A^{* L O C}$ und $A^{L O C}$ bzw. $W^{* L C C}$ und $W^{L(C)}$ eingesetzt werden. 


$$
\begin{aligned}
& \text { Rate }^{D L L, w}=\left(\text { rate }_{i j}^{D L L, w}\right)_{i, j=1, \ldots, n} \\
& \text { rate }_{i j}^{D L l, w}= \begin{cases}\left(a_{i j}^{G L O r} \cdot \sum_{k=1}^{n} r_{k i}^{* L O C}\right)+\left(a_{i j}^{* G L O r} \cdot \sum_{l=1}^{n} r_{j l}^{L O C}\right) & \text { wenn matvalgap } p_{i j}>0 \\
0 & \text { ansonsten }\end{cases}
\end{aligned}
$$

Eine rechnerisch aufwendigere Alternative zu der Bedeutungsbestimmungsmethode aus Formel (221), die sich auch in Formel (226) wieder findet, ist der Vergleich der Gesamtbedeutung der lokalen Struktur mit und ohne der zu bewertenden Kante unter Einbezug der angestrebten Gewichtung. Die Formeln (227), (228) und (229) stellen dieses alternative, gewissermaßen ,exaktere' Verfahren dar, welches die Bedeutung der Kante $e_{o p}^{D I l}$ über die Differenz der beiden Gesamtbedeutungen festlegt, wobei das Kantengewicht eins zu eins aus der auf die Knotenmenge der lokalen Struktur reduzierten und zeilen- bzw. spaltennormierten globalen Struktur entnommen wird (Funktion RATEEDILOP bzW. für alle direkten internen Lücken Funktion RATEDIGAP). Neben der Bedeutungsbestimmung für eine direkte interne Lücke eröffnet dieses Verfahren die Möglichkeit, die Bewertung von Wechselwirkungen von mehreren direkten internen Lücken zur Bestimmung der optimalen Kombination der in die lokale Struktur aufzunehmenden Kanten durchzuführen. Wechselwirkungen würden dabei über einen Vergleich der Bedeutungswerte für die Summe der Einzelbewertungen der direkten internen Lücken mit dem Wert aus der kombinierten Berechnung identifiziert und gereiht werden können. In Formel (229) können auch $A^{* L O C, e D I L-o p}$, $A^{L O C \text {,eDIL-op }}, A^{* I O C}$ und $A^{L O C}$ bzW. $W^{*} L O C, e D I L-o p, W^{L O C, e D I L-o p}, W^{* L O C}$ und $W^{L O C}$ die Matrizen $R^{* L O C, e D I L-o p}, R^{L, O C, e D I L-o p}, R^{* I O C}$ und $R^{L(C)}$ - von denen prinzipiell die Einheitsmatrix abzuziehen wäre - ersetzen, um nur bestimmte Effekte in die Bewertung einzubeziehen. 


$$
\begin{aligned}
& A^{* L O C, e D L L-o p}=\left(a_{i j}^{* L O C, e D I L-o p}\right)_{i, j=1, \ldots, n} \text { für } e_{o p}^{D I L}=\left(v_{o}, v_{p}\right) \text { mit matvalgap }{ }_{o p}>0
\end{aligned}
$$

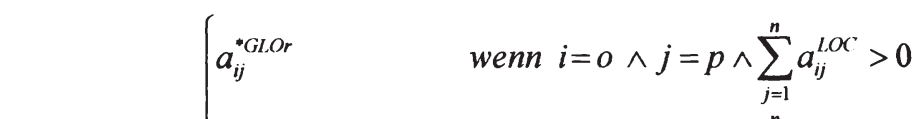

$$
\begin{aligned}
& a_{i j}^{*} L O C, e D I L-o p= \begin{cases}1 & \text { wenn } i=o \wedge j=p \wedge \sum_{j=1}^{n} a_{i j}^{L O C}=0 \\
a_{i j} L O C & \left(1-a_{i k}\right)\end{cases} \\
& a_{i j}^{* L O C} \cdot\left(1-a_{i k}^{\text {GLOr }}\right) \text { wenn } i=o \wedge k=p \\
& a_{i j}^{* L O C} \quad \text { wenn } i \neq o \wedge j \neq p \\
& A^{L O C, e D I L-o p}=\left(a_{i j}^{L O C, e D I L-o p}\right)_{i, j=1, \ldots, n} \text { für } e_{o p}^{D I I .}=\left(v_{o}, v_{p}\right) \text { mit matvalgap } p_{o p}>0 \\
& \left\{a_{i j}^{\text {GLOr }} \quad \text { wenn } i=o \wedge j=p \wedge \sum_{i=1}^{n} a_{i j}^{L O C}>0\right. \\
& a_{i j}^{L O C, e D I L-o p}= \begin{cases}1 \quad \text { wenn } i=o \wedge j=p \wedge \sum_{i=1}^{n} a_{i j}^{L O C}=0 \\
a_{j}^{L O C} \cdot\left(1-a_{k j}\right)\end{cases} \\
& a_{i j}^{L O C} \cdot\left(1-a_{k j}^{G L O r}\right) \text { wenn } i=o \wedge k=p \\
& a_{i j}^{L O C} \quad \text { wenn } i \neq o \wedge j \neq p \\
& \operatorname{rate}^{e D I L-o p}=\left(\sum_{i=1}^{n} \sum_{j=1}^{n} r_{i j}^{* L O C, e D I L-o p}+\sum_{j=1}^{n} \sum_{i=1}^{n} r_{i j}^{L O C, e D I L-o p}\right)-\left(\sum_{i=1}^{n} \sum_{j=1}^{n} r_{i j}^{* L O C}+\sum_{j=1}^{n} \sum_{i=1}^{n} r_{i j}^{L O C}\right)
\end{aligned}
$$

Es ist darauf hinzuweisen, dass die Berechnung gemäß Formel (229) auch negative Werte ergeben kann. Dieser Fall tritt ein, wenn der zusätzliche Effekt der normierten Einbettung einer Kante in die Verflechtungsstruktur geringer ist als der relative Verlust der Bedeutung der bereits bestehenden Kanten. Speziell gilt dies für Konstellationen in denen die zusätzliche Kante Nachfolger- bzw. Vorgängerknoten mit geringer Bedeutung verbindet bzw. in denen keine weiteren Verbindungen von den Nachfolger- oder zu den Vorgängerknoten bestehen. Geringe und negative Werte von rate $e^{e D L-o p}$ deuten demgemäß auf eine Verknüpfung der zusätzlichen Kante insbesondere mit ,schwachen Knoten' hin. Daraus resultiert eine geringere Multiplikatorwirkung, die u.U. auch zu einem leicht negativen Gesamteffekt der zusätzlichenałantefühten ksan:7Abhäningig von 
der gewählten Strategie für den Umgang mit schwachen Knoten können auch Kanten mit geringem bzw. leicht negativem Gesamteffekt von Interesse sein. Im Allgemeinen sind jedoch Kanten mit hohen Werten von rate ${ }^{e D l L-o p}$ von besonderer Relevanz für die Intensivierung der Austauschbeziehungen.

\subsection{Bewertung und Zielwerte für externe Lücken}

Eine auf die lokale Struktur (mitunter auch einschließlich ausgewählter oder sämtlicher direkten Lücken) bezogene Bewertung und Rangreihung von externen Lücken kann über ein Bedeutungsbestimmungsverfahren, wie in Formel (230) und Formel (231) gezeigt, realisiert werden. Ausgangsbasis bilden dabei die zeilen- bzw. spaltennormierten Matrizen zur nicht reduzierten globalen Struktur $A^{*}(G L O)$ bzw. $A^{G L O}$, aus denen bestimmte Elemente ohne abermalige Normierung zur Gewichtung der Knotenbedeutung bezogen auf die lokale Struktur eingesetzt werden. Das Beibehalten der ursprünglichen Normierung erscheint hierbei insofern vorteilhaft, als die externen Knoten bzw. Kanten in ihrer relativen Bedeutung als Vorgänger und/oder Nachfolger für die internen Knoten bzw. Kanten differenziert werden können, was bei einer zeilen- bzw. spaltenweisen Normierung der Gewichte von Kanten, die zwischen externen und internen Knoten verlaufen, nicht möglich wäre. Wie bei den vorangegangenen Formeln können auch in Formel (230) anstatt der um die Einheitsmatrix reduzierten Matrizen $R^{* L O C}$ und $R^{L O C}$ abhängig von der Bewertungsstrategie $A^{* L O C}$ und $A^{L O C}$ oder $W^{* L C}$ und $W^{L O C}$ verwendet werden.

$$
\operatorname{Rate}^{E X T, w}=\left(\text { rate }_{x y}^{E X T, w}\right)_{x, y=1, \ldots, z}
$$

wobei $v_{x}, v_{y} \in V^{G L O}, V^{L O C} \subset V^{G L O}, V^{E X T}=V^{G L O} \backslash V^{L O C},\left|V^{G L O}\right|=z>n=\left|V^{L O C}\right|(230)$

$$
\text { rate }_{x y}^{E X T, w}= \begin{cases}a_{x y}^{G L O} \cdot \sum_{k=1}^{n} r_{l x}^{* L O C} & \text { wenn } v_{x} \in V^{L O C} \wedge v_{y} \in V^{E X T} \\ a_{x y}^{* G L O} \cdot \sum_{l=1}^{n} r_{y l}^{L O C} & \text { wenn } v_{y} \in V^{L O C} \wedge v_{x} \in V^{E X T} \\ 0 & \text { ansonsten }\end{cases}
$$


Ausgehend von der Matrix Rate ${ }^{E X T, w}$ kann schließlich durch Summierung über die zu den jeweiligen externen Knoten gehörigen Zeilen und Spalten die relative Bedeutung der externen Knoten für die lokale Struktur aus rate ${ }^{E X T v, w}$ abgelesen werden (Funktion RATEEXTV,W). Eine Reihung der externen Knoten kann gemäß den Werten aus dem Vektor rate ${ }^{E X T v, w}$ erfolgen.

$$
\begin{aligned}
& \operatorname{rate}^{E X T v, w}=\left(\operatorname{rate}_{x}^{E X T v, w}\right)_{v_{x} \in V^{E I T}} \\
& \operatorname{rate}_{x}^{E X T v, w}=\sum_{y=1}^{z} \operatorname{rate}_{x y}^{E X T, w}+\sum_{y=1}^{z} \operatorname{rate}_{y x}^{E X T, w}
\end{aligned}
$$

Eine umfassendere Bedeutungsbestimmung für externe Knoten kann im Gegensatz zu der in den Formeln (230) und (231) vorgeschlagenen Vorgehensweise aus einem Vergleich der Gesamtbedeutung der lokalen Struktur mit der Gesamtbedeutung einer um den jeweils zu bewertenden externen Knoten erweiterten lokalen Struktur resultieren. In einem solchen exakteren, aber auch rechnerisch aufwendigeren Bedeutungsermittlungsverfahren, würde die Bedeutung des externen Knoten für die lokale Struktur über die Differenz der beiden Gesamtbedeutungen festgelegt werden. Die Gewichte der Kanten aus der globalen Struktur würden hierzu in einem ersten Schritt bezogen auf die - um den betrachteten externen Knoten erweiterten - Knoten der lokalen Struktur (Formel (232)) spalten- bzw. zeilennormiert werden $\left(A^{G L O r, E X P-x}, A^{* G L O r, E X P-x}\right)$, bevor die Zeilen und Spalten des betrachteten externen Knoten an die zur Normierung passenden erweiterten Matrizen $A^{* I O C, E X P-x}$ und $A^{L O C, E X P-x}$ angefügt werden. Nicht zu vergessen ist dabei, dass auch die Normierung der aus $A^{* L O C}$ und $A^{L O C}$ übernommenen Zellwerte durch die Erweiterung angepasst werden muss. Dementsprechend werden die übernommenen Zellwerte mit einem entsprechenden Faktor multipliziert, um eine einheitliche Zeilen- bzw. Spaltennormierung herzustellen. Daran anschließend werden - bei Bedeutungsbestimmung über eine unendliche Weglänge - die Spaltensummen von $R^{* L O C}$ und die Zeilensummen von $R^{L O C}$ sowie die Spaltensummen von $R^{* L O C, E X P-x}$ und die Zeilensummen von $R^{L O C . E X P-x}$ addiert. Die Differenz dieser beiden Summen (,Gesamtbedeutungen') 
ergeben die Bedeutung des betrachteten externen Knoten. Die Einheitsmatrizen sollten von den Inversen abgezogen werden. Davon kann allerdings auch abgesehen werden, nachdem dadurch das Ergebnis nicht verzerit wird. Die Erweiterungsmöglichkeiten dieses in den Formeln (233), (234) und (235) dargestellten Berechnungsverfahrens (Funktionen RATEEXTV und RATEEXTVLIST) sind vielfältig. So wäre es denkbar, mittels dieser Berechnung Effekte von mehreren Knoten auf die lokale Struktur zu quantifizieren. Will man bspw. die Wechselwirkungen mehrerer externer Knoten auf die Bedeutung messen, so kann zusätzlich zu den Bedeutungen der jeweiligen externen Knoten auch der Gesamtbedeutungsunterschied zu einer, um mehrere externe Knoten erweiterten, lokalen Struktur mit der Summe der Bedeutungen der zu betrachtenden Knoten verglichen werden. Über mehrere Rechenschritte könnte schließlich die ideale Kombination von externen Knoten für die lokale Struktur gefunden werden.

$$
\begin{aligned}
& \text { MatVal GLOr,EXP-x }=\left(\text { matval }_{i j}^{(\mathcal{G L O O}, E X P-x}\right)_{i, j=1, \ldots, n+1} \quad \text { für } v_{x} \in V^{E X T} \\
& \operatorname{matval}_{i j}^{G L O r, L X P-x}=\operatorname{matval}_{r c}^{G L O} \quad \text { wenn }\left(v_{i}=v_{r} \in V^{L O C} \wedge v_{j}=v_{s} \in V^{L O C}\right) \\
& \vee\left(v_{i}=v_{x} \in V^{E X I} \wedge v_{j}=v_{s} \in V^{L O C}\right) \\
& \vee\left(v_{i}=v_{r} \in V^{L O C} \wedge v_{j}=v_{x} \in V^{E X T}\right) \\
& A^{* L O C, E X P-x}=\left(a_{i j}^{* L O C, E X P-x}\right)_{i, j=1, \ldots, n+1} \text { für } v_{x} \in V^{E X T}
\end{aligned}
$$

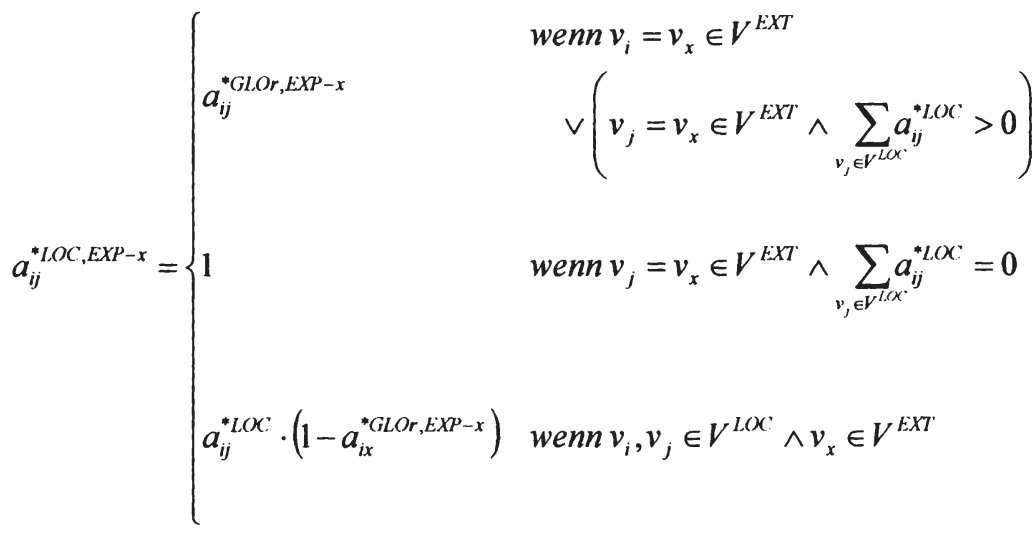




$$
\begin{aligned}
& A^{L O C, E X P-x}=\left(a_{i j}^{L O C, E X P-x}\right)_{i, j=1, \ldots, n+1} \quad \text { für } v_{x} \in V^{E X T} \\
& \int a_{i j}^{G L O r, E X P_{-x}} \quad \boldsymbol{w e n n}\left(v_{i}=v_{x} \in V^{E X T} \wedge \sum_{v_{i} \in V^{L O C}} a_{i j}^{L O C}>0\right) \\
& \vee v_{j}=v_{x} \in V^{E X T} \\
& a_{i j}^{L O C, E X P-x}=\left\{\begin{array}{l}
1 \quad v_{i}=v_{x} \in V^{E X T} \wedge \sum_{v_{i} \in V^{L O C}} a_{i j}^{L O C}=0
\end{array}\right. \\
& a_{i j}^{L O C} \cdot\left(1-a_{x j}^{G L O r, E X P-x}\right) \text { wenn } v_{i}, v_{j} \in V^{L O C} \wedge v_{x} \in V^{E X T} \\
& \operatorname{rate}^{E X T v}=\left(\text { rate }_{x}^{E X T v}\right)_{v_{x} \in V^{E T T}} \\
& \operatorname{rate}_{x}^{E X T v}=\left(\sum_{i=1}^{n+1} \sum_{j=1}^{n+1} r_{i j}^{{ }^{*} L O C, E X P-x}+\sum_{j=1}^{n+1} \sum_{i=1}^{n+1} r_{i j}^{L O C, E X P-x}\right)-\left(\sum_{i=1}^{n} \sum_{j=1}^{n} r_{i j}^{* L O C}+\sum_{j=1}^{n} \sum_{i=1}^{n} r_{i j}^{L O C}\right)^{(2)}
\end{aligned}
$$

Aus den Bewertungen der externen Knoten für die lokale Struktur kann - ähnlich wie bei den direkten internen Lücken - zur Übersicht ein Vektor generiert werden, der die externen Knoten, die über einer Schranke für die Bedeutung liegen, ausweist (Funktion EXTGAP). Der Wert für diese Schranke th lässt sich abermals über die bereits diskutierten Verteilungskennzahlen bestimmen. Formel (236) zeigt die Definition dieses Vektors für die Bewertungsmethode aus Formel (235), alternativ wäre auch eine Bestimmung gemäß Formel (231) möglich.

$$
\begin{aligned}
\operatorname{extGap}^{\text {binary }} & =\left(\operatorname{extgap}_{x}^{\text {binary }}\right)_{v_{x} \in V^{E I T}} \\
\operatorname{extgap}_{x}^{\text {binary }} & =\left\{\begin{array}{ll}
1 & \text { wenn rate } \\
0 & \text { andernfalls }
\end{array}>\right.\text { th }
\end{aligned}
$$

Eine Übersicht der Wirkungen eines externen Knoten $v_{x}$ auf die Knoten der lokalen Struktur kann aufbauend auf dem Ansatz aus Formel (235) über Formel (238) gewonnen werden (Funktionen RATEEXTVXSTAT und RATEEXTVXSTATLIST). Formel (239) weist die hierzu passende Bedeutung des, externen Knoten in 
der erweiterten lokalen Struktur getrennt aus. Formel (237) zeigt die für die Übersicht erforderliche Matrix $R^{L O C \cdot+O Z S}$, die eine an die Dimension von $R^{L, O C, E X P-x}$ angepasste Version von $R^{L O C}$ ist. Analog wird $R^{* I O C \cdot O Z S}$ aus $R^{* L O C}$ generiert. Während rate ${ }^{E X T v_{x} I N}$ aus Formel (238) die Veränderung der Bedeutung der internen Knoten als Vorgänger bei Hinzunahme des externen Knoten $v_{x}$ quantifiziert, misst rate $e^{E X T v_{x} O U / T}$ die Bedeutung der internen Knoten als Nachfolger bei Hinzunahme des externen Knoten $v_{x}$. Im Gegensatz dazu stellen rate ${ }^{E X T v_{x} I N 4 L O C}$. und rate ${ }^{E X T v_{x}, O U T A L O C}$ aus den Formeln (240) und (241) die direkte und indirekte Bedeutung des externen Knoten als Nachfolger bzw. als Vorgänger für die internen Knoten dar (Funktion RATEEXTVX4LOC). Formel (242) kombiniert die Nachfolger- und Vorgänger des externen Knoten in einer Maßzahl rate ${ }^{E X T v_{x}, 4 L O C}$.

$$
\begin{aligned}
& R^{L O C+0 Z S}=\left(r_{i j}^{L O C+0 Z S}\right)_{i, j=1, \ldots, n+1} \\
& r_{i j}^{L O C+O Z S}= \begin{cases}r_{i j}^{L O C} & \text { wenn } v_{i}, v_{j} \in V^{L O C} \\
0 & \text { wenn } v_{i} \in V^{E X T} \vee v_{j} \in V^{E X T}\end{cases} \\
& \operatorname{rate}^{E X I v_{x}}=\left(\text { rate }_{i n t}^{E X T v_{x}}\right)_{i n t=1, \ldots, n} \text { für } v_{i n t} \in V^{L(X} \wedge v_{x} \in V^{E X T} \\
& \text { rate }_{i n t}^{E X T v_{x}}=\text { rate }_{i n t}^{E X T v_{x}, I N}+\text { rate }_{i n t}^{E X T v_{x}, O U T} \\
& \text { wobei } \\
& \operatorname{rate}_{i n t}^{E X T v_{x}, I N}=\sum_{i=1}^{n+1}\left(r_{i n t}^{* L O C, E X P-x}-r_{i i n t}^{* L O C+0 Z S}\right) \\
& \text { rate }_{i n t}^{E X T v_{x}, O U T T}=\sum_{j=1}^{n+1}\left(r_{i n t j}^{L O C, L X P_{-x}}-r_{i n t j}^{L O C+0 Z S}\right) \\
& \text { rate }_{x}^{E X T v_{x}}=\sum_{i=1}^{n+1}\left(r_{i x}^{* L O C, E X P-x}-r_{i x}^{* L O C+0 Z S}\right)+\sum_{j=1}^{n+1}\left(r_{x j}^{L O C, E X P-x}-r_{x j}^{L O C+0 Z S}\right) \text { für } v_{x} \in V^{E X T} \\
& \operatorname{rate}^{E X T v_{x}, I N 4 L O C}=\left(\operatorname{rate}_{i n t}^{E X T v_{x}, I N 4 L O C}\right)_{i n t=1, \ldots, n} \text { für } v_{i n t} \in V^{L O C} \wedge v_{x} \in V^{E X T} \\
& =\left(r_{i n t x}^{* L, O C, E X P-x}\right)_{i m t=1, \ldots, n}
\end{aligned}
$$




$$
\begin{aligned}
\text { rate }^{E X T v_{x}, O t \pi 4 L O C} & =\left(\text { rate }_{i n t}^{E X T v_{x}, O U T 4 L O C}\right)_{i n t=1, \ldots, n} \quad \text { für } v_{i n t} \in V^{L O C} \wedge v_{x} \in V^{E X T} \\
& =\left(r_{x i n t}^{L O C, E X P-x}\right)_{i n t=1, \ldots, n} \\
r a t e^{E X T v_{x}, 4 L O C} & =\text { rate }^{E X T v_{x}, I N 4 L O C}+r a t e^{E X T v_{x}, O t / 4 L O C} \quad \text { für } v_{x} \in V^{E X T}
\end{aligned}
$$

Auch bei der Erweiterung der lokalen Struktur um externe Knoten und somit auch Kanten stellt sich die Frage, welche Zielwerte für die Verbindungen von oder zu den in der lokalen Struktur bestehenden Knoten anzustreben sind. Grundsätzlich kann bei dem Vorliegen von Absolutwerten in der globalen Struktur eine Nivellierung entsprechend den bereits diskutierten Nivellierungsansätzen erfolgen. Für jene Fälle, in denen eine Nivellierung von Absolutwerten nicht erwünscht ist oder ausschließlich Anteilswerte vorliegen, wird jedoch vorgeschlagen, die jeweiligen Zeilen- und Spaltenwerte aus einer um die zur Disposition stehenden externen Knoten erweiterten sowie zeilen- bzw. spaltennormierten lokalen Struktur als Zielwerte festzulegen. Selbstverständlich ist es auch möglich, diese Werte über einen Faktor zu relativieren.

Die Zielwerte einer betrachteten externen Lücke können - wie auch bei den direkten internen Lücken gezeigt - verwendet werden, um die notwendigen relativen Erhöhungen der Volumina der in der ursprünglichen lokalen Struktur bereits bestehenden jeweiligen Nachfolger- bzw. Vorgängerknoten, also deren Inputs bzw. Outputs, zur Erreichung der Zielwerte zu quantifizieren. Die in Formel (243) und Formel (244) präsentierten Vektoren $\Delta v o l^{*} E X T-x$ und $\Delta v l^{l X X-x}$ können diese Informationen über $A^{{ }^{*} L O C, E X P-x}$ und $A^{L O C, E X P-x}$ bereitstellen (Funktion RATEEXTVXVOL). Ebenso wie bei den direkten internen Lücken können $\Delta v o l^{t: X T-x}$ und $\Delta v o l^{* E X T-x}$ zu $\Delta v o l^{+E X T-x}$ addiert werden, um das durch die Kanten der externen Lücke induzierten Input- und Outputsteigerungserfordernis der jeweils verbundenen Nachfolger- und Vorgängerknoten bezogen auf die nicht erweiterte lokale Struktur zu bestimmen und mit anderen externen Lücken vergleichen zu können. Über den Vektor $\Delta v o l^{+E X T-x}$ kann z.B. die für die lokale Struktur bedeutendste externe Lücke mit dem geringsten oder höchsten (maximalen) 
lokalen Input- und Outputänderungsbedarf festgestellt werden. Zur Identifikation solcher externer Lücken mit alleinigem Bezug auf den (maximalen) Input- bzw. Outputänderungsbedarf sind $\Delta v o l^{E X T-x}$ bzw. $\Delta v o l^{* E X T-x}$ heranzuziehen.

$$
\begin{aligned}
& \Delta v o l^{* E X T-x}=\left(\Delta v o l_{i}^{* E X T-x}\right)_{i=1, \ldots, n} \quad \text { für } v_{i} \in V L O C \wedge v_{x} \in V E X T \\
& \Delta v o l_{i}^{*} E X T-x=\frac{a_{i x}^{* L O C, E X P-x}}{\left(1-a_{i x}^{* L O C, E X P-x}\right)} \\
& \Delta v \operatorname{vol}^{E X T-x}=\left(\Delta v o l_{j}^{E X T-x}\right)_{j=1, \ldots, n} \quad \text { für } v_{j} \in V^{L O C} \wedge v_{x} \in V E X T \\
& \Delta v \operatorname{vol}_{j}^{E X T-x}=\frac{a_{x j}^{L O C, E X P-x}}{\left(1-a_{x j}^{L O C, E X P-x}\right)}
\end{aligned}
$$




\section{Umlegungsphase}

Nachfolgend auf den Vergleich der lokalen Struktur, die sich entsprechend dem Zweck dieser Arbeit auf die Kollaborationsplattform bezieht, mit der globalen Struktur, d.h. der Produktionsstruktur der Gesamtwirtschaft bzw. Auszüge aus dieser, sowie auf die Bewertung der Knoten und Kanten der lokalen Struktur, stellt sich die Frage, wie die gewonnenen Kennzahlen von der Plattormebene auf die Ebene der Plattformteilnehmer umgelegt werden können. Es sind also die Bewertungen für die Güter bzw. Aktivitäten den an der Plattform teilnehmenden Unternehmen zuzuweisen, um auch auf dieser Ebene Handlungsempfehlungen zu ermöglichen.

\subsection{Generierung der Datenbasis}

Ein Ansatz zur Umlegung der Ergebnisse aus dem Vergleich mit der globalen Struktur kann auf Basis einer Matrix BClgugu bzw. BClauau verfolgt werden. Hierzu seien diese beiden Matrizen zunächst vorgestellt.

$$
\begin{aligned}
& B C I g g u_{u n t}=\left(b c i g g u_{u n t, c u}\right)_{\substack{c=1, \ldots, n g \\
u=1, \ldots, n g u}} \text { für unt }=1, \ldots, n u
\end{aligned}
$$

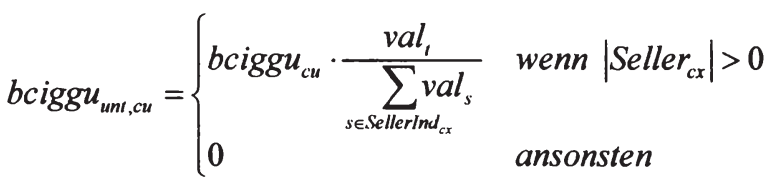

$$
\begin{aligned}
& \text { wobei } \\
& \text { Seller }_{c x}=\left\{\text { bciedgefruug }_{s}: \text { com }_{s c}=\operatorname{com}_{c} \wedge \text { buy }_{s x}=b_{b i z}\right\} \\
& \text { SellerInd }_{c x}=\left\{s \text { :bciedgefruug }_{s} \in \text { Seller }_{c x}\right\} \\
& \text { für } x: \sum_{i=1}^{x-1} n u p_{i}<u \leq \sum_{i=1}^{x} n u p_{i} \\
& \text { bciedgefruug, } \in \text { Seller }_{c x} \text { mit sell } t_{t u n t}=b i z_{u n t}
\end{aligned}
$$

Wie die Formeln (245) und (246) bzw. (247) und (248) zeigen, kann aufbauend auf BCledgeFRuug und BClggu bzw. BCledgeFRuua und BClaau die Matrix 
BClgugu bzw. BClauau bestimmt werden (Funktionen MBMAT und GUOAU). Während BClgugu die auf der Plattform transferierten Güter, deren Verkäufer, deren Käufer aber auch deren - geschätzte - Destinationen in der Produktion der von den Käufern bereitgestellten Gütern aufzeigt, stellt BClauau die Ausgangsaktivität der transferierten Leistung sowie deren - abermals geschätzte Destination (die Aktivität in welche die Leistung einfließt) in Verbindung mit dem verkaufenden und kaufenden Unternehmen auf der Plattorm dar. BClgugu liegt in der Dimension $n g u \times n g u$ vor, wobei $n g u$ abhängig ist von den an der Plattform teilnehmenden Unternehmen sowie den von diesen bereitgestellten Gütern. BClauau hat die Dimension nau $\times$ nau, nau wird dabei von der Anzahl der Plattformteilnehmer und deren Aktivitäten bestimmt.

$$
\begin{aligned}
& B C \lg u g u=\left(b c i g u g u_{a b}\right)_{\substack{a=1, \ldots, \ldots, n g u \\
b=1, \ldots, n g u}} \\
& b c i g u g u_{a b}=b c i g g u_{u n, c b} \\
& \text { wobei gilt: unt }=1 \wedge c=a \quad \text { wenn } a<\text { nup }_{1} \\
& a=\sum_{x=1}^{u n t-1} \text { nup }_{x}+c \text { ansonsten } \\
& B C \operatorname{laau}_{\text {unt }}=\left(\text { bciaau }_{\text {unt,cu }}\right)_{\substack{a=1, \ldots, \ldots a \\
u=1, \ldots, \text {,nau }}} \text { für unt }=1, \ldots, n u \\
& \text { bciaau }_{u n t, a u}= \begin{cases}\text { bciaau }_{a u} \cdot \frac{v a l_{t}}{\sum_{s \in \text { SellerInd }_{\alpha \mathrm{r}}} v a l_{s}} & \text { wenn } \mid \text { Seller }_{a x} \mid>0 \\
0 & \text { ansonsten }\end{cases} \\
& \text { wobei } \\
& \text { Seller }_{a x}=\left\{\text { bciedgefruua }_{s}: \text { act }_{s a}=a c t_{a} \wedge b_{u y} y_{s x}=b i z_{x}\right\} \\
& \text { SellerInd }_{a x}=\left\{s: \text { bciedgefruua }_{s} \in \text { Seller }_{a x}\right\} \\
& \text { für } x: \sum_{i=1}^{x-1} \text { nuap }_{i}<u \leq \sum_{i=1}^{x} \text { nuap }_{i} \\
& \text { bciedgefruua }_{t} \in \text { Seller }_{a x} \text { mit sell }{ }_{t u n t}=b i z_{u n t}
\end{aligned}
$$




$$
\begin{aligned}
& \text { BCIauau }=\left(\text { bciauau }_{c d}\right)_{\substack{c=1, \ldots, \ldots a u \\
d=1, \ldots, n a u}} \\
& \text { bciauau }_{c d}=\text { bciaau }_{u n t, k d} \\
& \text { wobei gilt }: \begin{array}{ll}
\text { unt }=1 \wedge k=c & \text { wenn } c<\text { nuap }_{1} \\
c=\sum_{x=1}^{u n t-1} \text { nuap }_{x}+k & \text { ansonsten }
\end{array}
\end{aligned}
$$

Nachdem BClgugu sowie BClauau nur einen komplexen Zugriff auf die teilnehmenden Unternehmen und/oder deren Güter bzw. Aktivitäten bieten, soll die Kantenliste BCleFgugu aus Formel (249) bzw. die Kantenliste BCleFauau aus Formel (250) eingesetzt werden, um einen einfacheren Zugriff zu ermöglichen (Funktion EDGEFRAME). Es ist darauf hinzuweisen, dass diese Listen sämtliche theoretisch möglichen Transaktionsbeziehungen beinhalten, d.h. für jeden Zellwert aus BCIgugu oder BCIauau existiert ein entsprechender Eintrag in der dazugehörigen Kantenliste (inklusive Zellen mit dem Wert 0). Zur computationalen Optimierung könnten Einträge (Transaktionsbeziehungen), die gemäß der Produktionsstruktur nicht auftreten können, herausgefiltert werden.

$$
\begin{aligned}
& B C l e F g u g u=\left(b c i e f g u g u_{k}\right)_{k=1, \ldots, n g u^{2}} \quad \text { für unt }, x=1, \ldots, n u, c, d=1, \ldots, n g \\
& \text { bciefgugu }_{k}=\left(\text { sell }_{k, u n t}, \operatorname{com}_{k, c}, \text { buy }_{k, x}, \text { destcom }_{k, d}, \text { val }_{k}\right) \\
& \text { wobei } \\
& \operatorname{sell}_{k, u n t}=\operatorname{sell}_{t, \text { unt }}=b i z_{u n t} \\
& \operatorname{com}_{k, c}=\operatorname{com}_{t, c}=\operatorname{com}_{c} \\
& b u y_{k, x}=b u y_{t, x}=b i z_{l, x}=b i z_{x} \\
& \operatorname{destcom}_{k, d}=\operatorname{com}_{l, d}=\operatorname{com}_{d} \\
& \left(\text { act }_{l, j}, \text { com }_{l, d}, \text { biz }_{l, x}\right) \in \text { BCIprofile }_{u n t}
\end{aligned}
$$

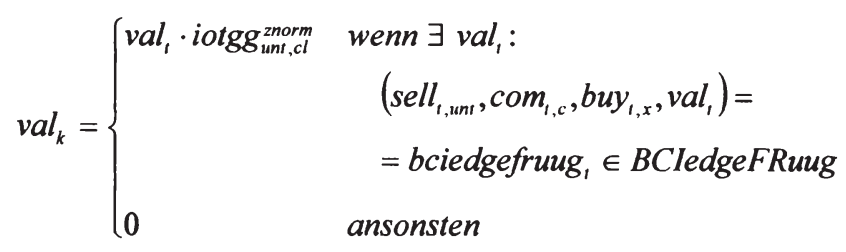




$$
\begin{aligned}
& \text { BCIeFauau }=\left(\text { bciefauau }_{k}\right)_{k=1, \ldots, n a u^{2}} \text { für unt }, x=1, \ldots, n u, a, d=1, \ldots, n a \\
& \text { bciefauau }_{k}=\left(\text { sell }_{k, u n t}, \text { act }_{k, a}, \text { buy }_{k, x}, \text { destact }_{k, d}, \text { val }_{k}\right) \\
& \text { wobei } \\
& \text { sell }_{k, u n t}=\text { sell }_{t, u n t}=b i i_{u n t} \\
& a c t_{k, a}=a c t_{t, a}=a c t_{a} \\
& b u y_{k, x}=b u y_{t, x}=b i z_{l, x}=b i z_{x} \\
& \text { destact }_{k, d}=a c t_{l, d}=a c t_{d} \\
& \left(\text { act }_{l, d}, \operatorname{com}_{l, j}, \text { biz }_{l, x}\right) \in \text { BCIprofile }_{u n t} \\
& \text { val }_{k}=\left\{\begin{array}{cc}
\text { val }_{t} \cdot \text { iotaa }_{u n t, a l}^{\text {znorm }} & \begin{array}{c}
\text { wenn } \exists \text { val }_{t}:\left(\text { sell }_{t, u n t}, \text { act }_{t, a}, \text { buy }_{t, x}, \text { val }_{t}\right)= \\
=\text { bciedgefruua }_{t} \in B C \text { edgeFRuua }
\end{array} \\
0 & \text { ansonsten }
\end{array}\right.
\end{aligned}
$$

Aufbauend auf BCIgugu (BCleFgugu) bzw. BClauau (BCleFauau) erfolgt die Umlegung der Kennzahlen zu den identifizierten Performanzlücken, direkten internen sowie externen Lücken bezogen auf Güter bzw. Aktivitäten auf die Plattformteilnehmer. Dabei wird auch die Klassifikation von Gütern bzw. Aktivitäten als ,schwache Knoten' in die Berechnung miteinbezogen. Die Matrizen die zur im Folgenden auf die Güterbetrachtung abstellenden - Umlegung der Kennzahlen auf die Teilnehmer herangezogen werden, sind Rate ${ }^{\cdot \text { potPerf }}$, PerfGap ${ }^{+ \text {binary }}$, Rate $^{\text {DIL }}$, DiGap ${ }^{\text {binary }}$, whNode ${ }^{I N}$, whNode ${ }^{O U T}$ und rate ${ }^{E X T v_{x}}$, welche auf Basis der Daten der Kollaborationsplattform über den Vergleich mit der gesamtwirtschaftlichen Produktionsstruktur entsprechend der oben vorgestellten Verfahrensschritte zuvor zu bestimmen sind. Substitute wie bspw. die über MatDist ermittelte Matrix PerfGap ${ }^{\text {binary }}$ u.ä. sind je nach gewünschter Schwerpunktsetzung oder Verfahrenswahl denkbar.

\subsection{Umlegung von Performanzlücken und internen strukturellen Lücken}

Formel (258) und Formel (258') zeigen - unterstützt durch die Formeln (251) bis (257) - wie Erkenntnisse aus dem Strukturvergleich bezogen auf die Güter der Kollaborationsplattform auf $B C l e F g u g u$ umgelegt werden können. Konkret wird 
BCleFgugu als BCleFgugu ${ }^{e x p}$ pro Eintrag um eine Kennzeichnung erweitert, die die Verbindung zweier Güter auf Teilnehmerebene als Performanzlücke, NichtPerformanzlücke (reguläre Beziehung) oder direkte interne Lücke klassifiziert und dabei auch die Priorität der jeweiligen Verbindung festlegt (Funktion ALLOCATEALL aufbauend auf DILLI, COMBDILINCUTRANS, MPONP und ALLOCATE). Kategorien zur Klassifikation sind $P^{D I L}, P$ und $P^{+}$für Performanzlücken, $N P^{D I L}$, $N P$ und $N P^{+}$für Nicht-Performanzlücken und $D I L^{i s o}, D I L^{-}, D I L^{\sim}, D I L^{-}$und $D I L^{o}$ für direkte interne Lücken. Das Superskript ${ }^{D I L}$ bezeichnet in diesem Zusammenhang eine Verbindung zwischen zwei Gütern, die auf der Teilnehmerebene nicht besteht und daher bevorzugt behandelt werden könnte; ${ }^{\text {iso }}$ soll hingegen Lücken aufzeigen, deren Schließung einen Teilnehmer aus der (einseitigen) Isolation auf der Plattform befreien kann. Diese Markierung wird in Formel (258) und (258') für direkte interne Lücken vergeben, kann aber auch als Sub-Klassifikation für Klassen die mit dem Superskript ${ }^{\text {DIL. }}$ versehen werden Einsatz finden. Die Superskripte und $^{+}$der Klassen zu Performanzlücken $(P)$ und Nicht-Performanzlücken $(N P)$ bzw. ${ }^{\text {iso }},{ }^{-}$, , $^{+}$und ${ }^{o}$ der Klassen zu direkten internen Lücken $(D I L)$ ermöglichen ebenfalls eine Priorisierung der identifizierten Lücken auf Teilnehmerebene, die sich aus einer jeweils festzulegenden Schranke ergibt. Unter den Klassen zu Performanzlücken bzw. Nicht-Performanzlücken haben Klassifikationen die mit dem Superskript ${ }^{D I L}$ versehen sind demnach eine höhere Priorität bei der Lückenschließung als Klassifikationen mit dem Superskript , welche wiederum dem Superskript vorzuziehen sind. Gleichfalls ist bei den direkten internen Lücken die Reihenfolge ${ }^{i s o}$, , , , ${ }^{+}$und ${ }^{o}$ als Indikator für die Priorität zu sehen, die - je nach Schwerpunktsetzung - bei allen Klassen auch abgeändert werden kann. Innerhalb jeder Klasse sollte eine weitere Reihung nach der Bewertung der jeweiligen Lücke auf Plattformebene erfolgen, um die Priorisierung bezogen auf die Teilnehmer mit der Relevanz der Verbindung zwischen zwei Gütern aus Sicht der Plattform zu kombinieren. Zwecks Umsetzung könnte hierzu bspw. eine weitere Spalte in BCleFgugu ${ }^{\text {exp }}$ mit der pro Güterverbindung passenden Bewertung angehängt werden oder aber zur Vermeidung von Michael Weber - 978-3-631-75376-7 
Redundanzen auf eine weitere Datenquelle verwiesen werden. Die Bewertung für die (Nicht-)Performanzlücken kann dabei - passend zu den bisherigen Ausführungen - aus $\mid$ Rate ${ }^{\text {potPerf }} \mid$ bezogen werden. Für direkte interne Lücken kann Rate $^{\text {IIL }}$ herangezogen werden. Will man die aus der Plattormperspektive bewerteten Verbindungen zusätzlich nach Maßgabe der an der jeweiligen Transaktion teilnehmenden Unternehmen gewichten, so könnte bspw. die nachfolgend beschriebene Teilmenge der an der Plattform teilnehmenden Unternehmen $\mathrm{Biz}^{\text {weak }}$ als Indikator für die Gewichtung eingesetzt werden. Es ist auch darauf hinzuweisen, dass die Wahl von Rate ${ }^{D I L 2}$, die die Bewertung der direkten internen Lücken bezogen auf reine oder gemischte Performanzlückenfolgen enthält, auch den Einsatz von dem darauf aufbauenden DiGap ${ }^{\text {binary2 }}$ in Formel (258) und (258') erforderlich macht. Grundsätzlich kann mittels DiGap ${ }^{\text {binary }}$ oder eben DiGap ${ }^{\text {binary2 }}$ über die Wahl des jeweiligen Schwellenwerts eine von der Bedeutung abhängige Selektion der in die Betrachtung einfließenden direkten internen Lücken erfolgen.

Eine Möglichkeit zur Festlegung einer Schranke zur Priorisierung der Performanzlücken auf Teilnehmerebene, $t h_{c d}^{P, \alpha}$, bietet Formel (251), die sich auf Verteilungskennzahlen zu Absolutwerten stützt. Eine analoge Herangehensweise liefert Formel (252) zur Schrankenbestimmung für Nicht-Performanzlücken (reguläre Verbindungen) auf Teilnehmerebene $t h_{c d}^{N P, \alpha}$. Je nach Schwerpunktsetzung kann die Reihung, die durch diese beiden Schranken (mit-)bestimmt wird, auch von anderen Werten (z.B. Anteilswerten) oder Relationen (unter Einbezug weiterer Kennzahlen) abhängig gemacht werden. Die Formeln (253) und (254) zeigen, wie (einseitig oder beidseitig) isolierte Teilnehmer, d.h. Teilnehmer die im betrachteten Zeitraum noch bei keiner Transaktion auf der Kollaborationsplattform Start- oder Endpunkt waren, zur besonderen Beachtung ausgewiesen werden können. Nach der Identifikation von isolierten Teilnehmern könnten diese innerhalb der als $P^{D I L}$ oder $N P^{D I L}$ klassifizierten Relationen herausgefiltert werden. DIL ${ }^{\text {iso-s }}$ (Formel (253)) und DIL iso-b (Formel (254)) sind Beispiele für eine solche Filterung bei direkten internen Lücken. Über Formel (257) können Unter- 
nehmen ( $B i z^{\text {weak }}$ ) bestimmt werden, deren Güter aus der Perspektive der Kollaborationsplattform vorwiegend als ,schwache Güter' betreffend deren Angebot oder Nachfrage klassifiziert werden und in der Reihung in Formel (258) und (258') besondere Beachtung finden sollen. Formel (255) und Formel (256) zeigen, wie die Einordnung jedes an der Plattform teilnehmenden Unternehmens anhand der Güterklassifikation nach Maßgabe von whNode ${ }^{I N}\left(B_{i z}{ }^{\text {weak-IN }}\right)$ bzw. whNode ${ }^{\text {OUT }}$

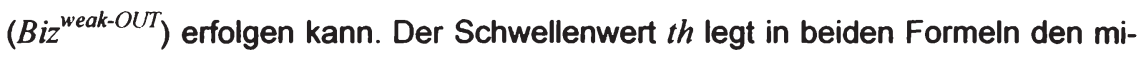
nimalen Anteil an schwachen Gütern pro Teilnehmer fest, ab dem der Teilnehmer als ,schwach' klassifiziert wird. Es sei darauf hingewiesen, dass $B i z^{\text {weak-IN }}$ und $\mathrm{Biz}^{\text {weak-OUT }}$ abhängig von der gewünschten Reihung auch direkt in Formel (258) und (258') bei der Priorisierung der Empfehlungen zu den identifizierten direkten internen Lücken an die Stelle des Käufers und Verkäufers oder aber auch umgekehrt gesetzt werden können. Das bedeutet, dass Teilnehmer, die vorwiegend über whNode ${ }^{\text {OUT }}$ als ,schwach' klassifizierte Güter auf der Plattform absetzen

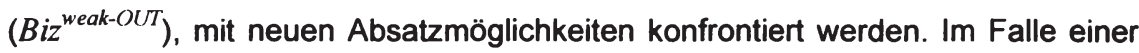
umgekehrten Vorgehensweise hingegen werden eben diesen Teilnehmern bisher unbeachtete Zuliefermöglichkeiten eröffnet, deren Erschließung die Einbettung der Teilnehmer in die Plattform bzw. deren Lieferketten erhöhen soll.

$$
\begin{aligned}
& B C I e F g u g u^{\text {P.cd }}=\left(\text { bciefgugu }_{t}^{\text {P.cd }}\right)_{t=1, \ldots, n P_{c d}} \text { für c, } d=1, \ldots, n g \\
& b c i e f g u g u_{t}^{P . c d}=\left\{b_{c i e f g u g u_{k}} \in B C l e F g u g u: \operatorname{com}_{k, c}=\operatorname{com}_{c}\right. \\
& \left.\wedge \operatorname{destcom}_{k, d}=\operatorname{com}_{d} \wedge \operatorname{val}_{k}>0 \wedge \text { perfgap }_{c d}^{+b i n a r y}=1\right\} \\
& t h_{c d}^{P, \alpha}=\operatorname{percentile}_{\alpha}\left(\left(\text { val }_{t}\right)_{t=1, \ldots, n P_{c d}}\right) \\
& B C l e F g u g u^{N P . c d}=\left(b c i e f g u g u_{t}^{N P . c d}\right)_{t=1, \ldots, n N P_{c d}} \text { für } c, d=1, \ldots, n g \\
& \text { bciefgugu } u_{t}^{N P . c d}=\left\{\text { bciefgugu }_{k} \in B C \text { CleFgugu }: \operatorname{com}_{k, c}=\operatorname{com}_{c}\right. \\
& \wedge \operatorname{destcom}_{k, d}=\operatorname{com}_{d} \wedge \operatorname{val}_{k}>0 \wedge \text { perfgap }_{c d}^{+ \text {bimary }}=0 \\
& \left.\wedge \operatorname{digap}_{c d}^{\text {binary }}=0\right\} \\
& t h_{c d}^{N P, \alpha}=\text { percentile }_{\alpha}\left(\left(\text { val }_{t}\right)_{t=1, \ldots, n N P_{c d}}\right)
\end{aligned}
$$




$$
\begin{aligned}
\text { Biz }{ }^{i s o-s} & =\left(b_{i z_{l}^{i s o-s}}\right)_{l=1, . ., n i s o s} \\
& =\left\{b i z_{b} \in B i z: \sum_{x=1}^{m u} b c i u u_{b x}=0\right\} \\
B i z^{i s o-b} & =\left(b i z_{l}^{i s o-b}\right)_{l=1, \ldots, \text { isob }} \\
& =\left\{b i z_{b} \in B i z: \sum_{x=1}^{n u} b c i u u_{x b}=0\right\}
\end{aligned}
$$

$$
\begin{aligned}
\text { Biz }^{\text {weak }-I N} & =\left(b_{i z}^{\text {weak }-I N}\right)_{l=1, \ldots, \text { weaklN }} \\
& =\left\{b i z_{b} \in B i z: \frac{\left|\operatorname{Com}_{b}^{\text {weak-IN }}\right|}{\left|\operatorname{Com}_{b}\right|}>t h\right\}
\end{aligned}
$$

wobei

$$
\operatorname{Com}_{b}=\left\{\operatorname{com}_{c} \in \operatorname{Com}:\left(\exists \text { bciprofile } u_{b, x}: \operatorname{com}_{b, x, c}=\operatorname{com}_{c}\right)\right\}
$$

Com $^{\text {weak }-I N}=\left\{\operatorname{com}_{c} \in\right.$ Com $:$ whnode $\left._{c}^{I N}>1\right\}$

$\operatorname{Com}_{b}^{\text {weak-IN }}=\operatorname{Com}_{b} \cap \mathrm{Com}^{\text {weak-IN }}$

$$
\begin{aligned}
\text { Biz weak-OUT } & =\left(b i z_{l}^{\text {weak-OUTT }}\right)_{l=1, \ldots, \text { meakl }} \\
& =\left\{b i z_{b} \in B i z: \frac{\left|\operatorname{Com}_{b}^{\text {weak-OUT }}\right|}{\left|\operatorname{Com}_{b}\right|}>\operatorname{th}\right\}
\end{aligned}
$$

wobei

$$
\begin{aligned}
& \text { Com }^{\text {weak-ourT }}=\left\{\operatorname{com}_{c} \in \text { Com }: \text { whnode } \text { OUTT }_{c}>1\right\} \\
& \text { Com }_{b}^{\text {weak-OUT }}=\text { Com }_{b} \cap \text { Com }^{\text {weak-OUT }}
\end{aligned}
$$

$$
\begin{aligned}
B i z^{\text {weak }} & =\left(b i z_{l}^{\text {weak }}\right)_{l=1, \ldots, \text { meeak }} \\
& =B i z^{\text {weak-IN }} \cup B i z^{\text {weak-OIIT }}
\end{aligned}
$$

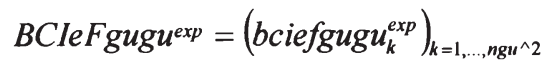

$b c i e f g u g u_{k}^{e x p}=\left(\operatorname{sell}_{k, u n t}, \operatorname{com}_{k, c}\right.$, buy $_{k, x}$, destcom $\left._{k, d}, v a_{k}, \operatorname{gap}_{k}\right)$ wobei

$$
\left(\operatorname{sell}_{k, u n t}, \operatorname{com}_{k, c}, \text { buy }_{k, x}, \text { destcom }_{k, d}, \text { val }_{k}\right)=\text { bciefgugu }_{k}
$$


sowie

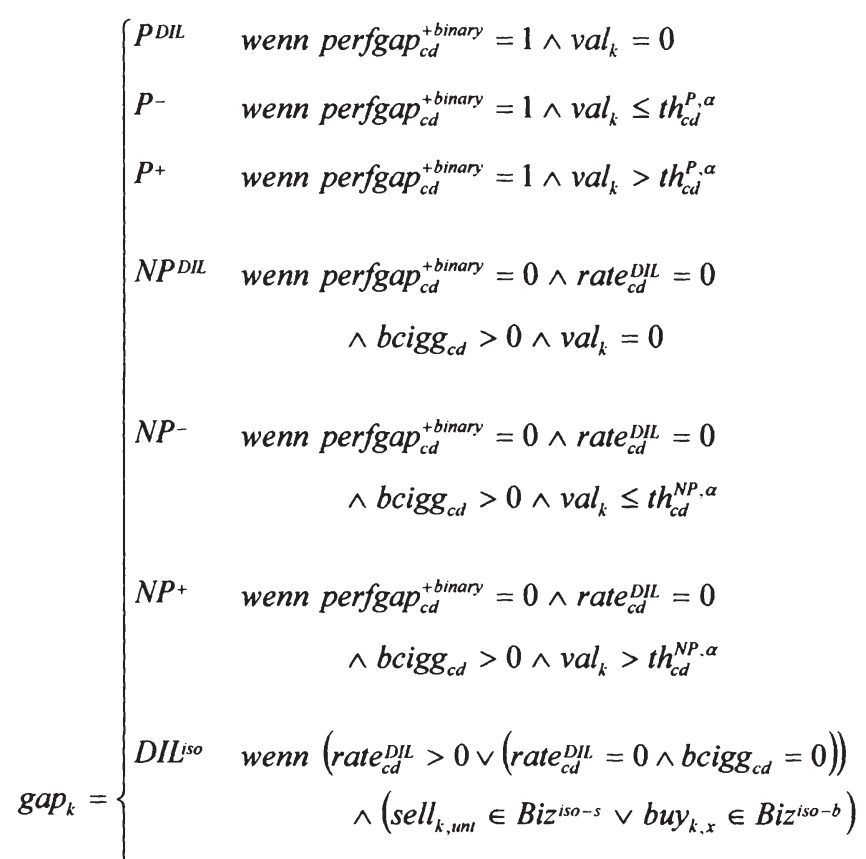

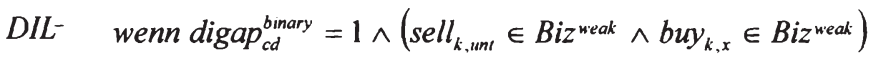

$$
\wedge\left(\text { sell }_{k, \text { unt }} \notin B i z^{i s o-s} \wedge b u y_{k, x} \notin B i z^{i s o-b}\right)
$$

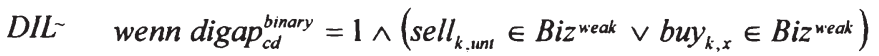

$$
\begin{aligned}
& \wedge-\left(\text { sell }_{k, u n t} \in B i z^{\text {ueak }} \wedge b u y_{k, x} \in B i z^{\text {weak }}\right) \\
& \wedge\left(\text { sell }_{k, u n t} \notin B i z^{i s o-s} \wedge b u y_{k . x} \notin B i z^{i s o-b}\right)
\end{aligned}
$$

DIL $L^{+}$wenn digap cd $^{\text {binary }}=1 \wedge\left(\right.$ sell $_{k, \text { unt }} \notin B i z^{\text {weak }} \wedge$ buy $\left.y_{k, x} \notin B i z^{\text {weak }}\right)$

$$
\wedge\left(\text { sell }_{k, \text { unt }} \notin B i z^{i s o-s} \wedge b u y_{k . x} \notin B i z^{i s o-b}\right)
$$

$D I L^{o} \quad$ wenn digap ${ }_{c d}^{\text {binary }}=0 \wedge\left(\right.$ rate $_{c d}^{D I L}>0 \vee\left(\right.$ rate $\left.\left._{c d}^{D L}=0 \wedge b c i g g_{c d}=0\right)\right)$

$$
\wedge\left(\text { sell }_{k, u n t} \notin B i z^{i s o-s} \wedge b u y_{k, x} \notin B i z^{i s o-b}\right)
$$




\subsection{Umlegung von externen Lücken}

Die Umlegung der Bewertungen zu den externen Knoten (Gütern oder Aktivitäten) hinsichtlich deren Bedeutung für die Kollaborationsplattform auf die Teilnehmer ermöglicht die Abschätzung der teilnehmerbezogenen Wirkung der Hinzunahme eines auf der Kollaborationsplattform nicht bereitgestellten Gutes oder einer nicht zur Verfügung stehenden Aktivität. Aufbauend auf rate $e^{E X T v_{x}, t L O C}$, welches über Formel (242) für die Plattform bestimmt wird, kann abhängig von dem Profil der Teilnehmer die Matrix RateBiz ${ }^{E X T}$ erstellt werden, in der für jeden Teilnehmer die mögliche Auswirkung der Erweiterung um ein zusätzliches Gut bzw. eine zusätzliche Aktivität auf Basis der von dem Teilnehmer bereitgestellten Güter bzw. Aktivitäten gemäß dem Profil festgehalten wird (Funktion ALLOCATEEXT). Formel (259) zeigt die Berechnung von RateBiz ${ }^{l X T}$ für die Güterperspektive.

$$
\begin{aligned}
& \text { RateBiz }{ }^{E X T}=\left(\text { ratebiz } z_{x j}^{E X T}\right)_{\substack{x=1, \ldots, \ldots(j-n g \\
j=1, \ldots, m u}} \\
& \text { ratebiz } z_{x j}^{E X T}=\sum_{\text {com }_{t m t} \in C o m_{j}} \text { rate }_{i n t}^{E X T v_{x},+L O C} \\
& \operatorname{Com}_{j}=\left\{\operatorname{com}_{c} \in \text { Com }:\left(\exists \text { bciprofileu } u_{j, l}: \operatorname{com}_{j, l, c}=\operatorname{com}_{c}\right)\right\}
\end{aligned}
$$

Eine Addition über die Bedeutungen der betrachteten, auf der Plattform fehlenden Güter bzw. Aktivitäten für die jeweils bereitgestellten Güter bzw. Aktivitäten der Teilnehmer kann nur einen grundsätzlichen Hinweis auf die Auswirkungen der Hinzunahme dieser Güter bzw. Aktivitäten geben. Verfeinerungen erscheinen daher erstrebenswert und können bspw. über den Einbezug der Transaktionsdaten erfolgen. Diese können dabei zur Gewichtung der Bedeutungen der fehlenden Güter bzw. Aktivitäten herangezogen werden (Funktion ALLOCATEEXTWEIGHT).

$$
\begin{aligned}
& \text { RateBiz } E X T, \text { sell }=\left(\text { ratebi } z_{x j}^{E X T, \text { sell }}\right)_{\substack{x=1, \ldots, n G-n g \\
j=1, \ldots, n u}}
\end{aligned}
$$

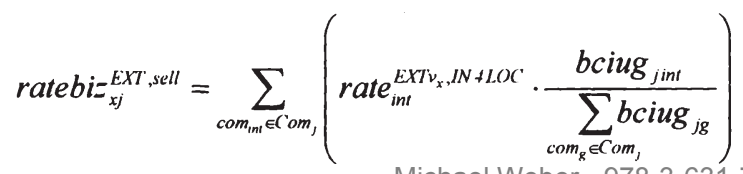




$$
\begin{aligned}
& \text { RateBiz }^{E X T, b u y}=\left(\text { ratebiz } z_{x j}^{E X T, b u y}\right)_{\substack{x=1, \ldots, n(j-n g \\
j=1, \ldots, m u}} \\
& \operatorname{ratebiz}_{x j}^{E X T, b u y}=\sum_{\text {com }_{m t} \in C_{0 m_{\jmath}}}\left(\text { rate }_{i n t}^{E X v_{x}, O U T+L O C} \cdot \frac{\sum_{v a l_{k} \in \operatorname{Val}^{\prime m},} v a l_{k}}{\sum_{g=1}^{n g} b c i g u_{g j}}\right) \\
& \text { mit } \\
& \text { Val }^{\text {int } j}=\left\{\text { val }_{k} \in \text { bciefgugu }_{k} \in\right. \text { BCleFgugu : } \\
& \left.\operatorname{destcom}_{k, d}=\operatorname{com}_{i n t} \wedge b u y_{k, x}=b i z_{j}\right\} \\
& \text { RateBiz }{ }^{k X T, t o t}=\left(\text { ratebiz } z_{x j}^{k X T, t o t}\right)_{\substack{x=1, \ldots, n G-n g \\
j=1, \ldots, n u}} \\
& \text { ratebiz } z_{x j}^{E X T, t o t}=\text { ratebiz } x_{x j}^{E X T, s e l l}+\text { ratebiz }_{x j}^{E X T, b u y}
\end{aligned}
$$

Formeln (260) bis (262) demonstrieren - abermals aus einer Güterperspektive einen solchen Gewichtungsansatz über die Transaktionsdaten der Teilnehmer, bei dem das Gewicht der zugeordneten Bedeutung des fehlenden Gutes für ein bereitgestelltes Gut dem Anteil dieses bereitgestellten Gutes an den gesamten Güterverkäufen bzw. -käufen (Formel (260) bzw. (261)) des an der Kollaborationsplattform teilnehmenden Unternehmens entspricht. Formel (262) bezieht sowohl die Güterverkäufe als auch die Güterkäufe in die Gewichtung ein. Im Gegensatz dazu bieten die Formeln (263) bis (265) - ebenfalls aus der Güterperspektive - einen Gewichtungsansatz, der sich auf die Verkaufs- oder Einkaufsanteile eines von einem Teilnehmer bereitgestellten Gutes in Relation zu dem jeweiligen gesamten Verkauf dieses Gutes oder Einkauf für das Gut (aller Teilnehmer) bezieht. Es wird bei diesem Ansatz folglich im Hinblick auf die Plattform und die Rolle eines Teilnehmers im Vergleich zu den anderen Teilnehmern gewichtet. Teilnehmer, die aktiver bei dem Verkauf eines Gutes oder dem Einkauf für ein Gut sind, profitieren entsprechend dieser Sichtweise von der Hinzunahme eines fehlenden Gutes stärker, weshalb die Bedeutung für diese Teilnehmer höher ist als bei Teilnehmern mit geringerer Transaktionsaktivität. Formel (263) zeigt die Gewichtung unter alleiniger Berücksichtigung der Verkaufsseite, während Formel (264) nur auf die Einkaufsseite abstellt. Formel (265) kombiniert beide Seiten, um eine umfassendere Gewichtung zu ermöglichen 


$$
\begin{aligned}
& \text { Rate Biz EXT,sell2 }=\left(\text { ratebiz }_{x j}^{E X T, \text { sell } 2}\right)_{\substack{x=1, \ldots, \ldots G-n g \\
j=1, \ldots, n u}}
\end{aligned}
$$

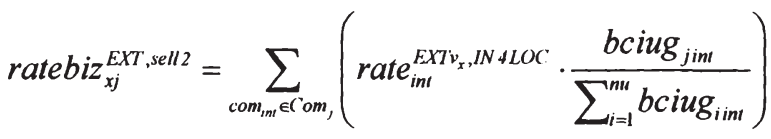

$$
\begin{aligned}
& \text { RateBiz } z^{E X T, b u y 2}=\left(\text { ratebiz } x_{x j}^{E X T, b u y 2}\right)_{\substack{x=1, \ldots, n G-n g \\
j=1, \ldots, n u}}
\end{aligned}
$$

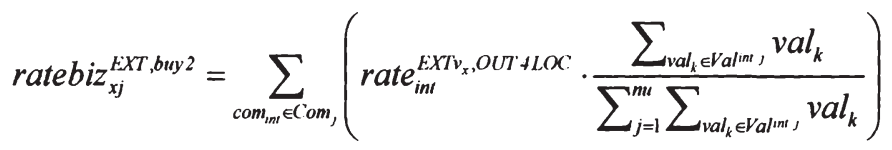

$$
\begin{aligned}
& \text { RateBiz } E X T, \text { tor } 2=\left(\text { ratebiz } x_{x j}^{E X T, t o t 2}\right)_{\substack{x=1, \ldots, \ldots, m i-n g \\
j=1, \ldots, m i}} \\
& \text { ratebiz } z_{x j}^{E X T, t o 12}=\text { ratebiz }_{x j}^{E X T, \text { sell } 2}+\text { ratebiz }_{x j}^{E X X, b u y 2}
\end{aligned}
$$

Mit der Übertragung der Bewertung der externen Güter bzw. Aktivitäten auf die Teilnehmer der Kollaborationsplattform sind die wesentlichen Schritte zur Umlegung der Bewertungsergebnisse auf die Teilnehmerebene abgeschlossen. Der hierin vorgestellte Ansatz bietet verschiedene Verfeinerungs- und Erweiterungsmöglichkeiten und kann somit auch als ein Grundgerüst für die Umlegung der gewonnenen Bewertungen und die teilnehmerbezogene Reihung der dazugehörigen Maßnahmen bzw. Empfehlungen gesehen werden. Nicht jede der auf Plattformebene vorliegenden Bewertungen eignet sich dabei für eine Umlegung auf Teilnehmerebene. So wird z.B. von einer direkten Übertragung der Ergebnisse von $\Delta \mathrm{Vol}^{D L L}$ und $\Delta v \mathrm{l}^{E X T}$ auf die Teilnehmerebene abgesehen. Ein alternativer Ansatz wäre die Berechnung ausgewählter Bewertungen nur unter Einschluss der Transaktionsdaten zu den Gütern bzw. Aktivitäten eines Teilnehmers neben den diese Güter bzw. Aktivitäten nicht berührenden Gütern bzw. Aktivitäten und dem anschließenden Herausfiltern dieser Bewertungen für den jeweiligen Teilnehmer. Dieser Ansatz wird allerdings aufgrund des damit verbundenen computationalen Aufwandes nicht empfohlen, auch wenn für die Umsetzung die bereits beschriebenen Bewertungskennzahlen für die Plattformebene (die lokale Struktur) nur geringfügig angepasst werden müssten. 


\section{Matchingphase}

Mit der Umlegung der Bewertungen von der Plattorm- auf die Teilnehmerebene können Transaktionen unter Einbezug der Teilnehmer- und Plattformperspektive gereiht und entsprechend dieser Reihung empfohlen werden. Ein solches Vorgehen würde allerdings die spezifischen Charakteristika der Teilnehmer nicht berücksichtigen und erscheint daher unzureichend, wenn das Ziel der Transaktionsempfehlungen die Herstellung von möglichst optimal zusammenpassenden Transaktionspartnerpaarungen ist. Intersubjektiv feststellbare Eigenschaften und subjektiv geäußerte Präferenzen der Teilnehmer müssen daher in das Modell einfließen, um der erwartbaren Transaktions(n)eig(n)ung der Teilnehmer auf Basis deren spezifischer Charakteristika bei Vorliegen einer grundsätzlich empfehlenswerten Transaktionsbeziehung gemäß der Erkenntnisse aus den vorangegangenen Phasen Rechnung tragen zu können. Hierfür sind - dem Modell vorgelagert - Erhebungen durchzuführen, die die Eigenschaften und Präferenzen der Teilnehmer festhalten bzw. regelmäßig aktualisieren und auf die für die Quantifizierung der Transaktions(n)eig(n)ung der jeweiligen Teilnehmerpaarungen zurückgegriffen werden kann. Im Sinne einer optimalen Eignungsberechnung sind die Erhebungen zu den Eigenschaften und Präferenzen pro Teilnehmer idealerweise auf dessen Güter bzw. Aktivitäten zu beziehen (in beide Transaktionsrichtungen - Input und Output). Ein sehr hoher Detaillierungsgrad der Präferenzen wäre erreicht, wenn diese bezogen auf die Inputfaktoren und Outputdestinationen gemäß der Produktionsstruktur der vom Teilnehmer bereitgestellten Güter bzw. Aktivitäten festgestellt werden können. In der Praxis wird eine solche Datenermittlung allerdings nur unter hohem Ressourcenaufwand realisierbar sein, weshalb auch güter- bzw. aktivitätsgruppenbezogene Erhebungen oder auch nur die Ermittlung von allgemeinen teilnehmerbezogenen Einkaufs- und Verkaufspräferenzen sowie Eigenschaften als Beispiele für mögliche Alternativen zu nennen sind. 


\section{1 Ähnlichkeits- und Distanzmaße}

Die Übereinstimmung der Ausprägungen der Präferenzen der Teilnehmer im Hinblick auf bestimmte Eigenschaften ist entscheidend für die Quantifizierung der Transaktions(n)eig(n)ung. Ein allgemeines Verfahren zur Bestimmung dieses Wertes soll im Folgenden auf Basis von Ähnlichkeits- und Distanzmaßen, die auf Grundlage der Ausführungen in [Fahrmeir et al. 1996] und [Backhaus et al. 2000] teilweise modifiziert in das Modell einfließen, definiert werden. Ähnlichkeits- und Distanzmaße werden z.B. in der Clusteranalyse zur Quantifizierung der Übereinstimmung oder - anders formuliert - Ähnlichkeit zwischen zwei Objekten oder zwischen zwei Mengen von Objekten eingesetzt, wobei bei steigender Ähnlichkeit der Wert für ein Ähnlichkeitsmaß anwächst, während der Wert eines Distanzmaßes sinkt. Es ist möglich, Ähnlichkeitsmaße, die üblicherweise im Intervall 0 bis 1 oder -1 bis 1 vorliegen, in ein Distanzmaß, welches häufig einen Wertebereich von 0 bis $\infty$ hat, zu transformieren. Zur Umrechnung eines Ähnlichkeitsmaßes $s_{i j}$ zu zwei Objekten, d.h. - entsprechend der bisherigen Ausführungen - Knoten bzw. deren Präferenzen, $v_{i}$ und $v_{j}$ zu einem Distanzmaß $d_{i j}$ kann je nach Intervall Formel (266) bzw. Formel (267) oder - für den umgekehrten Zweck - Formel (268) [Fahrmeir et al. 1996, S. 442] herangezogen werden (Funktion SWICHDISSIM). Zwei grundsätzliche Charakteristika von Distanzmaßen und Ähnlichkeitsmaßen, Symmetrie und die vollständige Übereinstimmung eines Objekts mit sich selbst, beschreibt Formel (269).

$$
\begin{gathered}
d_{i j}=1-s_{i j} \quad \text { für } 0 \leq s_{i j} \leq 1 \\
d_{i j}=\sqrt{2\left(1-s_{i j}\right)} \quad \text { für }-1 \leq s_{i j} \leq 1 \\
s_{i j}=1-\frac{d_{i j}}{\max _{i j}\left(d_{i j}\right)} \text { für } d_{i j} \geq 0 \\
d_{i j}=d_{j i}, s_{i j}=s_{j i} \\
d_{i i}=0, s_{i i}=1
\end{gathered}
$$


Die Formel zur Berechnung der Ähnlichkeit oder Distanz zwischen Objekten bzw. Objektmengen ist abhängig von der Werteskala der Objektmerkmale, wobei für jeden Skalentyp mindestens ein zu diesem Typ passendes Ähnlichkeits- oder Distanzmaß vorliegt. Nachfolgend sollen v.a. Ähnlichkeitsmaße zu den grundlegenden Skalentypen nominal (binär sowie mehrstufig nominal), ordinal und metrisch vorgestellt werden, die als generische Vorlage für die Variablen, in denen die Präferenzen gemessen werden, dienen. Abbildung 14 gibt eine Übersicht über die präsentierten Ähnlichkeits- bzw. Distanzmaße.

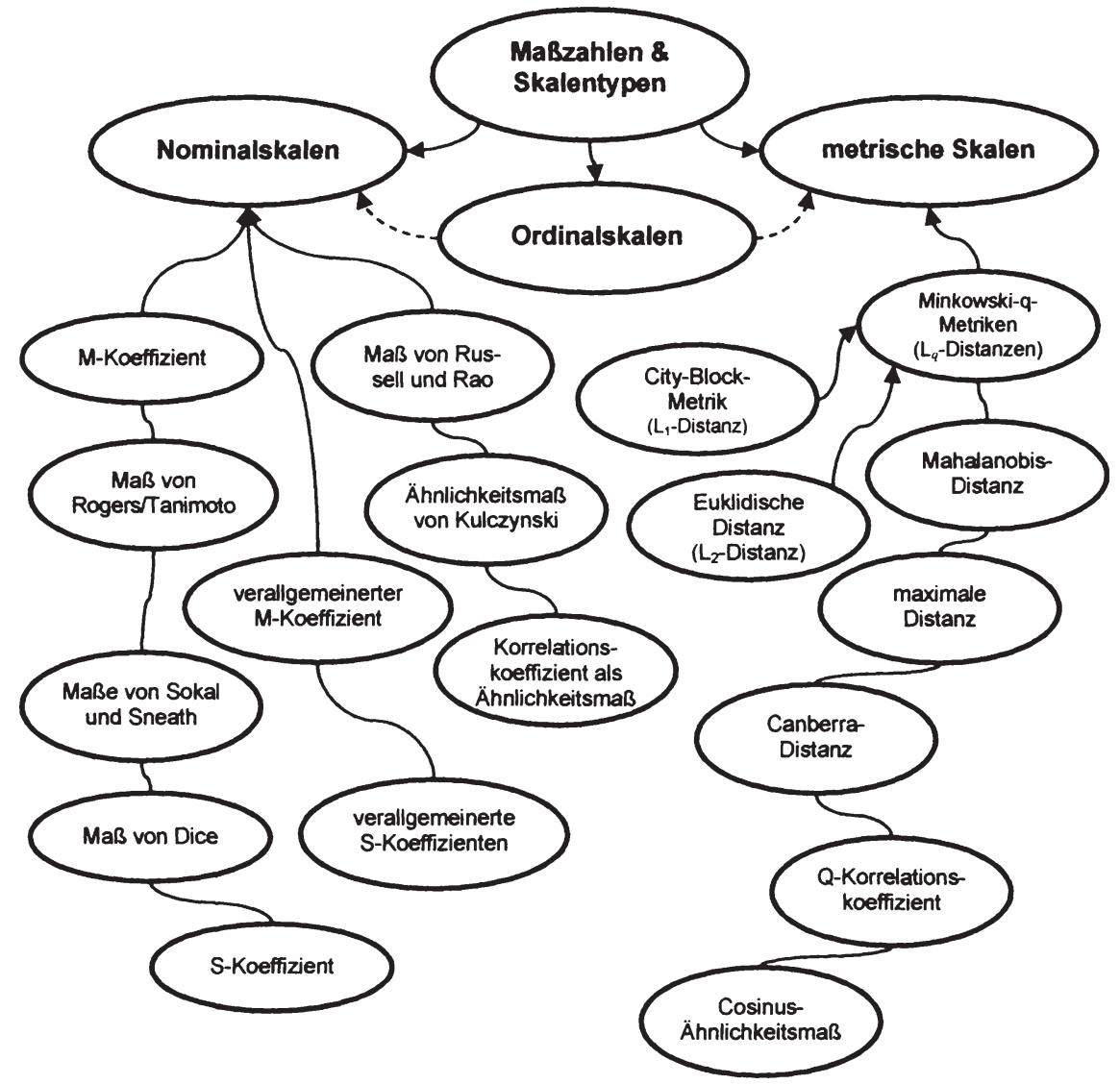

Abbildung 14: Maßzahlen für verschiedene Skalentypen 
Formel (270) zeigt die in der Präferenzmatrix $X^{\text {prefs }}$ zusammengestellten Präferenzvariablen $x_{k}^{t y p e}$, die zur Messung der Übereinstimmung eingesetzt werden sollen und über type $\in$ Type $=\{$ "bin", "nom", "ord", "met", "ref" $\}$ den jeweiligen Skalentypen zugeordnet sind. $x_{i k}^{t \text { tpe }}$ ist dabei der Wert der $k$-ten Präferenzvariable vom Skalentyp type $x_{k}^{t y p e}$ für Knoten $v_{i}$. Neben dem direkten paarweisen Vergleich von Objektmerkmalen ist es in manchen Fällen erforderlich, Ähnlichkeiten bzw. Distanzen durch ein indirektes Verfahren, d.h. durch den Vergleich mit einem von der Objektpaarung abhängigen Referenzwert, zu ermitteln. Aus diesem Grund beinhaltet die Präferenzmatrix $X^{\text {prefs }}$ zusätzlich zu den Variablen der Skalentypen binär nominal, mehrstufig nominal, ordinal und metrisch (intervall- oder verhältnisskaliert) auch Variablen vom Typ ,Referenzpräferenz', welcher im Rahmen dieser Arbeit erstmals vorgestellt und erläutert wird.

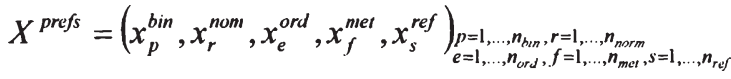

$$
\begin{aligned}
& x_{p}^{b i n}=\left(x_{i p}^{b i n}\right)_{i=1, \ldots, n} \\
& x_{r}^{\text {nom }}=\left(x_{i r}^{\text {nom }}\right)_{i=1, \ldots, n} \\
& x_{e}^{\text {ord }}=\left(x_{i e}^{\text {ord }}\right)_{i=1, \ldots, n} \\
& x_{f}^{m e t}=\left(x_{i f}^{m e t}\right)_{i=1, \ldots, n} \\
& x_{s}^{\text {ref }}=\left(x_{i s}^{r e f}\right)_{i=1, \ldots, n}
\end{aligned}
$$

\subsubsection{Binäre Nominaiskalen}

Der paarweise Vergleich von Knoten mit nominal skalierten, binären Präferenzvariablen stellt auf die Anzahl der Variablen ab, bei denen die Präferenzen der zu vergleichenden Knoten (nicht) übereinstimmen, wobei davon ausgegangen wird, dass ein Variablenwert von 1 das Vorhandensein einer Präferenz angibt, während 0 den gegenteiligen Fall anzeigt. Tabelle 32 stellt eine Kontingenztafel für den Vergleich zweier Knoten hinsichtlich deren binärer Variablen dar. 
Tabelle 32: Kontingenztafel für binäre Präferenzvariablen

Quelle: eigene Darstellung in Anlehnung an Fahrmeir et al. [1996, S. 443]

\begin{tabular}{|c|c|c|c|}
\hline & 1 & 0 & $\Sigma$ \\
\hline 1 & $a_{i j}$ & $c_{i j}$ & $a_{i j}+c_{i j}$ \\
\hline 0 & $b_{i j}$ & $d_{i j}$ & $b_{i j}+d_{i j}$ \\
\hline$\Sigma$ & $a_{i j}+b_{i j}$ & $c_{i j}+d_{i j}$ & $n_{\text {bin }}$ \\
\hline
\end{tabular}

Der Koeffizient $a_{i j}$, aus Tabelle 32 gibt die Anzahl der Präferenzen an, die bei Knoten $v_{i}$ und $v_{j}$ vorliegen. $b_{i j}$ quantifiziert hingegen die Anzahl an Präferenzen, die bei $v_{j}$, nicht aber bei $v_{i}$, gegeben sind. Der Koeffizient $c_{i j}$ steht wiederum für die Anzahl an Präferenzen, die $v_{i}$, nicht aber $v_{j}$, hat. Schließlich beschreibt $d_{i j}$ die Anzahl der binären Präferenzvariablen, die bei beiden betrachteten Knoten 0 betragen und daher einer gemeinsamen ,Nicht-Präferenz' entsprechen. Die in Tabelle 32 nicht beschriebene Summierung $a_{i j}+d_{i j}$ bzw. $b_{i j}+c_{i j}$ steht für die Anzahl der Übereinstimmungen bzw. die Anzahl der Nicht-Übereinstimmungen der Präferenzen des betrachteten Knotenpaares. Für das Verständnis der Generierung der Koeffizienten aus Tabelle 32 kann auch Formel (271) herangezogen werden.

$$
\begin{aligned}
& a_{i j}=\left|\left\{x_{k}^{b i n}, k=1, \ldots, n_{b i n}: x_{i k}^{b i n}=x_{j k}^{b i n}=1\right\}\right| \\
& b_{i j}=\left|\left\{x_{k}^{b i n}, k=1, \ldots, n_{b i n}:\left(x_{i k}^{b i n}=0 \wedge x_{j k}^{b i n}=1\right)\right\}\right| \\
& c_{i j}=\left|\left\{x_{k}^{b i n}, k=1, \ldots, n_{b i n}:\left(x_{i k}^{b i n}=1 \wedge x_{j k}^{b i n}=0\right)\right\}\right| \\
& d_{i j}=\left|\left\{x_{k}^{b i n}, k=1, \ldots, n_{b i n}: x_{i k}^{b i n}=x_{j k}^{b i n}=0\right\}\right|
\end{aligned}
$$

Basierend auf den über die Kontingenztafel ermittelten Häufigkeiten können unterschiedliche Ähnlichkeitsmaße errechnet werden. Die Mehrzahl dieser Maße beruht auf einem der drei folgend beschriebenen grundlegenden Muster, die in den Formeln (272), (273) und (274) definiert werden.

Der verallgemeinerte Matching- oder auch M-Koeffizient $s^{g M}$ wird in Formel (272) in Anlehnung an Fahrmeir et al. [1996, S. 444] erläutert. Der Koeffizient $s^{g M}$ beschreibt einen über $\gamma$ gewichteten Anteil derhëbereinstinamangent-žvischen den 
verglichenen Knoten (Objekten), wobei sowohl positive (Präferenz beiderseits 1) als auch negative (Präferenz beiderseits 0 ) Übereinstimmungen berücksichtigt werden. Dieses Ähnlichkeitsmaß ist demnach unabhängig von der Codierung der Variablenausprägungen. Wie in Formel (272) ersichtlich, wird die Anzahl an Nicht-Übereinstimmungen mit $(1-\gamma)$ gewichtet. Der Wert von $\gamma$ liegt zwischen 0 und 1, wobei die Gewichtung über $\gamma$ nur die Größenordnung der paarweisen Ähnlichkeiten einer Menge von Knoten (Objekten) ändert, nicht aber deren Reihenfolge.

$$
s_{i j}^{g M}=\frac{\gamma \cdot\left(a_{i j}+d_{i j}\right)}{\gamma \cdot\left(a_{i j}+d_{i j}\right)+(1-\gamma) \cdot\left(b_{i j}+c_{i j}\right)} \text { mit } 0<\gamma<1
$$

Der verallgemeinerte Similarity- oder auch S-Koeffizient $s^{g S, F}$ nach Fahrmeir et al. [1996, S. 445] kann über Formel (273) nachvollzogen werden. Mithilfe dieses Koeffizienten wird ein über $\gamma$ gewichteter Anteil der positiven Übereinstimmungen ermittelt, d.h. negative Übereinstimmungen werden nicht beachtet und die Codierung ist maßgeblich für das Ergebnis. Wie bei dem verallgemeinerten M-Koeffizienten gilt auch hier, dass $\gamma$ zwischen 0 und 1 liegt und nur die Größenordnung der Ähnlichkeiten, aber nicht deren Rangreihung durch $\gamma$ verändert wird. Im Allgemeinen stimmt die Reihenfolge, die durch den verallgemeinerten S-Koeffizienten entsteht, nicht mit jener des verallgemeinerten M-Koeffizienten überein.

$$
s_{i j}^{g S, F}=\frac{\gamma \cdot a_{i j}}{\gamma \cdot a_{i j}+(1-\gamma) \cdot\left(b_{i j}+c_{i j}\right)} \text { mit } 0<\gamma<1
$$

Der verallgemeinerte Similarity- oder auch S-Koeffizient $s^{g S, B}$ nach Backhaus et al. [2000, S. 333 mit Verweis auf Steinhausen \& Langer 1977] wird - ergänzt durch Indizes - in Formel (274) gezeigt. In dieser Version des verallgemeinerten S-Koeffizienten wird eine positive Übereinstimmung zwischen den Präferenzvariablen zweier Knoten immer mit 1 gewichtet, während eine negative Übereinstimmung bzw. eine Nicht-Übereinstimmung mit $\delta$ bzw. $\lambda$ geringer gewichtet werden kann. 


$$
s_{i j}^{g S, B}=\frac{a_{i j}+\delta \cdot d_{i j}}{a_{i j}+\delta \cdot d_{i j}+\lambda \cdot\left(b_{i j}+c_{i j}\right)} \text { mit } 0 \leq \delta, \lambda \leq 1
$$

Abhängig von der Wahl der Gewichte $\delta$ und $\lambda$ bzw. $\gamma$ sind über die Formel zu dem verallgemeinerten Similarity- bzw. Matchingkoeffizient spezifische Ähnlichkeitsmaße, die in der Literatur angeführt werden, berechenbar.

Der simple matching oder auch M-Koeffizient von Sokal \& Michener [1958] aus Formel (275) [Backhaus et al. 2000, S. 334; Fahrmeir et al. 1996, S. 444] kann über die Gewichte $\delta=1$ und $\lambda=1$ bei der Berechnung von $s^{g S, B}$ (Formel (274)) bzw. $\gamma=1 / 2$ bei der Ermittlung von $s^{g M}$ (Formel (272)) gewonnen werden. Der $M-K o e f f i z i e n t$ quantifiziert den ungewichteten Anteil der (positiven und negativen) Übereinstimmungen der Präferenzvariablen der paarweise zu vergleichenden Knoten.

$$
s_{i j}^{M}=\frac{a_{i j}+d_{i j}}{a_{i j}+d_{i j}+b_{i j}+c_{i j}}=\frac{a_{i j}+d_{i j}}{n_{b i n}}
$$

Kehrt man das Ähnlichkeitsmaß ,M-Koeffizient' zu einem Distanzmaß um, so entsteht die sogenannte ,Hamming distance' in einer normierten Fassung. Diese in Formel (276) angeführte Kennzahl beschreibt den ungewichteten Anteil der Nicht-Übereinstimmungen an allen betrachteten binären Variablen und wird häufig auch nur als Anzahl bestimmt.

$$
d_{i j}^{H}=1-s_{i j}^{M}=\frac{b_{i j}+c_{i j}}{a_{i j}+d_{i j}+b_{i j}+c_{i j}}=\frac{b_{i j}+c_{i j}}{n_{b i n}}
$$

Durch die Wahl von $\gamma=1 / 3$ zur Berechnung von $s^{g M}$ (Formel (272)) erhält man das Ähnlichkeitsmaß von Rogers \& Tanimoto, welches in Formel (277) [Fahrmeir et al. 1996 nach Rogers \& Tanimoto 1960] definiert wird. Bei diesem Ähnlichkeitsmaß fließen Nicht-Übereinstimmungen stärker in die Ermittlung der Maßzahl ein. 


$$
s_{i j}^{R T}=\frac{a_{i j}+d_{i j}}{a_{i j}+d_{i j}+2\left(b_{i j}+c_{i j}\right)}
$$

Die Wahl von $\gamma=2 / 3$ bei der Ermittlung des verallgemeinerten M-Koeffizienten $s^{g M}$ ermöglicht wiederum die Generierung des Ähnlichkeitsmaßes nach Sokal \& Sneath [Fahrmeir et al. 1996, S. 444 nach Sokal \& Sneath 1963]. Bei dieser in Formel (278) dargelegten Maßzahl werden im Gegensatz zu dem Ähnlichkeitsmaß von Rogers \& Tanimoto Übereinstimmungen stärker gewichtet.

$$
s_{i j}^{M, S S}=\frac{2\left(a_{i j}+d_{i j}\right)}{2\left(a_{i j}+d_{i j}\right)+b_{i j}+c_{i j}}
$$

Vergleichbar mit dem Ansatz von Rogers \& Tanimoto ist eine weitere Ähnlichkeitsmaßzahl von Sokal \& Sneath, die in Formel (279) [Fahrmeir et al. 1996, S. 445 nach Sokal \& Sneath 1963] definiert wird. Im Unterschied zu Rogers \& Tanimoto werden bei dieser Maßzahl allerdings negative Übereinstimmungen $\left(d_{i j}\right)$ nicht einbezogen. Die Maßzahl $s_{i j}^{s, S S}$ kann auch durch Setzen des Gewichtungsparameters $\gamma$ in Formel (273) für den verallgemeinerten S-Koeffizienten $s^{g S, F}$ auf $1 / 3$ ermittelt werden.

$$
s_{i j}^{S, s S}=\frac{a_{i j}}{a_{i j}+2\left(b_{i j}+c_{i j}\right)}
$$

Verwandt zu dem Ansatz, den Sokal \& Sneath in Formel (278) verfolgen, ist die Ähnlichkeitsmaßzahl von Dice, welche in Formel (280) [Fahrmeir et al. 1996, S. 445 nach Dice 1945; Backhaus et al. 2000, S. 334] beschrieben wird, wobei wie auch in Formel (279) negative Übereinstimmungen $\left(d_{i j}\right)$ aus der Berechnung genommen werden. Die Maßzahl von Dice kann über die verallgemeinerte S-Koeffizientenformel $s^{g S, B}$ nach Backhaus et al. (Formel (274)) mit den Gewichten $\delta=0$ und $\lambda=1 / 2$ bzw. über die verallgemeinerte S-Koeffizientenformel nach Fahrmeir et al. $s^{g S . F}$ (Formel (273)) mit dem Gewicht $\gamma=2 / 3$ gewonnen werden. 


$$
s_{i j}^{D}=\frac{2 a_{i j}}{2 a_{i j}+b_{i j}+c_{i j}}
$$

Der in Formel (281) [Backhaus et al. 2000, S. 334 nach Tanimoto 1957 bzw. Jaccard 1901; Fahrmeir et al. 1996; S. 445] beschriebene Tanimoto, Jaccard oder auch S-Koeffizient ist eine Maßzahl, die die Ähnlichkeit über den Anteil gemeinsamer Präferenzen (positive Übereinstimmungen) der paarweise verglichenen Knoten (Objekte) bezogen auf die Anzahl an Präferenzen, die mindestens von einem der beiden Knoten gewählt wurden, misst. Wie auch in den beiden vorangegangenen Formeln werden die negativen Übereinstimmungen $\left(d_{i j}\right)$ hierbei nicht berücksichtigt. Der S-Koeffizient kann über die beiden verallgemeinerten Formeln für den S-Koeffizienten generiert werden. Hierfür sind die Gewichte $\delta=0$ und $\lambda=1$ bei einer Berechnung über die verallgemeinerte S-Koeffizientenformel $s^{g S, B}$ nach Backhaus et al. (Formel (274)) bzw. $\gamma=1 / 2$ bei Einsatz der verallgemeinerten S-Koeffizientenformel $s^{g S, F}$ nach Fahrmeir et al. (Formel (273)) zu wählen .

$$
s_{i j}^{T J}=\frac{a_{i j}}{a_{i j}+b_{i j}+c_{i j}}
$$

Nicht dem verallgemeinerten S- oder M-Koeffizienten zuordenbar sind die in den Formeln (282), (283) und (284) vorgestellten Ähnlichkeitsmaße. Formel (282) [Backhaus et al. 2000, S. 334 nach Russell \& Rao 1940] präsentiert eine Maßzahl von Russell \& Rao, welche über den Anteil der positiven Übereinstimmungen an allen möglichen Vergleichsvariablen festgelegt wird.

$$
s_{i j}^{R R}=\frac{a_{i j}}{a_{i j}+d_{i j}+b_{i j}+c_{i j}}=\frac{a_{i j}}{n_{b i n}}
$$

Formel (283) wiederum zeigt das Ähnlichkeitsmaß von Kulczynski, welches dem Quotienten aus der Anzahl an positiven Übereinstimmungen und der Anzahl an Nicht-Übereinstimmungen entspricht [Backhaus et al. 2000, S. 334 nach Kulczynski 1927]. 


$$
s_{i j}^{K}=\frac{a_{i j}}{b_{i j}+c_{i j}}
$$

In der Literatur wird darüber hinaus auch ein Korrelationskoeffizient als Ähnlichkeitsmaß genannt [Fahrmeir et al. 1996, S. 446 u.a. nach Gower 1967]. Bei der Berechnung dieser in Formel (284) dargelegten Maßzahl ist zu beachten, dass die Matrix der binären Präferenzen (Submatrix $X^{b i n}=\left(x_{i p}^{b i n}\right)_{\substack{i=1, \ldots, n \\ p=1, \ldots, n_{h m}}}$ aus $X^{\text {prefs }}$ ) zunächst transponiert wird, d.h. die Knoten und Präferenzvariablen tauschen ihre Rollen, um die Korrelation zwischen den Knoten berechnen zu können. Hinzuweisen ist auf die Einschränkung, dass der Koeffizient $s_{i j}^{c o r r}$ nur dann definiert ist, wenn beide Knoten für die der Koeffizient berechnet wird mindestens einmal den Eintrag 0 und mindestens einmal den Eintrag 1 als Präferenzvariablenwert enthalten. Nachdem es sich bei dieser Maßzahl um einen Korrelationskoeffizienten handelt, reicht der Wertebereich von -1 bis 1 . Ein Wert von 1 bedeutet, dass das binäre Präferenzprofil der beiden verglichenen Knoten (Objekte) vollständig übereinstimmt, während bei einem Wert von -1 beide Präferenzprofile ausnahmslos komplementär sind.

$$
s_{i j}^{c o r r}=\frac{a_{i j} \cdot d_{i j}-b_{i j} \cdot c_{i j}}{\sqrt{\left(a_{i j}+c_{i j}\right) \cdot\left(b_{i j}+d_{i j}\right) \cdot\left(a_{i j}+b_{i j}\right) \cdot\left(c_{i j}+d_{i j}\right)}}
$$

Die Berechnung der hier diskutierten Ähnlichkeitsmaße für binäre Präferenzvariablen kann mittels der Funktion DIST.BINARYNEU erfolgen, die auf der Funktion DIST.BINARY aus dem R-Paket ,ade4' [Chessel et al. 2004] aufbaut.

\subsubsection{Mehrstufige Nominalskalen}

Haben die Präferenzvariablen mehr als zwei nominale Ausprägungen, so ist eine Erweiterung der oben beschriebenen Vorgehensweise zur Ermittlung der jeweiligen Ähnlichkeitsmaßzahlen erforderlich. Konkret müssen, wie auch bei Fahrmeir et al. [1996] und Backhaus et al. [2000] beschrieben, aber im Gegensatz zu folgenden Ausführungen nicht ausführlich spezifiziert und formalisiert, die 
mehrstufig nominal skalierten Präferenzvariablen $x_{k}^{\text {nom }}$ in binäre Hilfsvariablen (,Dummyvariablen') $x_{b i n, k, l}^{n o m}$ zerlegt werden, wobei deren Anzahl $n_{b i n, k}$ jeweils der Anzahl der nominalen Kategorien $c^{a} t_{k l}$ von $x_{k}^{\text {mom }}$ entspricht. Im Rahmen der Zerlegung wird für jede Kategorie $c a t_{k l}^{n o m}$ der mehrstufigen nominalen Präferenzvariable $x_{k}^{\text {nom }}$ eine eigene binäre Hilfspräferenzvariable $x_{b i n, k, l}^{\text {nom }}$ erzeugt. Der Wert dieser Hilfspräferenzvariable beträgt 1 , wenn die Ausprägung der nominalen Präferenzvariable der Kategorie $c a t_{k l}^{n o m}$ entspricht, und 0 andernfalls. $x_{b i n, k, i l}^{\text {nom }}$ aus Formel (285) ist der Wert der aus der Präferenzvariable $x_{k}^{\text {nom }}$ abgeleiteten binären Hilfsvariable $x_{b i n, k, l}^{\text {nom }}$ für den Knoten $v_{i}$.

$$
\begin{aligned}
x_{b i n, k, l}^{\text {nom }} & =\left(x_{b i n, k, i l}^{\text {nom }}\right)_{i=1, \ldots, n} \quad \text { für } k=1, \ldots, n_{\text {nom }} \wedge l=1, \ldots, n_{\text {bin }, k}=n c_{k}=\mid \text { Cat }_{k}^{\text {nom }} \mid \\
x_{b i n, k, l l}^{\text {nom }} & = \begin{cases}1 & \text { wenn } x_{i k}^{\text {nom }}=\text { cat } \\
0 & \text { ander }\end{cases}
\end{aligned}
$$

wobei cat $_{k l}^{\text {nom }} \in$ Cat $_{k}^{\text {nom }}=\left\{\right.$ cat $_{k 1}^{\text {nom }}, \ldots$, cat $\left._{k n c_{k}}^{\text {nom }}\right\}$

Anschließend an die Ermittlung der binären Hilfsvariablen $x_{b i n, k, l}^{n o m}, l=1, \ldots, n c_{k}$, kann die Kontingenztafel (vgl. Tabelle 32) für alle Präferenzvariablen $x_{k}^{\text {nom }}, k=1, \ldots, n_{\text {nom }}$, über alle abgeleiteten binären Hilfsvariablen entsprechend der Formel (286) erstellt werden. Auf Basis der ermittelten Kontingenztafel können die bereits vorgestellten Maßzahlen für binär skalierte Datentypen angewendet werden, um die Ähnlichkeit der zu vergleichenden Knoten (Objekte) zu quantifizieren (Funktion DIST.BINARYNEU).

$$
\begin{aligned}
& a_{i j}=\left|\left\{x_{b i, k, l}^{\text {nom }}, k=1, \ldots, n_{n o m} \wedge l=1, \ldots, n c_{k}: x_{b i n, k, i l}^{n o m}=x_{b i n, k, j l}^{n o m}=1\right\}\right| \\
& b_{i j}=\left|\left\{x_{b i n, k, l}^{\text {nom }}, k=1, \ldots, n_{\text {nom }} \wedge l=1, \ldots, n c_{k}:\left(x_{b i n, k, i l}^{n o m}=0 \wedge x_{b i n, k, j l}^{n o m}=1\right)\right\}\right| \\
& c_{i j}=\left|\left\{x_{b i n, k, l}^{n o m}, k=1, \ldots, n_{n o m} \wedge l=1, \ldots, n c_{k}:\left(x_{b i n, k, i l}^{n o m}=1 \wedge x_{b i n, k, j l}^{n o m}=0\right)\right\}\right|
\end{aligned}
$$

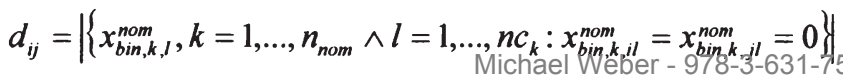


Dieses Vorgehen ist allerdings dann problematisch, wenn die Anzahl an nominalen Kategorien zwischen den mehrstufig nominalen Präferenzvariablen variiert, nachdem die Kategorien von Präferenzvariablen mit einer relativ höheren Anzahl an Kategorien schwächer gewichtet werden als jene der Präferenzvariablen mit einer verhältnismäßig geringeren Anzahl an Kategorien. Zur Vermeidung einer solchen Verzerrung sollte die Anzahl der Kategorien der einzelnen Variablen in die Berechnung einbezogen werden. Dies bedeutet, dass anstatt einer Kontingenztafel für alle abgeleiteten binären Variablen gemäß Formel (286) für jede mehrstufige nominale Variable $x_{k}^{\text {nom }}$ eine eigene Kontingenztafel für die zu der Variable gehörenden abgeleiteten binären Hilfsvariablen $x_{b i n, k, l}^{n o m}$ mit den Koeffizienten $a_{k, i j}, b_{k, i j}, c_{k, i j}, d_{k, i j}$ sowie $n_{b i n, k}$ entsprechend der Formel (287) zu erstellen ist.

$$
\begin{aligned}
a_{k, i j} & =\left|\left\{x_{b i n, k, l}^{n o m}, l=1, \ldots, n c_{k}: x_{b i n, k, i l}^{n o m}=x_{b i n, k, j l}^{n o m}=1\right\}\right| \\
b_{k, i j} & =\left|\left\{x_{b i n, k, l}^{n o m} l=1, \ldots, n c_{k}:\left(x_{b i n, k, l l}^{n o m}=0 \wedge_{b i n, k, j l}^{n o m}=1\right)\right\}\right| \\
c_{k, i j} & =\left|\left\{x_{b i n, k, l}^{n o m} l=1, \ldots, n c_{k}:\left(x_{b i n, k, l}^{n o m}=1 \wedge x_{b i n, k, j l}^{n o m}=0\right)\right\}\right| \\
d_{k, i j} & =\left|\left\{x_{b i n, k, l}^{n o m}, l=1, \ldots, n c_{k}: x_{b i n, k, i l}^{n o m}=x_{b i n, k, j l}^{n o m}=0\right\}\right| \\
n_{b i n, k} & =n c_{k}=\left|C a t_{k}^{n o m}\right|
\end{aligned}
$$

Zur Berücksichtigung der einzelnen Kontingenztabellen pro nominaler Präferenzvariable müssen die zuvor beschriebenen Formeln zur Berechnung von Ähnlichkeitsmaßzahlen angepasst werden. Beispielsweise zeigt Formel (289) die notwendigen Adaptierungen bei der Berechnung des gewichteten M-Koeffizienten für mehrstufige nominale Variablen. Das Attribut ,gewichtet' besagt, dass Verzerrungen durch die Gewichtung von Variablen mit unterschiedlicher Anzahl entzerrt werden. Formel (288) definiert den ungewichteten M-Koeffizienten für mehrstufige nominale Variablen auf Basis der Kontingenztafeln der einzelnen Präferenzvariablen (Formel (287)). Dies stellt einen Zwischenschritt bei der Herleitung von Formel (289) dar und wird zwecks Nachvollziehbarkeit angeführt. 


$$
s_{i j}^{M, m}=\frac{\sum_{k=1}^{n_{\text {nom }}}\left(a_{k, i j}+d_{k, i j}\right)}{\sum_{k=1}^{n_{\text {nom }}}\left(a_{k, i j}+d_{k, i j}+b_{k, i j}+c_{k, i j}\right)}=\frac{\sum_{k=1}^{n_{\text {nom }}}\left(a_{k, i j}+d_{k, i j}\right)}{\sum_{k=1}^{n_{\text {nom }}} n_{b i n, k}}
$$

Bei dem gewichteten M-Koeffizienten für mehrstufige nominale Variablen (Formel (289) in Anlehnung an Fahrmeir et al. [1996] bzw. Hyvärinen [1962]) erfolgt die Berücksichtigung der Anzahl der Kategorien der nominalen Variablen durch Multiplikation der Häufigkeit der (positiven und negativen) Übereinstimmungen der binären Hilfsvariablen einer Präferenzvariable mit der Anzahl der Kategorien dieser Präferenzvariable, d.h. der Anzahl ihrer binären Hilfsvariablen.

$$
\begin{aligned}
s_{i j}^{w M, m} & =\frac{\sum_{k=1}^{n_{\text {mom }}} n_{b i n, k} \cdot\left(a_{k, i j}+d_{k, i j}\right)}{\sum_{k=1}^{n_{\text {nom }}}\left(a_{k, i j}+d_{k, i j}+b_{k, i j}+c_{k, i j}\right)}=\frac{\sum_{k=1}^{n_{\text {nom }}} n_{b i n, k} \cdot\left(a_{k, i j}+d_{k, j}\right)}{\sum_{k=1}^{n_{n o m}} n_{b i n, k}} \\
& =\sum_{k=1}^{n_{\text {nom }}} \frac{n_{b i n, k}}{\sum_{h=1}^{n_{\text {nom }}} n_{b i n, h}} \cdot\left(a_{k, i j}+d_{k, i j}\right)
\end{aligned}
$$

Nachdem die Mehrzahl der oben vorgestellten Ähnlichkeitsmaßzahlen aus den Formeln zu den verallgemeinerten Maßzahlen $s^{g M}, s^{g S, F}$ und $s^{g S, B}$ abgeleitet werden können, sollen nachfolgend nur die Adaptierungen der dazugehörigen Formeln (272), (273) bzw. (274) zur Definition von gewichteten verallgemeinerten Koeffizienten für mehrstufige nominale Variablen beschrieben werden. Bei der Generierung von ungewichteten verallgemeinerten Koeffizienten für mehrstufige nominale Variablen aus den Kontingenztafeln nach Formel (287) entfällt in den Formeln (290) bis (292) die Multiplikation mit $n_{b i n, k}$ im Zähler.

Der gewichtete verallgemeinerte $M$-Koeffizient für mehrstufige nominale Variablen, der die Anzahl der Kategorien der nominalen Variablen berücksichtigt, kann demnach unter Einsatz der Formel (290) errechnet werden.

$$
s_{i j}^{\mathrm{wgM}, m}=\frac{\sum_{k=1}^{n_{\text {nom }}} n_{b i n, k} \cdot \gamma \cdot\left(a_{k, i j}+d_{k, i j}\right)}{\sum_{k=1}^{n_{\text {nom }}}\left(\gamma \cdot\left(a_{k, i j}+d_{k, i j}\right)+(1-\gamma) \cdot\left(b_{k, i j}+c_{k, i j}\right)\right)} \text { mit } 0<\gamma<1
$$


Formel (291) zeigt den gewichteten verallgemeinerten S-Koeffizient $s_{i j}^{\mathrm{wg} S, F, m}$ für mehrstufige nominale Variablen unter Berücksichtigung der Anzahl der Kategorien der nominalen Variablen.

$$
s_{i j}^{w g S, F, m}=\frac{\sum_{k=1}^{n_{n u m}} n_{b i n, k} \cdot \gamma \cdot a_{k, j}}{\sum_{k=1}^{n_{n o m}}\left(\gamma \cdot a_{k, j}+(1-\gamma) \cdot\left(b_{k, j}+c_{k, j}\right)\right)} \text { mit } 0<\gamma<1
$$

Der gewichtete verallgemeinerte S-Koeffizient $s_{i j}^{\mathrm{wg} S, B, m}$ für mehrstufige nominale Variablen, der - wie auch die beiden vorangegangenen Koeffizienten - die Anzahl der Kategorien der nominalen Variable berücksichtigt, kann Formel (292) entnommen werden.

$$
s_{i j}^{w g S, B, m}=\frac{\sum_{k=1}^{n_{n o m}} n_{b i n, k} \cdot\left(a_{k, i j}+\delta \cdot d_{k, i j}\right)}{\sum_{k=1}^{n_{m o m}}\left(a_{k, i j}+\delta \cdot d_{k, j j}+\lambda \cdot\left(b_{k, j}+c_{k, j}\right)\right)} \quad \text { mit } 0 \leq \delta, \lambda \leq 1
$$

\subsubsection{Ordinale Skalen}

Die Berechnung von Ähnlichkeits- bzw. Distanzmaßzahlen für ordinal skalierte Variablen kann entweder basierend auf dem Ansatz für mehrstufig nominal skalierte Variablen (Funktion DIST.BINARYNEU) oder aber - wie nachfolgend erläutert - unter Einsatz metrischer Verfahren (Funktionen zur Ermittlung von Distanzmatrizen aus den in Kapitel 10.1.4 genannten R-Paketen) erfolgen.

Bei der Wahl des ersteren Ansatzes ist zunächst für jede ordinale Präferenzvariable $x_{k}^{\text {ord }}$ pro ordinaler Kategorie $c a t_{k l}^{\text {ord }}$ eine binäre Hilfsvariable $x_{b i n, k, l}^{\text {ord }} \mathrm{zu}$ generieren. Hierbei ist zu beachten, dass die Kategorien jeweils nach ihrer Rangreihenfolge geordnet sind, d.h. $c a t_{k 1}^{\text {ord }}$ hat einen höheren Rang als $c a t_{k 2}^{\text {ord }}$ usw. Die Reihenfolge hat eine direkte Auswirkung auf die binäre Hilfsvariable $x_{b i n, k, l}^{\text {ord }}$, denn diese hat nur dann den Wert 1, wenn die Ausprägung der ordinalen Präferenzvariable $x_{k}^{\text {ord }}$ zumindest der Kategorie $c a t_{k l}^{\text {ord }}$ entspricht oder - anders 
formuliert - den Rang der Kategorie cat ${ }_{k l}^{\text {ord }}$ erreicht hat. Formel (293) definiert diese Zusammenhänge.

$$
\begin{aligned}
& x_{b i n, k, l}^{\text {ord }}=\left(x_{b i n, k, i l}^{\text {ord }}\right)_{i=1, \ldots, n} \quad \text { für } k=1, \ldots, n_{\text {ord }} \wedge l=1, \ldots, n c_{k}=\left|C a t_{k}^{\text {ord }}\right|
\end{aligned}
$$

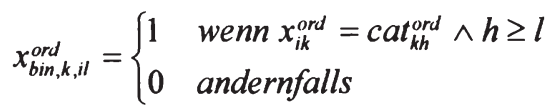

$$
\begin{aligned}
& \text { wobei } \text { cat }_{k l}^{\text {ord }} \in \text { Cat }_{k}^{\text {ord }}=\left\{\text { cat }_{k 1}^{\text {ord }}, \ldots, \text { cat }_{k n c_{k}}^{\text {ord }}\right\} \\
& \text { mit } \text { cat }_{k 1}^{\text {ord }} \prec \text { cat }{ }_{k 2}^{\text {ord }} \prec \ldots \prec \text { cat }{ }_{k n c_{k}}^{\text {ord }}
\end{aligned}
$$

Anschließend können die Berechnungsmethoden für mehrstufige Nominalvariablen gewichtet oder ungewichtet angewendet werden, wobei die obige Kritik zur ungewichteten Berechnung auch in diesem Zusammenhang aufrecht erhalten bleibt. Beispielsweise könnte der M-Koeffizient für mehrstufige Nominalvariablen entweder mit (Formeln (287) und (289)) oder ohne Gewichtung (Formeln (286) und (288)) gewählt werden, wobei - unabhängig von der gewählten Maßzahl - in Formel (286) bzw. (287) das Superskript nom durch das Superskript ord zu ersetzen ist.

Bei der Wahl eines metrischen Verfahrens zur Bestimmung der Ähnlichkeit von ordinal skalierten Variablen sind diese zunächst als metrisch zu interpretieren bzw. interpretierbar zu machen. Hierzu sind die geordneten Kategorien cat $_{k h}^{\text {ord }}, h=1, \ldots, n c_{k}$, der Variablen $x_{k}^{\text {ord }}$ in einen Index umzuwandeln, d.h. laufend durchzunummerieren, wie Formel (294) zeigt.

$$
\begin{aligned}
& x_{\text {mel }, k}^{\text {ord }}=\left(x_{\text {met }, i k}^{\text {ord }}\right)_{i=1, \ldots, n} \quad \text { für } k=1, \ldots, n_{\text {ord }} \\
& x_{\text {met }, i k}^{\text {ord }}=h \quad \text { wenn } x_{i k}^{\text {ord }}=c a t_{k h}^{\text {ord }}
\end{aligned}
$$

Zur Vermeidung von Verzerrungen, die durch die unterschiedliche Anzahlen von Kategorien bei verschiedenen Präferenzvariablen auftreten können, werden die Werte üblicherweise auf das Intervall $[0,1]$ über die Division durch die jeweilige Anzahl der Kategorien normiert (Formel (295)). 


$$
\begin{aligned}
& x_{\text {meiNorm }, k}^{\text {ord }}=\left(x_{\text {metNorm }, i k}^{\text {ord }}\right)_{i=1, \ldots, n} \text { für } k=1, \ldots, n_{\text {ord }} \\
& x_{\text {metNorm }, i k}^{\text {ord }}=\frac{x_{\text {mel }, i k}^{\text {ord }}}{n c_{k}}
\end{aligned}
$$

Im Anschluss an diese Prozedur sind Maßzahlen zur Quantifizierung der Übereinstimmung von metrischen Variablen, wie nachstehend spezifiziert, zu verwenden.

\subsubsection{Metrische Skalen}

Für metrisch skalierte Variablen erfolgt die Bestimmung der (Un-)Ähnlichkeit üblicherweise über Distanzmaße. Die wichtigsten sollen in den folgenden Ausführungen unter Bezug auf Fahrmeir et al. [1996] erläutert werden. Zuvor ist allerdings noch auf eine bedeutende Frage, die im Zusammenhang mit den Distanzmaßen zu stellen ist, hinzuweisen. Diese Frage betrifft die Einordnung eines Distanzmaßes als skalen- oder translationsinvariant. Skaleninvarianz beschreibt hierbei die Forderung, dass die berechnete Distanz zwischen den paarweise verglichenen Objekten (Knoten) unabhängig von der gewählten Maßeinheit der Objekteigenschaften ist. Sowohl bei metrischen intervallskalierten Variablen als auch bei metrischen verhältnisskalierten Variablen kann diese Forderung gestellt werden, nachdem deren Maßeinheiten grundsätzlich frei wählbar sind. Das Erfordernis der Translationsinvarianz, d.h. der Eigenschaft eines Distanzmaßes, nicht von der Wahl des Koordinatenursprungs abhängig zu sein, ist naturgemäß hingegen nur für metrische intervallskalierte Variablen von Relevanz, nachdem bei solchen Variablen neben der Maßeinheit auch die Wahl des Koordinatenursprungs frei ist.

Formel (296) [Fahrmeir et al. 1996, S. 448] zeigt den Ausgangspunkt der Minkowski- $q$-Metriken ( $L_{q}$-Distanzen). Abhängig von der Wahl von $q$ ergeben sich aus dieser Formel unterschiedliche metrische Distanzmaße wie die City-BlockMetrik (Manhattan-Metrik oder auch $\mathrm{L}_{1}$-Distanz, $q=1$ ) oder die Euklidische Distanz ( $\mathrm{L}_{2}$-Distanz, $q=2$ ). Letztere wird häufig auch als quadrierte Euklidische Distanz, d.h. als Summe der quadriertehnAbweichangen,3-Berectinet] Diese 
Variation bedeutet für Formel (296), dass die Quadratwurzel wegfällt. Eine quadrierte euklidische Distanz wird nicht mehr als $L_{q}$-Distanz klassifiziert. Während die City-Block-Metrik die Distanz als Summe der absoluten Abstände in den einzelnen Variablen misst, liefert die euklidische Distanz den kürzesten (direkten) Abstand zwischen zwei Knoten (Objekten) im durch deren Merkmale aufgespannten Koordinatensystem.

$$
d_{i j}^{L_{q}}=\left(\sum_{k=1}^{n_{m e t}}\left|x_{i k}^{m e t}-x_{j k}^{m e t}\right|^{q}\right)^{\frac{1}{q}} \quad \text { mit } q>1
$$

Die Minkowski- $q$-Metriken sind translations- aber nicht skaleninvariant, weshalb die Variablen vor der Distanzberechnung auf eine einheitliche Skala (Maßeinheit) gebracht werden müssen. Eine üblicherweise eingesetzte Transformationsprozedur ist eine Standardisierung, wie sie in Formel (297) beschrieben wird. Zur Vermeidung des Einflusses der Maßeinheiten auf die zu ermittelnde Distanz ist anschließend an die Transformation $z_{i k}^{m e t}$ anstatt $x_{i k}^{m e l}$ und $z_{j k}^{m e t}$ anstatt $x_{j k}^{m e t}$ in Formel (296) einzusetzen.

$$
\begin{aligned}
z_{i k}^{\text {met }} & =\frac{x_{i k}^{\text {met }}-\bar{x}_{k}^{\text {met }}}{s_{x_{k}^{m e t}}^{(q)}} \\
& w o b e i \\
\bar{x}_{k}^{\text {met }} & =\frac{1}{n} \cdot \sum_{i=1}^{n} x_{i k}^{\text {met }} \\
s_{x_{k}^{m e t}}^{(q)} & =\left(\frac{1}{n} \cdot \sum_{i=1}^{n}\left|x_{i k}^{\text {met }}-\bar{x}_{k}^{\text {met }}\right|^{q}\right)^{\frac{1}{q}}
\end{aligned}
$$

Die in Formel (298) [Fahrmeir et al. 1996, S. 450] definierte Mahalanobis-Distanz [Mahalanobis 1936] ist ein Beispiel für ein metrisches Distanzmaß, welches sowohl skalen- als auch translationsinvariant ist. Die Mahalanobis-Distanz berücksichtigt im Gegensatz zu den Minkowski-q-Metriken die Korrelation der Variablen. Sie entspricht der quadrierten euklidischen Distanz der über die 
Korrelationsmatrix transformierten (,entkorrelierten') Variablen. Eine Standardisierung ist für die Mahalanobis-Distanz nicht erforderlich.

$$
\begin{aligned}
d_{i j}^{M} & =\left(x_{i}^{m e t}-x_{j}^{m e t}\right)^{\prime} \cdot K^{-1} \cdot\left(x_{i}^{m e t}-x_{j}^{m e t}\right) \\
K & =\frac{1}{n} \sum_{i=1}^{n}\left(x_{i}^{m e t}-\bar{x}^{m e t}\right) \cdot\left(x_{i}^{m e t}-\bar{x}^{m e t}\right)^{\prime} \\
\bar{x}^{\text {met }} & =\frac{1}{n} \sum_{i=1}^{n} x_{i}^{m e t} \\
x_{i}^{\text {met }} & =\left(x_{i k}^{m e t}\right)_{k=1, \ldots, n_{\text {met }}}
\end{aligned}
$$

Eine einfache Methode zur Bestimmung der Distanz zweier Knoten (Objekte) stellt der größte absolute Abstand der einzelnen metrischen Variablen dar. Diese maximale Distanz wird in Formel (299) definiert. Analog dazu kann auch der minimale absolute Abstand als Distanzmaß herangezogen werden. Sowohl die minimale als auch die maximale Distanz sind zwar translations-, nicht jedoch skaleninvariant, weshalb hier, analog zu den Minkowski-q-Metriken, vor der Distanzberechnung eine Standardisierung der Variablen durchzuführen ist.

$$
d_{i j}^{\max }=\max _{k=1, \ldots, n_{m e t}}\left(\left|x_{i k}^{m e t}-x_{j k}^{m e t}\right|\right)
$$

Die in Formel (300) definierte Canberra-Distanz kann als relative ManhattanDistanz betrachtet werden, da sie ebenfalls aus den absoluten Abständen der einzelnen Variablen berechnet wird. Allerdings erfolgt die Summenbildung bei der Canberra-Distanz über die Anteile dieser absoluten Abstände jeweils bezogen auf den Absolutbetrag der Summe der Variablenwerte. Variablen, für die der Nenner 0 ergibt, werden in der Summe nicht berücksichtigt. Auch die CanberraDistanz ist translations-, aber nicht skaleninvariant. Mittels Standardisierung der Variablen kann dieses Problem behoben werden.

$$
d_{i j}^{C}=\sum_{k=1}^{n_{\text {mel }}} \frac{\left|x_{i k}^{m e r}-x_{j k}^{m e t}\right|}{\left|x_{i k}^{m e t}+x_{j k}^{m e t}\right|}
$$


Der Q-Korrelationskoeffizient aus Formel (301) [Backhaus et al. 2000] ist ein weiteres Maß für die Ähnlichkeit zweier Objekte (Knoten). Aufgrund der Berücksichtigung von Lage und Streuung in der Berechnung der Korrelation ist dieser Koeffizient skalen- und translationsinvariant.

$$
s_{i j}^{Q c o r r}=\frac{\sum_{k=1}^{n_{\text {met }}}\left(x_{i k}^{\text {met }}-\bar{x}_{i}^{\text {met }}\right) \cdot\left(x_{j k}^{\text {met }}-\bar{x}_{j}^{\text {met }}\right)}{\sqrt{\sum_{k=1}^{n_{\text {met }}}\left(x_{i k}^{\text {met }}-\bar{x}_{i}^{\text {met }}\right)^{2} \cdot \sum_{k=1}^{n_{\text {mel }}}\left(x_{j k}^{\text {met }}-\bar{x}_{j}^{\text {met }}\right)^{2}}}
$$

Verzichtet man in der Berechnung des Q-Korrelationskoeffizienten auf die Zentrierung, d.h. auf das Abziehen des Mittelwertes, erhält man das CosinusÄhnlichkeitsmaß (Formel (302)). Durch diese Maßnahme geht die Translationsinvarianz verloren, während die Skaleninvarianz erhalten bleibt. Das CosinusÄhnlichkeitsmaß kommt aufgrund seiner Interpretation als Cosinus des Winkels $\alpha$ zwischen $x_{i}^{\text {met }}$ und $x_{j}^{\text {met }}$ zum Einsatz. Durch Transformation in ein Distanzmaß, d.h. $1-s_{i j}^{\text {cos }}$, erhält man die Winkeldistanz. Bei Verwendung zentrierter oder gar standardisierter Variablen entspricht das Cosinus-Ähnlichkeitsmaß dem QKorrelationskoeffizienten.

$$
s_{i j}^{\cos }=\cos \alpha\left(x_{i}^{m e t}, x_{j}^{m e t}\right)=\frac{\sum_{k=1}^{n_{\text {mel }}}\left(x_{i k}^{\text {met }} \cdot x_{j k}^{m e t}\right)}{\sqrt{\sum_{k=1}^{n_{\text {met }}}\left(x_{i k}^{m e t}\right)^{2} \cdot \sum_{k=1}^{n_{\text {met }}}\left(x_{j k}^{\text {met }}\right)^{2}}}
$$

Abschließend zu den Ausführungen über Ähnlichkeits- bzw. Distanzmaße soll darauf hingewiesen werden, dass Berechnungsverfahren zu vielen dieser Maßzahlen in der statistischen Programmierumgebung $R$ [R 2007], bspw. in RPaketen wie ,stats' [R 2007], ,cluster' [Maechler et al. 2005] oder ,flexclust' [Leisch 2006], über spezielle Funktionen implementiert wurden.

\subsection{Referenzpräferenzvergleich - indirekter Vergleich zweier Objekte}

Die (Nicht-)Übereinstimmung zweier Objekte (Knoten) oder Objektmengen wird üblicherweise direkt über einen Vergleich der jeweiligen Variablenwerte ge- 
messen. Implizite Voraussetzung hierbei ist, dass das Merkmal, auf welches sich die Variable bezieht, von dem jeweils verglichenen Objektpaar (Knotenpaar) unabhängig ist, d.h. für jeden Knoten separat gemessen wird. Trifft diese Voraussetzung allerdings nicht zu, d.h. ist die Übereinstimmung zweier Objekte (Knoten) oder Objektmengen unter Einbezug eines Merkmals festzustellen, welches in Abhängigkeit zu dem verglichenen Paar steht, so ist ein direkter Vergleich der jeweiligen Variablenwerte nicht zielführend. Vielmehr erscheint ein indirekter Vergleich der Variablenwerte (Präferenzen) unter Einbindung des von der Objektpaarung abhängigen Merkmals (Referenzmerkmal) zweckmäßig. Ein solches indirektes Verfahren, welches nachfolgend als ,indirekter Präferenzvergleich' oder ,Referenzpräferenzvergleich' bezeichnet wird, eignet sich u.a. auch bei dem Vorliegen von dualen Präferenzen, deren Dualität sich aus der Betrachtung sowohl der Input- als auch der Output-Perspektive ergibt. Erforderlich ist hierbei eine vom (indirekten) Präferenzvergleich abhängige Funktion (Relations- oder Ergänzungsfunktion), die über den Referenzwert festhält, wie sich die Objektpaarung auf das in der Präferenz betrachtete, abhängige Referenzmerkmal auswirkt. Der Referenzpräferenzvergleich kann unabhängig vom Skalentyp wie folgt ablaufen:

1. In einem ersten Schritt ist der Wert des Referenzmerkmals $r e f_{k, i j}$ der beiden verglichenen Objekte $v_{i}$ und $v_{j}$ über die Relationsfunktion $f_{k}$ aus den Werten des zugrunde liegenden Merkmals $\operatorname{ref}_{k}\left(v_{i}\right)$ bzw. $\operatorname{ref}_{k}\left(v_{j}\right)$ zu bestimmen, wobei das Subskript $k$ den Bezug zur Präferenzvariable $x_{k}^{\text {ref }}$ herstellt (Formel (303)).

$$
r e f_{k, j}=f_{k}\left(r e f_{k}\left(v_{i}\right), r e f_{k}\left(v_{j}\right)\right) \text { für } k=1, \ldots, n_{r e f}
$$

2. Anschließend werden die Präferenzen beider Objekte jeweils mit diesem Referenzwert über eine zu dem Skalenniveau passende (Un-)Ähnlichkeitsmaßzahl verglichen (Formel (304) für eine Distanzmaßzahl), wobei das Skalenniveau der Referenz mit dem der Präferenzen übereinstimmen muss. 


$$
\begin{aligned}
\text { für } k & =1, \ldots, n_{r e f}: \\
d_{k, i j}^{r e f, i} & =d^{t y p e}\left(x_{i k}^{r e f}, r e f_{k, i j}\right) \\
d_{k, i j}^{r e f, j} & =d^{t y p e}\left(x_{j k}^{r e f}, r e f_{k, i j}\right)
\end{aligned}
$$

wobei type $\in$ Type \"ref"

3. Schließlich sind beide Vergleichsergebnisse zu einem Maß der indirekten (Nicht-)Übereinstimmung (u.U. gewichtet) zusammenzuführen (Formel (305)).

$$
d_{k, i j}^{r e f}=\omega \cdot d_{k, i j}^{r e f, i}+(1-\omega) \cdot d_{k, i j}^{\text {ref, }, j} \quad \text { mit } 0 \leq \omega \leq 1 \quad \text { für } k=1, \ldots, n_{\text {ref }}
$$

Falls für mehrere Präferenzvariablen ein Referenzpräferenzvergleich erforderlich ist, so sind die jeweiligen Maße der indirekten (Nicht-)Übereinstimmung - z.B. durch Mittelwertbildung (Formel (306)) - in ein Gesamt-(Un-)Ähnlichkeitsmaß zu integrieren. Dabei muss zuvor eine Normierung der Maßzahlen auf das Intervall $[0,1]$ durchgeführt werden. Alternativ kann bei Vorliegen von Präferenzvariablen auf unterschiedlichen Skalenniveaus zunächst eine Aggregation (Mittelwertbildung) pro Skalenniveau erfolgen (Formel (307)). Die so entstandenen Maßzahlen werden anschließend (bspw. durch eine mit der jeweiligen Anzahl an (Referenz-)Präferenzvariablen pro Skalentyp gewichtete Mittelwertbildung, Formel (308)) zu einer Gesamtmaßzahl zusammengefasst.

$$
\begin{aligned}
d_{i j}^{\text {ref }}=\frac{1}{n_{\text {ref }}} \cdot \sum_{k=1}^{n_{\text {ref }}} d_{k, i j}^{\text {ref }} \\
d_{i j}^{\text {ref }, t}=\frac{1}{n_{\text {ref }, t}} \cdot \sum_{k \in K_{t}} d_{k, i j}^{\text {ref, }} \\
t \in T y p e \backslash " r e f^{\prime \prime} \\
K_{t}=\left\{k \in\left\{1, \ldots, n_{\text {ref }}\right\}: x_{k}^{\text {ref, thpe }} \wedge \text { type }=t\right\} \\
n_{\text {ref }, t}=\left|K_{t}\right|
\end{aligned}
$$




$$
d_{i j}^{r e f}=\frac{1}{n_{r e f}} \cdot \sum_{t \in T}\left(n_{r e f, l} \cdot d_{i j}^{r e f, t}\right)
$$

Zur Veranschaulichung der Notwendigkeit und der Ausgestaltung eines Referenzpräferenzvergleichs können geografische Entfernungen als Beispiel herangezogen werden. Zwei Knoten $v_{i}$ und $v_{j}$ haben $x_{i k}^{r e f}$ bzw. $x_{j k}^{\text {ref }}$ als Präferenz für die maximale Reichweite ihrer Aktivitäten ${ }^{63}$. Nachdem ein direkter Vergleich der beiden Präferenzen geringe Aussagekraft bezüglich der Übereinstimmung der Aktivitätsradien beider Knoten hat, scheint ein indirekter Vergleich zweckmäßig. Entsprechend den obigen Ausführungen muss hierfür eine Referenz, die sich auf die Paarung $v_{i}$ und $v_{j}$ bezieht, festgelegt werden. Für das Beispiel wird daher ausgehend von der Kenntnis der Standorte von $v_{i}$ bzw. $v_{j}$ passend zu der Präferenzvariable die geografische Entfernung der beiden Knoten als Referenzwert bestimmt (Formel (309)). Dies kann z.B. aufbauend auf der Kenntnis der Breiten- und Längengrade $b_{i}, b_{j}, l_{i}$ und $l_{j}$ der Standorte von $v_{i}$ und $v_{j}$ auf Basis einer Funktion zur geografischen Entfernungsmessung - wie in Formel (310) nach [Meeus 2000] gezeigtt - erfolgen. Diese Funktion übernimmt somit in diesem Beispiel die Rolle der Relations- oder auch Ergänzungsfunktion.

Für den getrennten Vergleich der beiden Präferenzen mit dem Referenzwert kann anschließend bspw. eine spezielle Distanzfunktion gemäß Formel (311) verwendet werden (Funktion DISTCOMPREF). Die Bestimmung von $d_{k, i j}^{\text {comp ref }, j}$

63 Eine metrische Präferenzvariable mit einer Intervallskala von 0 bis $\infty$ könnte zu diesem Zweck definiert werden. Während $\infty$ für eine unbegrenzte Reisebereitschaft steht, zeigt ein Wert von 0 Unbeweglichkeit an.

64 Formel (310) stellt eine nicht exakte Entfernungsberechnungsmethode dar. Eine exaktere und WGS84 (World Geodetic System 1984) konforme Berechnung liefert die Funktion COORDIST. Mit der Funktion SELORTSDIST können Entfernungen aus einer Entfernungsdatenbank bzw. -tabelle abgerufen werden. D2DEZ hilft bei der Umwandlung geografischer Angaben zwischen einer Darstellung in Grad, Minuten und Sekunden und einer Dezimaldarstellupg 
erfolgt durch die Substitution von $x_{i k}^{\text {ref }}$ mit $x_{j k}^{\text {ref }}$ in Formel (311). Die Parameteros $\alpha, \beta$ und $\eta$ der speziellen Distanzfunktion aus Formel (311) sind dabei abhängig von den Annahmen zu den Auswirkungen der tatsächlichen Entfernung auf die Übereinstimmung zu wählen und beziehen sich auf die Präferenz des betrachteten Knoten. Nachdem es sich in Formel (311) um ein Distanzmaß handelt, ist ein niedriger Wert ein Indikator für eine hohe Übereinstimmung des Distanzradius eines Knoten mit der tatsächlichen Entfernung. Eine Gesamtbeurteilung

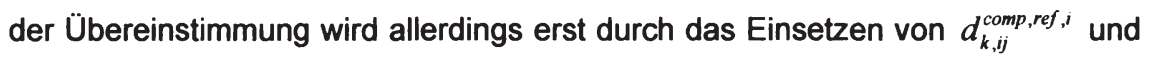

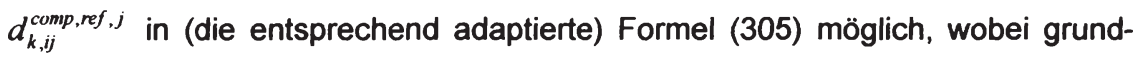
sätzlich von einer Gleichgewichtung $(\omega=0,5)$ auszugehen ist (Funktion WRAPINOUT).

$$
\begin{aligned}
& r e f_{k, i j}=d_{k, i j}^{g e o}=\operatorname{geodist}\left(v_{i}, v_{j}\right) \\
& \zeta=\arccos \left(\sin \left(b_{i}\right) \cdot \sin \left(b_{j}\right)+\cos \left(b_{i}\right) \cdot \cos \left(b_{j}\right) \cdot \cos \left(\left|l_{i}-l j\right|\right)\right) \\
& \text { geodist }\left(v_{i}, v_{j}\right)= \begin{cases}\zeta \cdot 6370 & \text { wenn } R A D \\
\frac{\zeta}{360} \cdot 40000 & \text { wenn } D E G\end{cases} \\
& d_{k, i j}^{\text {comp,ref,i }}= \begin{cases}0 & \text { wenn } r e f_{k, i j} \leq \alpha \cdot x_{i k}^{\text {ref }} \\
\beta \cdot\left(r e f_{k, i j}-\alpha \cdot x_{i k}^{r e f}\right) & \text { wenn } \alpha \cdot x_{i k}^{r e f}<r e f_{k, i j} \leq x_{i k}^{r e f} \\
\beta \cdot(1-\alpha) \cdot x_{i k}^{r e f}+\left(\operatorname{ref}_{k, i j}-x_{i k}^{r e f}\right)^{\eta} & \text { wenn ref } f_{k, i j}>x_{i k}^{r e f}\end{cases} \\
& \text { mit } 0<\alpha<1, \beta>0, \eta>1
\end{aligned}
$$

65 Diese Parameter könnten auch pro Knoten $v_{i}$ als $\alpha_{i}, \beta_{i}$ und $\eta_{i}$, pro Knoten $v_{i}$ und Präferenzvariable $x_{k}^{\text {ref }}$ als $\alpha_{i k}, \beta_{i k}$ und $\eta_{i k}$ oder unter zusätzlichem Einbezug der Dualităt der Präferenzen als $\alpha_{O U T \text {. ik, }} \beta_{\text {OUT,ik }}$ und $\eta_{\text {OUT.ik }}$ bzw. $\alpha_{I N . \text { ik }}, \beta_{I N . i k}$ und $\eta_{I N . i k}$ in Formel (311) einfließen. 


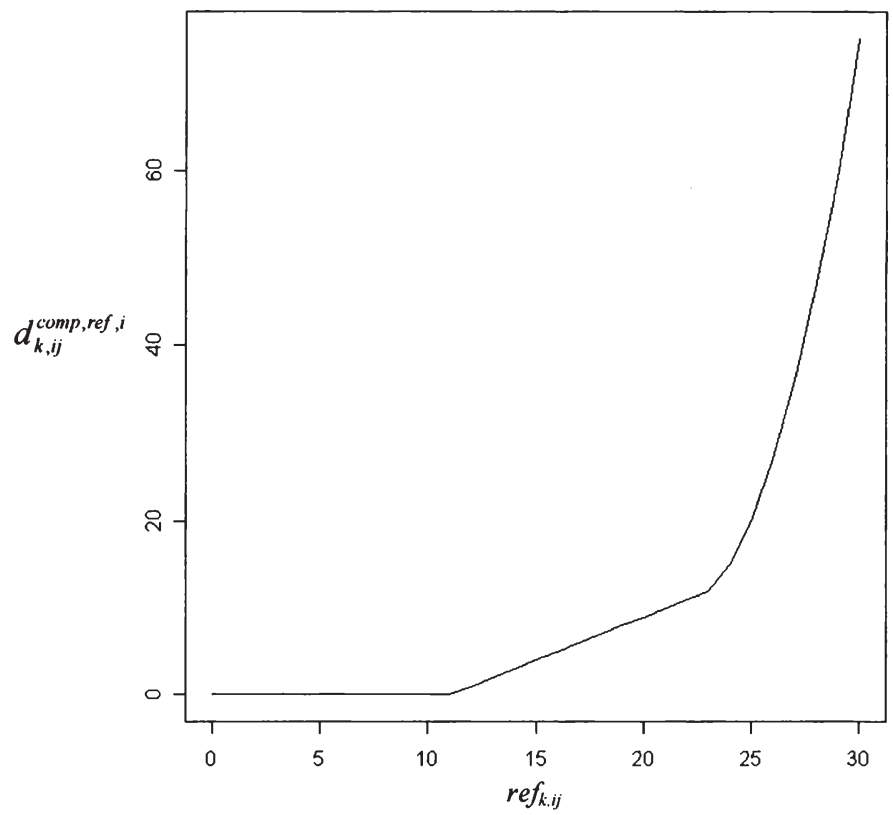

Abbildung 15: Beispiel für eine zusammengesetzte Distanzfunktion

Die zusammengesetzte Distanzfunktion in Formel (311) geht davon aus, dass die Präferenzvariable $x_{k}^{r e f}$ die maximale Reisebereitschaft der Knoten (Teilnehmer) angibt. In einem Entfernungsintervall, das bis zu einem durch $\alpha$ gesteuerten Anteil der Präferenz des Knoten reicht, wird die Distanz mit 0 festgesetzt, d.h. Präferenz und Referenz stimmen überein. In einem weiteren Intervall, welches den Bereich von $\alpha \cdot x_{i k}^{r e f}$ bis zur maximalen Reisebereitschaft abdeckt, wird die Distanz als mit $\beta$ gewichteter Abstand des Referenzwertes zur unteren Grenze des Intervalls ermittelt. Liegt die Distanz zwischen den beiden zu vergleichenden Knoten, d.h. der Referenzwert, über der Präferenz, also der maximalen Reisebereitschaft des betrachteten Knoten, so erfolgt die Berechnung der Distanz durch die Potenzierung des Referenz-Präferenz-Abstandes mit $\eta$. In diesem Be-

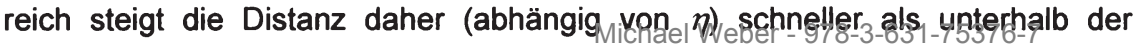


maximal tolerierten Entfernung. Abbildung 15 illustriert die spezielle Distanzfunktion aus Formel (311) mit den Werten $x_{i k}^{\text {ref }}=22, \alpha=0.5, \beta=1$ und $\eta=2$.

Alternativ könnte eine Distanzfunktion verwendet werden, die ebenfalls drei Intervalle, allerdings unter Zuweisung fixer Distanzwerte unterscheidet (Formel (312)). Die Anzahl der Intervalle ist prinzipiell frei wählbar, zumindest sollten aber die beiden Fälle (i) Referenzwert ist geringer als der maximal akzeptierte Wert (Distanzwert von 0) und (ii) Referenzwert ist höher als der maximal akzeptierte Wert (Distanzwert von 1) abgedeckt werden. Bei der Zusammenführung von $d_{k, i j}^{\text {comp,ref }, i}$ und $d_{k, i j}^{\text {comp,ref, } j}$ ergibt sich bei der Wahl dieser Alternative die Möglichkeit, für bestimmte Wertkombinationen von $d_{k, i j}^{\text {comp,ref,i }}$ und $d_{k, i j}^{\text {comp,ref,j }}$ den Wert des Maßes der indirekten (Nicht-)Übereinstimmung $d_{k, i j}^{\text {comp,ref }}$ vorzugeben.

$$
\begin{aligned}
d_{k, j}^{\text {comp }, \text { ref }, i} & = \begin{cases}0 & \text { wenn } r e f_{k, i j} \leq \alpha \cdot x_{i k}^{\text {ref }} \\
\varphi & \text { wenn } \alpha \cdot x_{i k}^{\text {ref }}<r e f_{k, i j} \leq x_{i k}^{\text {ref }} \\
1 & \text { wenn } r e f_{k, i j}>x_{i k}^{r e f}\end{cases} \\
\text { mit } 0 & <\alpha, \varphi<1
\end{aligned}
$$

Aus mehreren Subfunktionen zusammengesetzte (spezielle) Distanzfunktionen scheinen insbesondere für den Vergleich der Präferenzvariablen mit dem ermittelten Referenzwert geeignet zu sein. Formel (313) skizziert prototypisch eine solche zusammengesetzte Funktion.

$$
d_{k, i j}^{\text {comp,ref }}= \begin{cases}d_{k, i j}^{(1)} & \text { cond } 1 \\ d_{k, i j}^{(2)} & \text { cond } 2 \\ \cdots & \cdots \\ d_{k, i j}^{(D)} & \text { condD }\end{cases}
$$

Abgesehen von obigem Beispiel für den Referenzpräferenzvergleich für metrische Skalenniveaus mit zusammengesetzten oder aber auch einfachen Distanzfunktionen, sind auch nominale und ordinale Skalentypen für einen indirekten Vergleich zweier Objekte (Knoten)/geeignetber - 978-3-631-75376-7 


\subsection{Duale Präferenzen}

Nachdem in dieser Arbeit die (Nicht-)Übereinstimmung von Objekten (Knoten) bezogen auf eine Transaktionsbeziehung zu ermitteln ist, stellt sich die Frage, inwieweit duale Präferenzen, d.h. Präferenzen, die sowohl in einer Input- als auch in einer Outputbetrachtung vorliegen, mittels der vorgestellten Ähnlichkeitsund Distanzmaße behandelt werden können. Es ist sozusagen die (Un-)Ähnlichkeit zweier Objekte (Knoten) über zwei ,komplementäre' $X^{\text {prefs }}$, d.h. einerseits über die Präferenzmatrix der Objekte aus der Output- und andererseits über die korrespondierende Präferenzmatrix der Objekte aus der Inputperspektive, zu berechnen. Auf nur eine Präferenzvariable bezogen bedeutet dies, dass die (Un-)Ähnlichkeit aus dem Vergleich zweier unterschiedlicher Betrachtungen dieser Präferenzvariable bestimmt wird.

Ausgangsbasis für die Berechnung der Maßzahlen sind bei dem Vorliegen dualer Präferenzen folglich anstatt der Präferenzmatrix $X^{\text {prefs }}$ (Formel (270)) die Präferenzmatrizen $X^{\text {prefs,OITT }}$ und $X^{\text {prefs,IN }}$. Diese werden in Formel (314) beschrieben.

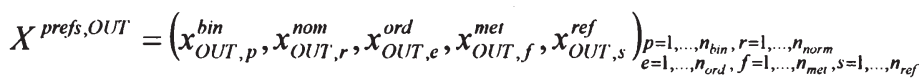

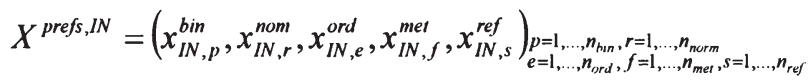

Ausgehend von diesen beiden Präferenzmatrizen muss die Notation in den oben vorgestellten Formeln angepasst werden. Dies ist bei kategoriell skalierten Variablen verhältnismäßig einfach, da hierfür nur in die Ermittlung der Koeffizienten der Kontingenztafel eingegriffen werden muss. Beispielsweise ändert sich die Berechnung der Koeffizienten für die einfache Kontingenztafel für binäre Präferenzvariablen aus Formel (271) entsprechend Formel (315). Kontingenztafeln für mehrstufig nominale und ordinale Präferenzvariablen sind analog zu adaptieren. 


$$
\begin{aligned}
& a_{i j}=\left|\left\{x_{I N, k}^{b i n}, x_{O U T, k}^{b i n}, k=1, \ldots, n_{b i n}: x_{O U T, i k}^{b i n}=x_{I N, j k}^{b i n}=1\right\}\right| \\
& b_{i j}=\left|\left\{x_{I N, k}^{b i n}, x_{O U T, k}^{b i n}, k=1, \ldots, n_{b i n}: x_{O U T, i k}^{b i n}=0 \wedge x_{I N, j k}^{b i n}=1\right\}\right| \\
& c_{i j}=\left|\left\{x_{I N, k}^{b i n}, x_{O U T, k}^{b i n}, k=1, \ldots, n_{b i n}: x_{O U T, i k}^{b i n}=1 \wedge x_{I N, j k}^{b i n}=0\right\}\right| \\
& d_{i j}=\left|\left\{x_{I N, k}^{b i n}, x_{O U T, k}^{b i n}, k=1, \ldots, n_{b i n}: x_{O U T, k}^{b i n}=x_{I N, j k}^{b i n}=0\right\}\right|
\end{aligned}
$$

Bei den Maßzahlen zur (Un-)Ähnlichkeit von metrisch skalierten Variablen muss hingegen direkt in die jeweilige Formel eingegriffen werden. Für die Minkowski-qMetriken aus Formel (296) bedeutet dies z.B. eine Anpassung der Notation gemäß Formel (316).

$$
d_{i j}^{L_{q}}=\left(\sum_{k=1}^{n_{m e j}}\left|x_{O U T, i k}^{m e t}-x_{I N, j k}^{m e r}\right|^{q}\right)^{\frac{1}{q}} \quad \text { mit } q>1
$$

Folgende Adaption des Referenzpräferenzvergleichs gemäß Formel (304) ist für den Einbezug dualer Präferenzen erforderlich (Formel (317)).

$$
\begin{aligned}
& \text { für } k=1, \ldots, n_{r e f}: \\
& d_{k, i j}^{r e f, i}=d^{\text {type }}\left(x_{O I r t, i k}^{r e f}, r e f_{k, i j}\right) \\
& d_{k, i j}^{r e f, j}=d^{\text {rype }}\left(x_{I N, j k}^{r e f}, r e f_{k, i j}\right)
\end{aligned}
$$

wobei type $\in$ Type \"ref"

Grundsätzlich sind zur Berücksichtigung von dualen Präferenzen bei den Maßzahlen zur Ermittlung der Ähnlichkeit anstatt der Variable $x_{i k}^{\text {type }}$ die entsprechende Variable $x_{O V I, i k}^{t y p e}$ aus der Präferenzmatrix $X^{\text {prefs,OIT }}$ und anstatt der Variable $x_{j k}^{t ; p e}$ die dazu passende Variable $x_{I N, j k}^{t j p e}$ aus der Matrix $X^{\text {prefs, IN }}$ zu setzen. Die Funktion WRAPINOUT dient der Einbeziehung dualer Präferenzen in die Berechnung von Ähnlichkeiten bzw. Distanzen. 


\subsection{Gesamtähnlichkeitsmaß}

Die Zusammenfassung der Ergebnisse der (Un-)Ähnlichkeitsmessung zweier Objekte (Knoten) über unterschiedliche Skalenniveaus in einer Maßzahl (,Gesamtähnlichkeitsmaßzahl' oder ,Matching-Score') trägt zur Erhöhung der Übersichtlichkeit bei. Für die Darstellung der Generierung von Matching-Scores werden dabei die Ähnlichkeiten oder Distanzen aller Typen von Präferenzvariablen als $S^{\text {prefs }}$ bzw. als $D^{\text {prefs }}$ bezeichnet. Formel (318) zeigt diese Matrizen, die für alle möglichen Paarungen von Objekten (Knoten) die (Un-)Ähnlichkeit erfassen. Typischerweise sind die Maßzahlen der (Nicht-)Übereinstimmung symmetrisch (Formel (319)), für duale Präferenzvergleiche gilt diese Symmetrie allerdings nicht.

$$
\begin{gathered}
D^{\text {prefs }}=\left(d_{i j}^{\text {bin }}, d_{i j}^{\text {nom }}, d_{i j}^{\text {ord }}, d_{i j}^{\text {met }}, d_{i j}^{\text {ref }}\right)_{i, j=1, \ldots, n} \\
S^{\text {prefs }}=\left(s_{i j}^{\text {bin }}, s_{i j}^{\text {nom }}, s_{i j}^{\text {ord }}, s_{i j}^{m e t}, s_{i j}^{r e f}\right)_{i, j=1, \ldots, n} \\
\text { für } i, j=1, \ldots, n: \\
\left(d_{i j}^{\text {bin }}, d_{i j}^{\text {nom }}, d_{i j}^{\text {ord }}, d_{i j}^{\text {met }}, d_{i j}^{\text {ref }}\right)=\left(d_{j i}^{\text {bin }}, d_{j i}^{\text {nom }}, d_{j i}^{\text {ord }}, d_{j i}^{\text {met }}, d_{j i}^{\text {ref }}\right) \\
\left(s_{i j}^{\text {bin }}, s_{i j}^{\text {nom }}, s_{i j}^{\text {ord }}, s_{i j}^{\text {met }}, s_{i j}^{\text {ref }}\right)=\left(s_{j i}^{\text {bin }}, s_{j i}^{\text {nom }}, s_{j i}^{\text {ord }}, s_{j i}^{\text {met }}, s_{j i}^{\text {ref }}\right)
\end{gathered}
$$

Aus einer der beiden Matrizen $S^{\text {prefs }}$ oder $D^{\text {prefs }}$ soll anschließend eine Matrix der Matching-Scores $M^{\text {prefs }}$ (Formel (320)) ermittelt werden, in der die (Un-)Ähnlichkeiten der binären, mehrstufig nominalen, ordinalen und metrischen Präferenzvariablengruppen inklusive der Gruppen zur Referenzpräferenz zu einem Matching-Score pro Paarung zusammengefasst werden (Funktion MATCHSCORE; ergänzt durch die Funktion VALEXCERPT zur Selektion der Knotenpaare innerhalb eines festgelegten Wertebereichs).

$$
M^{\text {prefs }}=\left(m_{i j}^{\text {prefs }}\right)_{i, j=1, \ldots, n}
$$

Die Koeffizienten der Matrix der Matching-Scores $m_{i j}^{\text {prefs }}$ können einerseits über eine Aggregation der Ähnlichkeits- bzw. Distanzmaße der Präferenzvariablen der unterschiedlichen Skalentypen oder andererseitselü̈be日7die-6Angleiclaung der 
Skalenniveaus der Präferenzvariablen ermittelt werden. Am Beispiel der Matrix der Ähnlichkeitsmaßzahlen $S^{\text {prefs }}$ werden in den folgenden Formeln diese beiden Ansätze in jeweils zwei Varianten demonstriert (vgl. hierzu z.B. [Fahrmeir et al. 1996] oder [Backhaus et al. 2000].

Unter der Voraussetzung einer Standardisierung der Präferenzvariablen kann eine Aggregation der pro Variablentyp berechneten Ähnlichkeitsmaße ungewichtet über den Mittelwert erfolgen, wie in Formel (321) gezeigt wird. Diese ungewichtete Variante kann aber auch durch eine gewichtete Mittelwertbildung ersetzt werden, die ebenso eine Standardisierung voraussetzt. Formel (322) zeigt eine solche Gewichtung auf Basis der Anzahl der Variablen pro Skalentyp.

$$
\begin{aligned}
m_{i j}^{\text {prefs }} & =\frac{1}{n_{t}} \cdot \sum_{\text {type } \in \text { Type }} s_{i j}^{\text {type }} \\
\text { eType } & =\left\{\text { type } \in \text { Type }: n_{\text {type }}>0\right\} \\
n_{t} & =|e T y p e| \\
m_{i j}^{\text {prefs }} & =\frac{1}{n_{\text {prefs }}} \cdot \sum_{\text {type } \in \text { Type }}\left(n_{\text {type }} \cdot s_{i j}^{t y p e}\right) \\
n_{\text {prefs }}= & n_{\text {bin }}+n_{\text {nom }}+n_{\text {ord }}+n_{\text {met }}+n_{\text {ref }}
\end{aligned}
$$

Der zweite Ansatz, die Angleichung der Skalenniveaus, kann grundsätzlich entweder über eine Absenkung oder aber über eine Anhebung des Skalenniveaus erfolgen. Die Variante einer Absenkung des Skalenniveaus führt zu einem Informationsverlust, dessen Ausmaß von dem Skalenniveau abhängig ist. Insbesondere metrische Variablen sind von diesem Problem betroffen. Als Beispiel kann eine Intervallbildung genannt werden, die bei metrisch skalierten Variablen direkte Auswirkungen auf die Informationstiefe hat. Im Gegensatz hierzu sind nominal skalierte Variablen von einer solchen Änderung des Skalenniveaus weniger betroffen, da diese zur Ermittlung der Ähnlichkeit ohnehin in binäre Hilfsvariablen zerlegt werden. Einen konkreten Vorschlag für eine Absenkung des Skalenniveaus zeigt Formel (323) [Fahrmeir et al. 1996, S. 452 nach Rubin 
1967]. Gemäß diesem Vorschlag wird die paarweise Gesamtähnlichkeit (Matching-Score) über einen erweiterten M-Koeffizienten berechnet. Die Erweiterung bezieht sich dabei auf die Anzahl der metrischen Variablen, in denen die metrischen Präferenzvariablen der Knoten (Objekte) $v_{i}$ und $v_{j}$ absolut weniger als $\Delta$ voneinander entfernt sind. Die Festlegung von $\Delta$ erfolgt willkürlich bzw. fallbezogen, eine Standardisierung der metrischen Variablen erscheint empfehlenswert.

Die Koeffizienten $a_{i j}, d_{i j}$ und $n_{b i n}$ aus Formel (323) beziehen sich auf die Kontingenztafel aller binären Präferenzvariablen (Formel (287)) sowie aller binären Hilfspräferenzvariablen zu den mehrstufig nominalen und ordinalen Präferenzvariablen.

$$
\begin{aligned}
m_{i j}^{\text {prefs }} & =\frac{a_{i j}+d_{i j}+n_{i j}^{<}}{n_{b i n}+n_{m e t}} \\
n_{i j}^{<} & =\left|\left\{x_{k}^{m e t}:\left|x_{i k}^{m e t}-x_{j k}^{m e t}\right|<\Delta\right\}\right|
\end{aligned}
$$

Eine Alternative zur Absenkung des Skalenniveaus ist die Verfeinerung von ,gröber' skalierten Variablen und die Anwendung von Distanz- bzw. Ähnlichkeitsmaßen für höhere Skalenniveaus. Dies bedeutet, dass ,gröber' skalierte Variablen als metrisch interpretiert werden und die Berechnung der (Nicht-)Übereinstimmung über ein metrisches Berechnungsverfahren erfolgt. Dieses Vorgehen ist problematisch, da eine solche Interpretation im Prinzip nicht zulässig ist, andernfalls wären wohl keine unterschiedlichen Ähnlichkeitsmaße für unterschiedliche Skalentypen erforderlich.

Allgemein kann festgehalten werden, dass die nun vorgestellten und in der Literatur diskutierten Ansätze zur Zusammenfassung von Variablen unterschiedlicher Skalentypen in gewisser Weise unbefriedigend sind, da jeder dieser Ansätze mehr oder weniger gravierende Schwachpunkte aufweist. Die geringste Problematik scheint dabei eine Aggregation über einen gewichteten Mittelwert 
aufzuwerfen, weshalb dieser Ansatz zur Ermittlung eines Matching-Scores für die Transaktionsempfehlungen des Modells vorgeschlagen wird.

\subsection{Präferenzprofile für externe Knoten}

Sind die Präferenzen neben den allgemeinen Profildaten der Teilnehmer bekannt, so kann deren Transaktions(n)eig(n)ung für unterschiedlichste Transaktionen mittels des Matching-Scores oder den einzelnen Maßzahlen zur (Nicht-) Übereinstimmung bestimmt werden. Dies gilt nicht nur für bestehende Teilnehmer an der Kollaborationsplattform, sondern hat auch für potenzielle Teilnehmer, d.h. Teilnahmekandidaten, Gültigkeit. Anders sind allerdings Situationen zu beurteilen, in denen es keinen konkreten Kandidaten für eine spezifische Transaktion gibt. In solchen Fällen liegen naturgemäß keine Informationen zu den Präferenzen vor, weshalb auf Basis des Transaktionsinhalts und der Präferenzen der theoretischen, bereits an der Plattform teilnehmenden Transaktionspartner idealerweise ein Präferenzprofil für etwaige zukünftige Teilnehmer festzulegen ist. Dieses Präferenzprofil sollte die Präferenzen der bestehenden Teilnehmer optimal ergänzen. Ein Verfahren zur Generierung von optimalen Präferenzprofilen für zukünftige Kandidaten soll nun beschrieben werden.

Die Grundidee des Verfahrens ist, über die Häufigkeitsverteilung der Ausprägungen der Präferenzvariable $x_{k}^{t y p e}$ für die Knoten $v_{i}, i=1, \ldots, n$, (bestehende Teilnehmer) die aussichtsreichsten Präferenzkonstellationen für externe Knoten (Teilnahmekandidaten) bezogen auf deren direkte Vorgänger bzw. Nachfolger (bestehende Teilnehmer) zu identifizieren. Zu diesem Zweck ist eine Aggregation der Präferenzen jener Knoten $v_{i}$ notwendig, die eine bestimmte Eigenschaft erfüllen. Eine solche Eigenschaft kann z.B. die Bereitstellung eines Gutes durch ein teilnehmendes Unternehmen entsprechend dessen Profildaten sein, welches in direkter Beziehung zu einem externen Gut des virtuellen Teilnahmekandidaten gemäß der Produktionsstruktur steht. Hierbei ist zwischen einer Input- und einer Outputperspektive zu trennen, um unterschiedliche ergänzende Präferenzprofile bezogen auf die Vorgänger und Nachfolger generieren zu können. Zusätzlich ist 
es möglich, die Vorgänger bzw. Nachfolger unterschiedlichen Teilmengen (Segmenten) - u.U. gewichtet - zuzuordnen, um ideale Präferenzkonstellationen für spezifische Zielgruppen innerhalb der Auswahl zu definieren.

Allgemein formuliert erfolgt eine Auswahl einer Teilmenge der Knoten $v_{i}$ mit der Eigenschaft $E\left(v_{i}\right)$, d.h. $v_{i}$ hat Eigenschaft $E$ (Formel (324)). Im Gegensatz steht $\neg E\left(v_{i}\right)$ für jene Menge an Knoten $v_{i}$, welche die Eigenschaft $E$ nicht aufweisen.

$$
V^{E}=\left\{v_{i} \in V: E\left(v_{i}\right)\right\}
$$

Je nachdem auf welcher Ebene die Präferenzwerte erhoben werden, d.h. auf der Ebene der Unternehmen, deren Aktivitäten oder Güter, können unterschiedliche Mengen gebildet werden. In diesem Zusammenhang ist zu betonen, dass eine weitere Verfeinerung eine Erhebung von Präferenzwerten bezogen auf den Input sowie die Destinationen des Output auf der Stufe der Unternehmen, Aktivitäten oder gar Güter wäre. Formel (325) stellt beispielhaft die Teilmenge von Unternehmen (in diesem Fall die internen Knoten) dar, die über ein oder mehrere Güter $\operatorname{com}_{c}$ gemäß dem Unternehmensprofil (siehe Formel (20) bis Formel (22)) mit dem externen Gut $c_{0}$ über $A^{\text {ILOC,EXP-X }}$ aus Formel (233) in Beziehung stehen.

$$
\begin{aligned}
V_{b i z}^{E X T-x}= & \left\{v_{i} \in V^{L O C}=\operatorname{Biz}:\left(v_{i}=\operatorname{biz}_{b}\right) \wedge\left(v_{x}=\operatorname{com}_{j} \in \operatorname{Com} V W=V E X T\right) \wedge\right. \\
& \left.\wedge\left(\exists \operatorname{com}_{c} \in \operatorname{Com}:\left(\left(\exists \text { bciprofile } u_{b, l}: \operatorname{com}_{b, l, c}=\operatorname{com}_{c}\right) \wedge a_{c j}^{* L O C . E X P-x}>0\right)\right)\right\}
\end{aligned}
$$

Im Unterschied zu Formel (325) zeigt Formel (326) die Definition der Teilmenge der internen Knoten auf Basis von Unternehmen in Kombination mit deren Gütern (Funktion EXTPARTNER). Bei beiden Formeln könnte man anstatt der Güter auch Aktivitäten heranziehen.

$$
\begin{aligned}
V_{b i z, c o m}^{E X T-x} & =\left\{\left(v_{i}, \operatorname{com}_{c}\right):\left(v_{i}=\operatorname{biz}_{b} \in B i z\right) \wedge\left(v_{x}=\operatorname{com}_{j} \in \operatorname{Com} V W=V^{E X T}\right) \wedge\right. \\
& \left.\wedge\left(\operatorname{com}_{c} \in \operatorname{Com}:\left(\exists \text { biprofile } u_{b, l}: \operatorname{com}_{b, l, c}=\operatorname{com}_{c}\right)\right) \wedge\left(a_{c j}^{* L O(, E X P-x}>0\right)\right\}
\end{aligned}
$$


Die Wahl des Verfahrens zur Aggregation der Ausprägungen der Präferenzvariable für die durch Eigenschaft $E$ definierte Knotenmenge $V^{E}$ ist abhängig von dem Skalentyp der Präferenzvariable. Bei den Aggregationsverfahren zu den verschiedenen Skalentypen $h\left(x_{k}^{i v p e}, v a l_{k q}^{t p p e}\right)$ können zwei grundlegende Alternativen unterschieden werden. Zum einen besteht die Möglichkeit, pro Präferenzausprägung die (relative) Anzahl der betrachteten Knoten, die diese Präferenzausprägung aufweisen, d.h., die (relative) Häufigkeitsverteilung der Präferenzvariable, zu ermitteln. Zum anderen kann darüber hinaus auch die jeweilige Bedeutung der Eigenschaft, die zur Auswahl der betrachteten Knoten herangezogen wurde, für diese Knoten zur Gewichtung eingesetzt werden. Demnach sollte z.B. die Bedeutung eines externen Knoten (Gut oder Aktivität) für einen internen Knoten (Teilnehmer oder Gut bzw. Aktivität eines teilnehmenden Unternehmens) zur Gewichtung der Präferenzen des internen Knoten in der Aggregation verwendet werden. Dadurch werden die Präferenzen eines internen Knoten, für den der externe Knoten eine größere Rolle spielt, in einem stärkeren Ausmaß berücksichtigt. Eine weitere Möglichkeit ergibt sich bei ordinalen und metrischen Skalen. Die bei diesen Skalentypen vorliegende Ordnung der Ausprägungen ermöglicht auch eine Betrachtung der kumulierten (relativen) Häufigkeiten. Darüber hinaus ist bei metrischen Skalen zu beachten, dass im Allgemeinen vor der Ermittlung der Häufigkeiten in Analogie zur Histogrammerstellung eine Zusammenfassung der Variablenausprägungen in Klassen erforderlich ist. Die Funktion AGGPREF ermöglicht die Aggregation der Präferenzen der Ausprägungen der Präferenzvariablen für die durch Eigenschaft $E$ definierte Knotenmenge $V^{E}$ mittels der in den folgenden Subkapiteln vorgestellten Verfahren.

\subsubsection{Binäre nominale Skalen}

Für binär skalierte Variablen zeigt Formel (327) die Ermittlung der absoluten Häufigkeit $h$, Formel (328) die der relative Häufigkeit $h^{\text {rel }}$ und Formel (329) den Einbezug der Bedeutung in die Aggregation der Präferenzausprägungen $h^{\text {rate }}$. 


$$
\begin{gathered}
h\left(x_{k}^{b i n}, v_{k q}^{b i n}\right)=\left|V_{b i n q}^{E}\right| \quad \text { für val } l_{k q}^{b i n} \in\{0,1\}, k=1, \ldots, n_{b i n} \\
\text { wobei } V_{b i n q}^{E}=\left\{v_{i} \in V^{E}: x_{i k}^{b i n}=v a l_{k q}^{b i n}\right\} \\
h^{\text {rel }}\left(x_{k}^{b i n}, v a l_{k q}^{b i n}\right)=\frac{h\left(x_{k}^{b i n}, v a l_{k q}^{b i n}\right)}{\left|V^{E}\right|} \text { für val } l_{k q}^{b i n} \in\{0,1\}, k=1, \ldots, n_{b i n} \\
h^{\text {rate }}\left(x_{k}^{b i n}, v_{k q}^{b i n}\right)=\sum_{v_{i} \in V_{\text {binq }}^{k}} \operatorname{rate}\left(E\left(v_{i}\right)\right) \text { für val } l_{k q}^{\text {bin }} \in\{0,1\}, k=1, \ldots, n_{b i n}
\end{gathered}
$$

\subsubsection{Mehrstufig nominale Skalen}

Die Berechnung von absoluten Häufigkeiten für mehrstufig nominal skalierte Variablen wird in Formel (330) definiert, während Formel (331) die Definition der relativen Häufigkeit und Formel (332) die Berücksichtigung der Bedeutung in der Aggregation für diesen Skalentyp demonstriert.

$$
\begin{aligned}
& h\left(x_{k}^{\text {nom }}, v a l_{k q}^{\text {nom }}\right)=\left|V_{\text {nomq }}^{E}\right| \quad \text { für val } l_{k q}^{\text {nom }}=\operatorname{cat}_{k q}^{\text {nom }} \in C a t_{k}^{\text {nom }}, k=1, \ldots, n_{\text {nom }} \\
& \text { wobei } V_{\text {nomq }}^{E}=\left\{v_{i} \in V^{E}: x_{i k}^{\text {nom }}=v a l_{k q}^{\text {nom }}\right\} \\
& \text { cat }_{k l}^{\text {nom }} \in \text { Cat }_{k}^{\text {nom }}=\left\{\text { cat }_{k 1}^{\text {nom }}, \ldots, \text { cat }_{k n c_{k}}^{\text {nom }}\right\} \\
& h^{\text {rel }}\left(x_{k}^{\text {nom }}, v a l_{k q}^{\text {nom }}\right)=\frac{h\left(x_{k}^{\text {nom }}, v a l_{k q}^{\text {nom }}\right)}{\left|V^{E}\right|} \text { für } v a l_{k q}^{n o m} \in \operatorname{Cat}_{k}^{\text {nom }}, k=1, \ldots, n_{\text {nom }} \\
& h^{\text {rate }}\left(x_{k}^{\text {nom }}, \operatorname{val}_{k q}^{\text {nom }}\right)=\sum_{v_{i} \in V_{\text {nom }}^{F}} \operatorname{rate}\left(E\left(v_{i}\right)\right) \text { für val }{ }_{k q}^{\text {nom }} \in \operatorname{Cat}_{k}^{\text {nom }}, k=1, \ldots, n_{\text {nom }}
\end{aligned}
$$

\subsubsection{Ordinale Skalen}

Für Ordinalskalen zeigt Formel (333) die Ermittlung der absoluten Häufigkeiten, Formel (334) die der relativen Häufigkeiten und Formel (335) die Aggregation der Variablenausprägungen mittels der Bedeutung der ausgewählten Eigenschaft. Formel (336) stellt darüber hinaus die kumulierten absoluten, Formel (337) die 
kumulierten relativen Häufigkeiten dar. In Formel (338) wird die kumulierte Aggregation über die Bedeutungen gezeigt.

$$
\begin{aligned}
& h\left(x_{k}^{\text {ord }}, \text { val }_{k q}^{\text {ord }}\right)=\left|V_{\text {ord } q}^{E}\right| \quad \text { für val }{ }_{k q}^{\text {ord }}=\text { cat }_{k q}^{\text {ord }} \in C a t_{k}^{\text {ord }}, k=1, \ldots, n_{\text {ord }} \\
& \text { wobei } V_{\text {ord } q}^{E}=\left\{v_{i} \in V^{E}: x_{i k}^{\text {ord }}=\text { val }_{k q}^{\text {ord }}\right\} \\
& \mathrm{Cat}_{k}^{\text {ord }}=\left\{\text { cat }_{k 1}^{\text {ord }}, \ldots, \text { cat }_{k n c_{k}}^{\text {ord }}\right\} \\
& \text { mit } \text { cat }_{k 1}^{\text {ord }} \prec c a t_{k 2}^{\text {ord }} \prec \ldots \prec c a t_{k n c_{k}}^{\text {ord }} \\
& h^{\text {rel }}\left(x_{k}^{\text {ord }}, v_{\text {al }}^{\text {ord }}\right)=\frac{h\left(x_{k}^{\text {ord }}, v_{k q}^{\text {ord }}\right)}{\left|V^{E}\right|} \text { für val } l_{k q}^{\text {ord }} \in \operatorname{Cat}_{k}^{\text {ord }}, k=1, \ldots, n_{\text {ord }} \\
& h^{\text {rate }}\left(x_{k}^{\text {ord }}, \text { val }_{k q}^{\text {ord }}\right)=\sum_{v_{i} \in V_{\text {ordq }}^{E}} \operatorname{rate}\left(E\left(v_{i}\right)\right) \text { für val }{ }_{k q}^{\text {ord }} \in \text { Cat }_{k}^{\text {ord }}, k=1, \ldots, n_{\text {ord }} \\
& h^{\text {cum }}\left(x_{k}^{\text {ord }}, v a l_{k q}^{\text {ord }}\right)=\sum_{f \geq q} h\left(x_{k}^{\text {ord }}, v a l_{k f}^{\text {ord }}\right) \quad \text { für val }{ }_{k f}^{\text {ord }}=\text { cat }_{k f}^{\text {ord }} \in C a t_{k}^{\text {ord }}, k=1, \ldots, n_{\text {ord }} \\
& h^{\text {cum }, \text { rel }}\left(x_{k}^{\text {ord }}, \text { val } k_{k q}^{\text {ord }}\right)=\sum_{f \geq q} h^{\text {rel }}\left(x_{k}^{\text {ord }}, \text { valord }\right) \\
& \text { für val ord }=\text { cat }_{k f}^{\text {ord }} \in \text { Cat }_{k}^{\text {ord }}, k=1, \ldots, n_{\text {ord }} \\
& h^{\text {cum, rate }}\left(x_{k}^{\text {ord }}, \text { val ord }\right)=\sum_{f \geq q} h^{\text {rate }}\left(x_{k}^{\text {ord }}, \text { valo ord }\right) \\
& \text { für val ord }=\text { cat }_{k q}^{\text {ord }} \in C a t_{k}^{\text {ord }}, k=1, \ldots, n_{\text {ord }}
\end{aligned}
$$

\subsubsection{Metrische Skalen}

Die Klassenbildung für metrisch skalierte Variablen kann über Formel (339) nachvollzogen werden. Die absoluten Häufigkeiten für diesen Skalentyp können mittels der Formel (340), die relativen Häufigkeiten durch Einsatz der Formel (341) berechnet werden. Formel (342) zeigt, wie die Bedeutungen der Eigen- 
schaften in die Aggregation einfließen können. Die kumulativen Varianten dieser drei Berechnungen liefern die Formeln (343), (344) sowie (345).

$$
\begin{aligned}
& b\left(x_{k}^{m e t}\right)=\frac{\max _{q}\left(v_{k q}^{m e t}\right)-\min _{q}\left(v_{k a l}^{m e t}\right)}{a_{E}} \text { für val }{ }_{k q}^{m e t} \in \operatorname{ValSet}_{k}^{m e t} \subseteq \mathfrak{R}, k=1, \ldots, n_{m e t} \\
& a_{E}=\left\lceil\log _{2}\left(\left|V^{E}\right|\right)\right\rceil \\
& \mathrm{Cat}_{k}^{m e t}=\left(\mathrm{cat}_{k t}^{\mathrm{met}}\right)_{t=1, \ldots, a_{E}}
\end{aligned}
$$

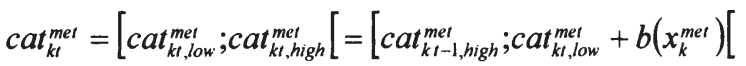

$$
\begin{aligned}
& \text { wobei }
\end{aligned}
$$

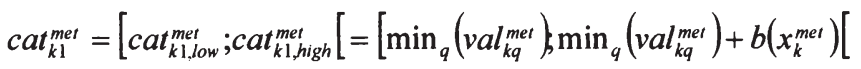

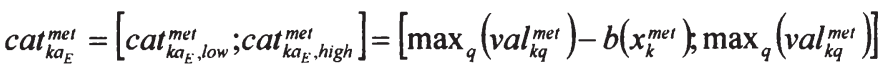

$$
\begin{aligned}
& h\left(x_{k}^{m e t}, v_{k q}^{m e t}\right)=\left|V_{l n t t}^{E}\right| \quad \text { für val }{ }_{k q}^{m e t} \in \operatorname{cat}_{k t}^{m e t}, k=1, \ldots, n_{\text {met }} \\
& \text { wobei } V_{\text {lntt }}^{E}=\left\{v_{i} \in V^{E}: x_{i k}^{\text {met }} \in \text { cat }_{k t}^{\text {met }}\right\} \\
& h^{\text {rel }}\left(x_{k}^{m e t}, v a l_{k q}^{m e t}\right)=\frac{h\left(x_{k}^{m e t}, v a l_{k q}^{m e t}\right)}{\left|V^{E}\right|} \text { für val } l_{k q}^{m e t} \in c a t_{k t}^{m e t}, k=1, \ldots, n_{m e t} \\
& h^{\text {rate }}\left(x_{k}^{\text {met }}, v a l_{k q}^{m e t}\right)=\sum_{v_{t} \in V_{m q^{t}}^{E}} \operatorname{rate}\left(E\left(v_{i}\right)\right) \text { für val }{ }_{k q}^{m e t} \in \operatorname{cat}_{k t}^{m e t}, k=1, \ldots, n_{m e t} \\
& h^{\text {cum }}\left(x_{k}^{m e t}, \text { val }_{k q}^{m e t}\right)=\left|V_{\text {metq }}^{E}\right| \quad \text { für val }{ }_{k y}^{m e t} \in \text { ValSet }_{k}^{\text {met }}, k=1, \ldots, n_{\text {met }} \\
& \text { wobei } V_{\text {metq }}^{E}=\left\{v_{i} \in V^{E}: x_{i k}^{\text {met }} \leq v a l_{k q}^{\text {met }}\right\} \\
& h^{\text {cum }, \text { rel }}\left(x_{k}^{m e t}, v a l_{k q}^{m e t}\right)=\frac{h^{\text {cum }}\left(x_{k}^{m e t}, v a l_{k q}^{m e t}\right)}{\left|V^{E}\right|} \text { für val }{ }_{k q}^{m e t} \in \text { ValSet }_{k}^{m e t}, k=1, \ldots, n_{m e t} \\
& h^{\text {cum }, \text { rate }}\left(x_{k}^{\text {met }}, \text { val }_{k q}^{\text {met }}\right)=\sum_{v_{i} \in V_{l m q}^{E}} \operatorname{rate}\left(E\left(v_{i}\right)\right) \text { für val } k_{k q}^{m e t} \in \operatorname{ValSet}_{k}^{m e t}, k=1, \ldots, n_{m e t}
\end{aligned}
$$




\subsubsection{Referenzpräferenzbestimmung}

Die idealen Präferenzausprägungen für externe Knoten, die indirekt mit den Präferenzen der internen Knoten verglichen werden, können sowohl für metrische als auch kategorielle Skalenniveaus über die nachfolgend vorgestellten speziellen Berechnungsverfahren bestimmt werden. Konkret sind dabei nicht nur die bevorzugte(n) Präferenzausprägung(en) $x_{k e}^{\text {ref }}$ des oder der externen Knoten $v_{e}$, sondern - diesem Schritt vorgelagert - die optimale(n) Ausprägung(en) $\operatorname{ref}_{k}\left(v_{e}\right)$ des Merkmals, das dem Referenzmerkmal zugrunde liegt, für den oder die externen Knoten zu identifizieren. Diese ergeben sich über die Referenzwerte $r e f_{k, e i}$ des oder der externen Knoten mit allen internen Knoten $v_{i}$ der durch die Eigenschaft $E$ definierten Teilmenge $V^{E}$.

\section{Metrisches Skalenniveau der Referenzpräferenzvariable}

Das Vorgehen bei metrischem Skalenniveau setzt sich aus vier Schritten zusammen. Zunächst sind aus der Menge $V^{E}$ jene internen Knoten zu finden, die durch ihre Präferenzen und Referenzwerte in Zusammenhang stehen (Funktion EXTRACTZ). Die Matrix $Z$, die in Formel (346) definiert wird, zeigt, ob interne Knoten über diese beiden Kriterien in Beziehung gesetzt werden können.

$$
\begin{aligned}
Z_{k}^{\text {ref }} & =\left(z_{k, i j}^{\text {ref }}\right)_{v_{i}, v_{j} \in V^{E}} \\
z_{k, i j}^{\text {ref }} & = \begin{cases}1 & \text { wenn } x_{i k}^{\text {ref }}+x_{j k}^{\text {ref }} \geq \text { ref }_{k, j j} \\
0 & \text { andernfalls }\end{cases}
\end{aligned}
$$

Aufbauend auf der Matrix $Z$ ist im nächsten Schritt die strukturelle Äquivalenz von

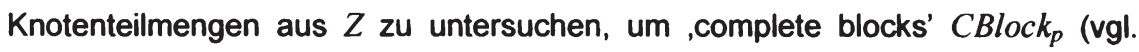
Tabelle 22) und dazugehörige Knotengruppen $C_{p} \subseteq V^{E}$ zu finden. Anforderungen an die Identifikation von vollständigen Blöcken sind hierbei, (i) dass pro Block die Menge der Knoten in der Vorgänger- und in der Nachfolgerrolle identisch ist, (ii) dass Blöcke, die in anderen, übergeordneten Blöcken aufgehen bzw. enthalten

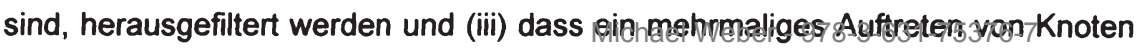


in unterschiedlichen Knotengruppen (Blöcken) zulässig ist, d.h. die Knotenteilmengen müssen nicht disjunkt sein. Formel (347) beschreibt die erforderlichen vollständigen Blöcke $C B l o c k_{p}$ und die zugrunde liegenden Knotenmengen $C_{p}$.

$$
\begin{aligned}
& C=\left\{C_{p} \subseteq V^{E}: C_{p} \times C_{p}=\text { CBlock } k_{p}\right\} \\
& \text { CBlock }_{p}=\left\{C_{p} \times C_{p}: \sum_{v_{a}, v_{b} \in C_{p}} z_{k, a b}^{r e f}=\left|C_{p}\right|^{2} \wedge C_{p} \subseteq V^{E}\right\}= \\
&=\left\{C_{p} \times C_{p}:\left(\forall\left(v_{a}, v_{b}\right) \in C_{p} \times C_{p}: z_{k, a b}^{r e f}=1\right) \wedge C_{p} \subseteq V^{E}\right\} \\
& \text { wobei } \\
& C_{p} \not C_{q} \text { für } C_{p}, C_{q} \subset C \\
& C_{p} \cap C_{q} \geq \varnothing \quad \text { für } C_{p}, C_{q} \subset C
\end{aligned}
$$

Die Vorgehensweise zur Ermittlung von vollständigen Blöcken kann wie folgt beschrieben werden. Bildet die Knotenmenge $V^{E}$ keinen vollständigen Block, so ist in allen Knotenteilmengen der Menge $V^{E}$ ausgehend von der Kardinalität 2 bis $\left|V^{E}\right|-1$ nach vollständigen Blöcken zu suchen. Für jede dieser Teilmengen der Knotenmenge $V^{E}$ ist demnach zu bestimmen, ob sie - unter Beachtung der zuvor beschriebenen Anforderungen - einen vollständigen Block ergeben, d.h. ob $\sum_{v_{u}, v_{b} \in C_{p}} z_{k, a b}^{r c f}=\left|C_{p}\right|^{2}$. Pro ausgewählter Knotenmenge $C_{p} \subset C$ wird anschließend überprüft, ob sie Teilmenge einer anderen Knotenmenge $C_{q} \subset C$ ist. Trifft dies zu, so wird die kleinere Knotenmenge aus der Menge $C$ der Knotenmengen, die vollständige Blöcke ergeben, entfernt. Zuletzt werden isolierte Knoten identifiziert, die in keiner der Knotengruppen für vollständige Blöcke in $C$ enthalten sind. Diese Spezialfälle werden ebenfalls als vollständige Blöcke in $C$ aufgenommen.

Bildet die Gesamtheit der Knoten der Menge $V^{E}$ einen vollständigen Block, d.h. $\sum_{v_{u}, v_{b} \in C_{p}} z_{k, a b}^{r e f}=\left|V^{E}\right|^{2}$, so ist dieses Verfahren nicht erforderlich, nachdem alle Knotenteilmengen, die konsequenterweise gleichermaßen vollständige Blöcke ergeben, 
komplett in dem übergeordneten vollständigen Block - der sämtliche Knoten einbezieht - enthalten sind. In Folge einer frühzeitigen Erkennung eines solchen Falles kann ebenso wie durch den gerichteten Einsatz des Blockbestimmungsverfahrens auf Knoten innerhalb zuvor ermittelter Cliquen die Performanz der Ermittlung von vollständigen Blöcken gemäß Formel (347) erhöht werden. Die Funktion FINDCBLOCKS dient der Identifikation aller vollständigen Blöcke.

Anschließend an die Blockbestimmung ist für jeden vollständigen Block CBlock jene Merkmalsausprägung eines fiktiven externen Knoten $r e f_{k}\left(v_{e}\right)$ zu bestimmen, die die Summe der Referenzwerte aller Knoten im Block mit dem externen Knoten minimiert. Die Nebenbedingung dieses Optimierungsverfahrens ist, dass alle Referenzwerte zwischen den internen und dem externen Knoten jeweils $\leq$ der Präferenz des jeweiligen internen Knoten sind. Darüber hinaus ist zu beachten, dass diese Referenzwerte über das Merkmal $\operatorname{ref}_{k}\left(v_{e}\right)$ des externen Knoten miteinander in Beziehung stehen. Dies bedeutet, dass sich mit einer Änderung von $r e f_{k, e p}$ auch alle anderen $r e f_{k, e i}$ ändern. Formel (348) stellt dieses Optimierungsproblem dar, das unter Verwendung der Funktion CALCREFPREF gelöst werden kann.

$$
\begin{aligned}
\sum_{v_{i} \in C_{p}} r e f_{k, e i} & \rightarrow \min \\
r e f_{k, e i} & \leq x_{i k}^{r e f} \\
r e f_{k, e i} & \leftrightarrow r e f_{k, e j} \\
\text { für } v_{i}, v_{j} & \in C_{p}
\end{aligned}
$$

Die passende Präferenz des externen Knoten ergibt sich schließlich in Anbetracht der Gruppe von internen Knoten, die gemäß dem vollständigen Block CBlock $_{p}$ strukturelle Äquivalenz aufweisen. Formel (349) definiert diese Präferenzfestlegung. 


$$
x_{e k}^{r e f} \geq \max _{v_{i} \in C_{p}}\left(r e f_{k, e i}\right)
$$

Eine Reihung der vorgeschlagenen Merkmale für externe Knoten kann nach der Größe des vollständigen Blocks oder der Summe der jeweiligen Bedeutung der Knoten des betrachteten Blocks erfolgen (Funktion ORDERETAPPEND).

\section{Kategorielle Skalenniveaus der Referenzpräferenzvariable}

Bei kategoriellen Skalenniveaus sind für jede Kategorie $\operatorname{cat}_{k l}^{k a t} \in \operatorname{Cat}_{k}^{k a t 66}$ mit Cat $_{k}^{k a t}=\left\{\right.$ cat $\left._{k 1}^{k a t}, \ldots, c a t_{k n k_{k}}^{k a t}\right\}$, die die kategorielle, d.h. binäre, mehrstufig nominale oder ordinale, Variable für den indirekten Präferenzvergleich $x_{k}^{\text {ref,kat }}$ (ReferenzPräferenzvariable) annehmen kann, folgende Schritte erforderlich.

Zuerst ist die Teilmenge der Knoten aus $V^{k}$ zu selektieren, die dieser Kategorie zugehören (d.h. diese Präferenz aufweisen), unabhängig von den Merkmalen dieser Knoten aus denen der Referenzwert ermittelt wird (Formel ((350)).

$$
V_{k l}^{r e f, k a t}=\left\{v_{i} \in V^{E}: x_{i k}^{r e f, k a t}=c a t_{k l}^{k a t}\right\}
$$

Danach ist anhand der ,reversen' Relationsfunktion ${ }^{67} f_{k}^{\text {rev }}$, mittels der präferierten paarweisen Referenzwerte $r e f_{k, i j}$ der (internen) Knoten aus der Teilmenge und deren jeweiligem Merkmal $\operatorname{ref}_{k}\left(v_{i}\right)$, aus welchem der Referenzwert $r e f_{k, i j}$ ermittelt wird, die ideale Ausprägung $\operatorname{ref}_{k}\left(v_{e}\right)$ des Merkmals des externen Knoten zu bestimmen (Formel (351)).

66 Die Wertemenge Cat kat bildet den Wertebereich für die Präferenzvariable, für das Merkmal der Objekte (Knoten), aus dem der Referenzwert ermittelt wird, und für den Referenzwert selbst.

67 Diese, reverse' Relationsfunktion stellt eine Umkehrung der für die Referenzwertberechnung erforderlichen Relationsfunktion dar, d.h. über die Kenntnis des Referenzwerts des Objektpaares und des Merkmalswerts eines Objekts kann über diese Funktion der Merkmalswert des zweiten Objekts oder ein Bereich für diesen bestimmt werden. 


$$
f_{k}^{r e v}\left(r e f_{k, j}, r e f_{k}\left(v_{i}\right)\right)=r e f_{k}\left(v_{e}\right)
$$

Die Ausprägung der dazu passenden Präferenz des externen Knoten ergibt sich dabei aus der Präferenz des jeweils aus der Teilmenge $V_{k l}^{\text {ref, } k a t}$ herangezogenen internen Knoten $x_{e k}^{r e f, k a t}=c a t_{k l}^{k a t}$.

Abschließend kann die Häufigkeit des Auftretens der Kombination eines Präferenzwerts mit einem bestimmten Merkmalswert des fiktiven externen Knoten ermittelt werden, um die Bedeutung dieser Präferenz- und Merkmalskombination bei einem externen Knoten für die internen Knoten (bzw. einer Auswahl dieser) festlegen zu können (Formel (352)).

$$
\begin{aligned}
& h\left(x_{k}^{r e f, k a t}, x_{e k}^{r e f, k a t}, r e f_{k}\left(v_{e}\right)\right)=\left|V_{k l q}^{r e f, k a t}\right| \\
& V_{k l q}^{\text {ref }, k a t}=\left\{v_{i} \in V_{k l}^{\text {ref, } k a t}: f_{k}^{r e f}\left(r e f_{k, i j}, r e f_{k}\left(v_{i}\right)\right)=c a t_{k q}^{k a t}\right\}
\end{aligned}
$$

Es sei darauf hingewiesen, dass bei dem Vorliegen mehrerer Präferenzwerte für ein und dieselbe Referenzpräferenzvariable eine weitere Aggregation erforderlich wäre.

Wurden mehrere zusammenhängende Präferenzvariablen definiert (abgestufte Präferenzen, z.B. über eine Rangreihung), so hat die Bestimmung der bevorzugten Präferenz und des bevorzugten Merkmalswerts des fiktiven externen Knoten unter Berücksichtigung aller zusammenhängenden Variablen zu erfolgen. Ein solcher Fall wirkt sich insbesondere auf den ersten der oben angeführten Schritte zum Umgang mit kategoriellen Referenzpräferenzvariablen bei der Präferenzprofilbestimmung von externen Knoten aus, d.h. der Selektion der Knotenteilmenge. Es sind dann alle Knoten auszuwählen, welche die zur Definition der Teilmenge verwendete Kategorie als Präferenzwert in einer der zusammenhängenden Präferenzvariablen haben. Im vierten Schritt sollte dann anstatt einer einfachen Häufigkeitsauszählung das jeweilige Niveau der Präferenzvariable berücksichtigt werden. 
Nach Abschluss der Ermittlung der idealen Präferenzvariablen für einen externen Knoten pro Skalenniveau können die Ergebnisse zu einem gesamten Präferenzprofil des fiktiven externen Knoten für jede Teilmenge $V^{E}$ zusammengetragen werden (Funktion EXTPREFPROFILE). Formel (353) zeigt ein einfaches Beispiel für eine solche Zusammenfassung, bei der die häufigste/bedeutungsstärkste Präferenzausprägung pro Präferenzvariable für einen externen Knoten auf Basis von absoluten Häufigkeiten erfolgt. Nachdem ein solches vereinfachendes Vorgehen durchaus kritisch zu betrachten ist, wird für den praktischen Einsatz empfohlen, anhand der (gemeinsamen) Verteilungen der Variablenausprägungen zielgruppenbezogen durch eine multivariate Vorgangsweise ideale Konstellationen von Präferenzvariablen für externe Knoten zu identifizieren.

$$
\begin{aligned}
& x_{e}^{p r e f s}=\left(x_{e p}^{b i n}, x_{e r}^{\text {nom }}, x_{e o}^{\text {ord }}, x_{e f}^{m e t}, x_{e s}^{r e f}\right)_{\substack{p=1, \ldots, n_{h i n}, r=1, \ldots, n_{n o r m} \\
o=1, \ldots, n_{\text {ord }}, f=1, \ldots, n_{\text {mel }}, s=1, \ldots, n_{r e f}}} \\
& x_{e p}^{b i n}=\max _{c a t_{p q}^{b i n} \in\{0,1\}}\left(h\left(x_{p}^{b i n}, c a t_{p q}^{b i n}\right)\right) \\
& x_{e r}^{\text {nom }}=\max _{c a r_{r q}^{n o m} \in C a t_{r}^{n o m}}\left(h\left(x_{r}^{\text {nom }}, \text { cat } r_{r q}^{\text {nom }}\right)\right) \\
& x_{e o}^{o r d}=\max _{c a a_{o q}^{o r d} \in C a_{o}^{\prime o r d}}\left(h\left(x_{o}^{\text {ord }}, c a t_{o q}^{\text {ord }}\right)\right) \\
& x_{e f}^{m e t}=\max _{c a t_{f q}^{m e} \in C a t_{f}^{m e t}}\left(h\left(x_{f}^{m e t}, c a t_{f q}^{m e t}\right)\right) \\
& x_{e s}^{r e f, m e t}=x_{e s}^{r e f}
\end{aligned}
$$

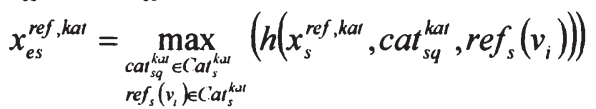

Aufbauend auf den Präferenzen der Plattformteilnehmer und den zuvor ermittelten Empfehlungen für Transaktionsbeziehungen auf der Plattform zeigen die Ausführungen zur Matchingphase Wege auf, wie bestmöglich zueinander passende Transaktionspartner für die Plattorm identifiziert werden können. Für den Vergleich der Präferenzprofile werden üblicherweise für unterschiedliche Skalenniveaus eingesetzte (Un-)Ähnlichkeitsmaßzahlen unterstützt und für den Zweck dieser Arbeit angepasst bzw. enweitert. Insbesondere wird eine duale Interpretation der Präferenzvariablen für die Maßzahlberechnung eingeführt, die den unterschiedlichen Rollen (Input-Output) der Plattformteilnehmer Rechnung 
trägt. Darüber hinaus ermöglichen die für die Matchingphase neu entwickelten Methoden einen indirekten Präferenzvergleich unter Berücksichtigung eines vom zu vergleichenden Objektpaar (z.B. Teilnehmer) abhängigen Referenzmerkmals (Referenzpräferenzvergleich). Die Matchingphase bietet einerseits die Bestimmung des Ausmaßes der Übereinstimmungen der Präferenzprofile für bestehende Teilnehmer, welches eine präferenzbezogene Reihung der Transaktionsempfehlungen möglich macht (Funktionen APPENDMATCH und ORDERNSELECT; grafische Darstellung über die Funktion PLOTEFFINAL basierend auf EDGEFR2MAT), und andererseits die Erstellung von ,optimalen' Präferenzprofilen für potenzielle neue Teilnehmer basierend auf den Präferenzkonstellationen der bestehenden Plattformteilnehmer, die bspw. bei der Suche nach neuen Teilnehmern Einsatz finden können. 
Michael Weber - 978-3-631-75376-7

Downloaded from PubFactory at 01/11/2019 05:44:37AM

via free access 


\section{Demonstrationsbeispiel}

Zur Veranschaulichung der Wirkungsweise des Transaktionssystemunterstützungsmodells soll nachfolgend für alle Phasen des Modells auf Basis volkswirtschaftsstatistischer Datenquellen sowie fiktiver, aber realitätsnaher Transaktionsdaten ein Beispiel zur Demonstration der in dieser Arbeit vorgeschlagenen Verfahren zur Generierung von Vorschlägen für die Optimierung der Zusammensetzung einer Kollaborationsplattform sowie die Unterstützung der Plattformteilnehmer beschrieben werden.

\subsection{Datenaufbereitung}

Den Ausgangspunkt bilden dabei als Strukturdaten die Aufkommens- und die Verwendungstabelle (um-)bewertet zu Anschaffungspreisen für die heimische Produktion und den heimischen Intermediärverbrauch in der Dimension 73 Güter x 73 Aktivitäten ${ }^{68}$, die daraus entsprechend der Gütertechnologieannahme mittels des Almon-Algorithmus abgeleitete und auf die 20 größten Spaltenwerte reduzierte Input-Output-Tabelle IOTgg69 (Appendix C, Klassifikationen gemäß Appendix B) sowie die nach realen Vorbildern generierten Profildaten zu den Plattformteilnehmern (BCIprofile, Tabelle 33) und deren auf der Plattform abgewickelten Transaktionen (BCledgeFRuug, Tabelle 34). Die Profildaten halten hierbei die Aktivitäten, in denen die Betriebe der Plattformteilnehmer (Unternehmen) tätig sind, ebenso fest wie die über die jeweiligen Aktivitäten produzierten Güter. Die Transaktionsdaten beinhalten Informationen zu den

68 Konkret handelt es sich um die eigens für die Zwecke dieser Arbeit zu Anschaffungspreisen umbewertete heimische Produktion zu Herstellungspreisen [Statistik Austria 2005, Tabelle 4 zuzüglich der auf Basis der Publikationstabelle 1 ermittelten Spannen, ohne Importanteile] und den ebenfalls für diese Arbeit errechneten heimischen Intermediärverbrauch zu Anschaffungspreisen, der auf Grundlage des Intermediärverbrauchs zu Anschaffungspreisen [Statistik Austria 2005, Tabelle 8] bestimmt wurde.

69 Zusätzlich wurden bei dieser Input-Output Tabelle nach der Kürzung auf die 20 größten Spaltenwerte die Diagonalelemente nullgesetzt und die Werte gerundet. 
Partnern (Unternehmen) der auf der Plattform abgewickelten Transaktionen, das jeweils verkaufte Gut (den Transaktionsgegenstand) und den Verkaufswert.

\subsubsection{Profildaten}

Tabelle 33 zeigt in der dritten Spalte die 15 an der Plattorm teilnehmenden Unternehmen, deren betriebliche Aktivitäten und die darin hergestellten Güter.

Tabelle 33: BCIprofile

\begin{tabular}{|c|c|c|c|}
\hline & Aktivität & Gut & Name \\
\hline 1 & $\mathrm{X02}$ & 02 & Forstwirtschaft $\mathrm{GmbH}$ \\
\hline 2 & $\mathrm{X} 17$ & 17 & Textil GmbH \\
\hline 3 & $\mathrm{X} 20$ & 20 & Holzverarbeitung GmbH \\
\hline 4 & $\mathrm{X} 22$ & 22 & Druck GmbH \\
\hline 5 & $\times 21$ & 21 & Papier und Chemie $\mathrm{GmbH}$ \\
\hline 6 & X24 & 24 & Papier und Chemie $\mathrm{GmbH}$ \\
\hline 7 & $\mathrm{X} 27$ & 27 & Metallverarbeitung $\mathrm{GmbH}$ \\
\hline 8 & $\mathrm{X} 45 \mathrm{~B}$ & 45B & Metallverarbeitung $\mathrm{GmbH}$ \\
\hline 9 & $\times 28$ & 28 & Metallerzeugung GmbH \\
\hline 10 & X45B & 45B & Metallerzeugung GmbH \\
\hline 11 & X29 & 29 & Maschinenbau GmbH \\
\hline 12 & $\times 45 A$ & $45 \mathrm{~A}$ & Hoch- und Tiefbau GmbH \\
\hline 13 & $\mathrm{X} 45 \mathrm{~B}$ & 45B & Bauinstallation $\mathrm{GmbH}$ \\
\hline 14 & $\times 55$ & 55 & Hotel GmbH \\
\hline 15 & X63B & $63 \mathrm{~B}$ & Reisebüro $\mathrm{GmbH}$ \\
\hline 16 & $X 72$ & 72 & EDV GmbH \\
\hline 17 & $X 52 A$ & $52 \mathrm{~A}$ & EDV GmbH \\
\hline 18 & $\mathrm{X} 74 \mathrm{~B}$ & $74 \mathrm{~B}$ & Architekturbüro OEG \\
\hline 19 & $\times 74 C$ & $74 \mathrm{C}$ & Werbeagentur $\mathrm{GmbH}$ \\
\hline
\end{tabular}

Vier dieser Plattformteilnehmer weisen jeweils zwei Aktivitäten auf, während die restlichen elf Teilnehmer nur einer Aktivität nachgehen. Auf der Plattform werden gemäß Tabelle 3317 Aktivitäten über die Teilnehmer grundsätzlich abgedeckt, die zur Vereinfachung für die Demonstration des Modells jeweils nur ein Gut

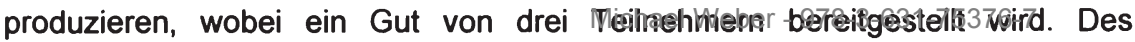


Weiteren werden zur Reduktion der Komplexität zwecks Darstellbarkeit, aber auch angesichts der Verfügbarkeit der Daten auf der gesamtwirtschaftlichen Ebene, die Betriebe der teilnehmenden Unternehmen vollständig einer Aktivität der (O)NACE-Zweistellerebene zugeordnet. Die Klassifikation entspricht hierbei der auf der (ठ)NACE aufbauenden Input-Output-Gliederung gemäß Statistik Austria [2005]. Gleiches gilt für die auf der Plattform angebotenen Güter, die auf der (ठ)CPA-Zweistellerebene in der Input-Output-Version gemäß Statistik Austria [2005] vorliegen. Kurzbezeichnungen für die Identifikatoren können dem Appendix B entnommen werden.

\subsubsection{Transaktionsdaten}

Tabelle 34 zeigt die Transaktionsdaten der Plattform, die das verkaufte Gut, den Verkäufer, den Käufer sowie den Transaktionswert in Tausend Geldeinheiten (GE) festhalten. Es zeigt sich, dass 16 Transaktionen auf der Plattform bereits stattgefunden haben.

Tabelle 34: BCledgeFRuug

\begin{tabular}{|c|c|c|c|c|}
\hline & Gut & Verkäufer & Käufer & $\begin{array}{c}\text { Wert } \\
\text { (in Tsd. GE) }\end{array}$ \\
\hline 1 & 02 & Forstwirtschaft GmbH & Holzverarbeitung $\mathrm{GmbH}$ & 28 \\
\hline 2 & 20 & Holzverarbeitung $\mathrm{GmbH}$ & Forstwirtschaft GmbH & 2 \\
\hline 3 & 22 & Druck GmbH & Papier und Chemie $\mathrm{GmbH}$ & 44 \\
\hline 4 & 22 & Druck GmbH & EDV GmbH & 20 \\
\hline 5 & 22 & Druck GmbH & Werbeagentur $\mathrm{GmbH}$ & 25 \\
\hline 6 & 21 & Papier und Chemie $\mathrm{GmbH}$ & Textil GmbH & 16 \\
\hline 7 & 20 & Holzverarbeitung GmbH & Papier und Chemie GmbH & 8 \\
\hline 8 & 27 & Metallverarbeitung $\mathrm{GmbH}$ & Metallerzeugung $\mathrm{GmbH}$ & 77 \\
\hline 9 & 27 & Metallverarbeitung $\mathrm{GmbH}$ & Maschinenbau $\mathrm{GmbH}$ & 35 \\
\hline 10 & 28 & Metallerzeugung $\mathrm{GmbH}$ & Metallverarbeitung $\mathrm{GmbH}$ & 5 \\
\hline 11 & 28 & Metallerzeugung $\mathrm{GmbH}$ & Maschinenbau GmbH & 84 \\
\hline 12 & 28 & Metallerzeugung $\mathrm{GmbH}$ & Hoch- und Tiefbau GmbH & 35 \\
\hline 13 & 28 & Metallerzeugung $\mathrm{GmbH}$ & Bauinstallation $\mathrm{GmbH}$ & 13 \\
\hline 14 & 29 & Maschinenbau GmbH & Metallerzeugung $\mathrm{GmbH}$ & 6 \\
\hline 15 & 29 & Maschinenbau GmbH & Bauinstallation $\mathrm{GmbH}$ & 22 \\
\hline 16 & 55 & Hotel GmbH & $\begin{array}{l}\text { Reisebüro GmbH } \\
\text { Michael Weber - } 978\end{array}$ & $\begin{array}{l}14 \\
5376-7\end{array}$ \\
\hline
\end{tabular}


Acht Unternehmen traten als Verkäufer auf, wobei drei von diesen allerdings nur eine Verkaufstransaktion durchgeführt haben. Als Käufer wurden zwölf Unternehmen auf der Plattform aktiv, wobei vier von diesen zwei Käufe tätigten, während die restlichen Unternehmen nur einen Kaufvorgang abwickelten. Insgesamt waren 14 an der Plattform teilnehmenden Unternehmen an den Transaktionen beteiligt, d.h. ein Unternehmen konnte keine Transaktion durchführen. Sechs Unternehmen treten sowohl in einer Verkäufer- als auch in einer Käuferrolle auf. Die Transaktionshöhe liegt in einem Bereich von 2 bis 84, der Durchschnittswert beträgt 27,12. Die Summe der Transaktionswerte ist 434. Das Unternehmen mit dem größten Anteil am Gesamtverkauf ist die Metallerzeugung $\mathrm{GmbH}$ (137), den höchsten Anteil auf der Käuferseite hat hingegen die Maschinenbau GmbH (119).

\subsubsection{Abgeleitete Datenquellen}

Die Matrixdarstellungen der Transaktionsverläufe wie die Matrix der abgesetzten Güter BClug (Tabelle 35), die Matrix der Teilnehmerverflechtungen BCluu (Tabelle 36) und die Matrix der Güterabnahme BCIgu (Tabelle 37) können gemäß Kapitel 5.4 unter Kenntnis der Transaktionsdaten gebildet werden.

Tabelle 35: BCIug

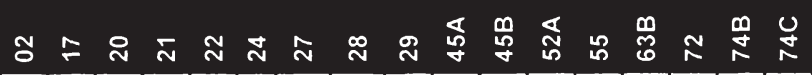

\begin{tabular}{|c|c|c|c|c|c|c|c|c|c|c|c|c|c|c|c|c|c|}
\hline Architekturbüro OEG & 0 & 0 & 0 & 0 & 0 & 0 & 0 & 0 & 0 & 0 & 0 & 0 & 0 & 0 & 0 & 0 & 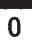 \\
\hline Bauinstallation $\mathrm{GmbH}$ & 0 & 0 & 0 & 0 & 0 & 0 & 0 & 0 & 0 & 0 & 0 & 0 & 0 & 0 & 0 & 0 & 0 \\
\hline Druck GmbH & 0 & 0 & 0 & 0 & 89 & 0 & 0 & 0 & 0 & 0 & 0 & 0 & 0 & 0 & 0 & 0 & 0 \\
\hline EDV GmbH & 0 & 0 & 0 & 0 & 0 & 0 & 0 & 0 & 0 & 0 & 0 & 0 & 0 & 0 & 0 & 0 & 0 \\
\hline Forstwirtschaft GmbH & 28 & 0 & 0 & 0 & 0 & 0 & 0 & 0 & 0 & 0 & 0 & 0 & 0 & 0 & 0 & 0 & 0 \\
\hline Hoch- und Tiefbau GmbH & 0 & 0 & 0 & 0 & 0 & 0 & 0 & 0 & 0 & 0 & 0 & 0 & 0 & 0 & 0 & 0 & 0 \\
\hline Holzverarbeitung GmbH & 0 & 0 & 10 & 0 & 0 & 0 & 0 & 0 & 0 & 0 & 0 & 0 & 0 & 0 & 0 & 0 & 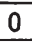 \\
\hline Hotel GmbH & 0 & 0 & 0 & 0 & 0 & 0 & 0 & 0 & 0 & 0 & 0 & 0 & 14 & 0 & 0 & 0 & 0 \\
\hline Maschinenbau GmbH & 0 & 0 & 0 & 0 & 0 & 0 & 0 & 0 & 28 & 0 & 0 & 0 & 0 & 0 & 0 & 0 & 0 \\
\hline Metallerzeugung GmbH & 0 & 0 & 0 & 0 & 0 & 0 & 0 & 137 & 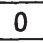 & 0 & 0 & 0 & 0 & 0 & 0 & 0 & 0 \\
\hline Metallverarbeitung $\mathrm{GmbH}$ & 0 & 0 & 0 & 0 & 0 & 0 & 112 & 0 & 0 & 0 & 0 & 0 & 0 & 0 & 0 & 0 & 0 \\
\hline Papier u. Chemie GmbH & 0 & 0 & 0 & 16 & 0 & 0 & 0 & 0 & 0 & 0 & 0 & 0 & 0 & 0 & 0 & 0 & 0 \\
\hline Reisebüro GmbH & 0 & 0 & 0 & 0 & 0 & 0 & 0 & 0 & 0 & 0 & 0 & 0 & 0 & 0 & 0 & 0 & 0 \\
\hline Tex & 0 & 0 & 0 & 0 & 0 & 0 & 0 & 0 & 0 & 0 & 0 & 0 & 0 & 0 & 0 & 0 & 0 \\
\hline Werbeagentur GmbH & 0 & 0 & 0 & 0 & 0 & 0 & 0 & 0 & 0 & 0 & 0 & -1 & 0 & 0 & 0 & 0 & 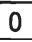 \\
\hline
\end{tabular}


Tabelle 36: BCIuu

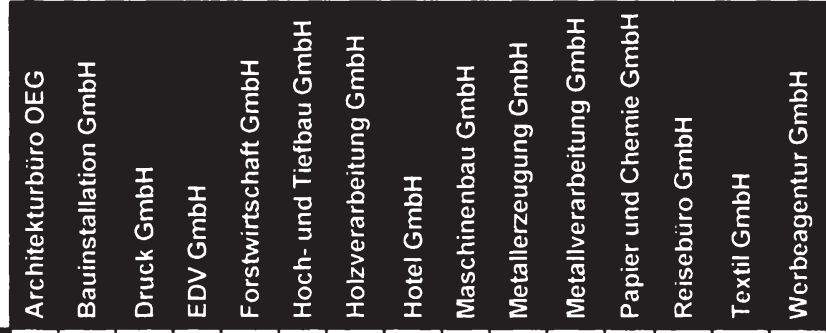

Architekturbüro OEG

Bauinstallation $\mathrm{GmbH}$ Druck $\mathrm{GmbH}$

EDV GmbH

Forstwirtschaft $\mathrm{GmbH}$ Hoch- und Tiefbau GmbH

Holzverarbeitung $\mathrm{GmbH}$ Hotel $\mathrm{GmbH}$

Maschinenbau $\mathrm{GmbH}$

Metallerzeugung $\mathrm{GmbH}$

Metallverarbeitung $\mathrm{GmbH}$

Papier und Chemie $\mathrm{GmbH}$

Reisebüro $\mathrm{GmbH}$

Textil GmbH

Werbeagentur $\mathrm{GmbH}$

\begin{tabular}{|c|c|c|c|c|c|c|c|c|c|c|c|c|c|c|}
\hline 0 & 0 & 0 & 0 & 0 & 0 & 0 & 0 & 0 & 0 & 0 & 0 & 0 & 0 & 0 \\
\hline 0 & 0 & 0 & 0 & 0 & 0 & 0 & 0 & 0 & 0 & 0 & 0 & 0 & 0 & 0 \\
\hline 0 & 0 & 0 & 20 & 0 & 0 & 0 & 0 & 0 & 0 & 0 & 44 & 0 & 0 & 25 \\
\hline 0 & 0 & 0 & 0 & 0 & 0 & 0 & 0 & 0 & 0 & 0 & 0 & 0 & 0 & 0 \\
\hline 0 & 0 & 0 & 0 & 0 & 0 & 28 & 0 & 0 & 0 & 0 & 0 & 0 & 0 & 0 \\
\hline 0 & 0 & 0 & 0 & 0 & 0 & 0 & 0 & 0 & 0 & 0 & 0 & 0 & 0 & 0 \\
\hline 0 & 0 & 0 & 0 & 2 & 0 & 0 & 0 & 0 & 0 & 0 & 8 & 0 & 0 & 0 \\
\hline 0 & 0 & 0 & 0 & 0 & 0 & 0 & 0 & 0 & 0 & 0 & 0 & 14 & 0 & 0 \\
\hline 0 & 22 & 0 & 0 & 0 & 0 & 0 & 0 & 0 & 6 & 0 & 0 & 0 & 0 & 0 \\
\hline 0 & 13 & 0 & 0 & 0 & 35 & 0 & 0 & 84 & 0 & 5 & 0 & 0 & 0 & 0 \\
\hline 0 & 0 & 0 & 0 & 0 & 0 & 0 & 0 & 35 & 77 & 0 & 0 & 0 & 0 & 0 \\
\hline 0 & 0 & 0 & 0 & 0 & 0 & 0 & 0 & 0 & 0 & 0 & 0 & 0 & 16 & 0 \\
\hline 0 & 0 & 0 & 0 & 0 & 0 & 0 & 0 & 0 & 0 & 0 & 0 & 0 & 0 & 0 \\
\hline 0 & 0 & 0 & 0 & 0 & 0 & 0 & 0 & 0 & 0 & 0 & 0 & 0 & 0 & 0 \\
\hline 0 & 0 & 0 & 0 & 0 & 0 & 0 & 0 & 0 & 0 & 0 & 0 & 0 & 0 & 0 \\
\hline
\end{tabular}

Tabelle 37: BCIgu

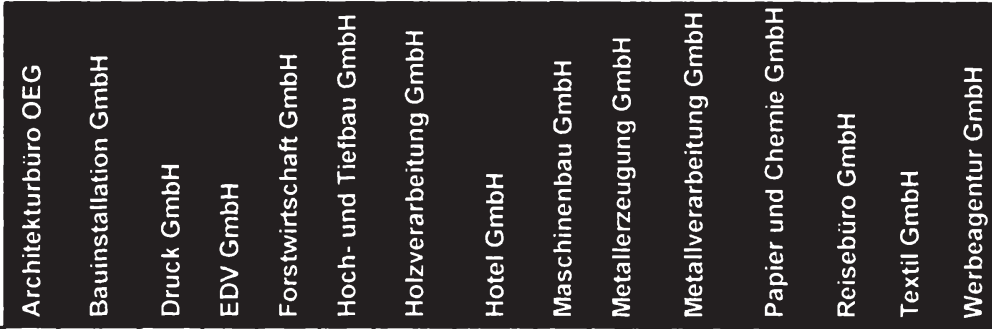

\begin{tabular}{|c|c|c|c|c|c|c|c|c|c|c|c|c|c|c|c|}
02 & 0 & 0 & 0 & 0 & 0 & 0 & 28 & 0 & 0 & 0 & 0 & 0 & 0 & 0 & 0 \\
\hline 17 & 0 & 0 & 0 & 0 & 0 & 0 & 0 & 0 & 0 & 0 & 0 & 0 & 0 & 0 & 0 \\
20 & 0 & 0 & 0 & 0 & 2 & 0 & 0 & 0 & 0 & 0 & 0 & 8 & 0 & 0 & 0 \\
\hline 21 & 0 & 0 & 0 & 0 & 0 & 0 & 0 & 0 & 0 & 0 & 0 & 0 & 0 & 16 & 0 \\
\hline 22 & 0 & 0 & 0 & 20 & 0 & 0 & 0 & 0 & 0 & 0 & 0 & 44 & 0 & 0 & 25 \\
24 & 0 & 0 & 0 & 0 & 0 & 0 & 0 & 0 & 0 & 0 & 0 & 0 & 0 & 0 & 0 \\
\hline 27 & 0 & 0 & 0 & 0 & 0 & 0 & 0 & 0 & 35 & 77 & 0 & 0 & 0 & 0 & 0 \\
28 & 0 & 13 & 0 & 0 & 0 & 35 & 0 & 0 & 84 & 0 & 5 & 0 & 0 & 0 & 0 \\
29 & 0 & 22 & 0 & 0 & 0 & 0 & 0 & 0 & 0 & 6 & 0 & 0 & 0 & 0 & 0 \\
\hline $5 \mathrm{~A}$ & 0 & 0 & 0 & 0 & 0 & 0 & 0 & 0 & 0 & 0 & 0 & 0 & 0 & 0 & 0 \\
\hline
\end{tabular}




\begin{tabular}{|c|c|c|c|c|c|c|c|c|c|c|c|c|c|c|c|}
\hline & 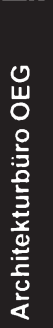 & 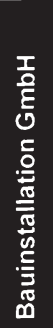 & 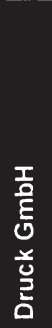 & 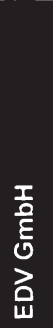 & 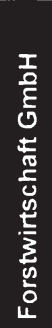 & 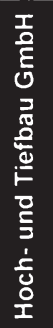 & 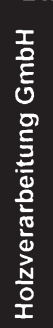 & $\begin{array}{l}\text { I } \\
\text { है } \\
\text { ㅇ } \\
\frac{\Phi}{\Xi} \\
\text { 오 }\end{array}$ & 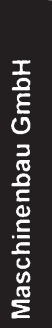 & 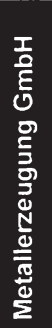 & 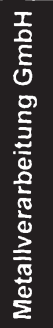 & 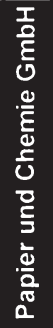 & 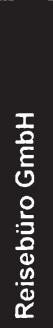 & 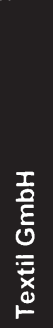 & 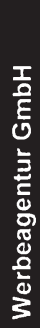 \\
\hline 45B & 0 & 0 & 0 & 0 & 0 & 0 & 0 & 0 & 0 & 0 & 0 & 0 & 0 & 0 & 0 \\
\hline $52 \mathrm{~A}$ & 0 & 0 & 0 & 0 & 0 & 0 & 0 & 0 & 0 & 0 & 0 & 0 & 0 & 0 & 0 \\
\hline 55 & 0 & 0 & 0 & 0 & 0 & 0 & 0 & 0 & 0 & 0 & 0 & 0 & 14 & 0 & 0 \\
\hline $63 \mathrm{~B}$ & 0 & 0 & 0 & 0 & 0 & 0 & 0 & 0 & 0 & 0 & 0 & 0 & 0 & 0 & 0 \\
\hline 72 & 0 & 0 & 0 & 0 & 0 & 0 & 0 & 0 & 0 & 0 & 0 & 0 & 0 & 0 & 0 \\
\hline $74 \mathrm{~B}$ & 0 & 0 & 0 & 0 & 0 & 0 & 0 & 0 & 0 & 0 & 0 & 0 & 0 & 0 & 0 \\
\hline $74 \mathrm{C}$ & 0 & 0 & 0 & 0 & 0 & 0 & 0 & 0 & 0 & 0 & 0 & 0 & 0 & 0 & 0 \\
\hline
\end{tabular}

BClaug (Tabelle 38), die Matrix der abgesetzten Güter mit Aktivitätsbezug, ist unter Einbezug der Profildaten erstellbar.

Tabelle 38: BCIaug

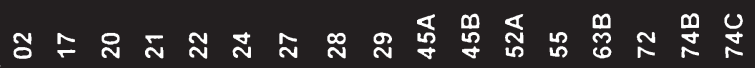

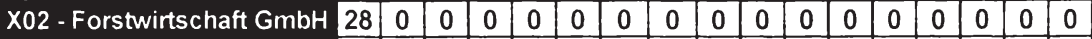

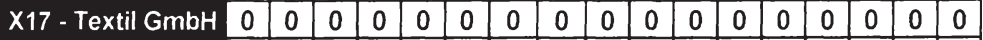

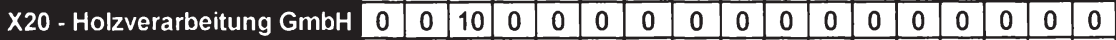

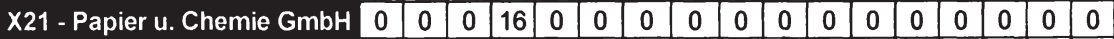

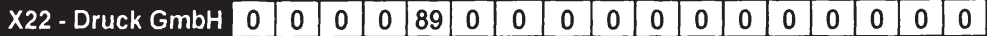

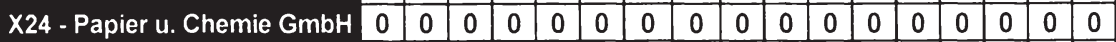

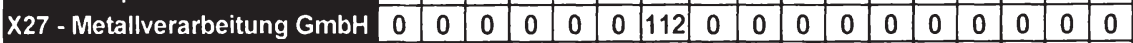

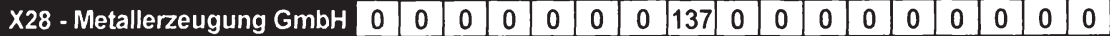

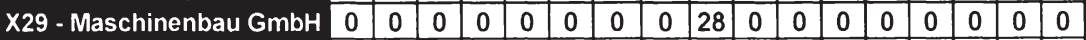

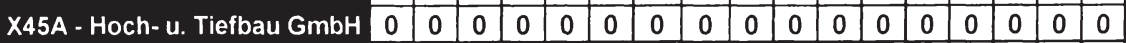

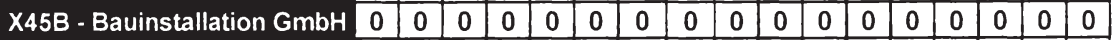

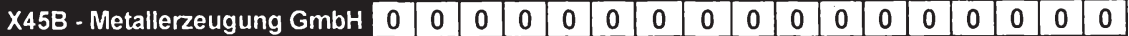

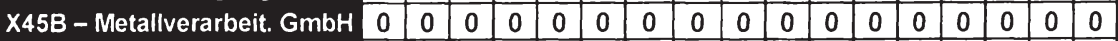

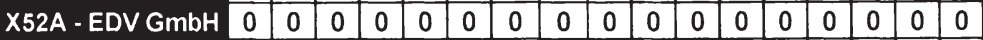

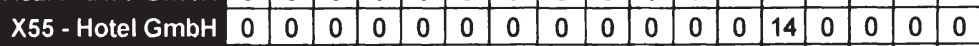

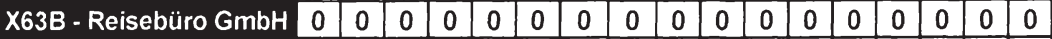

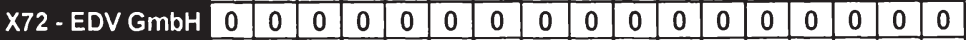

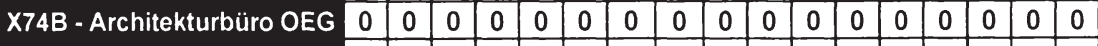

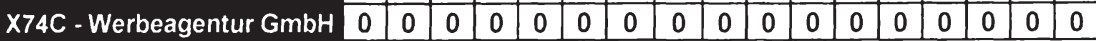


Eine einfache Interpretation der Teilnehmerverflechtungen auf Basis der Transaktionsdaten ohne Information über den Transaktionsinhalt ermöglicht Abbildung 16, die im Wesentlichen die Existenz von zwei untereinander interagierenden Gruppen von Teilnehmern erkennbar macht.

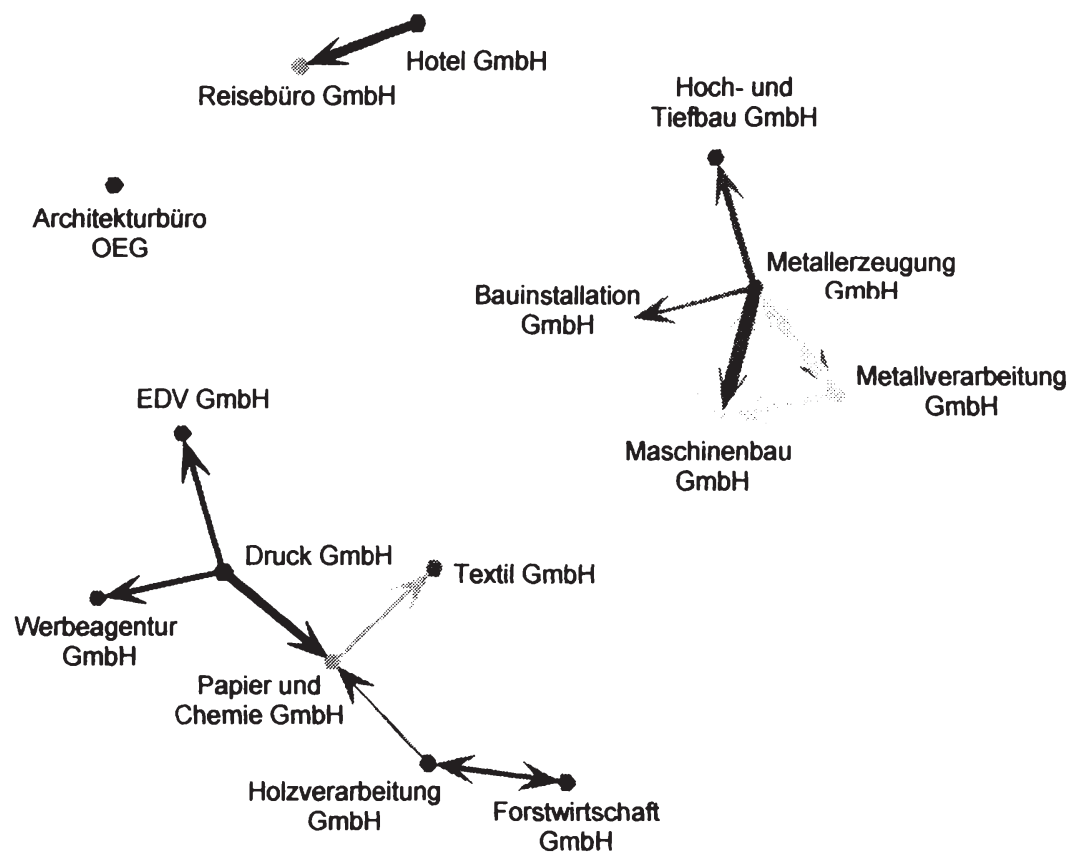

Abbildung 16: BCluu

Erweiterungen dieser einfach ableitbaren Matrizen können durch eine Verschränkung der Strukturdaten mit den Profil- und Transaktionsdaten in einem nächsten Schritt entsprechend den Formeln aus Kapitel 5.4 erfolgen. BCIgau (Tabelle 39), die Güter-Teilnehmer-Aktivitäten Matrix, kann dadurch ebenso generiert werden wie die Güter-Teilnehmer-Güter Matrix, BCIggu (Tabelle 40), und die Matrix zur Darstellung der Input-Output-Relationen auf der Kollaborationsplattform bezogen auf Güter, BCIgg (Tabelle 41). 
Tabelle 39: BCIgau

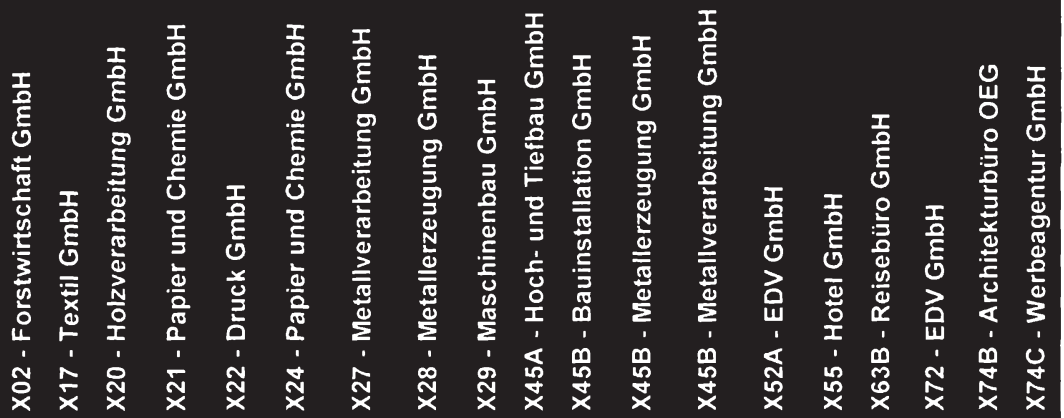

\begin{tabular}{l|l|l|l|l|l|l|l|l|l|l|l|l|l|l|l|l|l|l|l|}
02 & 0 & 0 & 28 & 0 & 0 & 0 & 0 & 0 & 0 & 0 & 0 & 0 & 0 & 0 & 0 & 0 & 0 & 0 & 0 \\
\hline 17 & 0 & 0 & 0 & 0 & 0 & 0 & 0 & 0 & 0 & 0 & 0 & 0 & 0 & 0 & 0 & 0 & 0 & 0 & 0 \\
\hline
\end{tabular}

20 \begin{tabular}{llllllllllllllllllll|l|l|l|l|} 
& 2 & 0 & 0 & 7,6 & 0 & 0,4 & 0 & 0 & 0 & 0 & 0 & 0 & 0 & 0 & 0 & 0 & 0 & 0 & 0 \\
\hline
\end{tabular}

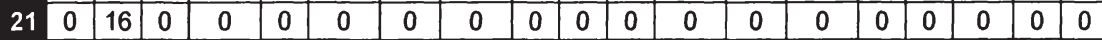

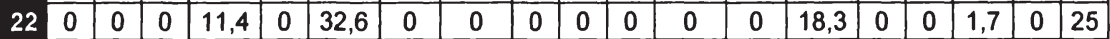

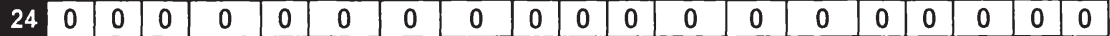

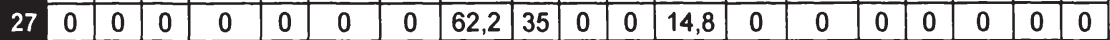

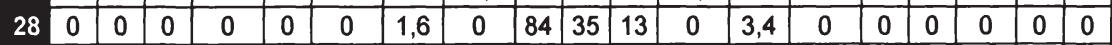

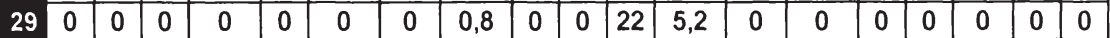

\begin{tabular}{|c|c|c|c|c|c|c|}
\hline 45A 0 & 0 & 0 & 0 & 0 & 0 & 0 \\
\hline
\end{tabular}

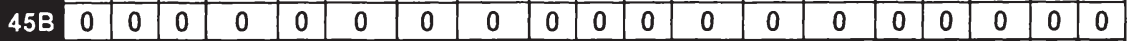

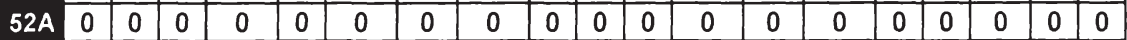

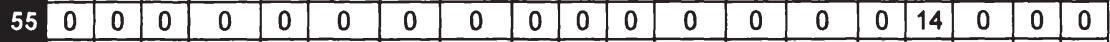

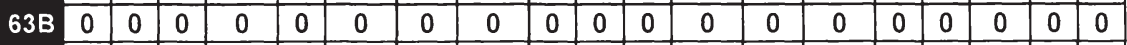

72 \begin{tabular}{lllllllllllllllll|l|l|l|l|l|} 
& 0 & 0 & 0 & 0 & 0 & 0 & 0 & 0 & 0 & 0 & 0 & 0 & 0 & 0 & 0 & 0 & 0 & 0 & 0 \\
\hline
\end{tabular}

\begin{tabular}{|c|c|c|c|c|c|c|c|c|c|c|c|c|c|c|c|c|c|c|}
\hline B 0 & 0 & 0 & 0 & 0 & 0 & 0 & 0 & 0 & 0 & 0 & 0 & 0 & 0 & 0 & 0 & 0 & 0 & 0 \\
\hline & 0 & 0 & 0 & 0 & 0 & 0 & 0 & 0 & 0 & 0 & 0 & 0 & 0 & 0 & 0 & 0 & 0 & \\
\hline
\end{tabular}

Tabelle 40: BCIggu

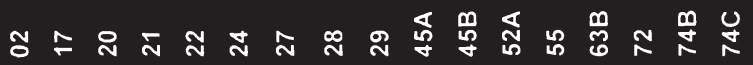

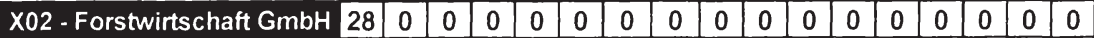

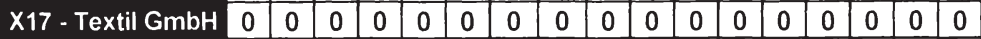

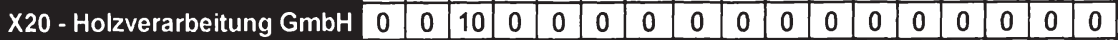

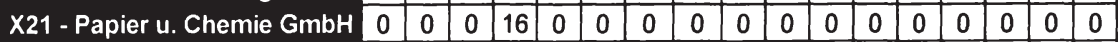

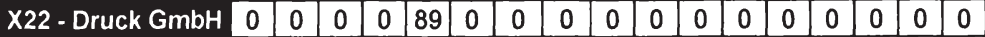

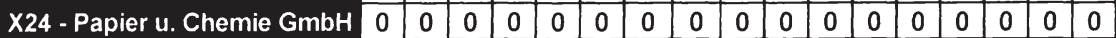

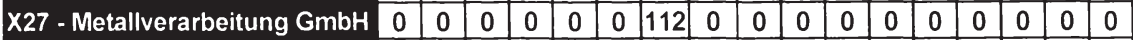

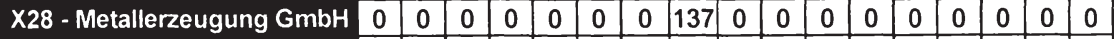

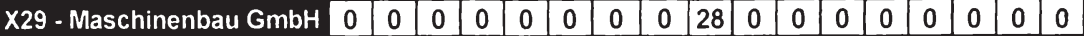

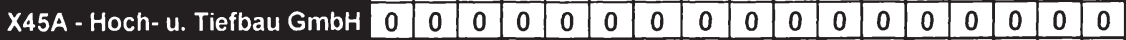

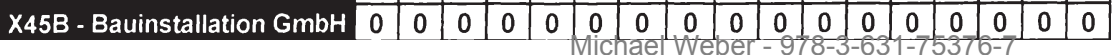




\begin{tabular}{|c|c|c|c|c|c|c|c|c|c|c|c|c|c|c|c|c|c|}
\hline & 8 & - & $\pi$ & $N$ & & $\mathbb{N}$ & N & $\stackrel{\sim}{\sim}$ & N & $\mathscr{8}$ & $\underset{8}{\infty}$ & శ్ & 号 & $\underset{\tilde{\omega}}{\infty}$ & $\mathbb{N}$ & & $\underset{N}{N}$ \\
\hline X45B - Metallerzeugung GmbH & 0 & 0 & 0 & 0 & 0 & 0 & 0 & 0 & 0 & 0 & 0 & 0 & 0 & 0 & 0 & 0 & 0 \\
\hline X45B - Metallverarbeit. GmbH & 0 & 0 & 0 & 0 & 0 & 0 & 0 & 0 & 0 & 0 & 0 & 0 & 0 & 0 & 0 & 0 & 0 \\
\hline X52A - EDV GmbH & 0 & 0 & 0 & 0 & 0 & 0 & 0 & 0 & 0 & 0 & 0 & 0 & 0 & 0 & 0 & 0 & 0 \\
\hline X55 - Hotel GmbH & 0 & 0 & 0 & 0 & 0 & 0 & 0 & 0 & 0 & 0 & 0 & 0 & 14 & 0 & 0 & 0 & 0 \\
\hline X63B - Reisebüro GmbH & 0 & 0 & 0 & 0 & 0 & 0 & 0 & 0 & 0 & 0 & 0 & 0 & 0 & 0 & 0 & 0 & 0 \\
\hline X72 - EDV GmbH & 0 & 0 & 0 & 0 & 0 & 0 & 0 & 0 & 0 & 0 & 0 & 0 & 0 & 0 & 0 & 0 & 0 \\
\hline X74B - Architekturbüro OEG & 0 & 0 & 0 & 0 & 0 & 0 & 0 & 0 & 0 & 0 & 0 & 0 & 0 & 0 & 0 & 0 & 0 \\
\hline X74C - Werbeagentur GmbH & 0 & 0 & 0 & 0 & 0 & 0 & 0 & 0 & 0 & 0 & 0 & 0 & 0 & 0 & 0 & 0 & 0 \\
\hline
\end{tabular}

Tabelle 41: BClgg

\section{ช}

\begin{tabular}{|c|c|c|c|c|c|c|c|c|c|c|c|c|c|c|c|c|c|}
\hline 02 & 0 & 0 & 28 & 0 & 0 & 0 & 0 & 0 & 0 & 0 & 0 & 0 & 0 & 0 & 0 & 0 & 0 \\
\hline 17 & 0 & 0 & 0 & 0 & 0 & 0 & 0 & 0 & 0 & 0 & 0 & 0 & 0 & 0 & 0 & 0 & 0 \\
\hline 20 & 2 & 0 & 0 & 8 & 0 & 0 & 0 & 0 & 0 & 0 & 0 & 0 & 0 & 0 & 0 & 0 & 0 \\
\hline 21 & 0 & 16 & 0 & 0 & 0 & 0 & 0 & 0 & 0 & 0 & 0 & 0 & 0 & 0 & 0 & 0 & 0 \\
\hline 22 & 0 & 0 & 0 & 11 & 0 & 33 & 0 & 0 & 0 & 0 & 0 & 18,6 & 0 & 0 & 1,4 & 0 & 25 \\
\hline 24 & 0 & 0 & 0 & 0 & 0 & 0 & 0 & 0 & 0 & 0 & 0 & 0 & 0 & 0 & 0 & 0 & 0 \\
\hline 27 & 0 & 0 & 0 & 0 & 0 & 0 & 0 & 62,2 & 35 & 0 & 14,8 & 0 & 0 & 0 & 0 & 0 & 0 \\
\hline 28 & 0 & 0 & 0 & 0 & 0 & 0 & 1,3 & 0 & 84 & 35 & 16,7 & 0 & 0 & 0 & 0 & 0 & 0 \\
\hline 29 & 0 & 0 & 0 & 0 & 0 & 0 & 0 & 0 & 0 & 0 & 28 & 0 & 0 & 0 & 0 & 0 & 0 \\
\hline $5 A$ & 0 & 0 & 0 & 0 & 0 & 0 & 0 & 0 & 0 & 0 & 0 & 0 & 0 & 0 & 0 & 0 & 0 \\
\hline $5 B$ & 0 & 0 & 0 & 0 & 0 & 0 & 0 & 0 & 0 & 0 & 0 & 0 & 0 & 0 & 0 & 0 & 0 \\
\hline $2 A$ & 0 & 0 & 0 & 0 & 0 & 0 & 0 & 0 & 0 & 0 & 0 & 0 & 0 & 0 & 0 & 0 & 0 \\
\hline 55 & 0 & 0 & 0 & 0 & 0 & 0 & 0 & 0 & 0 & 0 & 0 & 0 & 0 & 14 & 0 & 0 & 0 \\
\hline $3 B$ & 0 & 0 & 0 & 0 & 0 & 0 & 0 & 0 & 0 & 0 & 0 & 0 & 0 & 0 & 0 & 0 & 0 \\
72 & 0 & 0 & 0 & 0 & 0 & 0 & 0 & 0 & 0 & 0 & 0 & 0 & 0 & 0 & 0 & 0 & 0 \\
\hline $4 B$ & 0 & 0 & 0 & 0 & 0 & 0 & 0 & 0 & 0 & 0 & 0 & 0 & 0 & 0 & 0 & 0 & 0 \\
\hline $4 C$ & 0 & 0 & 0 & 0 & 0 & 0 & 0 & 0 & 0 & 0 & 0 & 0 & 0 & 0 & 0 & 0 & 0 \\
\hline
\end{tabular}

Einen Überblick über die auf der Plattform bereitgestellten Güter, deren Aktivitätsursprung sowie dem dazu passenden kumulierten Transaktionswert liefert BCIag (BCIsell) (Tabelle 42), während BCIga (BCIbuy) (Tabelle 43) die - zum Teil mittels der Strukturdaten abgeschätzte - Aktivitäten aufzeigt, die ,Destinationen' der über die Plattform bezogenen Güter sind. Beide Matrizen bieten somit einen Einblick in die Output- bzw. Input-Zusammenhänge zwischen Aktivitäten und Gütern aus einer rein plattformzentrierten Perspektive. 
Tabelle 42: BCIag (BCIsell)

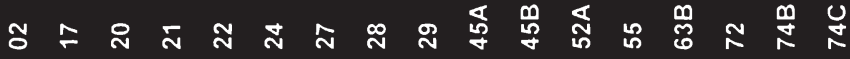

\begin{tabular}{|c|c|c|c|c|c|c|c|c|c|c|c|c|c|c|c|c|}
\hline $102 \quad 28$ & 0 & 0 & 0 & 0 & 0 & 0 & 0 & 0 & 0 & 0 & 0 & 0 & 0 & 0 & 0 & 0 \\
\hline$1 7 \longdiv { 0 }$ & 0 & 0 & 0 & 0 & 0 & 0 & 0 & 0 & 0 & 0 & 0 & 0 & 0 & 0 & 0 & 0 \\
\hline 200 & 0 & 10 & 0 & 0 & 0 & 0 & 0 & 0 & 0 & 0 & 0 & 0 & 0 & 0 & 0 & 0 \\
\hline $\begin{array}{ll}\times 21 & 0 \\
\end{array}$ & 0 & 0 & 16 & 0 & 0 & 0 & 0 & 0 & 0 & 0 & 0 & 0 & 0 & 0 & 0 & 0 \\
\hline 220 & 0 & 0 & 0 & \begin{tabular}{|l|}
89 \\
\end{tabular} & 0 & 0 & 0 & 0 & 0 & 0 & 0 & 0 & 0 & 0 & 0 & 0 \\
\hline X24 & 0 & 0 & 0 & 0 & 0 & 0 & 0 & 0 & 0 & 0 & 0 & 0 & 0 & 0 & 0 & 0 \\
\hline 0 & 0 & \begin{tabular}{|l|} 
\\
\end{tabular} & 0 & 0 & 0 & 112 & 0 & 0 & 0 & 0 & 0 & 0 & 0 & 0 & 0 & 0 \\
\hline$\times 28$ & 0 & 0 & 0 & 0 & 0 & 0 & 137 & 0 & 0 & 0 & 0 & 0 & 0 & 0 & 0 & 0 \\
\hline 0 & 0 & 0 & 0 & 0 & 0 & 0 & 0 & 28 & 0 & 0 & 0 & 0 & 0 & 0 & 0 & 0 \\
\hline 0 & 0 & 0 & 0 & 0 & 0 & 0 & 0 & 0 & 0 & 0 & 0 & 0 & 0 & 0 & 0 & 0 \\
\hline 0 & 0 & 0 & 0 & 0 & 0 & 0 & 0 & 0 & 0 & 0 & 0 & 0 & 0 & 0 & 0 & 0 \\
\hline 0 & 0 & 0 & 0 & 0 & 0 & 0 & 0 & 0 & 0 & 0 & 0 & 0 & 0 & 0 & 0 & 0 \\
\hline 0 & 0 & 0 & 0 & 0 & 0 & 0 & 0 & 0 & 0 & 0 & 0 & 14 & 0 & 0 & 0 & 0 \\
\hline 0 & 0 & 0 & 0 & 0 & 0 & 0 & 0 & 0 & 0 & 0 & 0 & 0 & 0 & 0 & 0 & 0 \\
\hline 0 & 0 & 0 & 0 & 0 & 0 & 0 & 0 & 0 & 0 & 0 & 0 & 0 & 0 & 0 & 0 & 0 \\
\hline 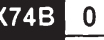 & 0 & 0 & 0 & 0 & 0 & 0 & 0 & 0 & 0 & 0 & 0 & 0 & 0 & 0 & 0 & 0 \\
\hline 0 & 0 & 0 & 0 & 0 & 0 & 0 & 0 & 0 & 0 & 0 & 0 & 0 & 0 & 0 & 0 & 0 \\
\hline
\end{tabular}

Tabelle 43: BCIga (BCIbuy)

\section{艾䒝}

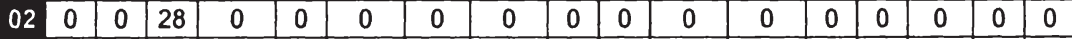

17 \begin{tabular}{l|l|l|l|l|l|l|l|l|l|l|l|l|l|l|l|l|}
\hline & 0 & 0 & 0 & 0 & 0 & 0 & 0 & 0 & 0 & 0 & 0 & 0 & 0 & 0 & 0 & 0 \\
\hline
\end{tabular}

20 \begin{tabular}{l|l|l|l|l|l|l|l|l|l|l|l|l|l|l|l|l|}
20 & 0 & 0 & 7,6 & 0 & 0,4 & 0 & 0 & 0 & 0 & 0 & 0 & 0 & 0 & 0 & 0 & 0 \\
\hline
\end{tabular}

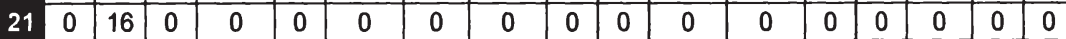

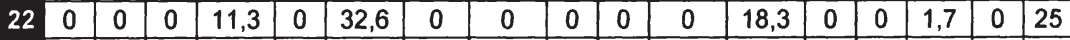

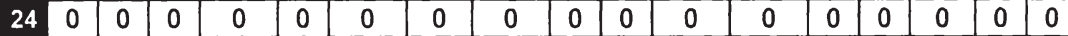

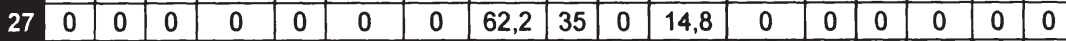

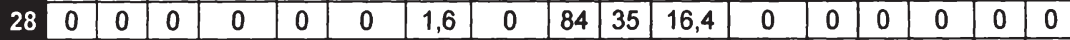

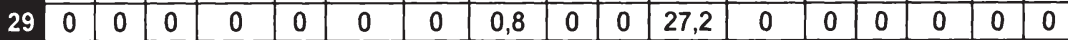

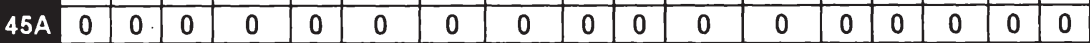

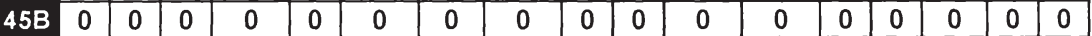

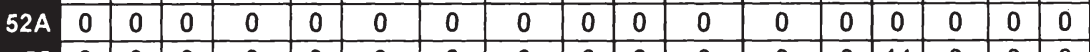

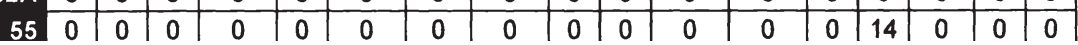




\subsection{Strukturanalyse}

Die Ergebnisse der Maßzahlberechnungen zu den Verflechtungseigenschaften der Matrix BCluu sowie der auf die lokale Struktur reduzierten Matrix rIOTgg gemäß Kapitel 6.2 können der Tabelle zu den Verflechtungsmaßzahlen (Tabelle 44), zu der Gradstatistik (Tabelle 45), zu den Gradindizes (Tabelle 46) und zu den Distanzindizes (Tabelle 47) entnommen werden.

Tabelle 44: Verflechtungsmaßzahlen

\begin{tabular}{|c|c|c|}
\hline & $B C 1 \%$ & $r l() T g g$ \\
\hline \multirow{2}{*}{$\begin{array}{r}\text { Anzahl gerichteter Kanten } \\
\text { Anzahl möglicher Kanten }\end{array}$} & 18 & 97 \\
\hline & 272 & 272 \\
\hline Dichte & 0,07 & 0,36 \\
\hline Wertedichte & 1,60 & $36.983,02$ \\
\hline Kantendurchschnittswert & 24,11 & $103.704,96$ \\
\hline $\begin{array}{r}\text { Durchschnittsgrad der Ein-bzw. Aus- } \\
\text { gangsgrade }\end{array}$ & 1,06 & 5,71 \\
\hline \multirow{2}{*}{$\begin{array}{l}\text { Durchschnittsgrad des gesamten Graphen } \\
\text { Standardabweichung der Eingangsgrade }\end{array}$} & 2,12 & 11,41 \\
\hline & 0,73 & 1,13 \\
\hline Standardabweichung der Ausgangsgrade & 1,51 & 3,95 \\
\hline Standardabweichung Gesamt & 1,49 & 4,04 \\
\hline \multirow{5}{*}{$\begin{array}{r}\text { Durchmesser (Diameter) } \\
\text { Durchschnittswert des Distanzindexwerts } \\
\text { der Vorläuferknoten } \\
\text { Standardabweichung des Distanzindex- } \\
\text { werts der Vorläuferknoten } \\
\text { Durchschnittswert des Distanzindexwerts } \\
\text { der Nachfolgerknoten } \\
\text { Standardabweichung des Distanzindex- } \\
\text { werts der Nachfolgerknoten }\end{array}$} & 3,00 & 3,00 \\
\hline & 0,07 & 0,54 \\
\hline & 0,10 & 0,23 \\
\hline & 0,07 & 0,52 \\
\hline & 0,05 & 0,04 \\
\hline
\end{tabular}

Der Vergleich der beiden Strukturen zeigt, dass die reduzierte gesamtwirtschaftliche Struktur rIOTgg mehr als fünfmal so viele Kanten aufweist als die für die Plattform errechnete Matrix BCIgg. Dies schlägt sich auch in der Dichte und bei den Durchschnittsgraden nieder. Nachdem die Plattform nur einen geringen Ausschnitt der Gesamtwirtschaft bietet, ist darüber hinaus der Unterschied der Wertedichten erheblich größer. Diese sind ohne Nivellierung nicht vergleichbar. Bei beiden Matrizen zeigt sich, dass die Variabilität der Ausgangsgrade größer ist als die Variabilität der Eingangsgrade, was darauf schließen lässt, dass die Güter (Knoten) in beiden Strukturen hinsichtlich der Anzahl der Vorgänger(-güter) 
homogener als im Hinblick auf die Anzahl der Nachfolger(-güter) sind. Der Durchmesser (Diameter), d.h. der größte Abstand zwischen zwei Knotenpaaren eines Graphen, beträgt sowohl für BClgg als auch für rIOTgg drei. Dies kann auch aus der Tabelle 48 für BCIgg bzw. der Tabelle 49 für rIOTgg abgelesen werden und ist ein Indikator für die maximale Entfernung und somit der Verbundenheit der Knoten eines Graphen. Die durchschnittlichen Distanzindexwerte der Vorläufer- und Nachfolgerknoten unterscheiden sich sowohl für BCIgg als auch für rIOTgg nur geringfügig bzw. gar nicht, während die Standardabweichungen für die Vorläuferknoten in beiden Fällen größer als für die Nachfolgerknoten sind. Wenig überraschend sind die Distanzindexwerte für die reduzierte gesamtwirtschaftliche Struktur weit höher als für die Plattform. Wie bereits in Kapitel 6.2 ausgeführt, bedeutet dies, dass die Güter in der reduzierten gesamtwirtschaftlichen Struktur im Durchschnitt mit einer größeren Anzahl von Nachfolger- bzw. Vorläuferknoten(-gütern) auf kürzeren Pfaden in Austauschbeziehungen stehen. Die Distanzindexwerte für die einzelnen Knoten (Güter) in ihrer jeweiligen Rolle können für beide Matrizen der Tabelle 47 entnommen werden.

Tabelle 45: Gradstatistik

\begin{tabular}{|c|c|c|c|c|c|c|c|c|c|}
\hline & \multirow{2}{*}{\multicolumn{3}{|c|}{$B(\operatorname{lgg}$}} & \multicolumn{3}{|c|}{ rloTgg } & \multicolumn{3}{|c|}{ Vergleich } \\
\hline & & & & $d^{\prime \prime}$ & $f^{\prime \prime \prime}$ & $d^{l 1 \cdot(1) l}$ & $\operatorname{comp} d^{\prime \prime}$ & $\operatorname{comp} d^{\prime \prime \prime}$ l & comp $d^{\prime \prime|l| l \mid}$ \\
\hline 02 & 1 & 1 & 2 & 7 & 2 & 9 & 0,14 & 0,50 & 0,22 \\
\hline 17 & 1 & 0 & 1 & 5 & 1 & 6 & 0,20 & 0 & 0,17 \\
\hline 20 & 1 & 2 & 3 & 6 & 5 & 11 & 0,17 & 0,40 & 0,27 \\
\hline 21 & 2 & 1 & 3 & 6 & 8 & 14 & 0,33 & 0,12 & 0,21 \\
\hline 22 & 0 & 5 & 5 & 5 & 12 & 17 & 0 & 0,42 & 0,29 \\
\hline 24 & 1 & 0 & 1 & 5 & 5 & 10 & 0,20 & 0 & 0,10 \\
\hline 27 & 1 & 3 & 4 & 5 & 4 & 9 & 0,20 & 0,75 & 0,44 \\
\hline 28 & 1 & 4 & 5 & 6 & 9 & 15 & 0,17 & 0,44 & 0,33 \\
\hline 29 & 2 & 1 & 3 & 6 & 8 & 14 & 0,33 & 0,12 & 0,21 \\
\hline $45 A$ & 1 & 0 & 1 & 6 & 6 & 12 & 0,17 & 0 & 0,08 \\
\hline $45 B$ & 3 & 0 & 3 & 9 & 5 & 14 & 0,33 & 0 & 0,21 \\
\hline $52 \mathrm{~A}$ & 1 & 0 & 1 & 6 & 0 & 6 & 0,17 & - & 0,17 \\
\hline 55 & 0 & 1 & 1 & 5 & 8 & 13 & 0 & 0,12 & 0,08 \\
\hline $63 B$ & 1 & 0 & 1 & 4 & 0 & 4 & 0,25 & - & 0,25 \\
\hline 72 & 1 & 0 & 1 & 6 & 13 & 19 & 0,17 & 0 & 0,05 \\
\hline $74 B$ & 0 & 0 & 0 & 6 & 1 & 7 & 0 & 0 & 0 \\
\hline $74 C$ & 1 & 0 & 1 & 4 & 10 & 14 & 0,25 & 0 & 0.07 \\
\hline
\end{tabular}


Tabelle 45 zeigt die Eingangs- und Ausgangsgrade der Knoten (Güter) der Plattform und der gesamtwirtschaftlichen Struktur einzeln und gesamt sowie eine jeweils dazugehörende Vergleichskennzahl. Ein Vergleich kann auch auf Basis der normierten Grade gemäß Tabelle 46 erfolgen.

Tabelle 46: Gradindizes

\begin{tabular}{|c|c|c|c|c|c|c|}
\hline & \multicolumn{3}{|c|}{$B C$ Igg } & \multicolumn{3}{|c|}{ r. } \\
\hline & $\operatorname{dind}^{\prime \prime}$ & dimat" I & dind $\left.\right|^{\prime-1) ! \mid}$ & $\| \operatorname{lin} d^{\prime \prime}$ & dind $t^{\prime \prime \prime}$ & 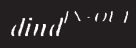 \\
\hline 02 & 0,06 & 0,06 & 0,13 & 0,44 & 0,13 & 0,56 \\
\hline 17 & 0,06 & 0 & 0,06 & 0,31 & 0,06 & 0,38 \\
\hline 20 & 0,06 & 0,13 & 0,19 & 0,38 & 0,31 & 0,69 \\
\hline 21 & 0,13 & 0,06 & 0,19 & 0,38 & 0,50 & 0,88 \\
\hline 22 & 0 & 0,31 & 0,31 & 0,31 & 0,75 & 1,06 \\
\hline 24 & 0,06 & 0 & 0,06 & 0,31 & 0,31 & 0,62 \\
\hline 27 & 0,06 & 0,19 & 0,25 & 0,31 & 0,25 & 0,56 \\
\hline 28 & 0,06 & 0,25 & 0,31 & 0,38 & 0,56 & 0,94 \\
\hline 29 & 0,13 & 0,06 & 0,19 & 0,38 & 0,50 & 0,88 \\
\hline $45 A$ & 0,06 & 0 & 0,06 & 0,38 & 0,38 & 0,75 \\
\hline $45 B$ & 0,19 & 0 & 0,19 & 0,56 & 0,31 & 0,88 \\
\hline $52 \mathrm{~A}$ & 0,06 & 0 & 0,06 & 0,38 & 0 & 0,38 \\
\hline 55 & 0 & 0,06 & 0,06 & 0,31 & 0,50 & 0,81 \\
\hline $63 B$ & 0,06 & 0 & 0,06 & 0,25 & 0 & 0,25 \\
\hline 72 & 0,06 & 0 & 0,06 & 0,38 & 0,81 & 1,19 \\
\hline $74 \mathrm{~B}$ & 0 & 0 & 0 & 0,38 & 0,06 & 0,44 \\
\hline $74 C$ & 0,06 & 0 & 0,06 & 0,25 & 0,63 & 0,88 \\
\hline
\end{tabular}

Tabelle 47: Distanzindizes

\begin{tabular}{|c|c|c|c|c|c|c|}
\hline & \multicolumn{2}{|c|}{ BC Is" } & \multicolumn{2}{|c|}{$r(0 T$ \%g } & \multicolumn{2}{|c|}{ Vergleich } \\
\hline & divsindl $^{\prime}$ & divindt" I & $\|^{\prime}$ & “ivinut" & comp divind $\|^{\prime}$ & comp divind" i \\
\hline 02 & 0,09 & 0,06 & 0,46 & 0,58 & 0,21 & 0,11 \\
\hline 17 & 0 & 0,13 & 0,39 & 0,51 & 0 & 0,24 \\
\hline 20 & 0,14 & 0,06 & 0,57 & 0,53 & 0,25 & 0,12 \\
\hline 21 & 0,06 & 0,14 & 0,67 & 0,51 & 0,09 & 0,28 \\
\hline 22 & 0,32 & 0 & 0,80 & 0,49 & 0,40 & 0 \\
\hline 24 & 0 & 0,06 & 0,59 & 0,49 & 0 & 0,13 \\
\hline 27 & 0,20 & 0,06 & 0,55 & 0,49 & 0,36 & 0,13 \\
\hline 28 & 0,25 & 0,06 & 0,70 & 0,51 & 0,36 & 0,12 \\
\hline 29 & 0,06 & 0,13 & 0,67 & 0,49 & 0,09 & 0,26 \\
\hline 45A & 0 & 0,08 & 0,62 & 0,56 & 0 & 0,15 \\
\hline 45B & 0 & 0,19 & 0,59 & 0,64 & 0 & 0,29 \\
\hline $52 A$ & 0 & 0,06 & 0 & 0,54 & & 0.12 \\
\hline
\end{tabular}


Demonstrationsbeispiel

\begin{tabular}{|c|c|c|c|c|c|c|}
\hline & \multicolumn{2}{|c|}{ BC Ig! } & \multicolumn{2}{|c|}{ r } & \multicolumn{2}{|c|}{ Vergleich } \\
\hline & distind $^{\prime \prime}$ & distind" I & distind $^{\prime \prime}$ & "listind"l" & comp distind ${ }^{\prime \prime}$ & comp distind" \\
\hline 55 & 0,06 & 0 & 0,67 & 0,51 & 0,09 & 0 \\
\hline $63 B$ & 0 & 0,06 & 0 & 0,45 & - & 0,14 \\
\hline 72 & 0 & 0,06 & 0,84 & 0,56 & 0 & 0,11 \\
\hline $74 B$ & 0 & 0 & 0,40 & 0,53 & 0 & 0 \\
\hline $74 C$ & 0 & 0,06 & 0,73 & 0,47 & 0 & 0,13 \\
\hline
\end{tabular}

\subsubsection{Erreichbarkeit und Distanzen}

Die Länge der Pfade bis zur erstmaligen Erreichbarkeit zwischen den Gütern (Knoten), die mit Bezug auf die bestehenden Kanten der jeweils betrachteten Matrix errechnet werden, zeigt die Tabelle 48 für BCIgg und die Tabelle 49 für rIOTgg. Beide Tabellen fließen in die Berechnung der obigen Statistiken ein. Selbstbezüglichkeit und Nicht-Erreichbarkeit werden in diesen Tabellen über die Zahl 0 ausgewiesen.

Tabelle 48: Distanzgraph zu BCIgg in Adjazenzmatrixform

\begin{tabular}{|c|c|c|c|c|c|c|c|c|c|c|c|c|c|c|c|c|c|}
\hline & $N$ & $\mp$ & 요 & $\check{N}$ & $\approx$ & 吕 & $\hat{N}$ & $\stackrel{\infty}{\sim}$ & I & $\frac{\pi}{8}$ & 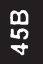 & ฟ్ & 号 & $\stackrel{m}{\mathscr{B}}$ & $\mathbb{N}$ & $\underset{N}{\stackrel{Q}{N}}$ & $\underset{N}{U}$ \\
\hline 02 & 0 & 3 & 1 & 2 & 0 & 0 & 0 & 0 & 0 & 0 & 0 & 0 & 0 & 0 & 0 & 0 & 0 \\
\hline 17 & 0 & 0 & 0 & 0 & 0 & 0 & 0 & 0 & 0 & 0 & 0 & 0 & 0 & 0 & 0 & 0 & 0 \\
\hline 20 & 1 & 2 & 0 & 1 & 0 & 0 & 0 & 0 & 0 & 0 & 0 & 0 & 0 & 0 & 0 & 0 & 0 \\
\hline 21 & 0 & 1 & 0 & 0 & 0 & 0 & 0 & 0 & 0 & 0 & 0 & 0 & 0 & 0 & 0 & 0 & 0 \\
\hline 22 & 0 & 2 & 0 & 1 & 0 & 1 & 0 & 0 & 0 & 0 & 0 & 1 & 0 & 0 & 1 & 0 & 1 \\
\hline 24 & 0 & 0 & 0 & 0 & 0 & 0 & 0 & 0 & 0 & 0 & 0 & 0 & 0 & 0 & 0 & 0 & 0 \\
\hline 27 & 0 & 0 & 0 & 0 & 0 & 0 & 0 & 1 & 1 & 2 & 1 & 0 & 0 & 0 & 0 & 0 & 0 \\
\hline 28 & 0 & 0 & 0 & 0 & 0 & 0 & 1 & 0 & 1 & 1 & 1 & 0 & 0 & 0 & 0 & 0 & 0 \\
\hline 29 & 0 & 0 & 0 & 0 & 0 & 0 & 0 & 0 & 0 & 0 & 1 & 0 & 0 & 0 & 0 & 0 & 0 \\
\hline $45 \mathrm{~A}$ & 0 & 0 & 0 & 0 & 0 & 0 & 0 & 0 & 0 & 0 & 0 & 0 & 0 & 0 & 0 & 0 & 0 \\
\hline $45 B$ & 0 & 0 & 0 & 0 & 0 & 0 & 0 & 0 & 0 & 0 & 0 & 0 & 0 & 0 & 0 & 0 & 0 \\
\hline $52 \mathrm{~A}$ & 0 & 0 & 0 & 0 & 0 & 0 & 0 & 0 & 0 & 0 & 0 & 0 & 0 & 0 & 0 & 0 & 0 \\
\hline 55 & 0 & 0 & 0 & 0 & 0 & 0 & 0 & 0 & 0 & 0 & 0 & 0 & 0 & 1 & 0 & 0 & 0 \\
\hline $63 B$ & 0 & 0 & 0 & 0 & 0 & 0 & 0 & 0 & 0 & 0 & 0 & 0 & 0 & 0 & 0 & 0 & 0 \\
\hline 72 & 0 & 0 & 0 & 0 & 0 & 0 & 0 & 0 & 0 & 0 & 0 & 0 & 0 & 0 & 0 & 0 & 0 \\
\hline $74 \mathrm{~B}$ & 0 & 0 & 0 & 0 & 0 & 0 & 0 & 0 & 0 & 0 & 0 & 0 & 0 & 0 & 0 & 0 & 0 \\
\hline $74 \mathrm{C}$ & 0 & 0 & 0 & 0 & 0 & 0 & 0 & 0 & 0 & 0 & 0 & 0 & 0 & 0 & 0 & 0 & 0 \\
\hline
\end{tabular}

Tabelle 48 zeigt 18 Pfade mit Weglänge 1, vier Pfade mit Weglänge 2 und einen Pfad mit Weglänge 3 für BClgg auf. 249 Knotenpaare sind auch indirekt nicht verbunden, weitere 17 Einträge mit dem Zellwert null beschreiben Selbstbezüg- 
lichkeit. Im Gegensatz hierzu sind in der gesamtwirtschaftlichen Struktur gemäß Tabelle 49 nur 49 Knotenpaare direkt und indirekt unverbunden. Neben den 97 direkten Verbindungen in rIOTgg existieren 143 indirekte Verbindungen, wobei 117 kürzeste Pfade die Weglänge 2 und 26 die Weglänge 3 aufweisen.

Tabelle 49: Distanzgraph zu rlOTgg in Adjazenzmatrixform

\section{ช}

\begin{tabular}{|l|l|l|l|l|l|l|l|l|l|l|l|l|l|l|l|l|l|}
\hline 02 & 0 & 2 & 1 & 1 & 2 & 2 & 2 & 3 & 3 & 2 & 2 & 2 & 3 & 3 & 2 & 2 & 3 \\
\hline 17 & 2 & 0 & 3 & 3 & 3 & 3 & 3 & 3 & 3 & 2 & 1 & 3 & 2 & 3 & 2 & 3 & 2 \\
\hline 20 & 1 & 2 & 0 & 1 & 2 & 2 & 1 & 2 & 2 & 1 & 1 & 2 & 2 & 3 & 2 & 2 & 2 \\
\hline 21 & 2 & 1 & 1 & 0 & 1 & 1 & 2 & 2 & 2 & 2 & 1 & 1 & 2 & 2 & 1 & 1 & 2 \\
\hline 22 & 1 & 1 & 2 & 1 & 0 & 1 & 2 & 2 & 1 & 1 & 2 & 1 & 1 & 1 & 1 & 1 & 1 \\
\hline 24 & 1 & 2 & 2 & 1 & 1 & 0 & 2 & 1 & 2 & 2 & 1 & 2 & 2 & 2 & 2 & 2 & 2 \\
\hline 27 & 2 & 2 & 2 & 2 & 2 & 2 & 0 & 1 & 1 & 1 & 1 & 2 & 2 & 3 & 2 & 2 & 2 \\
\hline 28 & 1 & 2 & 1 & 2 & 2 & 2 & 1 & 0 & 1 & 1 & 1 & 1 & 1 & 2 & 2 & 1 & 2 \\
\hline 29 & 1 & 1 & 1 & 1 & 1 & 1 & 1 & 2 & 0 & 2 & 1 & 2 & 2 & 2 & 2 & 2 & 2 \\
\hline $45 A$ & 1 & 2 & 1 & 2 & 2 & 2 & 2 & 1 & 2 & 0 & 1 & 2 & 1 & 2 & 1 & 2 & 2 \\
\hline $55 B$ & 1 & 2 & 2 & 2 & 2 & 2 & 2 & 2 & 2 & 1 & 0 & 2 & 1 & 2 & 1 & 2 & 1 \\
\hline 55 & 0 & 0 & 0 & 0 & 0 & 0 & 0 & 0 & 0 & 0 & 0 & 0 & 0 & 0 & 0 & 0 & 0 \\
\hline 72 & 2 & 2 & 2 & 2 & 2 & 2 & 1 & 1 & 2 & 1 & 1 & 0 & 1 & 1 & 1 & 1 \\
\hline 748 & 2 & 0 & 0 & 0 & 0 & 0 & 0 & 0 & 0 & 0 & 0 & 0 & 0 & 0 & 0 & 0 & 0 \\
\hline & 2 & 3 & 2 & 3 & 3 & 3 & 3 & 2 & 3 & 1 & 2 & 3 & 2 & 3 & 2 & 0 & 3 \\
\hline $74 C$ & 2 & 1 & 2 & 2 & 1 & 1 & 1 & 1 & 1 & 2 & 2 & 1 & 2 & 1 & 1 & 1 & 0 \\
\hline
\end{tabular}

\subsubsection{Identifikation und Bewertung von Cliquen}

Zur Identifikation und Bewertung von zusammenhängenden Subgraphen (Cliquen) kann der in Kapitel 6.4 beschriebene Ansatz von Hubbell [1965] verfolgt werden. Abbildung 17 zeigt die entsprechend der in Kapitel 8.3.2 vorgeschlagenen Modifikation des Ansatzes von Hubbell ermittelten Subgraphen für $B C l g g$. Der größte Subgraph besteht demgemäß aus den Gütern 02, 17, 20, 21 , $22,24,52 \mathrm{~A}, 72$, und $74 \mathrm{C}$, wobei das Gut 22 als Vorläuferknoten der anderen Güter des Subgraphen (mit Ausnahme von Gut 02 und Gut 17) eine zentrale Rolle in dieser Clique spielt. Der zweitgrößte Subgraph, der die Güter 27, 28, 29, 45A, und 45B umfasst, beinhaltet die höchsten Kantengewichte. Der dritte und 
letzte Subgraph enthält die Güter 55 und 63B. Das Gut 74B ist als unverbundener und somit isolierter Knoten ein Sonderfall.

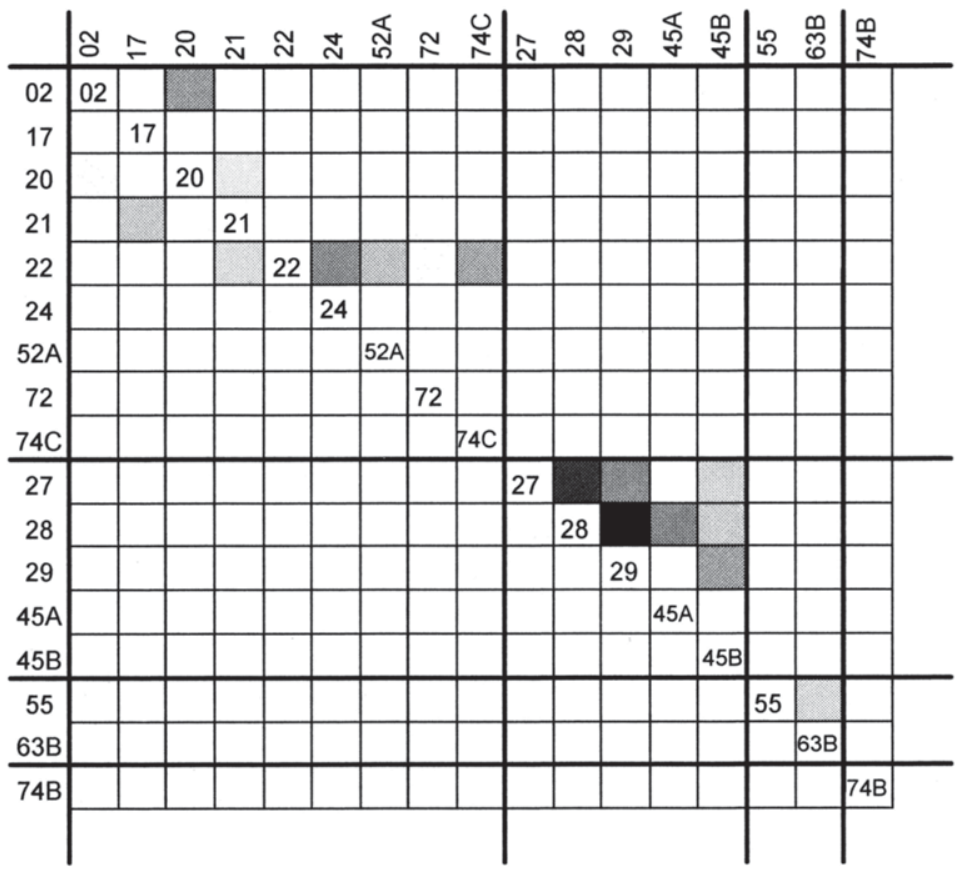

Abbildung 17: Cliquenidentifikation von BClgg

Abbildung 18 illustriert die identifizierten Subgraphen von BCIgg. Die Matrix rIOTgg kann im Gegensatz zur BClgg nicht in Subgraphen zerlegt werden, da sie selbst einen zusammenhängenden Graphen darstellt.

Die Zusammenhangsmaßzahl $\Theta_{(1-\alpha)}$, die eine untere Schranke des Assoziationsindex für die am stärksten verbundenen Knotenpaare angibt, beträgt bei $\alpha=0,15$ für die Matrix $B C \operatorname{lgg} 67,07$, für den größten Subgraph von $B C \operatorname{lgg} 29,77$, für den zweitgrößten Subgraph 73,19 und für rIOTgg 666.269,60. Der drittgrößte Subgraph von BClgg besteht aus nur einer Transaktion, weshalb kein Assoziationsindex ausgerechnet werden kann. Vergleicht man den ersten Subgraph von BCIgg mit dem zweiten, so zeigt sich, dass die Verbundenheit der Knotenpaare 
im zweiten Subgraph weitaus intensiver ist als im ersten. Dementsprechend tragen auch die Kanten des zweiten Subgraphen stärker zu der Zusammenhangsmaßzahl des Gesamtgraphen bei.
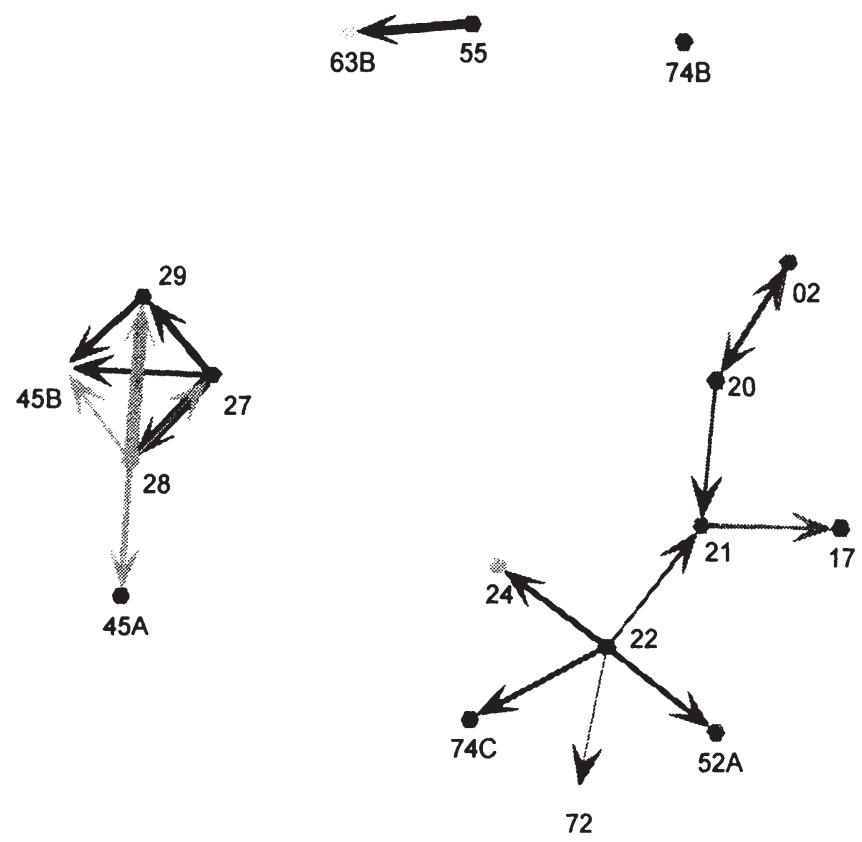

Abbildung 18: BClgg

\subsubsection{Blockmodeling}

Das Ergebnis der binären empirischen Blockmodellberechnung mit Fokus auf strukturelle Äquivalenz für BCIgg mit sechs Vorgänger- und sechs Nachfolgerknotengruppen zeigt Abbildung 19. In diesem Blockmodell (BM) konnten zwei vollständige Blöcke identifiziert werden. Dies sind jene Blöcke, die sich aus den Vorläuferknoten 27 und 28 sowie den Nachfolgerknoten 27, 29 und 45B bzw. aus dem Vorläuferknoten 22 sowie den Knoten 21, 24, 52A, 72 und $74 \mathrm{C}$ als Nachfolger zusammensetzen. Der zuerst genannte vollständige Block ist Teil des vorhin identifizierten Subgraphen 2, während der zuletzt genannte vollständige 
Block dem Subgraph 1 zuzuordnen ist. Die Blöcke mit den Vorgängerknoten 20 und 55 sowie den Nachfolgerknoten 63B und 02 bzw. den Vorgängerknoten 02 und 21 sowie den Nachfolgerknoten 17 und 20 sind im Gegensatz als regulär (zeilen- und spaltenfunktional) zu qualifizieren. Dies gilt auch für die Kombination der Knoten 27 und 28 sowie 28 und 45A. Bei den restlichen Blöcken handelt es sich um Nullblöcke, wobei zwei Ausnahmen in Abbildung 19 zu beobachten sind.

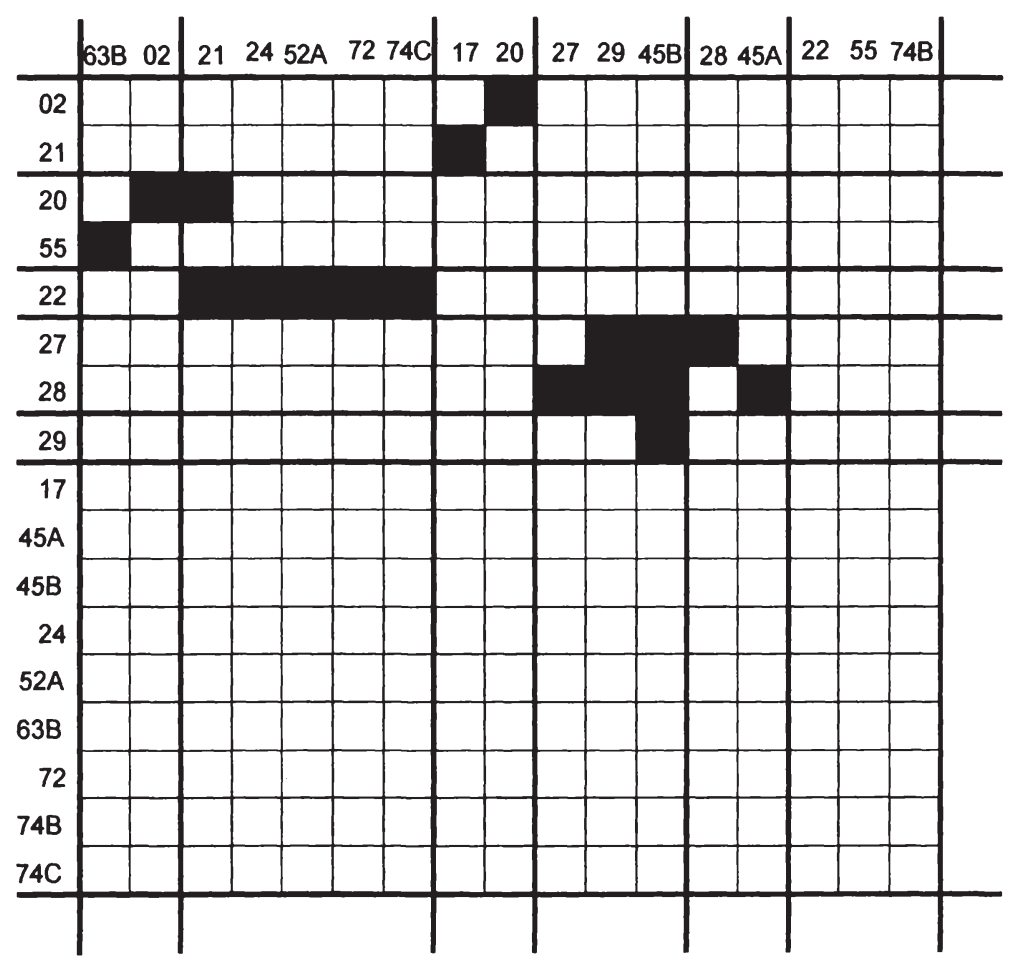

Abbildung 19: BM für BClgg mit 6 Vorgänger- und 6 Nachfolgerknotengruppen

Das Ergebnis der Blockmodellberechnung mit gewichteten Kanten mit Fokus auf Blöcke mit maximaler Homogenität wird in Abbildung 20 dargestellt. Im Unterschied zu der binären Blockmodellberechnung ergeben sich hierbei geringfügig modifizierte Blöcke bei sechs Vorgänger- und sechs Nachfolgerknotengruppen. 


\begin{tabular}{|c|c|c|c|c|c|c|c|c|c|c|c|c|c|c|c|c|c|}
\hline & $63 \mathrm{~B}$ & 21 & 24 & $52 \mathrm{~A}$ & $74 \mathrm{C}$ & 17 & 20 & $45 \mathrm{~B}$ & $45 \mathrm{~A}$ & 29 & 28 & 02 & 72 & 27 & 22 & 55 & 748 \\
\hline 02 & & & & & & & 28 & & & & & & & & & & \\
\hline 21 & & & & & & 16 & & & & & & & & & & & \\
\hline 20 & & 8 & & & & & & & & & & 2 & & & & & \\
\hline 55 & 14 & & & & & & & & & & & & & & & & \\
\hline 22 & & 11 & $\sqrt{33}$ & 18.6 & 25 & & & & & & & & 1.4 & & & & \\
\hline 27 & & & & & & & & 14.8 & & 36 & 62.2 & & & & & & \\
\hline 28 & & & & & & & & 16.7 & 35. & 84 & & & & 1.3 & & & \\
\hline 29 & & & & & & & & 28 & & & & & & & & & \\
\hline 17 & & & & & & & & & & & & & & & & & \\
\hline $45 \mathrm{~A}$ & & & & & & & & & & & & & & & & & \\
\hline $45 B$ & & & & & & & & & & & & & & & & & \\
\hline 24 & & & & & & & & & & & & & & & & & \\
\hline $52 \mathrm{~A}$ & & & & & & & & & & & & & & & & & \\
\hline 638 & & & & & & & & & & & & & & & & & \\
\hline 72 & & & & & & & & & & & & & & & & & \\
\hline 748 & & & & & & & & & & & & & & & & & \\
\hline $74 \mathrm{C}$ & & & & & & & & & & & & & & & & & \\
\hline
\end{tabular}

Abbildung 20: gewichtetes BM für BCIgg 6 Vorgänger- und 6 Nachfolgerknotengruppen

Die Zuordnung der Vorgängerknoten zu den Vorgängerknotengruppen bleibt unverändert, während sich die Zusammensetzung der Nachfolgerknotengruppen ändert. Aufgrund des äußerst geringen Kantengewichts fallen die Kanten $(20,02)$, $(22,72)$ und $(28,27)$ im Vergleich zu dem binären Blockmodell aus ihren jeweiligen Blöcken heraus, damit einhergehend sind die Spaltenknoten 02, 72 und 27 nicht mehr ihren ursprünglichen (binären) Knotengruppen zuzuordnen. Der Nachfolger- oder Spaltenknoten 21 wird aus Homogenitätsgründen mit dem Nachfolgerknoten 63B zu einer Knotengruppe zusammengefasst. Des Weiteren werden die Spaltenknotengruppen 27, 29 und 45B sowie 28 und 45A unter Ausschluss des Knoten 27 vermengt und entsprechend der Ähnlichkeit der Kantengewichte neu zusammengefasst. Dies bewirkt die neuen Spaltenknotengruppen 45B, 45A sowie 29 und 28 . 
Abbildung 21 stellt das binäre empirische Blockmodell unter Berücksichtigung von struktureller Äquivalenz für rIOTgg mit sechs Vorgänger- und sechs Nachfolgerknotengruppen dar.

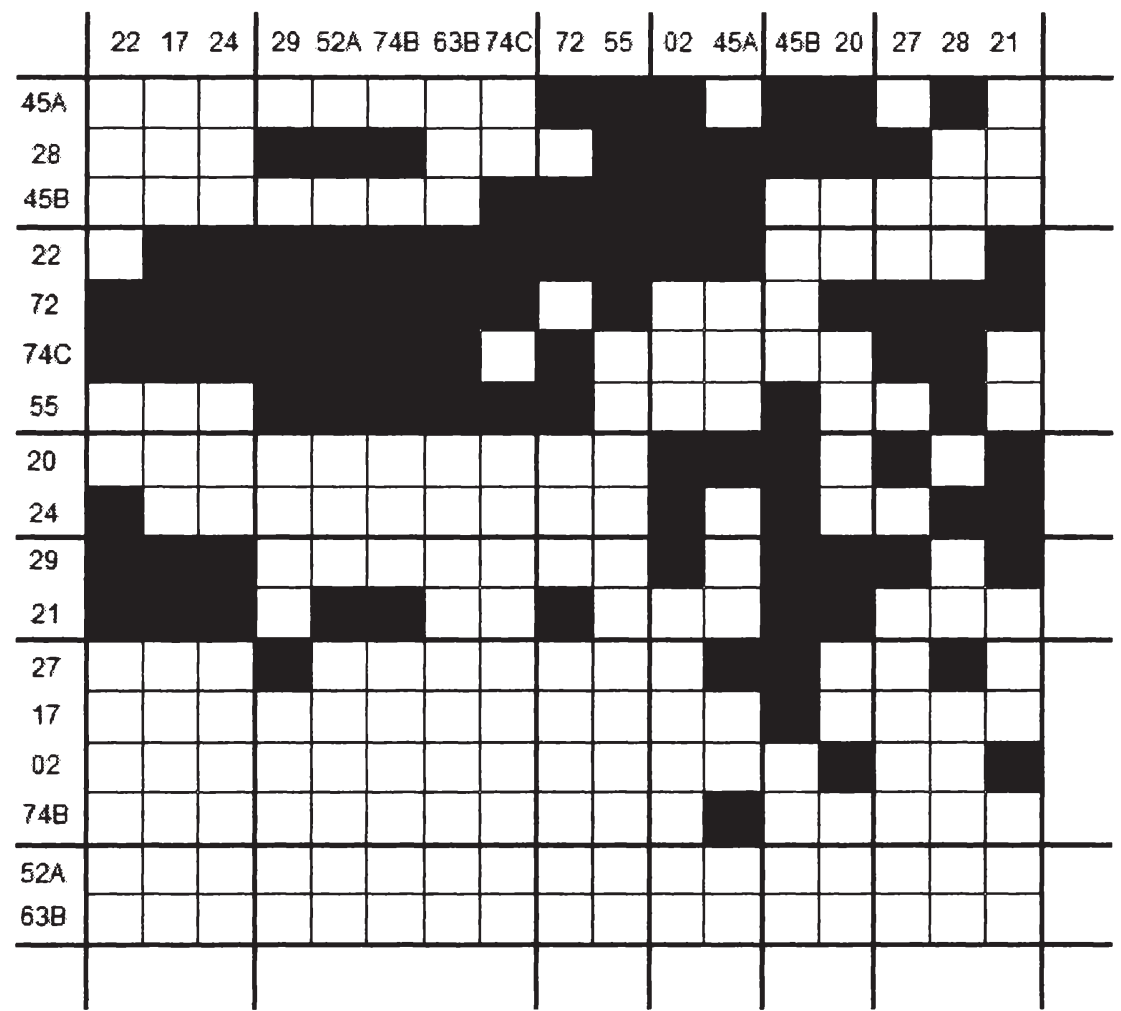

Abbildung 21: BM für rIOTgg mit 6 Vorgänger- und 6 Nachfolgerknotengruppen

Vergleicht man dieses Blockmodell nun mit dem binären empirischen Blockmodell für BClgg, so zeigt sich, dass die Gruppierung der Knoten unter Kenntnis der globalen Struktur zu einer unterschiedlichen Knotenzuordnung führt. Dies kann beispielsweise an dem in Abbildung 19 dargestellten vollständigen Block mit den Vorgängerknoten 27 und 28 sowie den Nachfolgerknoten 27, 29 und 45B demonstriert werden. Während der Knoten (das Gut) 27 in der reduzierten globalen Struktur rIOTgg als Vorgänger kaum zusätzliche Verbindungen zu den

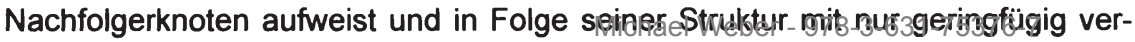


bundenen Vorgängerknoten wie 74B, 17 oder 02 gruppiert wird, erweist sich das Verbindungsmuster (die potenziellen Transaktionsverläufe) des Vorgängerknotens 28 in rIOTgg ähnlicher zu den Knoten 45A und 45B, wobei auch eine gewisse Vergleichbarkeit mit den Knoten 72 und 21 aus anderen Vorgängerknotengruppen gegeben ist. Der Nachfolgerknoten 29, der Teil der Nachfolgergruppe des vollständigen Blocks aus Abbildung 19 ist, kann aufgrund seiner Vorgänger- oder Nachfragestruktur in rIOTgg gemeinsam mit ähnlich strukturierten Knoten (Gütern) wie insbesondere $52 \mathrm{~A}$ und $74 \mathrm{~B}$, aber auch $63 \mathrm{~B}$ und $74 \mathrm{C}$ gruppiert werden und ist gleichzeitig Teil des größten vollständigen Blocks in rIOTgg, was die Bedeutung dieses Knotens als Nachfolger (Nachfrager) unterstreicht. Ebenso wie der Nachfolgerknoten 29 hat neben dem Knoten 27 speziell auch der Knoten 45B in der Nachfolgerrolle gemäß Abbildung 21 zusätzliches Potenzial. Beide Knoten sind in Folge der mangelnden gemeinsamen Zuordenbarkeit zu vollständigen Blöcken Teil unterschiedlicher Nachfolgerknotengruppen.

Der zweite vollständige Block aus Abbildung 19, der aus dem Vorgängerknoten 22 und den Nachfolgern 21, 24, 52A, 72 und $74 \mathrm{C}$ gebildet wird, kann ebenfalls zur Demonstration der Schlussfolgerungen eines Vergleichs der Knotengruppierungen für BCIgg und rIOTgg herangezogen werden. So zeigt sich, dass der Knoten 22 als Vorgänger eine ähnliche Absatzstruktur wie 72, 74C und auch 55 hat und gemeinsam mit diesen Knoten Teil des größten vollständigen Blocks von rIOTgg ist und dementsprechend großes Potenzial hat. Die Nachfolgerknoten $52 \mathrm{~A}$ und $74 \mathrm{C}$ sind ebenfalls diesem Block zugeordnet und haben demnach ebenso wie der ,benachbarte' Nachfolgerknoten 72, der mit dem Knoten 55 eine Gruppe bildet, oder dem Knoten 24, der als Nachfolger mit weiteren Knoten an einem weiteren vollständigen Block beteiligt ist, eine höhere Bedeutung als man unter alleiniger Beachtung des Blockmodells für die lokale Struktur schließen könnte. Auch das - etwas geringere - Potenzial des Nachfolgerknoten 21, der mit den Nachfolgern 27 und 28 eine lose zusammenhängende Knotengruppe bildet, die an keinem vollständigen Block beteiligt ist, 
soll in diesem Zusammenhang nicht unerwähnt bleiben. Allgemein kann festgestellt werden, dass der Vergleich der beiden binären empirischen Blockmodelle dieser zwei in Verbindung stehenden Strukturen die Möglichkeit eröffnet, Potenziale und Gemeinsamkeiten auf Basis der erfolgten Knotengruppierung und Kantenpartitionierung (einfacher) zu erkennen und dementsprechend (schneller) zu reagieren, d.h. gezielter Knoten zusammenzuführen und Verbindungen zu etablieren.

\begin{tabular}{|c|c|c|c|c|c|c|c|c|c|c|c|c|c|c|c|c|c|}
\hline & 02 & 17 & 72 & $74 \mathrm{~B}$ & 24 & $52 \mathrm{~A}$ & $63 \mathrm{~B}$ & $74 \mathrm{C}$ & 55 & 28 & 29 & $45 \mathrm{~A}$ & $45 \mathrm{~B}$ & 22 & 20 & 21 & 27 \\
\hline 02 & & & & & & & & & & & & & & & 335 & 220 & \\
\hline $74 \mathrm{C}$ & & 18 & 38 & 10 & 109 & 157 & 25 & & & 34 & 71 & & & 59 & & & 35 \\
\hline 72 & & 14 & & 26 & 46 & 102 & 14 & 63 & 59 & 48 & 118 & & & 17 & 28 & 31 & 27 \\
\hline 22 & 1 & 9 & 30 & 53 & 79 & 387 & 88 & 453 & 47 & & 56 & 46 & & & & 26 & \\
\hline 55 & & & 10 & 10 & & 52 & 292 & 6 & & 29 & 56 & & 25 & & & & \\
\hline 28 & 16 & & & 15 & & 60 & & & 64 & & 1003 & 461 & 294 & & 23 & & 107 \\
\hline 20 & 14 & & & & & & & & & & & 568 & 85 & & & 258 & 33 \\
\hline 29 & 11 & 16 & & & 51 & & & & & & & & 235 & 39 & 31 & 42 & 57 \\
\hline 21 & & 14 & 15 & 45 & 67 & 79 & & & & & & & 32 & 542 & 60 & & \\
\hline $74 \mathrm{~B}$ & & & & & & & & & & & & 409 & & & & & \\
\hline 27 & & & & & & & & & & 455 & 357 & 93 & 109 & & & & \\
\hline $45 \mathrm{~B}$ & 2 & & 14 & & & & & 4 & 183 & & & 218 & & & & & \\
\hline $45 A$ & 4 & & 11 & & & & & & 108 & 26 & & & 50 & & 36 & & \\
\hline 24 & 6 & & & & & & & & & 32 & & & 60 & 14 & & 34 & \\
\hline 17 & & & & & & & & & & & & & 42 & & & & \\
\hline $52 \mathrm{~A}$ & & & & & & & & & & & & & & & & & \\
\hline $63 \mathrm{~B}$ & & & & & & & & & & & & & & & & & \\
\hline
\end{tabular}

Abbildung 22: gewichtetes BM für rIOTgg 6 Vorgänger- und 6 Nachfolgerknotengruppen Abbildung 22 visualisiert das empirische Blockmodell für gewichtete Kanten im Hinblick auf Blöcke mit minimaler Heterogenität für rIOTgg. Abermals wurden die Knoten sechs Vorgänger- und sechs Nachfolgerknotengruppen zugeordnet, wo- 
bei aufgrund der Berücksichtigung von bewerteten Relationen neue Gruppierungen aufgetreten sind, die u.a. eingesetzt werden können, um Schwerpunkte bspw. bei etwaigen Empfehlungen für denkbare Verbindungen zu setzen, d.h. auf das Gewicht der Relationen einzugehen. Der Vergleich der gewichteten empirischen Blockmodelle für BCIgg (Abbildung 20) und rIOTgg (Abbildung 22, Werte gerundet, in Tausend) dient hierbei ebenso wie bei den binären empirischen Blockmodellen als Ausgangspunkt für die Ableitung von Schlussfolgerungen.

Für einen Vergleich können bspw. die Vorgängerknoten 27 und 28 herangezogen werden, die in Abbildung 20 jeweils einen Block mit den Nachfolgerknoten $45 B$ und $45 A$ sowie 29 und 28 bilden. In dem gewichteten empirischen Blockmodell für rIOTgg ist der Knoten 28 im Gegensatz zu dem korrespondierenden Blockmodell für BCIgg aufgrund der hohen Ähnlichkeit bezogen auf das Wertniveau unter zuzüglicher Beachtung der Struktur nun mit dem Vorgängerknoten 20 in einer Knotengruppe. Nachdem der Knoten 27 in rIOTgg Verbindungen mit einem vergleichsweise geringen Wertniveau zu weniger Nachfolgern als der Knoten $\mathbf{2 8}$ hat, wird dieser in dem Blockmodell aus Abbildung 22 mit anderen Vorgängerknoten als 28 gruppiert. Die Nachfolger 45A und 29, die in dem gewichteten Blockmodell für BCIgg noch unterschiedlichen Knotengruppen zugewiesen wurden, finden sich in Folge der vergleichbaren (hohen) Niveaus der Beziehungen zu dementsprechend wichtigen gemeinsamen Vorgängern in dem gewichteten Blockmodell für rIOTgg in einer gemeinsamen Gruppe wieder. Sie bilden zusammen mit den Vorgängern 28 und 20 den wertmäßig wichtigsten Block des Blockmodells welches der Abbildung 22 zugrunde liegt und sind dementsprechend dominante Nachfolger (Nachfrager). Die Nachfolgerknoten 45B bzw. 28 werden mit den Knoten 22 bzw. 55 gruppiert, mit denen sie die jeweils definierten Homogenitätsbedingungen bezüglich des Wertniveaus erfüllen.

Der Vergleich des zweiten markanten Blocks des Vorgängerknotens 22 mit den Nachfolgern 24, 52A und $74 \mathrm{C}$ aus dem gewichteten Blockmodell für $\mathrm{BClgg}$ mit

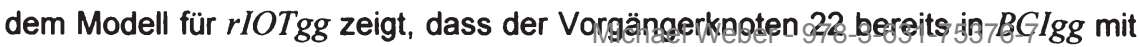


den herausstechenden Nachfolgern (Abnehmern) der wichtigsten Nachfolgerknotengruppe verbunden ist und im Hinblick auf die Wertrelationen - wie auch bei dem binären empirischen Blockmodell für rIOTgg - mit dem Vorgängerknoten 55 gruppiert werden kann. Die Nachfolgerknoten $52 \mathrm{~A}$ und $74 \mathrm{C}-$ sind sowohl in dem gewichteten Blockmodell für BClgg als auch in jenem für rIOTgg zusammengefasst, wobei in letzterem Modell 63B dieser Gruppierung hinzugezogen wird. Der Knoten 24 ist als Nachfolger nicht mehr in der selben Knotengruppe wie $52 \mathrm{~A}$ und $74 \mathrm{C}$, nachdem dessen Wertrelationen trotz vergleichbarer Beziehungsgeflechte zu den Vorgängerknoten geringer ist. Abschließend kann für den Vergleich von gewichteten empirischen Blockmodellen zweier zusammenhängender Strukturen festgestellt werden, dass es durch die Berücksichtigung von Kantengewichten möglich wird, neben den grundsätzlichen Verflechtungspotenzialen Knoten bzw. Kanten unter Beachtung der Wertrelationen zu gruppieren bzw. zu partitionieren. Dies kann insbesondere bei der Setzung von Prioritäten für Maßnahmen zur Strukturverbesserung, aber auch bei der Stabilisierung der lokalen Struktur von Vorteil sein.

\subsection{Strukturvergleich}

Der Vergleich der für Zwecke der Strukturanalyse erstellten binären und gewichteten empirischen Blockmodelle für die lokale Struktur (BClgg) und die reduzierte globale Struktur (rIOTgg) stellt bereits einen ersten Versuch dar, Aussagen über die Potenziale der Knoten bzw. Kanten der lokalen Struktur und erforderliche Maßnahmen zur Strukturverbesserung zu treffen. Nachfolgend soll zur weiteren Konkretisierung und höheren Detaillierung solcher Aussagen ein systematischer Strukturvergleich entsprechend der Ausführungen aus Kapitel 7 erfolgen. Tabelle 50 zeigt die Matrix MatValGap (in Tausend, gerundet), die über den Vergleich von BCIgg mit rIOTgg sowohl direkte interne strukturelle Lücken (positives Vorzeichen) als auch potenzielle Performanzlücken (negatives Vorzeichen) mit den Werten aus rIOTgg anzeigt. Selbstverständlich könnte die 
Matrix MatValGap alternativ in Listenform (als Kantenliste) angeschrieben werden, um die computergestützte Verarbeitung zu vereinfachen.

Tabelle 50: MatValGap

$\begin{array}{lllllllllllllllll}02 & 17 & 20 & 21 & 22 & 24 & 27 & 28 & 29 & 45 \mathrm{~A} & 45 \mathrm{~B} & 52 \mathrm{~A} & 55 & 63 \mathrm{~B} & 72 & 74 \mathrm{~B} & 74 \mathrm{C}\end{array}$

\begin{tabular}{|c|c|c|c|c|c|c|c|c|c|c|c|c|c|c|c|c|c|}
\hline 02 & 0 & 0 & -335 & 220 & 0 & 0 & 0 & 0 & 0 & 0 & 0 & 0 & 0 & 0 & 0 & 0 & 0 \\
\hline & 0 & 0 & 0 & 0 & 0 & 0 & 0 & 0 & 0 & 0 & 42 & 0 & 0 & 0 & 0 & 0 & 0 \\
\hline 2 & -14 & 0 & 0 & -258 & 0 & 0 & 33 & 0 & 0 & 568 & 85 & 0 & 0 & 0 & 0 & 0 & 0 \\
\hline & 0 & -14 & 60 & 0 & 542 & 67 & 0 & 0 & 0 & 0 & 32 & 79 & 0 & 0 & 15 & 45 & 0 \\
\hline 22 & 1 & 9 & 0 & -26 & 0 & -79 & 0 & 0 & 56 & 46 & 0 & -387 & 47 & 88 & -30 & 53 & -453 \\
\hline 24 & 6 & 0 & 0 & 34 & 14 & 0 & 0 & 32 & 0 & 0 & 60 & 0 & 0 & 0 & 0 & 0 & 0 \\
\hline 27 & 0 & 0 & 0 & 0 & 0 & 0 & 0 & -455 & -357 & 93 & -109 & 0 & 0 & 0 & 0 & 0 & 0 \\
\hline 28 & 16 & 0 & 23 & 0 & 0 & 0 & -107 & 0 & -1003 & -461 & -294 & 60 & 64 & 0 & 0 & 15 & 0 \\
\hline 29 & 11 & 16 & 31 & 42 & 39 & 51 & 57 & 0 & 0 & 0 & -235 & 0 & 0 & 0 & 0 & 0 & 0 \\
\hline $15 \mathrm{~A}$ & 4 & 0 & 36 & 0 & 0 & 0 & 0 & 26 & 0 & 0 & 50 & 0 & 108 & 0 & 11 & 0 & 0 \\
\hline $15 B$ & 2 & 0 & 0 & 0 & 0 & 0 & 0 & 0 & 0 & 218 & 0 & 0 & 183 & 0 & 14 & 0 & 4 \\
\hline $52 A$ & 0 & 0 & 0 & 0 & 0 & 0 & 0 & 0 & 0 & 0 & 0 & 0 & 0 & 0 & 0 & 0 & 0 \\
\hline & 0 & 0 & 0 & 0 & 0 & 0 & 0 & 29 & 56 & 0 & 25 & 52 & 0 & -292 & 10 & 10 & 6 \\
\hline 3 & 0 & 0 & 0 & 0 & 0 & 0 & 0 & 0 & 0 & 0 & 0 & 0 & 0 & 0 & 0 & 0 & 0 \\
\hline 7 & 0 & 14 & 28 & 31 & 17 & 46 & 27 & 48 & 118 & 0 & 0 & 102 & 59 & 14 & 0 & 26 & 63 \\
\hline & 0 & 0 & 0 & 0 & 0 & 0 & 0 & 0 & 0 & 409 & 0 & 0 & 0 & 0 & 0 & 0 & 0 \\
\hline 4 & 0 & 18 & 0 & 0 & 59 & 109 & 35 & 34 & 71 & 0 & 0 & 157 & 0 & 25 & 38 & 10 & 0 \\
\hline
\end{tabular}

Tabelle 51 und Tabelle 52 zeigen die Kantenliste GapLiTot zwecks Überblick in Matrixform. Sie ist das Endergebnis der Bestimmung von internen und externen Lückenfolgen (bis zu der Weglänge $n-1$ ) und gibt daher an, welcher Lückenfolgentyp (siehe Tabelle 30 und Tabelle 31) bei welcher Weglänge ein Knotenpaar erstmals verknüpfen kann. Lückenfolgetypen, die in einer Zelle (für ein Knotenpaar) nicht vorkommen, können das entsprechende Knotenpaar gar nicht in Beziehung setzen. 
Tabelle 51: Typen von Lückenfolgen mit dazugehöriger Weglänge I

\begin{tabular}{|c|c|c|c|c|c|c|c|c|c|}
\hline & 02 & 17 & 20 & 21 & 22 & 24 & 27 & 28 & 29 \\
\hline 02 & NOW:1 & $\begin{array}{l}\text { NOW:1 } \\
\text { PIL:2 } \\
\text { KIP:3 } \\
\text { KPI:4 }\end{array}$ & $\begin{array}{l}\text { PER:1 } \\
\text { KIL:2 } \\
\text { KPI:3 }\end{array}$ & $\begin{array}{l}\text { KIL:1 } \\
\text { PER:2 } \\
\text { KPI:3 }\end{array}$ & $\begin{array}{c}\text { NOW:1 } \\
\text { KIL:2 } \\
\text { KPI:3 }\end{array}$ & $\begin{array}{l}\text { NOW:1 } \\
\text { KIL:2 } \\
\text { KPI:3 }\end{array}$ & $\begin{array}{l}\text { NOW:1 } \\
\text { PIL:2 } \\
\text { KIL:3 } \\
\text { KPI:4 }\end{array}$ & $\begin{array}{l}\text { NOW:1 } \\
\mathrm{KPI}: 3\end{array}$ & $\begin{array}{c}\text { NOW:1 } \\
\mathrm{KPI}: 3\end{array}$ \\
\hline 17 & $\begin{array}{c}\text { NOW:1 } \\
\text { KIL:2 } \\
\text { KPI:4 }\end{array}$ & NOW:1 & $\begin{array}{l}\text { NOW:1 } \\
\mathrm{KPI}: 3\end{array}$ & $\begin{array}{c}\text { NOW:1 } \\
\text { KIL:3 } \\
\text { KPI:4 }\end{array}$ & $\begin{array}{c}\text { NOW:1 } \\
\text { EXT:2 } \\
\text { KIL:3 } \\
\text { KPI:5 }\end{array}$ & $\begin{array}{c}\text { NOW:1 } \\
\text { El4:2 } \\
\text { KIL:3 } \\
\text { KPI:4 }\end{array}$ & $\begin{array}{c}\text { NOW:1 } \\
\text { KIL:3 } \\
\text { KPI:4 }\end{array}$ & $\begin{array}{l}\text { NOW:1 } \\
\text { El4:2 } \\
\text { KIL:3 } \\
\text { KPI:4 }\end{array}$ & $\begin{array}{l}\text { NOW:1 } \\
\text { EXT:2 } \\
\text { KIL:3 } \\
\text { KPI:4 }\end{array}$ \\
\hline 20 & $\begin{array}{c}\text { PER:1 } \\
\text { KIL:2 } \\
\text { KPI:3 }\end{array}$ & $\begin{array}{l}\text { NOW:1 } \\
\text { PER:2 } \\
\text { KPI:3 }\end{array}$ & NOW:1 & $\begin{array}{l}\text { PER:1 } \\
\text { PIL:2 } \\
\text { KPI:3 }\end{array}$ & $\begin{array}{l}\text { NOW:1 } \\
\text { PIL:2 } \\
\text { KPI:3 }\end{array}$ & $\begin{array}{l}\text { NOW:1 } \\
\text { PIL:2 } \\
\text { KPI:3 }\end{array}$ & $\begin{array}{l}\text { KIL:1 } \\
\text { EXT:2 } \\
\text { KPI:3 }\end{array}$ & $\begin{array}{l}\text { NOW:1 } \\
K P I: 2\end{array}$ & $\begin{array}{l}\text { NOW:1 } \\
\text { PIL:2 } \\
\text { KPI:3 }\end{array}$ \\
\hline 21 & $\begin{array}{l}\text { NOW:1 } \\
\mathrm{KPI}: 2\end{array}$ & $\begin{array}{l}\text { PER:1 } \\
\text { KIL:2 } \\
\text { KPI:3 }\end{array}$ & $\begin{array}{l}\text { KIL:1 } \\
\text { KPI:3 }\end{array}$ & NOW:1 & $\begin{array}{l}\mathrm{KIL}: 1 \\
\mathrm{KPI}: 3\end{array}$ & $\begin{array}{l}\text { KIL:1 } \\
\text { KPI:2 }\end{array}$ & $\begin{array}{c}\text { NOW:1 } \\
\text { KIL:2 } \\
\text { KPI:3 } \\
\end{array}$ & $\begin{array}{c}\text { NOW:1 } \\
\text { KIL:2 } \\
\text { KPI:3 }\end{array}$ & $\begin{array}{l}\text { NOW:1 } \\
\text { KIL:2 } \\
\text { KPI:3 }\end{array}$ \\
\hline 22 & $\begin{array}{l}\text { KIL:1 } \\
\mathrm{KPI}: 2\end{array}$ & $\begin{array}{c}\text { KIL:1 } \\
\text { KPP:2 } \\
\text { KPI:3 }\end{array}$ & $\begin{array}{l}\text { NOW:1 } \\
\mathrm{KPI}: 2\end{array}$ & $\begin{array}{l}\text { PER:1 } \\
\text { KPI:2 }\end{array}$ & NOW:1 & $\begin{array}{l}\text { PER:1 } \\
\text { KPI:2 }\end{array}$ & $\begin{array}{l}\text { NOW:1 } \\
K P I: 2\end{array}$ & $\begin{array}{l}\text { NOW:1 } \\
K P I: 2\end{array}$ & $\begin{array}{l}\mathrm{KIL}: 1 \\
\mathrm{KPI}: 2\end{array}$ \\
\hline 24 & $\begin{array}{l}\text { KIL:1 } \\
\text { KPI:3 }\end{array}$ & $\begin{array}{l}\text { NOW:1 } \\
\mathrm{KPI}: 2\end{array}$ & $\begin{array}{l}\text { NOW:1 } \\
K P I: 2\end{array}$ & $\begin{array}{l}\text { KIL:1 } \\
\text { KPI:2 }\end{array}$ & $\begin{array}{l}\text { KIL:1 } \\
\text { KPI:3 }\end{array}$ & NOW:1 & $\begin{array}{l}\text { NOW:1 } \\
\text { PIL:2 } \\
\text { KPI:3 }\end{array}$ & $\begin{array}{l}\mathrm{KIL}: 1 \\
\mathrm{EI} 4 \mathrm{2} \\
\mathrm{KPI}: 3\end{array}$ & $\begin{array}{l}\text { NOW:1 } \\
\mathrm{KPI}: 2\end{array}$ \\
\hline 27 & $\begin{array}{l}\text { NOW:1 } \\
\mathrm{KPI}: 2\end{array}$ & $\begin{array}{l}\text { NOW:1 } \\
\text { PIL:2 } \\
\text { KPI:3 }\end{array}$ & $\begin{array}{l}\text { NOW:1 } \\
K P I: 2\end{array}$ & $\begin{array}{l}\text { NOW:1 } \\
\text { PIL:2 } \\
\text { KPI:3 }\end{array}$ & $\begin{array}{l}\text { NOW:1 } \\
\text { PIL:2 } \\
\text { KPI:3 }\end{array}$ & $\begin{array}{l}\text { NOW:1 } \\
\text { PIL:2 } \\
\text { KPI:3 }\end{array}$ & NOW:1 & $\begin{array}{c}\text { PER:1 } \\
\text { KIL:2 } \\
\text { KPI:3 }\end{array}$ & $\begin{array}{c}\text { PER:1 } \\
\text { KPI:3 }\end{array}$ \\
\hline 28 & $\begin{array}{l}\text { KIL:1 } \\
\text { PIL:2 } \\
\text { KPI:3 }\end{array}$ & $\begin{array}{l}\text { NOW:1 } \\
\text { PIL:2 } \\
\text { KPI:3 }\end{array}$ & $\begin{array}{l}\text { KIL:1 } \\
\text { PIL:2 } \\
\text { KPI:3 }\end{array}$ & $\begin{array}{l}\text { NOW:1 } \\
\mathrm{KPI}: 2\end{array}$ & $\begin{array}{c}\text { NOW:1 } \\
\text { PIL:2 } \\
\text { KPI:3 }\end{array}$ & $\begin{array}{l}\text { NOW:1 } \\
\text { PIL:2 } \\
\text { KPI:3 }\end{array}$ & $\begin{array}{c}\text { PER:1 } \\
\text { KPI:2 }\end{array}$ & NOW:1 & $\begin{array}{l}\text { PER:1 } \\
\text { KIP:2 } \\
\text { KPI:3 }\end{array}$ \\
\hline 29 & $\begin{array}{l}\text { KIL:1 } \\
\text { KPI:2 }\end{array}$ & $\begin{array}{l}\mathrm{KIL}: 1 \\
\mathrm{KPI}: 2\end{array}$ & $\begin{array}{l}\mathrm{KIL}: 1 \\
\mathrm{KPI}: 2\end{array}$ & $\begin{array}{l}\text { KIL:1 } \\
\text { KPI:2 }\end{array}$ & $\begin{array}{l}\text { KIL:1 } \\
\text { KPI:3 }\end{array}$ & $\begin{array}{l}\mathrm{KIL}: 1 \\
\mathrm{KPI}: 2\end{array}$ & $\begin{array}{l}\text { KIL:1 } \\
\text { KPI:3 }\end{array}$ & $\begin{array}{l}\text { NOW:1 } \\
K P I: 2\end{array}$ & NOW:1 \\
\hline $45 \mathrm{~A}$ & $\begin{array}{l}\mathrm{KIL}: 1 \\
\mathrm{KPI}: 2\end{array}$ & $\begin{array}{l}\text { NOW:1 } \\
\text { KIL:2 } \\
\text { KPI:3 }\end{array}$ & $\begin{array}{l}\mathrm{KIL}: 1 \\
\mathrm{KPI}: 2\end{array}$ & $\begin{array}{l}\text { NOW:1 } \\
K P I: 2\end{array}$ & $\begin{array}{c}\text { NOW:1 } \\
\text { KIL:2 } \\
\text { KPI:3 }\end{array}$ & $\begin{array}{c}\text { NOW:1 } \\
\text { KIL:2 } \\
\text { KPI:3 }\end{array}$ & $\begin{array}{c}\text { NOW:1 } \\
\mathrm{KPI}: 2\end{array}$ & $\begin{array}{l}\text { KIL:1 } \\
\text { KPI:3 }\end{array}$ & $\begin{array}{c}\text { NOW:1 } \\
\mathrm{KPI}: 2\end{array}$ \\
\hline 45B & $\begin{array}{l}\text { KIL:1 } \\
\text { KPI:3 }\end{array}$ & $\begin{array}{c}\text { NOW:1 } \\
\text { KIL:2 } \\
\text { KPI:3 }\end{array}$ & $\begin{array}{l}\text { NOW:1 } \\
K P I: 2\end{array}$ & $\begin{array}{c}\text { NOW:1 } \\
\text { KIL:2 } \\
\text { KPI:3 }\end{array}$ & $\begin{array}{c}\text { NOW:1 } \\
\text { KIL:2 } \\
\text { KPI:4 }\end{array}$ & $\begin{array}{l}\text { NOW:1 } \\
\text { KIL:2 } \\
\text { KPI:3 }\end{array}$ & $\begin{array}{c}\text { NOW:1 } \\
\text { KIL:2 } \\
\text { KPI:3 }\end{array}$ & $\begin{array}{c}\text { NOW:1 } \\
\text { KIL:2 } \\
\text { KPI:3 }\end{array}$ & $\begin{array}{c}\text { NOW:1 } \\
\text { KIL:2 } \\
\text { KPI:3 } \\
\end{array}$ \\
\hline $52 A$ & NOW:1 & NOW:1 & NOW:1 & NOW:1 & NOW:1 & NOW:1 & NOW:1 & NOW:1 & NOW:1 \\
\hline 55 & $\begin{array}{c}\text { NOW:1 } \\
\text { KIL:2 } \\
\text { KPI:3 }\end{array}$ & $\begin{array}{c}\text { NOW:1 } \\
\text { KIL:2 } \\
\text { KPI:3 }\end{array}$ & $\begin{array}{l}\text { NOW:1 } \\
\text { KIL:2 } \\
\text { KPI:3 }\end{array}$ & $\begin{array}{c}\text { NOW:1 } \\
\text { KIL:2 } \\
\text { KPI:3 }\end{array}$ & $\begin{array}{c}\text { NOW:1 } \\
\text { KIL:2 } \\
\text { KPI:3 }\end{array}$ & $\begin{array}{c}\text { NOW:1 } \\
\text { KIL:2 } \\
\text { KPI:3 }\end{array}$ & $\begin{array}{l}\text { NOW:1 } \\
\mathrm{KPI}: 2\end{array}$ & $\begin{array}{l}\text { KIL:1 } \\
\text { KPI:3 }\end{array}$ & $\begin{array}{l}\mathrm{KIL}: 1 \\
\mathrm{KPI}: 2\end{array}$ \\
\hline $63 B$ & $\begin{array}{c}\text { NOW:1 } \\
\text { El4:2 } \\
\text { El1:3 }\end{array}$ & $\begin{array}{c}\text { NOW:1 } \\
\text { El4:2 } \\
\text { El1:3 }\end{array}$ & $\begin{array}{l}\text { NOW:1 } \\
\text { EXT:2 } \\
\text { El1:3 }\end{array}$ & $\begin{array}{c}\text { NOW:1 } \\
\text { EXT:2 } \\
\text { El1:3 }\end{array}$ & $\begin{array}{c}\text { NOW:1 } \\
\text { EXT:2 } \\
\text { El2:3 } \\
\text { El1:4 }\end{array}$ & $\begin{array}{c}\text { NOW:1 } \\
\text { EXT:2 } \\
\text { El1:3 }\end{array}$ & $\begin{array}{c}\text { NOW:1 } \\
\text { El4:2 } \\
\text { El1:3 }\end{array}$ & $\begin{array}{c}\text { NOW:1 } \\
\text { El4:2 } \\
\text { El1:3 }\end{array}$ & $\begin{array}{c}\text { NOW:1 } \\
\text { EXT:2 } \\
\text { El1:3 }\end{array}$ \\
\hline 72 & $\begin{array}{c}\text { NOW:1 } \\
\text { KPI:2 }\end{array}$ & $\begin{array}{l}\mathrm{KIL}: 1 \\
\mathrm{KPI}: 2 \\
\end{array}$ & $\begin{array}{l}\mathrm{KIL}: 1 \\
\mathrm{KPI}: 3\end{array}$ & $\begin{array}{l}\mathrm{KIL}: 1 \\
\mathrm{KPI}: 2 \\
\end{array}$ & $\begin{array}{l}\mathrm{KIL}: 1 \\
\mathrm{KPI}: 3\end{array}$ & $\begin{array}{l}\mathrm{KIL}: 1 \\
\mathrm{KPI}: 2\end{array}$ & $\begin{array}{l}\mathrm{KIL}: 1 \\
\mathrm{KPI}: 2\end{array}$ & $\begin{array}{l}\mathrm{KIL}: 1 \\
\mathrm{KPI}: 2 \\
\end{array}$ & $\begin{array}{l}\mathrm{KIL}: 1 \\
\mathrm{KPI}: 2 \\
\end{array}$ \\
\hline $74 \mathrm{~B}$ & $\begin{array}{c}\text { NOW:1 } \\
\text { KIL:2 } \\
\text { KPI:3 }\end{array}$ & $\begin{array}{l}\text { NOW:1 } \\
\text { EXT:2 } \\
\text { KIL:3 } \\
\text { KPI:4 }\end{array}$ & $\begin{array}{l}\text { NOW:1 } \\
\text { KIL:2 } \\
\text { KPI:3 }\end{array}$ & $\begin{array}{l}\text { NOW:1 } \\
\text { EXT:2 } \\
\text { KPI:3 }\end{array}$ & $\begin{array}{c}\text { NOW:1 } \\
\text { EXT:2 } \\
\text { KIL:3 } \\
\text { KPI:4 }\end{array}$ & $\begin{array}{l}\text { NOW:1 } \\
\text { EXT:2 } \\
\text { KIL:3 } \\
\text { KPI:4 }\end{array}$ & $\begin{array}{l}\text { NOW:1 } \\
\text { EXT:2 } \\
\text { KPI:3 }\end{array}$ & $\begin{array}{l}\text { NOW:1 } \\
\text { KIL:2 } \\
\text { KPI:4 }\end{array}$ & $\begin{array}{l}\text { NOW:1 } \\
\text { EXT:2 } \\
\text { KPI:3 }\end{array}$ \\
\hline $74 C$ & $\begin{array}{l}\text { NOW:1 } \\
\text { KIL:2 }\end{array}$ & $\begin{array}{l}\text { KIL:1 } \\
\text { KPI:3 }\end{array}$ & $\begin{array}{l}\text { NOW:1 } \\
\text { KIL:2 }\end{array}$ & $\begin{array}{c}\text { NOW:1 } \\
\mathrm{KPI}: 2\end{array}$ & $\begin{array}{l}\text { KIL:1 } \\
\text { KPI:3 }\end{array}$ & $\begin{array}{l}\text { KIL:1 } \\
\text { KPI:2 }\end{array}$ & $\begin{array}{l}\text { KIL:1 } \\
\mathrm{KPI}: 2\end{array}$ & $\begin{array}{l}\mathrm{KIL}: 1 \\
\mathrm{KPI}: 2\end{array}$ & $\begin{array}{l}\text { KIL:1 } \\
\text { KPI:2 }\end{array}$ \\
\hline
\end{tabular}


Tabelle 52: Typen von Lückenfolgen mit dazugehöriger Weglänge II

\begin{tabular}{|c|c|c|c|c|c|c|c|c|}
\hline & $45 \mathrm{~A}$ & 45B & $52 A$ & 55 & $63 B$ & 72 & $74 \mathrm{~B}$ & $74 \mathrm{C}$ \\
\hline 02 & $\begin{array}{l}\text { NOW:1 } \\
\text { PIL:2 } \\
\text { KPI:3 }\end{array}$ & $\begin{array}{l}\text { NOW:1 } \\
\text { KPI:2 }\end{array}$ & $\begin{array}{c}\text { NOW:1 } \\
\text { KIL:2 } \\
\text { KPI:3 }\end{array}$ & $\begin{array}{c}\text { NOW:1 } \\
\text { El4:2 } \\
\text { KPI: } 3\end{array}$ & $\begin{array}{l}\text { NOW:1 } \\
\text { KIL:3 } \\
\text { KPI:4 }\end{array}$ & $\begin{array}{c}\text { NOW:1 } \\
\text { KIL:2 } \\
\text { KPI: } 3 \\
\end{array}$ & $\begin{array}{l}\text { NOW:1 } \\
\text { KIL:2 } \\
\text { KPI:3 }\end{array}$ & $\begin{array}{l}\text { NOW:1 } \\
\mathrm{KPI}: 3\end{array}$ \\
\hline 17 & $\begin{array}{c}\text { NOW:1 } \\
\text { KIL:2 } \\
\text { EI1:3 } \\
\text { KPI:4 }\end{array}$ & $\begin{array}{l}\text { KIL:1 } \\
\text { EXT:2 } \\
\text { KPI:4 }\end{array}$ & $\begin{array}{c}\text { NOW:1 } \\
\text { El4:2 } \\
\text { KIL:3 } \\
\text { KPI:4 }\end{array}$ & $\begin{array}{c}\text { NOW:1 } \\
\text { KIL:2 } \\
\text { KPI:5 }\end{array}$ & $\begin{array}{l}\text { NOW:1 } \\
\mathrm{KPI}: 3\end{array}$ & $\begin{array}{c}\text { NOW:1 } \\
\text { KIL:2 } \\
\text { KPI:4 }\end{array}$ & $\begin{array}{l}\text { NOW:1 } \\
\text { EXT:2 } \\
\text { KIL:3 } \\
\text { KPI:5 }\end{array}$ & $\begin{array}{l}\text { NOW:1 } \\
\text { KIL:2 } \\
\text { KPI:4 }\end{array}$ \\
\hline 20 & $\begin{array}{l}\mathrm{KIL}: 1 \\
\mathrm{KPI}: 3\end{array}$ & $\begin{array}{l}\mathrm{KIL}: 1 \\
\mathrm{KPI}: 2\end{array}$ & $\begin{array}{l}\text { NOW:1 } \\
\text { PIL:2 } \\
\text { KPI:3 }\end{array}$ & $\begin{array}{l}\text { NOW:1 } \\
\text { KIL:2 } \\
\text { KPI:3 }\end{array}$ & $\begin{array}{l}\text { NOW:1 } \\
\text { EXT:2 } \\
\text { KPI:3 }\end{array}$ & $\begin{array}{l}\text { NOW:1 } \\
\text { KPI:2 }\end{array}$ & $\begin{array}{l}\text { NOW:1 } \\
\text { PIL:2 } \\
\text { KPI:3 }\end{array}$ & $\begin{array}{l}\text { NOW:1 } \\
\text { KIL:2 } \\
\text { KPI:3 }\end{array}$ \\
\hline 21 & $\begin{array}{c}\text { NOW:1 } \\
\text { KIL:2 } \\
\text { KPI:3 } \\
\end{array}$ & $\begin{array}{l}\mathrm{KIL}: 1 \\
\mathrm{KPI}: 2\end{array}$ & $\begin{array}{l}\mathrm{KIL}: 1 \\
\mathrm{KPI}: 2\end{array}$ & $\begin{array}{c}\text { NOW:1 } \\
\text { KIL:2 } \\
\text { KPl:3 }\end{array}$ & $\begin{array}{c}\text { NOW:1 } \\
\text { KIL:2 } \\
\mathrm{KPI}: 3\end{array}$ & $\begin{array}{l}\mathrm{KIL}: 1 \\
\mathrm{KPI}: 2\end{array}$ & $\begin{array}{l}\text { KIL:1 } \\
\text { KPI:3 }\end{array}$ & $\begin{array}{l}\text { NOW:1 } \\
\mathrm{KPI}: 2\end{array}$ \\
\hline 22 & $\begin{array}{l}\text { KIL:1 } \\
\text { KPI:3 }\end{array}$ & $\begin{array}{l}\text { NOW:1 } \\
K P I: 2\end{array}$ & $\begin{array}{l}\text { PER:1 } \\
\mathrm{KPI}: 2\end{array}$ & $\begin{array}{l}\text { KIL:1 } \\
\mathrm{KPI}: 2\end{array}$ & $\begin{array}{l}\text { KIL:1 } \\
\text { PIL:2 } \\
\text { KPI:3 }\end{array}$ & $\begin{array}{l}\text { PER:1 } \\
\text { KPI:2 }\end{array}$ & $\begin{array}{l}\mathrm{KIL}: 1 \\
\mathrm{KPI}: 2\end{array}$ & $\begin{array}{l}\text { PER:1 } \\
\text { KPI:2 }\end{array}$ \\
\hline 24 & $\begin{array}{l}\text { NOW:1 } \\
\mathrm{KPI}: 2\end{array}$ & $\begin{array}{l}\mathrm{KIL}: 1 \\
\mathrm{KPI}: 2\end{array}$ & $\begin{array}{l}\text { NOW:1 } \\
\mathrm{KPI}: 2\end{array}$ & $\begin{array}{c}\text { NOW:1 } \\
\text { KIL:2 } \\
\text { KPI:3 }\end{array}$ & $\begin{array}{l}\text { NOW:1 } \\
\text { KIL:2 } \\
\mathrm{KPI}: 3\end{array}$ & $\begin{array}{l}\text { NOW:1 } \\
\text { KPI:2 }\end{array}$ & $\begin{array}{l}\text { NOW:1 } \\
\text { KIL:2 } \\
\text { KPI:3 }\end{array}$ & $\begin{array}{l}\text { NOW:1 } \\
\mathrm{KPI}: 2\end{array}$ \\
\hline 27 & $\begin{array}{l}\text { KIL:1 } \\
\text { PPL:2 } \\
\text { KPI:3 }\end{array}$ & $\begin{array}{l}\text { PER:1 } \\
\text { KIP:2 } \\
\text { KPP:3 } \\
\text { KPI:4 }\end{array}$ & $\begin{array}{c}\text { NOW:1 } \\
\text { PIL:2 } \\
\text { KPI:3 }\end{array}$ & $\begin{array}{l}\text { NOW:1 } \\
K P I: 2\end{array}$ & $\begin{array}{l}\text { NOW:1 } \\
\text { EXT:2 } \\
\text { KPI:3 }\end{array}$ & $\begin{array}{l}\text { NOW:1 } \\
\mathrm{KPI}: 2\end{array}$ & $\begin{array}{l}\text { NOW:1 } \\
\text { PIL:2 } \\
\text { KPI:3 }\end{array}$ & $\begin{array}{l}\text { NOW:1 } \\
\text { PIL:2 } \\
\text { KPI:3 }\end{array}$ \\
\hline 28 & $\begin{array}{l}\text { PER:1 } \\
\mathrm{KPI}: 2\end{array}$ & $\begin{array}{l}\text { PER:1 } \\
\text { KPP:2 } \\
\text { KPI:4 }\end{array}$ & $\begin{array}{l}\text { KIL:1 } \\
\text { KPI:3 }\end{array}$ & $\begin{array}{l}\text { KIL:1 } \\
\text { PIL:2 } \\
\text { KPI:3 }\end{array}$ & $\begin{array}{l}\text { NOW:1 } \\
\text { PIL:2 } \\
\text { KPI:3 }\end{array}$ & $\begin{array}{l}\text { NOW:1 } \\
\mathrm{KPI}: 2\end{array}$ & $\begin{array}{l}\text { KIL:1 } \\
\mathrm{KPI}: 3\end{array}$ & $\begin{array}{l}\text { NOW:1 } \\
\mathrm{KPI}: 2\end{array}$ \\
\hline 29 & $\begin{array}{l}\text { NOW:1 } \\
\text { KPI:2 }\end{array}$ & $\begin{array}{l}\text { PER:1 } \\
\text { KPI:2 }\end{array}$ & $\begin{array}{l}\text { NOW:1 } \\
\mathrm{KPI}: 2\end{array}$ & $\begin{array}{l}\text { NOW:1 } \\
K P I: 2\end{array}$ & $\begin{array}{l}\text { NOW:1 } \\
\text { KIL:2 } \\
\text { KPI:3 }\end{array}$ & $\begin{array}{l}\text { NOW:1 } \\
\text { KPI:2 }\end{array}$ & $\begin{array}{l}\text { NOW:1 } \\
\text { KIL:2 } \\
\text { KPI:3 }\end{array}$ & $\begin{array}{l}\text { NOW:1 } \\
\text { PIL:2 } \\
\text { KPI:3 }\end{array}$ \\
\hline $45 A$ & NOW:1 & $\begin{array}{l}\mathrm{KIL}: 1 \\
\mathrm{KPI}: 2\end{array}$ & $\begin{array}{l}\text { NOW:1 } \\
\text { KIL:2 } \\
\text { KPI:3 }\end{array}$ & $\begin{array}{l}\mathrm{KIL}: 1 \\
\mathrm{KPI}: 3\end{array}$ & $\begin{array}{l}\text { NOW:1 } \\
\text { KPI:2 }\end{array}$ & $\begin{array}{l}\text { KIL:1 } \\
\text { KPI:3 }\end{array}$ & $\begin{array}{l}\text { NOW:1 } \\
\text { KIL:2 } \\
\text { KPI:3 }\end{array}$ & $\begin{array}{l}\text { NOW:1 } \\
\text { KIL:2 } \\
\text { KPI:3 }\end{array}$ \\
\hline 45B & $\begin{array}{l}\text { KIL:1 } \\
\text { EI4:2 } \\
\text { KPI:3 }\end{array}$ & NOW:1 & $\begin{array}{c}\text { NOW:1 } \\
\text { KIL:2 } \\
\text { KPI:3 }\end{array}$ & $\begin{array}{l}\text { KIL:1 } \\
\mathrm{KPI}: 4\end{array}$ & $\begin{array}{l}\text { NOW:1 } \\
\text { KPI:2 }\end{array}$ & $\begin{array}{l}\mathrm{KIL}: 1 \\
\mathrm{KPI}: 3\end{array}$ & $\begin{array}{c}\text { NOW:1 } \\
\text { KIL:2 } \\
\text { KPI:4 }\end{array}$ & $\begin{array}{l}\text { KIL:1 } \\
\mathrm{KPI}: 3\end{array}$ \\
\hline $52 \mathrm{~A}$ & NOW:1 & NOW:1 & NOW:1 & NOW:1 & NOW:1 & NOW:1 & NOW:1 & NOW:1 \\
\hline 55 & $\begin{array}{l}\text { NOW:1 } \\
\text { KPI:2 }\end{array}$ & $\begin{array}{l}\text { KIL:1 } \\
\text { PIL:2 } \\
\text { KPI:3 }\end{array}$ & $\begin{array}{l}\mathrm{KIL}: 1 \\
\mathrm{KPI}: 3\end{array}$ & NOW:1 & $\begin{array}{l}\text { PER:1 } \\
\text { KIL:2 } \\
\text { KPI:4 }\end{array}$ & $\begin{array}{l}\mathrm{KIL}: 1 \\
\mathrm{KPI}: 3\end{array}$ & $\begin{array}{l}\text { KIL:1 } \\
\text { KPI:4 }\end{array}$ & $\begin{array}{l}\text { KIL:1 } \\
\text { KPI:3 }\end{array}$ \\
\hline $63 B$ & $\begin{array}{c}\text { NOW:1 } \\
\text { El4:2 } \\
\text { El1:3 }\end{array}$ & $\begin{array}{l}\text { NOW:1 } \\
\text { El1:3 }\end{array}$ & $\begin{array}{c}\text { NOW:1 } \\
\text { El4:2 } \\
\text { El1:3 }\end{array}$ & $\begin{array}{c}\text { NOW:1 } \\
\text { EXT:2 } \\
\text { EI2:3 } \\
\text { El1:4 }\end{array}$ & NOW:1 & $\begin{array}{c}\text { NOW:1 } \\
\text { El4:2 } \\
\text { El1:3 }\end{array}$ & $\begin{array}{c}\text { NOW:1 } \\
\text { EXT:2 } \\
\text { El2:3 } \\
\text { El1:4 }\end{array}$ & $\begin{array}{l}\text { NOW:1 } \\
\text { EXT:2 } \\
\text { El1:3 }\end{array}$ \\
\hline 72 & $\begin{array}{c}\text { NOW:1 } \\
\text { KPI:2 }\end{array}$ & $\begin{array}{l}\text { NOW:1 } \\
\text { KPI:2 }\end{array}$ & $\begin{array}{l}\text { KIL:1 } \\
\text { KPI:2 }\end{array}$ & $\begin{array}{l}\text { KIL:1 } \\
\text { KPI:3 }\end{array}$ & $\begin{array}{l}\text { KIL:1 } \\
\text { KPI:2 }\end{array}$ & NOW:1 & $\begin{array}{l}\text { KIL:1 } \\
\mathrm{KPI}: 3\end{array}$ & $\begin{array}{l}\mathrm{KIL}: 1 \\
\mathrm{KPI}: 2\end{array}$ \\
\hline $74 B$ & $\begin{array}{l}\text { KIL:1 } \\
\text { EXT:2 } \\
\text { KPI:3 }\end{array}$ & $\begin{array}{l}\text { NOW:1 } \\
\text { KIL:2 } \\
\text { KPI:3 }\end{array}$ & $\begin{array}{c}\text { NOW:1 } \\
\text { El4:2 } \\
\text { KIL:3 } \\
\text { KPI:4 }\end{array}$ & $\begin{array}{c}\text { NOW:1 } \\
\text { KIL:2 } \\
\text { KPI:4 }\end{array}$ & $\begin{array}{l}\text { NOW:1 } \\
\text { El4:2 } \\
\text { KPI:3 }\end{array}$ & $\begin{array}{c}\text { NOW:1 } \\
\text { KIL:2 } \\
\text { KPI:4 }\end{array}$ & NOW:1 & $\begin{array}{l}\text { NOW:1 } \\
\text { EXT:2 } \\
\text { KIL:3 } \\
\text { KPI:4 }\end{array}$ \\
\hline $74 \mathrm{C}$ & $\begin{array}{l}\text { NOW:1 } \\
\mathrm{KPI}: 2\end{array}$ & $\begin{array}{l}\text { NOW:1 } \\
K P I: 2\end{array}$ & $\begin{array}{l}\text { KIL:1 } \\
\text { KPI:2 }\end{array}$ & $\begin{array}{l}\text { NOW:1 } \\
\text { KIL:2 } \\
\text { KPI:3 }\end{array}$ & $\begin{array}{l}\mathrm{KIL}: 1 \\
\mathrm{KPI}: 3\end{array}$ & $\begin{array}{l}\text { KIL:1 } \\
\text { PIL:2 } \\
\text { KPI:3 }\end{array}$ & $\begin{array}{l}\mathrm{KIL}: 1 \\
\mathrm{KPI}: 3 \\
978=3=6\end{array}$ & NOW:1 \\
\hline
\end{tabular}


Das Knotenpaar $(17,74 B)$ kann zum Beispiel über die Lückenfolgetypen ,EXT', ,KIL' und 'KPl' erstmals bei den Weglängen 2,3 bzw. 5 verbunden werden. ,EXT:2' deutet darauf hin, dass die Knoten 17 und 74B mittels eines externen Knoten indirekt verknüpft werden können. ,KIL:3' sagt aus, dass dies auch über drei zusätzliche interne Kanten möglich ist, während ,KPI:5' die Schlussfolgerung zulässt, dass bei einer Weglänge von 5 neben ausschließlich zusätzlichen Kanten eine Verbindung auch durch eine Kombination von zusätzlichen und bestehenden Kanten möglich ist. ,NOW:1' besagt, dass es keine direkte Verbindungsmöglichkeit für diese beiden Knoten gibt. Weist eine Zelle nur diesen Lückenfolgetyp bei Weglänge 1 auf, so können die betroffenen Knoten nicht in Zusammenhang gebracht werden. Dies ist in Tabelle 51 und Tabelle 52 bspw. durch den Ausschluss von Selbstbezüglichkeit durchgehend in der Diagonale zu beobachten. Darüber hinaus dient der Knoten 52A für keinen der weiteren Knoten als Inputfaktor, weder direkt noch indirekt.

Die deskriptive Auswertung der in Tabelle 51 und Tabelle 52 dargelegten Zusammenhänge kann der Tabelle 53 entnommen werden, die wichtige Größen des Vergleichs zwischen lokaler und globaler Struktur gemäß Kapitel 7.6 hervorhebt. Tabelle 54 legt die auf diese Größen zurückzuführenden Kennzahlen nach Maßgabe der Berechnungsvorschläge aus Kapitel 7.7 dar.

Tabelle 53: Deskriptive Auswertung von GapLiTot

\begin{tabular}{|c|c|c|}
\hline & Erklärung & Wert \\
\hline |GiopliTor| & $\begin{array}{r}\text { rein theoretisch mögliche direkte Verbindungen } \\
\text { (inkl. Selbstbezüglichkeit) }\end{array}$ & 289 \\
\hline $\mid$ GiaplitillR| & bestehende direkte Verbindungen & 18 \\
\hline $\mid\left(i(i) L i^{h / l} \mid\right.$ & zusätzlich mögliche direkte Verbindungen & 79 \\
\hline 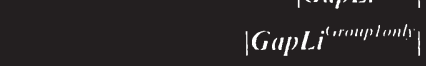 & $\begin{array}{r}\text { immer auch alleinig über bestehende Kanten } \\
\text { erreichbar }\end{array}$ & 0 \\
\hline 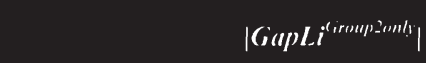 & $\begin{array}{r}\text { nur unter Einbezug von zusätzlichen Kanten } \\
\text { erreichbar }\end{array}$ & 217 \\
\hline 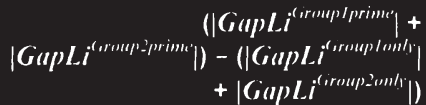 & $\begin{array}{r}\text { je nach Weglänge über bestehende Kanten, } \\
\text { zusätzliche Kanten oder in Kombination } \\
\text { erreichbar }\end{array}$ & 23 \\
\hline $\mid$ (iilp $L i^{\text {(iroupl/primus }} \mid$ & $\begin{array}{r}\text { primär über bestehende interne Kanten } \\
\text { erreichbar }\end{array}$ & 19 \\
\hline 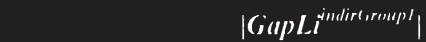 & davon indirekt & 1 \\
\hline 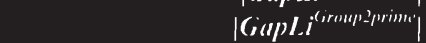 & primär über zusätzliche interne Kanten erreichbar & 221 \\
\hline 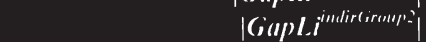 & Michael Weber - 978_davon indirekt & 142 \\
\hline
\end{tabular}




\begin{tabular}{|c|c|c|}
\hline & Erklärung & Wert \\
\hline 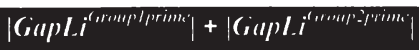 & intern prinzipiell erreichbar & 240 \\
\hline 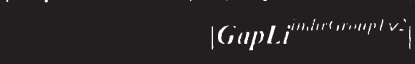 & $\begin{array}{r}\text { davon nur indirekt (Weglänge }>1 \text { ) intern } \\
\text { erreichbar }\end{array}$ & 143 \\
\hline \multirow{2}{*}{ 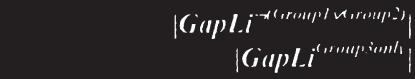 } & intern unerreichbar (inkl. Selbstbezüglichkeit) & 49 \\
\hline & nur unter Einbezug externer Knoten erreichbar & 16 \\
\hline 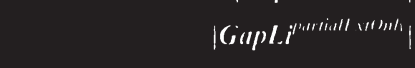 & $\begin{array}{r}\text { davon ausschließlich über partielle externe } \\
\text { Knotenfolgen erreichbar }\end{array}$ & 8 \\
\hline 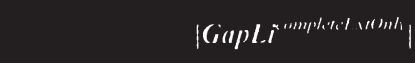 & $\begin{array}{r}\text { davon ausschließlich über komplette externe } \\
\text { Knotenfolgen erreichbar }\end{array}$ & 0 \\
\hline 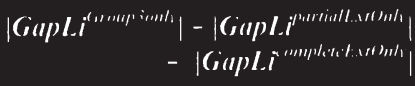 & $\begin{array}{r}\text { davon Weglängen mit kompletten und partiellen } \\
\text { externen Knotenfolgen }\end{array}$ & 8 \\
\hline 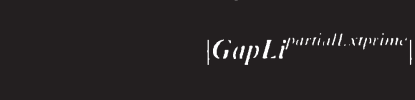 & $\begin{array}{r}\text { primär über partielle externe Knotenfolgen } \\
\text { erreichbar (ohne bestehende und zusätzliche } \\
\text { interne Kanten) }\end{array}$ & 8 \\
\hline 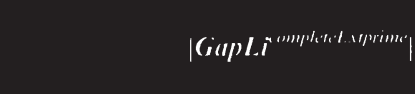 & $\begin{array}{r}\text { primär über komplette externe Knotenfolgen } \\
\text { erreichbar (ohne bestehende und zusätzliche } \\
\text { interne Kanten) }\end{array}$ & 8 \\
\hline 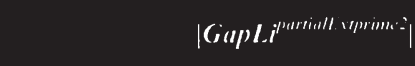 & $\begin{array}{r}\text { primär über partielle externe Knotenfolgen } \\
\text { erreichbar }\end{array}$ & 14 \\
\hline 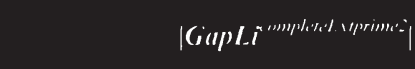 & $\begin{array}{r}\text { primär über komplette externe Knotenfolgen } \\
\text { erreichbar }\end{array}$ & 20 \\
\hline 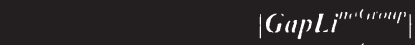 & absolut unerreichbar (inkl. Selbstbezüglichkeit) & 33 \\
\hline 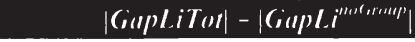 & intern oder extern erreichbar & 256 \\
\hline
\end{tabular}

Entsprechend dieser beiden Auswertungen ist insbesondere die Förderung zusätzlicher Kanten in der lokalen Struktur zur Verbesserung der Verflechtungen zu empfehlen, wie die Kennzahl share $e^{\text {indir(irouplv2 }}$ sowie deren Erweiterung in Bezug auf allgemeine Verbindungsmöglichkeiten zeigen. Die Hinzunahme externer Knoten spielt dementsprechend eine deutlich nachgeordnete Rolle, wie neben den soeben genannten Maßzahlen auch die Kennzahlen zu dem Verkürzungspotenzial durch externe Knoten share $e^{\text {partialExiShorter }}$ und share completeExiShorter $z$ zeigen.

Tabelle 54: Kennzahlen auf Basis der Auswertung von GapLiTot

\begin{tabular}{|c|c|c|}
\hline & Erklärung & Wert \\
\hline$\checkmark / h a r c^{\prime \prime R I}$ & Anteil intern bestehender Kanten am Kantenpotenzial & 0,186 \\
\hline$s / h 11 e^{n / 11}$ & $\begin{array}{r}\text { Anteil zusätzlich möglicher interner direkter Kanten am } \\
\text { Kantenpotenzial }\end{array}$ & 0,814 \\
\hline 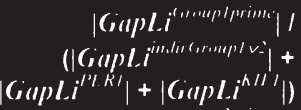 & $\begin{array}{r}\text { Anteil intern primär über bestehende Kanten } \\
\text { erreichbar }\end{array}$ & 0,079 \\
\hline 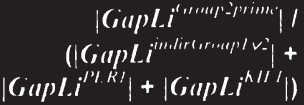 & Anteil intem primär über zusătzliche Kanten erreichbar & 0,921 \\
\hline 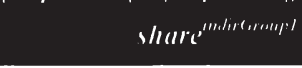 & Anteil intem primär indirekt über bestehende Kanten & 0,007 \\
\hline
\end{tabular}




\begin{tabular}{|c|c|c|}
\hline & Erklärung & Wert \\
\hline 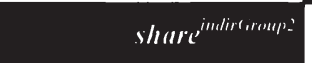 & $\begin{array}{r}\text { Anteil intern primär indirekt über zusätzliche Kanten } \\
\text { erreichbar }\end{array}$ & 0,993 \\
\hline 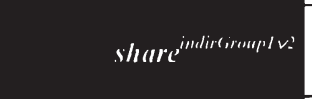 & $\begin{array}{r}\text { Anteil von nicht direkt aber intern indirekt zusammen- } \\
\text { hängenden Knotenpaaren an indirekten } \\
\text { Verbindungsmöglichkeiten }\end{array}$ & 0,899 \\
\hline share $e^{i m d i r t \text { rounp: }}$ & $\begin{array}{r}\text { Anteil von nur über zuzügliche externe Knoten (in- } \\
\text { direkt) verbindbaren Knotenpaaren an indirekten } \\
\text { Verbindungsmöglichkeiten }\end{array}$ & 0,101 \\
\hline 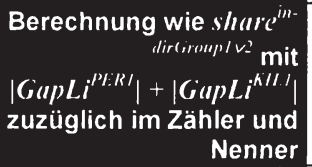 & $\begin{array}{r}\text { Anteil von intern zusammenhängenden Knotenpaaren } \\
\text { an allg. Verbindungsmöglichkeiten }\end{array}$ & 0,937 \\
\hline 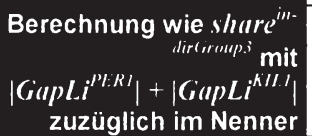 & $\begin{array}{r}\text { Anteil von nur über zuzügliche externe Knoten verbind- } \\
\text { baren Knotenpaaren an allg. Verbindungs- } \\
\text { möglichkeiten }\end{array}$ & 0,063 \\
\hline share purtiall:verl'rimi & $\begin{array}{r}\text { Anteil primärer externer partieller Knotenfolge an } \\
\text { externen Möglichkeiten }\end{array}$ & 0,5 \\
\hline share & $\begin{array}{r}\text { Anteil primärer externer kompletter Knotenfolge an } \\
\text { externen Möglichkeiten }\end{array}$ & 0,5 \\
\hline 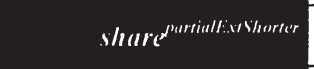 & $\begin{array}{r}\text { Verkürzungspotenzial interner indirekter Zusammen- } \\
\text { hänge durch partielle externe Knotenfolgen }\end{array}$ & 0,042 \\
\hline share completed washourter & $\begin{array}{r}\text { Verkürzungspotenzial interner indirekter Zusammen- } \\
\text { hänge durch komplette externe Knotenfolgen }\end{array}$ & 0,084 \\
\hline shore & Anteil unerreichbar (Selbstbezüglichkeit aufgehoben) & 0,114 \\
\hline 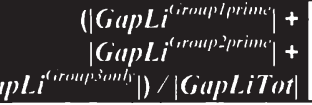 & Anteil erreichbar & 0,886 \\
\hline
\end{tabular}

Die detaillierte Bestimmung der Zusammensetzung der Lücken(-folgen) kann wie in den Kapiteln 7.8 und 7.9 beschrieben über eine erweiterte Kantenliste für die jeweilig zu betrachtende Weglänge erfolgen. Am Beispiel der Kantenliste für die Weglänge 2 (Edgeframe ${ }^{\text {oneho }}$ gemäß Formel (183)) werden nachfolgend Statistiken für die möglichen Beziehungen zu und von internen aber auch externen Knoten angeführt und erläutert. Zur Betrachtung der Lückenfolgen von internen Knoten wird zunächst die Teilmenge Edgeframe indir $_{\text {one }}$ (Formel (185))

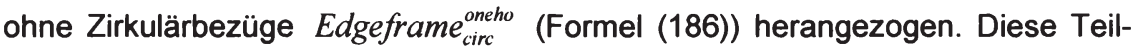
menge wird getrennt nach den Kategorien ,beidseitig neu', ,ausgangsseitig (verkaufsseitig) neu' und ,zielseitig (einkaufsseitig) neu' ausgewertet, wobei ,ausgangsseitig neu' bedeutet, dass nur die Kante, die von einem internen Ausgangsknoten wegführt, innerhalb des Weges neu ist, während ,zielseitig neu' 
jene Wege bezeichnet, die nur eine neue (zusätzlich mögliche) Kante zu dem Zielknoten enthalten. Die Kategorie ,beidseitig neu' umfasst dementsprechend Wege mit zwei, d.h. bei Weglänge 2 ausschließlich, neuen Kanten.

Tabelle 55 zeigt die deskriptive Statistik für die Kategorie ,beidseitig neu', welche für die Verbindungsknoten geordnet nach der Häufigkeit ihres Auftretens in der Kantenliste neben der absoluten und relativen Häufigkeit auch den Wertanteil der zu und von diesen Verbindungsknoten auf Basis der gesamtwirtschaftlichen Kantengewichte fließen könnte, angibt. Der Wertanteil dient als Indikator für die Relevanz des Knoten als Abnehmer (Inputanteil des Verbindungsknoten) bzw. Anbieter (Outputanteil des Verbindungsknoten) in den betrachteten Lückenfolgen und wird ungeachtet der Häufigkeit des Auftretens gleich bleibender Transaktionsarten (Kanten) als Anteil der Summe der Kantengewichte zu bzw. von dem Verbindungsknoten bezogen auf die jeweilige Gesamtsumme über alle Verbindungsknoten berechnet.

Tabelle 55: Beidseitig neue Kanten

\begin{tabular}{|c|c|c|c|c|}
\hline & Häufigkeit & $\begin{array}{l}\text { rel. Häufig- } \\
\text { keit }\end{array}$ & Inputanteil & Outputanteil \\
\hline 72 & 62 & 0,181 & 0,019 & 0,115 \\
\hline 22 & 34 & 0,099 & 0,147 & 0,058 \\
\hline 55 & 32 & 0,093 & 0,101 & 0,037 \\
\hline $74 C$ & 29 & 0,085 & 0,016 & 0,108 \\
\hline $45 A$ & 28 & 0,082 & 0,292 & 0,046 \\
\hline $45 B$ & 28 & 0,082 & 0,064 & 0,082 \\
\hline 29 & 27 & 0,079 & 0,066 & 0,048 \\
\hline 21 & 26 & 0,076 & 0,072 & 0,163 \\
\hline 28 & 24 & 0,070 & 0,037 & 0,035 \\
\hline 24 & 19 & 0,055 & 0,059 & 0,028 \\
\hline 20 & 14 & 0,041 & 0,039 & 0,133 \\
\hline 02 & 6 & 0,017 & 0,009 & 0,043 \\
\hline $74 B$ & 6 & 0,017 & 0,035 & 0,079 \\
\hline 17 & 4 & 0,012 & 0,012 & 0,008 \\
\hline 27 & 4 & 0,012 & 0,033 & 0,018 \\
\hline
\end{tabular}

Entsprechend der Tabelle 55 kommt der Knoten 72 am häufigsten in dieser Kategorie vor und weist dabei den größten Abstand zu dem nächstplatzierten Knoten auf. Der Inputanteil dieses Knotens ist vergleichsweise gering, nichtsdestotrotz hat der Knoten 72 einen hohen Outputahtedel Dero an fünfier 3 Stele 6 platzierte 
Knoten 45A hält den höchsten Inputanteil dieser Auswertung, während der an achter Stelle gereihte Knoten 21 den höchsten Outputanteil vorweisen kann. Dieser Knoten ist der einzige Knoten, der sowohl bei dem Inputanteil als auch bei dem Outputanteil über dem 3. Quartil von 0,069 bzw. 0,095 in dieser Kantenliste liegt.

Tabelle 56 und Tabelle 57 präsentieren die gleichen statistischen Maßzahlen wie Tabelle 55 für Lückenfolgen der Weglänge 2 mit nur ausgangsseitig bzw. zielseitig neuen (zusätzlichen internen) Kanten.

Tabelle 56: Nur ausgangsseitig neue Kanten

\section{Häufigkeit rel. Häufigkeit Inputanteil Outputanteil}

\begin{tabular}{|l|l|l|l|l|}
\hline 22 & 21 & 0,266 & 0,292 & 0,199 \\
28 & 19 & 0,241 & 0,073 & 0,380 \\
\cline { 2 - 5 } 27 & 11 & 0,139 & 0,066 & 0,187 \\
\hline 20 & 9 & 0,114 & 0,077 & 0,055 \\
\hline 02 & 6 & 0,076 & 0,017 & 0,068 \\
\hline 55 & 5 & 0,063 & 0,201 & 0,059 \\
21 & 4 & 0,051 & 0,143 & 0,003 \\
29 & 4 & 0,051 & 0,131 & 0,048 \\
\hline
\end{tabular}

Tabelle 57: Nur zielseitig neue Kanten

\begin{tabular}{|r|c|c|c|c|} 
& \multicolumn{2}{c}{ Häufigkeit } & $\begin{array}{c}\text { rel. Häufig- } \\
\text { keit }\end{array}$ & \multicolumn{2}{c|}{ Inputanteil } & Outputanteil \\
\hline $45 \mathrm{~B}$ & 15 & 0,185 & 0,151 & 0,102 \\
\cline { 3 - 5 } & 13 & 0,160 & 0,321 & 0,060 \\
21 & 12 & 0,148 & 0,067 & 0,203 \\
\cline { 2 - 5 } 72 & 12 & 0,148 & 0,007 & 0,139 \\
\hline $74 \mathrm{C}$ & 9 & 0,111 & 0,107 & 0,120 \\
\hline 28 & 5 & 0,062 & 0,108 & 0,043 \\
\hline $45 \mathrm{~A}$ & 5 & 0,062 & 0,109 & 0,051 \\
24 & 4 & 0,049 & 0,019 & 0,032 \\
\hline 20 & 3 & 0,037 & 0,079 & 0,166 \\
\hline 02 & 1 & 0,012 & 0,003 & 0,053 \\
17 & 1 & 0,012 & 0,003 & 0,010 \\
27 & 1 & 0,012 & 0,025 & 0,022 \\
\hline
\end{tabular}

In Tabelle 56 treten die Knoten 22 und 28 weit häufiger auf als die restlichen Knoten, wobei der Knoten 22 auch den höchsterMmputanteißeufiweist/and auch 
bezüglich dem Outputanteil an zweiter Stelle rangiert und diesbezüglich nur von Knoten 28 übertroffen wird. Das 3. Quartil für den Input- bzw. Outputanteil in Tabelle 56 beträgt 0,158 bzw. 0,190. Diese Werte werden nur von Knoten 22 überschritten. Die Spitzenwerte in Tabelle 57 werden von den Knoten 45B, 29, 21 und 72 erreicht. Den höchsten Inputanteil hat hierbei der Knoten 29, der höchste Outputanteil kann dem Knoten 21 zugeordnet werden. Die 3. Quartile für den Input- bzw. Outputanteil sind 0,108 bzw. 0,125 und werden von den Knoten 45B und 74C nur knapp verfehlt.

Zusammenfassend zeigen die auf interne Knoten bezogenen Kantenlisten für die Weglänge 2, inwieweit und welche Verbindungsknoten (Güter) für den Ausbau der Transaktionen zwischen bestehenden Teilnehmern bei dieser Weglänge in Frage kommen. Zur Veranschaulichung der mit diesen Verbindungsknoten (Gütern) in Beziehung setzbaren Ausgangs- und Zielknoten können Tabelle 58, Tabelle 59, Tabelle 60 und Tabelle 61 eingesetzt werden. Tabelle 58 zeigt die Anzahl der möglichen Verbindungen von den Ausgangs- (Zeilen) zu den Verbindungsknoten (Spalten) gemäß der Auswahl von Lückenfolgen mit beidseitig neuen Kanten aus der Kantenliste mit Weglänge 2. Die Verbindungsknoten 02 , 45B und 74B sind in dieser Tabelle jene Verbindungsknoten, welche die meisten Ausgangsknoten betreffen, wie die Häufigkeitszeile (\#) zeigt. Der Verbindungsknoten 72 ist jener Knoten, der die meisten weiterführenden Verbindungen in den 3-gliedrigen Transaktionswegen bei mehreren Ausgangsknoten aufweist. Die Summenspalte Tabelle 58 zeigt, inwieweit Ausgangsknoten prinzipiell Potenzial für weitere Transaktionsbeziehungen haben. Tabelle 59 beschreibt die Anzahl an möglichen Beziehungen von den Verbindungs- zu den Zielknoten. In dieser Tabelle tritt abermals der Verbindungsknoten 72 als jener Knoten hervor, welcher die meisten Zielknoten bedienen kann und wird dicht gefolgt von Knoten 74C. Tabelle 60 untersucht mögliche Transaktionsverläufe zwischen den Ausgangsund Verbindungsknoten innerhalb der Kategorie ,ausgangsseitig neu'. Der Verbindungsknoten 02 kann die meisten Ausgangsknoten in dieser Kategorie erreichen, während der Ausgangsknoten 02 am geringsten von nur ausgangsseitig 
Demonstrationsbeispiel

neuen Beziehungen profitieren kann. Der Ausgangsknoten 72 besitzt das vergleichsweise höchste Potenzial.

Tabelle 58: Ausgangs- zu Verbindungsknoten für beidseitig neue Kanten

\begin{tabular}{|c|c|c|c|c|c|c|c|c|c|c|c|c|c|c|c|c|}
\hline & ช & ₹ & ్ & $\bar{\sim}$ & $\mathbb{N}$ & N & $\hat{N}$ & $\stackrel{\sim}{\sim}$ & ฉి & 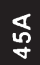 & $\stackrel{\mathscr{8}}{\mathscr{g}}$ & 另 & $\mathfrak{N}$ & $\frac{\mathscr{Q}}{N}$ & $\underset{N}{U}$ & $w$ \\
\hline 02 & 0 & 0 & 0 & 7 & 0 & 0 & 0 & 0 & 0 & 0 & 0 & 0 & 0 & 0 & 0 & 7 \\
\hline 17 & 0 & 0 & 0 & 0 & 0 & 0 & 0 & 0 & 0 & 0 & 5 & 0 & 0 & 0 & 0 & 5 \\
\hline 20 & 0 & 0 & 0 & 0 & 0 & 0 & 1 & 0 & 0 & 5 & 5 & 0 & 0 & 0 & 0 & 11 \\
\hline 21 & 0 & 0 & 3 & 0 & 7 & 4 & 0 & 0 & 0 & 0 & 5 & 0 & 12 & 1 & 0 & 32 \\
\hline 22 & 1 & 1 & 0 & 0 & 0 & 0 & 0 & 0 & 6 & 6 & 0 & 7 & 0 & 1 & 0 & 22 \\
\hline 24 & 1 & 0 & 0 & 6 & 7 & 0 & 0 & 5 & 0 & 0 & 5 & 0 & 0 & 0 & 0 & 24 \\
\hline 27 & 0 & 0 & 0 & 0 & 0 & 0 & 0 & 0 & 0 & 6 & 0 & 0 & 0 & 0 & 0 & 6 \\
\hline 28 & 1 & 0 & 3 & 0 & 0 & 0 & 0 & 0 & 0 & 0 & 0 & 6 & 0 & 1 & 0 & 11 \\
\hline 29 & 1 & 1 & 3 & 7 & 6 & 5 & 1 & 0 & 0 & 0 & 0 & 0 & 0 & 0 & 0 & 24 \\
\hline $45 \mathrm{~A}$ & 1 & 0 & 2 & 0 & 0 & 0 & 0 & 5 & 0 & 0 & 4 & 7 & 13 & 0 & 0 & 32 \\
\hline $45 B$ & 1 & 0 & 0 & 0 & 0 & 0 & 0 & 0 & 0 & 5 & 0 & 6 & 13 & 0 & 10 & 35 \\
\hline 55 & 0 & 0 & 0 & 0 & 0 & 0 & 0 & 4 & 7 & 0 & 4 & 0 & 12 & 1 & 10 & 38 \\
\hline 72 & 0 & 1 & 3 & 6 & 7 & 5 & 1 & 5 & 7 & 0 & 0 & 6 & 0 & 1 & 9 & 51 \\
\hline $74 B$ & 0 & 0 & 0 & 0 & 0 & 0 & 0 & 0 & 0 & 6 & 0 & 0 & 0 & 0 & 0 & 6 \\
\hline $74 \mathrm{C}$ & 0 & 1 & 0 & 0 & 7 & 5 & 1 & 5 & 7 & 0 & 0 & 0 & 12 & 1 & 0 & 39 \\
\hline \# & 6 & 4 & 5 & 4 & 5 & 4 & 4 & 5 & 4 & 5 & 6 & 5 & 5 & 6 & 3 & 71 \\
\hline
\end{tabular}

Tabelle 59: Verbindungs- zu Zielknoten für beidseitig neue Kanten

\begin{tabular}{|c|c|c|c|c|c|c|c|c|c|c|c|c|c|c|c|c|c|c|}
\hline & ช & 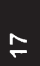 & ని & $\bar{N}$ & $\mathbb{N}$ & a & $\hat{N}$ & $\stackrel{\infty}{\sim}$ & : & \& & $\underset{\mathscr{O}}{\mathscr{8}}$ & శ్రీ & 号 & 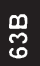 & $\mathfrak{N}$ & $\underset{\sim}{\mathbb{N}}$ & U & \# \\
\hline 02 & 0 & 0 & 0 & 6 & 0 & 0 & 0 & 0 & 0 & 0 & 0 & 0 & 0 & 0 & 0 & 0 & 0 & 1 \\
\hline 17 & 0 & 0 & 0 & 0 & 0 & 0 & 0 & 0 & 0 & 0 & 4 & 0 & 0 & 0 & 0 & 0 & 0 & 1 \\
\hline 20 & 0 & 0 & 0 & 0 & 0 & 0 & 5 & 0 & 0 & 4 & 5 & 0 & 0 & 0 & 0 & 0 & 0 & 3 \\
\hline 21 & 0 & 0 & 4 & 0 & 4 & 3 & 0 & 0 & 0 & 0 & 4 & 4 & 0 & 0 & 3 & 4 & 0 & 7 \\
\hline 22 & 5 & 5 & 0 & 0 & 0 & 0 & 0 & 0 & 4 & 5 & 0 & 0 & 5 & 5 & 0 & 5 & 0 & 7 \\
\hline 24 & 4 & 0 & 0 & 3 & 4 & 0 & 0 & 4 & 0 & 0 & 4 & 0 & 0 & 0 & 0 & 0 & 0 & 5 \\
\hline 27 & 0 & 0 & 0 & 0 & 0 & 0 & 0 & 0 & 0 & 4 & 0 & 0 & 0 & 0 & 0 & 0 & 0 & 1 \\
\hline 28 & 5 & 0 & 5 & 0 & 0 & 0 & 0 & 0 & 0 & 0 & 0 & 5 & 4 & 0 & 0 & 5 & 0 & 5 \\
\hline 29 & 4 & 4 & 4 & 4 & 3 & 4 & 4 & 0 & 0 & 0 & 0 & 0 & 0 & 0 & 0 & 0 & 0 & 7 \\
\hline $45 A$ & 5 & 0 & 4 & 0 & 0 & 0 & 0 & 5 & 0 & 0 & 4 & 0 & 5 & 0 & 5 & 0 & 0 & 6 \\
\hline $45 B$ & 6 & 0 & 0 & 0 & 0 & 0 & 0 & 0 & 0 & 5 & 0 & 0 & 5 & 0 & 6 & 0 & 6 & 5 \\
\hline 55 & 0 & 0 & 0 & 0 & 0 & 0 & 0 & 4 & 5 & 0 & 4 & 5 & 0 & 0 & 4 & 5 & 5 & 7 \\
\hline $7:$ & 0 & 5 & 5 & 4 & 5 & 5 & 5 & 5 & 5 & 0 & 0 & 5 & 4 & 5 & 0 & 5 & 4 & 13 \\
\hline $74 \mathrm{E}$ & 0 & 0 & 0 & 0 & 0 & 0 & 0 & 0 & 0 & 6 & 0 & 0 & 0 & 0 & 0 & 0 & 0 & 1 \\
\hline $74 \mathrm{C}$ & 0 & 3 & 0 & 0 & 3 & 3 & 3 & 3 & 3 & 0 & 0 & 3 & 0 & 3 & 2 & 3 & 0 & 10 \\
\hline$\Sigma$ & 29 & 17 & 22 & 17 & 19 & 15 & \begin{tabular}{|l|}
17 \\
\end{tabular} & 21 & 17 & 24 & 25 & 22 & 23 & 13 & 20 & 27 & 15 & 79 \\
\hline
\end{tabular}


Tabelle 60: Ausgangs- zu Verbindungsknoten für ausgangsseitig neue Kanten

\begin{tabular}{|c|c|c|c|c|c|c|c|c|c|}
\hline & $\approx$ & 오 & $\bar{N}$ & $\approx$ & $\hat{N}$ & $\stackrel{\infty}{\sim}$ & ㄱ & 号 & $\omega$ \\
\hline 02 & 0 & 0 & 1 & 0 & 0 & 0 & 0 & 0 & 1 \\
\hline 20 & 0 & 0 & 0 & 0 & 3 & 0 & 0 & 0 & 3 \\
\hline 21 & 0 & 1 & 0 & 4 & 0 & 0 & 0 & 0 & 5 \\
\hline 22 & 1 & 0 & 0 & 0 & 0 & 0 & 1 & 1 & 3 \\
\hline 24 & 1 & 0 & 1 & 4 & 0 & 4 & 0 & 0 & 10 \\
\hline 28 & 1 & 2 & 0 & 0 & 0 & 0 & 0 & 1 & 4 \\
\hline 29 & 1 & 2 & 1 & 5 & 2 & 0 & 0 & 0 & 11 \\
\hline $45 \mathrm{~A}$ & 1 & 2 & 0 & 0 & 0 & 3 & 0 & 1 & 7 \\
\hline $45 B$ & 1 & 0 & 0 & 0 & 0 & 0 & 0 & 1 & 2 \\
\hline 55 & 0 & 0 & 0 & 0 & 0 & 4 & 1 & 0 & 5 \\
\hline 72 & 0 & 2 & 1 & 4 & 3 & 4 & 1 & 1 & 16 \\
\hline $74 \mathrm{C}$ & 0 & 0 & 0 & 4 & 3 & 4 & 1 & 0 & 12 \\
\hline$\#$ & 6 & 5 & 4 & 5 & 4 & 5 & 4 & 5 & 38 \\
\hline
\end{tabular}

Tabelle 61 stellt die möglichen Transaktionsarten zwischen den Verbindungsund Zielknoten in der Kategorie ,zielseitig neu' dar. Der Verbindungsknoten 72 erweist sich auch in dieser Auswertung als jener Knoten, der die meisten Zielknoten erreichen kann. Gleichzeitig gehört der Zielknoten 72 ebenso wie der Zielknoten 02 zu den Knoten mit dem höchsten Potenzial unter den 3-gliedrigen Transaktionswegen mit nur zielseitig neuen (zusätzlich möglichen) Kanten.

Tabelle 61: Verbindungs- zu Zielknoten für zielseitig neue Kanten

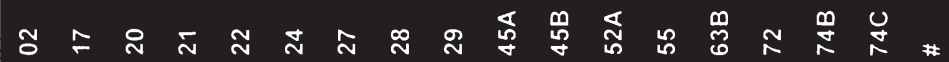

\begin{tabular}{|r|c|c|c|c|c|c|c|c|c|c|c|c|c|c|c|c|c|c|}
02 & 0 & 0 & 0 & 1 & 0 & 0 & 0 & 0 & 0 & 0 & 0 & 0 & 0 & 0 & 0 & 0 & 0 & 1 \\
17 & 0 & 0 & 0 & 0 & 0 & 0 & 0 & 0 & 0 & 0 & 1 & 0 & 0 & 0 & 0 & 0 & 0 & 1 \\
20 & 0 & 0 & 0 & 0 & 0 & 0 & 1 & 0 & 0 & 1 & 1 & 0 & 0 & 0 & 0 & 0 & 0 & 3 \\
21 & 0 & 0 & 1 & 0 & 1 & 2 & 0 & 0 & 0 & 0 & 2 & 2 & 0 & 0 & 2 & 2 & 0 & 7 \\
\hline 24 & 1 & 0 & 0 & 1 & 0 & 0 & 0 & 1 & 0 & 0 & 1 & 0 & 0 & 0 & 0 & 0 & 0 & 4 \\
27 & 0 & 0 & 0 & 0 & 0 & 0 & 0 & 0 & 0 & 1 & 0 & 0 & 0 & 0 & 0 & 0 & 0 & 1 \\
\hline 28 & 1 & 0 & 1 & 0 & 0 & 0 & 0 & 0 & 0 & 0 & 0 & 1 & 1 & 0 & 0 & 1 & 0 & 5 \\
29 & 2 & 2 & 2 & 2 & 2 & 2 & 1 & 0 & 0 & 0 & 0 & 0 & 0 & 0 & 0 & 0 & 0 & 7 \\
\hline $45 A$ & 1 & 0 & 1 & 0 & 0 & 0 & 0 & 0 & 0 & 0 & 1 & 0 & 1 & 0 & 1 & 0 & 0 & 5 \\
$45 B$ & 3 & 0 & 0 & 0 & 0 & 0 & 0 & 0 & 0 & 3 & 0 & 0 & 3 & 0 & 3 & 0 & 3 & 5 \\
72 & 0 & 1 & 1 & 1 & 0 & 1 & 1 & 1 & 1 & 0 & 0 & 1 & 1 & 1 & 0 & 1 & 1 & 12 \\
\hline $74 C$ & 0 & 1 & 0 & 0 & 0 & 1 & 1 & 1 & 1 & 0 & 0 & 1 & 0 & 1 & 1 & 1 & 0 & 9 \\
$\Sigma$ & 8 & 4 & 6 & 5 & 3 & 6 & 4 & 3 & 2 & 5 & 6 & 5 & 6 & 2 & 7 & 5 & 4 & 60 \\
\hline
\end{tabular}


Zwecks Analyse der Lückenfolgen über externe Verbindungsknoten wird die Teilmenge Edgeframe $e_{\text {ext }}^{\text {onho }}$ (Formel (184)) ohne Zirkulärbezüge Edgeframe circ $_{\text {one }}^{\text {E }}$ (Formel (186)) gebildet und mit den gleichen statistischen Maßzahlen untersucht, wie sie bereits für die Auswertung interner Lückenfolgen eingesetzt wurden.

Tabelle 62: Externe Verbindungsknoten

\begin{tabular}{|c|c|c|c|c|}
\hline & Häufigkeit & rel. Häufigkeit & Inputanteil & Outputanteil \\
\hline 65 & 144 & 0,100 & 0,086 & 0,085 \\
\hline 64 & 128 & 0,089 & 0,047 & 0,045 \\
\hline $70 A$ & 128 & 0,089 & 0,334 & 0,153 \\
\hline 74D & 112 & 0,078 & 0,020 & 0,072 \\
\hline $40 A$ & 98 & 0,068 & 0,010 & 0,040 \\
\hline 66 & 86 & 0,060 & 0,050 & 0,022 \\
\hline $74 A$ & 80 & 0,056 & 0,021 & 0,063 \\
\hline 23 & 79 & 0,055 & 0,007 & 0,036 \\
\hline 71 & 75 & 0,052 & 0,013 & 0,050 \\
\hline 25 & 68 & 0,047 & 0,021 & 0,023 \\
\hline $60 \mathrm{C}$ & 52 & 0,036 & 0,020 & 0,039 \\
\hline 51B & 44 & 0,031 & 0,008 & 0,028 \\
\hline $40 B$ & 40 & 0,028 & 0,003 & 0,017 \\
\hline 26 & 34 & 0,024 & 0,014 & 0,085 \\
\hline 31 & 30 & 0,021 & 0,047 & 0,017 \\
\hline $50 \mathrm{~B}$ & 30 & 0,021 & 0,017 & 0,009 \\
\hline 90 & 29 & 0,020 & 0,013 & 0,008 \\
\hline $92 \mathrm{~B}$ & 18 & 0,013 & 0,012 & 0,015 \\
\hline 36 & 17 & 0,012 & 0,052 & 0,001 \\
\hline $60 A$ & 16 & 0,011 & 0,015 & 0,005 \\
\hline $92 \mathrm{~A}$ & 16 & 0,011 & 0,015 & 0,007 \\
\hline 93 & 16 & 0,011 & 0,007 & 0,003 \\
\hline 01 & 13 & 0,009 & 0,022 & 0,010 \\
\hline 32 & 12 & 0,008 & 0,014 & 0,002 \\
\hline 15 & 11 & 0,008 & 0,042 & 0,080 \\
\hline 62 & 10 & 0,007 & 0,021 & 0,022 \\
\hline 14 & 8 & 0,006 & 0,005 & 0,035 \\
\hline 33 & 8 & 0,006 & 0,012 & 0,001 \\
\hline $52 B$ & 7 & 0,005 & 0,002 & 0,002 \\
\hline $63 A$ & 6 & 0,004 & 0,016 & 0,001 \\
\hline 91 & 6 & 0,004 & 0,024 & 0,000 \\
\hline 37 & 5 & 0,003 & 0,000 & 0,008 \\
\hline $60 \mathrm{~B}$ & 5 & 0,003 & 0,009 & 0,013 \\
\hline $63 C$ & 5 & 0,003 & 0,002 & 0,001 \\
\hline
\end{tabular}


Tabelle 62 beschreibt dementsprechend die Häufigkeiten und Wertanteile von externen Verbindungsknoten in 3-gliedrigen Transaktionsketten mit internen Ausgangs- und Zielknoten ohne Zirkulärbezug. Wie der Tabelle entnommen werden kann, können über den externen Verbindungsknoten 65 die meisten zusätzlichen Transaktionswege hergestellt werden, wobei die Bedeutung des Knoten als Abnehmer und Anbieter gemessen an dem Input- und Outputanteil beinahe identisch ist und jeweils den zweithöchsten Wert dieser Kantenliste darstellt. Der ex aequo mit dem Knoten 64 zweitgereihte Verbindungsknoten 70A liegt sowohl bezüglich dem Input- als auch dem Outputanteil mit großem Abstand an erster Stelle. Die 3. Quartile für die Wertanteile betragen 0,022 (Inputanteil) und 0,040 (Outputanteil).

Die Auswirkungen der Hinzunahme von externen Verbindungsknoten zur Erhöhung der Verflechtungen zwischen den bestehenden internen Knoten können mit Hilfe der Tabelle 63 und der Tabelle 64 betreffend der Ausgangsknoten sowie der Tabelle 65 in Bezug auf die Zielknoten analysiert werden.

Tabelle 63: Ausgangs- zu externen Verbindungsknoten I

\begin{tabular}{|c|c|c|c|c|c|c|c|c|c|c|c|c|c|c|c|c|c|}
\hline & 5 & & $\stackrel{2}{2}$ & $\approx$ & $\stackrel{\sim}{N}$ & $\ddot{N}$ & $\bar{m}$ & ల్ల & $\ddot{m}$ & $\ddot{m}$ & ले & ঠ্ণ & 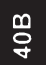 & $\stackrel{\infty}{\circ}$ & $\frac{\infty}{\omega n}$ & $\underset{\sim}{\mathbb{N}}$ & \\
\hline 02 & 1 & 0 & 0 & 0 & 0 & 0 & 0 & 0 & 0 & 0 & 0 & 0 & 0 & 0 & 0 & 0 & \\
\hline 17 & 0 & 0 & 0 & 8 & 9 & 0 & 0 & 0 & 0 & 0 & 0 & 0 & 0 & 0 & 0 & 1 & \\
\hline 20 & 2 & 0 & 0 & 0 & 0 & 0 & 0 & 0 & 0 & 4 & 0 & 0 & 0 & 0 & 0 & 0 & \\
\hline & 0 & 0 & 2 & 0 & 9 & 7 & 4 & 2 & 1 & 0 & 0 & 0 & 0 & 5 & 0 & 0 & \\
\hline & 0 & 0 & 2 & 0 & 0 & 0 & 4 & 0 & 1 & 3 & 0 & 12 & 5 & 0 & 8 & 0 & \\
\hline & 2 & 0 & 0 & 0 & 8 & 0 & 4 & 2 & 1 & 0 & 0 & 0 & 0 & 5 & 0 & 0 & \\
\hline & 0 & 0 & 0 & 0 & 9 & 6 & 4 & 0 & 1 & 0 & 0 & 0 & 0 & 5 & 0 & 1 & \\
\hline 28 & 2 & 2 & 0 & 13 & 8 & 0 & 4 & 2 & & 4 & 1 & 12 & 0 & 5 & 0 & 0 & \\
\hline 29 & 2 & 2 & 2 & 14 & 8 & 7 & 3 & 0 & & 0 & 1 & 12 & 6 & 5 & 0 & 1 & \\
\hline $45 A$ & 2 & 0 & 0 & 13 & 0 & 0 & 0 & 0 & 0 & 0 & 1 & 13 & 6 & 0 & 0 & 0 & \\
\hline $45 B$ & 2 & 0 & 0 & 0 & 0 & 0 & 0 & 2 & 0 & 0 & 0 & 0 & 6 & 0 & 0 & 0 & \\
\hline 55 & 0 & 0 & 1 & 0 & 0 & 0 & 0 & 2 & 0 & 0 & 0 & 12 & 5 & 0 & 9 & 1 & 3 \\
\hline $63 \mathrm{~B}$ & 0 & 0 & 0 & 0 & 0 & 0 & 0 & 0 & 0 & 0 & 0 & 0 & 0 & 0 & 9 & 0 & 2 \\
\hline 72 & 0 & 0 & 2 & 13 & 8 & 7 & 3 & 0 & 1 & 3 & 1 & 12 & 6 & 5 & 9 & 1 & 3 \\
\hline $74 \mathrm{~B}$ & 0 & 2 & 0 & 13 & 0 & 0 & 0 & 0 & 0 & 0 & 0 & 13 & 0 & 0 & 0 & 1 & 0 \\
\hline $74 \mathrm{C}$ & 0 & 2 & 2 & 13 & 9 & 7 & 4 & 2 & 1 & 3 & 1 & 12 & 6 & 0 & 9 & 1 & 0 \\
\hline$\#$ & 7 & 4 & 6 & 6 & 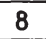 & 5 & 8 & 6 & 8 & 5 & 5 & 8 & 7 & 6 & 5 & 7 & \\
\hline
\end{tabular}


Tabelle 64: Ausgangs- zu externen Verbindungsknoten II

\begin{tabular}{|c|c|c|c|c|c|c|c|c|c|c|c|c|c|c|c|c|c|c|}
\hline & 옹 & $\begin{array}{l}ن \\
\check{c}\end{array}$ & ชิ & ભొ & U్ల & $\mathbb{E}$ & ๑ & 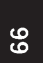 & $\stackrel{\sigma}{R}$ & 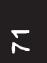 & $\underset{N}{N}$ & 沜 & 요 & $\bar{\sigma}$ & ๙ & 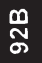 & ฮี & $\omega$ \\
\hline 02 & 0 & 0 & 0 & 0 & 0 & 0 & 0 & 0 & 0 & 0 & 0 & 0 & 0 & 0 & 0 & 0 & 0 & 1 \\
\hline 17 & 0 & 0 & 0 & 0 & 0 & 0 & 0 & 0 & 0 & 0 & 0 & 0 & 0 & 0 & 0 & 0 & 0 & 10 \\
\hline 20 & 0 & 0 & 0 & 0 & 0 & 0 & 0 & 0 & 16 & 0 & 0 & 0 & 0 & 0 & 0 & 0 & 2 & 24 \\
\hline 21 & 0 & 0 & 0 & 0 & 0 & 0 & 16 & 13 & 0 & 15 & 0 & 16 & 0 & 0 & 0 & 3 & 0 & 93 \\
\hline 22 & 1 & 8 & 2 & 1 & 1 & 16 & 16 & 13 & 16 & 15 & 16 & 16 & 5 & 1 & 2 & 2 & 2 & 168 \\
\hline 24 & 0 & 0 & 0 & 0 & 0 & 0 & 0 & 0 & 16 & 0 & 0 & 16 & 0 & 0 & 0 & 2 & 2 & 58 \\
\hline 27 & 0 & 0 & 0 & 0 & 0 & 0 & 0 & 0 & 0 & 0 & 0 & 0 & 0 & 0 & 0 & 0 & 0 & 28 \\
\hline 28 & 1 & 0 & 0 & 0 & 1 & 16 & 16 & 0 & 16 & 0 & 0 & 16 & 5 & 1 & 3 & 0 & 2 & 131 \\
\hline 29 & 1 & 0 & 0 & 1 & 0 & 0 & 0 & 0 & 16 & 0 & 0 & 0 & 5 & 0 & 0 & 0 & 2 & 89 \\
\hline $45 A$ & 1 & 8 & 0 & 1 & 0 & 16 & 16 & 0 & 16 & 0 & 0 & 0 & 5 & 1 & 3 & 0 & 0 & 105 \\
\hline $45 B$ & 0 & 9 & 0 & 1 & 0 & 16 & 16 & 0 & 16 & 0 & 16 & 0 & 5 & 1 & 3 & 3 & 2 & 101 \\
\hline 55 & 0 & 9 & 2 & 0 & 0 & 16 & 16 & 12 & 0 & 15 & 16 & 16 & 0 & 1 & 0 & 3 & 0 & 139 \\
\hline $63 \mathrm{~B}$ & 0 & 0 & 1 & 0 & 0 & 0 & 0 & 12 & 0 & 0 & 0 & 0 & 0 & 0 & 0 & 0 & 0 & 24 \\
\hline 72 & 1 & 9 & 2 & 1 & 1 & 16 & 16 & 12 & 0 & 15 & 16 & 16 & 0 & 1 & 3 & 3 & 2 & 188 \\
\hline $74 \mathrm{~B}$ & 0 & 0 & 2 & 0 & 1 & 16 & 16 & 12 & 16 & 0 & 0 & 0 & 0 & 0 & 0 & 0 & 0 & 92 \\
\hline $74 C$ & 0 & 9 & 1 & 1 & 1 & 16 & 16 & 12 & 0 & 15 & 16 & 16 & 4 & 0 & 2 & 2 & 2 & 185 \\
\hline$\#$ & 5 & 6 & 6 & 6 & 5 & 8 & 9 & 7 & 8 & 5 & 5 & 7 & 6 & 6 & 6 & 7 & 8 & 217 \\
\hline
\end{tabular}

Die Verteilung der Anzahl der potenziellen Nutznießer der externen Verbindungsknoten in Hinblick auf die Ausgangsknoten in Tabelle 63 und Tabelle 64 zeigt abermals die hohe Relevanz der externen Knoten 65, 70A und auch 64. Die Ausgangsknoten 72 und $74 \mathrm{C}$ weisen gemäß diesen beiden Tabellen das höchste Verbindungspotenzial auf. Aus der Perspektive der Zielknoten treten gemäß Tabelle 65 neben den externen Verbindungsknoten 65, 70A und 64 auch die Knoten 74A und 74D hervor, da diese ebenfalls die maximale Anzahl an erreichbaren Zielknoten bedienen können. Die internen Zielknoten 17 und 52A können durch die Erweiterung der lokalen Struktur um externe Verbindungsknoten insgesamt am stärksten profitieren. 
Tabelle 65: Extemer Verbindungs- zu Zielknoten

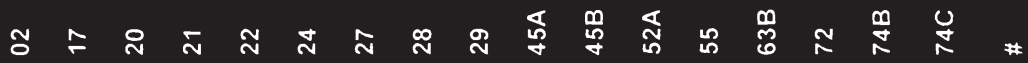

\begin{tabular}{c|c|c|c|c|c|c|c|c|c|c|c|c|c|c|c|c|c|c|}
01 & 6 & 0 & 0 & 0 & 0 & 0 & 0 & 0 & 0 & 0 & 0 & 0 & 7 & 0 & 0 & 0 & 0 & 2 \\
14 & 0 & 0 & 0 & 4 & 0 & 0 & 0 & 0 & 0 & 4 & 0 & 0 & 0 & 0 & 0 & 0 & 0 & 2 \\
15 & 0 & 0 & 0 & 0 & 0 & 6 & 0 & 0 & 0 & 0 & 0 & 0 & 5 & 0 & 0 & 0 & 0 & 2 \\
23 & 6 & 6 & 6 & 0 & 0 & 6 & 6 & 5 & 0 & 5 & 6 & 6 & 6 & 6 & 5 & 5 & 5 & 14 \\
\hline
\end{tabular}

\begin{tabular}{ll|l|l|l|l|l|l|l|l|l|l|l|l|l|l|l|l|l|}
25 & 0 & 0 & 0 & 0 & 8 & 7 & 0 & 7 & 7 & 8 & 8 & 8 & 0 & 0 & 7 & 8 & 0 & 9
\end{tabular}

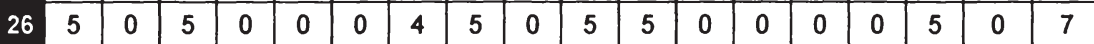

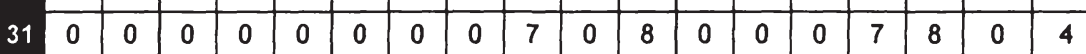

32

33

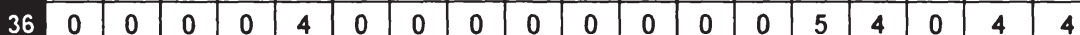

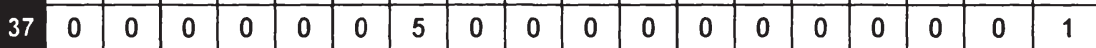

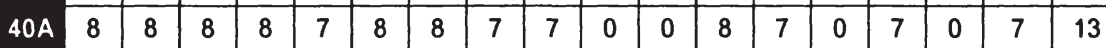

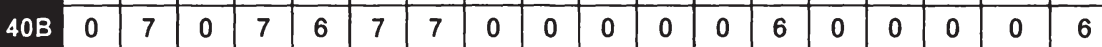

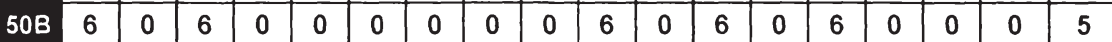

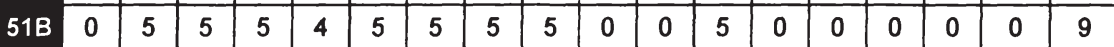

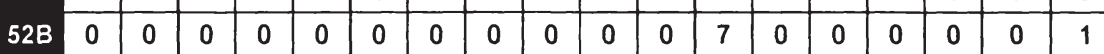

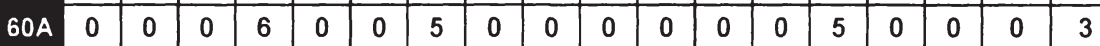

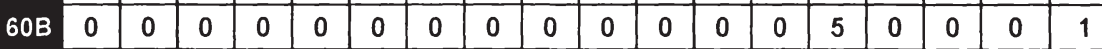

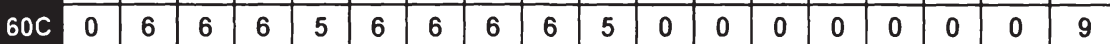

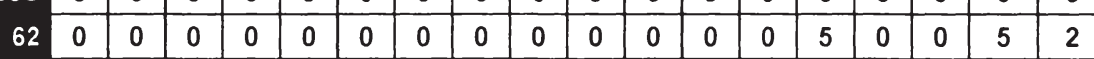

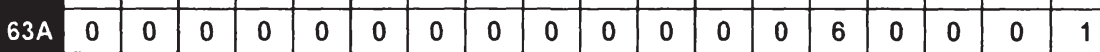

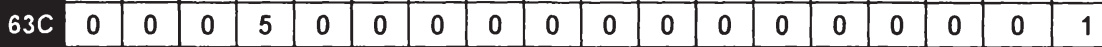

\begin{tabular}{|l|l|l|l|l|l|l|l|l|l|l|l|l|l|l|l|l|l|l|}
64 & 8 & 8 & 8 & 8 & 7 & 8 & 8 & 7 & 8 & 7 & 7 & 8 & 7 & 8 & 7 & 7 & 7 & 17 \\
\hline
\end{tabular}

\begin{tabular}{ll|l|l|l|l|l|l|l|l|l|l|l|l|l|l|l|l|l|}
65 & 9 & 9 & 9 & 8 & 8 & 9 & 9 & 8 & 9 & 8 & 8 & 9 & 8 & 9 & 8 & 8 & 8 & 17 \\
\hline
\end{tabular}

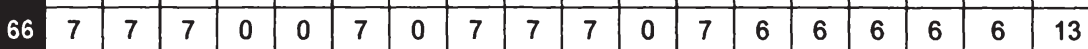

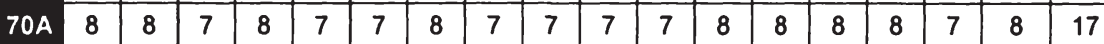

\begin{tabular}{ll|l|l|l|l|l|l|l|l|l|l|l|l|l|l|l|l|l|}
71 & 5 & 5 & 5 & 4 & 4 & 0 & 5 & 5 & 5 & 5 & 5 & 5 & 4 & 5 & 4 & 5 & 4 & 16
\end{tabular}

\begin{tabular}{ll|l|l|l|l|l|l|l|l|l|l|l|l|l|l|l|l|l|}
$74 A$ & 5 & 5 & 5 & 5 & 4 & 5 & 5 & 5 & 5 & 5 & 4 & 5 & 4 & 5 & 4 & 5 & 4 & 17 \\
\hline
\end{tabular}

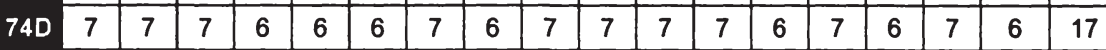

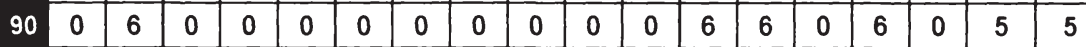

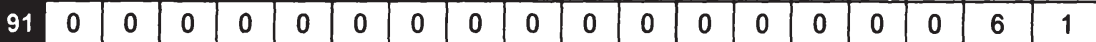

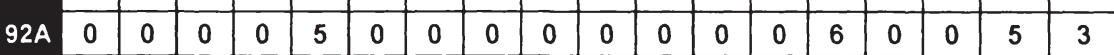

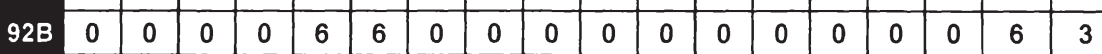

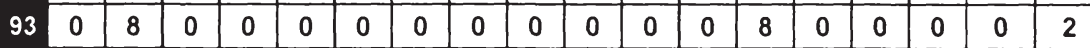

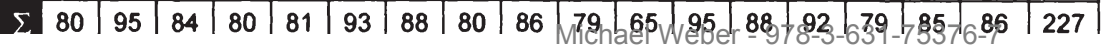


Der Vergleich der lokalen mit der globalen Struktur unter Modifikation der bereits in der Strukturanalysephase eingesetzten graphentheoretischen Kennzahlen hin zu einem komparativen Dichtemaß sowie den beiden komparativen Wertedichtemaßen kann, wie in Kapitel 7.10 beschrieben, die Strukturvergleichsphase nach der Identifikation und deskriptiven Analyse der Lückentypen sowie der Bestimmung maßgeblicher interner sowie externer Kanten bzw. Knoten abrunden und eine Brücke zu der nun anschließenden Lückenbewertungsphase bieten. Für die vorliegenden Datenquellen ist 0,186 die Kennzahl für das komparative Dichtemaß $o_{\text {comp }}, 4,474$ für das verbesserte Wertedichtemaß $o^{V A L I^{*}}, 4,314381 \mathrm{E}-05$ für das komparative Wertedichtemaß (das Gesamtgewichtemaß) für die lokale Knotenmenge $o_{\text {comp }}^{\text {VAL* }}$ und 8,838913E-05 für das komparative Wertedichtemaß bezogen auf die lokale Kantenmenge $o_{\text {compLE }}^{\text {VAl** }}$. Während die letztere Maßzahl zur Nivellierung der Kantengewichte der globalen Struktur eingesetzt werden kann, um aus der Menge der potenziellen Performanzlücken tatsächliche Performanzlücken zu bestimmen, ist die erstgenannte Kennzahl ein einfach berechenbarer Indikator für das Potenzial zur Erhöhung der Verflechtungen in der lokalen Struktur. Ein Wert von 0,186 zeigt dementsprechend relativ hohe Erweiterungsmöglichkeiten der vorliegenden lokalen Struktur an.

\subsection{Lückenbewertung}

Gemäß den in Kapitel 8 eingeführten Kennzahlen und Klassifikationsansätzen können die Knoten und Kanten bzw. ,Lücken' aufbauend auf den erfolgten Strukturvergleich umfassend bewertet werden.

\subsubsection{Identifikation und Bewertung von Performanzlücken}

Unter Einsatz des komparativen Wertedichtemaßes bezogen auf die lokale Kantenmenge $o_{\text {compLE }}^{\text {VAL** }}$ können zunächst tatsächliche Performanzlücken nach dem einfachen Bewertungsverfahren bestimmt und anschließend nach dem ermittelten Performanzlückenwert in Gruppen eingeteilt werden. Tabelle 66 zeigt die anhand des 1. und 3. Quartils des Performanzlügkegmerts 
bereiche eingeteilten 10 Performanzlücken der lokalen Struktur in der Matrix PerfGap ${ }^{\text {interval,3 }}$. Der niedrigsten (1) bzw. höchsten Klasse (3) werden jeweils 3 Performanzlücken zugewiesen, während der mittleren Kategorie 4 Performanzlücken zugeordnet werden. Bei letzteren handelt es sich um die Kanten $(28,27)$, $(28,45 A),(28,45 B)$ und $(55,63 B)$. Die Kanten $(02,20),(22,72)$ und $(28,29)$ zählen hingegen zu den schwächeren Performanzlücken, die Kanten $(20,21),(22,52 \mathrm{~A})$ und $(22,74 \mathrm{C})$ gehören zu den starken Performanzlücken. Der Vorgängerknoten 28 weist die höchste Anzahl an Performanzlücken (4) auf von denen 3 der mittleren Kategorie angehören. Der Vorgängerknoten 22 ist an drei Performanzlücken beteiligt, wovon zwei als starke Performanzlücken klassifiziert werden. In der Rolle als Nachfolger ist keiner der Knoten an mehr als einer Performanzlücke beteiligt.

Tabelle 66: PerfGap interval.3 $^{\text {. }}$

\begin{tabular}{|c|c|c|c|c|c|c|c|c|c|c|c|c|c|c|c|c|c|}
\hline & ชิ & $\approx$ & సి & $\bar{\sim}$ & $\approx$ & $\mathbb{N}$ & $\hat{N}$ & $\stackrel{\infty}{\sim}$ & 尺 & ङ & 臰 & శ్ర & 㖞 & 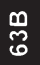 & $\mathfrak{N}$ & $\underset{\sim}{\stackrel{m}{N}}$ & $\frac{U}{N}$ \\
\hline 02 & 0 & 0 & 1 & 0 & 0 & 0 & 0 & 0 & 0 & 0 & 0 & 0 & 0 & 0 & 0 & 0 & 0 \\
\hline 17 & 0 & 0 & 0 & 0 & 0 & 0 & 0 & 0 & 0 & 0 & 0 & 0 & 0 & 0 & 0 & 0 & 0 \\
\hline 20 & 0 & 0 & 0 & 3 & 0 & 0 & 0 & 0 & 0 & 0 & 0 & 0 & 0 & 0 & 0 & 0 & 0 \\
\hline 21 & 0 & 0 & 0 & 0 & 0 & 0 & 0 & 0 & 0 & 0 & 0 & 0 & 0 & 0 & 0 & 0 & 0 \\
\hline 22 & 0 & 0 & 0 & 0 & 0 & 0 & 0 & 0 & 0 & 0 & 0 & 3 & 0 & 0 & 1 & 0 & 3 \\
\hline 24 & 0 & 0 & 0 & 0 & 0 & 0 & 0 & 0 & 0 & 0 & 0 & 0 & 0 & 0 & 0 & 0 & 0 \\
\hline 27 & 0 & 0 & 0 & 0 & 0 & 0 & 0 & 0 & 0 & 0 & 0 & 0 & 0 & 0 & 0 & 0 & 0 \\
\hline 28 & 0 & 0 & 0 & 0 & 0 & 0 & 2 & 0 & 1 & 2 & 2 & 0 & 0 & 0 & 0 & 0 & 0 \\
\hline 29 & 0 & 0 & 0 & 0 & 0 & 0 & 0 & 0 & 0 & 0 & 0 & 0 & 0 & 0 & 0 & 0 & 0 \\
\hline $45 A$ & 0 & 0 & 0 & 0 & 0 & 0 & 0 & 0 & 0 & 0 & 0 & 0 & 0 & 0 & 0 & 0 & 0 \\
\hline 45B & 0 & 0 & 0 & 0 & 0 & 0 & 0 & 0 & 0 & 0 & 0 & 0 & 0 & 0 & 0 & 0 & 0 \\
\hline $52 \mathrm{~A}$ & 0 & 0 & 0 & 0 & 0 & 0 & 0 & 0 & 0 & 0 & 0 & 0 & 0 & 0 & 0 & 0 & 0 \\
\hline 55 & 0 & 0 & 0 & 0 & 0 & 0 & 0 & 0 & 0 & 0 & 0 & 0 & 0 & 2 & 0 & 0 & 0 \\
\hline 3B & 0 & 0 & 0 & 0 & 0 & 0 & 0 & 0 & 0 & 0 & 0 & 0 & 0 & 0 & 0 & 0 & 0 \\
\hline 72 & 0 & 0 & 0 & 0 & 0 & 0 & 0 & 0 & 0 & 0 & 0 & 0 & 0 & 0 & 0 & 0 & 0 \\
\hline 4B & 0 & 0 & 0 & 0 & 0 & 0 & 0 & 0 & 0 & 0 & 0 & 0 & 0 & 0 & 0 & 0 & 0 \\
\hline $74 \mathrm{C}$ & 0 & 0 & 0 & 0 & 0 & 0 & 0 & 0 & 0 & 0 & 0 & 0 & 0 & 0 & 0 & 0 & 0 \\
\hline
\end{tabular}

Im Gegensatz zu der aus dem einfachen Bewertungsverfahren gewonnenen Matrix PerfGap ${ }^{\text {interval, } 3}$ aus Tabelle 66 zeigt hEabelles 67 gdie 3entsprechend dem 
komplexen Bewertungsverfahren berechnete Matrix PerfGap ${ }^{\text {PLinaryRATE }}$, die die indirekten Effekte bezogen auf die Nachfolger- und Vorgängerknoten bis zur Weglänge $n-1$ zusätzlich berücksichtigt. Vergleicht man beide Matrizen, so weist die Tabelle 67 zusätzlich die Kante $(27,29)$ als Performanzlücke auf, während die Kanten $(02,20),(28,29),(28,45 A),(28,45 B)$ und $(55,63 B)$ als Performanzlücken in Tabelle 67 wegfallen. Dementsprechend werden die Kanten $(20,21),(22,52 A),(22,72),(22,74 C)$ und $(28,27)$ von beiden Verfahren als Performanzlücken identifiziert. Auch das komplexe Bewertungsverfahren erkennt alle starken Performanzlücken (Kategorie 3). Der Knoten 28 ist in der Vorgängerrolle im komplexeren Verfahren nur noch mit einer Performanzlücke vertreten, der in der Vorgängerrolle hervorstechende Knoten 22 bleibt unverändert stark an Performanzlücken beteiligt.

Tabelle 67: PerfGap ${ }^{\text {PLbinanyRATE }}$

\section{ช}

\begin{tabular}{|r|l|l|l|l|l|l|l|l|l|l|l|l|l|l|l|l|l|}
02 & 0 & 0 & 0 & 0 & 0 & 0 & 0 & 0 & 0 & 0 & 0 & 0 & 0 & 0 & 0 & 0 & 0 \\
\hline 17 & 0 & 0 & 0 & 0 & 0 & 0 & 0 & 0 & 0 & 0 & 0 & 0 & 0 & 0 & 0 & 0 & 0 \\
\hline 20 & 0 & 0 & 0 & 1 & 0 & 0 & 0 & 0 & 0 & 0 & 0 & 0 & 0 & 0 & 0 & 0 & 0 \\
\hline 21 & 0 & 0 & 0 & 0 & 0 & 0 & 0 & 0 & 0 & 0 & 0 & 0 & 0 & 0 & 0 & 0 & 0 \\
\hline 22 & 0 & 0 & 0 & 0 & 0 & 0 & 0 & 0 & 0 & 0 & 0 & 1 & 0 & 0 & 1 & 0 & 1 \\
\hline 24 & 0 & 0 & 0 & 0 & 0 & 0 & 0 & 0 & 0 & 0 & 0 & 0 & 0 & 0 & 0 & 0 & 0 \\
\hline 27 & 0 & 0 & 0 & 0 & 0 & 0 & 0 & 0 & 1 & 0 & 0 & 0 & 0 & 0 & 0 & 0 & 0 \\
\hline 28 & 0 & 0 & 0 & 0 & 0 & 0 & 1 & 0 & 0 & 0 & 0 & 0 & 0 & 0 & 0 & 0 & 0 \\
\hline 29 & 0 & 0 & 0 & 0 & 0 & 0 & 0 & 0 & 0 & 0 & 0 & 0 & 0 & 0 & 0 & 0 & 0 \\
\hline 45A & 0 & 0 & 0 & 0 & 0 & 0 & 0 & 0 & 0 & 0 & 0 & 0 & 0 & 0 & 0 & 0 & 0 \\
\hline 45B & 0 & 0 & 0 & 0 & 0 & 0 & 0 & 0 & 0 & 0 & 0 & 0 & 0 & 0 & 0 & 0 & 0 \\
\hline $52 A$ & 0 & 0 & 0 & 0 & 0 & 0 & 0 & 0 & 0 & 0 & 0 & 0 & 0 & 0 & 0 & 0 & 0 \\
\hline 55 & 0 & 0 & 0 & 0 & 0 & 0 & 0 & 0 & 0 & 0 & 0 & 0 & 0 & 0 & 0 & 0 & 0 \\
\hline $63 B$ & 0 & 0 & 0 & 0 & 0 & 0 & 0 & 0 & 0 & 0 & 0 & 0 & 0 & 0 & 0 & 0 & 0 \\
\hline 72 & 0 & 0 & 0 & 0 & 0 & 0 & 0 & 0 & 0 & 0 & 0 & 0 & 0 & 0 & 0 & 0 & 0 \\
\hline $74 B$ & 0 & 0 & 0 & 0 & 0 & 0 & 0 & 0 & 0 & 0 & 0 & 0 & 0 & 0 & 0 & 0 & 0 \\
\hline $74 C$ & 0 & 0 & 0 & 0 & 0 & 0 & 0 & 0 & 0 & 0 & 0 & 0 & 0 & 0 & 0 & 0 & 0 \\
\hline
\end{tabular}

Tabelle 68 fasst die Kennzahlen zur Identifikation von ,schwachen Knoten', d.h. Knoten die relativ stark mit Performanzlücken in Verbindung zu bringen sind, zusammen. Alleinig der Knoten 20 ist als weakNode ${ }^{\text {OlT }}$, d.h. als schwacher Vorgängerknoten zu klassifizieren, während die Knngten 21 , 27-3-52A-4 und $74 \mathrm{C}$ als 
weakNode ${ }^{I N}$, d.h. schwache Nachfolgerknoten, einzuordnen sind. Es zeigt sich, dass der Knoten 22, der in vielen Performanzlücken eine Vorgängerrolle einnimmt nicht als schwacher Knoten einzustufen ist, sondern vielmehr die jeweiligen Nachfolger in den Performanzlückenbeziehungen. Berücksichtigt man neben den Performanzlückenanteilen der Knoten auch die Anteile der Knoten am Gesamtvolumen der lokalen Struktur, so ist der Knoten 72 zusätzlich als schwacher Nachfolgerknoten zu betrachten. Die Schranke zur Bestimmung der Vektoren weakNode ${ }^{O I T}$, weakNode ${ }^{I N}$ und weakNode ${ }^{T O T}$ wurde mit 0,05 festgesetzt. Knoten mit einem Wert von Null für pfgNode ${ }^{\text {OIT }}$ bzw. pfgNode ${ }^{I N}$ und shNode ${ }^{\text {OlT }}$ bzw. shNode ${ }^{I N}$ haben keine direkten Nachfolger- bzw. Vorgängerknoten. Bei isolierten Knoten sind alle vier Werte gleich Null.

Tabelle 68: schwache Knoten

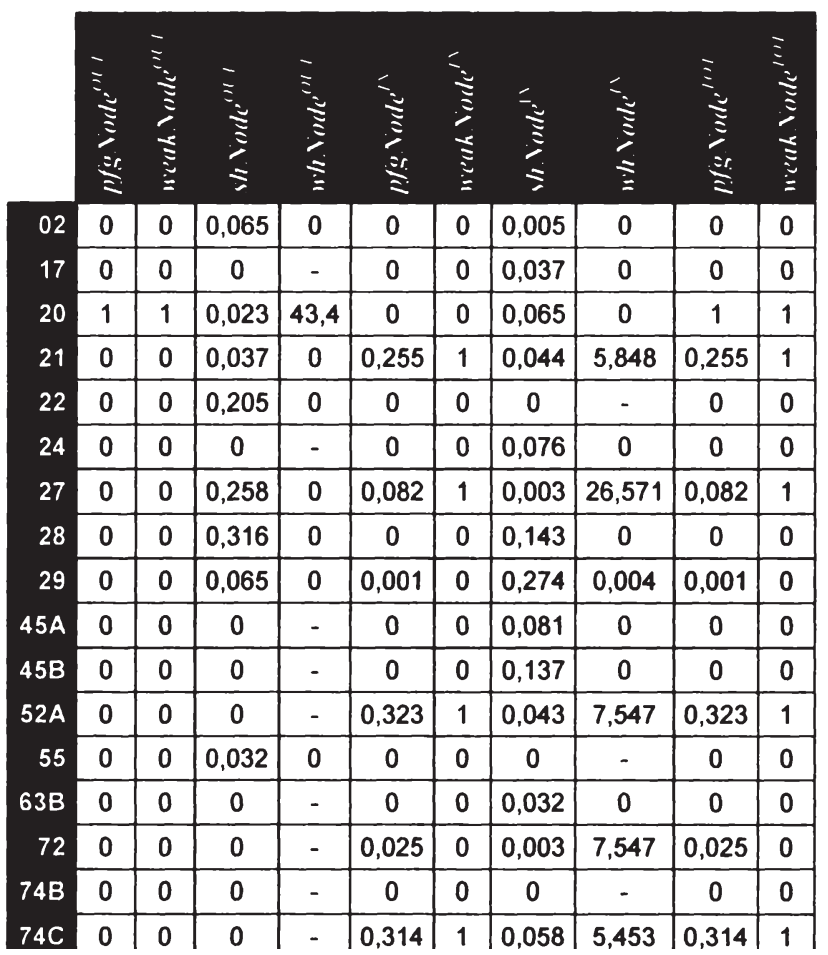




\subsubsection{Bewertung von direkten internen Lücken}

Während die Matrix Rate ${ }^{D L L}$ aus Tabelle 69 die Bewertung von direkten internen Lücken bezogen auf sämtliche Kanten- bzW. Lückenfolgen der Weglänge $n$ - 1 in der lokalen Struktur zeigt, stellt die Matrix Rate ${ }^{D L L 2}$ aus Tabelle 70 die Bewertung von direkten internen Lücken ausschließlich in Bezug auf Performanzlückenfolgen bzw. Folgen, die Performanzlücken enthalten, dar.

Tabelle 69: Rate $^{\text {DIL }}$

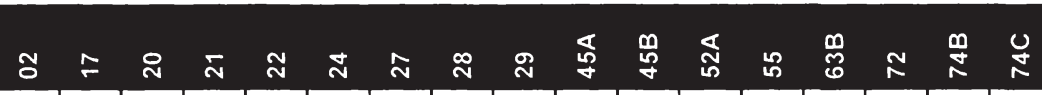

\begin{tabular}{|rc|c|c|c|c|c|c|c|c|c|c|c|c|c|c|c|c|}
\hline 02 & 0 & 0 & 0 & 1,2 & 0 & 0 & 0 & 0 & 0 & 0 & 0 & 0 & 0 & 0 & 0 & 0 & 0 \\
\hline 17 & 0 & 0 & 0 & 0 & 0 & 0 & 0 & 0 & 0 & 0 & 2,7 & 0 & 0 & 0 & 0 & 0 & 0 \\
20 & 0 & 0 & 0 & 0 & 0 & 0 & 5,0 & 0 & 0 & 1,0 & 1,0 & 0 & 0 & 0 & 0 & 0 & 0 \\
21 & 0 & 0 & 3,6 & 0 & 6,9 & 1,7 & 0 & 0 & 0 & 0 & 1,7 & 1,7 & 0 & 0 & 1,7 & 1,7 & 0 \\
\hline 22 & 1,8 & 0 & 0 & 0 & 0 & 0 & 0 & 0 & 0,5 & 0 & 0 & 0 & 1,0 & 0 & 0 & 0 & 0 \\
\hline 24 & 2,2 & 0 & 0 & 1,4 & 5,5 & 0 & 0 & 4,4 & 0 & 0 & 0,4 & 0 & 0 & 0 & 0 & 0 & 0 \\
27 & 0 & 0 & 0 & 0 & 0 & 0 & 0 & 0 & 0 & 0 & 0 & 0 & 0 & 0 & 0 & 0 & 0 \\
\hline 28 & 2,4 & 0 & 2,4 & 0 & 0 & 0 & 0 & 0 & 0 & 0 & 0 & 0,6 & 1,6 & 0 & 0 & 0,6 & 0 \\
\hline 29 & 3,1 & 1,3 & 3,1 & 2,3 & 6,4 & 1,3 & 5,3 & 0 & 0 & 0 & 0 & 0 & 0 & 0 & 0 & 0 & 0 \\
\hline $45 A$ & 2,2 & 0 & 2,2 & 0 & 0 & 0 & 0 & 4,4 & 0 & 0 & 0,4 & 0 & 1,4 & 0 & 0,4 & 0 & 0 \\
\hline $45 B$ & 4,4 & 0 & 0 & 0 & 0 & 0 & 0 & 0 & 0 & 2,6 & 0 & 0 & 3,6 & 0 & 2,6 & 0 & 2,6 \\
\hline $52 A$ & 0 & 0 & 0 & 0 & 0 & 0 & 0 & 0 & 0 & 0 & 0 & 0 & 0 & 0 & 0 & 0 & 0 \\
\hline 55 & 0 & 0 & 0 & 0 & 0 & 0 & 0 & 4,0 & 0,5 & 0 & 0 & 0 & 0 & 0 & 0 & 0 & 0 \\
\hline $63 B$ & 0 & 0 & 0 & 0 & 0 & 0 & 0 & 0 & 0 & 0 & 0 & 0 & 0 & 0 & 0 & 0 & 0 \\
72 & 0 & 0 & 1,9 & 1,0 & 5,2 & 0 & 4,0 & 4,0 & 0,5 & 0 & 0 & 0 & 1,0 & 0 & 0 & 0 & 0 \\
\hline $74 B$ & 0 & 0 & 0 & 0 & 0 & 0 & 0 & 0 & 0 & 0 & 0 & 0 & 0 & 0 & 0 & 0 & 0 \\
\hline $74 C$ & 0 & 0,3 & 0 & 0 & 5,4 & 0,3 & 4,3 & 4,3 & 0,8 & 0 & 0 & 0,3 & 0 & 0,3 & 0,3 & 0,3 & 0 \\
\hline
\end{tabular}

Die Auswahl der überdurchschnittlichen Werte von Rate ${ }^{I I L}$, d.h. der Werte, die über dem Schwellwert 1,81 (berechnet als arithmetisches Mittel der Bewertungen aller 79 direkten internen Lücken) liegen, ergibt eine Matrix DiGap ${ }^{\text {binary }}$ mit $31 \mathrm{zu}$ priorisierenden direkten internen Lücken. Dabei weisen die Knotenpaare mit dem Knoten 22 in der Nachfolgerrolle, d.h. $(21,22),(29,22),(24,22),(74 C, 22)$ und $(72,22)$, die höchsten Werte (gemäß Rate ${ }^{D I L}$ größer als 5, zum Teil sogar größer als 6) auf. Nachfolgerknoten 27 kommt mit den Vorgängerknoten 20 und 29 ebenfalls in zwei direkten internen Lücken mit einer Bewertung in Rate ${ }^{D I L}$ größer bzw. gleich 5 vor. Die Knoten 29 und 45B spielen in den meisten (konkret 5) 
direkten internen Lücken die Vorgängerrolle. Knoten 02 ist der häufigste Nachfolger in den identifizierten direkten internen Lücken (6 Mal).

Tabelle 70: Rate DIL.2 $^{2}$

\begin{tabular}{|c|c|c|c|c|c|c|c|c|c|c|c|c|c|c|c|c|c|}
\hline & ช & 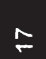 & సి & $\bar{N}$ & $\mathbb{N}$ & $\stackrel{\sim}{\sim}$ & $\hat{N}$ & $\stackrel{\infty}{\sim}$ & Iి & 歹 & 吕 & ๙్ర & 艿 & 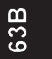 & N & $\underset{\sim}{\mathscr{\sim}}$ & $\underset{N}{S}$ \\
\hline 02 & 0 & 0 & 0 & 0 & 0 & 0 & 0 & 0 & 0 & 0 & 0 & 0 & 0 & 0 & 0 & 0 & 0 \\
\hline 17 & 0 & 0 & 0 & 0 & 0 & 0 & 0 & 0 & 0 & 0 & 1,5 & 0 & 0 & 0 & 0 & 0 & 0 \\
\hline 20 & 0 & 0 & 0 & 0 & 0 & 0 & 2,0 & 0 & 0 & 1,0 & 1,0 & 0 & 0 & 0 & 0 & 0 & 0 \\
\hline 21 & 0 & 0 & 2,3 & 0 & 3,6 & 1,5 & 0 & 0 & 0 & 0 & 1,5 & 1,5 & 0 & 0 & 1,5 & 1,5 & 0 \\
\hline 22 & 1,8 & 0 & 0 & 0 & 0 & 0 & 0 & 0 & 0 & 0 & 0 & 0 & 1,0 & 0 & 0 & 0 & 0 \\
\hline 24 & 1,5 & 0 & 0 & 0 & 1,8 & 0 & 0 & 3,6 & $\overline{0}$ & 0 & 0 & 0 & 0 & 0 & 0 & 0 & 0 \\
\hline 27 & 0 & 0 & 0 & 0 & 0 & 0 & 0 & 0 & 0 & 0 & 0 & 0 & 0 & 0 & 0 & 0 & 0 \\
\hline 28 & 1,8 & 0 & 0,8 & 0 & 0 & 0 & 0 & 0 & 0 & 0 & 0 & 0 & 1,0 & 0 & 0 & 0 & 0 \\
\hline 29 & 2,8 & 1,0 & 1,8 & 1,0 & 3,1 & 1,0 & 2,0 & 0 & 0 & 0 & 0 & 0 & 0 & 0 & 0 & 0 & 0 \\
\hline $45 A$ & 2,2 & 0 & 1,2 & 0 & 0 & 0 & 0 & 4,4 & 0 & 0 & 0,4 & 0 & 1,4 & 0 & 0,4 & 0 & 0 \\
\hline 45B & 3,0 & 0 & 0 & 0 & 0 & 0 & 0 & 0 & 0 & 1,2 & 0 & 0 & 2,2 & 0 & 1,2 & 0 & 1,2 \\
\hline $52 A$ & 0 & 0 & 0 & 0 & 0 & 0 & 0 & 0 & 0 & 0 & 0 & 0 & 0 & 0 & 0 & 0 & 0 \\
\hline 55 & 0 & 0 & 0 & 0 & 0 & 0 & 0 & 4,0 & 0 & 0 & 0 & 0 & 0 & 0 & 0 & 0 & 0 \\
\hline $63 B$ & 0 & 0 & 0 & 0 & 0 & 0 & 0 & 0 & 0 & 0 & 0 & 0 & 0 & 0 & 0 & 0 & 0 \\
\hline 72 & 0 & 0 & 0,9 & 0 & 2,2 & 0 & 1,0 & 4,0 & 0 & 0 & 0 & 0 & 1,0 & 0 & 0 & 0 & 0 \\
\hline $74 \mathrm{~B}$ & 0 & 0 & 0 & 0 & 0 & 0 & 0 & 0 & 0 & 0 & 0 & 0 & 0 & 0 & 0 & 0 & 0 \\
\hline $74 C$ & 0 & 0,3 & 0 & 0 & 2,4 & 0,3 & 1,3 & 4,3 & 0,1 & 0 & 0 & 0,3 & 0 & 0,3 & 0,3 & 0,3 & 0 \\
\hline
\end{tabular}

Betrachtet man hingegen die Matrix Rate ${ }^{\text {DIL2 }}$ (Tabelle 70) bzw. die daraus unter Berücksichtigung des als mittlere Bewertung aller direkten internen Lücken berechneten Schwellwerts 1,09 abgeleitete Matrix DiGap ${ }^{\text {binary2 }}$, so werden 33 bevorzugt zu behandelnde direkte interne Lücken herausgefiltert. Davon wurden 27 auch mit dem ersten Verfahren ermittelt, was einen hohen Grad an Übereinstimmung und damit Stabilität der Ergebnisse bedeutet. Auffallend sind auch hier die Knoten 28, 22 und 02, die nicht nur am häufigsten als Nachfolger in direkten internen Lücken vorkommen, sondern auch die höchsten Bewertungen aufweisen. Die Knoten 28 und 22 treten in jeweils 5 direkten internen Lücken als Nachfolger auf, der Knoten 02 sogar in 6 . Die höchsten Bewertungen zeigt der Nachfolgerknoten 28 mit 4 Werten größer bzw. gleich 4 und einem Wert größer als 3, dicht gefolgt von den Nachfolgerknoten 22 und 02. In der Vorgängerrolle zeichnet sich der Knoten 21 aus. Dieser Knoten scheint in 7 direkten internen Lücken auf. Die Vorgängerknoten 45B und 29, die in DiGap binary am häufigsten in 
direkten internen Lücken enthalten sind, liegen in DiGap ${ }^{\text {binary2 }}$ mit 5 bzw. 4 Vorkommen auf den Plätzen 2 und 3. Die beiden Verfahren liefern dementsprechend auch hinsichtlich der Priorisierung der direkten internen Lücken bzw. der darin auftretenden einzelnen Knoten vergleichbare Ergebnisse.

Die Tabelle 71 und die Tabelle 72 geben für jede direkte interne Lücke aus DiGap ${ }^{\text {binary }}$ den Input- $\left(\Delta \mathrm{Vol}^{l{ }^{I L L}}\right)$ bzw. Outputänderungsbedarf $\left(\Delta \mathrm{Vol}^{*} \mathrm{DIl}\right)$ der dazugehörigen Nachfolger- bzw. Vorgängerknoten für die lokale Struktur an. In Fällen in denen das Input- bzw. Outputvolumen des Nachfolger- bzw. Vorgängerknotens allerdings 0 ist, sind diese Werte sinnvollerweise als weiterer Indikator der relativen Bedeutung der Schließung dieser Lücke zu sehen. Dies gilt bspw. für die beiden höchsten Werte aus Tabelle 71, die die beiden direkten internen Lücken $(21,22)$ und $(45 B, 55)$ betreffen. Der mit dem Wert 0,42 dritthöchste Zielwert dieser Matrix der direkten internen Lücke $(28,02)$ besagt hingegen, dass der Nachfolgerknoten 02 sein gesamtes Inputvolumen bei Schließung dieser internen Lücke nach Maßgabe der Proportionen aus der reduzierten globalen Struktur um $42 \%$ erhöhen müsste.

Tabelle 71: $\Delta \mathrm{Vol}^{\mathrm{DIL}}$

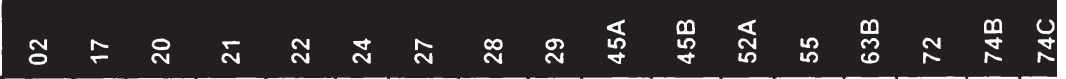

\begin{tabular}{|c|c|c|c|c|c|c|c|c|c|c|c|c|c|c|c|c|c|}
\hline 02 & 0 & 0 & 0 & 0 & 0 & 0 & 0 & 0 & 0 & 0 & 0 & 0 & 0 & 0 & 0 & 0 & 0 \\
\hline 17 & 0 & 0 & 0 & 0 & 0 & $\overline{0}$ & 0 & 0 & 0 & 0 & 0,1 & 0 & 0 & 0 & 0 & 0 & 0 \\
\hline 20 & 0 & 0 & 0 & 0 & 0 & 0 & 0,2 & 0 & 0 & 0 & 0 & $\overline{0}$ & 0 & 0 & 0 & 0 & 0 \\
\hline 21 & 0 & 0 & 0,1 & 0 & 4,2 & 0 & 0 & 0 & 0 & 0 & 0 & 0 & 0 & 0 & 0 & 0 & 0 \\
\hline 22 & 0 & 0 & 0 & 0 & 0 & 0 & 0 & 0 & 0 & 0 & 0 & 0 & 0 & 0 & 0 & 0 & 0 \\
\hline 24 & 0,1 & 0 & 0 & 0 & 0 & 0 & 0 & 0,1 & 0 & 0 & 0 & 0 & 0 & 0 & 0 & 0 & $\overline{0}$ \\
\hline 27 & 0 & 0 & 0 & 0 & 0 & 0 & 0 & 0 & 0 & 0 & 0 & 0 & 0 & 0 & 0 & 0 & $\overline{0}$ \\
\hline 28 & 0,4 & 0 & 0,1 & 0 & 0 & 0 & 0 & 0 & 0 & 0 & 0 & 0 & 0 & 0 & 0 & 0 & 0 \\
\hline 29 & 0,3 & 0 & 0,1 & 0,1 & 0,1 & 0 & 0,3 & 0 & 0 & 0 & 0 & 0 & 0 & 0 & 0 & 0 & 0 \\
\hline $45 \mathrm{~A}$ & 0,1 & 0 & 0,1 & 0 & 0 & 0 & 0 & 0 & 0 & 0 & 0 & 0 & 0 & 0 & 0 & 0 & 0 \\
\hline $45 B$ & 0 & 0 & 0 & 0 & 0 & 0 & 0 & 0 & 0 & 0,1 & 0 & 0 & 0,7 & 0 & 0,1 & 0 & 0 \\
\hline $52 \mathrm{~A}$ & 0 & 0 & 0 & 0 & 0 & 0 & 0 & 0 & 0 & 0 & 0 & 0 & 0 & 0 & 0 & 0 & 0 \\
\hline 55 & 0 & 0 & 0 & 0 & 0 & 0 & 0 & 0,1 & 0 & 0 & 0 & 0 & 0 & 0 & 0 & 0 & 0 \\
\hline $63 B$ & 0 & 0 & 0 & 0 & 0 & 0 & 0 & 0 & 0 & 0 & 0 & 0 & 0 & 0 & 0 & 0 & 0 \\
\hline 72 & 0 & 0 & 0,1 & 0 & 0 & 0 & 0,1 & 0,1 & 0 & 0 & 0 & 0 & 0 & 0 & 0 & 0 & 0 \\
\hline $74 B$ & 0 & 0 & 0 & 0 & 0 & 0 & 0 & 0 & 0 & 0 & 0 & 0 & 0 & 0 & 0 & 0 & 0 \\
\hline $74 \mathrm{C}$ & 0 & 0 & 0 & 0 & 0,1 & 0 & 0,2 & & id & $10 \mathrm{~V}$ & $b \notin$ & 08 & $3-03$ & 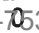 & 7007 & 0 & 0 \\
\hline
\end{tabular}


Die Matrix $\Delta \mathrm{Vol}^{*} D I L$ in Tabelle 72 für den Outputänderungsbedarf der Vorgängerknoten weist abermals die direkte interne Lücke $(21,22)$ mit dem höchsten Wert auf, welcher zugleich als Zielwert zu interpretieren ist. Der Wert der Kante $(17,45 \mathrm{~B})$ ist unbestimmt (Inf), nachdem dies die einzige Kante in der reduzierten globalen Struktur ist, die von dem Knoten 17 wegführt. Die Werte der direkten internen Lücken $(45 \mathrm{~B}, 45 \mathrm{~A})$ sowie $(45 \mathrm{~B}, 55)$ stechen bei der Betrachtung der Matrix $\Delta \mathrm{Vol}^{* D I L}$ ebenfalls (und letztere im Vergleich zu $\Delta \mathrm{Vol}^{I J L}$ abermals) hervor.

Tabelle 72: $\Delta \mathrm{Vol}^{*} \mathrm{DIL}$

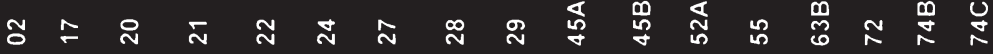

\begin{tabular}{ll|l|l|l|l|l|l|l|l|l|l|l|l|l|l|l|l|}
02 & 0 & 0 & 0 & 0 & 0 & 0 & 0 & 0 & 0 & 0 & 0 & 0 & 0 & 0 & 0 & 0 & 0 \\
17 & 0 & 0 & 0 & 0 & 0 & 0 & 0 & 0 & 0 & 0 & $\operatorname{lnf}$ & 0 & 0 & 0 & 0 & 0 & 0 \\
\hline
\end{tabular}

20

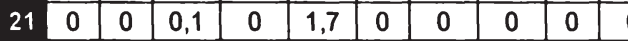

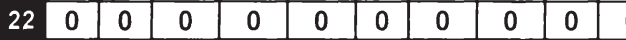

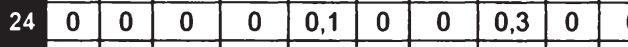

$27 \quad 000010$

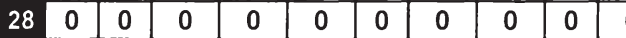

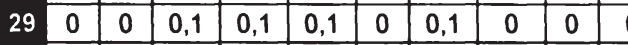

45A 00 O 0 0,2

45B

$52 \mathrm{~A}$

55

$63 \mathrm{~B}$

72

$74 \mathrm{~B}$

$74 \mathrm{C}$

\begin{tabular}{l|l|l|}
\hline & 0 & 0 \\
\hline & 0 & 0 \\
\hline
\end{tabular}

\begin{tabular}{l|l|l}
0 & 0 & 0 \\
\hline & 0 & 0
\end{tabular}

\begin{tabular}{ll|l|l|}
0 & 0 & 0 \\
0 & 0 & 0 \\
\hline
\end{tabular}

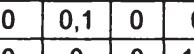

\begin{tabular}{l|l|l|l|l}
0 & 0 & 0 & 0 \\
0 & 0 & 0 & 0 \\
\hline & 0 & 0 & 0 \\
\hline
\end{tabular}

\begin{tabular}{l|l|l|}
\hline & 0 & 0 \\
\hline 0 & 0 & 0 \\
\hline & 0 & 0
\end{tabular}

\begin{tabular}{l|l}
0 & 0 \\
\hline & 0
\end{tabular}

\begin{tabular}{ll|l|l|}
0 & 0 & 0 \\
\hline & 0 & 0 & 0 \\
\hline
\end{tabular}

\begin{tabular}{llll}
0 & 0 & 0 \\
0 & 0 & 0 & 0 \\
\hline
\end{tabular}

\begin{tabular}{lllll}
0 & 0 & 0 & 0 \\
\hline & 0 & 0 & 0 & 0
\end{tabular}

\begin{tabular}{l|l|l}
0 & 0 & 0 \\
\hline 0 & 0 & 0 \\
\hline
\end{tabular}

\begin{tabular}{l|l|l|l}
0 & 0 & 0 & 1 \\
0 & 0 & 0 & 0
\end{tabular}

\begin{tabular}{l|l|l|}
0 & 0 \\
\hline & 0 & 0 \\
\hline
\end{tabular}

\begin{tabular}{ll|l|l|}
0 & 0 & 0 \\
0 & 0 & 0 \\
0 & 0 & 0
\end{tabular}

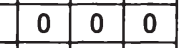

Tabelle 73 zeigt Rate ${ }^{D L, w}$, die Matrix der gewichteten Kantenbewertungen der direkten internen Lücken. Die Gewichte für die Bedeutungen der jeweiligen Startund Endknoten stammen hierbei aus den normierten Matrizen der reduzierten globalen Struktur $A^{G L O r}$ und $A^{* G L O r}$ und entsprechen somit den Zielwerten der direkten internen Lücken. Die höchste Bedeutung in dieser Matrix hat mit einem Wert von 4,67 die direkte interne Lücke zwischen den Knoten 21 und 22. Danach folgt die Lücke $(45 B, 55)$ mit der Bewertung 1,46. Die restlichen direkten internen 
Lücken weisen Werte kleiner 1 auf. Es fällt auf, dass zu dem Nachfolgerknoten 22 vergleichsweise hoch bewertete direkte interne Lücken führen.

Tabelle 73: Rate RIL.w $^{\text {DI. }}$

\begin{tabular}{|c|c|c|c|c|c|c|c|c|c|c|c|c|c|c|c|c|c|}
\hline & ชิ & $\mp$ & ิ & $\bar{\sim}$ & $\mathbb{N}$ & d & $\hat{\sim}$ & $\stackrel{\infty}{\sim}$ & ని & ఝ & $\underset{8}{\mathscr{8}}$ & ฟ్ర & 员 & $\stackrel{\infty}{\tilde{B}}$ & $\mathbb{N}$ & $\underset{\sim}{\stackrel{\infty}{N}}$ & $\frac{U}{N}$ \\
\hline 02 & 0 & 0 & 0 & 0,5 & 0 & 0 & 0 & 0 & 0 & 0 & 0 & 0 & 0 & 0 & 0 & 0 & 0 \\
\hline 17 & 0 & 0 & 0 & 0 & 0 & 0 & 0 & 0 & 0 & 0 & 0,1 & 0 & 0 & 0 & 0 & 0 & 0 \\
\hline 20 & 0 & 0 & 0 & 0 & 0 & 0 & 0,3 & 0 & 0 & 0,3 & 0,1 & 0 & 0 & 0 & 0 & 0 & 0 \\
\hline 21 & 0 & 0 & 0,3 & 0 & 4,7 & 0,3 & 0 & 0 & 0 & 0 & 0,1 & 0,2 & 0 & 0 & 0,2 & 0,5 & 0 \\
\hline 22 & 0 & 0 & 0 & 0 & 0 & 0 & 0 & 0 & 0 & 0 & 0 & 0 & 0 & 0 & 0 & 0 & 0 \\
\hline 24 & 0,1 & 0 & 0 & 0,3 & 0,5 & 0 & 0 & 0,9 & 0 & 0 & 0 & 0 & 0 & 0 & 0 & 0 & 0 \\
\hline 27 & 0 & 0 & 0 & 0 & 0 & 0 & 0 & 0 & 0 & 0 & 0 & 0 & 0 & 0 & 0 & 0 & 0 \\
\hline 28 & 0,2 & 0 & 0 & 0 & 0 & 0 & 0 & 0 & 0 & 0 & 0 & 0 & 0,1 & 0 & 0 & 0,1 & 0 \\
\hline 29 & 0,3 & 0,3 & 0,2 & 0,2 & 0,5 & 0,2 & 0,8 & 0 & 0 & 0 & 0 & 0 & 0 & 0 & 0 & 0 & 0 \\
\hline $45 \mathrm{~A}$ & 0,1 & 0 & 0,3 & 0 & 0 & 0 & 0 & 0,5 & 0 & 0 & 0 & 0 & 0,6 & 0 & 0 & 0 & 0 \\
\hline 45B & 0,1 & 0 & 0 & 0 & 0 & 0 & 0 & 0 & 0 & 0,3 & 0 & 0 & 1,5 & 0 & 0,3 & 0 & 0 \\
\hline $52 \mathrm{~A}$ & 0 & 0 & 0 & 0 & 0 & 0 & 0 & 0 & 0 & 0 & 0 & 0 & 0 & 0 & 0 & 0 & 0 \\
\hline 55 & 0 & 0 & 0 & 0 & 0 & 0 & 0 & 0,2 & 0,1 & 0 & 0 & 0 & 0 & 0 & 0 & 0 & 0 \\
\hline $63 B$ & 0 & 0 & 0 & 0 & 0 & 0 & 0 & 0 & 0 & 0 & 0 & 0 & 0 & 0 & 0 & 0 & 0 \\
\hline 72 & 0 & 0 & 0,1 & 0,1 & 0,2 & 0 & 0,2 & 0,3 & 0,1 & 0 & 0 & 0 & 0,1 & 0 & 0 & 0 & 0 \\
\hline $74 B$ & 0 & 0 & 0 & 0 & 0 & 0 & 0 & 0 & 0 & 0 & 0 & 0 & 0 & 0 & 0 & 0 & 0 \\
\hline $74 \mathrm{C}$ & 0 & 0,1 & 0 & 0 & 0,6 & 0,1 & 0,3 & 0,3 & 0,1 & 0 & 0 & 0,1 & 0 & 0 & 0,1 & 0 & 0 \\
\hline
\end{tabular}

Erfolgt die Bewertung der direkten internen Lücken einzeln über einen Vergleich der Gesamtbedeutungen der lokalen Struktur und der um die jeweilige Kante erweiterten lokalen Struktur, so erhält man rate ${ }^{e D I L-o p}$. Tabelle 74 gibt eine Übersicht über die Werte von rate ${ }^{e D I L-o p}$ für sämtliche direkte interne Lücken, die, wie am Ende von Kapitel 8.4 beschrieben, auch negativ sein können. Für die hier betrachtete lokale Struktur zeigt sich, dass die Schließung von jenen direkten internen Lücken, die zwei in sich eng verflochtene Cliquen bzw. zwei SubCliquen, die nur durch eine vergleichsweise schwach gewichtete Kante zusammenhängen, verbinden, den größten Bedeutungszuwachs hervorruft. Dementsprechend führen die direkten internen Lücken $(21,22)$ (Verbindung von Sub-Cliquen), $(29,22),(45 B, 02)$ und $(45 B, 55)$ (Verbindung von Cliquen) die nach rate ${ }^{\text {eDIL-op }}$ geordnete Liste aller direkten internen Lücken an. Am Ende der Liste befinden sich direkte interne Lücken wie z.B. $(20,45 A),(22,17)$ und $(20,45 B)$, an 
deren Nachfolgerknoten keine bestehenden Kanten anschließen, und die daher sogar leicht negativ bewertet werden.

Tabelle 74: Liste aller rate $e^{e D I L-o p}$

\begin{tabular}{|c|c|c|c|c|c|c|c|c|c|c|c|}
\hline & von & $\mathrm{zu}$ & . & & von & $\mathrm{zu}$ & (I)II. & & von & $\mathrm{zu}$ & $(I) / L$ \\
\hline 1. & 21 & 22 & 18,76 & 31. & 72 & $74 \mathrm{~B}$ & 3,02 & 61. & 29 & 24 & 0,29 \\
\hline 2. & 29 & 22 & 18,67 & 32. & 24 & 21 & 2,81 & 62. & 21 & 24 & 0,27 \\
\hline 3. & $45 B$ & 02 & 18,37 & 33. & $74 \mathrm{C}$ & 29 & 2,56 & 63. & 21 & 72 & 0,18 \\
\hline 4. & 45B & 55 & 14,13 & 34. & 21 & $74 B$ & 2,42 & 64. & 21 & $52 \mathrm{~A}$ & 0,13 \\
\hline 5. & $74 \mathrm{C}$ & 27 & 10,43 & 35. & 21 & 20 & 2,36 & 65. & 29 & 17 & 0,13 \\
\hline 6. & $45 \mathrm{~A}$ & 02 & 9,81 & 36. & 72 & 21 & 2,09 & 66. & 55 & 29 & 0,07 \\
\hline 7. & 24 & 02 & 9,26 & 37. & 72 & 29 & 2,03 & 67. & 28 & $52 \mathrm{~A}$ & 0,04 \\
\hline 8. & $45 \mathrm{~A}$ & 55 & 8,79 & 38. & 22 & 55 & 2,03 & 68. & 55 & $52 \mathrm{~A}$ & 0 \\
\hline 9. & 72 & 27 & 8,60 & 39. & 28 & $74 B$ & 1,99 & 69. & 55 & 72 & 0 \\
\hline 10. & $45 A$ & 20 & 8,31 & 40. & $74 C$ & 72 & 1,61 & 70. & 55 & $74 C$ & 0 \\
\hline 11. & 72 & 22 & 7,26 & 41. & 28 & 20 & 1,60 & 71. & 21 & $45 B$ & 0 \\
\hline 12. & $74 C$ & 22 & 7,00 & 42. & $74 \mathrm{C}$ & 24 & 1,59 & 72. & 22 & $63 B$ & $-0,01$ \\
\hline 13. & 29 & 02 & 6,97 & 43. & $45 A$ & 72 & 1,59 & 73. & 22 & 29 & $-0,01$ \\
\hline 14. & 24 & 22 & 6,91 & 44. & 55 & 28 & 1,50 & 74. & 22 & $45 \mathrm{~A}$ & $-0,03$ \\
\hline 15. & 72 & 28 & 6,90 & 45. & $74 \mathrm{C}$ & $52 A$ & 1,47 & 75. & 55 & 45B & $-0,04$ \\
\hline 16. & 24 & 28 & 6,67 & 46. & $45 A$ & 45B & 1,43 & 76. & 27 & $45 \mathrm{~A}$ & $-0,11$ \\
\hline 17. & $74 C$ & 28 & 6,62 & 47. & 24 & $45 B$ & 1,34 & 77. & 20 & 45B & $-0,17$ \\
\hline 18. & 20 & 27 & 6,20 & 48. & $74 \mathrm{C}$ & $63 B$ & 1,34 & 78. & 22 & 17 & $-0,18$ \\
\hline 19. & 72 & 55 & 6,03 & 49. & $74 \mathrm{C}$ & 17 & 1,17 & 79. & 20 & $45 \mathrm{~A}$ & $-0,83$ \\
\hline 20. & 72 & 20 & 5,42 & 50. & 72 & 24 & 1,15 & & & & \\
\hline 21. & 28 & 02 & 4,99 & 51. & 72 & $52 \mathrm{~A}$ & 1,14 & & & & \\
\hline 22. & 28 & 55 & 4,02 & 52. & 72 & $74 C$ & 1,14 & & & & \\
\hline 23. & $45 A$ & 28 & 3,99 & 53. & 72 & $63 \mathrm{~B}$ & 1,05 & & & & \\
\hline 24. & 45B & 72 & 3,88 & 54. & 55 & $74 B$ & 1,00 & & & & \\
\hline 25. & $45 B$ & $45 A$ & 3,77 & 55. & 22 & $74 \mathrm{~B}$ & 1,00 & & & & \\
\hline 26. & 17 & 45B & 3,77 & 56. & 72 & 17 & 0,93 & & & & \\
\hline 27. & 45B & $74 \mathrm{C}$ & 3,61 & 57. & $74 B$ & $45 A$ & 0,77 & & & & \\
\hline 28. & 29 & 20 & 3,43 & 58. & 29 & 21 & 0,42 & & & & \\
\hline 29. & $74 \mathrm{C}$ & $74 \mathrm{~B}$ & 3,28 & 59. & 22 & 02 & 0,40 & & & & \\
\hline 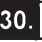 & 29 & 27 & 3,08 & 60. & 02 & 21 & 0,34 & & & & \\
\hline
\end{tabular}

\subsubsection{Bewertung von externen Lücken}

Die Bedeutungsbestimmung für externe Lücken kann entsprechend Kapitel 8.5 im Wesentlichen auf zwei Arten erfolgen. Tabelle 75 zeigt die sortierten Ergebnisse der Berechnung für die gewichtete Bedeutung sämtlicher externer Knoten rate $^{E X T v, w}$, während Tabelle 76 die sortierten Ergebnisse der Knotenbedeutung nach dem Berechnungsansatz rate ${ }^{E X X v}$ beschreibt, Biesers, bewertet_die externen 
Knoten einzeln über einen Vergleich der Gesamtbedeutungen der lokalen Struktur und der um den jeweiligen externen Knoten erweiterten lokalen Struktur. Im Unterschied zu der Rangliste der externen Verbindungsknoten aus dem Strukturvergleich (Tabelle 62) berücksichtigen beide Berechnungsmethoden zusätzlich externe Knoten, die am Anfang oder Ende von (mehrgliedrigen) Transaktionsketten stehen. Folglich können bei beiden Berechnungsmethoden im Gegensatz zu Tabelle 62 (weitere) externe Knoten in unterschiedlicher Rangfolge auftreten. Der externe Knoten 18 ist hierfür ein gutes Beispiel. Die Spitzenwerte in Tabelle 75 erzielen die externen Knoten 92A, 93, 36, 70A, 18 und 31. Vergleicht man nun dieses Resultat mit der Rangliste aus Tabelle 76, so zeigen sich erhebliche Unterschiede, die insbesondere durch die Gewichtung mit den Gesamtbedeutungen der internen Vorgänger und Nachfolger der externen Knoten bei der Ermittlung von rate ${ }^{E X T v, w}$ entstehen. Beispielsweise ist die hohe Bewertung des Knoten 92A darauf zurückzuführen, dass gemäß $A^{* G L O}$ dessen wichtigster interner Nachfolger der Knoten 22 ist, der seinerseits der zentrale Vorgängerknoten in der lokalen Struktur ist. Folglich wird die Bedeutung von Knoten 92A überdurchschnittlich hoch gewichtet. Der starke Einfluss der Gewichtung ist bei der Berechnung von rate ${ }^{k X T v}$ (Tabelle 76) nicht vorhanden, weshalb dieser Ansatz dann ratsam erscheint, wenn die bisherige lokale Struktur bei der Bewertung in geringerem Ausmaß berücksichtigt werden soll. Diese Methode rückt den zusätzlichen Beitrag des externen Knoten für die Transaktionsketten stärker in den Vordergrund. In Tabelle 76 erzielen die Knoten 65, 92B, 91 und 64 sehr hohe Bewertungen. Bei der Wahl des 0,75-Quantils als Schwellenwert für extGap $^{\text {binary }}$ sind die ersten 14 Knoten des sortierten rate ${ }^{\text {EXTv }}$ (Tabelle 76) externe Lücken. Die Auswahl von externen Knoten, die für die Erweiterung der lokalen Struktur in Frage kommen, kann natürlich auch aus einer Kombination der Bewertungsansätze unter Abwägung verschiedener Gesichtspunkte erfolgen. Für die weiteren Berechnungen wird der externe Knoten 70A zur Erweiterung bestimmt. 
Tabelle 75: rate EXTv.w $^{\text {. }}$

\section{EXTV $v_{x}$ Wert}

\begin{tabular}{|r|c|c|}
\hline 1. & $92 \mathrm{~A}$ & 3,95 \\
\hline 2. & 93 & 1,03 \\
\hline 3. & 36 & 0,99 \\
\hline 4. & $70 \mathrm{~A}$ & 0,95 \\
\hline $\mathbf{5}$ & 18 & 0,85 \\
\hline $\mathbf{6 .}$ & 31 & 0,84 \\
\hline $\mathbf{7 .}$ & 64 & 0,65 \\
\hline $\mathbf{8 .}$ & 65 & 0,63 \\
\hline $\mathbf{9 .}$ & $40 \mathrm{~A}$ & 0,62 \\
\hline 10. & 16 & 0,61 \\
\hline 11. & $40 \mathrm{~B}$ & 0,58 \\
\hline 12. & 32 & 0,56 \\
\hline 13. & 41 & 0,55 \\
\hline 14. & 25 & 0,55 \\
\hline
\end{tabular}

\section{EXT $v_{x}$ Wert}

$E X T v_{x}$ Wert

EXTv, Wert

\begin{tabular}{|c|c|c|c|c|c|c|c|}
\hline 5. $63 \mathrm{~A}$ & 0,50 & 29. & 26 & 0,25 & 43. & 62 & 0,11 \\
\hline
\end{tabular}

16. \begin{tabular}{l|l|l|}
\hline 71 & 0,49 & 30.
\end{tabular}

70B 0,47

30. $80 \quad 0,25$

31. $51 \mathrm{~B}$

44. $85 \mathrm{~A}-0,11$

\begin{tabular}{c|c|}
11 & 0,47 \\
\hline
\end{tabular}

9. $74 \mathrm{D} \quad 0,43$

32. 01

0,24

45. $70 \mathrm{~B} \quad 0,09$

\begin{tabular}{|c|c|} 
46. 61 & 0,09 \\
\hline
\end{tabular}

20. 35

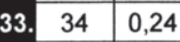

\begin{tabular}{|c|c|} 
47. $51 \mathrm{~A}$ & 0,09 \\
\hline
\end{tabular}

21. $60 \mathrm{C} \quad 0,41$

22. $74 \mathrm{~A}$

34. $92 \mathrm{~B} \quad 0,23$

48. $60 \mathrm{~B} \quad 0,08$

35. $90 \quad 0,23$

49. $40 \mathrm{C}$ - 0,07

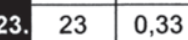

\begin{tabular}{l|l|l|}
36. & 66 & 0,20 \\
\hline
\end{tabular}

50. $15 \quad 0,07$

37. $60 \mathrm{~A}) 0,18$

51. $30 \quad 0,06$

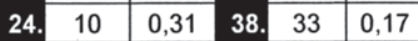

\begin{tabular}{|l|l|l|}
\hline 52. & 37 & 0,05 \\
\hline
\end{tabular}

25. $19 \quad 0,29$

26. 050,28

39. $52 \mathrm{~B} \quad 0,16$

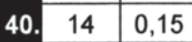

\begin{tabular}{|l|l|l|} 
53. 67 & 0,04 \\
\hline
\end{tabular}

27. $91 \quad 0,27$

\begin{tabular}{ll|l} 
28. $85 \mathrm{~B}$ & 0,27 \\
\hline
\end{tabular}

\begin{tabular}{|l|l|l|}
\hline 41. & 75 & 0,15 \\
\hline 42. & 73 & 0,12 \\
\hline
\end{tabular}

54. $63 \mathrm{C}$ - 0,04

55. $50 \mathrm{~A}$

56. 95 . 0 .

Tabelle 76: rate eXT $^{\text {E }}$

\section{EXTv. Wert}

\section{$E X T v_{x}$ Wert}

\section{EXTv Wert}

EXTV $v_{x}$ Wert

\begin{tabular}{|c|c|c|c|c|c|c|c|c|c|c|c|}
\hline 1. & 65 & 129,19 & 15. & 23 & 63,99 & 29. & 31 & 16,23 & 43. & 14 & 8,36 \\
\hline 2. & 92B & 114,48 & 1 & $60 \mathrm{~A}$ & 61,31 & 30. & $50 \mathrm{~B}$ & 14,29 & 44. & 05 & 7,65 \\
\hline & 91 & 107,58 & & 51B & 48,63 & 31. & $63 C$ & 13,66 & 45. & 61 & 7,36 \\
\hline & 64 & 101,62 & & 32 & 48,59 & 32. & 33 & 13,40 & 46. & $51 \mathrm{~A}$ & 6,84 \\
\hline & $40 \mathrm{~B}$ & 89,03 & & 01 & 38,66 & 33. & $85 \mathrm{~B}$ & 11,22 & 41 & $40 \mathrm{C}$ & 6,03 \\
\hline & $40 \mathrm{~A}$ & 86,55 & & 66 & 37,25 & 34 & 80 & 11,05 & 48. & 16 & 5,70 \\
\hline & $70 \mathrm{~A}$ & 86,08 & & 36 & 32,45 & 35. & 19 & 11,02 & 49. & 30 & 5,68 \\
\hline & $60 \mathrm{C}$ & 84,95 & 22. & 93 & 30,08 & 36. & 11 & 10,64 & 0. & 73 & 5,40 \\
\hline & 74D & 82,42 & & 37 & 29,41 & . & 41 & 9,97 & & 10 & 4,82 \\
\hline & $74 \mathrm{~A}$ & 79,96 & & 90 & 29,40 & ". & $70 \mathrm{~B}$ & 9,91 & 2. & 35 & 4,51 \\
\hline & 62 & 75,71 & & 26 & 26,97 & 39. & $85 \mathrm{~A}$ & 9,48 & 53. & 34 & 4,49 \\
\hline & 25 & 74,05 & & 15 & 19,02 & 40 & 75 & 9,13 & 54. & 67 & 3,83 \\
\hline 13 & $92 \mathrm{~A}$ & 66,94 & & $63 \mathrm{~A}$ & 18,58 & 41. & $60 \mathrm{~B}$ & 8,91 & 55. & $50 \mathrm{~A}$ & 3,63 \\
\hline & 71 & 65,79 & & 52B & 17,32 & 42 & 18 & 8,74 & & 95 & 0 \\
\hline
\end{tabular}

Ausgehend von der Wahl von 70A als Erweiterungsknoten $\left(v_{x} \in V^{E X T}\right)$ fasst Tabelle 77 die direkte und indirekte Bedeutung des externen Knoten 70A zusammen. Entsprechend dieser Werte ist die Bedeutung des externen Knoten 70A als Vorgänger für die bisherigen Knoten der lokalen Struktur beinahe doppelt so hoch wie die Bedeutung des externen Knoten als Nachfolger. Anders formuliert hat der Knoten 70A einen stärkeren Einfluss als Anbieter für die internen Knoten. 
Tabelle 78 zeigt die Verteilung dieser direkten und indirekten Effekte des externen Knoten auf die bisherigen Knoten der lokalen Struktur für dessen Rolle als Vorgänger (rate $e^{E X T v_{x}, O U T 4 L O C}$ ), Nachfolger (rate ${ }^{E X T v_{x} I N 4 L O C}$ ) und als Vorgänger und Nachfolger ( rate $^{E X T v_{x}, 4 L O C}$ ) für die internen Knoten. Tabelle 79 stellt eine Übersicht über die gesamten direkten und indirekten Bedeutungen der externen Knoten als Vorgänger (rate ${ }^{E X T v_{x_{x}} O U / T}$ ), als Nachfolger (rate $e^{E X I v_{x} I N}$ ) und kombiniert (rate $e^{E X T v_{x}}$ ) für alle internen Knoten dar. Der zur Erweiterung ausgewählte externe Knoten $70 \mathrm{~A}$ liegt auch in dieser Rangliste relativ weit vorne. Es fällt auf, dass dieser Knoten einer der wenigen externen Knoten ist, die sowohl aus Vorgänger- als auch aus Nachfolger-Perspektive unter den besten $20 \%$ liegen. Einige in der Gesamtliste sehr gut platzierte Knoten, wie z.B. die Knoten 91, 28 und 60A weisen zwar eine außergewöhnlich hohe Bedeutung als Nachfolger, nicht jedoch als Vorgänger auf und sind daher durch ein weniger ausgewogenes Vernetzungspotenzial gekennzeichnet.

Tabelle 77: Gesamtbedeutung des externen Knoten 70A für die internen Knoten

\begin{tabular}{|c|c|c|c|}
\hline & rate $e^{E X T \mathrm{ix}, O U T}$ & rate $^{E X T_{i}, I N}$ & rate $_{x}^{E X T v_{s}}$ \\
\hline $70 A$ & 15,11 & 8,93 & 24,04 \\
\hline
\end{tabular}

Tabelle 78: Verteilung der Bedeutung des externen Knoten 70A auf die internen Knoten

$\begin{array}{lllllllllllllllll}02 & 17 & 20 & 21 & 22 & 24 & 27 & 28 & 29 & 45 \mathrm{~A} & 45 \mathrm{~B} & 52 \mathrm{~A} & 55 & 63 \mathrm{~B} & 72 & 74 \mathrm{~B} & 74 \mathrm{C}\end{array}$

\begin{tabular}{|c|c|c|c|c|c|c|c|c|c|c|c|c|c|c|c|c|c|}
\hline rate $^{E X T i, O U T+1 . \%}$ & 0,5 & 0,8 & 0,5 & 0,8 & 1,0 & 1,0 & 0,9 & 0,9 & 0,9 & 0,9 & 0,9 & 1,0 & 1,0 & 1,0 & 1,0 & 1,0 & 1,0 \\
\hline rate $e^{E X T_{v}, I N+L O C}$ & 0,1 & 0 & 0,1 & 0 & 0,5 & 1,2 & 1,2 & 1,2 & 1,1 & 1,1 & 1,1 & 0 & 0 & 0 & 0 & 1,2 & 0 \\
\hline rate $e^{E X T_{p}, t h}$ & 0,6 & 0,8 & 0,6 & 0,8 & 1,5 & 2,2 & 2,1 & 2,1 & 2,0 & 2,0 & 2,0 & 1,0 & 1,0 & 1,0 & 1,0 & 2,2 & 1,0 \\
\hline
\end{tabular}

Tabelle 79: Gesamtbedeutungen der externen Knoten für die internen Knoten

\begin{tabular}{|c|c|c|c|c|}
\hline & Knoten & rate $^{E X w_{w}, 0 U T}$ & rate $^{E X T v_{,}, I N}$ & rate $_{x}^{E X T w_{x}}$ \\
\hline 1. & 91 & 0,01 & 55,22 & 55,23 \\
\hline 2. & 92B & 6,16 & 42,09 & 48,24 \\
\hline 3. & 62 & 0,54 & 38,46 & 39,00 \\
\hline 4. & 65 & 16,83 & 15,09 & 31,92 \\
\hline 5. & 64 & 14,39 & 13,69 & 28,08 \\
\hline 6. & $60 \mathrm{C}$ & 12,94 & 11,15 & 24,09 \\
\hline 7. & $70 \mathrm{~A}$ & 15,11 & 8,93 & 24,04 \\
\hline 8. & $60 \mathrm{~A}$ & 2,82 & 20,92 & 23,75 \\
\hline 9. & $74 \mathrm{~A}$ & 15,28 & 7,95 & 23,23 \\
\hline 10. & $40 \mathrm{~B}$ & 12,91 & 8,60 & 21,51 \\
\hline
\end{tabular}




\begin{tabular}{|c|c|c|c|c|}
\hline & Knoten & relle $e^{\prime \backslash 1, .11 !}$ & rate $e^{l \backslash /, ~}$ & rater, ${ }^{\prime 1 /}$ \\
\hline 11. & $74 \mathrm{D}$ & 15,82 & 4,50 & 20,32 \\
\hline 12. & $40 A$ & 15,14 & 5,09 & 20,23 \\
\hline 13. & 25 & 10,53 & 9,00 & 19,53 \\
\hline 14. & 71 & 15,56 & 2,69 & 18,25 \\
\hline 15. & $92 A$ & 6,16 & 12,01 & 18,18 \\
\hline 16. & 23 & 10,97 & 6,94 & 17,91 \\
\hline 17. & 32 & 1,03 & 16,30 & 17,33 \\
\hline 18. & $51 \mathrm{~B}$ & 12,34 & 4,32 & 16,66 \\
\hline 19. & 66 & 6,56 & 6,74 & 13,30 \\
\hline 20. & 36 & 6,17 & 5,12 & 11,29 \\
\hline 21. & 01 & 4,06 & 6,47 & 10,53 \\
\hline 22. & 93 & 2,16 & 7,29 & 9,44 \\
\hline 23. & 37 & 4,93 & 4,24 & 9,18 \\
\hline 24. & 90 & 2,30 & 6,78 & 9,08 \\
\hline 25. & 26 & 6,41 & 2,65 & 9,06 \\
\hline 26. & $85 \mathrm{~B}$ & 0 & 8,83 & 8,83 \\
\hline 27. & 19 & 0 & 8,82 & 8,82 \\
\hline 28. & 80 & 0 & 8,73 & 8,73 \\
\hline 29. & 11 & 0 & 8,38 & 8,38 \\
\hline 30. & $70 B$ & 0 & 8,33 & 8,33 \\
\hline 31. & 75 & 0 & 7,48 & 7,48 \\
\hline 32. & $85 A$ & 0 & 7,42 & 7,42 \\
\hline 33. & $63 A$ & 0,03 & 7,29 & 7,32 \\
\hline 34. & 41 & 0 & 7,29 & 7,29 \\
\hline 35. & $52 B$ & 0,06 & 7,05 & 7,11 \\
\hline 36. & $51 \mathrm{~A}$ & 0 & 6,41 & 6,41 \\
\hline 37. & 18 & 0 & 6,07 & 6,07 \\
\hline 38. & 61 & 0 & 6,04 & 6,04 \\
\hline 39. & 31 & 1,38 & 4,49 & 5,87 \\
\hline 40. & 15 & 2,16 & 3,35 & 5,51 \\
\hline 41. & 05 & 0 & 4,99 & 4,99 \\
\hline 42. & 33 & 1,00 & 3,80 & 4,80 \\
\hline 43. & $50 \mathrm{~B}$ & 1,53 & 2,81 & 4,33 \\
\hline 44. & 16 & 0 & 3,78 & 3,78 \\
\hline 45. & $40 \mathrm{C}$ & 0 & 3,74 & 3,74 \\
\hline 46. & 30 & 0 & 3,71 & 3,71 \\
\hline 47. & 73 & 0 & 3,70 & 3,70 \\
\hline 48. & $63 C$ & 0,07 & 3,33 & 3,40 \\
\hline 49. & 34 & 0 & 3,12 & 3,12 \\
\hline 50. & 60B & 0,41 & 2,55 & 2,95 \\
\hline
\end{tabular}




\begin{tabular}{|c|c|c|c|c|}
\hline & Knoten & $r a t e^{\prime \backslash R . d I I ~}$ & $r a t e^{\prime 1 / . ハ ~}$ & renter, \\
\hline 53. & $50 \mathrm{~A}$ & 0 & 2,40 & 2,40 \\
\hline 54. & 67 & 0 & 2,32 & 2,32 \\
\hline 55. & 10 & 0 & 2,31 & 2,31 \\
\hline 56. & 95 & 0 & 0 & 0 \\
\hline
\end{tabular}

Tabelle 80 beschreibt die Veränderung der bisherigen Bedeutung der internen Knoten durch Hinzunahme des externen Knoten 70A und seiner Kanten in ihrer Rolle als Nachfolger (rate $e_{i n t}^{E X X v_{x}, O U T}$ ), Vorgänger ( rate $_{i n t}^{E X T v_{x}, I N}$ ) und gesamt $\left(\right.$ rate $\left._{i n t}^{L X T v_{x}}\right)$. Während alle internen Knoten einen (wenn auch teilweise sehr geringen) Bedeutungszuwachs als Nachfolger aufweisen, gibt es hinsichtlich der Bedeutung als Vorgänger für einzelne interne Knoten Verluste durch die Einbindung des externen Knoten 70A (z.B. Knoten 22). Dies macht erneut ersichtlich, dass der Knoten 70A insbesondere als Vorgänger (Anbieter) in Erscheinung treten kann. Insgesamt hat die Erweiterung der lokalen Struktur aufgrund der starken Zuwächse der Vorgängerbedeutung der Knoten 28, 27, 45B und 45A dennoch eine stärkere positive Auswirkung auf die Bedeutung der internen Knoten als Vorgänger denn als Nachfolger.

Tabelle 80: relativer Bedeutungsgewinn/-verlust durch den externen Knoten

$\begin{array}{llllllllllllllllll}02 & 17 & 20 & 21 & 22 & 24 & 27 & 28 & 29 & 45 \mathrm{~A} & 45 \mathrm{~B} & 52 \mathrm{~A} & 55 & 63 \mathrm{~B} & 72 & 74 \mathrm{~B} & 74 \mathrm{C} & \Sigma\end{array}$

\begin{tabular}{|c|c|c|c|c|c|c|c|c|c|c|c|c|c|c|c|c|c|c|}
\hline Ith & 1,2 & 0 & 0,4 & $-0,2$ & $-1,6$ & 0,1 & 11,3 & 1,4 & 6,0 & 10,3 & 11,2 & 0 & $-0,1$ & 0 & 0 & 0,6 & 0 & 49,6 \\
\hline I & 0,0 & 0,2 & 0,1 & 0,1 & , & 0,2 & 0,1 & 0,3 & 0,5 & 0,5 & 0,4 & 4,4 & 2,0 & 2,1 & 0,9 & 0,2 & 0,3 & \\
\hline ratc & 0,2 & 0,2 & 0,5 & $-0,2$ & $-1,4$ & 0,3 & 11,4 & 1,7 & 6,5 & 10,8 & 11 , & 4,4 & 1,9 & 2,1 & 0,9 & 0,8 & 0,3 & \\
\hline
\end{tabular}

Tabelle 81 zeigt die erforderlichen relativen Anpassungen der Transaktionsvolumina der in der ursprünglichen lokalen Struktur bereits bestehenden Nachfolger- bzw. Vorgängerknoten zur Erreichung der aus der globalen Struktur abgeleiteten Zielwerte bei Eingliederung des externen Knoten 70A. Erwartungsgemäß ergibt sich für die internen Knoten vor allem das Erfordernis zur Änderung der Inputvolumina $\left(70 \mathrm{~A}-\Delta v o l^{t X T-x}\right)$. Hiervon sind insbesondere die Nachfolgerknoten 72 und 52A betroffen. Angebotsseitig $\left(70 \mathrm{~A}-\Delta v o l^{* E X T-x}\right)$ sind kaum nennenswerte Anpassungen notwendig. Handelt es sich bei dem hinzugenommenen externen Knoten 70A um den einzigen Vorgänger bzw Nachfolger 
eines internen Knoten, so ist der jeweilige Input- bzw. Outputänderungsbedarf unbestimmt, wie z.B. für den internen Nachfolgerknoten 55. Für den internen Knoten 74B, der in der ursprünglichen lokalen Struktur isoliert war, kann weder der Input- noch der Outputänderungbedarf ermittelt werden, da der neue Knoten $70 \mathrm{~A}$ für diesen internen Knoten den einzigen Nachfolger und auch Vorgänger darstellt.

Tabelle 81: Input- und Output-Änderungsbedarf der lokalen Struktur

$\begin{array}{lllllllllllllllll}02 & 17 & 20 & 21 & 22 & 24 & 27 & 28 & 29 & 45 A & 45 B & 52 A & 55 & 63 B & 72 & 74 B & 74 C\end{array}$

\begin{tabular}{|c|c|c|c|c|c|c|c|c|c|c|c|c|c|c|c|c|c|}
\hline $70 \mathrm{~A}-\Delta v \boldsymbol{v} l^{E X T-x}$ & 0,0 & 0,3 & 0,1 & 0,0 & Inf & 0,2 & 0,1 & 0,1 & 0,1 & 0,1 & 0,1 & 1,8 & Inf & 0,1 & 2,5 & Inf & 0,1 \\
\hline$A-\Delta v o l^{* E X T-x}$ & 0 & 0 & 0,1 & 0 & 0,0 & Inf & 0 & 0,0 & 0,1 & Inf & $\operatorname{lnf}$ & 0 & 0 & 0 & 0 & Inf & \\
\hline
\end{tabular}

\subsection{Umlegung der Lückenbewertung}

Zur Ermöglichung der Ableitung von Handlungsempfehlungen auf der Ebene der an der Plattform teilnehmenden Unternehmen ist die Umlegung der gewonnenen Lückenbewertungen von der Güterebene auf die Ebene der Plattformteilnehmer erforderlich.

\subsubsection{Umlegung der Bewertung interner Lücken}

Die Umlegung der Bewertung für interne Lücken erfolgt auf Basis der Matrix $B C$ Igugu bzw. der entsprechenden Kantenliste BCleFgugu, die sämtliche theoretisch möglichen Transaktionsbeziehungen zwischen Plattformteilnehmern gegliedert nach dem Transaktionsgut sowie dem Gut des Käufers, für dessen Produktion die Transaktion wahrscheinlich durchgeführt wird. Die nach Maßgabe von Kapitel 9 in Tabelle 82 dargestellte, auf die tatsächlich auf der Plattform ausgeführten Transaktionen reduzierte Kantenliste $B C l e F g u g u^{T R A N S}$ umfasst daher die Werte der Transaktionen zwischen den Unternehmen in ihrer Rolle als Verkäufer und Käufer auf der Plattform gegliedert nach dem Transaktionsgut (vGut) und dem wahrscheinlichen produktionsbezogenen Zweck der Transaktion (zGut). 
Abgesehen von der Spalte zGut entspricht die Kantenliste BCleFgugu ${ }^{\text {TRANS }}$ der Liste der Transaktionen BCledgeFRuug aus Tabelle 34.

Tabelle 82: BCleFgugu TRANS $^{\text {BR }}$

\begin{tabular}{|c|c|c|c|c|c|}
\hline & vGut & Verkäufer & zGut & Käufer & Wert \\
\hline 1 & 02 & Forstwirtschaft GmbH & 20 & Holzverarbeitung $\mathrm{GmbH}$ & 28 \\
\hline 2 & 20 & Holzverarbeitung $\mathrm{GmbH}$ & 02 & Forstwirtschaft GmbH & 2 \\
\hline 3 & 20 & Holzverarbeitung $\mathrm{GmbH}$ & 21 & Papier und Chemie $\mathrm{GmbH}$ & 8 \\
\hline 4 & 21 & Papier und Chemie GmbH & 17 & Textil GmbH & 16 \\
\hline 5 & 22 & Druck GmbH & 21 & Papier und Chemie GmbH & 11 \\
\hline$G$ & 22 & Druck GmbH & 24 & Papier und Chemie GmbH & 33 \\
\hline 7 & 22 & Druck GmbH & $52 \mathrm{~A}$ & EDV GmbH & 18,6 \\
\hline 8 & 22 & Druck $\mathrm{GmbH}$ & 72 & EDV GmbH & 1,4 \\
\hline 9 & 22 & Druck GmbH & $74 C$ & Werbeagentur $\mathrm{GmbH}$ & 25 \\
\hline 10 & 27 & Metallverarbeitung $\mathrm{GmbH}$ & 28 & Metallerzeugung $\mathrm{GmbH}$ & 62,2 \\
\hline 11 & 27 & Metallverarbeitung $\mathrm{GmbH}$ & 29 & Maschinenbau GmbH & 35 \\
\hline 12 & 27 & Metallverarbeitung $\mathrm{GmbH}$ & $45 B$ & Metallerzeugung $\mathrm{GmbH}$ & 14,8 \\
\hline 13 & 28 & Metallerzeugung $\mathrm{GmbH}$ & 27 & Metallverarbeitung $\mathrm{GmbH}$ & 1,3 \\
\hline 14 & 28 & Metallerzeugung $\mathrm{GmbH}$ & 29 & Maschinenbau GmbH & 84 \\
\hline 15 & 28 & Metallerzeugung $\mathrm{GmbH}$ & $45 \mathrm{~A}$ & Hoch- und Tiefbau GmbH & 35 \\
\hline 16 & 28 & Metallerzeugung $\mathrm{GmbH}$ & $45 B$ & Bauinstallation $\mathrm{GmbH}$ & 13 \\
\hline 17 & 28 & Metallerzeugung $\mathrm{GmbH}$ & $45 \mathrm{~B}$ & Metallverarbeitung $\mathrm{GmbH}$ & 3,7 \\
\hline 18 & 29 & Maschinenbau GmbH & $45 B$ & Bauinstallation $\mathrm{GmbH}$ & 22 \\
\hline 19 & 29 & Maschinenbau GmbH & $45 \mathrm{~B}$ & Metallerzeugung $\mathrm{GmbH}$ & 6 \\
\hline 2 & 55 & Hotel GmbH & $63 B$ & Reisebüro $\mathrm{GmbH}$ & 14 \\
\hline
\end{tabular}

Die Kantenliste $B C$ CleFgugu wird in weiterer Folge um eine Lückenkennzeichnung erweitert, die die Verbindung zweier Güter auf Unternehmensebene als reguläre Beziehung (Nicht-Performanzlücke, d.h. die bestehende Transaktion erreicht ein zufrieden stellendes Ausmaß), Performanzlücke oder direkte interne Lücke klassifiziert. Im Zuge der Klassifikation wird auch die Priorität der Schließung der jeweiligen Lücke angegeben. Die resultierende erweiterte Kantenliste BCleFgugu ${ }^{e x p}$ zeigt die Tabelle 88 weiter unten. Die Ermittlung der Lückenklassi- 
fikation bzw. der Priorisierung der Verbindungen (Lücken) innerhalb einer Klasse erfolgt über unterschiedliche Zwischenschritte, die nachfolgend erläutert werden.

Zunächst werden anhand der Transaktionsdaten ein- oder beidseitig isolierte Plattformteilnehmer ermittelt. Einseitig isolierte Teilnehmer sind definitionsgemäß Unternehmen, von denen entweder keine Transaktionen ausgehen ( $\mathrm{Biz}^{i s o-s}$ nach Tabelle 83) oder zu denen keine Transaktionen führen (Biz ${ }^{\text {iso-b }}$ nach Tabelle 84), während beidseitig isolierte Teilnehmer in keine der beiden möglichen Richtungen Transaktionen aufweisen und somit in der Schnittmenge der beiden Gruppen von einseitig isolierten Teilnehmern aufscheinen. Dies trifft gemäß der Tabelle 83 und der Tabelle 84 auf die Architekturbüro OEG zu.

Tabelle 83: $\mathrm{Biz}^{\text {iso-s }}$

\begin{tabular}{|c|c|c|c|c|c|c|c|}
\hline$B_{i}{ }^{\text {ism- }}$ & $\begin{array}{c}\text { Architektur } \\
\text { büro OEG }\end{array}$ & $\begin{array}{c}\text { Bauinstallation } \\
\mathrm{GmbH}\end{array}$ & EDV GmbH & $\begin{array}{c}\text { Hoch- und } \\
\text { Tiefbau GmbH }\end{array}$ & $\begin{array}{c}\text { Reisebüro } \\
\mathrm{GmbH}\end{array}$ & $\begin{array}{c}\text { Textil } \\
\mathrm{GmbH}\end{array}$ & $\begin{array}{c}\text { Werbeagentur } \\
\mathrm{GmbH}\end{array}$ \\
\hline
\end{tabular}

Tabelle 84: $\mathrm{Biz}^{i s o-b}$

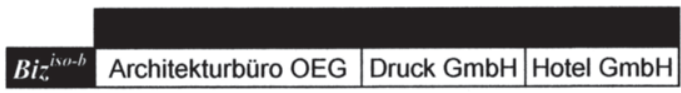

In einem weiteren Schritt werden die auf der Plattform verfügbaren Güter basierend auf whNode ${ }^{O C I}$ bzw. whNode ${ }^{I N}$ (Tabelle 68) in schwache und reguläre Güter unterteilt, um ,schwache' Plattformteilnehmer identifizieren zu können.

Tabelle 85: Com ${ }^{\text {weak-ouT }}$

20

Com ${ }^{\text {meah-olT } 43,4}$

Tabelle 86: Com $^{\text {weak-IN }}$

$\begin{array}{lllll}21 & 27 & 52 \mathrm{~A} & 72 & 74 \mathrm{C}\end{array}$

\begin{tabular}{l|l|l|l|l|l|} 
Com $^{\text {weuk-IN }}$ & 5,8 & 26,6 & 7,5 & 7,5 & 5,5 \\
\hline
\end{tabular}

Tabelle 87: $\mathrm{Biz}^{\text {weak }}$

\begin{tabular}{|c|c|c|c|}
\hline & $\begin{array}{c}\text { Papier und } \\
\text { Chemie GmbH }\end{array}$ & $\begin{array}{c}\text { Werbeagentur } \\
\text { GmbH }\end{array}$ & $\begin{array}{c}\text { Holzverarbeitung } \\
\text { GmbH }\end{array}$ \\
\hline Bizweak- & IN & IN & OUT \\
\hline
\end{tabular}


Tabelle 85 und Tabelle 86 listen die als schwache Vorgänger- und Nachfolgerknoten klassifizierten Güter der Plattform. Die Güter 27, 52A, 72, 21 und 74C sind demgemäß schwache Nachfolger, das Gut 20 ein schwacher Vorgänger. Plattformteilnehmer, die überwiegend schwache (Vorgänger-)Güter absetzen bzw. schwache (Nachfolger-)Güter beziehen, werden nun selbst als schwache Verkäufer (Biz ${ }^{\text {weak-OIIT }}$ in Tabelle 87 ) bzw. schwache Käufer (Biz ${ }^{\text {weak-IN }}$ in Tabelle 87) bezeichnet. Hierbei handelt es sich um die Unternehmen Holzverarbeitung $\mathrm{GmbH}$ auf Verkäuferseite sowie Papier und Chemie $\mathrm{GmbH}$ und Werbeagentur $\mathrm{GmbH}$ auf der Käuferseite.

Unter Einbezug dieser Differenzierung in schwache und reguläre Verkäufer und/oder Käufer bzw. in - aus Verkäufer- und/oder Käufersicht - isolierte Plattformteilnehmer einerseits und der Unterscheidung von Performanz- und NichtPerformanz-Lücken bzw. direkten internen Lücken (unter Verwendung der Matrizen PerfGap ${ }^{+ \text {binary }}$ bzw. DiGap ${ }^{\text {binary }}$ ) sowie der Bewertung der direkten internen Lücken auf Basis der Matrix Rate ${ }^{D L L}$ andererseits erfolgt nun die Klassifikation und Priorisierung der Transaktionsverläufe aus BCleFgugu. Tabelle 88 zeigt die um diese Information erweiterte Kantenliste BCleFgugu ${ }^{\text {exp }}$. Insgesamt werden 11 Performanzlücken, 9 Nicht-Performanzlücken und 101 direkte interne Lücken identifiziert. Von den 11 Performanzlücken, die beinahe zur Hälfte die Metallerzeugung $\mathrm{GmbH}$ als Verkäufer betreffen, wird aufgrund der festgelegten Schwellwerte nur eine Verbindung als weniger prioritär $\left(P^{\prime}\right)$ gekennzeichnet, während den anderen 10 Verbindungen mit dem Lückentyp $P$ eine höhere Priorität zugewiesen wird.

Die Nicht-Performanzlücken werden in zwei Prioritätsklassen untergliedert, da keine dieser Lücken der vorrangig zu behandelnden Kategorie $N P^{J I L}$ zugeordnet wird. Diese wäre dadurch gekennzeichnet, dass zwar die Verbindung zwischen dem Transaktionsgut und dem daraus produzierbaren Gut auf der Plattform im Vergleich zur Gesamtwirtschaft ausreichend stark ist, jedoch keine diese beiden Güter betreffenden Transaktionen zwischen den Plattformteilnehmern der ent-

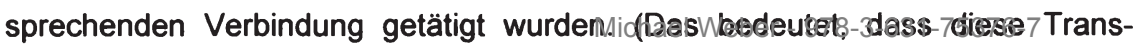


aktion(en) zwischen anderen Unternehmen erfolgt sein müssen.) 8 NichtPerformanzlücken werden als $N P^{-}$klassifiziert, da der Transaktionswert eine bestimmte Schranke unterschreitet. Die Metallverarbeitung $\mathrm{GmbH}(3 \mathrm{x})$ und die Druck $\mathrm{GmbH}(2 \mathrm{x})$ treten in dieser Kategorie häufig als Verkäufer auf, während die Metallerzeugung $\mathrm{GmbH}$ in drei von acht Verbindungen als Käufer vorkommt. Nur eine Nicht-Performanzlücke wird in die Klasse $N P^{+}$, die die geringste Priorität unter den Nicht-Performanzlücken hat, eingeordnet.

Eine Betrachtung der Verteilung der 101 direkten internen Lücken auf die fünf definierten Prioritätsklassen zeigt, dass die beiden Klassen mit der höchsten $\left(D I L^{i s g}\right)$ bzw. geringsten $\left(D I L^{\circ}\right)$ Priorität am häufigsten auftreten und gemeinsam mehr als drei Viertel der Lücken ausmachen. Etwa die Hälfte der direkten internen Lücken ist durch (zumindest einseitig) isolierte Plattformteilnehmer bedingt, während ca. $30 \%$ der Lücken dieses Lückentyps zwar grundsätzlich direkte interne Lücken darstellen, jedoch aufgrund ihrer geringen Bewertung in Rate ${ }^{D I L}$ als nicht prioritär zu behandelnd eingestuft werden. Die verbleibenden 20 direkten internen Lücken gliedern sich in eine Lücke der Kategorie $D I L, 11$ Lücken der Kategorie $D I L^{+}$und 8 Lücken der Kategorie $D I L^{\sim}$. DIL enthält direkte interne Lücken, die sowohl einen schwachen Käufer als auch einen schwachen Verkäufer betreffen. $D I L^{\sim}$ hingegen umfasst Lücken mit entweder einem schwachen Käufer oder einem schwachen Verkäufer, und die Lücken der Kategorie $D I L^{*}$ bestehen zwischen regulären (d.h. nicht als schwach klassifizierten) Unternehmen. Insgesamt sind in die meisten (knapp 85\%) der direkten internen Lücken, die aufgrund ihrer Bewertung nicht als $D I L^{o}$ klassifiziert werden, entweder (zumindest einseitig) isolierte oder schwache Plattformteilnehmer involviert. 
Tabelle 88: BCIeFgugu ${ }^{\text {exp }}$

vonAoG

vonUnt

nachAoG

nachUnt

Wert Lücke

\begin{tabular}{|c|c|c|c|c|c|c|}
\hline 1 & 02 & Forstwirtschaft GmbH & 20 & Holzverarbeitung $\mathrm{GmbH}$ & 28,00 & Pmin \\
\hline 2 & 20 & Holzverarbeitung $\mathrm{GmbH}$ & 21 & Papier und Chemie $\mathrm{GmbH}$ & 8,00 & Pmin \\
\hline 3 & 22 & Druck GmbH & $52 \mathrm{~A}$ & EDV GmbH & 18,55 & Pmin \\
\hline 4 & 22 & Druck GmbH & 72 & EDV GmbH & 1,45 & Pmin \\
\hline 5 & 22 & Druck GmbH & $74 \mathrm{C}$ & Werbeagentur $\mathrm{GmbH}$ & 25,00 & $P \min$ \\
\hline 6 & 28 & Metallerzeugung $\mathrm{GmbH}$ & 45B & Metallverarbeitung $\mathrm{GmbH}$ & 3,67 & Pmin \\
\hline 7 & 28 & Metallerzeugung $\mathrm{GmbH}$ & 27 & Metallverarbeitung $\mathrm{GmbH}$ & 1,33 & Pmin \\
\hline 8 & 28 & Metallerzeugung $\mathrm{GmbH}$ & $45 \mathrm{~A}$ & Hoch- und Tiefbau GmbH & 35,00 & Pmin \\
\hline 9 & 28 & Metallerzeugung $\mathrm{GmbH}$ & 29 & Maschinenbau GmbH & 84,00 & Pmin \\
\hline 10 & 55 & Hotel GmbH & 63B & Reisebüro GmbH & 14,00 & Pmin \\
\hline 11 & 28 & Metallerzeugung $\mathrm{GmbH}$ & 45B & Bauinstallation $\mathrm{GmbH}$ & 13,00 & Pplus \\
\hline 12 & 20 & Holzverarbeitung $\mathrm{GmbH}$ & 02 & Forstwirtschaft GmbH & 2,00 & NPmin \\
\hline 13 & 21 & Papier und Chemie GmbH & 17 & $\mathrm{imbH}$ & 16,00 & NPmin \\
\hline 14 & 22 & Druck & 21 & Papier und & 10,95 & NPmin \\
\hline 15 & 22 & Druck & 24 & Papier und Chemie $\mathrm{GmbH}$ & 33,05 & NPmin \\
\hline 16 & 27 & Metallver & 45B & Meta & 14,85 & NPmin \\
\hline 17 & 27 & Met & 29 & & 35,00 & NPmin \\
\hline 18 & 27 & Metallverarbeitung $\mathrm{GmbH}$ & 28 & Metallerzeugung $\mathrm{GmbH}$ & 62,15 & NPmin \\
\hline 19 & 29 & Maschinenbau GmbH & 45B & Metallerzeugung $\mathrm{GmbH}$ & 6,00 & NPmin \\
\hline 20 & 29 & Maschinenbau $\mathrm{GmbH}$ & 45B & Bauinstallation $\mathrm{GmbH}$ & 22,00 & NPplus \\
\hline 21 & 17 & Textil GmbH & 45B & Bauins & 0 & DILiso \\
\hline 22 & 17 & Textil GmbH & 45B & Metallerzeugung GmbH & 0 & DILiso \\
\hline 23 & 17 & Textil & 45B & Metallver & 0 & DILiso \\
\hline 24 & 21 & Papier und Chemie $\mathrm{GmbH}$ & 22 & 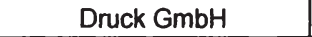 & 0 & DILiso \\
\hline 25 & 21 & Papier und Chemie $\mathrm{GmbH}$ & 74B & Architekturbüro OEG & 0 & DILiso \\
\hline 26 & 22 & Druck GmbH & $74 \mathrm{~B}$ & Architekturbüro OEG & 0 & DILiso \\
\hline 27 & 22 & Druck GmbH & 55 & Hotel GmbH & 0 & DILiso \\
\hline 28 & 24 & Papier und Chemie $\mathrm{GmbH}$ & 22 & Druck GmbH & 0 & DILiso \\
\hline 29 & 28 & Metallerzeugung $\mathrm{GmbH}$ & $74 \mathrm{~B}$ & Architekturbüro OEG & 0 & DILiso \\
\hline 30 & 28 & Metallerzeugung $\mathrm{GmbH}$ & 55 & Hotel GmbH & 0 & DILiso \\
\hline 31 & 29 & Maschinenbau GmbH & 22 & Druck GmbH & 0 & DILiso \\
\hline 32 & $45 A$ & Hoch- und Tiefbau GmbH & 45B & Bauinstallation $\mathrm{GmbH}$ & 0 & DILiso \\
\hline 33 & $45 A$ & Hoch- und Tiefbau GmbH & 45B & Metallerzeugung $\mathrm{GmbH}$ & 0 & DILiso \\
\hline 34 & $45 A$ & Hoch- und Tiefbau GmbH & 45B & Metallverarbeitung $\mathrm{GmbH}$ & 0 & DILiso \\
\hline 35 & $45 A$ & Hoch- und Tiefbau GmbH & 20 & Holzverarbeitung $\mathrm{GmbH}$ & 0 & DILiso \\
\hline 36 & $45 A$ & Hoch- und Tiefbau GmbH & 72 & EDV GmbH & 0 & DILiso \\
\hline 37 & $45 A$ & Hoch- und Tiefbau GmbH & 02 & Forstwirtschaft $\mathrm{GmbH}$ & 0 & DILiso \\
\hline 38 & $45 \mathrm{~A}$ & Hoch- und Tiefbau GmbH & 55 & Hotel GmbH & 0 & DILiso \\
\hline 39 & $45 A$ & Hoch- und Tiefbau GmbH & 28 & Metallerzeugung $\mathrm{GmbH}$ & 0 & DILiso \\
\hline 40 & 45B & Bauinstallation $\mathrm{GmbH}$ & $45 \mathrm{~A}$ & Hoch- und Tieftay GmbH & 537 & DILiso \\
\hline
\end{tabular}


vonAoG

vonUnt

nachAoG

nachUnt

Wert Lücke

\begin{tabular}{|c|c|c|c|c|c|c|}
\hline 41 & 45B & Bauinstallation $\mathrm{GmbH}$ & 72 & EDV GmbH & 0 & DILiso \\
\hline 42 & 45B & Bauinstallation $\mathrm{GmbH}$ & 02 & Forstwirtschaft GmbH & 0 & DILiso \\
\hline 43 & $45 B$ & Bauinstallation $\mathrm{GmbH}$ & 55 & Hotel GmbH & 0 & DILiso \\
\hline 44 & $45 B$ & Metallerzeugung GmbH & 55 & Hotel GmbH & 0 & DILiso \\
\hline 45 & $45 B$ & Metallverarbeitung GmbH & 55 & Hotel GmbH & 0 & DILiso \\
\hline 46 & $45 B$ & Bauinstallation GmbH & $74 C$ & Werbeagentur GmbH & 0 & DILiso \\
\hline 47 & 55 & Hotel GmbH & $74 \mathrm{~B}$ & Architekturbüro OEG & 0 & DILiso \\
\hline 48 & 72 & EDV GmbH & 21 & Papier und Chemie GmbH & 0 & DILiso \\
\hline 49 & 72 & EDV GmbH & 27 & Metallverarbeitung $\mathrm{GmbH}$ & 0 & DILiso \\
\hline 50 & 72 & EDV GmbH & 20 & Holzverarbeitung GmbH & 0 & DILiso \\
\hline 51 & 72 & EDV GmbH & 22 & Druck GmbH & 0 & DILiso \\
\hline 52 & 72 & EDV GmbH & 24 & Papier und Chemie GmbH & 0 & DILiso \\
\hline 53 & 72 & EDV GmbH & $52 A$ & EDV GmbH & 0 & DILiso \\
\hline 54 & 72 & EDV GmbH & $74 B$ & Architekturbüro OEG & 0 & DILiso \\
\hline 55 & 72 & EDV GmbH & 17 & Textil GmbH & 0 & DILiso \\
\hline 56 & 72 & EDV GmbH & 29 & Maschinenbau GmbH & 0 & DILiso \\
\hline 57 & 72 & EDV GmbH & 55 & Hotel GmbH & 0 & DILiso \\
\hline 58 & 72 & EDV GmbH & $63 B$ & Reisebüro GmbH & 0 & DILiso \\
\hline 59 & 72 & EDV GmbH & 28 & Metallerzeugung $\mathrm{GmbH}$ & 0 & DILiso \\
\hline 60 & 72 & EDV GmbH & $74 \mathrm{C}$ & Werbeagentur GmbH & 0 & DILiso \\
\hline 61 & $74 B$ & Architekturbüro OEG & $45 \mathrm{~A}$ & Hoch- und Tiefbau GmbH & 0 & DILiso \\
\hline 62 & $74 \mathrm{C}$ & Werbeagentur GmbH & 27 & Metallverarbeitung GmbH & 0 & DILiso \\
\hline 63 & $74 \mathrm{C}$ & Werbeagentur GmbH & 22 & Druck GmbH & 0 & DILiso \\
\hline 64 & $74 \mathrm{C}$ & Werbeagentur GmbH & 24 & Papier und Chemie GmbH & 0 & DILiso \\
\hline 65 & $74 \mathrm{C}$ & Werbeagentur GmbH & $52 \mathrm{~A}$ & EDV GmbH & 0 & DILiso \\
\hline 66 & $74 \mathrm{C}$ & Werbeagentur $\mathrm{GmbH}$ & 72 & EDV GmbH & 0 & DILiso \\
\hline 67 & $74 \mathrm{C}$ & Werbeagentur GmbH & $74 B$ & Architekturbüro OEG & 0 & DILiso \\
\hline 68 & $74 C$ & Werbeagentur $\mathrm{GmbH}$ & 17 & Textil GmbH & 0 & DILiso \\
\hline 69 & $74 C$ & Werbeagentur GmbH & 29 & Maschinenbau GmbH & 0 & DILiso \\
\hline 70 & $74 C$ & Werbeagentur GmbH & $63 B$ & Reisebüro GmbH & 0 & DILiso \\
\hline 71 & $74 \mathrm{C}$ & Werbeagentur GmbH & 28 & Metallerzeugung GmbH & 0 & DILiso \\
\hline 72 & 21 & Papier und Chemie GmbH & 20 & Holzverarbeitung GmbH & 0 & DILmin \\
\hline 73 & 20 & Holzverarbeitung GmbH & 27 & Metallverarbeitung GmbH & 0 & DILtilde \\
\hline 74 & 24 & Papier und Chemie $\mathrm{GmbH}$ & 02 & Forstwirtschaft GmbH & 0 & DILtilde \\
\hline 75 & 24 & Papier und Chemie GmbH & 28 & Metallerzeugung GmbH & 0 & DILtilde \\
\hline 76 & 28 & Metallerzeugung GmbH & 20 & Holzverarbeitung GmbH & 0 & DILtilde \\
\hline 77 & 29 & Maschinenbau GmbH & 21 & Papier und Chemie $\mathrm{GmbH}$ & 0 & DILtilde \\
\hline 78 & 29 & Maschinenbau GmbH & 20 & Holzverarbeitung GmbH & 0 & DILtilde \\
\hline 79 & $45 B$ & Metallerzeugung GmbH & $74 C$ & Werbeagentur GmbH & 0 & DILtilde \\
\hline 80 & $45 \mathrm{~B}$ & Metallverarbeitung GmbH & $74 \mathrm{C}$ & Werbeagentur GmbH & 0 & DILtilde \\
\hline 81 & 22 & Druck GmbH & 02 & Forstwirtschaft GmbH & 0 & DILplus \\
\hline
\end{tabular}


vonAoG

vonUnt

nachAoG

nachUnt

Wert Lücke

\begin{tabular}{|c|c|c|c|c|c|c|}
\hline 82 & 28 & Metallerzeugung GmbH & 02 & Forstwirtschaft GmbH & 0 & DILplus \\
\hline 83 & 29 & Maschinenbau GmbH & 27 & Metallverarbeitung $\mathrm{GmbH}$ & 0 & DILplus \\
\hline 84 & 29 & Maschinenbau GmbH & 02 & Forstwirtschaft $\mathrm{GmbH}$ & 0 & DILple \\
\hline 85 & 45B & Metallerzeugung $\mathrm{GmbH}$ & $45 \mathrm{~A}$ & Hoch- und Tiefbau GmbH & 0 & DILplu: \\
\hline 86 & 45B & Metallverarbeitung $\mathrm{GmbH}$ & $45 \mathrm{~A}$ & Hoch- und Tiefbau GmbH & 0 & DILplu \\
\hline 87 & 45B & Metallerzeugung $\mathrm{GmbH}$ & 72 & EDV GmbH & 0 & DILplt \\
\hline 88 & 45B & Metallverarbeitung $\mathrm{GmbH}$ & 72 & EDV GmbH & 0 & DILplt \\
\hline 89 & 45B & Metallerzeugung $\mathrm{GmbH}$ & 02 & Forstwirtschaft $\mathrm{GmbH}$ & 0 & DILpI \\
\hline 90 & 45B & Metallverarbeitung $\mathrm{GmbH}$ & 02 & Forstwirtschaft GmbH & 0 & DILpli \\
\hline 91 & 55 & Hotel GmbH & 28 & Metallerzeugung $\mathrm{GmbH}$ & 0 & DILplu \\
\hline 92 & 02 & Forstwirtschaft GmbH & 21 & Papier und Chemie $\mathrm{GmbH}$ & 0 & DILZ \\
\hline 93 & 20 & Holzverarbeitung $\mathrm{GmbH}$ & 45B & Bauinstallation $\mathrm{GmbH}$ & 0 & \\
\hline 94 & 20 & Holzverarbeitung $\mathrm{GmbH}$ & 45B & Metallerzeugung $\mathrm{GmbH}$ & 0 & DIL \\
\hline 95 & 20 & Holzverarbeitung $\mathrm{GmbH}$ & 45B & Metallverarbeitung $\mathrm{GmbH}$ & 0 & $D$ \\
\hline 96 & 20 & Holzverarbeitung GmbH & $45 \mathrm{~A}$ & Hoch- und Tiefbau GmbH & 0 & DILZ \\
\hline 97 & 21 & Papier und Chemie $\mathrm{GmbH}$ & 45B & Bauinstallation $\mathrm{GmbH}$ & 0 & DILze \\
\hline 98 & 21 & Papier und Chemie $\mathrm{GmbH}$ & 45B & Metallerzeugung $\mathrm{GmbH}$ & 0 & DILZ \\
\hline 99 & 21 & Papier und Chemie $\mathrm{GmbH}$ & 45B & Metallverarbeitung $\mathrm{GmbH}$ & 0 & DILze \\
\hline 100 & 21 & Papier und Chemie $\mathrm{GmbH}$ & 24 & Papier und Chemie $\mathrm{GmbH}$ & 0 & DII \\
\hline 01 & 21 & Papier und Chemie $\mathrm{GmbH}$ & $52 \mathrm{~A}$ & EDV GmbH & 0 & $\Delta$ \\
\hline 102 & 21 & Papier und Chemie $\mathrm{GmbH}$ & 72 & EDV GmbH & 0 & 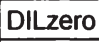 \\
\hline & 22 & & $45 \mathrm{~A}$ & Hoch- und Tiefbau GmbH & 0 & LIL \\
\hline 104 & 22 & Druck GmbH & 17 & & 0 & DILZ \\
\hline 105 & 22 & Druck GmbH & 29 & Maschinenbau GmbH & 0 & DILze \\
\hline 06 & 22 & Druck GmbH & $63 B$ & Reisebüro $\mathrm{GmbH}$ & 0 & DILZ \\
\hline 107 & 24 & Papier und Chemie $\mathrm{GmbH}$ & 21 & Papier und Chemie $\mathrm{GmbH}$ & 0 & DILZ \\
\hline 108 & 24 & Papier und Chemie $\mathrm{GmbH}$ & 45B & Bauinstallation $\mathrm{GmbH}$ & 0 & DILze \\
\hline 109 & 24 & Papier und Chemie $\mathrm{GmbH}$ & 45B & Metallerzeugung $\mathrm{GmbH}$ & 0 & DILze \\
\hline 110 & 24 & Papier und Chemie $\mathrm{GmbH}$ & 45B & Metallverarbeitung $\mathrm{GmbH}$ & 0 & DILze \\
\hline 111 & 27 & Metallverarbeitung $\mathrm{GmbH}$ & $45 A$ & Hoch- und Tiefbau GmbH & 0 & DILZ \\
\hline 12 & 28 & Metallerzeugung $\mathrm{GmbH}$ & $52 \mathrm{~A}$ & EDV GmbH & 0 & DILZ \\
\hline 113 & 29 & Maschinenbau GmbH & 24 & Papier und Chemie $\mathrm{GmbH}$ & 0 & DILz \\
\hline 114 & 29 & Maschinenbau GmbH & 17 & Textil GmbH & 0 & DILZ \\
\hline 115 & 55 & Hotel GmbH & 45B & Bauinstallation $\mathrm{GmbH}$ & 0 & DILze \\
\hline 116 & 55 & Hotel GmbH & 45B & Metallerzeugung $\mathrm{GmbH}$ & 0 & DILzer \\
\hline 117 & 55 & Hotel GmbH & 45B & Metallverarbeitung $\mathrm{GmbH}$ & 0 & DILze \\
\hline 118 & 55 & Hotel GmbH & $52 \mathrm{~A}$ & EDV GmbH & 0 & DILze \\
\hline 119 & 55 & Hotel $\mathrm{GmbH}$ & 72 & EDV GmbH & 0 & DILze \\
\hline 120 & 55 & Hotel GmbH & 29 & Maschinenbau GmbH & 0 & DILze \\
\hline 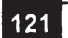 & 55 & Hotel GmbH & $74 \mathrm{C}$ & Werbeagentur GmbH & 0 & DILZ \\
\hline
\end{tabular}




\subsubsection{Umlegung der Effekte externer Knoten}

Die Umlegung der Effekte der Hinzunahme eines externen, d.h. auf der Plattform noch nicht bereitgestellten, Gutes auf die Ebene der Plattformteilnehmer und damit die Abschätzung der möglichen teilnehmerbezogenen Wirkung einer derartigen Plattformerweiterung kann auf der Grundlage der direkten und indirekten Vorgänger- und Nachfolgerbedeutung des externen Gutes aus rate ${ }^{E X T v_{x}, 4 L C C}$ sowie rate ${ }^{E X T v_{x}, I N 4 L O C}$ und rate $e^{E X T v_{x}, O U T 4 L O C}$ auf drei unterschiedliche Arten erfolgen. Tabelle 89 zeigt die anhand der Teilnehmerprofile in BCIprofile (Tabelle 33) aus rate ${ }^{E X T v_{x}, 4 L O C}$ (Tabelle 78) abgeleitete Bewertung der Effekte der Einbindung des externen Gutes 70A. Demzufolge würden die Unternehmen Metallverarbeitung $\mathrm{GmbH}$, Metallerzeugung $\mathrm{GmbH}$ und Papier und Chemie $\mathrm{GmbH}$ am stärksten von dieser Plattformerweiterung profitieren. An vierter Stelle der Nutznießer steht die ursprünglich völlig isolierte Architekturbüro $\mathrm{GmbH}$.

Tabelle 89: Bedeutung des externen Gutes 70A für die Plattformteilnehmer

\begin{tabular}{|c|c|c|}
\hline & Teilnehmer & Bedeutung 70A \\
\hline 6 & Metallverarbeitung $\mathrm{GmbH}$ & 4,112 \\
\hline 7 & Metallerzeugung GmbH & 4,082 \\
\hline 5 & Papier und Chemie GmbH & 3,012 \\
\hline 14 & Architekturbüro OEG & 2,221 \\
\hline 8 & Maschinenbau $\mathrm{GmbH}$ & 2,039 \\
\hline 9 & Hoch- und Tiefbau GmbH & 2,034 \\
\hline 10 & Bauinstallation $\mathrm{GmbH}$ & 2,004 \\
\hline 13 & EDV GmbH & 2,000 \\
\hline 4 & Druck GmbH & 1,468 \\
\hline 11 & Hotel GmbH & 1,000 \\
\hline 12 & Reisebüro $\mathrm{GmbH}$ & 1,000 \\
\hline 15 & Werbeagentur $\mathrm{GmbH}$ & 1,000 \\
\hline 2 & Textil GmbH & 0,844 \\
\hline 3 & Holzverarbeitung GmbH & 0,623 \\
\hline 1 & Forstwirtschaft $\mathrm{GmbH}$ & 0,604 \\
\hline
\end{tabular}

Wird die Bewertung der Auswirkungen der Hinzunahme des externen Gutes 70A für einen Plattformteilnehmer durch Gewichtung der Bedeutung des externen Gutes für ein bereitgestelltes Gut aus Tabelle 78 mit dem Anteil des bereit- 
gestellten Gutes an den gesamten Verkäufen bzw. Käufen des Plattformteilnehmers ermittelt, so erhält man Tabelle 90. Diese umfasst die Bewertung der Effekte der Plattformenweiterung für die bestehenden Teilnehmer als Verkäufer, Käufer und kombiniert. Neben der Metallverarbeitung $\mathrm{GmbH}$ und der Metallerzeugung $\mathrm{GmbH}$ befindet sich nun sowohl aus Käufer- als auch aus der kombinierten Perspektive die Maschinenbau GmbH unter den Top 3, während aus Verkäufersicht die Reisebüro $\mathrm{GmbH}$, die EDV $\mathrm{GmbH}$ und die Werbeagentur $\mathrm{GmbH}$ an der Spitze liegen. Bei der Interpretation der Bewertungen in Tabelle 90 ist zu beachten, dass die Bewertung der Auswirkung der Plattformerweiterung für (einseitig) isolierte Unternehmen nicht bzw. nur einseitig definiert ist.

Tabelle 90: gewichtete Bedeutung des externen Gutes 70A (Variante 1)

\begin{tabular}{|c|c|c|c|c|}
\hline & Teilnehmer & 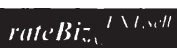 & 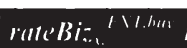 & 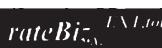 \\
\hline 1 & Forstwirtschaft GmbH & 0,080 & 0,524 & 0,604 \\
\hline 2 & Textil GmbH & - & 0,844 & - \\
\hline 3 & Holzverarbeitung $\mathrm{GmbH}$ & 0,080 & 0,543 & 0,623 \\
\hline 4 & Druck GmbH & 0,468 & - & - \\
\hline 5 & Papier und Chemie $\mathrm{GmbH}$ & 0,000 & 0,927 & 0,927 \\
\hline 6 & Metallverarbeitung $\mathrm{GmbH}$ & 1,226 & 0,877 & 2,103 \\
\hline 7 & Metallerzeugung $\mathrm{GmbH}$ & 1,195 & 0,881 & 2,076 \\
\hline 8 & Maschinenbau GmbH & 1,163 & 0,876 & 2,039 \\
\hline 9 & Hoch- und Tiefbau GmbH & - & 0,880 & - \\
\hline 10 & Bauinstallation $\mathrm{GmbH}$ & - & 0,875 & - \\
\hline 11 & Hotel GmbH & 0,000 & - & - \\
\hline 12 & Reisebüro $\mathrm{GmbH}$ & - & 1,000 & - \\
\hline 13 & EDV GmbH & - & 1,000 & - \\
\hline 14 & Architekturbüro OEG & - & - & - \\
\hline 15 & Werbeagentur $\mathrm{GmbH}$ & - & 1,000 & - \\
\hline
\end{tabular}

Eine weitere Möglichkeit zur Einbeziehung der Verkaufs- und Einkaufszahlen der Plattform (d.h. der Transaktionsdaten) in die Bewertung der Effekte einer Plattformerweiterung besteht in der Gewichtung der Bedeutung des Erweiterungskandidaten für ein bereitgestelltes Gut aus Tabelle 78 mit dem Verkaufs- bzw. Einkaufsanteil des bereitgestellten Gutes an den gesamten Verkäufen bzw. Käufen dieses Gutes auf der Plattform. Dadurch ergibt sich eine stärkere Gewichtung für Teilnehmer, die bisher bei dem Verkauf bzw. Kauf des bereitgestellten Gutes aktiver sind. Tabelle 91 zejgt das Ergebnjs dieser-Bew/ertung für 
die Hinzunahme des Gutes 70A. Aus Käufer- und kombinierter Perspektive profitiert nach dieser Bewertung vor allem die Maschinenbau $\mathrm{GmbH}$, während aus Verkäufersicht die EDV $\mathrm{GmbH}$ und die Papier und Chemie $\mathrm{GmbH}$ den höchsten Nutzen ziehen. Bei dieser Methode zur Bewertung der Wirkung der Hinzunahme eines externen Gutes ist zu berücksichtigen, dass die Bewertung für Unternehmen, deren bereitgestellte Güter bisher auf der Plattform gar nicht verbzw. gekauft wurden, nicht bzw. nur einseitig definiert ist.

Tabelle 91: gewichtete Bedeutung des externen Gutes 70A (Variante 2)

\begin{tabular}{|c|c|c|c|c|}
\hline & Teilnehmer & ratcBi-.., & 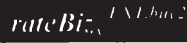 & 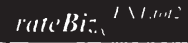 \\
\hline 1 & Forstwirtschaft GmbH & 0,080 & 0,524 & 0,604 \\
\hline 2 & Textil GmbH & - & 0,844 & - \\
\hline 3 & Holzverarbeitung GmbH & 0,080 & 0,543 & 0,623 \\
\hline 4 & Druck GmbH & 0,468 & - & - \\
\hline 5 & Papier und Chemie $\mathrm{GmbH}$ & - & 1,801 & - \\
\hline 6 & Metallverarbeitung $\mathrm{GmbH}$ & - & 0,936 & - \\
\hline 7 & Metallerzeugung GmbH & - & 1,190 & - \\
\hline 8 & Maschinenbau GmbH & 1,163 & 0,876 & 2,039 \\
\hline 9 & Hoch- und Tiefbau GmbH & - & 0,880 & - \\
\hline 10 & Bauinstallation $\mathrm{GmbH}$ & - & 0,515 & - \\
\hline 11 & Hotel GmbH & 0,000 & - & - \\
\hline 12 & Reisebüro $\mathrm{GmbH}$ & - & 1,000 & - \\
\hline 13 & EDV GmbH & - & 2,000 & - \\
\hline 14 & Architekturbüro OEG & - & - & - \\
\hline 15 & Werbeagentur $\mathrm{GmbH}$ & - & 1,000 & - \\
\hline
\end{tabular}

In Anbetracht der Tabelle 89, Tabelle 90 und Tabelle 91 zeigt sich, dass mit den in dieser Arbeit vorgeschlagenen Metriken unterschiedlichen Schwerpunktsetzungen bei der Umlegung der Bewertungen des erwartbaren Effekts von externen Knoten auf die Plattformteilnehmer Rechnung getragen werden kann. Jede dieser Umlegungsvarianten ermöglicht eine teilnehmerbezogene Quantifizierung einer Erweiterung der lokalen Struktur, um ein von einem neuen (oder auch bestehenden) Teilnehmer bereitzustellendes Gut und trägt somit auch zur Konkretisierung der möglichen Fördermaßnahmen für die Plattformteilnehmer bei. 


\subsection{Matching}

Im Sinne optimaler Transaktionsempfehlungen soll in einem letzten Schritt ergänzend zu der Umlegung der Bewertungen auch auf die spezifischen Charakteristika der möglichen Transaktionspartner eingegangen werden, um für jede Paarung die Transaktions(n)eig(n)ung auf Basis zuvor erhobener Präferenzdaten (BCIpreferences) festzustellen. Darüber hinaus ist es auch von Interesse, für etwaige zukünftige Teilnehmer, die ein externes Gut bzw. eine externe Aktivität in die Plattform mit einbringen sollen, ein ideales Präferenzprofil zu erstellen, um mit diesem aktiv (potenzielle) Teilnehmer anzuwerben bzw. hinsichtlich ihrer Eignung zu evaluieren.

\subsubsection{Präferenzstruktur}

Ausgehend von den Charakteristika und Präferenzen der Plattformteilnehmer, die in Tabelle 92, Tabelle 93 und Tabelle 94 beschrieben werden, kann entsprechend der Ausführungen aus den Kapiteln 10.1 bis 10.4 die Transaktions(n)eig(n)ung mittels der vorgestellten Distanz- bzw. Ähnlichkeitsmaße für jede Paarung ermittelt werden. Die Charakteristika und Präferenzen liegen für dieses Demonstrationsbeispiel pro bereitgestelltem Gut eines Teilnehmers im Hinblick auf den Einkauf für dieses Gut (,IN') sowie den Verkauf des jeweiligen Gutes (,OUT') vor. Konkret geben Tabelle 92, Tabelle 93 und Tabelle 94 pro Gut jedes Plattformteilnehmers Auskunft über die geografische Lage sowie diverse Präferenzwerte, die in binär nominalen, mehrstufig nominalen, ordinalen, metrischen sowie Referenzpräferenz-Variablen für die Einkaufs- (,IN') und Verkaufsperspektive (,OUT') festgehalten sind. Denkbare Beispiele für binäre Merkmale wären die Notwendigkeit (,IN') bzw. das Angebot (,OUT') einer erweiterten Gewährleistung, einer Stornierungsmöglichkeit oder auch einer speziellen Lieferkondition. Ein Beispiel für ein mehrstufig nominales Merkmal wären verschiedene Zahlungskonditionen (z.B. Vorkasse, Nachnahme, Bartering, Lieferantenkredit). Beispiele für mögliche ordinal messbare Merkmale wären unterschiedliche Kategorien von Lieferzeiten (z.B. bis 2 Wochen, bis 4 Wochen, über 4 Wochen) oder verschiedene Stufen des/eBartering ${ }_{3}\left(z_{3} B_{-7} b_{3} \Phi_{6}-1 / 3\right.$ des 
Preises, bis $2 / 3$ des Preises, über $2 / 3$ des Preises). Ein Beispiel für metrische Variablen könnte die gewünschte maximale (,IN') bzw. angebotene minimale (,OUT') Lieferzeit sein. Die Referenzpräferenzvariable könnte wiederum in der Einkaufsperspektive (,IN') die maximal akzeptierte Entfernung eines Verkäufers angeben, während in der Verkaufsperspektive (,OUT') damit die maximale Reichweite der Verkaufsaktivitäten beschrieben werden kann (der Wert ,Inf' steht dabei für Indifferenz im Hinblick auf die Entfernung eines möglichen Transaktionspartners).

Tabelle 92: BCIpreferences I

\begin{tabular}{|c|c|c|c|c|c|c|c|c|c|}
\hline & Gut & Teilnehmer-ID & PLZ & Ort & $\begin{array}{l}\text { binNOM. } \\
\text { IN }\end{array}$ & $\begin{array}{l}\text { mehrst- } \\
\text { NOM1-IN }\end{array}$ & $\begin{array}{l}\text { mehrst- } \\
\text { NOM2-IN }\end{array}$ & $\begin{array}{l}\text { mehrst- } \\
\text { NOM3.IN }\end{array}$ & $\begin{array}{l}\text { mehrst- } \\
\text { NOM4-IN }\end{array}$ \\
\hline & 02 & $\begin{array}{c}\text { Forstwirtschaft } \\
\mathrm{GmbH}\end{array}$ & A-8630 & Mariazell & 0 & 1 & 1 & 1 & 1 \\
\hline 2 & 17 & Textil GmbH & A-9715 & Stuben & 1 & 0 & 0 & 0 & 1 \\
\hline 3 & 20 & $\begin{array}{c}\text { Holzverarbeitung } \\
\text { GmbH }\end{array}$ & A-4371 & Dimbach & 0 & 1 & 0 & 0 & 0 \\
\hline 4 & 22 & Druck GmbH & D-81825 & München & 1 & 1 & 0 & 0 & 0 \\
\hline 5 & 21 & $\begin{array}{l}\text { Papier und Che- } \\
\text { mie GmbH }\end{array}$ & A-1180 & Wien & 1 & 1 & 1 & 0 & 1 \\
\hline 6 & 24 & $\begin{array}{c}\text { Papier und Che- } \\
\text { mie GmbH }\end{array}$ & A-1180 & Wien & 1 & 0 & 1 & 0 & 0 \\
\hline 7 & 27 & $\begin{array}{c}\text { Metallverarbeitung } \\
\mathrm{GmbH}\end{array}$ & A-1030 & Wien & 0 & 1 & 1 & 0 & 1 \\
\hline 8 & $45 \mathrm{~B}$ & $\begin{array}{c}\text { Metallverarbeitung } \\
\mathrm{GmbH}\end{array}$ & $A-1030$ & Wien & 1 & 0 & 1 & 0 & 1 \\
\hline 9 & 28 & \begin{tabular}{|c|}
$\begin{array}{c}\text { Metallerzeugung } \\
\mathrm{GmbH}\end{array}$ \\
\end{tabular} & A-4040 & $\operatorname{Linz}$ & 1 & 1 & 1 & 0 & 0 \\
\hline 10 & $45 B$ & \begin{tabular}{|c|} 
Metallerzeugung \\
GmbH
\end{tabular} & $A-4040$ & Linz & 1 & 0 & 1 & 0 & 1 \\
\hline 11 & 29 & $\begin{array}{c}\text { Maschinenbau } \\
\text { GmbH }\end{array}$ & D-73540 & Heubach & 0 & 0 & 0 & 0 & 1 \\
\hline 12 & $45 \mathrm{~A}$ & $\begin{array}{c}\text { Hoch- und Tieffau } \\
\text { GmbH }\end{array}$ & A-1150 & Wien & 0 & 0 & 1 & 0 & 0 \\
\hline 13 & 45B & $\begin{array}{c}\text { Bauinstallation } \\
\text { GmbH }\end{array}$ & $\mathrm{H}-9400$ & Sopron & 0 & 1 & 0 & 0 & 0 \\
\hline 14 & 55 & Hotel GmbH & A-9500 & \begin{tabular}{|l} 
Villach \\
\end{tabular} & 0 & 0 & 1 & 1 & 0 \\
\hline 15 & 63B & Reisebüro $\mathrm{GmbH}$ & $A-1190$ & Wien & 1 & 0 & 1 & 1 & 0 \\
\hline 16 & 72 & EDV GmbH & D-82140 & Olching & 1 & 0 & 0 & 1 & 1 \\
\hline 17 & $52 \mathrm{~A}$ & EDV GmbH & $D-82140$ & Olching & 0 & 0 & 1 & 1 & 0 \\
\hline 18 & $74 B$ & $\begin{array}{c}\text { Architekturbüro } \\
\text { OEG }\end{array}$ & A-8010 & Graz & 1 & 0 & 1 & 0 & 0 \\
\hline 19 & $74 \mathrm{C}$ & $\begin{array}{c}\text { Werbeagentur } \\
\text { GmbH }\end{array}$ & A-1010 & Wien & 1 & 0 & 1 & 1 & 0 \\
\hline
\end{tabular}


Tabelle 93: BCIpreferences /I

\begin{tabular}{|c|c|c|c|c|c|c|c|c|}
\hline & Gut & Teilnehmer-ID & ORD-IN & METR-IN & $\begin{array}{l}\text { REFPREF - } \\
\text { IN }\end{array}$ & ORD-OUT & METR-OUT & $\begin{array}{l}\text { REFPREF- } \\
\text { OUT }\end{array}$ \\
\hline 1 & 02 & Forstwirtschaft $\mathrm{GmbH}$ & 2 & 60 & 100 & 3 & 18 & 600 \\
\hline 2 & 17 & Textil GmbH & 2 & 6 & 2100 & 1 & 96 & 800 \\
\hline 3 & 20 & $\begin{array}{c}\text { Holzverarbeitung } \\
\text { GmbH }\end{array}$ & 1 & 69 & 250 & 2 & 79 & 1000 \\
\hline 4 & 22 & Druck GmbH & 3 & 52 & 200 & 1 & 40 & 500 \\
\hline 5 & 21 & $\begin{array}{l}\text { Papier und Chemie } \\
\text { GmbH }\end{array}$ & 1 & 34 & 400 & 2 & 26 & 250 \\
\hline 6 & 24 & $\begin{array}{l}\text { Papier und Chemie } \\
\text { GmbH }\end{array}$ & 1 & 99 & 80 & 2 & 30 & 400 \\
\hline 7 & 27 & $\begin{array}{l}\text { Metallverarbeitung } \\
\text { GmbH }\end{array}$ & 3 & 25 & 250 & 2 & 27 & 85 \\
\hline 8 & $45 \mathrm{~B}$ & $\begin{array}{c}\text { Metallverarbeitung } \\
\text { GmbH }\end{array}$ & 1 & 1 & 200 & 1 & 38 & 50 \\
\hline 9 & 28 & $\begin{array}{c}\text { Metallerzeugung } \\
\text { GmbH }\end{array}$ & 1 & 62 & 60 & 1 & 54 & 140 \\
\hline 10 & $45 B$ & $\begin{array}{c}\text { Metallerzeugung } \\
\text { GmbH }\end{array}$ & 2 & 22 & 90 & 2 & 59 & 155 \\
\hline 11 & 29 & Maschinenbau $\mathrm{GmbH}$ & 3 & 21 & 100 & 2 & 5 & 600 \\
\hline 12 & $45 \mathrm{~A}$ & $\begin{array}{c}\text { Hoch- und Tiefbau } \\
\text { GmbH }\end{array}$ & 3 & 19 & 200 & 2 & 88 & 300 \\
\hline 13 & $45 \mathrm{~B}$ & Bauinstallation $\mathrm{GmbH}$ & 1 & 59 & 200 & 2 & 77 & 250 \\
\hline 14 & 55 & Hotel GmbH & 1 & 15 & 200 & 3 & 9 & 210 \\
\hline 15 & $63 \mathrm{~B}$ & Reisebüro $\mathrm{GmbH}$ & 2 & 88 & Inf & 2 & 3 & 50 \\
\hline 16 & 72 & EDV GmbH & 1 & 86 & 100 & 3 & 89 & 300 \\
\hline 17 & $52 \mathrm{~A}$ & EDV GmbH & 1 & 63 & 250 & 3 & 4 & 50 \\
\hline 18 & $74 \mathrm{~B}$ & Architekturbüro OEG & 2 & 18 & 100 & 3 & 97 & $\operatorname{lnf}$ \\
\hline 19 & $74 \mathrm{Cl}$ & Werbeagentur $\mathrm{GmbH}$ & 1 & 89 & 100 & 3 & 71 & Inf \\
\hline
\end{tabular}

Tabelle 94: BCIpreferences III

\begin{tabular}{|c|c|c|c|c|c|c|c|}
\hline & Gut & Teilnehmer-ID & $\begin{array}{l}\text { binNOM- } \\
\text { OUT }\end{array}$ & $\begin{array}{l}\text { mehrst- } \\
\text { NOM1-OUT }\end{array}$ & $\begin{array}{l}\text { mehrst- } \\
\text { NOM2-OUT }\end{array}$ & $\begin{array}{l}\text { mehrst- } \\
\text { NOM3-OUT }\end{array}$ & $\begin{array}{l}\text { mehrst- } \\
\text { NOM4-OUT }\end{array}$ \\
\hline 1 & 02 & Forstwirtschaft GmbH & 0 & 0 & 0 & 1 & 0 \\
\hline 2 & 17 & Textil GmbH & 1 & 0 & 0 & 0 & 1 \\
\hline 3 & 20 & $\begin{array}{c}\text { Holzverarbeitung } \\
\text { GmbH }\end{array}$ & 1 & 0 & 0 & 1 & 0 \\
\hline 4 & 22 & Druck GmbH & 1 & 1 & 0 & 0 & 1 \\
\hline 5 & 21 & $\begin{array}{l}\text { Papier und Chemie } \\
\text { GmbH }\end{array}$ & 1 & 0 & 0 & 0 & 1 \\
\hline 6 & 24 & $\begin{array}{c}\text { Papier und Chemie } \\
\text { GmbH }\end{array}$ & 0 & 0 & 1 & 1 & 0 \\
\hline 7 & 27 & $\begin{array}{c}\text { Metallverarbeitung } \\
\text { GmbH } \\
\end{array}$ & 0 & 1 & 1 & 0 & 1 \\
\hline 8 & $45 B$ & $\begin{array}{l}\text { Metallverarbeitung } \\
\text { GmbH }\end{array}$ & 1 & 1 & 0 & 1 & 0 \\
\hline 9 & 28 & $\begin{array}{c}\text { Metallerzeugung } \\
\text { GmbH }\end{array}$ & 1 & 0 & 0 & 1 & 0 \\
\hline
\end{tabular}




\begin{tabular}{|c|c|c|c|c|c|c|}
\hline Gut & Teilnehmer-ID & $\begin{array}{l}\text { binNOM- } \\
\text { OUT }\end{array}$ & $\begin{array}{l}\text { mehrst- } \\
\text { NOM1-OUT }\end{array}$ & $\begin{array}{l}\text { mehrst- } \\
\text { NOM2-OUT }\end{array}$ & $\begin{array}{l}\text { mehrst- } \\
\text { NOM3-OUT }\end{array}$ & $\begin{array}{l}\text { Inehrst- } \\
\text { NOM4-OUT }\end{array}$ \\
\hline $1045 B$ & $\begin{array}{c}\text { Metallerzeugung } \\
\text { GmbH }\end{array}$ & 0 & 0 & 1 & 0 & 1 \\
\hline 1129 & Maschinenbau GmbH & 0 & 0 & 0 & 1 & 0 \\
\hline $45 \mathrm{~A}$ & $\begin{array}{c}\text { Hoch- und Tiefbau } \\
\text { GmbH }\end{array}$ & 1 & 0 & 0 & 1 & 1 \\
\hline $1345 \mathrm{~B}$ & Bauinstallation $\mathrm{GmbH}$ & 0 & 0 & 1 & 1 & 0 \\
\hline $14 \quad 55$ & Hotel GmbH & 1 & 0 & 0 & 0 & 1 \\
\hline $1563 \mathrm{~B}$ & Reisebüro GmbH & 0 & 1 & 1 & 0 & 0 \\
\hline $16 \quad 72$ & EDV GmbH & 1 & 0 & 0 & 1 & 0 \\
\hline $1752 \mathrm{~A}$ & EDV GmbH & 0 & 1 & 0 & 0 & 0 \\
\hline $1874 \mathrm{~B}$ & Architekturbüro OEG & 0 & 1 & 0 & 0 & 0 \\
\hline $1974 \mathrm{C}$ & Werbeagentur $\mathrm{GmbH}$ & 0 & 0 & 0 & 1 & 0 \\
\hline
\end{tabular}

\subsubsection{Gesamtähnlichkeitsmaß für Plattformteilnehmer}

Tabelle 95 zeigt das Ergebnis der Anwendung der Ähnlichkeits- bzw. Distanzmaße für die binär nominalen, mehrstufig nominalen, ordinalen, metrischen und Referenzpräferenz-Variablen (letztere unter zusätzlicher Berücksichtigung der Standortinformation als Referenzmerkmal) gemäß den Kapiteln 10.1 bis 10.4 zusammengefasst in einem Gesamtähnlichkeitsmaß über alle Variablentypen der Beobachtungen aus Tabelle 92 und deren Fortsetzung in Tabelle 93 und Tabelle 94 (Zeilen 1-19). Als Maßzahl für die binären nominalen Variablen wurde hierbei der simple matching oder auch M-Koeffizient von Sokal \& Michener [1958] verwendet, während für die mehrstufig nominalen sowie die ordinalen Variablen das Ähnlichkeitsmaß von Russell \& Rao [1940] für die jeweilig generierten binären Hilfsvariablen und die jeweilig erweiterte Kontingenztafel eingesetzt wurde. Für die metrische Variable wurde die Euklidische Distanz (Minkowski- $q$-Metrik mit $q=2$ ) als Distanzmaß gewählt und später in ein Ähnlichkeitsmaß transformiert. Des Weiteren wurde auch ein indirekter Vergleich der Gut-TeilnehmerKombinationen unter Zuzug eines Referenzmerkmals durchgeführt. Konkret wurden hierfür die (metrischen) Werte der Referenzpräferenzvariable, die Präferenzen bezüglich der Entfernung der Transaktionspartner angeben, mit der Standortinformation (PLZ bzw. Ort) als Referenzmerkmal zur Berechnung gemäß Kapitel 10.2 verwendet. Nachdem die Präferenzwerte sowohl in einer Input- als

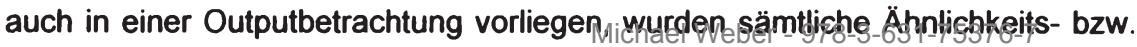


Demonstrationsbeispiel

Distanzmaße entsprechend der in Kapitel 10.3 vorgestellten Berechnungsmethode für duale Präferenzen ermittelt und letztlich mit gleicher Gewichtung in ein Gesamtähnlichkeitsmaß nach Kapitel 10.4 überführt (Tabelle 95).

Tabelle 95: Gesamtähnlichkeitsmaß

$\begin{array}{lllllllllllllllllll}1 & 2 & 3 & 4 & 5 & 6 & 7 & 8 & 9 & 10 & 11 & 12 & 13 & 14 & 15 & 16 & 17 & 18 & 19\end{array}$

\begin{tabular}{|r|l|l|l|l|l|l|l|l|l|l|l|l|l|l|l|l|l|l|l|}
\hline 1 & 1,0 & 0,5 & 0,5 & 0,5 & 0,4 & 0,2 & 0,8 & 0,4 & 0,3 & 0,5 & 0,8 & 0,8 & 0,5 & 0,6 & 0,4 & 0,3 & 0,6 & 0,5 & 0,3 \\
\hline 2 & 0,4 & 1,0 & 0,3 & 0,5 & 0,5 & 0,6 & 0,3 & 0,5 & 0,5 & 0,5 & 0,3 & 0,2 & 0,3 & 0,2 & 0,6 & 0,6 & 0,3 & 0,4 & 0,6 \\
\hline 3 & 0,5 & 0,6 & 1,0 & 0,6 & 0,5 & 0,6 & 0,4 & 0,4 & 0,6 & 0,6 & 0,3 & 0,4 & 0,4 & 0,3 & 0,7 & 0,6 & 0,4 & 0,6 & 0,6 \\
\hline 4 & 0,4 & 0,6 & 0,4 & 1,0 & 0,7 & 0,4 & 0,5 & 0,6 & 0,6 & 0,6 & 0,4 & 0,3 & 0,4 & 0,4 & 0,5 & 0,6 & 0,4 & 0,5 & 0,5 \\
\hline 5 & 0,5 & 0,7 & 0,3 & 0,6 & 1,0 & 0,5 & 0,6 & 0,6 & 0,5 & 0,7 & 0,4 & 0,5 & 0,3 & 0,4 & 0,6 & 0,5 & 0,3 & 0,7 & 0,5 \\
6 & 0,7 & 0,5 & 0,5 & 0,4 & 0,4 & 1,0 & 0,7 & 0,4 & 0,4 & 0,5 & 0,6 & 0,7 & 0,5 & 0,7 & 0,5 & 0,3 & 0,6 & 0,5 & 0,4 \\
\hline 7 & 0,8 & 0,5 & 0,6 & 0,4 & 0,5 & 0,3 & 1,0 & 0,5 & 0,4 & 0,6 & 0,6 & 0,7 & 0,6 & 0,6 & 0,4 & 0,2 & 0,5 & 0,5 & 0,3 \\
8 & 0,5 & 0,5 & 0,4 & 0,5 & 0,6 & 0,5 & 0,4 & 1,0 & 0,6 & 0,6 & 0,2 & 0,4 & 0,4 & 0,4 & 0,6 & 0,4 & 0,3 & 0,6 & 0,5 \\
\hline 9 & 0,4 & 0,5 & 0,4 & 0,6 & 0,6 & 0,5 & 0,3 & 0,5 & 1,0 & 0,5 & 0,3 & 0,3 & 0,4 & 0,4 & 0,6 & 0,6 & 0,4 & 0,5 & 0,6 \\
\hline 10 & 0,8 & 0,4 & 0,6 & 0,5 & 0,5 & 0,4 & 0,7 & 0,4 & 0,4 & 1,0 & 0,6 & 0,7 & 0,6 & 0,6 & 0,5 & 0,4 & 0,6 & 0,5 & 0,4 \\
\hline 11 & 0,6 & 0,5 & 0,5 & 0,4 & 0,3 & 0,1 & 0,6 & 0,4 & 0,2 & 0,4 & 1,0 & 0,6 & 0,4 & 0,6 & 0,4 & 0,3 & 0,5 & 0,4 & 0,2 \\
12 & 0,5 & 0,6 & 0,4 & 0,6 & 0,5 & 0,6 & 0,4 & 0,5 & 0,5 & 0,6 & 0,3 & 1,0 & 0,3 & 0,3 & 0,8 & 0,6 & 0,4 & 0,6 & 0,7 \\
13 & 0,8 & 0,4 & 0,6 & 0,4 & 0,4 & 0,4 & 0,6 & 0,3 & 0,4 & 0,4 & 0,5 & 0,6 & 1,0 & 0,6 & 0,6 & 0,4 & 0,6 & 0,4 & 0,5 \\
14 & 0,4 & 0,7 & 0,3 & 0,7 & 0,6 & 0,4 & 0,6 & 0,6 & 0,5 & 0,7 & 0,6 & 0,6 & 0,3 & 1,0 & 0,5 & 0,5 & 0,3 & 0,7 & 0,4 \\
15 & 0,7 & 0,5 & 0,5 & 0,4 & 0,4 & 0,3 & 0,8 & 0,5 & 0,4 & 0,5 & 0,5 & 0,7 & 0,5 & 0,6 & 1,0 & 0,1 & 0,4 & 0,5 & 0,3 \\
16 & 0,5 & 0,5 & 0,4 & 0,7 & 0,5 & 0,5 & 0,5 & 0,4 & 0,5 & 0,6 & 0,5 & 0,4 & 0,3 & 0,3 & 0,7 & 1,0 & 0,4 & 0,5 & 0,6 \\
17 & 0,6 & 0,5 & 0,5 & 0,6 & 0,3 & 0,1 & 0,7 & 0,3 & 0,3 & 0,4 & 0,8 & 0,7 & 0,4 & 0,5 & 0,2 & 0,2 & 1,0 & 0,4 & 0,1 \\
18 & 0,7 & 0,3 & 0,6 & 0,6 & 0,3 & 0,4 & 0,7 & 0,2 & 0,4 & 0,3 & 0,6 & 0,6 & 0,6 & 0,3 & 0,5 & 0,3 & 0,5 & 1,0 & 0,4 \\
\hline 19 & 0,7 & 0,4 & 0,6 & 0,5 & 0,3 & 0,3 & 0,7 & 0,3 & 0,4 & 0,4 & 0,6 & 0,7 & 0,6 & 0,5 & 0,5 & 0,4 & 0,6 & 0,4 & 1,0 \\
\hline
\end{tabular}

Abbildung 23 stellt die Verteilung des Gesamtähnlichkeitsmaßes (ohne Selbstbezüglichkeit) als Histogramm dar. Drei Viertel der Transaktionspaarungen weisen eine Gesamtähnlichkeit unter 0,58 auf, die maximale Gesamtähnlichkeit liegt bei 0,80 . Nur etwa $8 \%$ der Transaktionspaarungen zeigen eine Gesamtähnlichkeit größer als 0,7. 


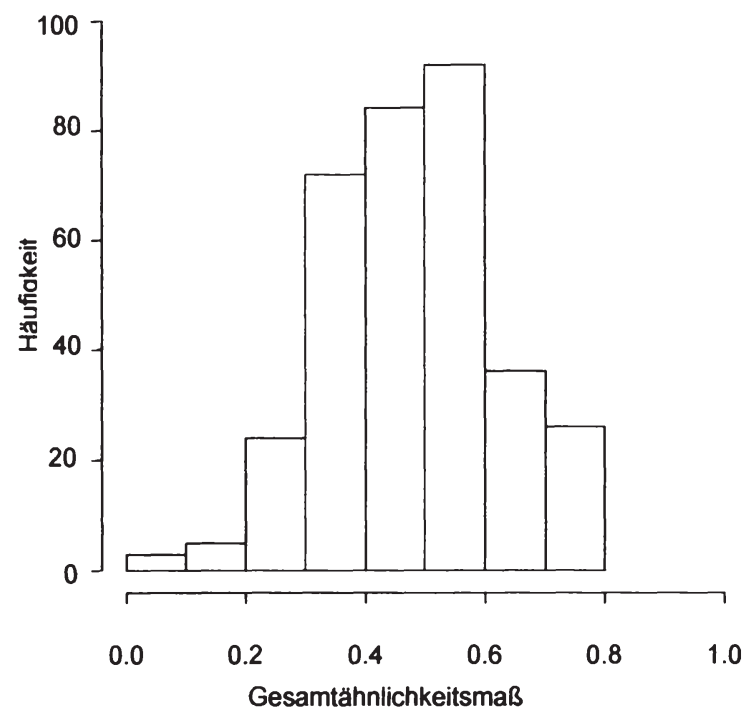

Abbildung 23: Histogramm für das Gesamtähnlichkeitsmaß

\subsubsection{Präferenzprofil für potenzielle Teilnehmer}

Das ideale Profil der Präferenzen eines potenziellen bzw. fiktiven Teilnehmers, der die Plattform um ein externes Gut (eine externe Aktivität) erweitern soll, wurde nach Maßgabe von Kapitel 10.5 aufbauend auf der Präferenzstruktur jener bestehenden Teilnehmer, die gemäß der bisherigen Analysen bzw. Prognosen eine Transaktionsbeziehung mit diesem externen Gut eingehen könnten, ermittelt. Hierbei wurden - je nach Variable - auch die Bedeutungen der in Frage kommenden bestehenden Teilnehmer in die Auswahl der jeweilig gewünschten Präferenz des Erweiterungskandidaten einbezogen, was speziell in mittels Häufigkeit unentscheidbaren Fällen, die Präferenzermittlung vereinfachte. Tabelle 96 und deren Fortsetzung, Tabelle 97, zeigen die Ergebnisse, die aus der Anwendung der in Kapitel 10.5.1 bis 10.5.5 beschriebenen Metriken für den Erweiterungsknoten $\left(v_{x} \in V^{E X 7}\right) 70 \mathrm{~A}$ resultieren. Besonderes Augenmerk sei hierbei auf die Interpretation und Generierung der Referenzpräferenzwerte für einen potenziellen Bereitsteller des Gutes 70A gelegt. Der rein in Hinblick auf geografische Distanzen ermittelte ideale Standort des Bereitstellers, von Gut 70A 
liegt gemäß den Präferenzen sowohl der möglichen Nachfrager des Gutes 70A als auch der denkbaren Anbieter für das Gut 70A entsprechend der Koordinaten in Tabelle 97 südlich von A-3100 St. Pölten. Bei der Berechnung dieses Standortes konnten dabei sämtliche Präferenzen der Anbieter, nicht jedoch der Nachfrager berücksichtigt werden, da bei letzteren divergierende bzw. inkompatible Interessenkonstellationen auftraten, welche eine Auswahl der in Frage kommenden Nachfrager für die Standortbestimmung erforderten ${ }^{70}$. Diese Auswahl umfasst 14 der 19 möglichen Nachfrager für Gut 70 A aus Tabelle 92 sowie Tabelle 93 und Tabelle 94, die unter allen möglichen Gruppierungen gemeinsam die höchste Gesamtbedeutung für die Plattform erreichen. Für beide Transaktionsrichtungen konnten im Anschluss an die Standortbestimmung Referenzpräferenzwerte unter Berücksichtigung der möglichen Anbieter und Nachfrager ermittelt werden.

Tabelle 96: 70A Präferenzwerte OUT und IN I

binNOM mehrstNOM1 mehrstNOM2 mehrstNOM3 mehrstNOM4

\begin{tabular}{|r|l|l|l|l|l|}
\hline OUT & 1 & 0 & 1 & 0 & 0 \\
\hline IN & 0 & 0 & 0 & 1 & 0 \\
\hline
\end{tabular}

Tabelle 97: 70A Präferenzwerte OUT und IN II

ORD METR REFPREF Koordinaten4Ext1 Koordinaten4Ext2

\begin{tabular}{|r|c|c|c|c|c|}
\hline OUT & 1 & 11 & 191 & 47,9605088 & 15,3975952 \\
\hline IN 2 & 86 & 379 & 48,1004973 & 15,7340695 \\
\hline
\end{tabular}

\subsubsection{Erweiterung der Transaktionsempfehlungen}

Mit der Kenntnis des Gesamtähnlichkeitsmaßes für sämtliche Transaktionskonstellationen kann zum Abschluss des Demonstrationsbeispiels die Kantenliste der klassifizierten Transaktionsverläufe $B C l e F g u g u^{e x p}$ aus Tabelle 88 um das zum jeweiligen Transaktionsverlauf passende Gesamtähnlichkeitsmaß enweitert

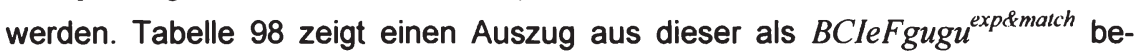
zeichneten Kantenliste, wobei für jede Lückenkategorie zwecks Übersichtlichkeit nur die drei jeweils höchsten Gesamtähnlichkeiten dargestellt werden. Während

70 Konkret leitet sich dies - wie in Kapitel 10.5 .5 beschrieben - aus unterschiedlichen ,complete blocks' CBlock, und dazugehörigen Knotengruppeg-C. 
die Performanzlücken ( $P$ und $P$ ) im Demonstrationsbeispiel mit maximalen Gesamtähnlichkeiten von etwa 0,5 bzw. 0,4 eine relativ schwache Übereinstimmung der Präferenzen zeigen, sind insbesondere die isolierten direkten internen Lücken $\left(D I L^{i s}\right)$ mit Werten um 0,7 durch eine vergleichsweise hohe Gesamtähnlichkeit der betroffenen potenziellen Transaktionspartner gekennzeichnet. Abbildung 24 legt die Transaktionsempfehlungen für den Auszug aus Tabelle 98 zwecks Übersichtlichkeit auch grafisch dar.

Tabelle 98: Auswahl aus BCleFgugu exp\&maich

\begin{tabular}{|c|c|c|c|c|c|c|c|}
\hline & $\begin{array}{l}\text { von- } \\
\text { AoG }\end{array}$ & vonUnt & $\begin{array}{l}\text { nach- } \\
\text { AoG }\end{array}$ & nachUnt & Wert & gap & $\begin{array}{l}\text { Pref- } \\
\text { Sim }\end{array}$ \\
\hline 4 & 22 & Druck GmbH & 72 & EDV GmbH & 1,45 & Pmin & 0,554 \\
\hline 10 & 55 & Hotel GmbH & $63 B$ & Reisebüro $\mathrm{GmbH}$ & 14 & Pmin & 0,533 \\
\hline 2 & 20 & Holzverarbeitung $\mathrm{GmbH}$ & 21 & Papier und Chemie GmbH & 8 & Pmin & 0,506 \\
\hline 11 & 28 & Metallerzeugung GmbH & 45B & Bauinstallation $\mathrm{GmbH}$ & 13 & Pplus & 0,388 \\
\hline 13 & 21 & Papier und Chemie $\mathrm{GmbH}$ & 17 & Textil GmbH & 16 & NPmin & 0,708 \\
\hline 14 & 22 & Druck GmbH & 21 & Papier und Chemie GmbH & 11 & NPmin & 0,687 \\
\hline 16 & 27 & Metallverarbeitung $\mathrm{GmbH}$ & 45B & Metallerzeugung GmbH & 14,8 & NPmin & 0,582 \\
\hline 20 & 29 & Maschinenbau GmbH & 45B & Bauinstallation $\mathrm{GmbH}$ & 22 & NPplus & 0,443 \\
\hline 42 & 45B & Bauinstallation $\mathrm{GmbH}$ & 02 & Forstwirtschaft GmbH & 0 & DILiso & 0,765 \\
\hline 58 & 72 & EDV GmbH & $63 \mathrm{~B}$ & Reisebüro GmbH & 0 & DILiso & 0,743 \\
\hline 51 & 72 & EDV GmbH & 22 & Druck GmbH & 0 & DILiso & 0,723 \\
\hline 72 & 21 & Papier und Chemie GmbH & 20 & Holzverarbeitung $\mathrm{GmbH}$ & 0 & DILmin & 0,310 \\
\hline 74 & 24 & Papier und Chemie $\mathrm{GmbH}$ & 02 & Forstwirtschaft GmbH & 0 & DILtilde & 0,737 \\
\hline 80 & 45B & Metallverarbeitung $\mathrm{GmbH}$ & $74 \mathrm{C}$ & Werbeagentur GmbH & 0 & DILtilde & 0,544 \\
\hline 78 & 29 & Maschinenbau GmbH & 20 & Holzverarbeitung GmbH & 0 & DILtilde & 0,463 \\
\hline 89 & 45B & Metallerzeugung GmbH & 02 & Forstwirtschaft GmbH & 0 & DILplus & 0,798 \\
\hline 85 & 45B & Metallerzeugung $\mathrm{GmbH}$ & $45 \mathrm{~A}$ & Hoch- und Tieftau GmbH & 0 & DILplus & 0,667 \\
\hline 83 & 29 & Maschinenbau GmbH & 27 & Metallverarbeitung $\mathrm{GmbH}$ & 0 & DILplus & 0,633 \\
\hline 98 & 21 & Papier und Chemie GmbH & 45B & Metallerzeugung $\mathrm{GmbH}$ & 0 & DILzero & 0,738 \\
\hline 111 & 27 & Metallverarbeitung $\mathrm{GmbH}$ & $45 \mathrm{~A}$ & Hoch- und Tiefbau GmbH & 0 & DILzero & 0,733 \\
\hline 116 & 55 & Hotel GmbH & 45B & Metallerzeugung $\mathrm{GmbH}$ & 0 & DILzero & 0,715 \\
\hline
\end{tabular}

Die abschließende Erweiterung der Kantenliste ermöglicht eine Verfeinerung der Priorisierung von Transaktionsempfehlungen bzw. Transaktionsfördermaßnahmen auf der Ebene der Plattformteilnehmer. Es kann dadurch eine weitere Selektion von aussichtsreichen Empfehlungen für Transaktionsbeziehungen erfolgen, die zusätzlich zu der grundsätzlichen wechselseitigen Relevanz der (potenziellen) Transaktionspartner basierend auf Input-Output-Beziehungen auch die Transaktions(n)eig(n)ung der Partner berücksichtigt. 


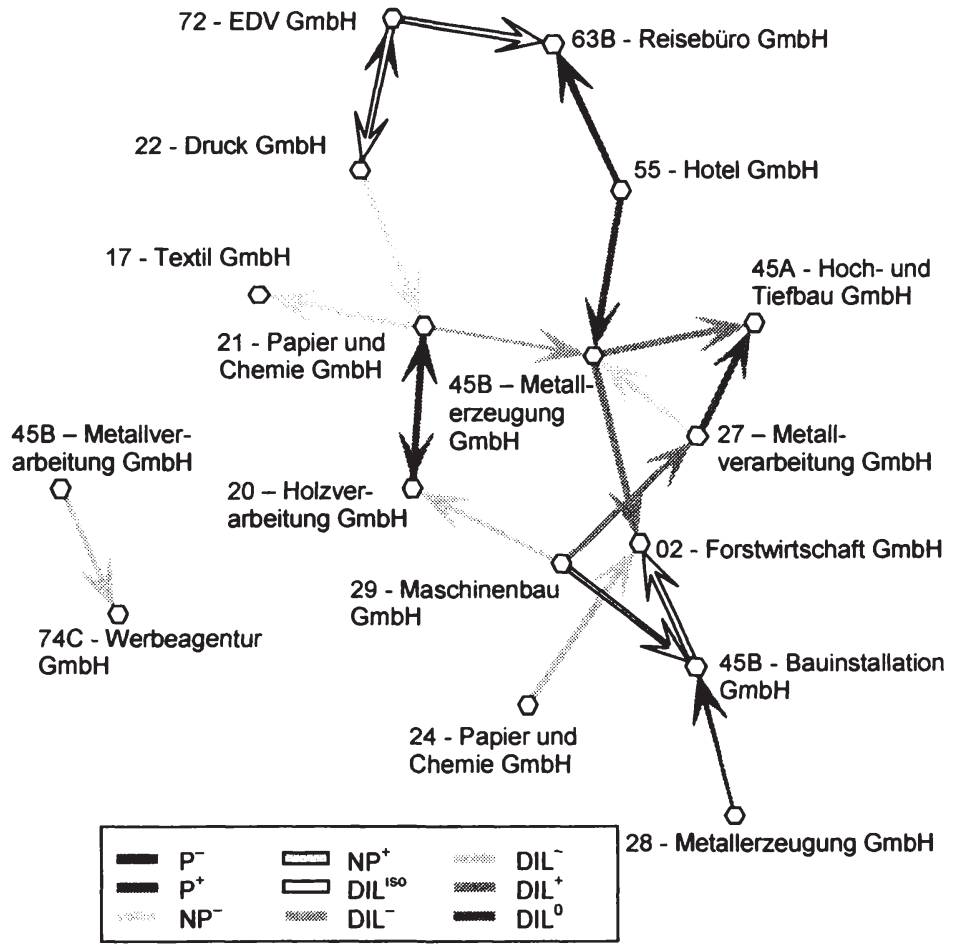

Abbildung 24: Darstellung des Auszuges aus BCleFgugu ${ }^{\text {exp \&match }}$

\subsection{Diskussion}

Zum Abschluss des Demonstrationsbeispiels werden nachfolgend die mittels der vorgestellten Methoden erzielten Ergebnisse für alle Phasen des Modells kurz zusammengefasst und diskutiert. Ausgangspunkt des Beispiels ist (i) eine Konfiguration einer fiktiven Plattform mit 15 Teilnehmern, 17 Aktivitäten und Gütern sowie 19 unterschiedlichen Kombinationen von Teilnehmern mit Gütern bzw. Aktivitäten, (ii) modifizierte gesamtwirtschaftliche Datensätze, die die zugrundeliegende Volkswirtschaft repräsentieren, und (iii) 16 realwirtschaftlich abgeleitete Transaktionsverläufe, die als historische Transaktionsbeziehungen auf der Plattform fungieren. Zusätzlich wird für das Beispiel pro Gut jedes Teilnehmers eine eigene (duale) Präferenzstrukturaangenemnees.3-631-75376-7 
Unter Einsatz der aus dieser Basis abgeleiteten Datenquellen zeigen die Auswertungen der auf der Ebene von Gütern durchgeführten Strukturanalysephase, dass die Verflechtungen auf der Plattform als eine nicht-integrale relationale Struktur bezeichnet werden können. Nach der alleinigen Analyse der Struktur der Plattform handelt es sich bei dieser Aussage zunächst noch um eine Annahme, die durch den geringen Anteil der direkt und indirekt verbundenen Knotenpaare (Güter) getroffen werden kann. Die Gegenüberstellung der Analyse der Plattformstruktur mit der Analyse der reduzierten gesamtwirtschaftlichen Struktur bestätigt schließlich diese Annahme und zeigt, dass die Plattform nach Maßgabe der reduzierten gesamtwirtschaftlichen Struktur ein enormes Verflechtungspotenzial (Faktor 5) in sich birgt. Dieser Schluss kann anhand verschiedenster Maßzahlen wie bspw. der Dichte oder Durchschnittsgrade abgeleitet werden und unterstreicht die Notwendigkeit einer detaillierteren Auswertung zur Identifikation der Schwachstellen und Ableitung von Gegenmaßnahmen.

Im Zuge der Strukturanalyse können außerdem drei voneinander unabhängige (unverbundene) Beziehungsgeflechte (Cliquen) innerhalb der Plattorm gefunden werden, die sich aus 9, 5 bzw. 2 Gütern zusammensetzen, wobei die Verbundenheit in dem mittleren Beziehungsgeflecht am höchsten ist. In der größten Clique nimmt Gut 22 eine besondere Position als Vorläuferknoten ein. Das Gut 74B ist vollständig isoliert. Die Blockmodellberechnung spiegelt dieses Ergebnis für die Plattform wider und gibt in der Gegenüberstellung mit dem Blockmodell für die reduzierte gesamtwirtschaftliche Struktur erstmalig Aufschluss über konkrete Güter, die als Ansatzpunkt für das Ausschöpfen des Verflechtungspotenzials besonders geeignet sind. Dementsprechend empfehlen sich die Güter 27, 29, 45A und 45B besonders als Nachfolger, d.h. als Güter, über welche die Nachfrage auf der Plattform erhöht werden kann, und die Güter 20, 22, 28, 72 und $74 \mathrm{C}$ als Vorgänger, d.h. als Güter, die angebotsseitig die Plattform beleben können.

Ein systematischer Vergleich der beiden Strukturen erfolgt in der Strukturvergleichsphase, in der mittels der für diese Arbeit eingeführten Lückentypologie 
anhand deskriptiver Maßzahlen festgestellt wird, dass zur Strukturoptimierung primär die Förderung zusätzlicher Beziehungen zwischen Gütern auf der Plattform zu empfehlen ist, während die Erweiterung der Plattform mit externen Gütern eine nachgeordnete Rolle spielt. Beispiele für in diesem Zusammenhang relevante Kennzahlen sind der Anteil zusätzlich möglicher interner direkter Kanten am Kantenpotenzial, der Anteil von nicht direkt aber intern indirekt zusammenhängenden Knotenpaaren an indirekten Verbindungsmöglichkeiten oder das Verkürzungspotenzial durch externe Knotenfolgen. Im Anschluss wird die Relevanz der einzelnen Güter als Abnehmer bzw. Anbieter in den zusätzlich möglichen Transaktionsbeziehungen für die Weglänge 2 ermittelt, um festzustellen, welche Güter für den Ausbau der Transaktionen zwischen bestehenden Teilnehmern bei dieser Weglänge in Frage kommen. In der Analyse der beidseitig neuen Transaktionswege treten die Güter 21, 45A und 72 bzw. auch 02 , $45 \mathrm{~B}, 74 \mathrm{~B}$ und $74 \mathrm{C}$ als wichtige Verbindungsgüter hervor, wobei das Gut 72 von spezieller Bedeutung ist. Bei Betrachtung der nur ausgangsseitig (verkaufsseitig) neuen Transaktionswege der Weglänge 2 zeigen die Güter 02,22 und 28 das höchste Verbindungspotenzial, während das Gut 72 in dieser Kategorie insgesamt am stärksten von zusätzlichen Transaktionswegen profitieren kann. Hingegen spielen in zielseitig (einkaufsseitig) neuen Transaktionswegen die Güter 02, 21, 29, 45B, 72 und 74C die größte Rolle als Verbindungsglieder. Fasst man diese Auswertungen zusammen, so sind v.a. die Güter $02,45 \mathrm{~B}$ und 72 als verbindende Güter zur Generierung neuer Transaktionsbeziehungen der Weglänge 2 zu nennen, wobei das Gut 72 in nahezu allen Analysen Spitzenwerte erzielt. Im Rahmen der Untersuchung der Transaktionswege mit Weglänge 2 mit externen Verbindungsgütern können die externen Güter 64,65 und $70 \mathrm{~A}$ als besonders relevante Verbindungsgüter identifiziert werden. Die internen Güter 72 und $74 \mathrm{C}$ profitieren gemäß dieser Auswertung ausgangsseitig am stärksten, während zielseitig die Güter 17 und 52A die meisten Vorteile aus der Erweiterung mit externen Gütern ziehen. Eine nur einseitige Betrachtung der Relevanz von externen Gütern zeigt, dass $74 \mathrm{~A}$ und $74 \mathrm{D}$ speziell aus der zielseitigen (verkaufsseitigen) Perspektive zusätzlich nennenswert sind. Die abschließend in 
der Strukturvergleichsphase berechneten komparativen Dichtemaßzahlen unterstreichen einmal mehr das hohe Erweiterungspotenzial der Plattform.

Unter Berücksichtigung dieser Maßzahlen werden in der Lückenbewertungsphase tatsächliche Performanzlücken unter Einsatz unterschiedlicher Bewertungsverfahren bestimmt. Die Kanten oder Güterbeziehungen $(20,21)$, $(22,52 \mathrm{~A}),(22,72),(22,74 \mathrm{C})$ und $(28,27)$ treten hierbei als kritische Performanzlücken hervor, d.h. diese Verbindungen sind unter den bestehenden Relationen auf der Plattform im Vergleich zur Gesamtwirtschaft besonders schwach ausgeprägt. Bei der Einbeziehung von indirekten Effekten in die Analyse ist die Kante $(27,29)$ zusätzlich als Performanzlücke hervorzuheben, nachdem diese vergleichsweise schwach in bedeutende mehrgliedrige Transaktionsketten eingebettet ist. Als ,schwache Knoten' (Güter), d.h. als Güter die in ursächlichem Zusammenhang mit Performanzlücken stehen, können das Gut 20 in der Rolle als Bereitsteller sowie die Güter 21, 27, 52A, 72 und $74 \mathrm{C}$ in ihrer Rolle als Abnehmer identifiziert werden. Die Bewertungsverfahren für direkte interne Lücken ergeben, dass einige vielversprechende, aber bislang fehlende Austauschbeziehungen zwischen Gütern speziell von den Gütern 21, 45B bzw. 29 ausgehen oder zu den Gütern 02 bzw. 22 hinführen. Im besonderen sind die Paarungen $(21,22),(29,22),(24,22),(74 C, 22),(72,22),(45 B, 55),(45 B, 45 A)$, $(24,02),(29,02),(45 A, 02)$ und $(45 B, 02)$ von Gütern zusätzlich anzustreben. Im Rahmen der Bewertungen zeigt sich, dass die Schließung von jenen direkten internen Lücken, die zwei in sich eng verflochtene Cliquen bzw. zwei SubCliquen, die nur durch eine vergleichsweise schwach gewichtete Austauschbeziehung zusammenhängen, verbinden, den größten Bedeutungszuwachs für die Plattform hervorruft. Diese Verbindungen haben einen dementsprechend hohen Rang in der Prioritätenliste zur Schließung der 79 identifizierten direkten internen Lücken. Die Bestimmung der Bedeutung der Hinzunahme von externen Gütern zeigt, dass die Güter 18, 31, 36, 64, 65, 70A, 91, 92A und 93 aussichtsreiche Erweiterungskandidaten für die Plattorm sind. Nachdem das externe Gut $70 \mathrm{~A}$ in vielerlei Hinsicht bei den Bewertungen hervorsticht, wird es als $\mathrm{Er}$ weiterungskandidat für die Plattform ausgewählth Es ist einess_der wengigen Güter, 
welches sowohl als potenzieller ,Anbieter' als auch als möglicher ,Nachfrager' unter den besten $20 \%$ der gereihten externen Güter liegt, wobei die Bedeutung des Gutes 70A für die Ausweitung des Angebots auf der Plattform höher als für die Nachfrage ist. Dementsprechend sollten sich in Folge der Eingliederung des Gutes 70A vor allem die Inputvolumina der betreffenden internen Güter erhöhen, wie auch die Zielwertberechnungen unterstreichen.

Nach der Umlegung der Ergebnisse von der Ebene der Plattform (güterbezogen) auf die Ebene der Plattformteilnehmer erweisen sich die Papier und Chemie $\mathrm{GmbH}$ und die Werbeagentur $\mathrm{GmbH}$ als schwache Käufer sowie die Holzverarbeitung $\mathrm{GmbH}$ als schwacher Verkäufer innerhalb der Plattform. Rein verkaufsseitig isoliert sind die Bauinstallation $\mathrm{GmbH}$, die EDV $\mathrm{GmbH}$, die Hoch- und Tiefbau $\mathrm{GmbH}$, die Reisebüro $\mathrm{GmbH}$, die Textil $\mathrm{GmbH}$ und die Werbeagentur $\mathrm{GmbH}$. Nur einkaufsseitig isoliert sind hingegen die Druck $\mathrm{GmbH}$ und die Hotel $\mathrm{GmbH}$. Die Architekturbüro OEG ist innerhalb der Plattform in beiden Transaktionsrichtungen isoliert. Tabelle 88 beschreibt die durch die Umlegung erreichte Klassifizierung der Transaktionsempfehlungen, wobei die Reihung der Empfehlungen gemäß der in Kapitel 9.2 vorgeschlagenen Klassenhierarchie erfolgt. Dies bedeutet, dass zunächst interne Performanzlücken, interne NichtPerformanzlücken und letztlich direkte interne Lücken in den verschiedenen Subkategorien zu priorisieren sind. Selbstverständlich können abhängig von der gewünschten Plattformoptimierungsstrategie abweichende Prioritäten gesetzt werden.

Eine Reihung der Empfehlungen für Transaktionsbeziehungen innerhalb der einzelnen Kategorien kann anhand der in der Matchingphase ermittelten Maßzahl zur Transaktions(n)eig(n)ung, welche auf der dualen Präferenzstruktur der Teilnehmer aufbaut, erfolgen. Entsprechend der daraus entstehenden erweiterten Transaktionsempfehlungsliste, die in Tabelle 98 auszugsweise dargestellt wird, können pro Kategorie die aussichtsreichsten Transaktionspaarungen gefunden und dann zur Realisierung vorgeschlagen oder auf eine andere Weise gefördert werden. So wäre bspw. die Erhöhung des Transaktions- 
werts bei Transaktionen zwischen dem schwachen Verkäufer Holzverarbeitung $\mathrm{GmbH}$ und dem schwachen Käufer Papier und Chemie GmbH mit dem Transaktionsinhalt Gut $20\left(P^{-}\right)$im Sinne der obigen Ausführungen sowohl aus Plattformperspektive als auch aus der Perspektive der Teilnehmer vorteilhaft. Im Gegensatz hierzu sind Maßnahmen zur Unterstützung der Transaktion zwischen der Papier und Chemie $\mathrm{GmbH}$ und der Textil $\mathrm{GmbH}$ vergleichsweise weniger vorrangig $(N P-)$, nichtsdestotrotz weisen die beiden Transaktionspartner im $\mathrm{Be}$ zug auf den Transaktionsinhalt und den geschätzten Transaktionszweck eine hohe Übereinstimmung der Präferenzen auf. Die Etablierung von Transaktionen zwischen der auf der Plattform verkaufsseitig isolierten Bauinstallation $\mathrm{GmbH}$ und der Forstwirtschaft $\mathrm{GmbH}$ aus der Kategorie $D I L^{\text {iso }}$ erscheint empfehlenswert im Hinblick auf die stärkere Einbindung bestehender Teilnehmer in die Plattform und hat eine höhere Priorität als die weiteren Subkategorien der direkten internen Lücken. Nachdem in dem Demonstrationsbeispiel keine Analogie von bestehenden Transaktionsverläufen zwischen Teilnehmern für vergleichbare bisher nicht im Austausch befindliche Teilnehmer auftritt, bleiben die Kategorien $P^{D I L}$ und $N P^{D I L}$ leer. Insgesamt führt die Analyse dennoch zu zahlreichen Empfehlungen für die Teilnehmer an der Kollaborationsplattform, die dazu beitragen, die Lücken in den Verflechtungen im Vergleich zur gesamtwirtschaftlichen Struktur zu schließen.

Die Umlegung der Effekte der Aufnahme des Erweiterungskandidaten 70A in die Plattform ergibt, dass insbesondere die Teilnehmer Metallverarbeitung $\mathrm{GmbH}$, Metallerzeugung $\mathrm{GmbH}$, Papier und Chemie $\mathrm{GmbH}$, Maschinenbau $\mathrm{GmbH}$ und Architekturbüro $\mathrm{GmbH}$ am stärksten von der Integration dieses Gutes profitieren. Rein aus der Verkaufsperspektive können zudem die Reisebüro $\mathrm{GmbH}$, die EDV $\mathrm{GmbH}$ sowie die Werbeagentur $\mathrm{GmbH}$ einen hohen Nutzen aus der Hinzunahme des Gutes 70A ziehen. Mit dem Wissen um die durch die Erweiterung bevorteilten Teilnehmer wird anschließend in der Matchingphase ein ideales Präferenzprofil für einen möglichen zukünftigen Teilnehmer ermittelt. Dieses Präferenzprofil kann nun verwendet werden, um zielgerichtet bestmöglich 
passende neue Teilnehmer außerhalb der Plattform zu finden. Die Verfahren des Transaktionssystemunterstützungsmodells sind demgemäß nicht nur geeignet eine vorhandene nicht-integrale Struktur unter Zuhilfenahme globaler bzw. gesamtwirtschaftlicher Verflechtungsinformationen zu vervollständigen, sondern können diese auch gezielt durch Integration externer Teilnehmer weiterentwickeln. 


\section{Rückblick und Ausblick}

Die Reduktion relativer Intransparenz durch verbesserte (lokale) Informationsnutzung zur Vereinfachung und Beschleunigung der individuellen ökonomischen Koordination von Wirtschaftstreibenden bzw. zur Ermöglichung von ,selbstorganisierenden Wertschöpfungsnetzen' basierend auf umfangreichen Produktionsstrukturerhebungen ist der Leitgedanke der in dieser Arbeit beschrieben Infrastrukturleistung. Das vorgeschlagene Modell für zwischenbetriebliche Kollaborationsplattformen verfolgt dabei die Zielsetzung, Komplexität im Wirtschaftsleben reduzieren zu helfen und insbesondere Transaktionen durch spezifische Informationsverteilung mittels eines hierfür entwickelten Rahmenwerks zur Generierung von Empfehlungen (auf Teilnehmer- und Plattormebene) zu unterstützen und reiht sich dadurch in die F\&E Aktivitäten im Bereich der ,Business Collaboration Infrastructures' [Österle et al. 2000] und der ,digitalen Ókosysteme' für KMU [Nachira et al. 2007; Dini et al. 2005] ein. Der Kern dieser Arbeit widmet sich demgemäß der Entwicklung von Methoden zur Verbesserung der Einbettung von Unternehmen in wirtschaftliche Verflechtungen und zur Optimierung deren Wertgenerierung. Diesbezügliche Unterstützungsmaßnahmen könnten speziell für kleine und mittlere Unternehmen mit tendenziell knapperen Ressourcen in Zukunft von Vorteil sein. Der vorgestellte Ansatz steht dabei gewissermaßen im Gegensatz zu der Vorgehensweise des ,collaborative filtering' [Herlocker et al. 2004] in der für einen spezifischen Kunden auf Basis dessen Präferenzen bzw. Warenkorbs unter Rückgriff auf vergleichbare Präferenzen bzw. Warenkörbe aus dem Kundenstamm eine Vorhersage für möglicherweise relevante Güter getroffen wird. Während diese Praktik im Kontext der Interaktion eines Unternehmens mit dem Endverbraucher (B2C) - bspw. im Buchhandel - geeignet und ausreichend erscheint, ist dieses Vorgehen allerdings in Bezug auf Transaktionen zwischen Betrieben (bzw. Aktivitätsbereichen) verschiedener Unternehmen (B2B) unzureichend. Dies lässt sich damit begründen, dass die Präferenzen bzw. die bisherigen Transaktionsverläufe (sozusagen der kumulierte bisherige Warenkorb) nicht primär Empfehlungen motivieren können, sondern 
eher den Charakter einer wichtigen Zusatzinformation haben. Die entscheidende Vorbedingung für zwischenbetriebliche Transaktionsempfehlungen ist vielmehr die Kenntnis der Input-Output-Struktur der Betriebe aller beteiligten Unternehmen, welche in dieser Arbeit über das Wissen um die von den Teilnehmern bereitgestellten Güter bzw. Aktivitäten unter Zuhilfenahme der gesamtwirtschaftlichen Input-Output-Struktur ${ }^{71}$ geschätzt wird. Dies ermöglicht die Identifikation von Lücken in den Wertschöpfungsnetzen sowohl auf der kollektiven als auch auf der individuellen Ebene, welche gezielt zur Schließung vorgeschlagen werden können.
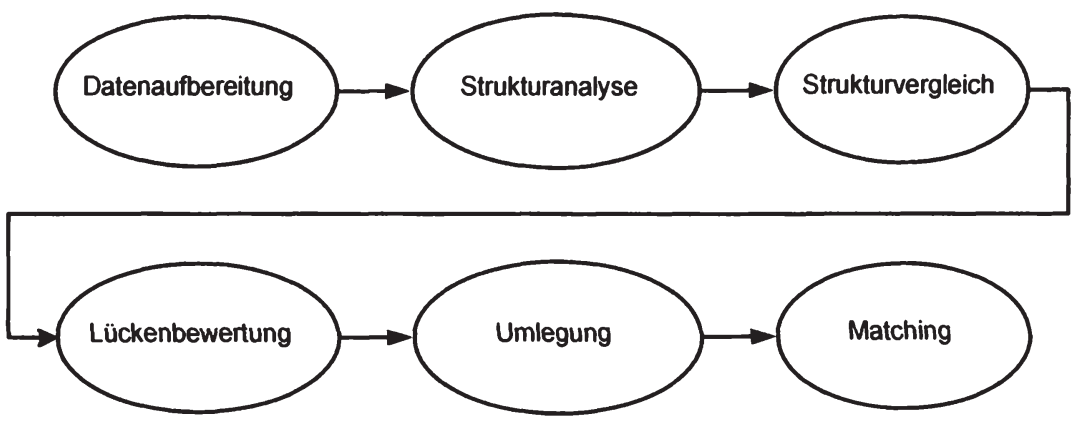

Abbildung 25: Überblick über die Modellphasen

Auf die konkrete Vorgehensweise und auf hilfreiche Maßzahlen und Berechnungsverfahren zur Umsetzung der angestrebten Transaktionssystemunterstützung wird in dieser Arbeit in den Erläuterungen zu den sechs Modellphasen beginnend mit Kapitel 5 detailliert eingegangen. Den Ausgangspunkt bildet dabei die Sammlung bzw. Erstellung der grundlegenden Datenquellen in der Phase der Datenaufbereitung (Kapitel 5). Hierzu gehören insbesondere die Profildaten zu den Teilnehmern in Verbindung mit Daten zu deren Präferenzstruktur, die Daten zu den bisherigen Transaktionsverläufen sowie umfassende gesamtwirtschaftliche Strukturdaten, die einen Vergleich mit der Kollaborationsplattform zulassen, 
nachdem die Transaktionsdaten der Plattform einen Ausschnitt der gesamtwirtschaftlichen Daten darstellen. Aufbauend auf diesen Datenquellen ist es möglich, verschiedene Varianten von ,Sell'-Matrizen, ,Buy'-Matrizen, erweiterte ,Buy'Matrizen sowie Matrizen zu den Input-Output-Relationen auf der Kollaborationsplattorm anzufertigen. Es bleibt zu betonen, dass für diesen Zweck spezifische Annahmen im Hinblick auf die Vergleichbarkeit der Datenquellen bzw. die Zulässigkeit von Zuschätzungen getroffen werden und darüber hinaus eingangs eine Bereinigung der eingesetzten Daten durchgeführt wird.

Nach der Phase der Datenaufbereitung hat die Strukturanalysephase (Kapitel 6) die Aufgabe, die Verflechtungseigenschaften der Transaktions- sowie der gesamtwirtschaftlichen Strukturdaten zu analysieren. Hierfür werden verschiedene Kennzahlen der Graphentheorie und der (sozialen) Netzwerkanalyse eingesetzt, um sowohl direkte als auch indirekte Zusammenhänge in den Daten zu untersuchen. Mit Kapitel 6 wird darüber hinaus die Terminologie der Graphentheorie eingeführt, wodurch eine Abstraktion von Gütern, Aktivitäten oder Teilnehmern und deren Beziehungen durch den Einsatz der übergeordneten Begriffe (Vorgänger- und Nachfolger-)Knoten sowie Kanten für den weiteren Verlauf der Arbeit erfolgen kann. In den Ausführungen zu der Strukturanalysephase werden auch interessante Querverbindungen zwischen der auf dem Perron-Frobenius Theorem basierenden Leontief-Inverse, der sozialen Netzwerkanalyse und dem PageRank ${ }^{\mathrm{TM}}$ Algorithmus hergestellt, die in dieser Form und Kausalität neu erscheinen. Des Weiteren bildet die Berücksichtigung von Kantengewichten bei der Untersuchung von Verflechtungseigenschaften einen Schwerpunkt innerhalb der Strukturanalysephase. Kapitel 6 beinhaltet daher neben den etablierten Beiträgen zu dieser Thematik auch neue Ansätze für das Clustering, die Partitionierung und im Zuge dessen die Visualisierung von relationalen Datensätzen unter Berücksichtigung der Kantengewichte. Beiträge zu diesem aktuellen Thema des wertbasierten Blockmodeling konnte ausgehend von der Arbeit an Kapitel 6 inzwischen entwickelt und publiziert werden [Weber \& Denk 2007; Weber 2007; Denk \& Weber 2007; Weber \& Denk 2008]. 
Nachfolgend auf die Strukturanalyse kann mit Hilfe der Methoden aus Kapitel 7 ein Strukturvergleich zwischen den Transaktionsdaten der Plattorm und den gesamtwirtschaftlichen Verflechtungsdaten durchgeführt werden. Zu diesem Zweck wird in Kapitel 7 eine Lückentypologie eingeführt und ebenso wie die darauf basierenden Arten von Lückenfolgen erläutert. Die Strukturvergleichsphase gibt Aufschluss über die Vorgehensweise bei der Identifikation von Lücken und Lückenfolgen und stellt des Weiteren Kennzahlen sowie Möglichkeiten zur deskriptiven Auswertung der Lücken und deren Folgen im Rahmen des Strukturvergleichs vor. Die auf der Ebene der Plattform (d.h. güter- oder aktivitätsbezogen) erfolgende Identifikation von Lücken (und Lückenfolgen) ermöglicht gemeinsam mit deren deskriptiver statistischer Auswertung bereits erste aufschlussreiche Aussagen über denkbare Handlungsempfehlungen zur Ausschöpfung des Potenzials der Kollaborationsplattform. Zusätzlich wird im Zuge der Strukturvergleichsphase aufgezeigt, wie konkretere Aussagen und Auswertungen für Transaktionsketten mit einer bestimmten Länge getroffen werden können. Für die Generierung und Priorisierung von Empfehlungen, die einerseits einen hohen Detaillierungsgrad aufweisen und andererseits in der Lage sind, größere Zusammenhänge zu berücksichtigen, sind Folgerungen aus dem Strukturvergleich alleine allerdings nicht ausreichend.

Die in Kapitel 8 behandelte Lückenbewertung liefert daher Berechnungsverfahren für die spezifische Bestimmung der Bedeutung der verschiedenen Lückentypen aus der Perspektive der Plattform. In Folge der Bedeutungsbestimmung kann indirekt die Priorität der Schließung von Lücken festgelegt und des Weiteren auch ein Zielwert für die jeweilige Maßnahme der Lückenschließung angegeben werden. Nachdem sich diese Berechnungen nicht auf die Ebene der Plattformteilnehmer beziehen, ist es notwendig, die bewerteten Lücken auf die Teilnehmer der Plattform umzulegen. Kapitel 9 beschreibt, wie in dieser Umlegungsphase die Bewertungen zu den Lückentypen abhängig von deren Relevanz auf die Ebene der (jeweils betroffenen) Teilnehmer transferiert werden können, um Empfehlungen für Transaktionen auch auf diesem individuellen Niveau zu ermöglichen. Eine abschließervdeeZusałzinformation-zzu den 
gewonnenen Transaktionsempfehlungen erfolgt durch Berücksichtigung der Präferenzdaten nach Maßgabe von Kapitel 10. Die in diesem Kapitel beschriebene Matchingphase ermöglicht die Berechnung eines Gesamtähnlichkeitsmaßes zur Feststellung der Transaktions(n)eig(n)ung der jeweiligen Teilnehmerpaarung pro Transaktionsempfehlung. Hierbei werden speziell auch die mit der jeweiligen Stellung (Rolle) eines Teilnehmers variierenden Präferenzen berücksichtigt. Darüber hinaus wird in Kapitel 10 gezeigt, wie mit Rückgriff auf die bisherigen Berechnungen ein Präferenzprofil für potenzielle zukünftige Teilnehmer erstellt werden kann, um die Kollaborationsplattform nicht nur intern zu optimieren, sondern auch gezielt zu erweitern. Kapitel 11 dient abschließend der Illustration der Funktionsweise und der erfolgten Umsetzung des entwickelten Modells zur Generierung von Transaktionsempfehlungen auf verschiedenen Ebenen einer Plattform anhand eines (fiktiven) Demonstrationsbeispiels.

Mittels der vorgestellten Modellphasen und deren Illustration versucht diese Arbeit einen Weg aufzuzeigen, wie Leistungen von Unternehmen - insbesondere KMU - durch Umgehung bzw. Linderung typischer Probleme des Zustandekommens von Transaktionen, wie nicht kongruente Wahrnehmungsfelder, Kommunikationsverläufe und Transaktionskonfigurationen, gezielter miteinander verknüpft werden können. Angestrebt werden dabei sogenannte integrale Strukturen, d.h. Strukturen, in denen alle möglichen und beidseitig vorteilhaften Transaktionen auch tatsächlich umsetzbar sind. Die Umsetzung dieses Modells in eine Infrastrukturleistung auf einer Kollaborationsplattform eröffnet die Möglichkeit, insbesondere die regionalen Verflechtungen von KMU (auch grenzüberschreitend) zu erhöhen und gleichzeitig deren betriebswirtschaftliche Situation zu vereinfachen, was dementsprechend aus gesamtwirtschaftlicher wie auch einzelwirtschaftlicher Sicht wünschenswert ist und von Wirtschaftsforschern häufig gefordert wird. Diese Arbeit stellt sich zu diesem Zweck der Frage, inwieweit transaktionsrelevante und -fördernde Informationen generiert und so verteilt werden können, dass sowohl die Identifikation als auch die Schließung der Lücken der Transaktionsgeflechte, die in Folge der nicht-integralen Struktur auftreten, realisierbar werden. Für TransaktionssystemearderenzZielsetzung die Er- 
möglichung und Förderung von wechselseitigen Transaktionen zwischen den Teilnehmern ist, ist diese Fragestellung von höchster Relevanz. Im Bewusstsein des Vorherrschens von nicht-integralen Strukturen kann die Beantwortung daher nicht alleinig durch das Bereitstellen einer Plattform zur Abwicklung von Transaktionen erfolgen, sondern sollte vielmehr eine Komponente zur Aufdeckung und Schließung der Lücken im Sinne der Schaffung von integralen, zumindest aber integraleren, Strukturen innerhalb des Systems beinhalten. Das vorgestellte Modell und die daraus generierbaren transaktionsrelevanten und fördernden Informationen wie insbesondere Transaktionsempfehlungen stellen eine solche Komponente zur Verfügung und geben dementsprechend eine Antwort auf die zuvor gestellte Frage nach den Möglichkeiten der Generierung und Verteilung von relevanten Informationen zur Lückenschließung.

Der vorgestellte Ansatz zur Umsetzung einer umfassenden Transaktionssystemunterstützung setzt - neben den Informationen zu dem Profil, den Präferenzen sowie den plattformbezogenen Transaktionsverläufen der beteiligten Akteure eine umfangreiche und detaillierte Kenntnis ökonomischer Zusammenhänge voraus, deren Datengrundlage zum Zeitpunkt des Verfassens dieser Arbeit ungeachtet der technologischen und methodischen Fortschritte nur unter hohem Ressourcenaufwand bereitgestellt werden kann. Nichtsdestotrotz erscheint die hierin ausgearbeitete Infrastrukturleistung im Hinblick auf die zu erwartenden positiven Effekte gezielter Transaktionsempfehlungen für die Schaffung zunehmend integraler Wertschöpfungsnetze als ein verfolgenswerter Ansatz zur Unterstützung wirtschaftlicher Akteure. Dies bedingt den weiteren Ausbau und letztlich die Verwirklichung der in dieser Arbeit dargelegten Konzepte und Methoden für die Ausgestaltung eines Systems zur Generierung von Empfehlungen für zwischenbetriebliche Transaktionen als gesamtwirtschaftliche Infrastrukturleistung. 


\section{Literaturverzeichnis}

Almon, C. (2000). Product-to-Product Tables via Product-Technology with No Negative Flows. Economic Systems Research, 12(1), 27-43.

Alt, R., \& Österle, H. (2003). Real-Time Business. Lösungen, Bausteine und Potenziale des Business Networking. Berlin: Springer.

Arrow, K. J. (1984). On the Agenda of Organizations. In: K. J. Arrow (Hrsg.), The Economics of Information (S. 167-184). Collected Papers of Kenneth J. Arrow. Cambridge, MA: Belknap Press of Harvard University Press.

Arrow, K. J., \& Debreu, G. (1954). The Existence of an Equilibrium for a Competitive Economy. Econometrica, 22, 265-290.

Backhaus, K., Erichson, B., Plinke, W., \& Weiber, R. (2000). Multivariate AnaIysemethoden: Eine anwendungsorientierte Einführung. Berlin: Springer.

Batagelj, V. (1997). Notes on blockmodeling. Social Networks, 19(2), 143-155.

Batagelj, V., Ferligoj, A., \& Doreian, P. (1992a). Direct and indirect methods for structural equivalence. Social Networks, 14(1-2), 63-90.

Batagelj, V., Doreian, P., \& Ferligoj, A. (1992b). An optimizational approach to regular equivalence. Social Networks, 14(1-2), 121-135.

Bateson, G. (1971). The cybernetics of "self": a theory of alcoholism. Psychiatry, 34(1), 1-18.

Berners-Lee, T., Hendler, J., \& Lassila, O. (2001). The Semantic Web. Scientific American, (May 2001). Abgerufen am 01. September 2005 von http:/hwww.sciam.com/article.cfm?articlelD=00048144-10D2-1C7084A9809EC588EF21

Boisot, M., \& Canals, A. (2004). Data, information and knowledge: have we got it right? Joumal of Evolutionary Economics, 14(1), 43-67. 
Borgatti, S. P., \& Everett, M. G. (1992). Regular blockmodels of multiway, multimode matrices. Social Networks, 14(1-2), 91-120.

Breiger, R. L., Boorman, S. A., \& Arabie, P. (1975). An Algorithm for Clustering Relational Data with Applications to Social Network Analysis and Comparison with Multidimensional Scaling. Joumal of Mathematical Psychology, 12, 329-383.

Brin, S., \& Page, L. (1998). The Anatomy of a Large-Scale Hypertextual Web Search Engine. Computer Networks and ISDN Systems, 30(1-7), 107-117.

Brusco, M., \& Steinley, D. (2006). Inducing a blockmodel structure of two-mode binary data using seriation procedures. Joumal of Mathematical Psychology, 50, 468-477.

Burt, R. S. (1976). Positions in Networks. Social Forces, 55(1), 93-122.

Butts, C. T. (2007). sna: Tools for Social Network Analysis. Abgerufen am 01. April 2007 von http://erzuli.ss.uci.edu/R.stuff

Chessel, D., Dufour, A., \& Thioulouse, J. (2004). The ade4 package - I: One-table methods. $R$ News, 4, 5-10.

Damásio, A. R. (1999). The Feeling of What Happens: Body and Emotion in the Making of Consciousness. New York: Harcourt Brace \& Company.

Denk, M., \& Fröschl, K. A. (2004). East of Neuchâtel: A Universal Model for the Representation of Statistical Taxonomy Systems (M. Hatzopoulos \& Y. Manolopoulos, Hrsg.). Proceedings of the 16th Intemational Conference on Scientific and Statistical Database Management 21-23 June 2004, 373-382.

Denk, M., \& Weber, M. (2007). Valued two-mode blockmodeling for input-output analysis. Abstract booklet of Applied Statistics 2007, Bled, Slovenia, September 23-26, 2007.

Destatis (2005). Informationsportal der statistischen Ämter Deutschlands zu eStatistik.core. Abgerufen am 17. Mai 2006 von http://www.statspez.de/core 
Dice, L. R. (1945). Measures of the amount of ecologic association between species. Joumal of Ecology, 26(3), 297-302.

Diestel, R. (1997). Graph Theory. Heidelberg: Springer.

Dini, P., Darking, M., Rathbone, N., Vidal, M., Hernandez, P., Ferronato, P., u. a. (2005). The Digital Ecosystems Research Vision: 2010 and Beyond. Workshops on Digital Ecosystems. Abgerufen am 30. Juni 2006 von http://www.digitalecosystems.org/events/2005.05/ de_position_paper_vf.pdf

Doreian, P., Batagelj, V., \& Ferligoj, A. (1994). Partitioning networks based on generalized concepts of equivalence. The Joumal of Mathematical Sociology, 19(1), 1-27.

Doreian, P., Batagelj, V., \& Ferligoj, A. (2004). Generalized blockmodeling of twomode network data. Social Networks, 26(1), 29-53.

Doreian, P., Batagelj, V., \& Ferligoj, A. (2005). Generalized Blockmodeling. Cambridge: University Press.

du Bois-Reymond, E. H. (1912). Über die Grenzen des Naturerkennens. In der zweiten allgemeinen Sitzung der 45. Versammlung Deutscher Naturforscher und Ärzte zu Leipzig am 14. August 1872 gehaltener Vortrag. In: E. du Bois-Reymond (Hrsg.), Reden von Emil du Bois-Reymond in zwei Bänden (S. 441-473). Leipzig: Veit \& Comp. Abgerufen am 30. Dezember 2006, von http://vlp.mpiwgberlin.mpg.de/pdf/lit28636_Hi.pdf

Durkheim, É. (1977). Über soziale Arbeitsteilung. Frankfurt (M): Suhrkamp.

Eurostat (1995). Europäisches System volkswirtschaftlicher Gesamtrechnungen. Luxemburg: Europ. Gemeinschaften. Abgerufen am 31. Juli 2006, von http://circa.europa.eu/irc/dsis/ nfaccount/info/data/esa95/de/titelde.htm

Eurostat (2007). Eurostat Input-Output Manual (Draft). In: European Commission Methodologies and Working Papers. Luxemburg: Europ. Gemeinschaften.

Everitt, B. S. (1974). Cluster analysis. London: Heinemann. 
Fahrmeir, L., Hamerle, A., \& Tutz, G. (Hrsg.). (1996). Multivariate statistische Verfahren. Berlin: de Gruyter.

Ferrar, W. L. (1951). Finite matrices. London: Oxford University Press.

Festinger, L. (1949). The Analysis of Sociograms Using Matrix Algebra. Human Relations, 2(2), 153-158.

Fleisch, E. (2001). Betriebswirtschaftliche Perspektiven des Ubiquitous Computing. In: H. U. Buhl, A. Huther, \& B. Reitwiesner (Hrsg.), Information Age Economy. 5. Intemationale Tagung Wirtschaftsinformatik 2001. Heidelberg: Physica-Verlag.

Flichy, P. (1994). Tele - Geschichte der modemen Telekommunikation. Frankfurt (M): Campus.

Forsyth, E., \& Katz, L. (1946). A Matrix Approach to the Analysis of Sociometric Data: Preliminary Report. Sociometry, 9(4), 340-347.

Freeman, L. C. (1977). A Set of Measures of Centrality Based on Betweenness. Sociometry, 40(1), 35-41.

Freeman, L. C. (1979). Centrality in social networks: I. Conceptual clarification. Social Networks, 1, 215-239.

Frobenius, F. G. (1912). Über Matrizen aus nicht negativen Elementen. Sitzungsberichte der Königlich Preussischen Akademie der Wissenschaften. Sitzung der physikalisch-mathematischen Classe vom 23. Mai 1912, 26, 456477.

Gödel, K. (1931). Über formal unentscheidbare Sätze der Principia Mathematica und verwandter Systeme I. Monatshefte für Mathematik und Physik, 38, 173-198.

Gower, J. C. (1967). A Comparison of Some Methods of Cluster Analysis. Biometrics, 23(4), 623-637.

Groves, R. M., Fowler, F. J. J., Couper, M. P., Lepkowski, J. M., Singer, E., \& Tourangeau, R. (2004). Survey Methodologjycthoboken, Nof.\&loter Wiley \& Sons. 
Habermas, J. (1981). Theorie des kommunikativen Handelns. Frankfurt (M): Suhrkamp.

Handschuh, S., \& Staab, S. (2003). Annotation for the Semantic Web. Amsterdam: IOS Press.

Harary, F. (1959). Status and Contrastatus. Sociometry, 22(1), 23-43.

Harary, F. (1969). Graph theory. Reading, MA: Addison-Wesley.

Hayek, F. A. (1937). Economics and Knowledge. Economica, 4(13), 33-54.

Hayek, F. A. (1945). The Use of Knowledge in Society. American Economic Review, 35(4), 519-530.

Herlocker, J. L., Konstan, J. A., Terveen, L. G., \& Riedl, J. T. (2004). Evaluating Collaborative Filtering Recommender Systems. ACM Transactions on Information Systems, 22(1), 5-53.

Herrmann-Pillath, C. (2002). Grundriß der Evolutionsökonomik. München: Wilhelm Fink Verlag.

Hilbert, D. (1900). Mathematische Probleme. Nachrichten von der Königl. Gesellschaft der Wissenschaften zu Göttingen. Mathematisch-Physikalische Klasse, 1900(3), 253-297.

Hilbert, D. (1930). Naturerkennen und Logik. Die Naturwissenschaften, 18, 959963.

Holub, H., \& Schnabl, H. (1985). Input-Output-Rechnung: Input-Output-Tabellen. München Wien: Oldenbourg.

Holub, H., \& Schnabl, H. (1994). Input-Output-Rechnung: Input-Output-Analyse. München Wien: Oldenbourg.

Hubbell, C. H. (1965). An Input-Output Approach to Clique Identification. Sociometry, 28(4), 377-399. 
Hulliger, B. (1998). Linking of Classifications by Linear Mappings. Joumal of Official Statistics, 14(3), 255-266.

Hyvärinen, L. (1962). Classification of qualitative data. BIT Numerical Mathematics, 2(2), 83-89.

Jaccard, P. (1901). Distribution de la flore alpine dans le Bassin des Dranes et dans quelques régions voisines. Bulletin de la Société Vaudoise des Sciences Naturelles, 37, 241-272.

Jochimsen, R., \& Gustafsson, K. (1977). Infrastruktur. Grundlage der marktwirtschaftlichen Entwicklung. In: U. E. Simonis (Hrsg.), Infrastruktur - Theorie und Politik (S. 38-53). Köln: Kiepenheuer.

Katz, L. (1953). A new status index derived from sociometric analysis. Psychometrika, 18(1), 39-43.

Katzenbeisser, W. (1978). Graphentheoretische Interpretationen des InputOutput-Modells. Wien: Wirtschaftsuniversität Wien.

Kimbrough, S. O., \& Wu, D. J. (Hrsg.). (2005). Formal Modelling in Electronic Commerce (Intemational Handbooks on Information Systems). Berlin: Springer.

Kish, L. (1965). Survey Sampling. New York: John Wiley. \&. Sons.

Knight, F. H. (1921). Risk, Uncertainty and Profit. Boston, MA: Houghton Mifflin Company.

Köhler, E. (2002a). Gödel und der Wiener Kreis. In: E. Köhler, P. Weibel, M. Stöltzner, B. Buldt, C. Klein, \& W. DePauli-Schimanovich-Göttig (Hrsg.), Kurt Gödel - Wahrheit und Beweisbarkeit 1. Dokumente und historische Analysen (S. 83-108). Wien: öbv \& hpt.

Köhler, E. (2002b). Gödels Jahre in Princeton. In: E. Köhler, P. Weibel, M. Stöltzner, B. Buldt, C. Klein, \& W. DePauli-Schimanovich-Göttig (Hrsg.), Kurt Gödel - Wahrheit und Beweisbarkeit 1. Dokumente und historische Analysen (S. 149-184). Wien: öbv \& hpt. 
Kreibich, R. (1986). Die Wissenschaftsgesellschaft. Von Galilei zur High-TechRevolution. Frankfurt (M): Suhrkamp.

Kulczynski, S. (1927). Die Pflanzenassoziationen der Pieninen. Bulletin Intemational de l'Academie Polonaise des Sciences et des Lettres, Classe des Sciences mathematiques et naturelles, Serie B, Supplement II, 57-203.

Leisch, F. (2006). A Toolbox for K-Centroids Cluster Analysis. Computational Statistics and Data Analysis, 51(2), 526-544.

Leontief, W. W. (1928). Die Wirtschaft als Kreislauf. In: Archiv für Sozialwissenschaft und Sozialpolitik (S. 577-623).

Leontief, W. W. (1936). Quantitative Input and Output Relations in the Economic System of the United States. The Review of Economic Statistics, 18(3), 105-125.

Leontief, W. W. (1937). Interrelation of Prices, Output, Savings and Investment. The Review of Economic Statistics, 19(3), 109-132.

Leontief, W. W. (1941). The Structure of American Economy, 1919-1939: An Empirical Application of Equilibrium Analysis. Cambridge, MA: Harvard University Press.

Leontief, W. W. (1944). Output, Employment, Consumption, and Investment. The Quaterly Joumal of Economics, 58(2), 290-314.

Leontief, W. W. (1946). Exports, Imports, Domestic Output, and Employment. The Quaterly Joumal of Economics, 60(2), 171-193.

Lin, N. (1976). Foundations of Social Research. New York: McGraw-Hill.

Lorrain, F., \& White, H. C. (1971). Structural equivalence of individuals in social networks. Joumal of Mathematical Sociology, 1, 49-80.

Luce, R. D., \& Perry, A. D. (1949). A method of matrix analysis of group structure. Psychometrika, 14(2), 95-116.

Luhmann, N. (1988). Die Wirtschaft der Gesellschaft. Frankfurt (M): Suhrkamp. 
Lyberg, L. E., Biemer, P., Collins, M., De Leeuw, E. D., Dippo, C., Schwarz, N., u. a. (Hrsg.). (1997). Survey Measurement and Process Quality. New York: John Wiley \& Sons.

Machlup, F. (1962). The Production and Distribution of Knowledge in the United States. Princeton: University Press.

Maechler, M., Rousseeuw, P., Struyf, A., \& Hubert, M. (2005). Cluster Analysis Basics and Extensions. nicht publiziert.

Mahalanobis, P. C. (1936). On the generalized distance in statistics. Proceedings of the National Institute of Science of India, 2(1), 49-55.

Malone, T. W., \& Crowston, K. (1994). The interdisciplinary study of coordination. ACM Computing Surveys, 26(1), 87-119 .

Malone, T. W., \& Rockart, J. F. (1991). Computers, networks and corporation. Scientific American, 265(3), 128-136.

Meeus, J. (2000). Astronomical Algorithms. Richmond: Willmann-Bell.

Metcalfe, J. S. (2002). Knowledge of growth and the growth of knowledge. Journal of Evolutionary Economics, 12(1), 3-15.

Metcalfe, J. S. (2003). Equilibrium and Evolutionary Foundations of Competition and Technology Policy: New Perspectives on the Division of Labour and the Innovation Process. In: P. Pelikán \& G. Wegner (Hrsg.), The Evolutionary Analysis of Economic Policy. Cheltenham: Edward Elgar.

Minsky, M. (Hrsg.). (1968). Semantic Information Processing. Cambridge, MA: MIT Press.

Mirowski, P. (1992). What Were von Neumann and Morgenstern Trying to Accomplish? In: R. E. Weintraub (Hrsg.), Toward a History of Game Theory. Durham, NC: Duke University Press.

Mirowski, P. (2002). Machine Dreams. Economics Becomes a Cyborg Science. Cambridge: University Press. 
Morgenstern, O. (1928). Wirtschaftsprognose. Eine Untersuchung ihrer Voraussetzungen und Möglichkeiten. Wien: Springer.

Morgenstern, O. (1934). Die Grenzen der Wirtschaftspolitik. Wien: Springer.

Nachira, F. (2007). Digital Ecosystems and FP7. Abgerufen am 01. März 2007 von http://www.digital-ecosystems.org/fp7/fp7-overview.html

Nachira, F., Nicolai, A., Dini, P., Le Louarn, M., \& Rivera León, L. (Hrsg.). (2007). Digital Business Ecosystems. Luxembourg: Office for Official Publications of the European Communities. Abgerufen am 14 November 2007 von http://www. digital-ecosystems.org/book/de-book2007.html

Ósterle, H., Fleisch, E., \& Alt, R. (2000). Business Networking. Shaping Enterprise Relationships on the Intemet. Berlin Heidelberg: Springer.

Page, L. (2001). United States Patent 6,285,999: Method for node ranking in a linked database. Abgerufen am 15. Oktober 2006 von http://patft.uspto.gov/ netacgi/nph-Parser?patentnumber=6,285,999

Page, L., Brin, S., Motwani, R., \& Winograd, T. (1998). The pagerank citation ranking: Bringing order to the web. Technical report, Stanford Digital Library Technologies Project, 1998.

Perron, O. (1907). Zur Theorie der Matrices. Mathematische Annalen, 64(2), 248263.

Pfaff, D., \& Skiera, B. (2002). Ubiquitous Computing - Abgrenzung, Merkmale und Auswirkungen aus betriebswirtschaftlicher Sicht. In: B. Britzelmaier, S. Geberl, \& S. Weinmann (Hrsg.), Der Mensch im Netz - Ubiquitous Computing.: 4. Liechtensteiner Wirtschaftsinformatik-Symposium.

Polanyi, M. (1958). Personal knowledge: towards a post-critical philosophy. Chicago, IL: University of Chicago Press. 
R Development Core Team (2007). R: A Language and Environment for Statistical Computing. R Foundation for Statistical Computing. Vienna, Austria. Abgerufen am 01. April 2007 von http://www.R-project.org

Rainer, N., \& Richter, J. (1992). Some aspects of the analytical use of descriptive make and absorption tables. Economic Systems Research, 4(2).

Reichmayr, C. (2003). Collaboration und WebServices. Architekturen, Portale, Techniken und Beispiele. Berlin: Springer.

Rogers, D. J., \& Tanimoto, T. T. (1960). A Computer Program for Classifying Plants. Science, 132(3434), 1115-1118.

Rosen, R. (1991). Life Itself - A Comprehensive Enquiry into the Nature, Origin and Fabrication of Life. New York: Columbia University Press.

Rubin, J. (1967). Optimal classification into groups: an approach for solving the taxonomy problem. Joumal of Theoretical Biology, 15(1), 103-144.

Russell, P. F., \& Rao, T. R. (1940). On habitat and association of species of Anopheline larvae in south-eastern Madras. Joumal of the Malaria Institute of India, 3, 153-178.

Schumpeter, J. A. (1942). Capitalism, Socialism, and Democracy. New York: Harper.

Scott, J. (1991). Social Network Analysis: A Handbook. London: SAGE Publications.

Screpanti, E., \& Zamagni, S. (1995). An Outline of the History of Economic Thought. Oxford: University Press.

Seeley, J. R. (1949). The net of reciprocal influence: A problem in treating sociometric data. Canadian Joumal of Psychology, 3, 234-240.

Shannon, C. E. (1948). A Mathematical Theory of Communication. The Bell System Technical Joumal, 27(July; October), 379-423; 623-656. Abgerufen am 
30. Dezember 2006 von http://cm.bell-labs.com/cm/ms/what/shannonday/shannon1948.pdf

Smith, A. (1776). An Inquiry into the Nature and Causes of the Wealth of Nations. Edinburgh. Abgerufen am 31. Dezember 2006 von http://www.adamsmith.org/ smith/won-index.htm

Smoryński, C. (2002). Gödels Unvollständigkeitssätze. In: B. Buldt, E. Köhler, M. Stöltzner, P. Weibel, \& W. DePauli-Schimanovich-Göttig (Hrsg.), Kurt Gödel Wahrheit und Beweisbarkeit 2. Kompendium zum Werk (S. 147-159). Wien: öbv \& hpt.

Sokal, R. R., \& Michener, C. D. (1958). A statistical method for evaluating systematic relationships. University of Kansas Science Bulletin, 38, 1409-1438.

Sokal, R. R., \& Sneath, P. H. A. (1963). Principles of Numerical Taxonomy. San Francisco: W.H. Freeman.

Statistik Austria (2003). Systematik der Wirtschaftstätigkeiten ONACE 2003. Wien: Verlag Österreich.

Statistik Austria (2004). Grundsystematik der Güter OCPA 2002. Wien: Verlag Österreich.

Statistik Austria (2005). Aufkommens- und Verwendungstabelle 2001. Wien: Verlag Österreich.

Steenge, A. E., \& Konijn, P. J. A. (1992). A New Approach to Irreducibility in Multisectoral Models with Joint Production. Economic Systems Research, 4(2), 125131.

Stehr, N. (2001). Wissen und Wirtschaften. Frankfurt (M): Suhrkamp.

Steinhausen, D., \& Langer, K. (1977). Clusteranalyse: Einführung in Methoden und Verfahren der automatischen Klassifikation. Berlin: de Gruyter.

Stuckenschmidt, H., \& van Harmelen, F. (2004). Information Sharing on the Semantic Web. Berlin: Springer. Michael Weber - 978-3-631-75376-7 
Tanimoto, T. T. (1957). IBM Internal report (IBM Technical Report Series).

Tapscott, D. (1995). The Digital Economy. New York: McGraw-Hill.

Taylor, M. (1969). Influence Structures. Sociometry, 32(4), 490-502.

United Nations (1993). System of National Accounts 1993, Ser.F, No.2, Rev.4. New York: United Nations.

United Nations (2004). Updates and Amendments to the System of National Accounts 1993, Ser.F, No.2, Rev.4, Add.1. New York: United Nations.

Visser, U. (2004). Intelligent Information Integration for the Semantic Web. Berlin: Springer.

von Neumann, J. (1928). Zur Theorie der Gesellschaftsspiele. Mathematische Annalen, 100(1), 295-320.

von Neumann, J., \& Morgenstern, O. (1944). Theory of Games and Economic Behavior. Princeton: University Press.

Wasserman, S., \& Faust, K. (1994). Social Network Analysis: Methods and Applications. Cambridge: University Press.

Weber, M. (2007). Introducing blockmodeling to input-output analysis. Online Proceedings of the 16th Intemational Input-Output Conference, Istanbul, Turkey, July 2-6, 2007. Abgerufen am 15. August 2007 von http://www.iioa.org/Conference/16th-downable\%20paper.html

Weber, M., \& Denk, M. (2007). Two-mode blockmodeling for valued graphs. Abstract Booklet of the 31st Annual Conference of the German Classification Society on Data Analysis, Machine Leaming, and Applications, March 7-9, 2007, Freiburg i. Br., Germany, 202.

Weber, M., \& Denk, M. (2008). Valued blockmodeling for input-output applications. Abstract Booklet of the Workshop on Blockmodeling, January 10, 2008, Ljubljana, Slovenia, 10-11. 
Weber, M., \& Fröschl, K. A. (2006). Das Semantic Web als Innovation in der ökonomischen Koordination. In: T. Pellegrini \& A. Blumauer (Hrsg.), Semantic Web: Wege zur vernetzten Wissensgesellschaft (S. 89-113). Berlin Heidelberg: Springer.

Weiser, M. (1991). The Computer for the 21st Century. Scientific American, 265(3), 94-104.

White, D. R., \& Reitz, K. P. (1983). Graph and Semigroup Homomorphisms on Networks of Relations. Social Networks, 5, 193-234.

White, H. C., Boorman, S. A., \& Breiger, R. L. (1976). Social Structure from Multiple Networks. I. Blockmodels of Roles and Positions. American Joumal of Sociology, 81(4), 730-779.

Whitehead, A. N., \& Russell, B. (1910). Principia Mathematica. Cambridge: University Press. Abgerufen am 30. Dezember 2006 von http://name.umdl.umich. edu/AAT3201.0001.001

Wielandt, H. (1950). Unzerlegbare, nicht negative Matrizen. Mathematische Zeitschrift, 52(1), 642-648.

Wigand, R., Picot, A., \& Reichwald, R. (1997). Information, Organization and Management: Expanding Markets and Corporate Boundaries. Chichester: John Wiley \& Sons.

Wilson, T. D. (2002). The nonsense of knowledge management. Information Research, 8(1), Paper Nr. 144.

Witt, U. (2003). The Evolving Economy: Essays on the Evolutionary Approach to Economics. Cheltenham: Edward Elgar.

Žiberna, A. (2006). blockmodeling: An R package for generalized and classical blockmodeling of valued networks.

Žiberna, A. (2007). Generalized blockmodeling of valued networks. Social Networks, 29(1), 105-126. 
Michael Weber - 978-3-631-75376-7

Downloaded from PubFactory at 01/11/2019 05:44:37AM

via free access 


\section{Appendix}

\section{Appendix A: Funktionsübersicht}

Tabelle 99: Funktionen für die Datenaufbereitung

\begin{tabular}{|c|c|c|c|}
\hline Name & Parameter & Ergebnis & Beschreibung \\
\hline ALMON & $\begin{array}{l}\text { VWuse, } \\
\text { VWmake, } \\
\text { threshold }\end{array}$ & $\begin{array}{l}\text { Güter x Güter } \\
\text { Matrix (IOTgg) }\end{array}$ & $\begin{array}{l}\text { Algorithmus von Almon zur Generie- } \\
\text { rung von IOT in der Dimension Güter x } \\
\text { Güter ohne negative Koeffizienten. }\end{array}$ \\
\hline SHRINK & $\begin{array}{l}\text { Matrix, Redukti- } \\
\text { onsfaktor fact }\end{array}$ & $\begin{array}{l}\text { auf fact Spalten- } \\
\text { werte reduzierte } \\
\text { Matrix }\end{array}$ & $\begin{array}{l}\text { Funktion zur Kürzung jeder Spalte einer } \\
\text { Matrix auf die fact größten Werte. } \\
\text { Diese Funktion wird auch in der Struk- } \\
\text { turanalysephase eingesetzt. }\end{array}$ \\
\hline MAKEBCIMAT & $\begin{array}{l}\text { BCledgeFRuug, } \\
\text { BCIprofile, } a, b\end{array}$ & $\begin{array}{l}\text { BCIuu, BCIug, } \\
\text { BCIau, BCIgu, } \\
\text { BClua }\end{array}$ & $\begin{array}{l}\text { Umwandlung der Transaktionslisten } \\
\text { unter Einsatz der Profildaten zur Ge- } \\
\text { nerierung bestimmter abgeleiteter } \\
\text { Datenquellen (je nach Wahl der Para- } \\
\text { meter } a, b) \text {. }\end{array}$ \\
\hline MAKEBClAUG & $\begin{array}{l}\text { BCIedgeFRuug } \\
\text { oder } \\
\text { BCledgeFRuua, } \\
\text { BClprofile, } a, b \text {, } \\
\text { uname }\end{array}$ & $\begin{array}{l}\text { BClaug, BClsell } \\
\text { (BCIag) }\end{array}$ & $\begin{array}{l}\text { Wie MAKEBCIMAT wobei mit dieser } \\
\text { Funktion die Aktivität des Unter- } \\
\text { nehmens zugeordnet wird bzw. die } \\
\text { Aktivität von den Unternehmen los- } \\
\text { gelöst betrachtet werden kann (Para- } \\
\text { meter uname). }\end{array}$ \\
\hline $\begin{array}{l}\text { ESTIMATE- } \\
\text { GOA4U }\end{array}$ & $\begin{array}{l}\text { BCIgu oder } \\
\text { BCIau, IOTgg } \\
\text { oder VWuse oder } \\
\text { IOTaa, } \\
\text { BCIprofile, typ, } \\
\text { uname }\end{array}$ & $\begin{array}{l}\text { BCIggu, BClgau, } \\
\text { BClaau, BCIgg, } \\
\text { BClaa, BCIbuy } \\
\text { (BCIga) }\end{array}$ & $\begin{array}{l}\text { Proportionale Zuweisung der er- } \\
\text { worbenen Leistungen auf passende } \\
\text { Güter bzw. Aktivităten des Teilnehmers } \\
\text { nach Maßgabe der Strukturdaten. }\end{array}$ \\
\hline
\end{tabular}

Tabelle 100: Funktionen für die Strukturanalyse

\begin{tabular}{|c|c|c|c|}
\hline Name & Parameter & Ergebnis & Beschreibung \\
\hline GSTAT & $\begin{array}{l}\text { Adjazenzmatrix } \\
\text { (für lokale Struktur; } \\
\text { z.B. BCIgg), } \\
\text { optionale Adja- } \\
\text { zenzmatrix für den } \\
\text { Vergleich (für } \\
\text { globale Struktur; } \\
\text { z.B. IOTgg), diag }\end{array}$ & $\begin{array}{l}\text { allgem. graphen- } \\
\text { theoretische Kenn- } \\
\text { zahlen, Grad- } \\
\text { statistiken, Grad- } \\
\text { indizes, Distanz- } \\
\text { indizes, kompara- } \\
\text { tive Dichtemaße }\end{array}$ & $\begin{array}{l}\text { Ermittlung graphentheoretischer Kenn- } \\
\text { zahlen (inkl. Gradstatistiken, Grad- } \\
\text { indizes, Distanzindizes) für die } \\
\text { Adjazenzmatrix. Werden zwei Matrizen } \\
\text { angegeben, so werden die Kennzahlen } \\
\text { für beide Graphen gegenübergestellt } \\
\text { und zusätzlich komparative Dichtemaße } \\
\text { ausgegeben. diag dient als Schalter für } \\
\text { den Umgang mit Selbstbezüglichkeit. } \\
\text { Diese Funktion wird auch in der Struk- } \\
\text { turvergleichsphase eingesetzt. }\end{array}$ \\
\hline GEOD & $\begin{array}{l}\text { Adjazenzmatrix } \\
\text { (z.B. BCIgg oder } \\
\text { IOTgg), dia }\end{array}$ & $D I S T$ & $\begin{array}{l}\text { Berechnung der Länge des kürzesten } \\
\text { Pfades für jedes Knotenpaar der Ad- } \\
\text { jazenzmatrix. Der Parameter dia steuert } \\
\text { den Einbezug von Selbstbezüglichkeit. }\end{array}$ \\
\hline
\end{tabular}




\begin{tabular}{|c|c|c|c|}
\hline Name & Parameter & Ergebnis & Beschreibung \\
\hline GEODCOUNT & $\begin{array}{l}\text { Adjazenzmatrix } \\
\text { (BCIgg, IOTgg), } \\
\text { dia, diaout }\end{array}$ & DISTc & $\begin{array}{l}\text { Ermittlung der Anzahl der kürzesten } \\
\text { Verbindungen für jedes Knotenpaar der } \\
\text { Adjazenzmatrix. Der Parameter dia hat } \\
\text { dieselbe Funktion wie in GEOD. }\end{array}$ \\
\hline SUMMAT & $\begin{array}{l}\text { Adjazenzmatrix } \\
\text { (z.B. BCIgg, } \\
\text { IOTgg), dia }\end{array}$ & Smat & $\begin{array}{l}\text { Berechnung der Anzahl der Ver- } \\
\text { bindungen bis zur Weglănge }(n-1) \text { für } \\
\text { jedes Knotenpaar der Adjazenzmatrix. } \\
\text { Parameter dia siehe GEOD. }\end{array}$ \\
\hline REACH & $\begin{array}{l}\text { Adjazenzmatrix } \\
\text { (z.B. BClgg, } \\
\text { IOTgg), dia }\end{array}$ & Rmat & $\begin{array}{l}\text { Bestimmung der Erreichbarkeit für alle } \\
\text { Knotenpaare der Adjazenzmatrix unter } \\
\text { Einsatz von SUMMAT. Parameter dia } \\
\text { siehe GEOD. }\end{array}$ \\
\hline MATPOWER & $\begin{array}{l}\text { Adjazenzmatrix, } p \text {, } \\
\text { circular }\end{array}$ & Mat $^{p \text { circ }}$, Mat $^{p}$ & $\begin{array}{l}\text { Matrixpotenzierung unter Ausschluss } \\
\text { von Schleifen. Der Parameter } p \text { ist der } \\
\text { Exponent, circular bestimmt die Aus- } \\
\text { gabe von zirkulären Bezügen in der } \\
\text { Ergebnismatrix. }\end{array}$ \\
\hline COMPKLIK & $\begin{array}{l}\text { Adjazenzmatrix } \\
\text { (z.B. BClgg), typ }\end{array}$ & klik & $\begin{array}{l}\text { Identifikation von zusammenhängenden } \\
\text { Subgraphen für die Adjazenzmatrix auf } \\
\text { Basis einer symmetrischen Asso- } \\
\text { ziationsmatrix und der entsprechenden } \\
\text { Klassifikation der Knotenpaare als stark } \\
\text { bzw. schwach zusammenhängend. Der } \\
\text { Parameter typ gibt an, ob der Original- } \\
\text { ansatz nach Hubbell oder dessen } \\
\text { Adaption verwendet werden soll. } \\
\text { Diese Funktion wird auch in der Lü- } \\
\text { ckenbewertungsphase eingesetzt. }\end{array}$ \\
\hline COMPZMG & $\begin{array}{l}\text { Adjazenzmatrix } \\
\text { (z.B. BCIgg), } \\
\text { alpha }\end{array}$ & $\Theta_{(1-\alpha)}$ & $\begin{array}{l}\text { Berechnung der Zusammenhangs- } \\
\text { maßzahl des durch die Adjazenzmatrix } \\
\text { definierten Graphen als ( } 1 \text { - alpha)- } \\
\text { Perzentil des Assoziationsindex. }\end{array}$ \\
\hline $\begin{array}{l}\text { ORDERED- } \\
\text { PLOTKLIK }\end{array}$ & $\begin{array}{l}\text { Adjazenzmatrix } \\
\text { (z.B. BClgg), klik, } \\
\text { typ }\end{array}$ & Grafik & $\begin{array}{l}\text { Grafische Darstellung der gemäß der } \\
\text { identifizierten Cliquen (Parameter klik) } \\
\text { permutierten Adjazenzmatrix. Para- } \\
\text { meter typ siehe comPKLIK. Diese } \\
\text { Funktion wird auch in der Lücken- } \\
\text { bewertungsphase eingesetzt. }\end{array}$ \\
\hline FS & $\begin{array}{l}\text { Adjazenzmatrix } \\
\text { (z.B. BCIgg), fsu, } \\
\text { fso, adj, nodia }\end{array}$ & FmatVal & $\begin{array}{l}\text { Reduktion der Adjazenzmatrix auf Ver- } \\
\text { bindungen im über fsu und fso spezi- } \\
\text { fizierten Wertebereich. Der Parameter } \\
\text { adj steuert, ob die reduzierte Matrix } \\
\text { binär ausgegeben werden soll. Der } \\
\text { Parameter nodia gibt an, ob die Dia- } \\
\text { gonale der Ergebnismatrix } 0 \text { gesetzt } \\
\text { werden soll. }\end{array}$ \\
\hline PLOTIO & $\begin{array}{l}\text { Adjazenzmatrix } \\
\text { (z.B. BClgg) }\end{array}$ & Graph & $\begin{array}{l}\text { Darstellung der Adjazenzmatrix als } \\
\text { Graph. }\end{array}$ \\
\hline CHECKBT & $\begin{array}{l}\text { Adjazenzmatrix } \\
\text { (z.B. BCIgg bzw. } \\
\text { Ausschnitt (Block) } \\
\text { daraus) }\end{array}$ & $\begin{array}{l}\text { Blocktyp inkl. not- } \\
\text { wendiger Änderun- } \\
\text { gen }\end{array}$ & $\begin{array}{l}\text { Überprüfung welchem generalisierten } \\
\text { Blocktyp die Adjazenzmatrix entspricht } \\
\text { und, falls keine Zuordnung möglich ist, } \\
\text { Ermittlung der erforderlichen Ände- } \\
\text { rungen (Löschen von Nullzeilen bzw. } \\
\text { Nullspalten) um einen Blocktyp zu- } \\
\text { ordnen zu können. }\end{array}$ \\
\hline
\end{tabular}




\begin{tabular}{|c|c|c|c|}
\hline Name & Parameter & Ergebnis & Beschreibung \\
\hline BLOCKMODELING & $\begin{array}{l}\text { Adjazenzmatrix } \\
\text { (z.B. BCIgg), } \\
\text { Anzahl Zeilen- } \\
\text { cluster, Anzahl } \\
\text { Spaltencluster, } \\
\text { Startlösung Zeilen- } \\
\text { cluster (optional), } \\
\text { Startlosung Spal- } \\
\text { tencluster } \\
\text { (optional), } \\
\text { nOptim }\end{array}$ & $\begin{array}{l}\text { Blockmodell (um- } \\
\text { fasst eine Liste } \\
\text { aller untersuchten } \\
\text { Clusterings sowie } \\
\text { für das optimale } \\
\text { Clustering jedes } \\
\text { Optimierungs- } \\
\text { laufes Zeilen- und } \\
\text { Spaltencluster, } \\
\text { ideales und empi- } \\
\text { risches Block- } \\
\text { modell, Ziel- } \\
\text { funktionswert (= } \\
\text { Diskrepanz) und } \\
\text { Beiträge der } \\
\text { Blöcke zum Ziel- } \\
\text { funktionswert) } \\
\end{array}$ & $\begin{array}{l}\text { Berechnung eines two-mode Block- } \\
\text { modells für die Adjazenzmatrix basie- } \\
\text { rend auf struktureller Äquivalenz mit } \\
\text { vorgegebener Anzahl an Zeilen- und } \\
\text { Spaltenclustem ausgehend von einer } \\
\text { zufällig generierten oder vorgegebenen } \\
\text { Startzuordnung zu Clustern. Der Para- } \\
\text { meter nOptim spezifiziert die Anzahl der } \\
\text { Optimierungsläufe. }\end{array}$ \\
\hline PUTVINC & $\begin{array}{l}\text { Adjazenzmatrix } \\
\text { (z.B. BCIgg), } \\
\text { Anzahl Zeilen- } \\
\text { cluster, Anzahl } \\
\text { Spaltencluster, } \\
\text { graphics }\end{array}$ & $\begin{array}{l}\text { Zeilen- und Spal- } \\
\text { tenclustering }\end{array}$ & $\begin{array}{l}\text { Generierung eines zufälligen Clustering } \\
\text { der Zeilen und Spalten der Adja- } \\
\text { zenzmatrix mit vorgegebenen Cluster- } \\
\text { anzahlen. Wird in BLOCKMODELING zur } \\
\text { Erstellung einer Zufallsstartlosung ein- } \\
\text { gesetzt. Der Parameter graphics gibt } \\
\text { an, ob das erzeugte Clustering auch } \\
\text { grafisch dargestellt werden soll. }\end{array}$ \\
\hline $\begin{array}{l}\text { EVALUATE- } \\
\text { CLUSTERING }\end{array}$ & $\begin{array}{l}\text { Adjazenzmatrix } \\
\text { (z.B. BCIgg), } \\
\text { Anzahl Zeilen- } \\
\text { cluster, Anzahl } \\
\text { Spaltencluster, } \\
\text { Zeilenclustering, } \\
\text { Spaltenclustering, } \\
\text { Liste der schon } \\
\text { überprüften } \\
\text { Cluster- } \\
\text { zuordnungen } \\
\text { (optional) }\end{array}$ & $\begin{array}{l}\text { Blockmodell (siehe } \\
\text { BLOCKMODELING; } \\
\text { insb. ideales und } \\
\text { empirisches Block- } \\
\text { modell, Ziel- } \\
\text { funktionswert und } \\
\text { Beiträge der } \\
\text { Blöcke zum Ziel- } \\
\text { funktionswert) }\end{array}$ & $\begin{array}{l}\text { Berechnung des idealen und empiri- } \\
\text { schen Blockmodells, des Zielfunktions- } \\
\text { wertes und der Beiträge der einzelnen } \\
\text { Blöcke zur Zielfunktion für die an- } \\
\text { gegebene Clusterzuordnung der } \\
\text { Adjazenzmatrix. }\end{array}$ \\
\hline ITERATE & $\begin{array}{l}\text { Blockmodell, ind, } \\
\text { iterations }\end{array}$ & $\begin{array}{l}\text { Blockmodell (siehe } \\
\text { BLOCKMODELING } \\
\text { Und EVALUATE- } \\
\text { CLUSTERING) }\end{array}$ & $\begin{array}{l}\text { Steuerung des iterativen Prozesses zur } \\
\text { Untersuchung von Clusterzuordnungen } \\
\text { in der Nachbarschaft des angegebenen } \\
\text { Blockmodells (Ergebnis von } \\
\text { EVALUATECLUSTERING). Der Parameter } \\
\text { ind gibt den Laufindex der aktuellen } \\
\text { Iteration an, während iterations die } \\
\text { maximale Anzahl an Iterationsschritten } \\
\text { festlegt. }\end{array}$ \\
\hline
\end{tabular}




\begin{tabular}{|l|l|l|l|}
\hline \multicolumn{1}{|c}{ Name } & \multicolumn{2}{c|}{ Eargebnis } & \multicolumn{1}{c|}{ Beschreibung } \\
\hline EXPLORESHIFT & $\begin{array}{l}\text { Blockmodell, Index } \\
\text { des Blockmodells } \\
\text { in der Liste der } \\
\text { bereits unter- } \\
\text { suchten Block- } \\
\text { modelle }\end{array}$ & $\begin{array}{l}\text { (lokal optimales) } \\
\text { Blockmodell (siehe } \\
\text { BLOCKMODELING } \\
\text { und EVALUATE- } \\
\text { CLUSTERING), } \\
\text { Index dieses } \\
\text { Blockmodells in } \\
\text { der Liste der } \\
\text { bereits unter- } \\
\text { suchten Block- } \\
\text { modelle }\end{array}$ & $\begin{array}{l}\text { Ermittlung eines lokal optimalen Block- } \\
\text { modells durch Generierung und Unter- } \\
\text { suchung der Nachbarschaft des an- } \\
\text { gegebenen Blockmodells (der aktuellen } \\
\text { Losung) mittels (einzelnem) Ver- } \\
\text { schieben jedes Zeilen- und jedes } \\
\text { Spaltenknoten in alle verfügbaren alter- } \\
\text { nativen Zeilen- bzw. Spaltencluster. }\end{array}$ \\
\hline DRAWCLU & $\begin{array}{l}\text { Adjazenzmatrix } \\
\text { (z.B. BCIgg), } \\
\text { Zeilen- und Spal- } \\
\text { tenclustering }\end{array}$ & Grafik & $\begin{array}{l}\text { Grafische Darstellung des an- } \\
\text { gegebenen Blockmodells für die } \\
\text { Adjazenzmatrix. }\end{array}$ \\
\hline
\end{tabular}

Tabelle 101: Funktionen für den Strukturvergleich

\begin{tabular}{|c|c|c|c|}
\hline Name & Parameter & Ergebnis & Beschreibung \\
\hline STRUK & $\begin{array}{l}\text { Adjazenzmatrix } \\
\text { (für lokale Struktur; } \\
\text { z.B. BCIgg), } \\
\text { Adjazenzmatrix für } \\
\text { den Vergleich (für } \\
\text { globale Struktur; } \\
\text { z.B. IOTgg), } \\
\text { wwdim }\end{array}$ & MatValGap & $\begin{array}{l}\text { Ermittlung der Matrix MatValGap zur } \\
\text { Bestimmung interner struktureller Lü- } \\
\text { cken der lokalen Struktur durch Ver- } \\
\text { gleich der lokalen Verflechtungsdaten } \\
\text { aus BCIgg mit den globalen Struktur- } \\
\text { daten aus IOTgg. Der Parameter } \\
\text { vwdim gibt an, ob die Ergebnismatrix } \\
\text { MatValGap in der Dimension der } \\
\text { globalen oder der lokalen Ver- } \\
\text { flechtungsdaten ausgegeben werden } \\
\text { soll. }\end{array}$ \\
\hline EDGEFRAME & $\begin{array}{l}\text { Adjazenzmatrix } \\
\text { (z.B. MatValGap, } \\
\text { BClgugu), split }\end{array}$ & $\begin{array}{l}\text { Kantenliste (z.B. } \\
\text { EdgeframeMat- } \\
\text { ValGap, } \\
\text { BCleFgugu) }\end{array}$ & $\begin{array}{l}\text { Umwandlung einer Adjazenzmatrix in } \\
\text { eine Kantenliste. Der Parameter split } \\
\text { steuert bei Adjazenzmatrizen mit } \\
\text {,kombinierten' Knoten (z.B. die Kom- } \\
\text { bination Gut-Unternehmen in BCIgu- } \\
g u \text { ), ob diese im Zuge der Kanten- } \\
\text { listenerstellung in ihre Bestandteile } \\
\text { aufgesplittet werden sollen. } \\
\text { Diese Funktion wird auch in der Um- } \\
\text { legungsphase eingesetzt. }\end{array}$ \\
\hline EDGEMIX & $\begin{array}{l}\text { Kantenliste 1, } \\
\text { Kantenliste 2, } \\
\text { NAinkl }\end{array}$ & $\begin{array}{l}\text { zusammen- } \\
\text { geführte Kanten- } \\
\text { liste }\end{array}$ & $\begin{array}{l}\text { Zusammenführung zweier Kantenlisten } \\
\text { durch Verknüpfung der Nachfolger- } \\
\text { knoten der } 1 \text {. Kantenliste mit den Vor- } \\
\text { gängerknoten der } 2 \text {. Kantenliste. Der } \\
\text { Parameter NAinkl gibt an, ob für, Ver- } \\
\text { bindungsknoten', die nur in einer der } \\
\text { beiden Kantenlisten vorkommen, ein } \\
\text { Eintrag in der zusammengeführten } \\
\text { Kantenliste erstellt werden soll. }\end{array}$ \\
\hline
\end{tabular}




\begin{tabular}{|c|c|c|c|}
\hline Name & Parameter & Ergebnis & Beschreibung \\
\hline MATGAPCOMP & $\begin{array}{l}\text { MatValGap, pow, } \\
\text { ARR, diaoff, circu- } \\
\text { lar }\end{array}$ & $\begin{array}{l}\text { Matrix bzw. Liste } \\
\text { von Matrizen der } \\
\text { Lückenfolgen- } \\
\text { klassifikation } \\
\text { (MatGapComp } \\
\text { bzw. } \\
\text { list }_{p-1 \text { pow }}(\text { Mat- } \\
\left.\left.\text { GapComp } p^{p}\right)\right)\end{array}$ & $\begin{array}{l}\text { Bestimmung der internen Lückenfolge- } \\
\text { typen, die die jeweiligen Knotenpaare } \\
\text { der Ausgangsmatrix Mat( } \text { Val)Gap auf } \\
\text { der Weglänge pow bzw. auf den Weg- } \\
\text { längen } 1 \text { bis pow verbinden. Der Para- } \\
\text { meter ARR steuert, ob die Klassifikation } \\
\text { der internen Lückenfolgen nur für Weg- } \\
\text { länge pow oder für die Weglängen } 1 \text { bis } \\
\text { pow erfolgt. diaoff dient als Schalter für } \\
\text { den Umgang mit Selbstbezüglichkeit. } \\
\text { circular bestimmt die Berücksichtigung } \\
\text { von zirkulären Bezügen. }\end{array}$ \\
\hline STAFETTE & $\begin{array}{l}\text { matgapcomp, } \\
\text { listout, interm }\end{array}$ & $\begin{array}{l}\text { Stafette (z.B. } \\
\text { GapLi, GapLiExt, } \\
\text { GapLiTot) }\end{array}$ & $\begin{array}{l}\text { Ermittlung einer speziellen Distanz- } \\
\text { matrix, die für jedes Knotenpaar angibt, } \\
\text { welche Typen von Lückenfolgen die } \\
\text { jeweiligen Knoten bei welcher Weg- } \\
\text { länge das erste Mal in Beziehung } \\
\text { setzen, auf Basis einer mittels MAT- } \\
\text { GAPCoMP bestimmten Liste von Matri- } \\
\text { zen von (internen und/oder extemen) } \\
\text { Lückenfolgetypen (Parameter mat- } \\
\text { gapcomp). listout steuert dabei das } \\
\text { Ausgabeformat (Distanzmatrix oder } \\
\text { Kantenliste). intem gibt an, ob auch } \\
\text { externe Lückenfolgetypen berück- } \\
\text { sichtigt werden sollen. }\end{array}$ \\
\hline MATEXTCOMP & $\begin{array}{l}\text { Adjazenzmatrix } \\
\text { (für globale Struk- } \\
\text { tur; z.B. IOTgg), } \\
\text { locN, pow, ARR, } \\
\text { combVlocVglo, } \\
\text { diaoff, circular }\end{array}$ & $\begin{array}{l}\text { Matrix bzw. Liste } \\
\text { von Matrizen der } \\
\text { Anzahlen von } \\
\text { extemen Lücken- } \\
\text { folgen }\end{array}$ & $\begin{array}{l}\text { Bestimmung von externen Lücken- } \\
\text { folgen durch Ermittlung der Anzahl der } \\
\text { Wege, die die jeweiligen Knotenpaare } \\
\text { der lokalen Struktur (bzw. auch der } \\
\text { lokalen und globalen Struktur) auf der } \\
\text { Weglänge pow bzw. auf den Weg- } \\
\text { längen } 1 \text { bis pow verbinden. locN defi- } \\
\text { niert die Namen der lokalen Knoten. } \\
\text { combVlocVglo gibt an, ob nur Wege } \\
\text { betrachtet werden sollen, die mit in- } \\
\text { ternen Knoten beginnen und enden. } \\
\text { Die Parameter pow, ARR, diaoff und } \\
\text { circular erfüllen den gleichen Zweck wie } \\
\text { in MATGAPCOMP. }\end{array}$ \\
\hline DETEREXT & $\begin{array}{l}\text { Adjazenzmatrix } \\
\text { (für globale Struk- } \\
\text { tur, z.B. IOTgg), } \\
\text { MatValGap, pow, } \\
\text { ARR, diaoff, } \\
\text { circular }\end{array}$ & $\begin{array}{l}\text { Matrix bzw. Liste } \\
\text { von Matrizen der } \\
\text { Lückenfolgen- } \\
\text { klassifikation }\end{array}$ & $\begin{array}{l}\text { Bestimmung der extermen Lückenfolge- } \\
\text { typen, die die jeweiligen Knotenpaare } \\
\text { der Ausgangsmatrix MatValGap auf } \\
\text { der Weglänge pow bzw. auf den Weg- } \\
\text { längen } 1 \text { bis pow verbinden. Die Para- } \\
\text { meter pow, ARR, diaoff und circular } \\
\text { erfüllen den gleichen Zweck wie in } \\
\text { MATGAPCOMP. }\end{array}$ \\
\hline
\end{tabular}




\begin{tabular}{|c|c|c|c|}
\hline Name & Parameter & Ergebnis & Beschreibung \\
\hline DETERINTEXT & $\begin{array}{l}\text { Adjazenzmatrix } \\
\text { (für globale Struk- } \\
\text { tur, z.B. IOTgg), } \\
\text { MatValGap, pow, } \\
\text { ARR, diaoff, } \\
\text { circular }\end{array}$ & $\begin{array}{l}\text { Matrix bzw. Liste } \\
\text { von Matrizen der } \\
\text { Lückenfolgen- } \\
\text { klassifikation }\end{array}$ & $\begin{array}{l}\text { Bestimmung der internen und externen } \\
\text { Lückenfolgetypen, die die jeweiligen } \\
\text { Knotenpaare der Ausgangsmatrix } \\
\text { MatValGap auf der Weglänge pow } \\
\text { bzw. auf den Weglängen } 1 \text { bis pow } \\
\text { verbinden. Die Parameter pow, ARR, } \\
\text { diaoff und circular erfüllen den gleichen } \\
\text { Zweck wie in MATGAPCOMP. }\end{array}$ \\
\hline TYPESTAT & $\begin{array}{l}\text { Stafette der in- } \\
\text { ternen Lücken- } \\
\text { folgen (GapLi), } \\
\text { Stafette der ex- } \\
\text { ternen Lücken- } \\
\text { folgen (GapLiExt), } \\
\text { ratio }\end{array}$ & $\begin{array}{l}\text { Kardinalitäten und } \\
\text { Anteilsmaßzahlen } \\
\text { zur Lückenstruktur }\end{array}$ & $\begin{array}{l}\text { Berechnung von einfachen Häufig- } \\
\text { keiten (Kardinalitäten der Lückenfolge- } \\
\text { klassen) und Kennzahlen zur Lücken- } \\
\text { struktur (z.B. direktes Verbindungs- } \\
\text { potenzial, Potenzial über indirekte } \\
\text { (interne und/oder externe) Wirkungs- } \\
\text { ketten, Verkürzungspotenzial). Der } \\
\text { Parameter ratio steuert ob nur } \\
\text { Kardinalitäten oder auch die Anteils- } \\
\text { kennzahlen zur Lückenstruktur aus- } \\
\text { gegeben werden sollen. }\end{array}$ \\
\hline STRUHO & $\begin{array}{l}\text { Adjazenzmatrix } \\
\text { (für globale Struk- } \\
\text { tur, z.B. IOTgg), } \\
\text { Profildaten (z.B. } \\
\text { BCIprofile), } \\
\text { entspr. Kantenliste } \\
\text { (BCledgeFRuug } \\
\text { oder BCledge- } \\
\text { FRuua), typ, sel }\end{array}$ & $\begin{array}{l}\text { Kantenliste ex- } \\
\text { terner und in- } \\
\text { direkter interner } \\
\text { Lücken für Weg- } \\
\text { länge } 2 \text { bzw. } 3 \\
\text { (z.B. } \\
\text { Edgeframe } \\
\text { Edgeframe }^{\text {owoho }} \text { ') }\end{array}$ & $\begin{array}{l}\text { Detaillierte Ermittlung externer und } \\
\text { indirekter interner Lücken für die Weg- } \\
\text { länge } 2 \text { bzw. } 3 \text {. Der Parameter typ gibt } \\
\text { an, ob Lücken auf Basis von Gütern } \\
\text { oder Aktivitäten ermittelt werden sollen. } \\
\text { sel definiert, welche Start- und Ziel- } \\
\text { knotenmenge verwendet wird (lokal } \\
\text { oder global) und ob ein oder zwei ex- } \\
\text { terne Verbindungsknoten eingesetzt } \\
\text { werden (d.h. Weglänge } 2 \text { oder 3). }\end{array}$ \\
\hline STRUHOMU & $\begin{array}{l}\text { VWuse, VWmake, } \\
\text { Profildaten (z.B. } \\
\text { BCIprofile), } \\
\text { entsprechende } \\
\text { Kantenliste (BCI- } \\
\text { edgeFRuug oder } \\
\text { BCledgeFRuua), } \\
\text { startuse }\end{array}$ & 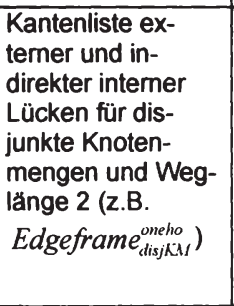 & $\begin{array}{l}\text { Detaillierte Ermittlung externer und } \\
\text { indirekter interner Lücken für disjunkte } \\
\text { Knotenmengen und Weglänge } 2 \text {. } \\
\text { Anstatt einer Adjazenzmatrix wie in der } \\
\text { Funktion STRUHO werden hier daher } \\
\text { eine Use- und eine Make-Matrix be- } \\
\text { nötigt. Der Parameter startuse gibt an, } \\
\text { ob das Verknüpfungsmuster Use-Make } \\
\text { oder Make-Use angewendet werden } \\
\text { soll. }\end{array}$ \\
\hline $\begin{array}{l}\text { EDGESTAT- } \\
\text { ONEHO }\end{array}$ & Kantenliste & Kennzahlen & $\begin{array}{l}\text { Berechnung deskriptiver Statistiken } \\
\text { (absolute und relative Häufigkeiten, } \\
\text { Input-Wertanteil, Output-Wertanteil) für } \\
\text { Verbindungsknoten in einer Kantenliste } \\
\text { von Lückenfolgen der Weglänge } 2 \text {. }\end{array}$ \\
\hline $\begin{array}{l}\text { EDGESTAT- } \\
\text { ONEHOTAB }\end{array}$ & Kantenliste & $\begin{array}{l}\text { Adjazenzmatrix mit } \\
\text { Häufigkeiten }\end{array}$ & $\begin{array}{l}\text { Ermittlung der Anzahlen der Lücken- } \\
\text { folgen der Weglänge 2, die die Knoten- } \\
\text { paare der angegebenen Kantenliste } \\
\text { verbinden. }\end{array}$ \\
\hline
\end{tabular}


Tabelle 102: Funktionen für die Lückenbewertung

\begin{tabular}{|c|c|c|c|}
\hline Name & Parameter & Ergebnis & Beschreibung \\
\hline $\begin{array}{l}\text { SIMPLE- } \\
\text { PERFGAP }\end{array}$ & 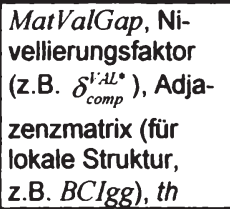 & $\begin{array}{l}\text { Adjazenzmatrix mit } \\
\text { klassifizierten } \\
\text { Performanzlücken } \\
\text { (z.B. PerfGap }^{\text {binary }} \text {, } \\
\text { PerfGap }\end{array}$ & $\begin{array}{l}\text { Bestimmung von Performanzlücken mit } \\
\text { Hilfe der einfachen Bewertungsverfahren. } \\
\text { th gibt dabei die Schranke(n) für die } \\
\text { Klassifikation von Kanten als Performanz- } \\
\text { lücken an. }\end{array}$ \\
\hline $\begin{array}{l}\text { RATEPERF- } \\
\text { GAP }\end{array}$ & $\begin{array}{l}\text { MatValGap, Ad- } \\
\text { jazenzmatrix (für } \\
\text { lokale Struktur, } \\
\text { z.B. BCIgg), type, } \\
\text { direction, bin }\end{array}$ & $\begin{array}{l}\text { Adjazenzmatrix mit } \\
\text { klassifizierten } \\
\left(\text { PerfGap }{ }^{\text {binary }}\right) \\
\text { oder bewerteten } \\
\text { (Rate }^{\text {poIPerf }} \text { ) Per- } \\
\text { formanzlücken }\end{array}$ & $\begin{array}{l}\text { Bestimmung von Performanzlücken mit } \\
\text { Hilfe der komplexen Bewertungsver- } \\
\text { fahren. Der Parameter type steuert, ob } \\
\text { die Bewertung der Performanzlücken } \\
\text { unter Einbezug nur direkter (Matrizen } A \text {, } \\
\left.A^{*}\right) \text {, auch indirekter }\left(W, W^{*}\right) \text {, oder aller } \\
\text { indirekter }\left(R, R^{*}\right) \text { Wirkungen ermittelt } \\
\text { wird. direction gibt an, ob die Berechnung } \\
\text { in Bezug auf Nachfolger- oder Vor- } \\
\text { găngerknoten bzw. beide erfolgen soll. } \\
\text { bin bestimmt, ob die Bewertung der Per- } \\
\text { formanzlücken oder deren Klassifikation } \\
\text { ausgegeben werden soll. }\end{array}$ \\
\hline WhL & $\begin{array}{l}\text { zeilen- oder spal- } \\
\text { tennormierte Adja- } \\
\text { zenzmatrix }\left(A, A^{*}\right) \text {, } \\
\text { valAV, diaoff, } \\
\text { circular, pow }\end{array}$ & $\begin{array}{l}\text { Adjazenzmatrix mit } \\
\text { den Werten der } \\
\text { spezifizierten } W \text { - } \\
\text { Reihe }\left(W^{\text {wll }}\right. \\
\left.W^{\text {wl.cover }}, W^{\text {w.l.mer2 }}\right)\end{array}$ & $\begin{array}{l}\text { Berechnung der W-Reihe der an- } \\
\text { gegebenen Adjazenzmatrix. pow legt die } \\
\text { maximale Weglänge fest (Defaultwert ist } \\
n-1 \text { ). valAV definiert ob nur durch- } \\
\text { schnittliche Wirkungen berücksichtigt } \\
\text { werden sollen. diaoff und circular wie in } \\
\text { MATGAPCOMP. }\end{array}$ \\
\hline WDIST & $\begin{array}{l}\text { zeilen- oder spal- } \\
\text { tennormierte Ad- } \\
\text { jazenzmatrix }(A \text {, } \\
\left.A^{*}\right), \text { shortestAV, } \\
\text { indirOnly, diaoff, } \\
\text { circular }\end{array}$ & $\begin{array}{l}\text { Adjazenzmatrix mit } \\
\text { den Werten der } \\
\text { speziellen W- } \\
\text { Reihe }\left(W^{\text {DIST }}\right. \\
\left.W^{\text {DIST.aver }}\right)\end{array}$ & $\begin{array}{l}\text { Berechnung der speziellen W-Reihe der } \\
\text { angegebenen Adjazenzmatrix, die nur } \\
\text { Effekte bis zur jeweiligen ersten Erreich- } \\
\text { barkeit der Knotenpaare berücksichtigt. } \\
\text { indirOnly steuert die Einbeziehung direk- } \\
\text { ter Effekte. shortestAV ist vergleichbar } \\
\text { mit valAV in Wh. diaoff und circular wie } \\
\text { in MATGAPCOMP. }\end{array}$ \\
\hline BVTH & $\begin{array}{l}\text { zeilen- oder spal- } \\
\text { tennormierte Adja- } \\
\text { zenzmatrix }\left(A, A^{*}\right) \text {, } \\
\text { th, pmax, diaoff, } \\
\text { circular }\end{array}$ & $\begin{array}{l}\text { Adjazenzmatrix mit } \\
\text { den Werten der } \\
\text { speziellen } W \text { - } \\
\text { Reihe }\left(W^{b(t h)}\right)\end{array}$ & $\begin{array}{l}\text { Berechnung der speziellen W-Reihe der } \\
\text { angegebenen Adjazenzmatrix, die die } \\
\text { Weglänge so festlegt, dass nur indirekte } \\
\text { Effekte, die den Schwellwert th über- } \\
\text { schreiten, berücksichtigt werden. pmax } \\
\text { gibt eine Obergrenze für die Weglänge } \\
\text { an. diaoff und circular wie in MATGAP. } \\
\text { COMP. }\end{array}$ \\
\hline $\begin{array}{l}\text { SMAT- } \\
\text { WEIGHTING }\end{array}$ & $\begin{array}{l}\text { zeilen- oder spal- } \\
\text { tennormierte Adja- } \\
\text { zenzmatrix }\left(A, A^{*}\right)\end{array}$ & $\begin{array}{l}\text { Liste von Adja- } \\
\text { zenzmatrizen mit } \\
\text { Werten der Leon- } \\
\text { tief-Inverse für alle } \\
\text { vollständigen } \\
\text { Blöcke } \\
\end{array}$ & $\begin{array}{l}\text { Identifikation von allen vollständigen } \\
\text { Blöcken der angegebenen Adjazenz- } \\
\text { matrix und Berechnung der jeweiligen } \\
\text { Leontief-Inversen zur Bedeutungs- } \\
\text { bestimmung. }\end{array}$ \\
\hline
\end{tabular}




\begin{tabular}{|c|c|c|c|}
\hline Name & Parameter & Ergebnis & Beschreibung \\
\hline $\begin{array}{l}\text { MATPOWER- } \\
\text { CUM }\end{array}$ & $\begin{array}{l}\text { Matrix, pow, } \\
\text { areaR, areaC, } \\
\text { diaoff, circular }\end{array}$ & $\begin{array}{l}\text { kumulierte pow-te } \\
\text { Potenzmatrix }\end{array}$ & $\begin{array}{l}\text { Erweiterung der Funktion SUMMAT. Be- } \\
\text { rechnung der kumulierten pow-ten Po- } \\
\text { tenz der angegebenen Matrix. areaR und } \\
\text { areaC dienen zur Nullsetzung von be- } \\
\text { stimmten Bereichen der Matrix. diaoff und } \\
\text { circular wie in MATGAPCOMP. }\end{array}$ \\
\hline WEAKNODES & $\begin{array}{l}\text { Adjazenzmatrix } \\
\text { (für lokale Struktur, } \\
\text { z.B. BCIgg), dazu } \\
\text { ermittelte Per- } \\
\text { formanz-lücken } \\
\text { (z.B. Perf- } \\
\text { Gap binary), Be- } \\
\text { wertung dieser } \\
\text { Performanzlücken } \\
\text { (z.B. Rate } \\
\text { thoiPerf), } \\
\text { threction, type } \\
\end{array}$ & $\begin{array}{l}\text { Knotenbewertung } \\
\text { od. -klassifikation } \\
\text { (pfgNode, weak- } \\
\text { Node, shNode, } \\
\text { whNode, jeweils } \\
\text { mit Superskript }{ }^{I N} \text {, } \\
\text { OUT } \text { oder }^{T O T} \text { ) }\end{array}$ & $\begin{array}{l}\text { Bewertung bzw. Klassifikation der Knoten } \\
\text { der angegebenen Adjazenzmatrix hin- } \\
\text { sichtlich ihrer Eigenschaft als ,schwache } \\
\text { Knoten'. direction wie in RATEPERFGAP. } \\
\text { type gibt an, welche Art der Bewertung } \\
\text { bzw. Klassifikation verwendet werden } \\
\text { soll. th legt die Schranke für die Klassi- } \\
\text { fikation fest. }\end{array}$ \\
\hline RATEDIGAP & $\begin{array}{l}\text { Adjazenzmatrix } \\
\text { (für lokale Struktur, } \\
\text { z.B. BCIgg), } \\
\text { Adjazenzmatrix } \\
\text { (für globale Struk- } \\
\text { tur, z.B. IOTgg), } \\
\text { Kennzeichnung } \\
\text { der Performanz- } \\
\text { lücken (Perf- } \\
\text { Gap binary, Mat- } \\
\text { ValGap, method, } \\
\text { th, type, bin } \\
\end{array}$ & 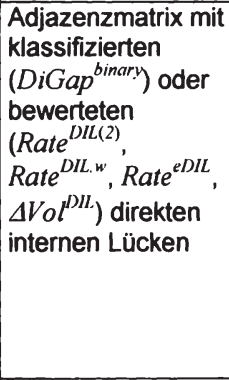 & $\begin{array}{l}\text { Bewertung und Kategorisierung von } \\
\text { direkten internen Lücken. method gibt an, } \\
\text { nach welchem Verfahren die Bewertung } \\
\text { erfolgen soll (z.B. mit/ohne Ein- } \\
\text { schränkung auf Performanzlückenfolgen, } \\
\text { unter Einbeziehung des Input- bzw. Out- } \\
\text { put-Anpassungsbedarfs, als Input- } \\
\text { /Output-Anpassungsbedarf oder über die } \\
\text { Differenz der Gesamtbedeutungen mit } \\
\text { und ohne der zu bewertenden Kan- } \\
\text { te/Lücke). type und bin wie in RATEPERF- } \\
\text { GAP. th wie in SIMPLEPERFGAP. }\end{array}$ \\
\hline RATEEDILOP & $\begin{array}{l}\text { Adjazenzmatrix } \\
\text { (für lokale Struktur, } \\
\text { z.B. BCIgg), } \\
\text { Adjazenzmatrix } \\
\text { (für globale Struk- } \\
\text { tur, z.B. IOTgg), } \\
\text { MatValGap, zu } \\
\text { bewertende Lücke } \\
e_{o p}^{D L L} \text { (angegeben } \\
\text { durch Zeilen- und } \\
\text { Spaltenindex } O \\
\text { und } p \text { ), type } \\
\end{array}$ & $\begin{array}{l}\text { Bewertung der } \\
\text { direkten internen } \\
\text { Lücke (rate } \text { (rDIL-op }^{\text {el }} \text { ) }\end{array}$ & $\begin{array}{l}\text { Bewertung einer direkten internen Lücke } \\
\text { durch das ,exaktere' Verfahren der Diffe- } \\
\text { renzbildung der Gesamtbedeutungen der } \\
\text { Adjazenzmatrix mit und ohne der zu } \\
\text { bewertenden Lücke/Kante. type wie in } \\
\text { RATEPERFGAP. }\end{array}$ \\
\hline RATEEXTV,W & $\begin{array}{l}\text { Adjazenzmatrix } \\
\text { (für lokale Struktur, } \\
\text { z.B. BCIgg), } \\
\text { Adjazenzmatrix } \\
\text { (für globale Struk- } \\
\text { tur, z.B. IOTgg) } \\
\end{array}$ & $\begin{array}{l}\text { Vektor der Be- } \\
\text { deutung der ex- } \\
\text { ternen Knoten } \\
\left(\text { rate }^{E X T v, w^{\prime}}\right)\end{array}$ & $\begin{array}{l}\text { Berechnung der relativen Bedeutung der } \\
\text { externen Knoten für die lokale Struktur. }\end{array}$ \\
\hline
\end{tabular}




\begin{tabular}{|c|c|c|c|}
\hline Name & Parameter & Ergebnis & Beschreibung \\
\hline RATEEXTV & $\begin{array}{l}\text { Adjazenzmatrix } \\
\text { (für lokale Struktur, } \\
\text { z.B. BClgg), } \\
\text { Adjazenzmatrix } \\
\text { (für globale Struk- } \\
\text { tur, z.B. IOTgg), } \\
\text { extemer Knoten } v_{x}\end{array}$ & $\begin{array}{l}\text { Bedeutung des } \\
\text { externen Knoten } \\
\left(\text { rate }_{x}^{k i T v}\right)\end{array}$ & $\begin{array}{l}\text { Berechnung der Bedeutung eines ex- } \\
\text { temen Knoten durch Vergleich der Ge- } \\
\text { samtbedeutung der lokalen Struktur mit } \\
\text { und ohne den zu bewertenden extemen } \\
\text { Knoten. }\end{array}$ \\
\hline $\begin{array}{l}\text { RATEEXTV- } \\
\text { LIST }\end{array}$ & $\begin{array}{l}\text { Adjazenzmatrix } \\
\text { (für lokale Struktur, } \\
\text { z.B. BClgg), } \\
\text { Adjazenzmatrix } \\
\text { (für globale Struk- } \\
\text { tur, z.B. IOTgg) } \\
\end{array}$ & $\begin{array}{l}\text { Vektor der Be- } \\
\text { deutung der ex- } \\
\text { ternen Knoten } \\
\left(\text { rate }^{\text {EITv }}\right)\end{array}$ & $\begin{array}{l}\text { Berechnung der Bedeutung der extemen } \\
\text { Knoten durch Vergleich der Gesamt- } \\
\text { bedeutung der lokalen Struktur mit und } \\
\text { ohne den jeweils zu bewertenden ex- } \\
\text { ternen Knoten. }\end{array}$ \\
\hline EXTGAP & $\begin{array}{l}\text { Bewertung der } \\
\text { externen Knoten } \\
\left(\text { z.B. rate } \text { riTv,w }^{E . T_{v}},\right. \\
\left.\text { rate }^{E I T v}\right) \text {, th }\end{array}$ & $\begin{array}{l}\text { Vektor der Klassi- } \\
\text { fikation der ex- } \\
\text { ternen Knoten als } \\
\text { exteme Lücken } \\
\left(\operatorname{extGap}^{\text {binany }}\right)\end{array}$ & $\begin{array}{l}\text { Ermittlung der Klassifikation der externen } \\
\text { Knoten als externe Lücken basierend auf } \\
\text { einer zuvor berechneten Bewertung und } \\
\text { einem Schwellwert th. }\end{array}$ \\
\hline $\begin{array}{l}\text { RATEEXTVX- } \\
\text { STAT }\end{array}$ & $\begin{array}{l}\text { Adjazenzmatrix } \\
\text { (für lokale Struktur, } \\
\text { z.B. BCIgg), } \\
\text { Adjazenzmatrix } \\
\text { (für globale Struk- } \\
\text { tur, z.B. IOTgg), } \\
\text { extemer Knoten } \\
v_{x} \text {, direction } \\
\end{array}$ & $\begin{array}{l}\text { Bedeutungsver- } \\
\text { änderung der } \\
\text { internen Knoten } \\
\left(\text { rate }^{E I T v_{x}},\right. \\
\text { rate }^{E: 17 v_{x}, N} \text { oder } \\
\text { rate }^{E .17 v_{x}, O l . T} \text { ) }\end{array}$ & $\begin{array}{l}\text { Bestimmung der Veränderung der Be- } \\
\text { deutung der internen Knoten als Vor- } \\
\text { gänger bzw. Nachfolger bei Hinzunahme } \\
\text { des angegebenen externen Knoten. } \\
\text { direction legt fest, ob die Vorgänger-, } \\
\text { Nachfolger- oder Gesamtperspektive } \\
\text { eingenommen werden soll. }\end{array}$ \\
\hline $\begin{array}{l}\text { RATEEXTVX- } \\
\text { STATLIST }\end{array}$ & $\begin{array}{l}\text { Adjazenzmatrix } \\
\text { (für lokale Struktur, } \\
\text { z.B. BCIgg), } \\
\text { Adjazenzmatrix } \\
\text { (für globale Struk- } \\
\text { tur, z.B. IOTgg), } \\
\text { direction }\end{array}$ & $\begin{array}{l}\text { Liste der Be- } \\
\text { deutungsver- } \\
\text { änderung der } \\
\text { internen Knoten }\end{array}$ & $\begin{array}{l}\text { Wie RATEEXTVXSTAT jedoch für alle } \\
\text { externen Knoten. }\end{array}$ \\
\hline $\begin{array}{l}\text { RATEEXTVX- } \\
\text { 4LOC }\end{array}$ & $\begin{array}{l}\text { Adjazenzmatrix } \\
\text { (für lokale Struktur, } \\
\text { z.B. BClgg), } \\
\text { Adjazenzmatrix } \\
\text { (für globale Struk- } \\
\text { tur, z.B. IOTgg), } \\
\text { extemer Knoten } \\
v_{x} \text {, direction }\end{array}$ & 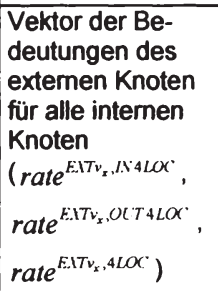 & $\begin{array}{l}\text { Ermittlung der Bedeutung des an- } \\
\text { gegebenen externen Knoten als Nach- } \\
\text { folger bzw. Vorgănger (oder beides) für } \\
\text { die intemen Knoten. direction wie in RATE- } \\
\text { EXTVXSTAT. }\end{array}$ \\
\hline $\begin{array}{l}\text { RATEEXTVX- } \\
\text { VOL }\end{array}$ & $\begin{array}{l}\text { Adjazenzmatrix } \\
\text { (für lokale Struktur, } \\
\text { z.B. BClgg), } \\
\text { Adjazenzmatrix } \\
\text { (für globale Struk- } \\
\text { tur, z.B. IOTgg), } \\
\text { externer Knoten } \\
v_{x} \text {, direction }\end{array}$ & $\begin{array}{l}\text { Vektor der er- } \\
\text { forderlichen An- } \\
\text { passungen } \\
\left(\Delta v o l^{E . T \times x} \text { oder }\right. \\
\left.\Delta v o l^{E .1 T \times}\right)\end{array}$ & $\begin{array}{l}\text { Berechnung der erforderlichen Input- } \\
\text { bzw. Outputanpassungen der intemen } \\
\text { Knoten bei Einbeziehung des an- } \\
\text { gegebenen externen Knoten. direction } \\
\text { steuert, ob die Input- oder Output- } \\
\text { Anpassung ermittelt wird. }\end{array}$ \\
\hline
\end{tabular}


Tabelle 103: Funktionen für die Umlegung

\begin{tabular}{|c|c|c|c|}
\hline Name & Parameter & Ergebnis & Beschreibung \\
\hline MBMAT & $\begin{array}{l}\text { Kantenliste der Transaktions- } \\
\text { daten (BCledgeFRuug od. } \\
\text { BCledgeFRuua), Profildaten } \\
\text { (BCIprofile), typ }\end{array}$ & $\begin{array}{l}\text { Adjazenzmatrix } \\
\text { mit ,kombi- } \\
\text { nierten' Vor- } \\
\text { gängerknoten } \\
\text { (z.B. BCIguu) }\end{array}$ & $\begin{array}{l}\text { Erstellung einer Transaktions- } \\
\text { matrix der Dimension Gut-Unter- } \\
\text { nehmen (bzw. Aktivität-Unterneh- } \\
\text { men) x Unternehmen unter Be- } \\
\text { rücksichtigung der Transaktions- } \\
\text { daten (BCledgeFRuugla) und der } \\
\text { Profildaten (BCIprofile). Der Para- } \\
\text { meter typ gibt an, ob sich die Be- } \\
\text { rechnung auf Güter oder Aktivi- } \\
\text { täten bezieht. }\end{array}$ \\
\hline GUOAU & $\begin{array}{l}\text { Adjazenzmatrix (für lokale } \\
\text { Struktur, in der Dimension } \\
\text { Gut-Unternehmen (bzw. } \\
\text { Aktivităt-Unternehmen) x } \\
\text { Unternehmen, z.B. BCIguu), } \\
\text { Adjazentmatrix (für globale } \\
\text { Struktur, z.B. IOTgg), Profil- } \\
\text { daten (BCIprofile), uname, } \\
\text { typ }\end{array}$ & $\begin{array}{l}\text { Adjazenzmatrix } \\
\text { mit kombi- } \\
\text { nierten Vor- } \\
\text { gänger- und } \\
\text { Nachfolger- } \\
\text { knoten (z.B. } \\
\text { BCIgugu) }\end{array}$ & $\begin{array}{l}\text { Erstellung einer quadratischen } \\
\text { Transaktionsmatrix mit kombinier- } \\
\text { ten Knoten Gut-Unternehmen bzw. } \\
\text { Aktivität-Unternehmen durch Zu- } \\
\text { schätzung des Ziel-Gutes bzw. der } \\
\text { Ziel-Aktivität einer Transaktion } \\
\text { anhand der gesamtwirtschaftlichen } \\
\text { Strukturdaten. Der Parameter } \\
\text { uname bestimmt die Knoten- } \\
\text { beschriftung. typ hat die gleiche } \\
\text { Funktion wie in MBMAT. }\end{array}$ \\
\hline DILLI & $\begin{array}{l}\text { MatValGap, Profildaten } \\
\text { (BCIprofile), typ }\end{array}$ & $\begin{array}{l}\text { Kantenliste der } \\
\text { direkten inter- } \\
\text { nen Lücken } \\
\text { (Ausschnitt v. } \\
\text { BCleFgugu) } \\
\end{array}$ & $\begin{array}{l}\text { Erstellung einer Kantenliste der } \\
\text { direkten internen Lücken aus der } \\
\text { Matrix MatValGap. typ hat die } \\
\text { gleiche Funktion wie in MBMAT. }\end{array}$ \\
\hline $\begin{array}{l}\text { COMBDIL- } \\
\text { INCUTRANS }\end{array}$ & $\begin{array}{l}\text { Kantenliste der direkten } \\
\text { internen Lücken, Kantenliste } \\
\text { der lokalen Transaktionen }\end{array}$ & $\begin{array}{l}\text { Kantenliste der } \\
\text { internen Lücken } \\
(B C I e F g u g u)\end{array}$ & $\begin{array}{l}\text { Kombination der Kantenlisten der } \\
\text { direkten intemen Lücken und der } \\
\text { lokalen Transaktionen (d.h. der } \\
\text { Performanz- und Nicht-Performanz- } \\
\text { Lücken) zu einer kompletten } \\
\text { Kantenliste der internen Lücken. }\end{array}$ \\
\hline MPONP & $\begin{array}{l}\text { Kantenliste der lokalen } \\
\text { Transaktionen und direkten } \\
\text { internen Lücken in kombi- } \\
\text { nierter Dimension (z.B. } \\
\text { BCleFgugu), identifizierte } \\
\text { Performanzlücken (z.B. } \\
\text { PerfGap } \\
\text { direkary }) \text {, identifizierte } \\
\text { DiGapterne Lücken (z.B. }\end{array}$ & $\begin{array}{l}\text { Kantenliste } \\
\text { bezogen auf } \\
\text { Güter bzw. } \\
\text { Aktivitäten }\end{array}$ & $\begin{array}{l}\text { Extraktion der in der angegebenen } \\
\text { kombinierten Kantenliste vor- } \\
\text { kommenden Güter- bzw. Aktivi- } \\
\text { tätenpaare }\end{array}$ \\
\hline
\end{tabular}




\begin{tabular}{|c|c|c|c|}
\hline Name & Parameter & Ergebnis & Beschreibung \\
\hline ALLOCATE & 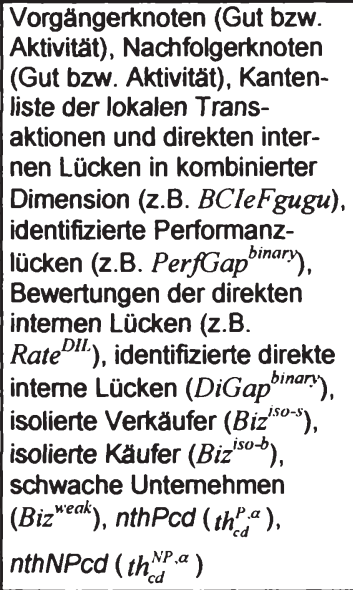 & $\begin{array}{l}\text { um die Lücken- } \\
\text { kennzeichnung } \\
\text { erweiterte } \\
\text { Kantenliste für } \\
\text { Transaktionen } \\
\text { zwischen Platt- } \\
\text { formteil- } \\
\text { nehmern, die } \\
\text { das angege- } \\
\text { bene interne } \\
\text { Güter- bzw. } \\
\text { Aktivitätenpaar } \\
\text { betreffen (Aus- } \\
\text { schnitt aus } \\
\text { BCleFgugu }\end{array}$ & $\begin{array}{l}\text { Umlegung der auf Ebene der Platt- } \\
\text { form bestimmten Lücken auf die } \\
\text { Ebene der teilnehmenden Unter- } \\
\text { nehmen für ein angegebenes } \\
\text { (Güter- oder Aktivitäten-) Knoten- } \\
\text { paar unter zusätzlicher Berück- } \\
\text { sichtigung von Unternehmen als } \\
\text { schwach' bzw. ,isoliert'. Die Para- } \\
\text { meter nthPcd und nthNPcd sind } \\
\text { Schwellwerte für die Klassifikation } \\
\text { von Performanz- bzw. Nicht- } \\
\text { Performanz-Lücken in die } \\
\text { jeweiligen Subkategorien. }\end{array}$ \\
\hline $\begin{array}{l}\text { ALLOCATE- } \\
\text { ALL }\end{array}$ & 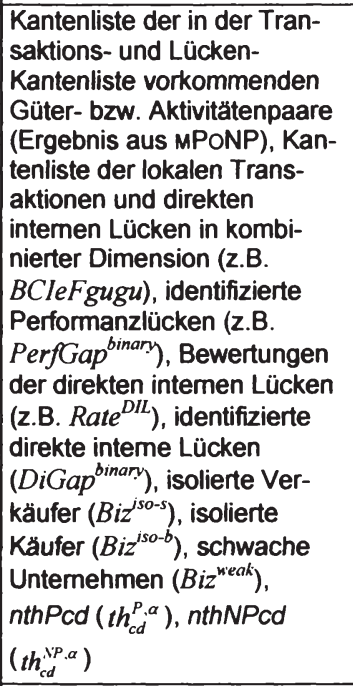 & $\begin{array}{l}\text { um die Lücken- } \\
\text { kennzeichnung } \\
\text { enweiterte } \\
\text { Kantenliste für } \\
\text { interne Lücken } \\
\text { zwischen Platt- } \\
\text { formteilnehmern } \\
\left(\text { BCIeFgugu }{ }^{\text {exp }}\right)\end{array}$ & $\begin{array}{l}\text { Wie ALLOCATE, jedoch für alle in der } \\
\text { lokalen Transaktions- und Lücken- } \\
\text { Kantenliste vorkommenden Güter- } \\
\text { bzw. Aktivitätenpaare. }\end{array}$ \\
\hline $\begin{array}{l}\text { ALLOCATE- } \\
\text { EXT }\end{array}$ & $\begin{array}{l}\text { Profildaten (BCIProfile), } \\
\text { Bewertung der extermen } \\
\text { Knoten (rate EXTvx.tLOC), ex- } \\
\text { terner Knoten } v_{x}, \text { typ }\end{array}$ & $\begin{array}{l}\text { Bewertung des } \\
\text { extemen Kno- } \\
\text { ten für die } \\
\text { Unternehmen } \\
\left(\text { RateBiz }{ }^{E X T}\right)\end{array}$ & $\begin{array}{l}\text { Umlegung der Bewertung des } \\
\text { spezifizierten externen Knoten } v_{x} \\
\text { für die internen Knoten aus } \\
\text { rate } \\
\text { EXTvx.4LOC von der Ebene der } \\
\text { Güter bzw. Aktivitäten auf die } \\
\text { Ebene der Unternehmen unter } \\
\text { Verwendung der Profilinformation. } \\
\text { typ hat die gleiche Funktion wie in } \\
\text { MBMAT. }\end{array}$ \\
\hline
\end{tabular}




\begin{tabular}{|c|c|c|c|}
\hline Name & Parameter & Ergebnis & Beschreibung \\
\hline $\begin{array}{l}\text { ALLOCATE- } \\
\text { EXTWEIGHT }\end{array}$ & $\begin{array}{l}\text { Profildaten (BCIprofile), } \\
\text { Bewertung der externen } \\
\text { Knoten (rate } E X T v_{x} I N 4 L O C \\
\text { rate } \\
\text { matrix aus Angebots } \\
\text { matrix a } \text {, Adjazenz- } \\
\text { Nachfrageperspektive (Di- } \\
\text { mension Unternehmen x } \\
\text { Gut/Aktivität oder umgekehrt, } \\
\text { z.B. BCIug), enweiterte } \\
\text { Kantenliste der internen } \\
\text { Lücken inkl. Lückenklassi- } \\
\text { fikation (BCIeFgugu }{ }^{E X P} \text { ), } \\
\text { externer Knoten } v_{x}, \text { typ, } \\
\text { direction, weight }\end{array}$ & 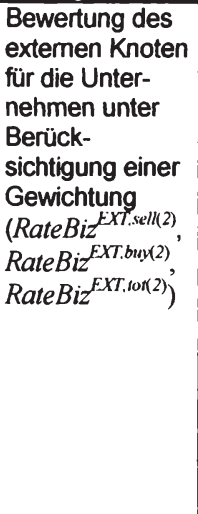 & $\begin{array}{l}\text { Umlegung der Bewertung des } \\
\text { spezifizierten externen Knoten } v_{x} \\
\text { für die internen Knoten aus } \\
\text { rate } E X X v x \text {.IN4LOC bzw. } \\
\text { rate } \\
\text { Güter bzw.OUT4l.OC von der Ebene der } \\
\text { Ebene der Unternehten auf die } \\
\text { Gut-IAktivität-Unternehmens- } \\
\text { Kombination) unter Verwendung } \\
\text { der Profilinformation. typ hat die } \\
\text { gleiche Funktion wie in MBMAT. } \\
\text { direction gibt an, welche Perspek- } \\
\text { tive (Angebot, Nachfrage, Gesamt) } \\
\text { die Bewertung einnehmen soll. } \\
\text { weight fungiert als Schalter } \\
\text { zwischen zwei Gewichtungs- } \\
\text { varianten. }\end{array}$ \\
\hline
\end{tabular}

Tabelle 104: Funktionen für das Matching

\begin{tabular}{|c|c|c|c|}
\hline Name & Parameter & Ergebnis & Beschreibung \\
\hline $\begin{array}{l}\text { DIST.BINARY- } \\
\text { NEU }\end{array}$ & $\begin{array}{l}\text { binäre Datenmatrix } \\
\text { (z.B. } 2 \text { duale (d.h. IN } \\
\text { und OUT) binäre Prä- } \\
\text { ferenzvariablen aus } \\
\text { BCIpreferences }{ }^{\text {bin }} \text { ), } \\
\text { method, gamma, delta, } \\
\text { lambda }\end{array}$ & $\begin{array}{l}\text { Ähnlichkeits- } \\
\text { matrix }\end{array}$ & $\begin{array}{l}\text { Berechnung einer Ahnlichkeitsmatrix } \\
\text { zur angegebenen binären Daten- } \\
\text { matrix. method definiert das zu ver- } \\
\text { wendende Ähnlichkeits- bzw. Dis- } \\
\text { tanzmaß. gamma, delta und lambda } \\
\text { sind methodenspezifische Parameter. }\end{array}$ \\
\hline MAKEBIN & $\begin{array}{l}\text { mehrstufig nominale } \\
\text { oder ordinale, duale } \\
\text { Präferenzvariablen IN } \\
\text { und OUT, ord }\end{array}$ & $\begin{array}{l}\text { binäre Hilfs- } \\
\text { variablen zu den } \\
\text { angegebenen } \\
\text { Variablen (eben- } \\
\text { falls gegliedert in } \\
\text { IN und OUT) }\end{array}$ & $\begin{array}{l}\text { Transformation der angegebenen } \\
\text { mehrstufig nominalen oder ordinalen } \\
\text { Variablen in entsprechende binäre } \\
\text { Hilfsvariablen als Basis zur An- } \\
\text { wendung von DIST.BINARYNEU oder } \\
\text { metrischen Distanz- bzw. Ähnlich- } \\
\text { keitsmaßen. ord bestimmt, ob es sich } \\
\text { um ordinale oder mehrstufig nominale } \\
\text { Variablen handelt. }\end{array}$ \\
\hline GIVECORD & Ort, daten & $\begin{array}{l}\text { geografische } \\
\text { Position des } \\
\text { Ortes in Grad } \\
\text { (Dezimaldar- } \\
\text { stellung) }\end{array}$ & $\begin{array}{l}\text { Ausgabe der geografischen Position } \\
\text { des angegebenen Ortes in Grad } \\
\text { (Dezimaldarstellung) aus der Ent- } \\
\text { fernungsdatenbank daten. }\end{array}$ \\
\hline COORDIST & $\begin{array}{l}\text { Standort } 1 \text { (geo- } \\
\text { grafische Breite und } \\
\text { Länge), Standort } 2 \\
\text { (geografische Breite } \\
\text { und Länge) }\end{array}$ & $\begin{array}{l}\text { Entfernung der } \\
\text { Standorte in } \mathrm{km}\end{array}$ & $\begin{array}{l}\text { Ermittlung der geografischen Ent- } \\
\text { fernung von Standort } 1 \text { und Standort } \\
2 \text { in } \mathrm{km} \text {. }\end{array}$ \\
\hline COORDPLDIST & $\begin{array}{l}\text { Ausgangsposition } \\
\text { (geografische Breite } \\
\text { und Länge), Richtung } \\
\text { (Winkel in Grad), Dis- } \\
\text { tanz in km }\end{array}$ & Zielposition & $\begin{array}{l}\text { Ermittlung der Zielposition, die in der } \\
\text { angegebenen Richtung und Ent- } \\
\text { fernung von der Ausgangsposition } \\
\text { liegt. }\end{array}$ \\
\hline
\end{tabular}




\begin{tabular}{|c|c|c|c|}
\hline Name & Parameter & Ergebnis & Beschreibung \\
\hline D2DEZ & $\begin{array}{l}\text { geografische Längen- } \\
\text { oder Breitenangabe in } \\
\text { Grad, Minuten und } \\
\text { Sekunden }\end{array}$ & $\begin{array}{l}\text { geografische } \\
\text { Längen- oder } \\
\text { Breitenangabe in } \\
\text { Dezimaldar- } \\
\text { stellung }\end{array}$ & $\begin{array}{l}\text { Umwandlung einer geografischen } \\
\text { Längen- od. Breitenangabe von einer } \\
\text { Darstellung in Grad, Minuten und } \\
\text { Sekunden in Dezimaldarstellung. }\end{array}$ \\
\hline SELORTSDIST & $\begin{array}{l}\text { Name Ort 1, Name Ort } \\
\text { 2, Entfernungs- } \\
\text { datenbank daten }\end{array}$ & $\begin{array}{l}\text { Entfemung der } \\
\text { Orte in } \mathrm{km}\end{array}$ & $\begin{array}{l}\text { Ausgabe der geografischen Ent- } \\
\text { fernung zwischen den Orten } 1 \text { und } 2 \\
\text { basierend auf der Entfernungs- } \\
\text { datenbank daten. }\end{array}$ \\
\hline DISTCOMPREF & 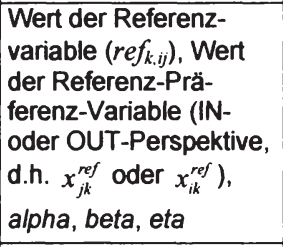 & $\begin{array}{l}\text { Distanz zwischen } \\
\text { ref } f_{k, i j} \text { und } x_{i k}^{r e f} \\
\text { bzw. } x_{j k}^{r e f} \\
\left(d_{k, i j}^{\text {comp ref,i bzw. }}\right. \\
\left.d_{k, i j}^{\text {comp ref, }, j}\right)\end{array}$ & $\begin{array}{l}\text { Vergleich des Wertes der Referenz- } \\
\text { Präferenz-Variable mit dem (Refe- } \\
\text { renz-)Wert der Referenzvariable für } \\
\text { ein Knotenpaar mit einer Distanz- } \\
\text { funktion. alpha, beta und eta sind } \\
\text { Parameter der zusammengesetzten } \\
\text { Distanzfunktion für den Referenz- } \\
\text { Präferenz-Vergleich. }\end{array}$ \\
\hline DISTREFPREF & 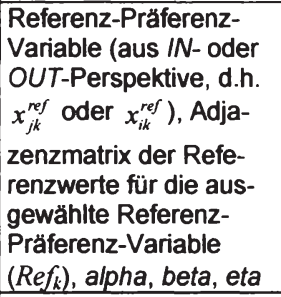 & $\begin{array}{l}\text { Distanzmatrix } \\
\left(D_{k}^{r e f}\right)\end{array}$ & $\begin{array}{l}\text { Ermittlung der indirekten Distanz der } \\
\text { Referenz-Präferenzen über den Ver- } \\
\text { gleich der Werte der Referenz-Pră- } \\
\text { ferenz-Variable mit den (Referenz-) } \\
\text { Werten der Referenzvariable mit einer } \\
\text { Distanzfunktion aus einer der dualen } \\
\text { Perspektiven. alpha, beta und eta wie } \\
\text { in DISTCOMPREF. }\end{array}$ \\
\hline WRAPINOUT & $\begin{array}{l}\text { duale Präferenzvari- } \\
\text { ablen IN und OUT, } \\
\text { function, metric, scale }\end{array}$ & $\begin{array}{l}\text { Distanz- bzw. } \\
\text { Ähnlichkeits- } \\
\text { matrix (z.B. } S^{\text {bin }}, \\
S^{\text {nom }}, D^{\text {met }}, D^{\text {ref }} \text { ) }\end{array}$ & $\begin{array}{l}\text { Ermittlung einer Ähnlichkeits- bzw. } \\
\text { Distanzmatrix für die angegebenen } \\
\text { dualen Präferenzvariablen, die das- } \\
\text { selbe Skalenniveau scale haben } \\
\text { müssen. function definiert, welche R- } \\
\text { Funktion zur Berechnung der } \\
\text { Distanzen bzw. Ähnlichkeiten ein- } \\
\text { gesetzt werden soll. metric spezifiziert } \\
\text { das zu verwendende Distanz- bzw. } \\
\text { Ähnlichkeitsmaß. } \\
\end{array}$ \\
\hline SWTCHDISSIM & $\begin{array}{l}\text { Distanz- oder Åhnlich- } \\
\left.\text { keitsmatrix (z.B. } D^{\text {mel }}\right) \text {, } \\
\text { direc }\end{array}$ & $\begin{array}{l}\text { Ähnlichkeits- } \\
\text { bzw. Distanz- } \\
\text { matrix }\end{array}$ & $\begin{array}{l}\text { Umwandlung von Åhnlichkeits- in } \\
\text { Distanzmaße bzw. von Distanz- in } \\
\text { Ähnlichkeitsmaße. Der Parameter } \\
\text { direc fungiert als Richtungsschalter, } \\
\text { der steuert ob von Ähnlichkeits- auf } \\
\text { Distanzmaße oder umgekehrt um- } \\
\text { gewandelt wird. }\end{array}$ \\
\hline MATCHSCORE & $\begin{array}{l}\text { zu kombinierende } \\
\text { Distanz- oder Ähnlich- } \\
\text { keitsmatrizen (z.B. } S^{\text {bi }} \text {, } \\
\left.S^{\text {nom }}, S^{\text {met }}, S^{\text {ref }}\right)\end{array}$ & $\begin{array}{l}\text { Gesamt-Distanz- } \\
\text { oder -Ähnlich- } \\
\text { keits-Matrix } \\
\left(M^{\text {prefs }}\right)\end{array}$ & $\begin{array}{l}\text { Zusammenfassung der einzelnen } \\
\text { Distanz- oder Ähnlichkeitsmaße in ein } \\
\text { Gesamt-Distanz- oder -Ähnlich- } \\
\text { keitsmaß. }\end{array}$ \\
\hline VALEXCERPT & $\begin{array}{l}\text { Distanz- oder Åhnlich- } \\
\text { keitsmatrix (z.B. S bin } \\
\left.D^{\text {met }}, M^{\text {prefs }}\right), \text { loVal, } \\
\text { hiVal }\end{array}$ & \begin{tabular}{l|} 
Kantenliste von \\
Knotenpaaren mit \\
Distanz bzw. \\
Ähnlichkeit im \\
vorgegebenen \\
Bereich \\
\end{tabular} & $\begin{array}{l}\text { Extraktion der Knotenpaare mit einer } \\
\text { Distanz oder Ännlichkeit im durch die } \\
\text { Parameter loVal und hiVal fest- } \\
\text { gelegten Intervall und Ausgabe als } \\
\text { Kantenliste. }\end{array}$ \\
\hline
\end{tabular}




\begin{tabular}{|c|c|c|c|}
\hline Name & Parameter & Ergebnis & Beschreibung \\
\hline EXTPARTNER & $\begin{array}{l}\text { Adjazenzmatrix (um } \\
\text { externen Knoten er- } \\
\text { weitert, z.B. } A^{* L O C . X X \text { - }} \\
{ }^{*} \text { ), extemer Knoten } \\
\text { (Erweiterungskandidat, } \\
\left.v_{x}\right), \text { Profildaten (BCI- } \\
\text { profile), typ, direction }\end{array}$ & $\begin{array}{l}\text { Vektor der (Be- } \\
\text { zeichnungen der) } \\
\text { kombinierten } \\
\text { internen Knoten, } \\
\text { die Vorgänger } \\
\text { bzw. Nachfolger } \\
\text { des externen } \\
\text { Knoten sind }\left(V^{t}\right)\end{array}$ & $\begin{array}{l}\text { Identifikation der Gut- bzw. Aktivität- } \\
\text { Unternehmens-Kombinationen, die } \\
\text { mit dem externen Knoten (Gut oder } \\
\text { Aktivität) in Verbindung stehen, über } \\
\text { die erweiterte Adjazenzmatrix und die } \\
\text { Profilinformation. typ unterscheidet } \\
\text { zwischen der Verwendung von } \\
\text { Gütern und Aktivitäten. direction gibt } \\
\text { an, ob der externe Knoten als Vor- } \\
\text { gänger oder Nachfolger betrachtet } \\
\text { werden soll. }\end{array}$ \\
\hline AGGPREF & $\begin{array}{l}\text { duale Präferenz- } \\
\text { variablen IN und OUT, } \\
\text { Vektor der internen } \\
\text { Vorgänger des ex- } \\
\text { ternen Knoten, Vektor } \\
\text { der internen Nach- } \\
\text { folger des externen } \\
\text { Knoten (Ergebnis von } \\
\text { EXTPARTNER), scale, } \\
\text { freq, weight, cum, } \\
\text { optional: Bedeutung } \\
\text { des externen Knoten } \\
\text { für die internen Knoten } \\
\text { (dual, d.h. } \\
\text { rate } \\
\text { rate } \\
\text { rXTix.OITT4LOC und }\end{array}$ & $\begin{array}{l}\text { Bewertungen der } \\
\text { möglichen Aus- } \\
\text { prägungen der } \\
\text { dualen Prä- } \\
\text { ferenzvariablen } \\
\text { des externen } \\
\text { Knoten }(\mathrm{z} . \mathrm{B} . \\
\left.h^{\text {rel }}\left(x_{k}^{\text {bin }}, \text { val }_{k q}^{\text {bin }}\right)\right)\end{array}$ & $\begin{array}{l}\text { Ermittlung der Bewertung der mög- } \\
\text { lichen Ausprägungen der an- } \\
\text { gegebenen dualen Präferenz- } \\
\text { variablen des externen Knoten. scale } \\
\text { gibt das Skalenniveau der Präferenz- } \\
\text { variablen an (für Referenz-Präferenz- } \\
\text { Variablen ist die Funktion AGGREF- } \\
\text { PREF einzusetzen). freq, weight und } \\
\text { cum bestimmen die Bewertungs- } \\
\text { methode. freq definiert, ob relative } \\
\text { oder absolute Häufigkeiten verwendet } \\
\text { werden. Metrische Präferenzvariablen } \\
\text { werden automatisch kategorisiert. } \\
\text { weight legt fest, ob die Bedeutung } \\
\text { des extemen Knoten für die } \\
\text { jeweiligen internen Knoten zur Ge- } \\
\text { wichtung herangezogen werden soll. } \\
\text { cum ist ein Schalter für kumulierte } \\
\text { Bewertungen. }\end{array}$ \\
\hline EXTRACTZ & $\begin{array}{l}\text { Referenz-Präferenz- } \\
\text { Variablen (IN oder } \\
\text { OUT), entspr. Refe- } \\
\text { renzvariablen, Vektor } \\
\text { der internen Vorgänger } \\
\text { des externen Knoten } \\
\text { (bzw. umgekehr, Teil- } \\
\text { ergebnis von EXT- } \\
\text { PARTNER) } \\
\end{array}$ & $\begin{array}{l}\text { binäre Adja- } \\
\text { zenzmatrix } Z\end{array}$ & $\begin{array}{l}\text { Berechnung der binären Adjazenz- } \\
\text { matrix } Z \text {, die angibt, welche Knoten- } \\
\text { paare aus der Menge } V^{t} \text { aufgrund } \\
\text { ihrer Referenzwerte und Präferenzen } \\
\text { zusammenpassen. }\end{array}$ \\
\hline $\begin{array}{l}\text { FIND- } \\
\text { CBLOCKS }\end{array}$ & $\begin{array}{l}\text { (binäre) Adjazenz- } \\
\text { matrix (z.B. Z) }\end{array}$ & $\begin{array}{l}\text { Liste der voll- } \\
\text { ständigen Blöcke } \\
\text { CBlock }_{p} \text { (an- } \\
\text { gegeben als } \\
\text { Vektor der Kno- } \\
\text { tennamen) } \\
\end{array}$ & $\begin{array}{l}\text { Identifikation der vollstăndigen Blöcke } \\
\text { der Adjazenzmatrix. }\end{array}$ \\
\hline
\end{tabular}




\begin{tabular}{|c|c|c|c|}
\hline Name & Parameter & Ergebnis & Beschreibung \\
\hline $\begin{array}{l}\text { CALC- } \\
\text { REFPREF }\end{array}$ & $\begin{array}{l}\text { Liste der vollständigen } \\
\text { Blöcke CBlock } \text { (Er- } \\
\text { gebnis von FIND- } \\
\text { CBLOCKs), Referenz- } \\
\text { Präferenz-Variablen } \\
\text { (IN oder OUT), entspr. } \\
\text { Vektor d. intemen } \\
\text { Vorgänger des ex- } \\
\text { ternen Knoten (bzw. } \\
\text { umgekehrt, Teiler- } \\
\text { gebnis von } \\
\text { EXTPARTNER), Ent- } \\
\text { femungsdatenbank }\end{array}$ & $\begin{array}{l}\text { Liste mit optima- } \\
\text { len Koordinaten } \\
\text { und optimalem } \\
\text { Wert der Refe- } \\
\text { renz-Präferenz- } \\
\text { Variable des } \\
\text { externen Knoten } \\
\text { für jeden voll- } \\
\text { ständigen Block }\end{array}$ & $\begin{array}{l}\text { Berechnung der optimalen Ko- } \\
\text { ordinaten und optimalen Werte der } \\
\text { Referenz-Präferenz-Variable des } \\
\text { externen Knoten für jeden voll- } \\
\text { ständigen Block. }\end{array}$ \\
\hline $\begin{array}{l}\text { ORDERET- } \\
\text { APPEND }\end{array}$ & $\begin{array}{l}\text { Liste der vollständigen } \\
\text { Blocke mit optimalen } \\
\text { Koordinaten und } \\
\text { optimalem Präferenz- } \\
\text { wert (Ergebnis von } \\
\text { CALCREFPREF), Re- } \\
\text { ferenz-Präferenz- } \\
\text { Variable (IN oder } \\
\text { OUT), Bedeutung des } \\
\text { externen Knoten für die } \\
\text { internen Knoten } \\
\text { (passend zur Referenz- } \\
\text { Präferenzvariable } \\
\text { entweder } \\
\text { rate } \\
\text { rate }^{E X T T_{x} . O U T A L O C} \text { oder } \\
\end{array}$ & $\begin{array}{l}\text { sortierte Liste der } \\
\text { vollständigen } \\
\text { Blöcke erweitert } \\
\text { um die Be- } \\
\text { wertung der } \\
\text { Bedeutung der } \\
\text { Blöcke }\end{array}$ & $\begin{array}{l}\text { Ermittlung der Bedeutung der Blöcke } \\
\text { sowie Erweiterung der Liste um diese } \\
\text { Bedeutung und anschließende } \\
\text { Reihung der Blöcke nach der Be- } \\
\text { deutung. }\end{array}$ \\
\hline AGGREFPREF & 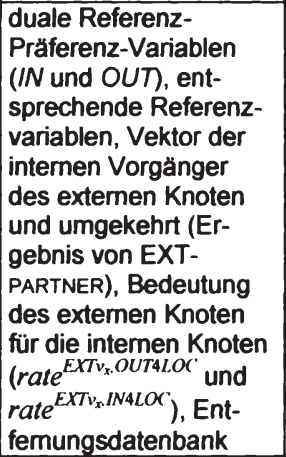 & $\begin{array}{l}\text { optimale Werte } \\
\text { der dualen Refe- } \\
\text { renz-Präferenz- } \\
\text { Variablen und der } \\
\text { entsprechenden } \\
\text { Referenz- } \\
\text { Variable }\end{array}$ & $\begin{array}{l}\text { Ermittlung der optimalen Ausprägung } \\
\text { der dualen Referenz-Präferenz- } \\
\text { Variablen und der entsprechenden } \\
\text { Referenz-Variable für den externen } \\
\text { Knoten unter Verwendung der Funkti- } \\
\text { onen EXTRACTZ, FINDCBLOCKS, CALC- } \\
\text { REFPREF und ORDERETAPPEND, } \\
\text { wobei jeweils die Ergebnisse für den } \\
\text { vollständigen Block mit der größten } \\
\text { Bedeutung ausgewählt werden. (Fo- } \\
\text { kussiert auf metrische Referenz- } \\
\text { Prăferenz-Variablen.) }\end{array}$ \\
\hline $\begin{array}{l}\text { EXTPREF- } \\
\text { PROFILE }\end{array}$ & $\begin{array}{l}\text { Bewertungen der mög- } \\
\text { lichen Ausprägungen } \\
\text { der dualen (binären, } \\
\text { mehrstufig nominalen, } \\
\text { ordinalen und/oder } \\
\text { metrischen) Präferenz- } \\
\text { variablen des externen } \\
\text { Knoten (Ergebnis von } \\
\text { AGGPREF), optimale } \\
\text { Werte der dualen Refe- } \\
\text { renz-Präferenz- }\end{array}$ & $\begin{array}{l}\text { optimales Präfe- } \\
\text { renzprofil für } \\
\text { einen externen } \\
\text { Knoten }\end{array}$ & $\begin{array}{l}\text { Ableitung eines optimalen Präferenz- } \\
\text { profils für den selektierten externen } \\
\text { Knoten basierend auf den Ergeb- } \\
\text { nissen von AGGPREF und AGG- } \\
\text { REFPREF. }\end{array}$ \\
\hline
\end{tabular}




\begin{tabular}{|c|c|c|c|}
\hline Name & Parameter & Ergebnis & Beschreibung \\
\hline & $\begin{array}{l}\text { Variablen und der } \\
\text { entsprechenden Re- } \\
\text { ferenz-Variable (Er- } \\
\text { gebnis von AG- } \\
\text { GREFPREF) }\end{array}$ & & \\
\hline $\begin{array}{l}\text { APPEND- } \\
\text { MATCH }\end{array}$ & $\begin{array}{l}\text { Kantenliste der in- } \\
\text { temen Lücken mit } \\
\text { Lückenklassifikation } \text { (z.B. BCleFgugu } \\
\text { Matrix der Match- } \\
\text { ingscores }\left(\text { z.B. } \text { M }^{\text {press }} \text { ), }\right. \\
\text { Präferenzdaten } \\
\text { (BCIpref) }\end{array}$ & $\begin{array}{l}\text { erweiterte Kan- } \\
\text { tenliste mit } \\
\text { Lückenklassi- } \\
\text { fikation und } \\
\text { Matchingscore } \\
(\text { (z.B. BCleF- } \\
\text { gugu } \text { exp\&malch }^{\text {exp }}\end{array}$ & $\begin{array}{l}\text { Zusammenführung der um die Lü- } \\
\text { ckenklassifikation erweiterten Kanten- } \\
\text { liste der internen Lücken mit den } \\
\text { Gesamtähnlichkeiten der Präferenzen } \\
\text { der Unternehmen. }\end{array}$ \\
\hline $\begin{array}{l}\text { ORDERN- } \\
\text { SELECT }\end{array}$ & $\begin{array}{l}\text { erweiterte Kantenliste } \\
\text { der internen Lücken } \\
\text { (inkl. Lückenklassi- } \\
\text { fikation und Mat- } \\
\text { chingscore, z.B. } \\
\text { BCleFgugu } \\
\text { d.h. Ergebnatch } \\
\text { APPENDMATCH), upto, } \\
\text { decreasing }\end{array}$ & $\begin{array}{l}\text { sortierte und } \\
\text { reduzierte er- } \\
\text { weiterte Kanten- } \\
\text { liste }\end{array}$ & $\begin{array}{l}\text { Präferenzbezogene Reihung und } \\
\text { Auswahl der upto (aufgrund ihrer } \\
\text { Präferenzprofile) am besten zu- } \\
\text { einander passenden Knotenpaare } \\
\text { innerhalb jeder Lückenkategorie. upto } \\
\text { legt fest wie viele Lücken pro Kate- } \\
\text { gorie ausgewählt werden sollen. } \\
\text { decreasing spezifiziert die Sortier- } \\
\text { richtung. }\end{array}$ \\
\hline EDGEFR2MAT & $\begin{array}{l}\text { erweiterte Kantenliste } \\
\text { (inkl. Matchingscore, } \\
\text { z.B. BCleF- } \\
\text { gugu exp\&match } \text { ), spalte, } \\
\text { entsprechende Ad- } \\
\text { jazenzmatrix (z.B. } \\
\text { BCIgugu) }\end{array}$ & $\begin{array}{l}\text { Adjazenzmatrix } \\
\text { mit Werten aus } \\
\text { der erweiterten } \\
\text { Kantenliste (i.A. } \\
\text { Matchingscore) }\end{array}$ & $\begin{array}{l}\text { Generierung einer Adjazenzmatrix mit } \\
\text { der Dimension der angegebenen } \\
\text { Adjazenzmatrix und Werten aus der } \\
\text { enweiterten Kantenliste (üblicherweise } \\
\text { des Matchingscores), wobei spalte } \\
\text { angibt, in welcher Spalte der Kanten- } \\
\text { liste sich die zu verwendenden Werte } \\
\text { befinden. }\end{array}$ \\
\hline PLOTEFFINAL & $\begin{array}{l}\text { erweiterte Kantenliste } \\
\text { (inkl. Matchingscore), } \\
\text { entsprechende Adja- } \\
\text { zenzmatrix (z.B. } \\
\text { BCIgugu) }\end{array}$ & Grafik & $\begin{array}{l}\text { Grafische Darstellung der erweiterten } \\
\text { Kantenliste. }\end{array}$ \\
\hline
\end{tabular}




\section{Appendix B: Klassifikationen}

Tabelle 105: Aktivitäten nach der auf der ÓNACE aufbauenden IO-Gliederung Quelle: Kurzbezeichnungen für Aktivitäten gemäß den Tabellen in Statistik Austria [2005]

\begin{tabular}{|c|c|}
\hline & Bezeichnung \\
\hline x01 & Landwirtschaft, Jagd \\
\hline $\mathrm{x} 02$ & Forstwirtschaft \\
\hline X05 & Fischerei und Fischzucht \\
\hline $\mathrm{x} 10$ & Kohlenbergbau, Torfgewinnung \\
\hline $\mathrm{x} 11$ & Erdöl- und Erdgas-, Erzbergbau (1) \\
\hline $\mathrm{x} 14$ & Gewinnung von Steinen und Erden, sonstiger Bergbau \\
\hline$\times 15$ & H. v. Nahrungs- u. Genussmitteln und Getränken \\
\hline $\mathrm{x} 16$ & Tabakverarbeitung \\
\hline $\mathrm{x} 17$ & H. v. Textilien und Textilwaren (ohne Bekleidung) \\
\hline$\times 18$ & H. v. Bekleidung \\
\hline $\mathrm{x} 19$ & Ledererzeugung und -verarbeitung \\
\hline$\times 20$ & Be- u. Verarbeitung von Holz (ohne H. v. Möbeln) \\
\hline$\times 21$ & H. u. Verarbeitung von Papier und Pappe \\
\hline$\times 22$ & Verlagswesen, Druckerei, Vervielfältigung \\
\hline$\times 23$ & Kokerei, Mineralölverarbeitung \\
\hline$\times 24$ & H. v. Chemikalien und chemischen Erzeugnissen \\
\hline$\times 25$ & H. v. Gummi- und Kunststoffwaren \\
\hline$\times 26$ & H. u. Bearbeitung v. Glas, H. v. W. a. Steinen u. Erden \\
\hline$\times 27$ & Metallerzeugung und -bearbeitung \\
\hline X28 & H. v. Metallerzeugnissen \\
\hline$\times 29$ & Maschinenbau \\
\hline$\times 30$ & H. v. Büromaschinen, EDV-Geräten \\
\hline$\times 31$ & H. v. Gerăten der Elektrizitätserzeugung, -verteilung \\
\hline$\times 32$ & Rundfunk-, Fernseh- u. Nachrichtentechnik \\
\hline$\times 33$ & Medizin-, Mess- u. Regelungstechnik; Optik \\
\hline$\times 34$ & H. v. Kraftwagen und Kraftwagenteilen \\
\hline$\times 35$ & Sonstiger Fahrzeugbau \\
\hline$\times 36$ & H. v. sonstigen Erzeugnissen \\
\hline$\times 37$ & Rückgewinnung (Recycling) \\
\hline$X 40 A$ & Elektrizitätsversorgung \\
\hline $\mathrm{X} 40 \mathrm{~B}$ & Gasversorgung \\
\hline $\mathrm{X} 40 \mathrm{C}$ & Fernwärmeversorgung \\
\hline $\mathrm{X} 41$ & Wasserversorgung \\
\hline$X 45 A$ & Hoch- und Tieftoau \\
\hline X45B & Bauinstallation, Ausbau- und Bauhilfsgewerbe \\
\hline$X 50 A$ & Kfz-Handel; Tankstellen \\
\hline
\end{tabular}




\begin{tabular}{|c|c|}
\hline & Bezeichnung \\
\hline$\times 50 B$ & Reparatur von $\mathrm{Kfz}$ \\
\hline$\times 51 A$ & Großhandel \\
\hline X51B & Handelsvermittlung \\
\hline$\times 52 A$ & Einzelhandel \\
\hline X52B & Reparatur von Gebrauchsgütern \\
\hline X55 & Beherbergungs- und Gaststättenwesen \\
\hline X60A & Eisenbahnen \\
\hline X60B & So. Landverkehr, Transport in Rohrfernleitungen \\
\hline $\mathrm{X} 60 \mathrm{C}$ & Güterbeförderung im Straßenverkehr \\
\hline X61 & Schifffahrt \\
\hline$x 62$ & Flugverkehr \\
\hline X63A & Hilfs- u. Nebentätigkeiten für den Verkehr \\
\hline X63B & Reisebüros und -veranstalter \\
\hline$\times 63 C$ & Speditionen, sonstige Verkehrsvermittlung \\
\hline X64 & Nachrichtenübermittlung \\
\hline X65 & Kreditwesen \\
\hline X66 & Versicherungswesen \\
\hline X67 & Mit dem Kredit- u. Versicherungswesen verbund. Tätigkeiten \\
\hline$\times 70 A$ & Vermietung von Realitäten \\
\hline$\times 708$ & Vermittlung und Verwaltung von Realitäten \\
\hline X71 & Vermietung beweglicher Sachen ohne Personal \\
\hline$\times 72$ & Datenverarbeitung und Datenbanken \\
\hline$\times 73$ & Forschung und Entwicklung \\
\hline$X 74 A$ & Rechts- und Unternehmensberatung, Marktforschung \\
\hline X74B & Architektur- u. Ingenieurbüros \\
\hline$\times 74 C$ & Werbewesen \\
\hline$\times 740$ & Sonstige unternehmensbezogene DL \\
\hline$\times 75$ & Offentliche Verwaltung, Sozialversicherung \\
\hline$\times 80$ & Unterrichtswesen \\
\hline X85A & Gesundheits- u. Veterinärwesen \\
\hline X85B & Sozialwesen \\
\hline $\mathbf{x} 90$ & Abwasser- u. Abfallbeseitigung u. sonstige Entsorgung \\
\hline X91 & Interessenvertretungen, Vereine \\
\hline X92A & Kultur, Sport und Unterhaltung \\
\hline X92B & Hörfunk - und Femsehanstalten, Kinos \\
\hline X93 & Erbringung von sonstigen $\mathrm{DL}$ \\
\hline X95 & Private Haushalte \\
\hline
\end{tabular}


Tabelle 106: Güter nach der auf der OCPA aufbauenden 1O-Gliederung

Quelle: Kurzbezeichnungen für Güter gemäß den Tabellen in Statistik Austria [2005]

\begin{tabular}{|c|c|}
\hline & Bezeichnung \\
\hline 01 & Erzeugnisse d. Landwirtschaft und Jagd \\
\hline 02 & Forstwirtschaftliche Erzeugnisse \\
\hline 05 & Fische und Fischereierzeugnisse \\
\hline 10 & Kohle und Torf \\
\hline 11 & Erdöl und Erdgas, Erze (1) \\
\hline 14 & Steine und Erden \\
\hline 15 & Nahrungs- und Futtermittel sowie Getränke \\
\hline 16 & Tabakerzeugnisse \\
\hline 17 & Textilien \\
\hline 18 & Bekleidung \\
\hline 19 & Leder und Lederwaren \\
\hline 20 & Holz sowie Holz-, Kork- und Flechtwaren \\
\hline 21 & Papier, Pappe und Waren daraus \\
\hline 22 & Verlags- und Druckerzeugnisse \\
\hline 23 & Mineralölerzeugnisse \\
\hline 24 & Chemische Erzeugnisse \\
\hline 25 & Gummi- und Kunststoffwaren \\
\hline 26 & Glas, Keramik, bearbeitete Steine und Erden \\
\hline 27 & Metalle und Halbzeug daraus \\
\hline 28 & Metallerzeugnisse \\
\hline 29 & Maschinen \\
\hline 30 & Büromaschinen, EDV-Geräte und -Einrichtungen \\
\hline 31 & Geräte der Elektrizitätserzeugung und -verteilung \\
\hline 32 & Nachrtechn., Rundfunk- u. FS-Geräte, elektr. Bauteile \\
\hline 33 & Medizinisch-, mess-, regeltechnische u. opt. Erz.; Uhren \\
\hline 34 & Kraftwagen und Kraftwagenteile \\
\hline 35 & Sonstige Fahrzeuge \\
\hline 36 & Möbel, Schmuck, Musikinstrumente, Sportgeräte u.a. \\
\hline 37 & Dienstleistungen der Rückgewinnung \\
\hline 40A & Elektrischer Strom und DL der Elektrizitätsversorgung \\
\hline $40 B$ & Gase und DL der Gasversorgung \\
\hline $40 \mathrm{C}$ & Fernwärme und $\mathrm{DL}$ der Fernwärmeversorgung \\
\hline 41 & Wasser und DL der Wasserversorgung \\
\hline 45A & Hoch- u. Tiefbauarbeiten \\
\hline 45B & Bauinstallationsarbeiten, sonstige Bauarbeiten \\
\hline $50 A$ & Handelsleistungen mit Kfz, Tankstellenleistungen \\
\hline 508 & Reparaturarbeiten an Kfz \\
\hline $51 \mathrm{~A}$ & Großhandelsleistungen \\
\hline $51 B$ & Handelsvermittlungsleistungen \\
\hline
\end{tabular}




\section{Bezeichnung}

52A Einzelhandelsleistungen

52B Reparaturarbeiten an Gebrauchsgütem

55 Beherbergungs- und Gaststättendienstleistungen

60A Eisenbahndienstleistungen

$60 \mathrm{~B}$ So. Landverkehrsleist., Transportleist. in Rohrfernleit.

60C Güterbeförderungsleistungen im Straßenverkehr

61 Schifffahrtsleistungen

62 Luftfahrtleistungen

63A DL bezüglich Hilfs- u. Nebentätigkeiten für den Verkehr

63B DL von Reisebüros u. -veranstaltern

63C Speditionsleistungen und sonst. Verkehrsvermittlungsl.

64 Nachrichtenübermittlungsdienstleistungen

65 DL der Kreditinstitute

66 DL der Versicherungen (ohne Sozialversicherung)

67 DL des Kredit- $u$. Versicherungshilfswesens

70A DL der Vermietung von Realitäten

70B DL der Vermittlung und Verwaltung von Realitäten

71 DL der Vermietung beweglicher Sachen ohne Personal

72 DL der EDV und von Datenbanken

73 Forschungs- und Entwicklungsleistungen

74A Rechts- $u$. Unternehmensberat.-, Marktforsch.leistungen

74B Architektur- $u$. Ingenieurbüroleistungen

$74 \mathrm{C}$ Werbeleistungen

74D Sonstige unternehmensbezogene DL

75 DL der öffentl. Verwaltung, Verteidigung u. Sozialversich.

80 Erziehungs- und Unterrichtsdienstleistungen

85A DL des Gesundheits- und Veterinärwesens

858 DL des Sozialwesens

90 Abwasser-, Abfallbeseitigungs- u. so. Entsorgungsleist.

91 DL v. Interessenvertretungen, Kirchen u.a.

92A Sonstige Kultur-, Sport- und Unterhaltungs-DL

92B DL v. Hörfunk- u. Fernsehanstalten, Kinos

93 Sonstige Dienstleistungen

95 Dienstleistungen privater Haushalte 


\section{Appendix C: IOTgg}

Tabelle 107: IOTgg (Werte in Tsd. GE) I

\begin{tabular}{|c|c|c|c|c|c|c|c|c|c|c|c|c|c|}
\hline & 01 & 02 & 05 & 10 & 11 & 14 & 15 & 16 & 17 & 18 & 19 & 20 & 21 \\
\hline 01 & 0 & 9075 & 0 & 0 & 0 & 0 & 3027946 & 0 & 0 & 0 & \begin{tabular}{|l|}
37718 \\
\end{tabular} & 0 & \\
\hline 02 & 31740 & 0 & 0 & 0 & 0 & 0 & 0 & 0 & 0 & 2352 & 0 & 335061 & 219682 \\
\hline 05 & 0 & 0 & 0 & 0 & 0 & 0 & 0 & 0 & 0 & 0 & 0 & 0 & 0 \\
\hline 10 & 0 & 0 & 0 & 0 & 0 & 0 & 0 & 0 & 0 & 0 & 0 & 0 & 0 \\
\hline 11 & 0 & 0 & 0 & 0 & 0 & 0 & 0 & 0 & 0 & 0 & 0 & 0 & 0 \\
\hline 14 & 0 & 0 & 0 & 0 & 0 & 0 & 0 & 0 & 0 & 0 & 0 & 0 & 33323 \\
\hline 15 & 258185 & 0 & 2370 & 0 & 0 & 0 & 0 & 1476 & 0 & 0 & 0 & 0 & 0 \\
\hline 16 & 0 & 0 & 0 & 0 & 0 & 0 & 0 & 0 & 0 & 0 & 0 & 0 & 0 \\
\hline 17 & 0 & 0 & 0 & 0 & 0 & 0 & 0 & 0 & 0 & 53618 & 6981 & 0 & 0 \\
\hline 18 & 0 & 0 & 0 & 0 & 0 & 0 & 0 & 0 & 0 & 0 & 0 & 0 & 0 \\
\hline 19 & 0 & 0 & 0 & 0 & 0 & 0 & 0 & 0 & 0 & 0 & 0 & 0 & 0 \\
\hline 20 & \begin{tabular}{|l|}
13881 \\
\end{tabular} & 13817 & 170 & 228 & 1303 & 0 & 0 & 0 & 0 & 0 & 0 & 0 & 257571 \\
\hline 21 & 0 & 0 & 0 & 0 & 0 & 0 & 140756 & 32222 & 14348 & 6597 & 7458 & \begin{tabular}{|l|l|}
59520 \\
\end{tabular} & 0 \\
\hline 22 & 0 & 1078 & 95 & 0 & 0 & 0 & \begin{tabular}{|l|l|}
96671 \\
\end{tabular} & 8784 & \begin{tabular}{|l|}
8988 \\
\end{tabular} & 7178 & 6428 & 0 & 26183 \\
\hline 23 & 131681 & 19844 & 315 & \begin{tabular}{|l|}
469 \\
\end{tabular} & 3224 & 39346 & 61598 & 0 & 10459 & 0 & 0 & 34469 & 0 \\
\hline 24 & \begin{tabular}{|l|}
58349 \\
\end{tabular} & 5604 & 0 & 0 & 6195 & 0 & 0 & 1541 & 0 & 0 & 3919 & 0 & 34311 \\
\hline 25 & 10361 & 0 & 129 & \begin{tabular}{|l|}
464 \\
\end{tabular} & 0 & 0 & 62733 & 0 & 0 & 0 & 12933 & 0 & 0 \\
\hline 26 & 20067 & 1793 & 254 & 0 & 1073 & 20838 & 0 & 0 & 0 & 0 & 0 & 30980 & 0 \\
\hline 27 & 0 & 0 & 0 & 103 & 0 & 0 & 0 & 0 & 0 & 0 & 0 & 0 & 0 \\
\hline 28 & 20966 & 15663 & 454 & 453 & 19958 & 11792 & 0 & 3709 & 0 & 7514 & 13414 & 22647 & 0 \\
\hline 29 & 80235 & 11395 & 581 & 1628 & 31667 & 24535 & 48490 & 0 & 15598 & 6985 & 5193 & 31431 & 42305 \\
\hline 30 & 0 & 0 & 0 & 0 & 0 & 0 & 0 & 0 & 0 & 0 & 0 & 0 & 0 \\
\hline 31 & 0 & 0 & 0 & 0 & 1609 & 0 & 0 & 0 & 0 & 0 & $\overline{7}$ & 0 & 0 \\
\hline 32 & 0 & 0 & 0 & 0 & 0 & 0 & 0 & 0 & 0 & 0 & 0 & 0 & 0 \\
\hline 33 & 0 & 0 & 0 & 0 & 0 & 0 & 0 & 0 & 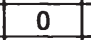 & 0 & 0 & 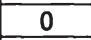 & 0 \\
\hline 34 & 0 & 0 & 0 & 0 & 0 & 0 & 0 & 0 & 0 & 0 & 0 & 0 & 0 \\
\hline 35 & 0 & 0 & 0 & 0 & 0 & 0 & c & 0 & 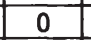 & 0 & 0 & 0 & 0 \\
\hline 36 & 0 & 0 & 0 & 93 & 0 & 0 & 0 & 0 & 0 & 0 & 0 & 0 & 0 \\
\hline 37 & 0 & 0 & 0 & 0 & 0 & 0 & 0 & 0 & 0 & 0 & 0 & 0 & 0 \\
\hline $40 \mathrm{~A}$ & \begin{tabular}{|l|}
63137 \\
\end{tabular} & 3665 & 447 & 1007 & 5645 & 47617 & 79080 & 1176 & 28004 & \begin{tabular}{|l|}
5019 \\
\end{tabular} & 7550 & 64357 & 57331 \\
\hline $40 B$ & 0 & 0 & 0 & 136 & 11659 & 20312 & 120085 & 1156 & 15278 & 0 & 0 & 0 & 108132 \\
\hline $40 \mathrm{C}$ & 0 & 0 & 0 & 0 & 0 & 0 & 0 & 0 & 0 & 0 & 0 & 0 & 0 \\
\hline 41 & 0 & 0 & 0 & 0 & $\sqrt{2}$ & 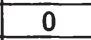 & & 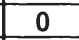 & 0 & 0 & 0 & 0 & 0 \\
\hline 45A & \begin{tabular}{|l|}
43703 \\
\end{tabular} & 3912 & 509 & 0 & 6058 & 0 & 0 & 0 & 0 & 0 & 3315 & 36276 & 0 \\
\hline $45 B$ & 27601 & 1914 & 259 & 0 & \begin{tabular}{|l|}
1336 \\
\end{tabular} & 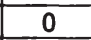 & 0 & 0 & 0 & 0 & 0 & 0 & 0 \\
\hline $50 \mathrm{~A}$ & 0 & 0 & 0 & 0 & 0 & 0 & 0 & 0 & 0 & 0 & 0 & 0 & 0 \\
\hline $50 B$ & 27883 & 1869 & 100 & 117 & 4343 & 10890 & 0 & 0 & 0 & 0 & 0 & 23063 & 0 \\
\hline $51 \mathrm{~A}$ & 0 & 0 & 0 & 0 & 0 & 0 & 0 & 0 & 0 & 0 & 0 & 0 & 0 \\
\hline $51 \mathrm{~B}$ & 0 & 0 & 0 & 0 & 0 & 0 & 0 & 0 & 39238 & \begin{tabular}{|l|}
17693 \\
\end{tabular} & 11741 & 46685 & 38001 \\
\hline $52 \mathrm{~A}$ & 0 & 0 & 0 & 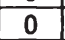 & 0 & 0 & 0 & 0 & 0 & 0 & 0 & 0 & 0 \\
\hline $52 \mathrm{~B}$ & 0 & 0 & 0 & 0 & 0 & 0 & 0 & 0 & 0 & 0 & 0 & 0 & 0 \\
\hline 55 & 0 & 0 & 0 & 0 & 0 & 0 & 53431 & 2061 & 0 & \begin{tabular}{|l|}
4451 \\
\end{tabular} & 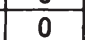 & 0 & 0 \\
\hline $60 \mathrm{~A}$ & 0 & 0 & 0 & 0 & 0 & 15826 & 0 & 0 & 0 & 0 & 0 & 0 & 28631 \\
\hline 608 & 0 & 0 & 0 & 0 & 0 & 0 & 0 & 0 & 0 & 0 & 0 & 0 & 0 \\
\hline $60 \mathrm{C}$ & 0 & 0 & 0 & 0 & 0 & 101662 & 3799 & 0 & 363 & 8 & 30 & 58672 & 184784 \\
\hline
\end{tabular}




\begin{tabular}{|r|c|c|c|c|c|c|c|c|c|c|c|c|c|} 
& \multicolumn{1}{c}{01} & 02 & 05 & 10 & 11 & 14 & 15 & 16 & 17 & 18 & 19 & 20 & 21 \\
\hline 61 & 0 & 0 & 0 & 0 & 0 & 0 & 0 & 0 & 0 & 0 & 0 & 0 & 0 \\
\hline 62 & 0 & 0 & 0 & 0 & 0 & 0 & 0 & 709 & 0 & 0 & 0 & 0 & 0 \\
\hline $63 \mathrm{~A}$ & 0 & 0 & 0 & 0 & 0 & 0 & 0 & 673 & 0 & 0 & 0 & 0 & 0 \\
\hline $63 \mathrm{~B}$ & 0 & 0 & 0 & 0 & 0 & 0 & 0 & 0 & 0 & 0 & 0 & 0 & 0 \\
\hline $63 \mathrm{C}$ & 0 & 0 & 0 & 0 & 0 & 11308 & 0 & 0 & 0 & 0 & 0 & 0 & 20976 \\
\hline 64 & 22296 & 2103 & 212 & 142 & 833 & 9859 & 0 & 624 & 11011 & 9383 & 4287 & 24107 & 21818 \\
\hline 65 & 38749 & 16361 & 235 & 625 & 4419 & 17962 & 157492 & 5663 & 35312 & 15977 & 13471 & 81213 & 73616 \\
\hline 66 & 30613 & 2579 & 289 & 417 & 0 & 8320 & 0 & 2464 & 6873 & 3130 & 0 & 27185 & 0 \\
67 & 0 & 0 & 0 & 0 & 0 & 0 & 0 & 0 & 0 & 0 & 0 & 0 & 0 \\
\hline $70 \mathrm{~A}$ & 0 & 1314 & 158 & 344 & 10922 & 20610 & 116594 & 2708 & 22121 & 23924 & 4452 & 34666 & 21260 \\
\hline $70 \mathrm{~B}$ & 0 & 0 & 0 & 0 & 0 & 0 & 0 & 0 & 0 & 0 & 0 & 0 & 0 \\
\hline 71 & 33239 & 11609 & 262 & 213 & 0 & 15325 & 79227 & 1357 & 12469 & 2619 & 0 & 22465 & 60950 \\
\hline 72 & 0 & 0 & 0 & 323 & 1169 & 0 & 84813 & 7992 & 14462 & 4384 & 3594 & 27722 & 31322 \\
\hline 73 & 0 & 0 & 0 & 0 & 0 & 0 & 0 & 0 & 0 & 0 & 0 & 0 & 0 \\
\hline $74 A$ & 0 & 1305 & 171 & 745 & 1095 & 17591 & 136199 & 4750 & 18438 & 16535 & 13719 & 42010 & 40331 \\
\hline $74 B$ & 0 & 0 & 0 & 0 & 7542 & 20600 & 0 & 0 & 0 & 0 & 0 & 0 & 0 \\
\hline $74 \mathrm{C}$ & 0 & 0 & 0 & 364 & 0 & 9440 & 113758 & 11375 & 17999 & 5375 & 5505 & 0 & 0 \\
\hline $74 \mathrm{D}$ & 9930 & 1441 & 126 & 267 & 9183 & 14172 & 110615 & 9218 & 24601 & 6275 & 5785 & 41747 & 48257 \\
\hline 75 & 0 & 0 & 0 & 0 & 0 & 0 & 0 & 0 & 0 & 0 & 0 & 0 & 0 \\
\hline 80 & 0 & 0 & 0 & 0 & 0 & 0 & 0 & 0 & 0 & 0 & 0 & 0 & 0 \\
\hline $85 \mathrm{~A}$ & 96768 & 0 & 0 & 0 & 0 & 0 & 0 & 0 & 0 & 0 & 0 & 0 & 0 \\
\hline $85 \mathrm{~B}$ & 0 & 0 & 0 & 0 & 0 & 0 & 0 & 0 & 0 & 0 & 0 & 0 & 0 \\
\hline 90 & 0 & 0 & 0 & 404 & 0 & 0 & 47260 & 774 & 9858 & 3560 & 3608 & 0 & 0 \\
\hline 91 & 0 & 0 & 0 & 0 & 0 & 0 & 0 & 0 & 0 & 0 & 0 & 0 & 0 \\
\hline $92 \mathrm{~A}$ & 0 & 0 & 0 & 0 & 0 & 0 & 0 & 0 & 0 & 0 & 0 & 0 & 0 \\
\hline $92 \mathrm{~B}$ & 0 & 0 & 0 & 0 & 0 & 0 & 57705 & 0 & 0 & 0 & 0 & 0 & 0 \\
\hline 93 & 0 & 0 & 0 & 0 & 0 & 0 & 0 & 0 & 13116 & 0 & 0 & 0 & 0 \\
\hline 95 & 0 & 0 & 0 & 0 & 0 & 0 & 0 & 0 & 0 & 0 & 0 & 0 & 0 \\
\hline
\end{tabular}

Tabelle 108: IOTgg (Werte in Tsd. GE) II

\begin{tabular}{|c|c|c|c|c|c|c|c|c|c|c|c|c|}
\hline & 22 & 23 & 24 & 25 & 26 & 27 & 28 & 29 & 30 & 31 & 32 & 33 \\
\hline 01 & 0 & 0 & 0 & 0 & 0 & 0 & 0 & 0 & 0 & 0 & 0 & 0 \\
\hline 02 & 0 & 0 & 0 & 0 & 0 & 0 & 0 & 0 & 0 & 0 & 0 & 0 \\
\hline 05 & 0 & 0 & 0 & 0 & 0 & 0 & 0 & 0 & 0 & 0 & 0 & 0 \\
10 & 0 & 0 & 0 & 0 & 0 & 0 & 0 & 0 & 0 & 0 & 0 & 0 \\
\hline 11 & 0 & 89273 & 0 & 0 & 0 & 0 & 0 & 0 & 0 & 0 & 0 & 0 \\
14 & 0 & 0 & 0 & 0 & 235383 & 0 & 0 & 0 & 0 & 0 & 0 & 0 \\
15 & 0 & 0 & 65687 & 0 & 0 & 0 & 0 & 0 & 0 & 0 & 0 & 0 \\
16 & 0 & 0 & 0 & 0 & 0 & 0 & 0 & 0 & 0 & 0 & 0 & 0 \\
17 & 0 & 0 & 0 & 26465 & 0 & 0 & 0 & 0 & 0 & 0 & 0 & 0 \\
18 & 0 & 0 & 0 & 0 & 0 & 0 & 0 & 0 & 0 & 0 & 0 & 0 \\
19 & 0 & 0 & 0 & 0 & 0 & 0 & 0 & 0 & 0 & 0 & 0 & 0 \\
\hline 20 & 0 & 0 & 0 & 0 & 0 & 33056 & 0 & 0 & 0 & 0 & 0 & 0 \\
21 & 542302 & 0 & 66859 & 33345 & 30570 & 0 & 0 & 0 & 0 & 21873 & 18357 & 7243 \\
22 & 0 & 0 & 79027 & 0 & 0 & 0 & 0 & 55703 & 0 & 31102 & 0 & 15025 \\
\hline 23 & 0 & 0 & 36602 & 0 & 51092 & 119584 & 31532 & 0 & 0 & 0 & 0 & 0 \\
\hline 24 & 13676 & 0 & 0 & 58165 & 0 & 0 & 31654 & 0 & 1567 & 42246 & 37140 & 13148 \\
25 & 25559 & 3219 & 42361 & 0 & 0 & 0 & 88322 & 181126 & 9277 & 53520 & 49509 & 14890 \\
\hline 26 & 0 & 0 & 0 & 22427 & 0 & 28793 & 38732 & 0 & 0 & 30976 & 15349 & 16782 \\
\hline 27 & 0 & 0 & 0 & 21067 & 27917 & 0 & 455006 & 356783 & 3867 & 64869 & 0 & 17557 \\
\hline
\end{tabular}




\begin{tabular}{|c|c|c|c|c|c|c|c|c|c|c|c|c|}
\hline & 22 & 23 & 24 & 25 & 26 & 27 & 28 & 29 & 30 & 31 & 32 & 33 \\
\hline 28 & 0 & 3628 & 0 & 45429 & 0 & 107267 & 0 & 1003469 & 14772 & 300702 & 76596 & 48082 \\
\hline 29 & 38724 & 35682 & 50516 & 35823 & 53547 & 56666 & 0 & 0 & 4190 & 59241 & 0 & 10850 \\
\hline 30 & 0 & 0 & 0 & 0 & 0 & 0 & 0 & 0 & 0 & 0 & 0 & 0 \\
\hline 31 & 0 & 0 & 0 & 0 & 0 & 0 & 0 & 121373 & 4266 & 0 & 44333 & 37156 \\
\hline 32 & 0 & 0 & 0 & 0 & 0 & 0 & 0 & 37730 & 124985 & 0 & 0 & 0 \\
\hline 33 & 0 & 0 & 0 & 0 & 0 & 0 & 0 & 0 & 2981 & 0 & 15903 & 0 \\
\hline 34 & 0 & 0 & 0 & 0 & 0 & 0 & 0 & 0 & 0 & 0 & 0 & 0 \\
\hline 35 & 0 & 0 & 0 & 0 & 0 & 0 & 0 & 0 & 0 & 0 & 17867 & 0 \\
\hline 36 & 11549 & 0 & 0 & 0 & 0 & 0 & 0 & 0 & 0 & 0 & 0 & 0 \\
\hline 37 & 0 & 0 & 0 & 51847 & 0 & 183819 & 0 & 0 & 0 & 0 & 0 & 0 \\
\hline $40 \mathrm{~A}$ & 34637 & 0 & 86564 & 63893 & 75711 & 81805 & 50340 & 46669 & 0 & 28609 & 20903 & 8202 \\
\hline $40 B$ & 12291 & 0 & 110520 & 0 & 73249 & 91244 & 0 & 0 & 0 & 0 & 0 & 0 \\
\hline $40 \mathrm{C}$ & 0 & 0 & 0 & 0 & 0 & 0 & 0 & 0 & 0 & 0 & 0 & 0 \\
\hline 41 & 0 & 0 & 0 & 0 & 0 & 0 & 0 & 0 & 0 & 0 & 0 & 0 \\
\hline $45 \mathrm{~A}$ & 0 & 7218 & 0 & 0 & 0 & 0 & 25688 & 0 & 0 & 0 & 0 & 0 \\
\hline 45B & 0 & 0 & 0 & 0 & 0 & 0 & 0 & 0 & 0 & 0 & 13565 & 0 \\
\hline $50 \mathrm{~A}$ & 0 & 0 & 0 & 0 & 0 & 0 & 0 & 0 & 0 & 0 & 0 & 0 \\
\hline $50 \mathrm{~B}$ & 0 & 0 & 0 & 0 & 27428 & 0 & 0 & 0 & 0 & 0 & 0 & 0 \\
\hline $51 \mathrm{~A}$ & 0 & 0 & 0 & 0 & 0 & 0 & 0 & 0 & 0 & 0 & 0 & 0 \\
\hline $51 \mathrm{~B}$ & 10495 & 2882 & 95811 & 26291 & 24179 & 50167 & 91762 & 156669 & 3474 & 31339 & 0 & 18225 \\
\hline $52 A$ & 0 & 0 & 0 & 0 & 0 & 0 & 0 & 0 & 0 & 0 & 0 & 0 \\
\hline $52 \mathrm{~B}$ & 0 & 0 & 0 & 0 & 0 & 0 & 0 & 0 & 0 & 0 & 0 & 0 \\
\hline 55 & 0 & 0 & 0 & 0 & 0 & 0 & 29156 & 55589 & 2562 & 0 & 13220 & 0 \\
\hline $60 \mathrm{~A}$ & 0 & 0 & 0 & 0 & 27699 & 24545 & 0 & 0 & 0 & 0 & 0 & 0 \\
\hline $60 \mathrm{~B}$ & 0 & 0 & 0 & 0 & 0 & 0 & 0 & 0 & 0 & 0 & 0 & 0 \\
\hline $60 \mathrm{C}$ & 60499 & 9976 & 134795 & 47005 & 176098 & 152024 & 90458 & 84532 & 0 & 31833 & 16278 & 0 \\
\hline 61 & 0 & 0 & 0 & 0 & 0 & 0 & 0 & 0 & 0 & 0 & 0 & 0 \\
\hline 62 & 0 & 0 & 0 & 0 & 0 & 0 & 0 & 0 & 0 & 0 & 0 & 0 \\
\hline $63 \mathrm{~A}$ & 0 & 18052 & 0 & 0 & 0 & 0 & 0 & 0 & 0 & 0 & 0 & 0 \\
\hline $63 \mathrm{~B}$ & 0 & 0 & 0 & 0 & 0 & 0 & 0 & 0 & 0 & 0 & 0 & 0 \\
\hline $63 C$ & 0 & 0 & 0 & 0 & 0 & 0 & 0 & 0 & 0 & 0 & 0 & 0 \\
\hline 64 & 53181 & 2831 & 37229 & 21685 & 0 & 26395 & 45705 & 90606 & 6435 & 25559 & 8491 & 14708 \\
\hline 65 & 68770 & 42690 & 101964 & 49874 & 70372 & 97922 & 114542 & 188670 & 9476 & 72106 & 60468 & 28355 \\
\hline 66 & 0 & 8512 & 34318 & 0 & 26628 & 0 & 24306 & 38629 & 1573 & 19658 & 0 & 0 \\
\hline 67 & 0 & 0 & 0 & 0 & 0 & 0 & 0 & 0 & 0 & 0 & 0 & 0 \\
\hline $70 \mathrm{~A}$ & 82940 & 25435 & 51277 & 47155 & 52549 & 33697 & 86444 & 124160 & 2590 & 58028 & 0 & 21134 \\
\hline $70 \mathrm{~B}$ & 0 & 0 & 0 & 0 & 0 & 0 & 0 & 0 & 0 & 0 & 0 & 0 \\
\hline 71 & 38138 & 10877 & 0 & 22904 & 45050 & 32518 & 42467 & 66159 & 1967 & 0 & 21158 & 10447 \\
\hline 72 & 16694 & 13696 & 46139 & 19860 & 41566 & 26799 & 47583 & 117766 & 45902 & 55194 & 0 & 23350 \\
\hline 73 & 0 & 3043 & 0 & 0 & 0 & 0 & 0 & 0 & 0 & 0 & 0 & 0 \\
\hline $74 \mathrm{~A}$ & 39755 & 5046 & 86634 & 32182 & 52849 & 42241 & 71201 & 127873 & 3148 & 33915 & 14534 & 23233 \\
\hline $74 \mathrm{~B}$ & 0 & 20579 & 0 & 0 & 0 & 0 & 0 & 0 & 0 & 0 & 0 & 0 \\
\hline $74 \mathrm{C}$ & 59104 & 11536 & 108509 & 23696 & 25787 & 34935 & 34036 & 70848 & 6538 & 26719 & 13929 & 13852 \\
\hline 74D & 89095 & 12835 & 101902 & 25347 & 54940 & 84103 & 176046 & 261979 & 11061 & 76485 & 69133 & 18062 \\
\hline 75 & 0 & 0 & 0 & 0 & 0 & 0 & 0 & 0 & 0 & 0 & 0 & 0 \\
\hline 80 & 0 & 0 & 0 & 0 & 0 & 0 & 0 & 0 & 0 & 0 & 0 & 0 \\
\hline $85 \mathrm{~A}$ & 0 & 0 & 0 & 0 & 0 & 0 & 0 & 0 & 0 & 0 & 0 & 0 \\
\hline $85 B$ & 0 & 0 & 0 & 0 & 0 & 0 & 0 & 0 & 0 & 0 & 0 & 0 \\
\hline 90 & 0 & 0 & 0 & 0 & 0 & 0 & 0 & 0 & 0 & 0 & 17565 & 0 \\
\hline 91 & 0 & 0 & 0 & 0 & 0 & 0 & 0 & 0 & 0 & 0 & 0 & 0 \\
\hline
\end{tabular}




\begin{tabular}{|r|c|c|c|c|c|c|c|c|c|c|c|c|c|}
\hline & 22 & 23 & 24 & 25 & 26 & 27 & 28 & 29 & 30 & 31 & 32 & 33 \\
\hline $92 \mathrm{~A}$ & 114447 & 0 & 0 & 0 & 0 & 0 & 0 & 0 & 0 & 0 & 0 & 0 \\
\hline $92 \mathrm{~B}$ & 35131 & 0 & 38165 & 0 & 0 & 0 & 0 & 0 & 0 & 0 & 0 & 0 \\
\hline 93 & 0 & 0 & 0 & 0 & 0 & 0 & 0 & 0 & 0 & 0 & 0 & 0 \\
\hline 95 & 0 & 0 & 0 & 0 & 0 & 0 & 0 & 0 & 0 & 0 & 0 & 0 \\
\hline
\end{tabular}

Tabelle 109: IOTgg (Werte in Tsd. GE) III

\begin{tabular}{|c|c|c|c|c|c|c|c|c|c|c|c|c|}
\hline & 34 & 35 & 36 & 37 & $40 \mathrm{~A}$ & $40 B$ & $40 \mathrm{C}$ & 41 & $45 A$ & $45 B$ & $50 \mathrm{~A}$ & $50 \mathrm{~B}$ \\
\hline 01 & 0 & 0 & 0 & 0 & 0 & 0 & 0 & 0 & 0 & 0 & 0 & 0 \\
\hline 02 & 0 & 0 & 0 & 0 & 0 & 0 & 0 & 0 & 0 & 0 & 0 & 0 \\
\hline 05 & 0 & 0 & 0 & 0 & 0 & 0 & 0 & 0 & 0 & 0 & 0 & 0 \\
\hline 10 & 0 & 0 & 0 & 0 & 37020 & 0 & 0 & 0 & 0 & 0 & 0 & 0 \\
\hline 11 & 0 & 0 & 0 & 0 & 0 & 296876 & 0 & 0 & 0 & 0 & 0 & 0 \\
\hline 14 & 0 & 0 & 0 & 0 & 0 & 0 & 0 & 0 & 732254 & 0 & 0 & 0 \\
\hline 15 & 0 & 0 & 0 & 0 & 0 & 0 & 0 & 0 & 0 & 0 & 0 & 0 \\
\hline 16 & 0 & 0 & 0 & 0 & 0 & 0 & 0 & 0 & 0 & 0 & 0 & 0 \\
\hline 17 & 0 & 0 & 0 & 0 & 0 & 0 & 0 & 0 & 0 & 41672 & 0 & 0 \\
\hline 18 & 0 & 0 & 0 & 0 & 0 & 0 & 0 & 0 & 0 & 0 & 0 & 0 \\
\hline 19 & 0 & 0 & 0 & 0 & 0 & 0 & 0 & 0 & 0 & 0 & 0 & 0 \\
\hline 20 & 0 & 9660 & 532753 & 0 & 0 & 0 & 1249 & 0 & 568373 & 85015 & 0 & 0 \\
\hline 21 & 0 & 0 & 0 & 0 & 0 & 0 & 0 & 0 & 0 & 31767 & 0 & 9795 \\
\hline 22 & 26337 & 0 & 26434 & 0 & 13120 & 2249 & 0 & 0 & 45748 & 0 & 172674 & 0 \\
\hline 23 & 0 & 0 & 0 & 6137 & 12390 & 0 & 39145 & 2339 & 216546 & 40881 & 21881 & 27615 \\
\hline 24 & 33826 & 0 & 0 & 0 & 0 & 0 & 0 & 0 & 0 & 59602 & 0 & 9691 \\
\hline 25 & 57267 & 15114 & 89013 & 17217 & 0 & 0 & 1203 & 1899 & 40932 & 30454 & 14369 & 18600 \\
\hline 26 & 26731 & 0 & 38598 & 0 & 0 & 0 & 0 & 0 & 1391177 & 361034 & 0 & 8934 \\
\hline 27 & 117030 & 40122 & 0 & 30021 & 0 & 0 & 0 & 2555 & 92875 & 108690 & 0 & 17774 \\
\hline 28 & 104329 & 92252 & 34051 & 885 & 9250 & 0 & 4720 & 8985 & 461405 & 294485 & 21053 & 59150 \\
\hline 29 & 160339 & 88444 & 0 & 2210 & 13177 & 11360 & 9510 & 12524 & 0 & 235131 & 0 & 81692 \\
\hline 30 & 0 & 0 & 0 & 0 & 0 & 0 & 0 & 0 & 0 & 0 & 0 & 0 \\
\hline 31 & 25383 & 0 & 23262 & 0 & 61048 & 0 & 1330 & 2324 & 0 & 225624 & 0 & 16592 \\
\hline 32 & 21795 & 0 & 0 & 0 & 0 & 0 & 0 & 0 & 0 & 0 & 0 & 0 \\
\hline 33 & 0 & 0 & 0 & 0 & 0 & 0 & 0 & 0 & 0 & 0 & 0 & 0 \\
\hline 34 & 0 & 0 & 0 & 0 & 0 & 0 & 0 & 0 & 0 & 0 & 23157 & 86981 \\
\hline 35 & 0 & 0 & 0 & 0 & 0 & 0 & 0 & 0 & 0 & 0 & 0 & 22644 \\
\hline 36 & 29505 & 8769 & 0 & 0 & 0 & 1350 & 0 & 0 & 0 & 0 & 0 & 0 \\
\hline 37 & 0 & 0 & 0 & 0 & 0 & 0 & 0 & 0 & 0 & 0 & 0 & 0 \\
\hline $40 A$ & 37727 & 0 & 35105 & 7595 & 0 & 0 & 0 & 13951 & 0 & 0 & 15311 & 14743 \\
\hline $40 B$ & 0 & 0 & 0 & 0 & 0 & 0 & 76706 & 0 & 0 & 0 & 0 & 0 \\
\hline $40 \mathrm{C}$ & 0 & 0 & 0 & 0 & 0 & 0 & 0 & 0 & 0 & 0 & 0 & 0 \\
\hline 41 & 0 & 0 & 0 & 0 & 0 & 0 & 0 & 0 & 0 & 0 & 0 & 0 \\
\hline $45 A$ & 0 & 0 & 0 & 1034 & 24733 & 2901 & 1866 & 16039 & 0 & 49913 & 0 & 0 \\
\hline 45B & 0 & 0 & 0 & 0 & 0 & 2492 & 0 & 15138 & 218463 & 0 & 0 & 0 \\
\hline $50 A$ & 0 & 0 & 0 & 0 & 0 & 0 & 0 & 0 & 0 & 0 & 0 & 0 \\
\hline $50 B$ & 38877 & 0 & 0 & 0 & 11661 & 0 & 0 & 0 & 68210 & 0 & 77038 & 0 \\
\hline $51 \mathrm{~A}$ & 0 & 0 & 0 & 0 & 0 & 0 & 0 & 0 & 0 & 0 & 0 & 0 \\
\hline $51 \mathrm{~B}$ & 0 & 6679 & 34422 & 1930 & 0 & 1797 & 1874 & 0 & 0 & 0 & 11036 & 0 \\
\hline $52 A$ & 0 & 0 & 0 & 0 & 0 & 0 & 0 & 0 & 0 & 0 & 0 & 0 \\
\hline $52 B$ & 0 & 0 & 0 & 0 & 0 & 0 & 0 & 0 & 0 & 0 & 0 & 0 \\
\hline 55 & 0 & 5341 & 0 & 0 & 14029 & 1770 & 0 & 2465 & 0 & 25483 & 0 & 0 \\
\hline $60 A$ & 0 & 0 & 0 & 1412 & 0 & 0 & 0 & 0 & 0 & 0 & 0 & 0 \\
\hline
\end{tabular}




\begin{tabular}{|c|c|c|c|c|c|c|c|c|c|c|c|c|}
\hline & 34 & 35 & 36 & 37 & $40 A$ & $40 B$ & $40 \mathrm{C}$ & 41 & $45 \mathrm{~A}$ & $45 B$ & $50 \mathrm{~A}$ & $50 \mathrm{~B}$ \\
\hline $60 \mathrm{~B}$ & 0 & 0 & 0 & 0 & 0 & 0 & 0 & 0 & 0 & 0 & 0 & 0 \\
\hline $60 \mathrm{C}$ & 0 & 9668 & 30176 & 9106 & 0 & 0 & 9314 & 0 & 66009 & 0 & 25992 & 0 \\
\hline 61 & 0 & 0 & 0 & 0 & 0 & 0 & 0 & 0 & 0 & 0 & 0 & 0 \\
\hline 62 & 0 & 0 & 0 & 0 & 0 & 0 & 0 & 0 & 0 & 0 & 0 & 0 \\
\hline 63A & 0 & 0 & 0 & 0 & 0 & 55999 & 0 & 0 & 0 & 0 & 13786 & 0 \\
\hline $63 \mathrm{~B}$ & 0 & 0 & 0 & 0 & 0 & 0 & 0 & 0 & 0 & 0 & 0 & 0 \\
\hline $63 \mathrm{C}$ & 0 & 0 & 0 & 1033 & 0 & 0 & 0 & 0 & 0 & 0 & 0 & 0 \\
\hline 64 & 35679 & 7342 & 22710 & 936 & 21031 & 3713 & 3349 & 3492 & 54190 & 32164 & 64120 & 0 \\
\hline 65 & 100774 & 23690 & 72548 & 3235 & 75985 & 20518 & 12698 & 9600 & 178437 & 116819 & 68066 & 48773 \\
\hline 66 & 0 & \begin{tabular}{|l|}
5892 \\
\end{tabular} & 20039 & 0 & 20920 & 1696 & \begin{tabular}{|l|}
5959 \\
\end{tabular} & 2985 & 48114 & 0 & 37427 & 0 \\
\hline 67 & 0 & 0 & 0 & 0 & 0 & 0 & 0 & 0 & 0 & 0 & 0 & 0 \\
\hline $70 A$ & 63085 & 11062 & 49950 & 2309 & 9501 & 2073 & 18045 & 0 & 191766 & 85140 & 114209 & 67220 \\
\hline $70 \mathrm{~B}$ & 0 & 0 & 0 & 0 & 0 & 0 & 0 & 0 & 0 & 0 & 0 & 0 \\
\hline 71 & 74201 & 9119 & 34001 & 1065 & 9987 & 3103 & 8664 & 2423 & 239345 & 46059 & 56058 & 32123 \\
\hline 72 & 31453 & 19578 & 34547 & 933 & 30352 & 8969 & 14356 & 3612 & 0 & 0 & 39906 & 41449 \\
\hline 73 & 0 & 0 & 0 & 0 & 0 & 0 & 0 & 0 & 0 & 0 & 0 & 0 \\
\hline $74 A$ & 65001 & 12475 & 50915 & 1350 & 0 & 4924 & 5196 & 5618 & 102332 & \begin{tabular}{|l}
97647 \\
\end{tabular} & 69045 & 12385 \\
\hline $74 \mathrm{~B}$ & 0 & 0 & 0 & 0 & 8891 & 0 & 0 & 0 & 409174 & 0 & 0 & 0 \\
\hline $74 \mathrm{C}$ & 0 & \begin{tabular}{|l|}
9896 \\
\end{tabular} & 30902 & 956 & 16472 & 2408 & 2052 & 2734 & 0 & 0 & 49077 & 0 \\
\hline 74D & 97603 & 37512 & 76352 & 2726 & 30772 & 19368 & 23610 & 8120 & 145128 & \begin{tabular}{|l|}
83597 \\
\end{tabular} & 44140 & 28542 \\
\hline 75 & 0 & 0 & 0 & 0 & 0 & 0 & 0 & 0 & 0 & 0 & 0 & 0 \\
\hline 80 & 0 & 0 & 0 & 0 & 0 & 0 & 0 & 0 & 0 & 0 & 0 & 0 \\
\hline $85 A$ & 0 & 0 & 0 & 0 & 0 & 0 & 0 & 0 & 0 & 0 & 0 & 0 \\
\hline $85 \mathrm{~B}$ & 0 & 0 & 0 & 0 & 0 & 0 & 0 & 0 & 0 & 0 & 0 & 0 \\
\hline 90 & 0 & 5301 & 22974 & 0 & 11683 & 1641 & 0 & 4672 & 0 & 0 & 0 & 23334 \\
\hline 91 & 0 & 0 & 0 & 0 & 0 & 0 & 0 & 0 & 0 & 0 & 0 & 0 \\
\hline $92 A$ & 0 & 0 & 0 & 0 & 0 & 0 & 0 & 0 & 0 & 0 & 0 & 0 \\
\hline $92 B$ & 0 & 0 & 0 & 0 & 0 & 0 & 0 & 0 & 0 & 0 & 18988 & 0 \\
\hline 93 & 0 & 0 & 0 & 0 & 0 & 0 & 0 & 0 & 0 & 0 & 0 & 0 \\
\hline 95 & 0 & 0 & 0 & 0 & 0 & 0 & 0 & 0 & 0 & 0 & 0 & 0 \\
\hline
\end{tabular}

Tabelle 110: IOTgg (Werte in Tsd. GE) IV

\begin{tabular}{|c|c|c|c|c|c|c|c|c|c|c|c|c|}
\hline & $51 \mathrm{~A}$ & $51 \mathrm{~B}$ & $52 \mathrm{~A}$ & $52 \mathrm{~B}$ & 55 & $60 \mathrm{~A}$ & $60 \mathrm{~B}$ & $60 \mathrm{C}$ & 61 & 62 & $63 A$ & $63 B$ \\
\hline 01 & 0 & 0 & 0 & 0 & 215459 & 0 & 0 & 0 & 0 & 0 & 0 & 0 \\
\hline 02 & 0 & 0 & 0 & 0 & 0 & 0 & 0 & 0 & 0 & 0 & 0 & 0 \\
\hline 05 & 0 & 0 & 0 & 0 & 0 & 0 & 0 & 0 & 0 & 0 & 0 & 0 \\
\hline 10 & 0 & 0 & 0 & 0 & 0 & 0 & 0 & 0 & 0 & 0 & 0 & 0 \\
\hline 11 & 0 & 0 & 0 & 0 & 0 & 0 & 0 & 0 & 0 & 0 & 0 & 0 \\
\hline 14 & 0 & 0 & 0 & 0 & 0 & 8870 & 0 & 0 & 0 & 0 & 0 & 0 \\
\hline 15 & 0 & 0 & 0 & 0 & 1703484 & 10029 & 0 & 0 & \begin{tabular}{|l|}
706 \\
\end{tabular} & 16889 & 0 & 0 \\
\hline 16 & 0 & 0 & 0 & 0 & 0 & 0 & 0 & 0 & 0 & 0 & 0 & 0 \\
\hline 17 & 0 & 0 & 0 & 2776 & 0 & 0 & 0 & 0 & 0 & 0 & 0 & 0 \\
\hline 18 & 0 & 0 & 0 & 0 & 0 & 0 & 0 & 0 & 0 & 10322 & 0 & 0 \\
\hline 19 & 0 & 0 & 0 & 1825 & 0 & 0 & 0 & 0 & 0 & 0 & 0 & 0 \\
\hline 20 & 0 & 0 & 0 & 0 & 0 & 0 & 0 & 0 & 0 & 0 & 0 & 0 \\
\hline 21 & 0 & 0 & 79104 & 0 & 0 & 0 & 0 & 0 & 340 & 0 & 0 & 0 \\
\hline 22 & 639365 & \begin{tabular}{|l|}
5870 \\
\end{tabular} & 386903 & 0 & 46730 & 0 & 34306 & 32143 & 689 & 50716 & 12591 & 87624 \\
\hline 23 & 109312 & 12571 & 101687 & 0 & 124473 & 21567 & 76847 & 499324 & 2885 & 131781 & 6930 & 12733 \\
\hline 24 & 0 & 0 & 0 & 0 & 0 & 0 & 0 & 0 & 0 & 0 & 0 & 0 \\
\hline 25 & 143388 & 0 & 70602 & 4455 & 0 & 0 & 20379 & 27852 & 0 & 0 & 27 & 0 \\
\hline
\end{tabular}




\begin{tabular}{|c|c|c|c|c|c|c|c|c|c|c|c|c|}
\hline & $51 \mathrm{~A}$ & $51 \mathrm{~B}$ & $52 \mathrm{~A}$ & $52 \mathrm{~B}$ & 55 & $60 A$ & $60 \mathrm{~B}$ & $60 \mathrm{C}$ & 61 & 62 & $63 \mathrm{~A}$ & $63 \mathrm{~B}$ \\
\hline 26 & 0 & 0 & 0 & 0 & 0 & 0 & 0 & 0 & 0 & 0 & 0 & 0 \\
\hline 27 & 0 & 0 & 0 & 9898 & 0 & 69876 & 0 & 0 & 0 & 0 & 0 & 0 \\
\hline 28 & 161672 & 0 & 60170 & 0 & 64055 & 0 & 17199 & 0 & 430 & 0 & 0 & 0 \\
\hline 29 & 129523 & 0 & 0 & 3794 & 0 & 0 & 21521 & 0 & 0 & 0 & 5321 & 0 \\
\hline 30 & 0 & 0 & 0 & 0 & 0 & 0 & 0 & 0 & 0 & 0 & 0 & 0 \\
\hline 31 & 0 & 0 & 0 & 8144 & 0 & 0 & 19158 & 0 & 0 & 0 & 0 & 0 \\
\hline 32 & 0 & 0 & 0 & 5460 & 0 & 0 & 0 & 0 & 0 & 0 & 0 & 0 \\
\hline 33 & 0 & 0 & 0 & 0 & 0 & 0 & 0 & 0 & 0 & 0 & 0 & 0 \\
\hline 34 & 0 & 0 & 0 & 0 & 0 & 0 & 0 & 0 & 0 & 0 & 0 & 0 \\
\hline 35 & 0 & 0 & 0 & 0 & 0 & 8398 & 21126 & 0 & 6443 & 77238 & 0 & 0 \\
\hline 36 & 0 & 0 & 0 & 0 & 0 & 0 & 0 & 0 & 0 & 0 & 0 & 5163 \\
\hline 37 & 0 & 0 & 0 & 0 & 0 & 0 & 0 & 0 & 0 & 0 & 0 & 0 \\
\hline $40 A$ & 0 & 9995 & 181299 & 2397 & 236932 & 186786 & 47993 & 0 & 0 & 12471 & 17984 & 0 \\
\hline $40 B$ & 0 & 0 & 0 & 0 & 41466 & 7703 & 0 & 0 & 0 & 0 & 7411 & 0 \\
\hline $40 \mathrm{C}$ & 0 & 0 & 0 & 0 & 0 & 0 & 0 & 0 & 0 & 0 & 5173 & 0 \\
\hline 41 & 0 & 0 & 0 & 0 & 0 & 0 & 0 & 0 & 0 & 0 & 0 & 0 \\
\hline 45A & 0 & 0 & 0 & 0 & 108248 & 60137 & 25449 & 29902 & 1074 & 0 & 110360 & 0 \\
\hline $45 B$ & 100593 & 0 & 0 & 0 & 182716 & 7238 & 0 & 28460 & 1118 & 0 & 57259 & 0 \\
\hline $50 A$ & 0 & 0 & 0 & 0 & 0 & 0 & 0 & 0 & 0 & 0 & 0 & 0 \\
\hline $50 B$ & 0 & 0 & 102719 & 0 & 0 & 0 & 62301 & 364818 & 345 & 0 & 12573 & 9720 \\
\hline $51 \mathrm{~A}$ & 0 & 0 & 0 & 0 & 0 & 0 & 0 & 0 & 0 & 0 & 0 & 0 \\
\hline $51 B$ & 404441 & 0 & 80509 & 2914 & 0 & 0 & 0 & 0 & 0 & 0 & 0 & 0 \\
\hline $52 A$ & 0 & 0 & 0 & 0 & 0 & 0 & 0 & 0 & 0 & 0 & 0 & 0 \\
\hline $52 B$ & 0 & 0 & 49183 & 0 & 0 & 0 & 0 & 0 & 0 & 0 & 0 & 0 \\
\hline 55 & 164432 & 48418 & 51740 & 1849 & 0 & 11147 & 0 & 56637 & 14030 & 78668 & 0 & 292116 \\
\hline $60 A$ & 0 & 6450 & 0 & 0 & 0 & 0 & 0 & 0 & 0 & 0 & 0 & 60804 \\
\hline $60 B$ & 0 & 5716 & 0 & 0 & 0 & 10381 & 0 & 0 & 0 & 0 & 21399 & 289085 \\
\hline $60 \mathrm{C}$ & 468720 & 0 & 0 & 0 & 0 & 0 & 0 & 0 & 0 & 0 & 0 & 0 \\
\hline 61 & 0 & 0 & 0 & 0 & 0 & 0 & 0 & 0 & 0 & 0 & 0 & 0 \\
\hline 62 & 0 & 19428 & 0 & 0 & 0 & 0 & 0 & 31385 & 0 & 0 & 0 & 478246 \\
\hline $63 A$ & 162023 & 17737 & 0 & 0 & 0 & 0 & 15854 & 50830 & 2946 & 213658 & 0 & 12145 \\
\hline $63 B$ & 0 & 10451 & 0 & 0 & 0 & 28520 & 0 & 0 & 0 & 93755 & 0 & 0 \\
\hline $63 \mathrm{C}$ & 0 & 0 & 0 & 0 & 0 & 17755 & 0 & 0 & 0 & 0 & 0 & 0 \\
\hline 64 & 558669 & 39590 & 283935 & 2423 & 96456 & 9552 & 19412 & 37827 & 1143 & 33215 & 9233 & 32487 \\
\hline 65 & 560887 & 45395 & 330579 & 5721 & 206872 & 30426 & 34440 & 112194 & 1409 & 32284 & 20323 & 36246 \\
\hline 66 & 205790 & 19388 & \begin{tabular}{|l|}
177028 \\
\end{tabular} & 0 & 64602 & 0 & 34266 & 145709 & 635 & 16687 & 10063 & 5626 \\
\hline 67 & 0 & 0 & 0 & 0 & 0 & 0 & 0 & 0 & 0 & 0 & 0 & 0 \\
\hline $70 A$ & 653652 & 55342 & 1472614 & 15595 & 683933 & 0 & 38365 & 98494 & 1398 & 35448 & 56687 & 39515 \\
\hline $70 B$ & 0 & 0 & 0 & 0 & 0 & 0 & 0 & 0 & 0 & 0 & 0 & 0 \\
\hline 71 & 380803 & 31701 & 174643 & 3370 & 148182 & 84031 & 39554 & 92245 & 328 & 18257 & 12448 & 6788 \\
\hline 72 & 372537 & 31574 & 102090 & 1643 & 59262 & 17378 & 22131 & 91773 & 681 & 19174 & 6862 & 14096 \\
\hline 73 & 0 & 0 & 0 & 0 & 0 & 0 & 0 & 0 & 0 & 0 & 0 & 0 \\
\hline $74 A$ & 513766 & 37698 & 406311 & 3213 & 149332 & 6524 & 34305 & 79169 & 566 & 11168 & 9862 & 20481 \\
\hline $74 B$ & 0 & 0 & 0 & 2468 & 0 & 0 & 0 & 0 & 0 & 11943 & 0 & 0 \\
\hline $74 \mathrm{C}$ & 227636 & 9008 & 157375 & 1819 & 0 & 0 & 0 & 20528 & 0 & 18432 & 6342 & 24696 \\
\hline 74D & 385532 & 25650 & 183862 & 1918 & 115821 & 7023 & 34470 & 80843 & 8563 & 16382 & 15408 & 26904 \\
\hline 75 & 0 & 0 & 0 & 0 & 0 & 0 & 0 & 0 & 0 & 0 & 0 & 0 \\
\hline 80 & 0 & 0 & 0 & 0 & 0 & 0 & 0 & 0 & 0 & 0 & 0 & 0 \\
\hline $85 A$ & 0 & 0 & 0 & 0 & 0 & 0 & 0 & 0 & 0 & 0 & 0 & 0 \\
\hline $85 B$ & 0 & 0 & 0 & 0 & 0 & 0 & 0 & 0 & 0 & 0 & 0 & 0 \\
\hline
\end{tabular}




\begin{tabular}{|r|c|c|c|c|c|c|c|c|c|c|c|c|c|} 
& $51 \mathrm{~A}$ & $51 \mathrm{~B}$ & $52 \mathrm{~A}$ & $52 \mathrm{~B}$ & 55 & $60 \mathrm{~A}$ & $60 \mathrm{~B}$ & $60 \mathrm{C}$ & 61 & 62 & $63 \mathrm{~A}$ & $63 \mathrm{~B}$ \\
90 & 94860 & 12402 & 45400 & 0 & 97935 & 0 & 0 & 34690 & 0 & 0 & 0 & 0 \\
91 & 0 & 0 & 0 & 0 & 0 & 0 & 0 & 0 & 0 & 0 & 0 & 0 \\
$92 \mathrm{~A}$ & 0 & 0 & 0 & 0 & 0 & 0 & 0 & 0 & 0 & 0 & 0 & 3529 \\
$92 \mathrm{~B}$ & 0 & 0 & 0 & 0 & 0 & 0 & 0 & 0 & 0 & 0 & 0 & 0 \\
93 & 0 & 0 & 0 & 0 & 52649 & 0 & 0 & 0 & 0 & 0 & 0 & 0 \\
95 & 0 & 0 & 0 & 0 & 0 & 0 & 0 & 0 & 0 & 0 & 0 & 0 \\
\hline
\end{tabular}

Tabelle 111: IOTgg (Werte in Tsd. GE) $V$

\begin{tabular}{|c|c|c|c|c|c|c|c|c|c|c|c|c|}
\hline & $63 \mathrm{C}$ & 64 & 65 & 66 & 67 & $70 A$ & $70 \mathrm{~B}$ & 71 & 72 & 73 & $74 A$ & 748 \\
\hline 01 & 0 & 0 & 0 & 0 & 0 & 0 & 0 & 0 & 0 & 0 & 0 & \\
\hline 02 & 0 & 0 & 0 & 0 & 0 & 0 & 0 & 0 & 0 & 0 & 0 & 0 \\
\hline 05 & 0 & 0 & 0 & 0 & 0 & 0 & 0 & 0 & 0 & 0 & 0 & 0 \\
\hline 10 & 0 & 0 & 0 & 0 & 0 & 0 & 0 & 0 & 0 & 0 & 0 & 0 \\
\hline 11 & 0 & 0 & 0 & 0 & 0 & 0 & 0 & 0 & 0 & 0 & 0 & 0 \\
\hline 14 & 0 & 0 & 0 & 0 & 0 & 19134 & 0 & 0 & 0 & 0 & 0 & 0 \\
\hline 15 & 0 & 0 & 20427 & 0 & 0 & 0 & 0 & 0 & 0 & 0 & 0 & 0 \\
\hline 16 & 0 & 0 & 0 & 0 & 0 & 0 & 0 & 0 & 0 & 0 & 0 & 0 \\
\hline 17 & 0 & 0 & 0 & 0 & 0 & 0 & 0 & 0 & 0 & 0 & 0 & 0 \\
\hline 18 & 0 & 0 & 0 & 0 & 0 & 0 & 0 & 0 & 0 & 0 & 0 & 0 \\
\hline 19 & 0 & 0 & 0 & 0 & 0 & 0 & 0 & 0 & 0 & 0 & 0 & 0 \\
\hline 20 & 0 & 0 & 0 & 0 & 0 & 65500 & 0 & 0 & 0 & 0 & 0 & 0 \\
\hline 21 & 4004 & 0 & 30223 & \begin{tabular}{|c|}
10883 \\
\end{tabular} & 2459 & 0 & 5706 & 7698 & 14850 & 6000 & 0 & 44686 \\
\hline 22 & 11705 & 140562 & 264349 & 117536 & 19052 & 17188 & 51827 & 78134 & 30177 & 14254 & 66458 & 52574 \\
\hline 23 & 0 & 51489 & 0 & 0 & 1490 & 0 & 4212 & 26863 & \begin{tabular}{|l|l|}
14748 \\
\end{tabular} & 2947 & 11686 & 16089 \\
\hline 24 & 0 & 0 & 0 & 0 & 0 & 18691 & 0 & 0 & 0 & 44422 & 0 & 0 \\
\hline 25 & 3665 & 0 & 0 & \begin{tabular}{|l|}
12523 \\
\end{tabular} & 2193 & 0 & 0 & 9216 & 11115 & 0 & 0 & 24869 \\
\hline 26 & 0 & 0 & 0 & 0 & 0 & 163654 & 0 & 0 & 0 & 0 & 0 & 9858 \\
\hline 27 & 0 & 0 & 0 & 0 & 0 & 0 & 0 & 0 & 0 & 0 & 0 & 0 \\
\hline 28 & 2767 & 48608 & 18378 & 0 & 1603 & 76694 & \begin{tabular}{|l|}
2682 \\
\end{tabular} & 0 & 0 & 7831 & 0 & 15398 \\
\hline 29 & 0 & 0 & 0 & 0 & 0 & 40159 & 0 & 0 & 0 & 0 & 0 & 0 \\
\hline 30 & 0 & 0 & 0 & 0 & 0 & 0 & 0 & 0 & 0 & 0 & 0 & 0 \\
\hline 31 & 0 & 80184 & 0 & 0 & 0 & 22175 & 4416 & 0 & 11443 & 0 & 0 & 14177 \\
\hline 32 & 0 & 88126 & 0 & 0 & 0 & 0 & 0 & 0 & 0 & 0 & 0 & 11865 \\
\hline 33 & 0 & 0 & 0 & 0 & 0 & 0 & 0 & 0 & 0 & 0 & 0 & 17206 \\
\hline 34 & 0 & 0 & 0 & 0 & 0 & 0 & 0 & 0 & 0 & 0 & 0 & 0 \\
\hline 35 & 0 & 0 & 0 & 0 & 0 & 0 & 0 & 0 & 0 & 0 & 0 & 0 \\
\hline 36 & 0 & 30922 & 0 & 0 & 0 & 0 & 0 & 6353 & \begin{tabular}{|l|}
9699 \\
\end{tabular} & 2747 & 15946 & 0 \\
\hline 37 & 0 & 0 & 0 & 0 & 0 & 0 & 0 & 0 & 0 & 0 & 0 & 0 \\
\hline $40 \mathrm{~A}$ & 7427 & \begin{tabular}{|l}
78090 \\
\end{tabular} & 36683 & 0 & 2292 & 139538 & 2602 & 16273 & 12055 & 3268 & 0 & 0 \\
\hline $40 \mathrm{~B}$ & 1314 & 0 & 0 & 0 & 0 & 0 & 2990 & 0 & 0 & 0 & 12057 & 0 \\
\hline $40 \mathrm{C}$ & 0 & 0 & 0 & 0 & 0 & 0 & 0 & 0 & 0 & 0 & 0 & 0 \\
\hline 41 & 0 & 0 & 0 & 0 & 0 & 238554 & 0 & 0 & 0 & 0 & 0 & 0 \\
\hline 45A & 0 & \begin{tabular}{|c|c|}
64630 \\
\end{tabular} & 112896 & 0 & 0 & 1859028 & 12386 & 0 & 11425 & 0 & 0 & 0 \\
\hline $45 B$ & 0 & \begin{tabular}{|l|}
33119 \\
\end{tabular} & 97706 & 0 & 0 & 2105734 & 5320 & 0 & 13781 & 0 & 12812 & 0 \\
\hline $50 \mathrm{~A}$ & 0 & 0 & 0 & 0 & 0 & 0 & 0 & 0 & 0 & 0 & 0 & 0 \\
\hline 508 & 0 & 0 & 0 & 0 & 2156 & 0 & 0 & 33998 & 0 & 3477 & 32052 & 0 \\
\hline $51 \mathrm{~A}$ & 0 & 0 & 0 & 0 & 0 & 0 & 0 & 0 & 0 & 0 & 0 & 0 \\
\hline $51 \mathrm{~B}$ & 0 & 0 & 0 & 0 & 0 & 0 & 0 & 0 & 0 & 0 & 0 & 0 \\
\hline $52 \mathrm{~A}$ & 0 & 0 & 0 & 0 & 0 & 0 & 0 & 0 & 0 & 0 & 0 & 0 \\
\hline $52 B$ & 0 & 0 & 0 & 0 & 0 & 0 & 0 & 0 & 0 & 0 & 0 & 0 \\
\hline
\end{tabular}




\begin{tabular}{|r|c|c|c|c|c|c|c|c|c|c|c|c|}
\hline & $63 \mathrm{C}$ & 64 & 65 & 66 & 67 & \multicolumn{1}{c}{$70 \mathrm{~A}$} & $70 \mathrm{~B}$ & 71 & 72 & 73 & $74 \mathrm{~A}$ & $74 \mathrm{~B}$ \\
\hline 55 & 0 & 32743 & 47000 & 100104 & 1453 & 0 & 0 & 13411 & 9751 & 3760 & 29685 & 9857 \\
\hline $60 \mathrm{~A}$ & 0 & 29164 & 0 & 11606 & 0 & 0 & 0 & 0 & 0 & 0 & 0 & 0 \\
\hline $60 \mathrm{~B}$ & 0 & 0 & 0 & 0 & 1184 & 0 & 0 & 0 & 0 & 0 & 0 & 0 \\
\hline $60 \mathrm{C}$ & 0 & 0 & 0 & 0 & 0 & 0 & 0 & 0 & 0 & 0 & 0 & 0 \\
\hline 61 & 0 & 0 & 0 & 0 & 0 & 0 & 0 & 0 & 0 & 0 & 0 & 0 \\
\hline 62 & 0 & 0 & 0 & 48310 & 0 & 0 & 0 & 6731 & 0 & 0 & 16528 & 0 \\
\hline $63 \mathrm{~A}$ & 9068 & 0 & 0 & 0 & 0 & 0 & 0 & 5839 & 0 & 0 & 0 & 0 \\
\hline $63 \mathrm{~B}$ & 0 & 0 & 0 & 20669 & 0 & 0 & 0 & 0 & 0 & 0 & 0 & 0 \\
\hline $63 \mathrm{C}$ & 0 & 0 & 0 & 0 & 0 & 0 & 0 & 0 & 0 & 0 & 0 & 0 \\
\hline 64 & 40690 & 0 & 319203 & 151482 & 20469 & 0 & 18247 & 27783 & 77609 & 6941 & 111044 & 37051 \\
\hline 65 & 22661 & 100627 & 0 & 90808 & 46681 & 784504 & 32888 & 63709 & 101519 & 11221 & 115903 & 50196 \\
\hline 66 & 3740 & 0 & 45164 & 0 & 1574 & 146316 & 5988 & 39632 & 33900 & 4425 & 62880 & 11701 \\
\hline 67 & 0 & 0 & 143406 & 474285 & 0 & 0 & 0 & 0 & 0 & 0 & 0 & 0 \\
\hline $70 \mathrm{~A}$ & 62396 & 132934 & 232007 & 95944 & 19879 & 0 & 71115 & 119415 & 294333 & 23575 & 180345 & 60130 \\
\hline $70 \mathrm{~B}$ & 0 & 0 & 0 & 0 & 0 & 738645 & 0 & 0 & 0 & 0 & 0 & 0 \\
\hline 71 & 44811 & 54808 & 173487 & 189982 & 10758 & 21477 & 6841 & 0 & 141429 & 5312 & 76904 & 15107 \\
\hline 72 & 4002 & 60023 & 232611 & 263976 & 11072 & 0 & 7305 & 28894 & 0 & 14775 & 136478 & 25524 \\
\hline 73 & 0 & 0 & 0 & 11268 & 0 & 0 & 0 & 0 & 0 & 0 & 0 & 0 \\
\hline $74 \mathrm{~A}$ & 16830 & 57553 & 164168 & 117251 & 11145 & 0 & 12438 & 64835 & 75627 & 5819 & 0 & 19886 \\
\hline $74 \mathrm{~B}$ & 1935 & 40805 & 44069 & 39225 & 0 & 83340 & 5582 & 0 & 0 & 0 & 0 & 0 \\
\hline $74 \mathrm{C}$ & 8495 & 174158 & 253217 & 91471 & 6807 & 0 & 5618 & 31706 & 38429 & 10221 & 23509 & 9731 \\
\hline $74 \mathrm{D}$ & 34578 & 63527 & 262559 & 113342 & 11489 & 355660 & 88004 & 63180 & 115953 & 31913 & 81379 & 20764 \\
\hline 75 & 0 & 0 & 0 & 0 & 0 & 0 & 0 & 0 & 0 & 0 & 10929 & 0 \\
\hline 80 & 0 & 0 & 0 & 0 & 0 & 0 & 0 & 0 & 0 & 0 & 0 & 0 \\
\hline $85 \mathrm{~A}$ & 0 & 0 & 0 & 0 & 0 & 0 & 0 & 0 & 0 & 0 & 0 & 0 \\
\hline $85 \mathrm{~B}$ & 0 & 0 & 0 & 0 & 0 & 0 & 0 & 0 & 0 & 0 & 0 & 0 \\
\hline 90 & 3302 & 0 & 35553 & 74884 & 7053 & 1063301 & 0 & 14251 & 13185 & 4202 & 29932 & 0 \\
\hline 91 & 1294 & 0 & 0 & 0 & 0 & 0 & 0 & 0 & 0 & 0 & 15787 & 0 \\
\hline $92 \mathrm{~A}$ & 0 & 0 & 0 & 0 & 0 & 0 & 0 & 0 & 0 & 0 & 0 & 0 \\
\hline 928 & 0 & 0 & 0 & 0 & 0 & 0 & 0 & 0 & 0 & 0 & 0 & 0 \\
\hline 93 & 0 & 0 & 0 & 0 & 0 & 0 & 0 & 0 & 0 & 0 & 0 & 0 \\
\hline 95 & 0 & 0 & 0 & 0 & 0 & 0 & 0 & 0 & 0 & 0 & 0 & 0 \\
\hline
\end{tabular}

Tabelle 112: IOTgg (Werte in Tsd. GE) VI

\begin{tabular}{|c|c|c|c|c|c|c|c|c|c|c|c|c|}
\hline & $74 \mathrm{C}$ & 740 & 75 & 80 & $85 \mathrm{~A}$ & $85 B$ & 90 & 91 & $92 \mathrm{~A}$ & $92 \mathrm{~B}$ & 93 & 95 \\
\hline 01 & 0 & 0 & 0 & 0 & 0 & 38717 & 0 & 0 & 0 & 0 & 0 & 0 \\
\hline 02 & 0 & 0 & 0 & 0 & 0 & 0 & 0 & 0 & 0 & 0 & 0 & 0 \\
\hline 05 & 0 & 0 & 0 & 0 & 0 & 0 & 0 & 0 & 0 & 0 & 0 & 0 \\
\hline 10 & 0 & 0 & 0 & 0 & 0 & 0 & 0 & 0 & 0 & 0 & 0 & 0 \\
\hline 11 & 0 & 0 & 0 & 0 & 0 & 0 & 0 & 0 & 0 & 0 & 0 & 0 \\
\hline 14 & 0 & 0 & 0 & 0 & 0 & 0 & 0 & 0 & 0 & 0 & 0 & 0 \\
\hline 15 & 0 & 0 & 0 & 70500 & 118560 & 292809 & 0 & 21689 & 0 & 0 & 0 & 0 \\
\hline 16 & 0 & 0 & 0 & 0 & 0 & 0 & 0 & 0 & 0 & 0 & 0 & 0 \\
\hline 17 & 0 & 0 & 0 & 0 & 0 & 45183 & 0 & 0 & 0 & 0 & 0 & 0 \\
\hline 18 & 0 & 0 & 0 & 0 & 0 & 0 & 0 & 0 & 0 & 0 & 0 & 0 \\
\hline 19 & 0 & 0 & 0 & 0 & 0 & 0 & 0 & 0 & 0 & 0 & 0 & 0 \\
\hline 20 & 0 & 0 & 0 & 0 & 0 & 0 & 0 & 0 & 0 & 0 & 10222 & 0 \\
\hline 21 & 0 & 24886 & 0 & 0 & 0 & 0 & 0 & 0 & 0 & 3926 & 0 & 0 \\
\hline 22 & 452667 & 102609 & 184854 & 35512 & 85999 & 28787 & 12646 & 176233 & 21616 & 4831 & 19852 & 0 \\
\hline 23 & 4109 & 20653 & 95372 & 62303 & 88404 & 67122 & 35551 & 47927 & 14605 & 0 & 13770 & 0 \\
\hline
\end{tabular}




\begin{tabular}{|c|c|c|c|c|c|c|c|c|c|c|c|c|}
\hline & $74 C$ & $74 \mathrm{D}$ & 75 & 80 & $85 A$ & $85 B$ & 90 & 91 & $92 \mathrm{~A}$ & $92 B$ & 93 & 95 \\
\hline 24 & 0 & 25794 & 0 & 15513 & 95787 & 0 & 0 & 0 & 0 & 7659 & 14021 & 10 \\
\hline 25 & 0 & 19902 & 0 & 0 & 52417 & 0 & 0 & 0 & 0 & 7753 & 0 & 0 \\
\hline 26 & 0 & 0 & 0 & 0 & 0 & 0 & 0 & 0 & 0 & 0 & 0 & 0 \\
\hline 27 & 0 & 0 & 0 & 0 & 0 & 0 & 0 & 0 & 0 & 0 & 0 & 0 \\
\hline 28 & 0 & 8424 & 76494 & 29980 & 0 & 37843 & 9561 & 11508 & 9602 & 0 & 10558 & 8 \\
\hline 29 & 0 & 0 & 0 & 0 & 0 & 0 & 10669 & 0 & 0 & 0 & 6944 & 0 \\
\hline 30 & 0 & 0 & 0 & 0 & 0 & 0 & 0 & 0 & 0 & 0 & 0 & 0 \\
\hline 31 & 0 & 0 & 67436 & 15004 & 0 & 0 & 10881 & 0 & 9522 & 3349 & 0 & 0 \\
\hline 32 & 0 & 0 & 0 & 0 & 0 & 0 & 0 & 0 & 0 & 12094 & 0 & 0 \\
\hline 33 & 0 & 0 & 0 & 0 & 0 & 0 & 0 & 0 & 0 & 0 & 0 & 0 \\
\hline 34 & 0 & 0 & 0 & 0 & 0 & 0 & 0 & 0 & 0 & 0 & 0 & 0 \\
\hline 35 & 0 & 0 & 0 & 0 & 0 & 0 & 0 & 0 & 0 & 0 & 0 & 0 \\
\hline 36 & 4483 & 0 & 0 & 0 & 0 & 0 & 0 & 0 & 0 & 0 & 0 & 0 \\
\hline 37 & 0 & 0 & 0 & 0 & 0 & 0 & 0 & 0 & 0 & 0 & 0 & 0 \\
\hline $40 \mathrm{~A}$ & 7092 & 15894 & 113752 & 81999 & 78955 & 101302 & 33484 & 47836 & 57182 & 10690 & 22901 & 10 \\
\hline $40 B$ & 0 & 0 & 0 & 76710 & 68723 & 78601 & 0 & 15294 & 33250 & 0 & 8521 & 0 \\
\hline $40 \mathrm{C}$ & 0 & 0 & 57034 & 26452 & 0 & 33105 & 0 & 0 & 9691 & 0 & 0 & 0 \\
\hline 41 & 0 & 0 & 0 & 0 & 0 & 0 & 15396 & 0 & 0 & 0 & 0 & 0 \\
\hline $45 \mathrm{~A}$ & 0 & 0 & 187135 & 85971 & 53148 & 71901 & 105451 & 34031 & 37720 & 0 & 0 & 0 \\
\hline $45 B$ & 4165 & 0 & 145722 & 82696 & 54618 & 79676 & 9028 & 30601 & 36681 & 23893 & 6303 & 0 \\
\hline $50 A$ & 0 & 0 & 0 & 0 & 0 & 0 & 0 & 0 & 0 & 0 & 0 & 0 \\
\hline $50 \mathrm{~B}$ & 0 & 18501 & 43664 & 0 & 0 & 0 & 35132 & 19987 & 0 & 0 & 0 & 0 \\
\hline $51 \mathrm{~A}$ & 0 & 0 & 0 & 0 & 0 & 0 & 0 & 0 & 0 & 0 & 0 & 0 \\
\hline $51 B$ & 0 & 0 & 0 & 0 & 0 & 0 & 0 & 0 & 0 & 0 & 0 & 0 \\
\hline $52 \mathrm{~A}$ & 0 & 0 & 0 & 0 & 0 & 0 & 0 & 0 & 0 & 0 & 0 & 0 \\
\hline $52 \mathrm{~B}$ & 0 & 0 & 0 & 0 & 0 & 0 & 0 & 0 & 0 & 0 & 0 & 0 \\
\hline 55 & 6489 & 14902 & 67353 & 30301 & 0 & 50017 & 0 & 25642 & 0 & 6332 & 0 & 0 \\
\hline $60 A$ & 0 & 0 & 0 & 0 & 0 & 0 & 0 & 0 & 0 & 0 & 0 & 0 \\
\hline $60 \mathrm{~B}$ & 0 & 0 & 0 & 0 & 0 & 0 & 0 & 0 & 0 & 0 & 0 & 0 \\
\hline $60 \mathrm{C}$ & 0 & 0 & 0 & 0 & 0 & 0 & 50764 & 0 & 0 & 0 & 0 & 0 \\
\hline 61 & 0 & 0 & 0 & 0 & 0 & 0 & 0 & 0 & 0 & 0 & 0 & 0 \\
\hline 62 & 4367 & 0 & 0 & 0 & 0 & 0 & 0 & 0 & 0 & 3514 & 0 & 0 \\
\hline $63 A$ & 0 & 0 & 0 & 0 & 0 & 0 & 13967 & 0 & 0 & 0 & 0 & 0 \\
\hline $63 B$ & 0 & 0 & 0 & 0 & 0 & 0 & 0 & 0 & 0 & 0 & 0 & 0 \\
\hline $63 C$ & 0 & 0 & 0 & 0 & 0 & 0 & 0 & 0 & 0 & 0 & 0 & 0 \\
\hline 64 & 74226 & 95314 & 233079 & 37904 & \begin{tabular}{|l|}
114151 \\
\end{tabular} & 44173 & 0 & 84632 & 54891 & 35084 & 12169 & 9 \\
\hline 65 & 78767 & 105416 & 311346 & 95699 & 116886 & 59481 & 33015 & 64379 & 58220 & 16484 & 33784 & 0 \\
\hline 66 & 14486 & 35958 & 0 & 0 & 44648 & 0 & 19432 & 14751 & 15870 & 0 & 10246 & 0 \\
\hline 67 & 0 & 0 & 0 & 0 & 0 & 0 & 0 & 0 & 0 & 0 & 0 & 0 \\
\hline $70 \mathrm{~A}$ & 71904 & 127332 & 828237 & 176233 & 320931 & 111729 & 43800 & 80850 & 102390 & 40543 & 77630 & 0 \\
\hline $70 \mathrm{~B}$ & 0 & 0 & 0 & 0 & 0 & 0 & 0 & 0 & 0 & 0 & 0 & 0 \\
\hline 71 & 44047 & 44395 & 210288 & 35632 & 68762 & 48968 & 31866 & 47720 & 40032 & 19243 & 15050 & 0 \\
\hline 72 & 62793 & 52362 & 0 & 19839 & 52782 & 0 & 0 & 26987 & 29378 & 6054 & 13802 & 0 \\
\hline 73 & 0 & 0 & 0 & 0 & 0 & 0 & 0 & 0 & 0 & 0 & 0 & 0 \\
\hline $74 \mathrm{~A}$ & 44783 & 78984 & 55154 & 0 & 123282 & 0 & 18544 & 42660 & 39718 & 19512 & 30188 & 0 \\
\hline $74 B$ & 0 & 0 & 143913 & 0 & 0 & 0 & 0 & 0 & 0 & 0 & 0 & 0 \\
\hline $74 \mathrm{C}$ & 0 & 22953 & 50168 & 15476 & 0 & 0 & 14749 & 35304 & 63026 & 95884 & 7551 & 0 \\
\hline $74 \mathrm{D}$ & 53099 & 0 & 159189 & 86912 & 192024 & 104394 & 63517 & 79859 & 94367 & 97385 & 25413 & 3 \\
\hline 75 & 0 & 0 & 0 & 0 & 0 & 0 & 0 & 0 & 0 & 0 & 0 & 0 \\
\hline 80 & 0 & 0 & 0 & 0 & 0 & 0 & 0 & 0 & 0 & 0 & 0 & 0 \\
\hline
\end{tabular}




\begin{tabular}{|c|c|c|c|c|c|c|c|c|c|c|c|c|}
\hline & $74 C$ & $74 \mathrm{D}$ & 75 & 80 & $85 A$ & $85 \mathrm{~B}$ & 90 & 91 & $92 \mathrm{~A}$ & $92 \mathrm{~B}$ & 93 & 95 \\
\hline $85 \mathrm{~A}$ & 0 & 0 & 0 & 0 & 0 & 103744 & 0 & 0 & 0 & 0 & 0 & 0 \\
\hline $85 B$ & 0 & 0 & 335093 & 0 & 44772 & 0 & 0 & 0 & 0 & 0 & 0 & 0 \\
\hline 90 & 15840 & 37990 & 0 & 20063 & 67999 & 32160 & 0 & 14463 & 15469 & 0 & 8393 & 0 \\
\hline 91 & 4703 & 8709 & 0 & 0 & 0 & 0 & 0 & 0 & 0 & 0 & 0 & 0 \\
\hline $92 \mathrm{~A}$ & 29458 & 0 & 0 & 0 & 0 & 0 & 0 & 0 & 0 & 8937 & 0 & 0 \\
\hline $92 \mathrm{~B}$ & 255797 & 0 & 0 & 0 & 0 & 0 & 0 & 0 & 0 & 0 & 0 & 0 \\
\hline 93 & 0 & 0 & 0 & 0 & 0 & 0 & 0 & 0 & 0 & 0 & 0 & 0 \\
\hline 95 & 0 & 0 & 0 & 0 & 0 & 0 & 0 & 0 & 0 & 0 & 0 & 0 \\
\hline
\end{tabular}




\section{Forschungsergebnlsse der Wirtschaftsunlversität Wien}

Herausgeber: Wirtschaftsuniversităt Wien vertreten durch a.o. Univ. Prof. Dr. Barbara Sporn

Band 1 Stefan Felder: Frequenzallokation in der Telekommunikation. Ökonomische Analyse der Vergabe von Frequenzen unter besonderer Berūcksichtigung der UMTS-Auktionen. 2004.

Band 2 Thomas Haller: Marketing im liberalisierten Strommarkt. Kommunikation und Produktplanung im Privatkundenmarkt. 2005.

Band 3 Alexander Stremitzer: Agency Theory: Methodology, Analysis. A Structured Approach to Writing Contracts. 2005.

Band 4 Gūnther Sedlacek: Analyse der Studiendauer und des Studienabbruch-Risikos. Unter Verwendung der statistischen Methoden der Ereignisanalyse. 2004.

Band 5 Monika Knassmüller: Unternehmensleitbilder im Vergleich. Sinn- und Bedeutungsrahmen deutschsprachiger Unternehmensleitbilder - Versuch einer empirischen (Re-)Konstruktion. 2005.

Band 6 Matthias Fink: Erfolgsfaktor Selbstverpflichtung bei vertrauensbasierten Kooperationen. Mit einem empirischen Befund. 2005.

Band 7 Michael Gerhard Kraft: Ökonomie zwischen Wissenschaft und Ethik. Eine dogmenhistorische Untersuchung von Léon M.E. Walras bis Milton Friedman. 2005.

Band 8 Ingrid Zechmeister: Mental Health Care Financing in the Process of Change. Challenges and Approaches for Austria. 2005.

Band 9 Sarah Meisenberger: Strukturierte Organisationen und Wissen. 2005.

Band 10 Anne-Katrin Neyer: Multinational teams in the European Commission and the European Parliament. 2005.

Band 11 Birgit Trukeschitz: Im Dienst Sozialer Dienste. Ökonomische Analyse der Beschäftigung in sozialen Dienstleistungseinrichtungen des Nonprofit Sektors. 2006

Band 12 Marcus Kölling: Interkulturelles Wissensmanagement. Deutschland Ost und West. 2006.

Band 13 Ulrich Berger: The Economics of Two-way Interconnection. 2006.

Band 14 Susanne Guth: Interoperability of DRM Systems. Exchanging and Processing XML-based Rights Expressions. 2006.

Band 15 Bernhard Klement: Ökonomische Kriterien und Anreizmechanismen für eine effiziente Förderung von industrieller Forschung und Innovation. Mit einer empirischen Quantifizierung der Hebeleffekte von F\&E-Förderinstrumenten in Österreich. 2006.

Band 16 Markus Imgrund: Wege aus der Insolvenz. Eine Analyse der Fortführung und Sanierung insolventer Klein- und Mittelbetriebe unter besonderer Berücksichtigung des Konfigurationsansatzes. 2007.

Band 17 Nicolas Knotzer: Product Recommendations in E-Commerce Retailing Applications. 2008.

Band 18 Astrid Dickinger: Perceived Quality of Mobile Services. A Segment-Specific Analysis. 2007.

Band 19 Nadine Wiedermann-Ondrej: Hybride Finanzierungsinstrumente in der nationalen und internationalen Besteuerung der USA. 2008.

Band 20 Helmut Sorger: Entscheidungsorientiertes Risikomanagement in der Industrieunternehmung. 2008.

Band 21 Martin Rietsch: Messung und Analyse des ökonomischen Wechselkursrisikos aus Unternehmenssicht: Ein stochastischer Simulationsansatz. 2008. 
Band 22 Hans Christian Mantler: Makroökonomische Effizienz des Finanzsektors. Herleitung eines theoretischen Modells und Schătzung der Wachstumsimplikationen für die Marktwirtschaften und Transformationsökonomien Europas. 2008.

Band 23 Youri Tacoun: La théorie de la valeur de Christian von Ehrenfels. 2008.

Band 24 Monika Koller: Longitudinale Betrachtung der Kognitiven Dissonanz. Eine Tagebuchstudie zur Reiseentscheidung. 2008.

Band 25 Marcus Scheiblecker: The Austrian Business Cycle in the European Context. 2008.

Band 26 Aida Numic: Multinational Teams in European and American Companies. 2008.

Band 27 Ulrike Bauernfeind: User Satisfaction with Personalised Internet Applications. 2008.

Band 28 Reinhold Schod: Systematische Analyse und Bewertung komplexer Supply Chain Prozesse bei dynamischer Festlegung des Auftragsentkopplungspunkts. 2008.

Band 29 Bianca Gusenbauer: Öffentlich-private Finanzierung von Infrastruktur in Entwicklungsländern und deren Beitrag zur Armutsreduktion. Fallstudien in Vietnam und auf den Philippinen. 2009.

Band 30 Elisabeth Salomon: Hybrides Management in sino-österreichischen Joint Ventures in China aus österreichischer Perspektive. 2009.

Band 31 Katharina Mader: Gender Budgeting: Ein emanzipatorisches, finanzpolitisches und demokratiepolitisches Instrument. 2009.

Band 32 Michael Weber: Die Generierung von Empfehlungen für zwischenbetriebliche Transaktionen als gesamtwirtschaftliche Infrastrukturleistung. 2009.

Band 33 Lisa Gimpl-Heersink: Joint Pricing and Inventory Control under Reference Price Effects. 2009.

www.peterlang.de 